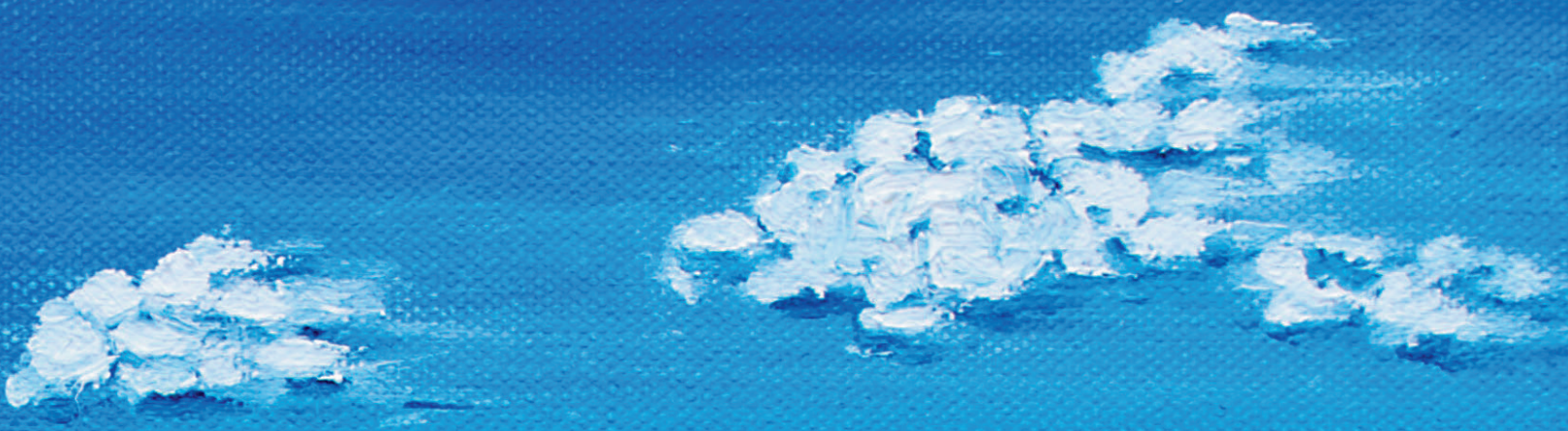

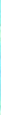
L

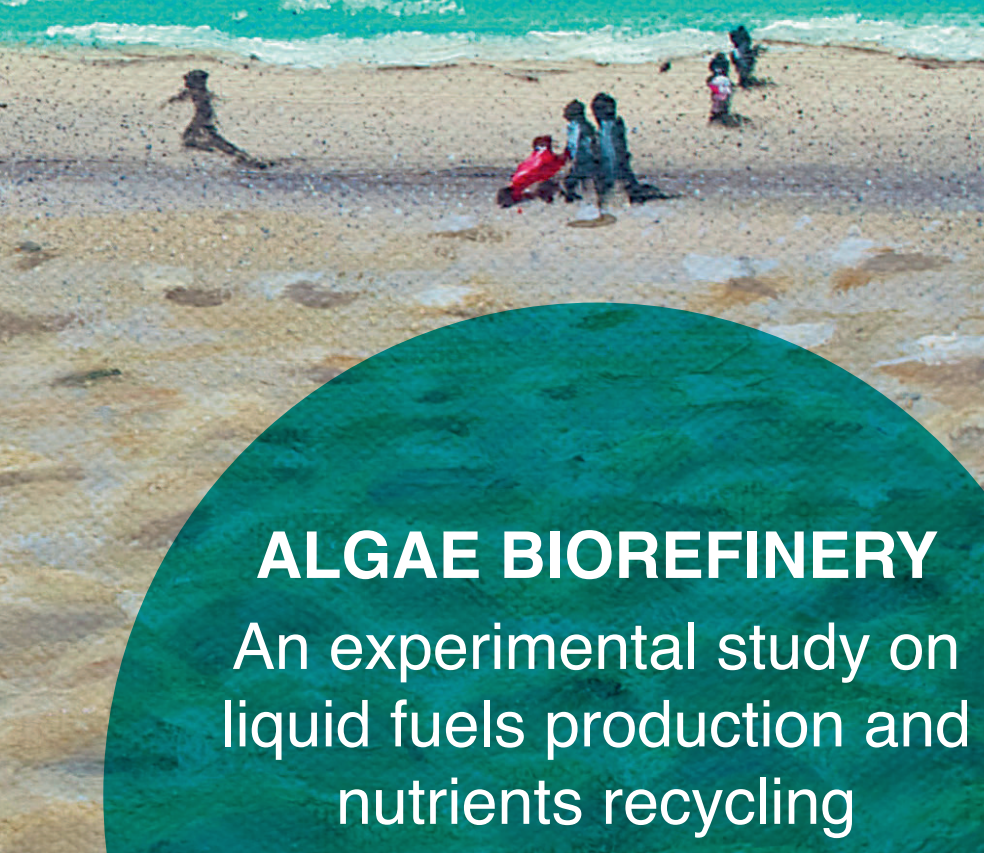

Laura Garcia Alba 


\section{ALGAE BIOREFINERY}

An experimental study on liquid fuels production and nutrients recycling 


\section{Promotion Committee:}

$\begin{array}{lll}\text { Chairman: } & \text { prof. dr. G. van der Steenhoven } & \text { University of Twente } \\ \text { Promotor: } & \text { prof. dr. S.R.A. Kersten } & \text { University of Twente } \\ \text { Assistant promotor: } & \text { dr. ir. D.W.F. Brilman } & \text { University of Twente } \\ \text { Members: } & \text { prof. dr. G. Mul } & \text { University of Twente } \\ & \text { prof. dr. ir. H. van den Berg } & \text { University of Twente } \\ & \text { prof. dr. P.E. Savage } & \text { University of Michigan } \\ & \text { prof. dr. D. Fabbri } & \text { University of Bologna } \\ & \text { dr. ir. A.M. Verschoor } & \text { Duplaco/Wetsus }\end{array}$

The research described in this thesis was financially supported by the province of Overijssel via the Green Energy Initiative of the University of Twente.

\section{Cover design:}

"Un paseo por la playa" by Angel Garcia Vela (coast of Noordwijk, NL).

Design by Cenk Aytekin (http://www.cenkaytekin.com/).

Algae Biorefinery:

An experimental study on liquid fuels production and nutrients recycling ISBN: 978-90-365-3552-6

DOI: $10.3990 / 1.9789036535526$

URL: http://dx.doi.org/10.3990/1.9789036535526

Printed by Gildeprint, Enschede, The Netherlands

(C) 2013 Laura Garcia Alba, Enschede, The Netherlands 


\title{
ALGAE BIOREFINERY
}

\section{AN EXPERIMENTAL STUDY ON LIQUID FUELS PRODUCTION AND NUTRIENTS RECYCLING}

\author{
DISSERTATION \\ to obtain \\ the degree of doctor at the University of Twente, \\ on the authority of the rector magnificus, \\ prof. dr. H. Brinksma, \\ on account of the decision of the graduation committee, \\ to be publicly defended \\ on Friday $21^{\text {st }}$ of June 2013 at 16:45 \\ by

\section{Laura Garcia Alba} \\ born on September $26^{\text {th }}, 1985$ \\ in Tarragona, Catalonia, Spain
}


This thesis has been approved by:

$\begin{array}{ll}\text { Prof. dr. S.R.A. Kersten } & \text { (promotor) } \\ \text { Dr. ir. D.W.F. Brilman } & \text { (assistant promotor) }\end{array}$ 
A mis padres Dulce y Angel por todo vuestro amor y paciencia

"Celebro que estéis bien"

(Francisco Alba Lago, mi abuelo) 



\section{Contents}

Chapter 1 Introduction

Chapter 2 Hydrothermal treatment (HTT) of microalgae:

Evaluation of the process as conversion method in an algae biorefinery concept

Appendix A

Chapter 3 Hydrotreatment of hydrothermal liquefaction oil from microalgae: Preliminary results

Appendix B

Chapter 4 Experimental and economical evaluation of supercritical $\mathrm{CO}_{2}$ extraction of oil from microalgae

Chapter 5 Microalgae growth on the aqueous phase from hydrothermal liquefaction of the same microalgae

Chapter 6 Recycling nutrients in algae biorefinery 205

Appendix D

Chapter 7 Summary and Outlook

Samenvatting 253

Resum 259

Publications list 265

About the Author 



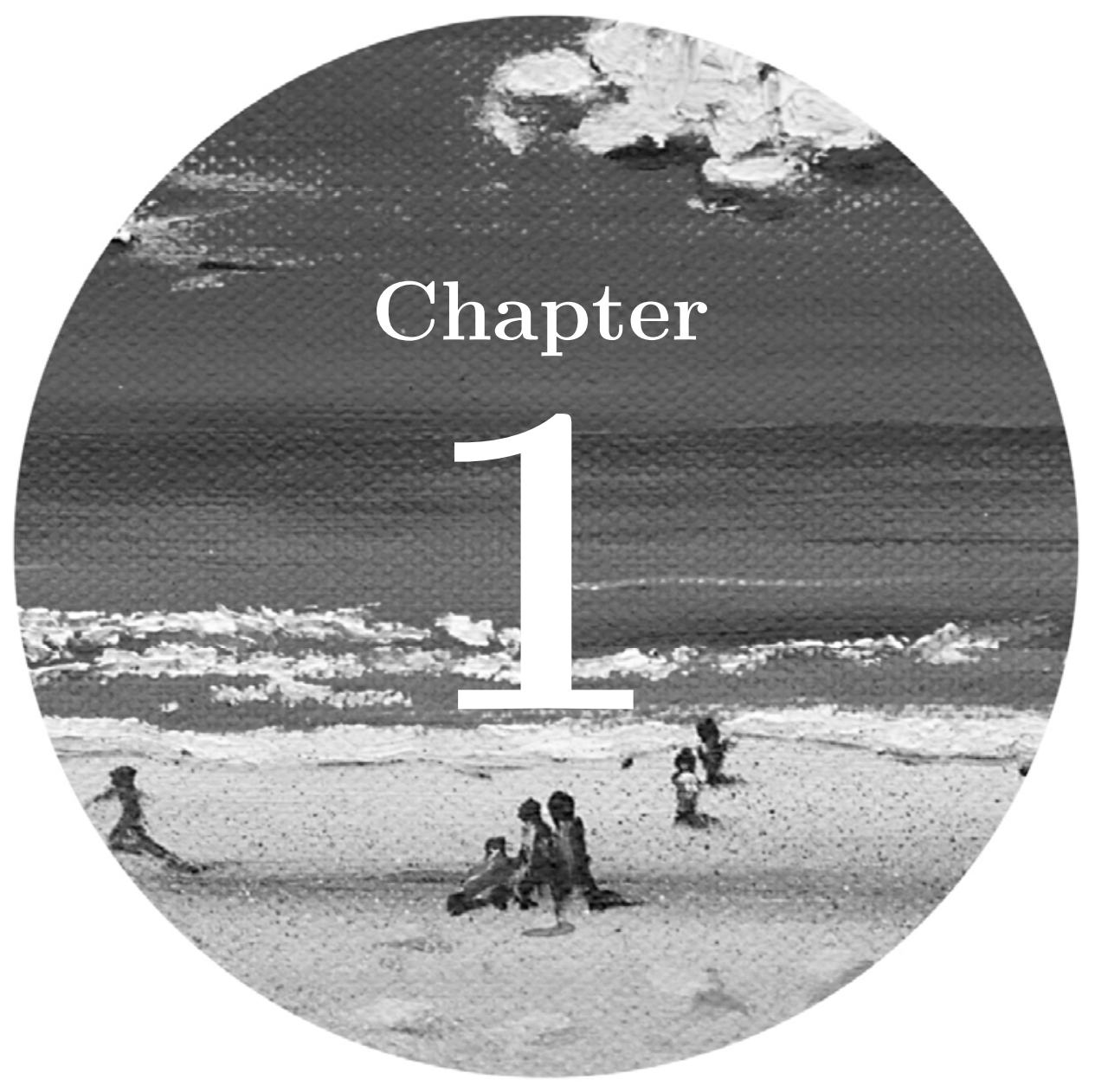

Introduction 


\begin{abstract}
The research described in thesis deals with microalgae as renewable source for the production of liquid biofuels within a biorefinery configuration. In this chapter, first the research context is presented followed by a summary of the aspects that highlight the potential of microalgae for the production of biofuels and biochemicals. Subsequently, the concept of biorefinery is introduced showing how that has been defined for algae. In addition, we present a brief overview of the past and recent developments in the frame of algae-to-fuels to underline the commercialization potential of this type of biomass. Lastly, the scope and outline of this thesis are given.
\end{abstract}




\section{Research context}

\subsection{The need for sustainable development}

In the context of sustainability, if we want future generations to remember us for our accomplishments, and not for our mistakes, drastic changes have to be made. Industry, academia and many other social institutions are joining efforts to lessen the self-created footprints which might not be drastically affecting today's society, however will be the challenges of the near future. Society is facing the ongoing rise of three aspects that are closely linked to each other:

The continuous growth of the world's population and its economies $\rightarrow$ increase in energy demand $\rightarrow$ increased consumption of fossil fuels.

Hence, conventional fossil fuels (coal, conventional natural gas and petroleum) reserves are decreasing and will eventually be exhausted. This results in a regular increase (on average) of fossil fuel prices and discussions on security of supply, which further contributes to more economical and political conflicts. In addition, the increase of anthropogenic $\mathrm{CO}_{2}$ in the atmosphere and emissions is a proven fact, ${ }^{1}$ driven by the extensive use of fossil fuels together with deforestation.

On the other hand, the recent "shale gas revolution" should not be overlooked. ${ }^{2}$ That term was given for the phenomenon that emerged in the United States and refers to the increased prospects of shale or unconventional natural gas supply (gas defined as unconventional in the sense of its less accessible location compare to that of conventional natural gas). Recently, in the U.S., shale gas has become available in larger quantities due to technological developments for its extraction and other legal aspects favoring its progress. Experts have indicated that reserves of this gas are very large and well distributed over the globe which would lead to a more diversified energy supply. However, environmental concerns have also grown regarding the hydraulic fracturing recovery process or also known as fracking. It requires large amounts of water and it also uses chemicals that can leak and lead to contamination of ground water. Moreover, its input on GHG emissions is still under debate since the real amount of methane that leaks during processing is not clear.

It might be a solution in the short term, but it is still a finite source of which realistic prospects are still unclear. Additionally, there is a general disagreement between experts in the field on its sustainability. That general uncertainty could delay its deployment. If 
that happens and, in parallel, the investments on renewables are lessen due to the current shale gas revolution, it might be too late for a solution to climate change. Therefore, the development of renewable alternatives -for heat, power, transportation fuels and chemicals- is still essential and shall continue. Probably, the situation will be that both renewables and shale gas will contribute together to a greener future.

The same as for the energy sector, linked aspects appear also for the food sector in the framework of depleting resources:

The continuous growth of the world's population and its economies $\rightarrow$ increase in food demand $\rightarrow$ increased consumption of fertilizers.

Fossil fuels are not the only depleting natural source. Existing rock phosphate reserves, mined to recover phosphorous, could be exhausted in the next 50-100 years. ${ }^{3}$ At the same time, the production of nitrogen for mineral fertilizers (the Haber process) requires significant amounts of depleting fossil energy. Phosphorous, together with nitrogen and potassium, are combined into mineral fertilizers which are essential for food production. Therefore, both depletion of fossil fuels and phosphate rock reserves will contribute to the increase of food price. Yet an important difference exists between oil and phosphorus: oil can be replaced by other non-carbon forms of energy while, in food production, there is no substitute for phosphorus and, hence, it can only be reused to alleviate its depleting trend. ${ }^{3}$ All these highlight the importance of recycling and closing the material cycles.

\subsection{The path towards sustainable development}

Production of heat and power in a sustainable manner can be achieved by the use of several renewable resources such as hydro, tidal, wind, geothermal and solar. Yet it is likely that for the coming decades there will be a demand for liquid fuels in the sector of long distance transportation (mainly aviation, shipping and trucks) as they are more efficiently stored and transported than any other energy source. Moreover, crude oil makes up a much larger share of the current global energy demand ( $\sim 41.2 \%$ as reported recently by the International Energy Agency ${ }^{4}$ for 2010), and it will remain like this unless new breakthrough technological developments emerge in the field of electricity.

Among the alternative sources, biomass is the only renewable source that can directly store energy in the form of carbon containing molecules. The main building block of chemicals and fuels is carbon. Consequently, biomass can be an optimal source of carbon towards a more sustainable development for the production of bio-based fuels and 
chemicals. With the utilization of biomass (or its products), greenhouse gas emissions are significantly reduced, and yet more, a carbon neutral cycle can be achieved when its consumption and production occur in short cycles. Biofuels production can contribute to diversify income and fuel supply sources thereby increasing the security of energy supply. ${ }^{5}$ Despite the abovementioned and many other benefits, biomass should be carefully managed to minimize the fossil energy input and environmental footprints (related to e.g. land and water use, and biodiversity) derived from its utilization. Clearly, we do not want to have the same negative impacts or create new ones as those we are trying to alleviate or avoid.

There are different types of biomass from which both gaseous (e.g. $\mathrm{H}_{2}$ and $\mathrm{CH}_{4}$ ) and liquid biofuels (the focus of this research) can be produced. First generation biofuels are mainly produced from food and oil crops (e.g. sugar cane and rapeseed) and animal fats. The most common representatives, biodiesel and bioethanol, have already been introduced to the markets. ${ }^{5,6}$ However, with them, a well-known concern, with both economical and ethical nuances, always arises: their competition with food and feed industries for the use of biomass and agricultural land which, eventually, will have negative effects on food commodity prices.

Development of the so-called second generation biofuels aimed to overcome the above issue. The feedstock is generally lignocellulosic-based biomass (wood and wood waste, agricultural and forestry residues, waste from industry, etc.) which can be converted to liquid fuels such as pyrolysis oil. If upgraded, pyrolysis oil has the potential to be coprocessed in standard refinery units. $^{7}$ However its direct use (to replace crude oil) is still limited due to its complex composition.

Fuels from aquatic organisms like algae are often considered the third generation biofuels. $^{8,9}$ In the recent years, the interest on algae as energy source has grown exponentially. This is illustrated in Figure 1 showing, over time, the amount of publications available in the literature database of Scopus using as keywords "algae for fuels". From 2007, the number of publications on that topic exhibited a remarkable increase. 


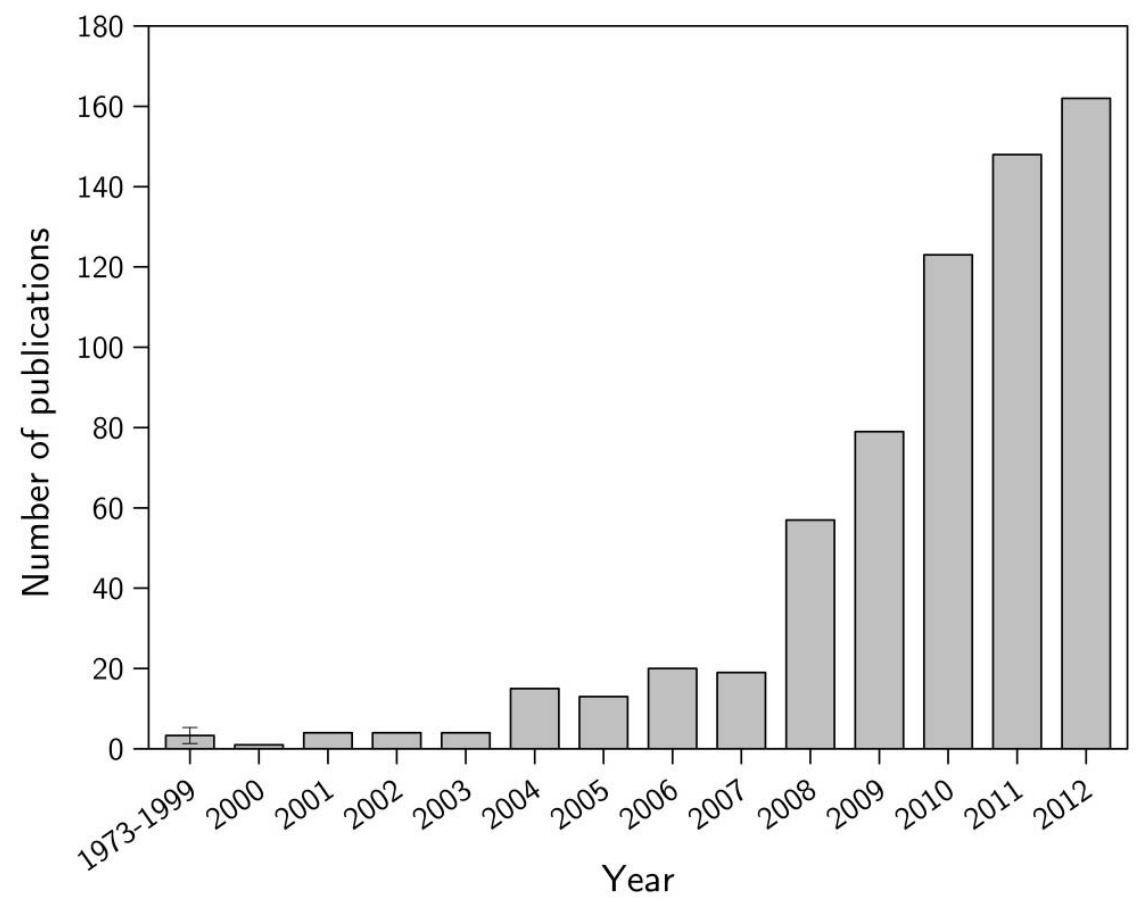

Figure 1. Number of publications related to "algae for fuels" over time (source: Scopus)

In 2008, Groeneveld ${ }^{10}$ stated "the biomass availability for the first generation of biofuels based on food products is very limited ( $<10 \mathrm{EJ} / \mathrm{y})$. The availability for the second generation biofuels based on agricultural and forestry wastes biomass is significant (ca. $100 \mathrm{EJ} / \mathrm{y})$. Growing algae for the third generation biofuels is still very much in the research phase, but it has an even bigger potential". The next section will show the aspects that highlight that suggested potential of algal biomass for biofuels.

\section{Microalgae: tiny factories converting solar energy into chemical energy}

Two kinds of algae exist: macroalgae (which can be up to $60 \mathrm{~m}$ in length), also known as seaweed and microalgae ( 1-50 $\mu \mathrm{m}$ in size). Although macroalgae can have advantages above microalgae, as being easier to harvest, they grow slower and have lower lipid content than microalgae. As the reader will recognize within the course of this thesis, those two factors are important for the production of biofuels. Therefore the research was restricted to the use of microalgae. For the purpose of this document, the term algae will be used to refer solely to microalgae.

Microalgae are plant-like organisms without roots or leaves and are too small to be seen clearly with the unaided eye. They can grow naturally in lakes, oceans, ponds and any 
other location where moisture is available; free-floating in water or attached to surfaces like rocks. They are eukaryotic organisms, although prokaryotic (with lack of nucleus) cyanobacteria are also considered microalgae. Up to date, the size of the whole algae biodiversity is unknown. Only a few tens of thousands (out of an upper limit that has been mentioned to be as high as 10 million $^{11}$ ) of species have been described and classified by numerous families, classes, orders and genera. Although changes to the existing taxonomy happen on a regular basis, algae are commonly divided into the following main groups: green algae (like Desmodesmus sp., species used in this research), red algae, diatoms, brown algae, gold algae, yellow-green algae and cyanobacteria (or blue algae). Most species are photoautotrophic; using inorganic substances as nutrients and light as energy source, but many can also use organic substances for growing in the light or dark (photoheterotrophic and heterotrophic growth respectively). ${ }^{5,12,13}$

The three main building blocks of microalgae are proteins, lipids and carbohydrates and their fraction relative to the total biochemical composition is highly species and growth conditions dependent. The main difference compared to terrestrial plants is the lack of lignin. A range for each of the fractions, which shows the broad compositional variability, was given by Williams and Laurens ${ }^{14}$ : 15-60\% lipids, 20-60\% proteins and 10-50\% carbohydrates (the rest can be e.g. nucleic acids accounting for 3-5\%). Carbohydrates (both monomers and polymers) have structural and metabolic functions in the cells and, at the same time, they serve as precursors for the synthesis of other biochemicals. Green algae, for instance, contain starch (consisting of amylose and amylopectin) as an energy store, while red algae are known to be a source of gelling polysaccharides not found in plants (e.g. agar). Proteins also have metabolic and structural roles. All photosynthetically active pigments (chlorophylls, carotenoids and phycobilins) are associated with proteins, which are responsible for a variety of specific functions in light harvesting and electron transfer. ${ }^{15}$ Lipids serve for both to store energy and as structural components of the cell. Under optimal growth conditions, algae synthesize fatty acids principally for esterification into glycerol-based membrane lipids (hence structural role). The major membrane lipids are glycolipids and phospholipids. Upon nutrients starvation (e.g. N) and other environmental stresses (e.g. temperature, light) many algae alter their lipid biosynthetic pathways towards the formation and accumulation of neutral lipids (mainly in the form of triacylglycerols) serving as storage form of carbon and energy. After that, they are deposited in densely packed lipid bodies located in the cytoplasm of the algal cell. This brief description on algae composition is adapted from other more extended studies. ${ }^{14-16}$ The reader can refer to those scientific reports as well as to the work 
presented by $\mathrm{Hu}$ et al. ${ }^{17}$ and Greenwell et al. ${ }^{18}$ for more details on algal lipids and their biosynthesis.

Rosenberg et al. ${ }^{19}$ stated, "microalgae are unique because they combine the renewable energy-capturing ability of photosynthesis with the high yields of controlled microbial cultivation, making them potentially valuable organisms for economical, industrial-scale production processes in the $21^{\text {st }}$ century". Many advantages have been mentioned in the literature, 5, 6, 20 mainly pointing at those that make algae better than terrestrial plants and, in particular, those that make algae better than crops for biodiesel. The most outstanding aspects are:

- In any growth related feature considered -photosynthetic efficiency, daily or yearly productivities, or lipid yield per area- algae have shown better performance than any other biomass source. Although the photosynthetic efficiency estimates of microalgae vary (1-3\% up to the theoretical maximum of $10 \%),{ }^{20,}{ }^{21}$ it is generally reported higher than terrestrial plants. Compared with conventional crop plants, which are usually harvested once or twice a year, microalgae have a very short harvesting cycle (in the range of hours to days, depending on the process) ${ }^{6}$;

- The previous point denotes that algae require less land for cultivation and, even better, they can be grown on non-arable land. Therefore, the competition for arable land is greatly reduced without compromising the production of food, feed and other products derived from crops. Moreover, co-production of food and fuel from microalgae increases the food production potential;

- Non-arable land means that they can grow in a wide range of climates (e.g. marginal lands like the desert) and water environments (fresh or saline water, wastewater, etc.). Furthermore, wastewater treatment with algae has a dual benefit: the cells clean the wastewater and the water itself serves as source of nutrients for the cells. If desired, they can utilize the $\mathrm{CO}_{2}$ from industrial flue gases;

- Their biochemical composition can be tuned by varying growth conditions (e.g. enhanced lipid accumulation by nitrogen starvation). Consequently, many products (fuels but also fine chemicals and bulk products) can be obtained from algae (discussed in the next section) thereby exhibiting a large number of commercial applications.

Many claim that microalgae is the only source of bio-based diesel-like fuels (i.e. biodiesel from transesterification of lipids and green diesel or renewable diesel from hydrogenation 
of lipids) with sufficient potential to replace fossil diesel. ${ }^{22,23}$ This becomes evident when comparing with the other renewable alternatives being conventional oil crops (Figure 2). With the data reported by Schenk et al. (for 2008), ${ }^{6}$ Figure 2 was constructed showing the oil yield and the area that would be required to cover the global oil demand from different traditional crops and algae (as reported by Schenk et al.: algae productivity of $10 \mathrm{~g} / \mathrm{m}^{2} /$ day and $30 \mathrm{wt} \%$ oil content being in close range to that produced by Seambiotic, Israel). Only an equivalent of $21 \%$ of the global arable land would be required for algae and its actual land claim could be even smaller when cultivating on non-arable land. The oil content of algae and oil crops is not that different (e.g. from 18 wt\% in soybean Glycine max L. to $48 \mathrm{wt} \%$ in castor Ricinus communis ${ }^{5}$. The difference lies on the biomass productivity, being much higher for algae, and hence resulting in higher oil yields.

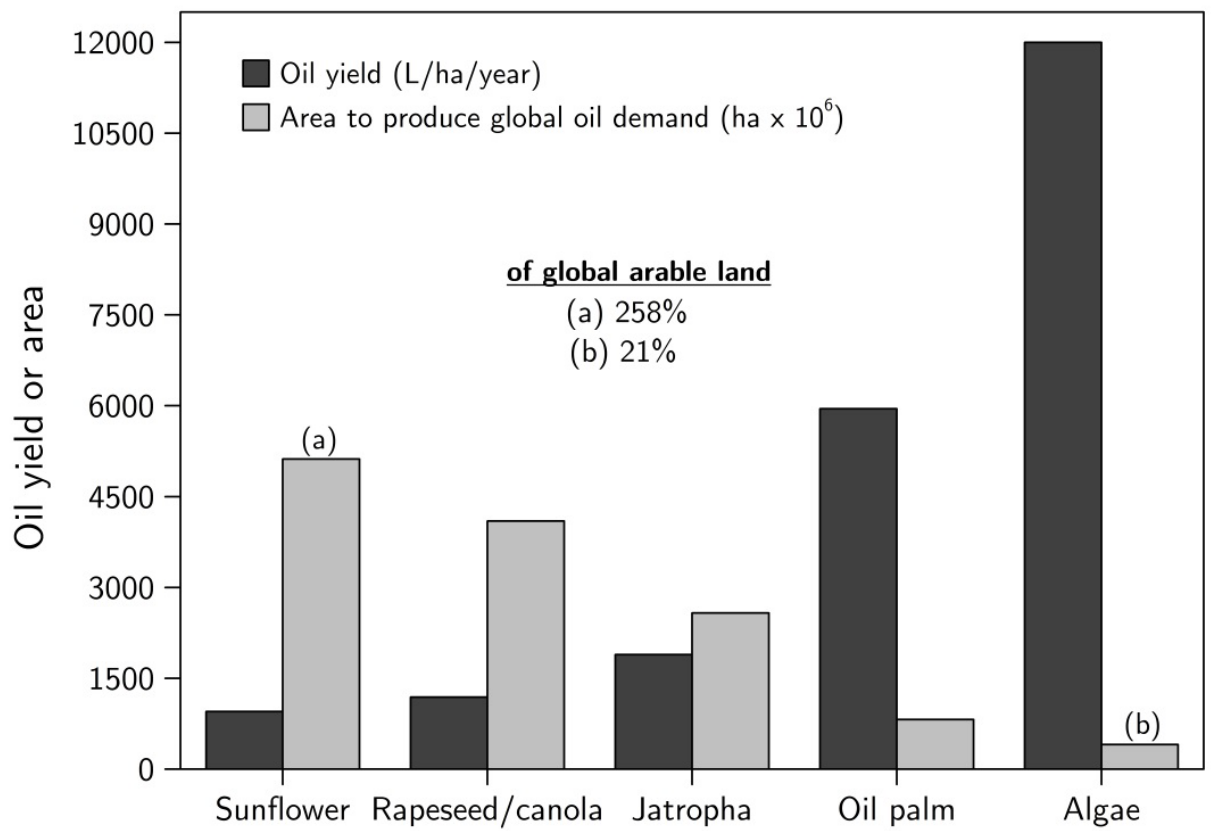

Figure 2. Oil production and area required for different crops and algae (data taken from Schenk et al. ${ }^{6}$ )

Despite the numerous advantages, the environmental impacts (apart from land also e.g. water use, GHG emissions, etc.) associated with algae production should be carefully measured and compared to that of terrestrial plants. For instance, water evaporation can be an issue when cultivating algae in open systems. However, the water footprint is still unclear. Regardless of cultivation location, some stated that the impact on water utilization is lower than for terrestrial $\mathrm{crops}^{20}{ }^{20}$ while others reported the opposite but without considering the use of wastewater, ${ }^{24}$ and again others reported a mixed performance $^{25}$ (including wastewater supply). Furthermore, as will be shown in this 
thesis, the nitrogen and phosphorus present in algae can end up in the products obtained after conversion. The presence of nitrogen in the produced bioliquids can be a major disadvantages in view of undesirable $\mathrm{NO}_{\mathrm{x}}$ emissions upon their combustion.

Finally, although a lot of products can be obtained (due to their broad composition spectrum) from algae and many advantages haven been stated, there are simply to many algal strains to arrive at general conclusions about economic viability and sustainability. Each of them can differ significantly in lipid profile, photosynthetic ability, growth rate, growth medium requirements, resistance to contaminants, cell wall resistance to disruption, etc. ${ }^{26}$ This highlights the importance of having (and thus developing) flexible extraction/conversion processes for use in biorefinery configurations.

\section{From microalgae to biofuels and biochemicals: the biorefinery concept}

The term "biorefinery" was established in the 1990s and one of its given definitions is "fully integrated systems of sustainable, environmentally and resource-friendly technologies for the comprehensive material and energetic utilization as well as exploitation of biological raw materials in form of green and residue biomass from a targeted sustainable regional land utilization". ${ }^{27}$ In an algae-based biorefinery, several extraction/conversion routes are technically feasible to co-produce high value-added products and feed/food ingredients, together with energy carrier products. Following the given biorefinery definition, all of this must be integrated efficiently in a way that we maximize the utilization of the biomass aiming for the maximum economic and environmental benefits. Many believe (including the author) that the biorefinery approach is the key for the success of the algae-to-biofuels concept.

Several algae biorefinery configurations have been defined. Yet in all concepts, four main stages can be identified (Figure 3): algae cultivation, dewatering, algae (fresh and residues) processing, and recycling (nutrients, $\mathrm{CO}_{2}$ and water). The reader may refer to the following extensive reviews - $5,14,28$ - which include the state of the art of the technologies used in each of the steps shown in Figure 3, and to others ${ }^{29-31}{ }_{-}$which provide a more critical perspective of the whole chain trying to identify the main challenges. In this introduction, only a brief description will be given, focusing on the aspects that are important for a biorefinery setting. 


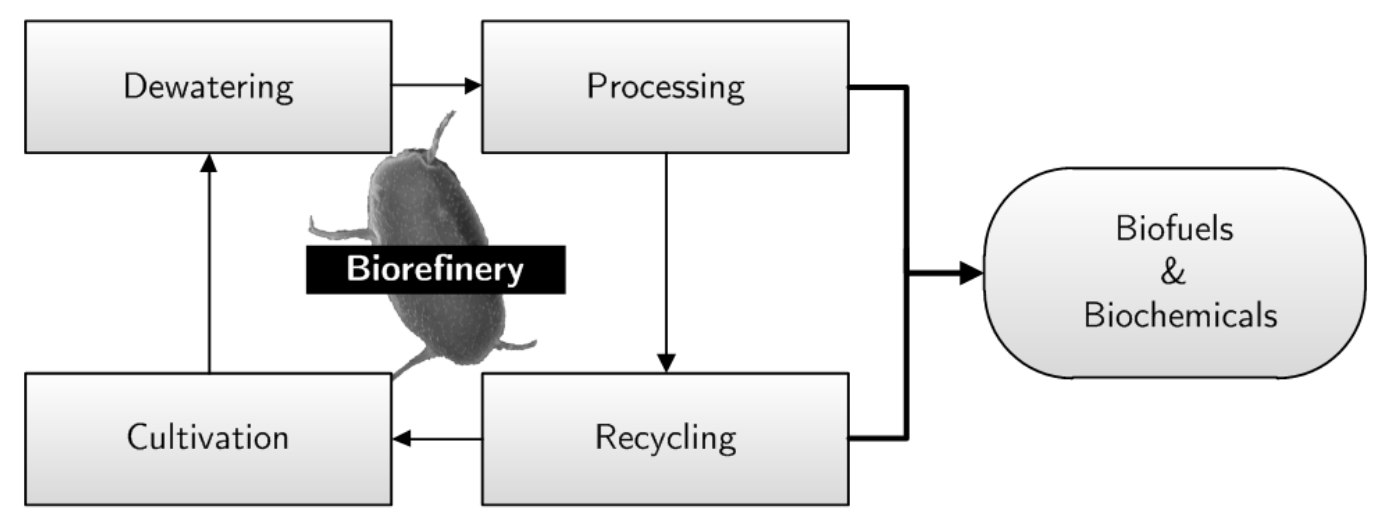

Figure 3. Algae biorefinery for biofuels and biochemicals

\subsection{Cultivation, dewatering and recycling}

Algae cultivation technologies were clearly defined in the work by Slade and Bauen ${ }^{12}$ : "there are two main cultivation systems for photoautotrophic algae: raceway ponds and photobioreactors (PBRs). A typical raceway pond has a closed loop oval channel ( 0.25$0.4 \mathrm{~m}$ deep) open to the air, and mixed with a paddle wheel to circulate the water and prevent sedimentation. In PBRs the culture medium is enclosed in a transparent array of tubes or plates and the micro-algal broth is circulated from a central reservoir". There is always debate on which of the two systems is more effective and/or economic. So far the general trend is that PBR systems performe better on algae productivity as the culture environment is highly controlled but tend to be more expensive (see ${ }^{12,14}$ ). A third option is the so-called hybrid system which basically combines both technologies in series. In this two-stage strategy a high-quality algal stream from the PBR, where the cells were grown under controlled and constant conditions, is fed to the open pond system where stress conditions are applied (e.g. $\mathrm{N}$ starvation) to increase the lipid content. A continuous and pure stream of the desired algal species is supplied to the pond with a much higher density (i.e. rate) of the targeted algae in comparison to contaminants entering the system. ${ }^{30}$ In this way, the risk of contamination is much lower. The reduced contamination also allows for cultivation of other species than would not grow in an open environment otherwise. The patented ALDUO ${ }^{\mathrm{TM}}$ hybrid technology of Cellana, a company producing algae-based bioproducts for health, feed, and fuel applications, underlines the economic potential of this approach. ${ }^{32}$

Due to the microscopic size of the microalgal cells and the large volumes of water, dewatering is a challenging area. Other key properties of microalgae which influence their separation are their shape (rods, spheres, chains or filaments) and the surface charge (usually negative). ${ }^{33}$ Many dewatering techniques have been described and studied: 
filtration, centrifugation, gravity sedimentation, flocculation, flotation, etc. Most of the times, more than one separation step is required. Therefore, the various techniques have to be strategically combined in the most efficient way possible. Rawat et al. ${ }^{30}$ reported that harvesting can contribute up to $20-30 \%$ of the total cost of algal biomass production. Therefore, to minimize the costs in a biorefinery setting for fuels, processes that can handle wet biomass are advantageous.

It is difficult to provide a value for the current cost of microalgae production because all life cycle analysis studies and techno-economical evaluations encountered are based on different assumptions and system boundaries. Regardless of that, both cultivation and dewatering are of the most expensive steps in any biorefinery configuration. A very recent study by Slade and Bauen ${ }^{12}$ (2013) reported the costs of algal biomass production in a PBR and a raceway pond, combining data from the literature with discussion with experts. The costs reported included cultivation and harvesting process steps, without favorable inputs like co-products or wastewater treatment. Table 1 shows the results adapted from the data given by Slade and Bauen

Table 1. Algae production costs adapted from Slade and Bauen ${ }^{12}$

\begin{tabular}{ccccccc}
\hline Scenario & $\begin{array}{c}\text { Biomass }^{\mathrm{a}} \\
\text { productivity } \\
\left(\mathbf{g} / \mathbf{m}^{2} / \text { day }\right)\end{array}$ & $\begin{array}{c}\text { Power } \\
\text { consumption } \\
\left(\mathbf{W} / \mathbf{m}^{2}\right)\end{array}$ & $\begin{array}{c}\text { Area } \\
(\mathbf{h a})\end{array}$ & $\begin{array}{c}\text { Water } \\
\text { evaporation } \\
\left(\mathbf{L} / \mathbf{m}^{2} / \mathbf{d a y}\right)\end{array}$ & $\begin{array}{c}\text { Costs of water, } \\
\mathbf{C O}_{2} \text { and } \\
\text { nutrients }\end{array}$ & $\begin{array}{c}\text { Algae } \\
\text { production } \\
\mathbf{c o s t}(\mathbf{\epsilon} / \mathbf{k g})\end{array}$ \\
\hline $\mathrm{RP}^{\mathrm{b}}-\mathrm{BC}$ & 10 & 1 & 400 & 10 & Included & 1.8 \\
$\mathrm{RP}^{\mathrm{b}}-\mathrm{PC}^{\mathrm{d}}$ & 20 & 1 & 400 & 10 & Excluded & 0.4 \\
\hline PBR-BC & 20 & 500 & 10 & 0.5 & Included & 10.0 \\
PBR-PC & 40 & 50 & 10 & 0.5 & Excluded & 3.8 \\
\hline
\end{tabular}

${ }^{\text {a }} 300$ operating days. ${ }^{\mathrm{b}}$ Raceway pond. ${ }^{\mathrm{c}}$ Base case. ${ }^{\mathrm{d}}$ Projected case.

The projected case values obtained for the raceway pond could be cost competitive with that of plant crops (from the cheapest case $0.22 € / \mathrm{kg}$ for rapeseed meal to the most expensive case $1.02 € / \mathrm{kg}$ for Soybean oil, according to latest monthly price and policy update report of $\mathrm{FAO}^{34}$ ). Their results showed that the costs can be cut by more than half, if a cheap source of nutrients, $\mathrm{CO}_{2}$ and water is introduced. Slade and Bauen ${ }^{12}$ stated that finding those low cost sources is a very demanding requirement, and it could dramatically restrict the number of locations available. However, in our view, it is possible to avoid that (or at least alleviate it) by defining a biorefinery configuration which allows for standalone operation with recycling of streams as key factor (more information within this thesis and in Chapter 7). 


\subsection{Processing}

By algae processing, we are not only referring to the production of fuels but also to any other product of interest that could be extracted from the algal building blocks; lipids, carbohydrates and proteins. If strategically established, co-production next to fuels could provide economic benefits to the biorefinery system. Many compounds from algae can be used as food supplements, animal feed, fine organic chemicals for pharmaceuticals, pigments, etc. Some examples are omega-3 fatty acids, eicosapentanoic acid (EPA), decosahexanoic acid (DHA) and chlorophyll. ${ }^{35}$ In the work by Foley et al., ${ }^{16}$ a comprehensive scheme is given showing the products that can be generated from each of the algal fractions. Another interesting application is the production of bioplastics from the carbohydrates fraction. For instance, Cereplast $^{36}$ is a company currently producing algae-based plastic products. Important platform chemicals can also be produced. Kim et al. ${ }^{37}$ demonstrated the facile single-step conversion of agar (polysaccharide in red algae) into HMF and its furfural derivatives in the presence of a solid Brønsted acid and obtained higher yields than that with land plant-based polymeric carbohydrates such as starch and cellulose.

A large number of applications for high-added value products can be found, however, important is to evaluate the volume of the various targeted markets in the biorefinery concept selected. The market volumes of high added value products and fuels are quite different and incompatible in size. If we aim for a significant replacement of fossil transportation fuels, algae production must take place on significant, large scale. In that situation, the high-added value co-products must have broad applications to avoid immediately saturating their respective markets and ensure that there is no overlapping of co-product markets (overlapping by e.g. aiming for markets that are covered by lignocellulosic based refineries). ${ }^{16}$

As previously mentioned, the focus of this thesis is on the production of liquid fuels from microalgae. In Figure 4, the main algae biofuel conversion technologies are listed, showing only the primary fuel products obtained from each. As direct secretion (see Figure 4), we could also consider the ability of some algal strains to naturally discharge oil out of the cells (e.g. Botryococcus braunii), but still a solvent would be needed to recover that oil. The reader can find the state of the art of these technologies in the work by Suali and Sarbatly $^{28}$ (one of the most recent reviews). For a more extensive review on the thermochemical routes, the reader is referred to López Barreiro et al. ${ }^{38}$ 


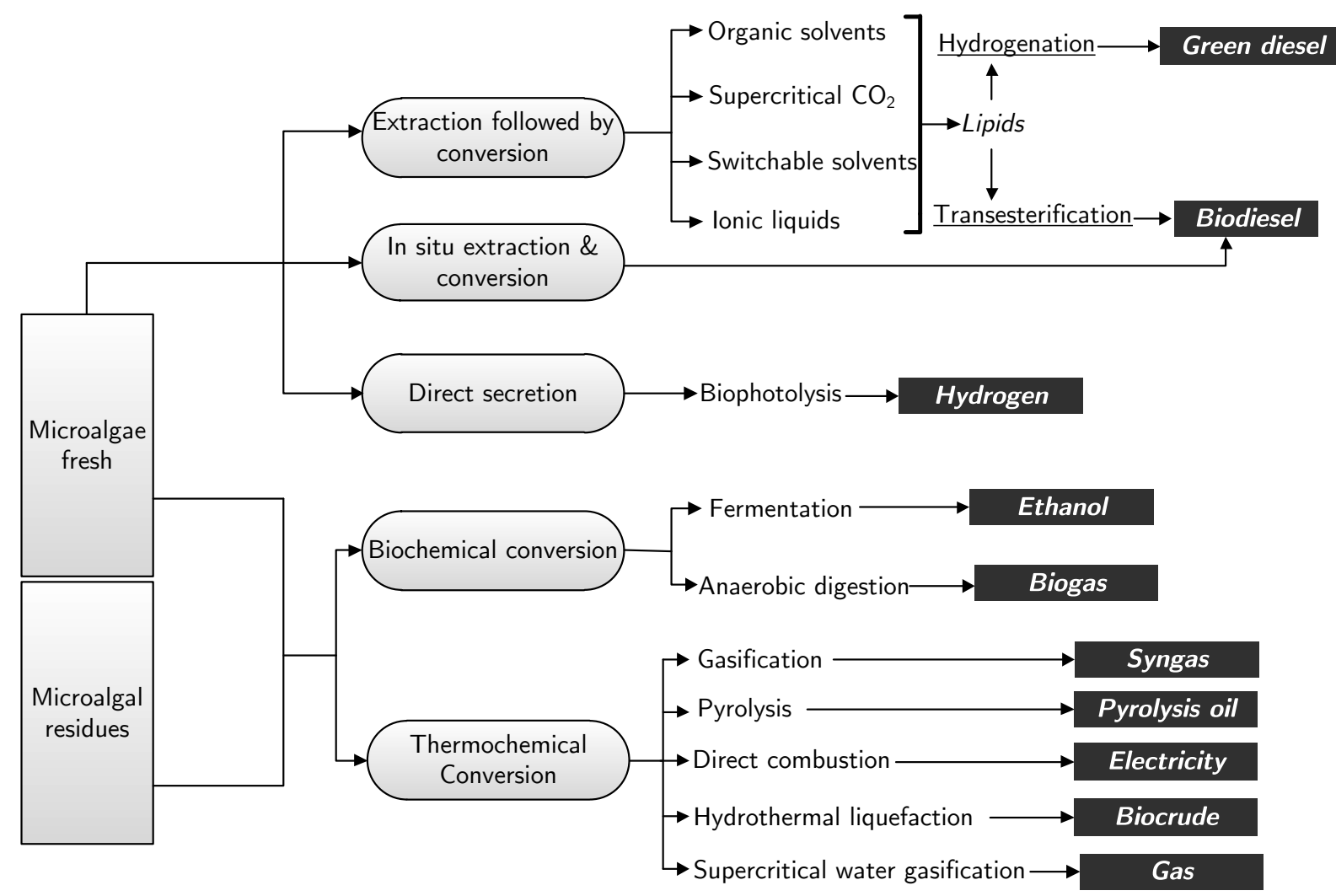

Figure 4. Biofuels (gaseous and liquids) from microalgae

Wet biomass handling processes, like hydrothermal liquefaction and supercritical water gasification (also known as hydrothermal gasification), benefit from the fact that complete dewatering of the algal stream is not required, thereby saving in related drying costs. Jin et al. ${ }^{39}$ defined hydrothermal conversion as "a potentially useful technology by mimicking nature -rapid conversion of biomass and $\mathrm{CO}_{2}$ into chemicals and fuels under hydrothermal conditions". With that, they referred to the fact that hydrothermal conversion, and especially for the case of algae, actually might resemble to the formation of fossil fuels, which occurs by the conversion of dead zooplankton and algae being buried underneath sedimentary rock and undergo intense heat and pressure in the earth crust. During hydrothermal liquefaction, water plays a very important role and has properties that differ greatly from the properties of water at ambient conditions. Remarkable changes occur with the density, dielectric constant and ionic product of water, with increasing temperature and pressure. The water becomes an organic-like solvent able to dissolve non-polar compounds and it basically can serve as reactant, catalyst and solvent. More information on water properties under hydrothermal conditions is presented in the work by Akiya and Savage. ${ }^{40}$ An important part of this thesis is devoted to hydrothermal liquefaction of microalgal biomass. 
In Figure 4, the most representative extraction methods are shown, such as supercritical $\mathrm{CO}_{2}$ extraction, a process that have been studied in this research. A cell disruption method prior to extraction of lipids and the other algal fractions (e.g. proteins) might be required, depending on the robustness of the cell wall and the location of the targeted compounds within the cells. Many techniques have been described in the literature such as mechanical (milling, pressing, etc.), ultrasonic, pulsed electric field, enzymatic, supersonic flow fluid processing, etc. ${ }^{18,41,42}$ If implemented in a biorefinery setting, cost should be carefully evaluated as, in general, cell disruption methods are energy intensive. It is difficult to provide a realistic value for the required energy input for cell disruption because it is largely dependent on the algal species used and the slurry concentration. Bead milling, one of the most energy demanding methods, can required up to $30 \mathrm{MJ} / \mathrm{kg}$ dry weight algae (for a dry weight concentration of $12.4 \mathrm{wt} \%$ ). ${ }^{43}$

In the context being described, algal strain selection can be also of importance. If a diesel-like fuel is the main product targeted, algae with high lipid content will be desired. One of the strategies extensively studied has been the cultivation under stress conditions (with nitrogen deprivation the most commonly used method), leading to higher lipid accumulation within the cells. However, this is generally accompanied by a decrease in growth rate with the risk of ending with a lower overall lipid productivity. Therefore, a two-step batch wise process is usually applied for lipid production. In the first stage the algae are grown under optimal conditions for bulk production and, when sufficient biomass is produced, a second stage is implemented with stress conditions to stimulate lipids accumulation. The economics of this strategy should be carefully evaluated and compared to the option of using an algal strain that naturally produces a relatively high amount of lipids, while showing a reasonable growth rate (e.g. Nannochloropsis). As stated by Yang et al., ${ }^{44}$ a trade-off must be made between growth rate and lipid content when choosing the more suitable microalgal species for diesel-like fuels production.

Many researchers have described the "perfect" algal species as that one that grows fast in a cost-effective cultivation system with high lipid content and that is not too difficult to harvest. Up till now, there has not been a consensus as to the best strain and it might very well be that it does not exist. Therefore, there should be always a balance between the algal species chosen and the processes selected to treat them. Their performance should be evaluated in an integrated biorefinery approach to find the right balance in economics, energy balance and environmental impact over the full cycle of cultivation, processing, products and nutrients recycling potential. 


\section{Commercialization potential of microalgae}

Man has utilized algae (both micro- and macro-) for hundreds of years for food, feed, remedies, and fertilizers. Ancient records show that people collected macroalgae for food as long ago as 500 B.C. in China and one thousand years later in Europe. ${ }^{45}$ The current worldwide microalgae manufacturing infrastructure, as reported by Wijffels and Barbosa $^{21}$ in 2010, is devoted to extraction of high-value products used for food and feed ingredients and it has a size of $\sim 5000$ metric tons of dry algal biomass. The history on algal biofuels development does not go so long back and it has been well documented in the recent U.S. National algal biofuels technology roadmap reported by the Biomass Program of the U.S. Department of Energy (DOE). ${ }^{46}$

The concept of using algae to make fuels started more than 50 years ago with the idea of using the carbohydrate fraction for methane gas production through anaerobic digestion. ${ }^{47}$ Ten years before that, scientist discovered the ability of many microalgal species to accumulate lipids by tuning their growth conditions. However, it was not until the oil crisis of the early 1970s when the concept of utilizing that lipid fraction for fuels gained interest. It was then, in 1978, when the U.S. Department of Energy (DOE) founded the well-known Aquatic Species Program, ${ }^{48}$ one of the most comprehensive research efforts to date on fuels from microalgae which lasted till 1996. The program was successful in demonstrating the feasibility of algal culture as a source of oil and resulted in important advances in the technology. More than 3,000 strains of microalgae were isolated and characterized, generating a large knowledge on algal physiology, biochemistry and for further genetic engineering. However, they concluded at the end of the project that the production of algal biofuels was not economically feasible as, at that time, the price estimates were not cost-competitive with petroleum. From 1990 to 1999, another large research program was developed in Japan known as "Biological $\mathrm{CO}_{2}$ Fixation and Utilization" which gave the same conclusion with respect the economics. ${ }^{21}$

Despite that outcome, the interest in developing algal feedstocks for biofuels production continued due to the constant rising of petroleum prices, environmental concerns and the need to reduce the dependence on foreign oil (especially for the U.S.). Therefore, in 2008 again the DOE's Biomass Program hosted a roadmapping workshop where more than 200 experts from many fields gathered and from which an extensive report was published; the 2010 National Algal Biofuels Technology Roadmap. More than providing a definitive conclusion, that work indicated the critical challenges in developing the concept at a commercial scale. Co-production of high-value products and fuels was pointed as the 
key for the economical success, ${ }^{35}$ concluding that the future of algal biofuels has a great potential but that more research is needed.

In that context, considerable investments are being made and several companies are recently emerging with the aim of reaching a commercial scale, most of which are based in U.S. The work by Singh and $\mathrm{Gu}^{35}$ and, more recently, Menetrez ${ }^{49}$ give a good overview of all algae production companies around the world, most of them engaged in the growth of algae for biofuels. Here only three examples will be given, to illustrate the status in commercialization.

- Algenol Biofuels, Inc.50: a company in Freeport (Texas) that produces ethanol directly discharged from the algal cells themselves. Algenol enhances cyanobacteria to produce ethanol by overexpressing fermentation pathway enzymes, channeling the majority of photosynthetically fixed carbon into ethanol production. They recently announced a production of 9,000 gallons of ethanol per acre per year (more than $84,000 \mathrm{~L} /$ ha/year). They received a DOE grant of $\$ 25,000,000$ and $\$ 33,915,478$ in nonfederal funding;

- Sapphire Energy, Inc. ${ }^{51}$ : Located in Columbus (New Mexico) received a DOE grant of $\$ 50,000,000$ and $\$ 85,064,206$ in nonfederal funding. This company aims to produce what they call "green crude oil" by converting algae through a thermal process. This biocrude can be further refined into other drop-in transportation fuels. In 2010, they began the construction of a green crude algal farm in New Mexico (121 ha) and claim that is currently operating at a commercial test phase;

- Solazyme, Inc. ${ }^{52}:$ A company in Riverside (Pennsylvania) that produces microalgae under heterotrophic conditions to increase their oil content which received a DOE grant of $\$ 21,765,738$ and $\$ 3,857,111$ in nonfederal funding. They claim to use sugars from plant waste organic material and standard industrial fermentation equipment. After recovery of the oil (method not disclosed), they subsequently process it by different means into biodiesel, renewable diesel (by the Honeywell UOP/Eni Ecofining process) and jet fuel. In 2010, they announced the demonstration of their naval distillate fuel on ships. 


\section{Scope and outline of the thesis}

Many researchers see algae as the ultimate renewable source for biofuels and, even further, as the ultimate alternative for depleting resources of fossil diesel. As example of that, Demirbaş ${ }^{23}$ stated:

"Billions of years ago the earth atmosphere was filled with $\mathrm{CO}_{2}$. Thus there was no life on earth. Life on earth started with Cyanobacterium and algae. These humble photosynthetic organisms sucked the atmospheric $\mathrm{CO}_{2}$ and started releasing oxygen. As a result, the levels of $\mathrm{CO}_{2}$ started decreasing to such an extent that life evolved on earth. Once again these smallest organisms are poised to save us from the threat of global warming."

However, there are also more critical points of view. For instance, Lam and Lee ${ }^{29}$ indicated:

"Based on the maturity of current technology, the true potential of microalgae biofuel towards energy security and its feasibility for commercialization are still questionable. At the current stage, microalgal biomass is still not a viable choice for commercial biofuels production due to the extensive energy input compared to current terrestrial energy crops."

That general disagreement often results from studies based on a large number of assumptions and speculations. This points out the need of more experimental assessments to know the real potential of algae. For that reason, the aim of this research is to experimentally evaluate the potential of several technologies and concepts to be introduced within an algae biorefinery configuration. Figure 5 shows the focus of the various chapters of this thesis. The basic scheme of an algae-based biorefinery could be divided into two main disciplines - engineering and biology- which in many occasions have to merge for better understanding of the whole chain.

The main driving force of the ongoing "algae fever" is that microalgae perform photosynthesis in the most efficient way on earth. That directly relates to biomass availability which is one of the most important factors when producing biofuels aiming to potentially cover a significant fraction of the current fossil fuel energy demand. For the same purpose, equally relevant is to be able to convert that biomass into a large volume of fuel per area showing the importance of investigating algal processing techniques that 
can achieve that (in this thesis Chapters 2, 3 and 4) while minimizing the inputs of energy and materials (in this thesis, Chapters 5 and 6).

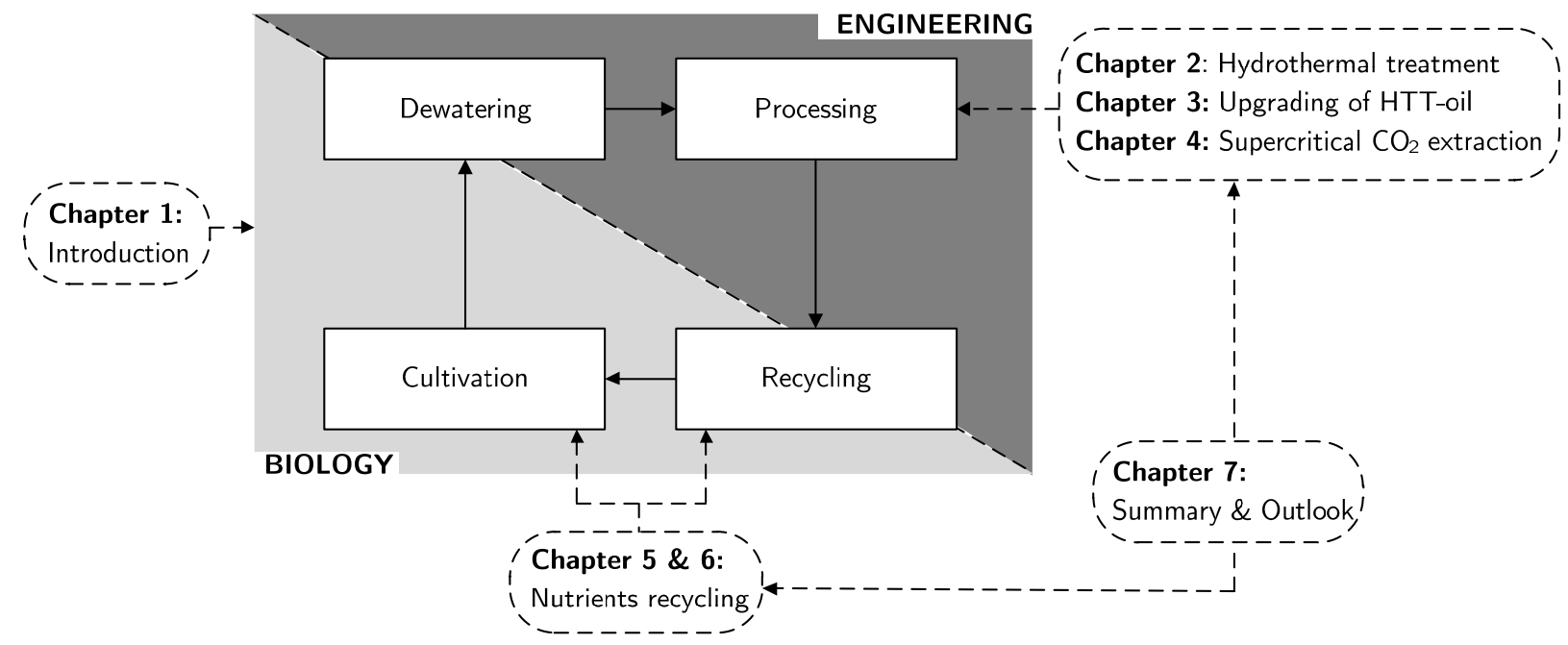

Figure 5. Scheme of the thesis outline

In Chapter 2, the hydrothermal treatment (HTT) process to convert microalgae into a crude oil is studied. The effect of a wide range of temperatures $\left(175-450^{\circ} \mathrm{C}\right)$ and reaction times (up to $60 \mathrm{~min}$ ) on the product yields and composition was evaluated, aiming to find the optimal set of conditions in terms of oil yield and quality. Regarding that last feature, a detailed molecular characterization of the oils was performed (in collaboration with the University of Bologna) intending to elucidate the HTT reaction mechanisms on a molecular level. An Appendix at the end of Chapter 2 provides the details of that work.

From Chapter 2, the need for an improved HTT oil quality became clear. Therefore, in Chapter 3, we evaluated various possible upgrading methods, aiming to produce a fuel, at least suitable for co-processing in standard refinery units to transportation fuels, and containing less nitrogen and oxygen. The focus of this chapter was on the hydrotreatment process as most suitable candidate for the production of a hydrocarbon rich fuel, possibly even direct suitable as liquid transportation fuel. Besides treating the HTT liquefaction oil, also extraction technologies were evaluated for denitrogenation.

Chapter 4 provides and experimental and economical study of supercritical $\mathrm{CO}_{2}$ extraction for the recovery of lipid-oil from microalgae. A wide range of extraction conditions were studied, both from dry and wet microalgal biomass, together with a comprehensive characterization of the oils extracted to find their quality as feedstock for diesel-like fuels. 
Finally, the concept of growing algae by recycling the nutrients present in the output water stream (named: aqueous phase) from hydrothermal treatment of the same algae is presented in Chapters 5 and 6. In Chapter 5, first the proof of concept was carried out. Subsequently, the effect of aqueous phase dilution ratio on growth performance was evaluated with the characteristic that the aqueous phase was diluted with either only water or water enriched with standard growth medium, while keeping the same total $\mathrm{N}$ concentration as that in the standard medium. After that, we studied the multiple recyclability potential of aqueous phase (AP) containing nutrients in Chapter 6 .

To close up, in Chapter 7, a possible algae biorefinery configuration is presented where all the technologies and concepts studied in the previous chapters are integrated. Along with that, the summary of the thesis is given. In addition, the biorefinery proposed is evaluated in terms of energy requirements while, at the same time, we try to point at the aspects where following-up research may need to focus in the future.

\section{Bibliography}

[1] D.J. Hofmann, J.H. Butler, P.P. Tans, A new look at atmospheric carbon dioxide, Atmos. Environ., 43(12), 2084-2086, 2009.

[2] P. Stevens, The 'shale sas revolution': Developments and changes, Chatham house, 2012.

[3] D. Cordell, J. Drangert, S. White, The story of phosphorus: Global food security and food for thought, Global Environ. Change, 19(2), 292-305, 2009.

[4] International Energy Agency, http://www.iea.org/publications/freepublications/publication/kwes.pdf, (accessed 13-4-2013).

[5] T.M. Mata, A.A. Martins, N.S. Caetano, Microalgae for biodiesel production and other applications: A review, Renewable Sustainable Energy Rev., 14(1), 217-232, 2010.

[6] P. Schenk, S. Thomas-Hall, E. Stephens, U. Marx, J. Mussgnug, C. Posten, O. Kruse, B. Hankamer, Second generation biofuels: High-efficiency microalgae for biodiesel production, Bioenergy Res., 1(1), 2043, 2008.

[7] F. de Miguel Mercader, M.J. Groeneveld, S.R.A. Kersten, N.W.J. Way, C.J. Schaverien, J.A. Hogendoorn, Production of advanced biofuels: Co-processing of upgraded pyrolysis oil in standard refinery units, Appl. Catal., B, 96(1-2), 57-66, 2010.

[8] M.F. Demirbas, Biofuels from algae for sustainable development, Appl. Energy, 88(10), 3473-3480, 2011.

[9] J.A.V. Costa, M.G. de Morais, The role of biochemical engineering in the production of biofuels from microalgae, Bioresour. Technol., 102(1), 2-9, 2011.

[10] M.J. Groeneveld, The change from fossil to solar and bifuels needs our energy. Inaugural Lecture. University of Twente, Enschede, 2008, Available at: http://doc.utwente.nl/67339/.

[11] C. Paul, M. Min, C. Yifeng, W. Liang, L. Yecong, C. Qin, Review of the biological and engineering aspects of algae to fuels approach, Int. J. Agric. Biol. Eng., 2(4), 1-30, 2009. 
[12] R. Slade, A. Bauen, Micro-algae cultivation for biofuels: Cost, energy balance, environmental impacts and future prospects, Biomass Bioenergy.

[13] T.J. Lundquist, I.C. Woertz, N.W.T. Quinn, J.R. Benemann, Realistic technology and engineering assessment of algae biofuel production, Energy Biosciences Institute, Berkeley, California, 2010.

[14] P.J.1.B. Williams, L.M.L. Laurens, Microalgae as biodiesel \& biomass feedstocks: Review \& analysis of the biochemistry, energetics \& economics, Energy Environ. Sci., 3(5), 554-590, 2010.

[15] A. Richmond, Handbook of microalgal culture: biotechnology and applied phycology, John Wiley \& Sons, Ames, 2008.

[16] P.M. Foley, E.S. Beach, J.B. Zimmerman, Algae as a source of renewable chemicals: opportunities and challenges, Green Chem., 13(6), 1399-1405, 2011.

[17] Q. Hu, M. Sommerfeld, E. Jarvis, M. Ghirardi, M. Posewitz, M. Seibert, A. Darzins, Microalgal triacylglycerols as feedstocks for biofuel production: perspectives and advances, Plant J., 54(4), 621-639, 2008.

[18] H.C. Greenwell, L.M.L. Laurens, R.J. Shields, R.W. Lovitt, K.J. Flynn, Placing microalgae on the biofuels priority list: a review of the technological challenges, J. R. Soc., Interface, 7, 703-726, 2009.

[19] J.N. Rosenberg, G.A. Oyler, L. Wilkinson, M.J. Betenbaugh, A green light for engineered algae: redirecting metabolism to fuel a biotechnology revolution, Curr. Opin. Biotechnol., 19(5), 430-436, 2008.

[20] L. Brennan, P. Owende, Biofuels from microalgae - A review of technologies for production, processing, and extractions of biofuels and co-products, Renewable Sustainable Energy Rev., 14(2), 557$577,2010$.

[21] R.H. Wijffels, M.J. Barbosa, An outlook on microalgal biofuels, Science, 329(5993), 796-799, 2010.

[22] Y. Chisti, Biodiesel from microalgae, Biotechnol. Adv., 25(3), 294-306, 2007.

[23] A. Demirbas, Use of algae as biofuel sources, Energy Convers. Manage., 51(12), 2738-2749, 2010.

[24] A.F. Clarens, E.P. Resurreccion, M.A. White, L.M. Colosi, Environmental life cycle comparison of algae to other bioenergy feedstocks, Environ. Sci. Technol., 44(5), 1813-1819, 2010.

[25] A.F. Clarens, H. Nassau, E.P. Resurreccion, M.A. White, L.M. Colosi, Environmental impacts of algae-derived biodiesel and bioelectricity for transportation, Environ. Sci. Technol., 45(17), 7554-7560, 2011.

[26] D.R. Georgianna, S.P. Mayfield, Exploiting diversity and synthetic biology for the production of algal biofuels, Nature, 488(7411), 329-335, 2012.

[27] B. Kamm, P.R. Gruber, M. Kamm, Biorefineries - industrial processes and products, Wiley, 2010.

[28] E. Suali, R. Sarbatly, Conversion of microalgae to biofuel, Renewable Sustainable Energy Rev., 16(6), 4316-4342, 2012.

[29] M.K. Lam, K.T. Lee, Microalgae biofuels: A critical review of issues, problems and the way forward, Biotechnol. Adv., 30(3), 673-690, 2012.

[30] I. Rawat, R. Ranjith Kumar, T. Mutanda, F. Bux, Biodiesel from microalgae: A critical evaluation from laboratory to large scale production, Appl. Energy, 103(0), 444-467, 2013.

[31] A. Singh, S.I. Olsen, A critical review of biochemical conversion, sustainability and life cycle assessment of algal biofuels, Appl. Energy, 88(10), 3548-3555, 2011.

[32] Cellana, http://cellana.com/our-technology/ip/, (accessed 13-4-2013).

[33] S. Fon Sing, A. Isdepsky, M. Borowitzka, N. Moheimani, Production of biofuels from microalgae, Mitig. Adapt. Strat. Gl., 18(1), 47-72, 2013.

[34] Food and Agriculture Organization of the United Nations 
http://www.fao.org/worldfoodsituation/wfs-home/en/, (accessed 13-4-2013).

[35] J. Singh, S. Gu, Commercialization potential of microalgae for biofuels production, Renewable Sustainable Energy Rev., 14(9), 2596-2610, 2010.

[36] Cereplast, http://www.cereplast.com/, (accessed 13-4-2013).

[37] B. Kim, J. Jeong, S. Shin, D. Lee, S. Kim, H.-J. Yoon, J.K. Cho, Facile single-step conversion of macroalgal polymeric carbohydrates into biofuels, ChemSusChem, 3(11), 1273-1275, 2010.

[38] D. López Barreiro, W. Prins, F. Ronsse, W. Brilman, Hydrothermal liquefaction (HTL) of microalgae for biofuel production: State of the art review and future prospects, Biomass Bioenergy.

[39] F. Jin, X. Zeng, Z. Jing, H. Enomoto, A potentially useful technology by mimicking nature-Rapid conversion of biomass and $\mathrm{CO}_{2}$ into chemicals and fuels under hydrothermal conditions, Ind. Eng. Chem. Res., 51(30), 9921-9937, 2012.

[40] N. Akiya, P.E. Savage, Roles of water for chemical reactions in high-temperature water, Chem. Rev., 102(8), 2725-2750, 2002.

[41] M. Vanthoor-Koopmans, R.H. Wijffels, M.J. Barbosa, M.H.M. Eppink, Biorefinery of microalgae for food and fuel, Bioresour. Technol., 135(0), 142-149, 2013.

[42] K. Boer, N. Moheimani, M. Borowitzka, P. Bahri, Extraction and conversion pathways for microalgae to biodiesel: a review focused on energy consumption, J. Appl. Phycol., 24(6), 1681-1698, 2012.

[43] J. Doucha, K. Lívanský, Influence of processing parameters on disintegration of Chlorella cells in various types of homogenizers, Appl. Microbiol. Biotechnol., 81(3), 431-440, 2008.

[44] J. Yang, M. Xu, X. Zhang, Q. Hu, M. Sommerfeld, Y. Chen, Life-cycle analysis on biodiesel production from microalgae: Water footprint and nutrients balance, Bioresour. Technol., 102(1), 159-165, 2011.

[45] L. Barsanti, P. Gualtieri, Algae: anatomy, biochemistry, and biotechnology, Taylor \& Francis, 2005.

[46] U.S. DOE, National algal biofuels technology roadmap, Office of Energy Efficiency and Renewable Energy, Biomass Program, 2010.

[47] C.G. Golueke, W.J. Oswald, H.B. Gotaas, Anaerobic digestion of algae, Appl. Microbiol., 5(1), 47-55, 1957.

[48] J. Sheehan, T. Dunahay, J.R. Benemann, P. Roesler, A look back at the U.S. Department of Energy's aquatic species program: biodiesel from algae, U.S. Department of energy (DOE), Golden, Colorado, 1998.

[49] M.Y. Menetrez, An overview of algae biofuel production and potential environmental impact, Environ. Sci. Technol., 46(13), 7073-7085, 2012.

[50] Algenol biofuels, http://www.algenolbiofuels.com/, (accessed 13-4-2013).

[51] Sapphire energy, http://www.sapphireenergy.com/, (accessed 13-4-2013).

[52] Solazyme, http://solazyme.com/, (accessed 13-4-2013). 



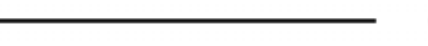

rar 


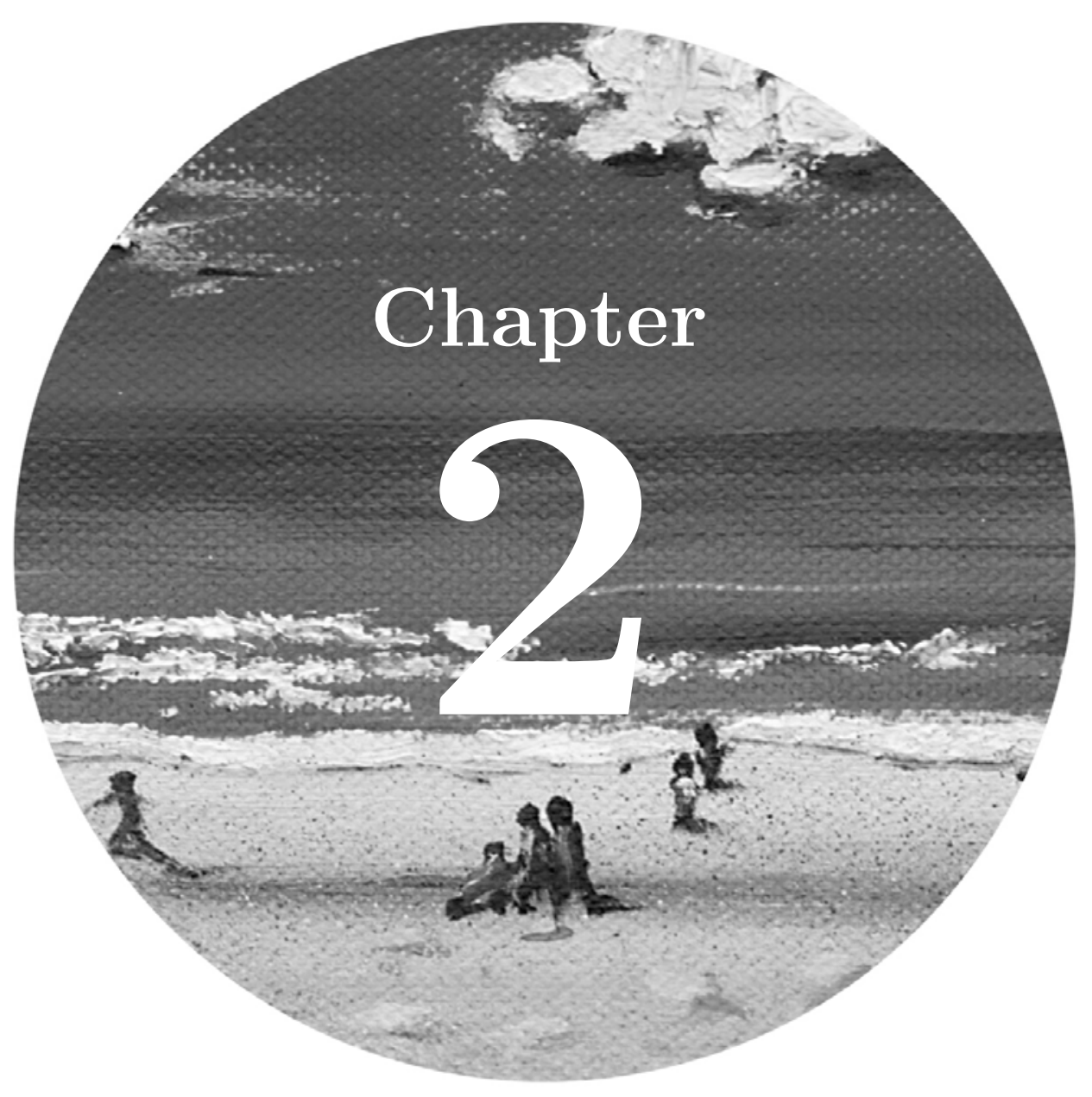

Hydrothermal treatment (HTT) of microalgae: Evaluation of the process as conversion method in an algae biorefinery concept 


\begin{abstract}
The hydrothermal treatment (HTT) technology is evaluated for its potential as process to convert algae- and algal-debris into a liquid fuel, within a sustainable algae biorefinery concept in which, next to fuels (gaseous and liquid), high value products are coproduced, nutrients and water are recycled and the use of fossil energy is minimized. In this work, the freshwater microalgae Desmodesmus sp. was used as feedstock. HTT was investigated over a very wide range of temperatures (175$450^{\circ} \mathrm{C}$ ) and reaction time (up to 60 minutes), using a batch reactor system. The different product phases were quantified and analyzed. The maximum oil yield (49 wt\%) was obtained at $375^{\circ} \mathrm{C}$ and 5 min reaction time, recovering $75 \%$ of the algal calorific value into the oil and an energy densification from 22 to $36 \mathrm{MJ} / \mathrm{kg}$. At increasing temperature both the oil yield as well as the nitrogen content in the oil increased, necessitating a further investigation on the molecular composition of the oil. This was performed in an adjacent collaborative paper (in Appendix of this chapter) with special attention to the nitrogen-containing compounds and to gain insight in the liquefaction mechanism. A pioneering visual inspection of the cells after HTT showed that a large step increase in the $\mathrm{HTT}$ oil yield, when going from $225^{\circ} \mathrm{C}$ to $250^{\circ} \mathrm{C}$ at 5 min reaction time, coincided with a major cell wall rupture under these conditions. Additionally, it was found that the oil composition, by extractive recovery after $\mathrm{HTT}$ below $250^{\circ} \mathrm{C}$, did change with temperature, even though the algal cells were visually still unbroken. Finally, the possibilities of recycling growth nutrients became evident by analyzing the aqueous fractions obtained after HTT. From the results obtained, we concluded that HTT is most suited as post-treatment technology in an algae biorefinery system, after the wet extraction of high valuable products, as protein-rich food/feed ingredients and lipids.
\end{abstract}




\section{Introduction}

The link between the development of new growing economies and the depletion of fossil fuels has forced society to develop new and sustainable ways for meeting their energy demands. Biomass is the only current renewable carbon source that can be directly used for chemicals and liquid fuels. ${ }^{1}$ Therefore, biomass is considered as an optional long-term sustainable and $\mathrm{CO}_{2}$-neutral energy source. Currently, microalgae have been identified as a potential bio-energy source exhibiting alleged advantages for fuel production:

- Higher growth rates than terrestrial biomass sources;

- The ability to fix $\mathrm{CO}_{2}$ to organic substances using solar energy while growing in a wide variety of climates and lands;

- No direct competition for agricultural land;

- Capable of storing solar energy into energy-rich compounds such as lipids.

Various conversion and extraction routes have been followed for the production of liquid fuels from microalgae. Lipid extraction -combined with transesterification- to produce biodiesel is one of the most common methods. ${ }^{2-4}$ To our knowledge, algal lipid extraction for fuel production has not yet been proven at large scale. This extraction approach would be most useful if the process could be optimized in order to use wet algal biomass and low-toxicity solvents.

Other thermo-chemical processes such as combustion, pyrolysis and dry conventional gasification have been also reported. ${ }^{5-7}$ In these processes, the use of dry biomass is required, which leads to a significant increase in energy costs due to the need for a drying step before the conversion step. ${ }^{8}$ Therefore, a wet biomass-handling process, such as hydrothermal liquefaction (HTL) is attractive for the production of liquid fuels from wet microalgae, as it circumvents the need for complete water removal and associated high energy cost for thermal drying. Algae concentrations of about $5-20 \%$ seem to be suitable for hydrothermal treatment which can be achieved using less than $5 \%$ of the energy costs required for complete drying (by means of thermal drying).$^{9}$ In this research, we look at the feasibility of an algae biorefinery concept (ABC) comprising four main stages: microalgae growth and harvesting, fuel production, residues processing and nutrients recovery and recycling. We consider the HTL process as a promising candidate to be included in our algae biorefinery concept. 
Hydrothermal liquefaction has been widely described as a process involving the reaction of biomass in water at subcritical temperatures (below $374^{\circ} \mathrm{C}$ ) and high pressure (above water vapor pressure) for a certain reaction time with or without the use of a catalyst. ${ }^{10}$ Due to the fact that, in this research, experiments at above $374^{\circ} \mathrm{C}$ have been also performed, the term "hydrothermal treatment (HTT)" will be used rather than "hydrothermal liquefaction" as this generally refers to thermal processing at subcritical temperatures.

Several studies into the hydrothermal conversion of lignocellulosic biomass have been performed in the past ${ }^{11-13}$ aiming to obtain various gas and liquid fuel products. One of the pioneering investigations for which this conversion method was applied using microalgae was reported by Dote et al. ${ }^{14}$ High hydrocarbon content microalgae, Botryococcus Braunii, were used for that study and a maximum oil yield of $64 \mathrm{wt} \%$ was obtained at $300^{\circ} \mathrm{C}$ during 60 min reaction time and adding sodium carbonate. Mainly thanks to the composition of Botryococcus Braunii, a high oil yield with significantly high heating value (40-50 MJ/kg) was obtained. Beyond Botryococcus Brauni, which is known as "slow growing organism" with relatively low resistance to biological contaminants, necessitating highly controlled culture conditions, ${ }^{15}$ large efforts has been focused on the hydrothermal conversion of other types of microalgae, characterized by fast growth or advantageous biological features. Hydrothermal liquefaction of microalgae has been applied in a wide variety of process conditions such as: using a variety of solvents (water, hydrogen donor solvents and organic solvents ${ }^{16,17}$ ), with or without metal catalyst or alkali salts $\left(\mathrm{Na}_{2} \mathrm{CO}_{3}\right.$ and $\mathrm{KOH}$ are commonly used) and for both low and high lipid content microalgae (e.g. Duanaliella tertiolecta and Nannochloropsis sp. respectively). The broad spectrum as covered by these studies on the hydrothermal treatment of microalgae is illustrated in Table 1.

As shown in Table 1 and also claimed by Zou et al., ${ }^{8}$ the physical and chemical properties of algal bio-oils are strongly dependent on the algal feedstock used and on the process conditions. If the biochemical composition is changed (e.g. change in protein content leading to the broad range of nitrogen content of the various algae strains listed in Table 1), a different type of oil will result. In addition, the data in Table 1 suggests that higher lipid content microalgae lead to higher oil yields as reported by Biller et al. ${ }^{18}$ after direct comparison between low and high lipid content algae. Recent investigations are focusing on liquefaction combined with hydro-treating using $\mathrm{H}_{2}$-gas, a hydrogen donor (such as formic acid) or with $\mathrm{Ni} / \mathrm{REHY}$ (zeolite) catalyst. ${ }^{10,19,20}$ 
Table 1. Overview of several studies on hydrothermal treatment of microalgae

\begin{tabular}{|c|c|c|c|c|c|c|c|c|}
\hline \multirow[b]{2}{*}{ Reference } & \multicolumn{3}{|c|}{ Algae } & \multicolumn{3}{|c|}{ Process conditions } & \multirow{2}{*}{$\begin{array}{c}\text { Max. oil } \\
\text { yield (wt\%) }\end{array}$} & \multirow{2}{*}{$\begin{array}{c}\mathrm{N} \text { in oil } \\
(\mathrm{wt} \%)\end{array}$} \\
\hline & Species & $\begin{array}{c}\mathrm{N} \\
(\text { dry wt\%) }\end{array}$ & $\begin{array}{l}\text { Lipids } \\
\text { (wt\%) }\end{array}$ & $\mathrm{T}\left({ }^{\circ} \mathrm{C}\right)$ & $\begin{array}{l}\text { R. time } \\
(\min )\end{array}$ & $\begin{array}{l}\text { Metal catalyst } \\
\text { and alkali salts }\end{array}$ & & \\
\hline Dote $1994^{14}$ & Botryococcus braunii & 2.8 & Up to $60^{\mathrm{a}}$ & $200-340$ & 60 & $\mathrm{Na}_{2} \mathrm{CO}_{3}(0-5 \%)$ & 64.0 & 0.70 \\
\hline Minowa $1995^{21}$ & Dunaliella tertiolecta & 9.8 & 20.5 & $200-340$ & 5 and 60 & $\mathrm{Na}_{2} \mathrm{CO}_{3}(0-5 \%)$ & 43.8 & 6.70 \\
\hline Matsui $1997^{17}$ & Spirulina & 4.8 & 12.0 & 300 and 340 & 30 and 60 & $\mathrm{Fe}(\mathrm{CO})_{5}-\mathrm{S}$ & 61.0 & 6.80 \\
\hline Yang $2004^{22}$ & Microcystis viridis & 9.5 & Not found & 300 and 340 & 30 and 60 & $\mathrm{Na}_{2} \mathrm{CO}_{3}(0-5 \%)$ & 33.0 & 7.10 \\
\hline Ross $2010^{10}$ & $\begin{array}{l}\text { Chlorella vulgaris and } \\
\text { Spirulina }\end{array}$ & 9.2 and 10.9 & 25 and $5^{\mathrm{a}}$ & 300 and 350 & 60 & $\begin{array}{c}\mathrm{KOH}, \mathrm{Na}_{2} \mathrm{CO}_{3}, \\
\mathrm{CH}_{3} \mathrm{COOH} \text { and } \\
\mathrm{HCOOH}\end{array}$ & 27.3 and 20.0 & 4.9 and 4.6 \\
\hline Brown $2010^{23}$ & Nannochloropsis sp. & 6.4 & 28 & $200-500$ & 60 & No cat. & 43.0 & 3.9 \\
\hline Zhou $2010^{24}$ & Enteromorpha prolifera & 3.65 & $1.5-5^{\mathrm{a}}$ & $220-320$ & $5-60$ & $\mathrm{Na}_{2} \mathrm{CO}_{3}(0-5 \%)$ & 23.0 & 5.76 \\
\hline Zou $2010^{8}$ & Dunaliella tertiolecta & 1.99 & 2.9 & $280-380$ & $10-90$ & $\mathrm{Na}_{2} \mathrm{CO}_{3}$ & 25.8 & 3.71 \\
\hline Duan $2010^{19}$ & Nannochloropsis sp. & 6.32 & 28 & 350 & 60 & $\begin{array}{l}\text { No cat., } \mathrm{Pd} / \mathrm{C}, \\
\mathrm{Pt} / \mathrm{C}, \mathrm{Ru} / \mathrm{C} \\
\mathrm{Ni} / \mathrm{SiO}_{2}-\mathrm{Al}_{2} \mathrm{O}_{3}, \\
\mathrm{CoMo} / \mathrm{\gamma}-\mathrm{Al}_{2} \mathrm{O}_{3} \\
\text { and zeolite }\end{array}$ & 57.0 & 3.88 \\
\hline Biller $2011^{18}$ & $\begin{array}{c}\text { Chlorella vulgaris, } \\
\text { Nannochloropsis } \\
\text { occulata, Porphyridium } \\
\text { cruentum and Spirulina }\end{array}$ & $\begin{array}{c}8.2,8.6,8.0 \\
\text { and } 11.2\end{array}$ & $\begin{array}{c}25,32,8 \text { and } \\
5\end{array}$ & 350 & 60 & $\begin{array}{c}\text { No cat., } \mathrm{Na}_{2} \mathrm{CO}_{3} \\
\text { and } \mathrm{HCOOH}\end{array}$ & $\begin{array}{c}35.8,34.3, \sim 20 \\
\text { and } 29\end{array}$ & $\begin{array}{l}5.6,4.1 \\
5.4 \text { and } 7\end{array}$ \\
\hline
\end{tabular}

${ }^{\mathrm{a}}$ General value reported in other literature. Not available in the referred article. ${ }^{\mathrm{b}}$ Under inert He and high pressure reducing conditions (with $\mathrm{H}_{2}$ ). 
One particularly important aspect in our algae biorefinery concept proposed is the recycling of nutrients ( $\mathrm{N}$ and $\mathrm{P}$ ) as initially proposed by Minowa et al. ${ }^{25}$ Only a few publications ${ }^{10,18}$ have reported the nitrogen distribution over the various products after thermal processing which would allow the evaluation of its recycling. Insights here are essential, since a high fossil energy input is required in the microalgae cultivation (for the production of the growth nutrients) and harvesting steps. ${ }^{9}$ In addition, phosphorous in particular is a finite resource of which reserves are being depleted..$^{26,27}$

In this study on HTT, all the product fractions were recovered and analyzed in order to achieve a proper mass balance closure, based on experimental data, whereas in most of the hydrothermal treatment studies using microalgae reported in literature, the water phase or the gas phase was typically calculated by difference. It is important to point out that we consider the product trends reliable only when the mass balances exceed $95 \%$ closure. We studied the hydrothermal treatment of microalgae over a wide range of reaction conditions aiming to find the maximum oil production, the correlation between oil yield and algal cells behavior under high temperature and the composition ('quality') of the oil obtained. With those results, the ultimate goal is to enable the evaluation of the suitability of this process as an algae conversion method in an algae biorefinery concept. The last feature, related to the composition of the oil, is extensively studied to elucidate the HTT reaction mechanisms on a molecular level. This was carried out under a collaborative framework with the University of Bologna and the work is presented in the Appendix of this chapter.

\section{Experimental}

\subsection{Materials}

The fresh water microalgae species Desmodesmus sp. was used for this research and purchased from Ingrepro B.V. (The Netherlands) where they were grown in an open raceway system. Desmodesmus sp. is a resistant type of algae with a strong cell wall and with typical cell dimensions of about 6 to $8 \mu \mathrm{m}$ length and $3 \mu \mathrm{m}$ width as shown in Figure 1.

The proximate and ultimate analyses of this type of microalgae are listed in Table 2. Desmodesmus sp. slurry was dried at $105^{\circ} \mathrm{C}$ during $24 \mathrm{~h}$ in order to determine the water content. After that, the dry residue was further treated at $550^{\circ} \mathrm{C}$ under oxidizing 
conditions during $5 \mathrm{~h}$ to measure the ash content. The $\mathrm{C}, \mathrm{H}$ and $\mathrm{N}$ contents of the dry algae (dry ash free, d.a.f.) were measured in duplicate using an elemental analyzer (Thermo Scientific Flash 2000, CHN-S) and the higher heating value (HHV) was calculated according to Boie's formula described in Section 2.5.

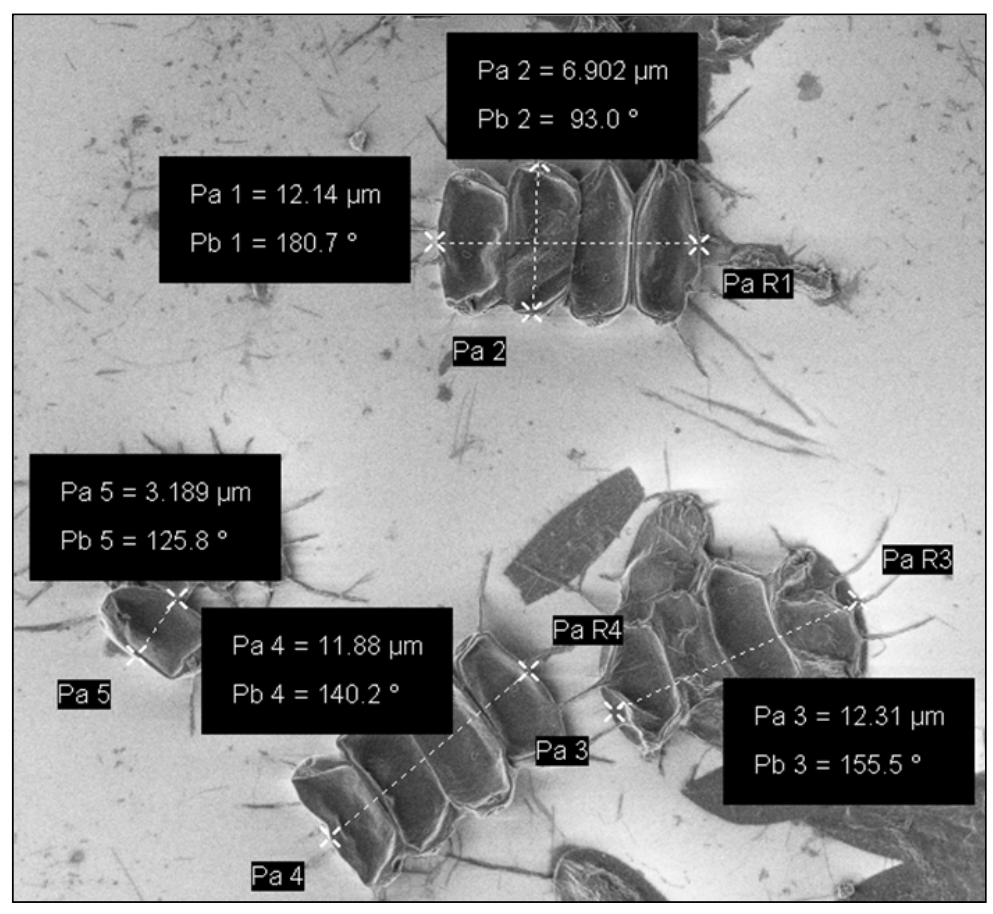

Figure 1. SEM picture of fresh air-dried Desmodesmus sp. including dimensions

Table 2. Microalgae Desmodesmus sp. analysis

\begin{tabular}{cccccc}
\hline $\begin{array}{c}\text { Feedstock properties } \\
(\mathbf{w t} \%)\end{array}$ & \multicolumn{2}{c}{$\begin{array}{c}\text { Bio-chemical composition } \\
(\mathbf{w t} \%)^{\mathrm{c}}\end{array}$} & \multicolumn{2}{c}{$\begin{array}{c}\text { Elemental composition } \\
(\mathbf{w t} \%)^{\mathrm{d}}\end{array}$} \\
\hline Water content & 90.62 & Protein & $38-44$ & $\mathrm{C}$ & 51.96 \\
Dry residue content & 9.38 & Lipid & $10-14$ & $\mathrm{H}$ & 7.31 \\
Organic content $^{\mathrm{a}}$ & 8.64 & Fiber & $10-13$ & $\mathrm{~N}$ & 6.86 \\
Ash content $^{\mathrm{b}}$ & 7.83 & Carbohydrate & $13-20$ & $\mathrm{O}^{\mathrm{e}}$ & 33.87 \\
& & & & $\mathrm{HHV}(\mathrm{MJ} / \mathrm{kg})$ & 23.44 \\
\hline
\end{tabular}

${ }^{\mathrm{a}}$ Defined as g algae (d.a.f.) / g algae solution. ${ }^{\mathrm{b}}$ On dry solid basis. ${ }^{\mathrm{c}}$ As given by supplier. ${ }^{\mathrm{d}}$ Dry algae ash free (d.a.f.). ${ }^{\text {e }}$ Calculated by difference as $100-($ ash $+\mathrm{C}+\mathrm{H}+\mathrm{N})$.

The Bligh and Dyer $(\mathrm{B} \& \mathrm{D})$ method $^{28}$ was selected as standard extraction technique for the recovery of lipid oil from our wet microalgae. In this method, a mixture of chloroform, methanol and water is used creating a biphasic system where the lipids are dissolved in the chloroform bottom phase. After phase separation and chloroform evaporation, the oil was obtained. An additional washing step with an aqueous $\mathrm{Na}_{2} \mathrm{SO}_{4}$ 
solution (suggested by Hara and Radin ${ }^{29}$ ) was combined with the B\&D method in order to remove any extracted non-lipid material from the chloroform phase. This extraction resulted in a lipid oil yield of $12.2 \mathrm{wt} \%$ which was in the range of the lipid content reported by the supplier.

\subsection{Hydrothermal treatment (HTT) and products separation}

HTT experiments were carried out using a $45 \mathrm{ml}$ internal volume, stainless steel autoclave, consisting of a cylinder with top and bottom openings. The inner temperature was measured by a thermocouple inserted through an orifice on the bottom lid, and the pressure via a gas connection from the top lid to a pressure transmitter. In this manner, both signals could be monitored by an external computer located outside the safety room where the complete set-up was located. The desired temperature for the thermal treatment inside the reactor was reached by immersion in a fluidized sand bed heated by an electrical oven and pre-heated air which was also used for the fluidization of the bed. The heating time to the experimental temperature for all the experiments was 6 to 7 minutes. After a certain reaction time at the corresponding temperature, fast quenching ( 1-2 $\mathrm{min})$ was performed by inserting the autoclave in a water bath. Three additional pneumatic devices - a piston, a shaker and a rotating arm- allowed the movement of the autoclave being controlled outside the high pressure box. The complete scheme of the set-up is shown in Figure 2.

A typical experiment starts by loading into the autoclave a pre-weighed amount of feedstock containing a dry algae concentration of 7-8 wt\% (d.a.f. basis as defined in Section 2.1). Typically, $20 \mathrm{~g}$ of algae solution was fed into the reactor. However, for the experiments at higher temperatures (those above $375^{\circ} \mathrm{C}$ ), the sample volume was adjusted, based on the density of pure water (since $92 \%$ of the algae solution is water) at the specific reaction temperature and maximum pressure bearable by the reactor (300 bar). After adding the sample, the autoclave was tightly closed and assembled to the shaker connected to the pneumatic piston.

Prior to each experiment, a leakage test with 80 bar of helium was performed by connecting a gas pipe with a gate valve from the reactor to the helium bottle. This also served to flush the air initially present inside the reactor. Next, the reactor was pressurized with 5 bar of helium in order to facilitate product gas collection, especially for those experiments at low temperature producing less than 1 bar of gas. Finally, the 
gas pipe was disconnected and the rest of the experiment was controlled from outside the safety chamber via the control panel.

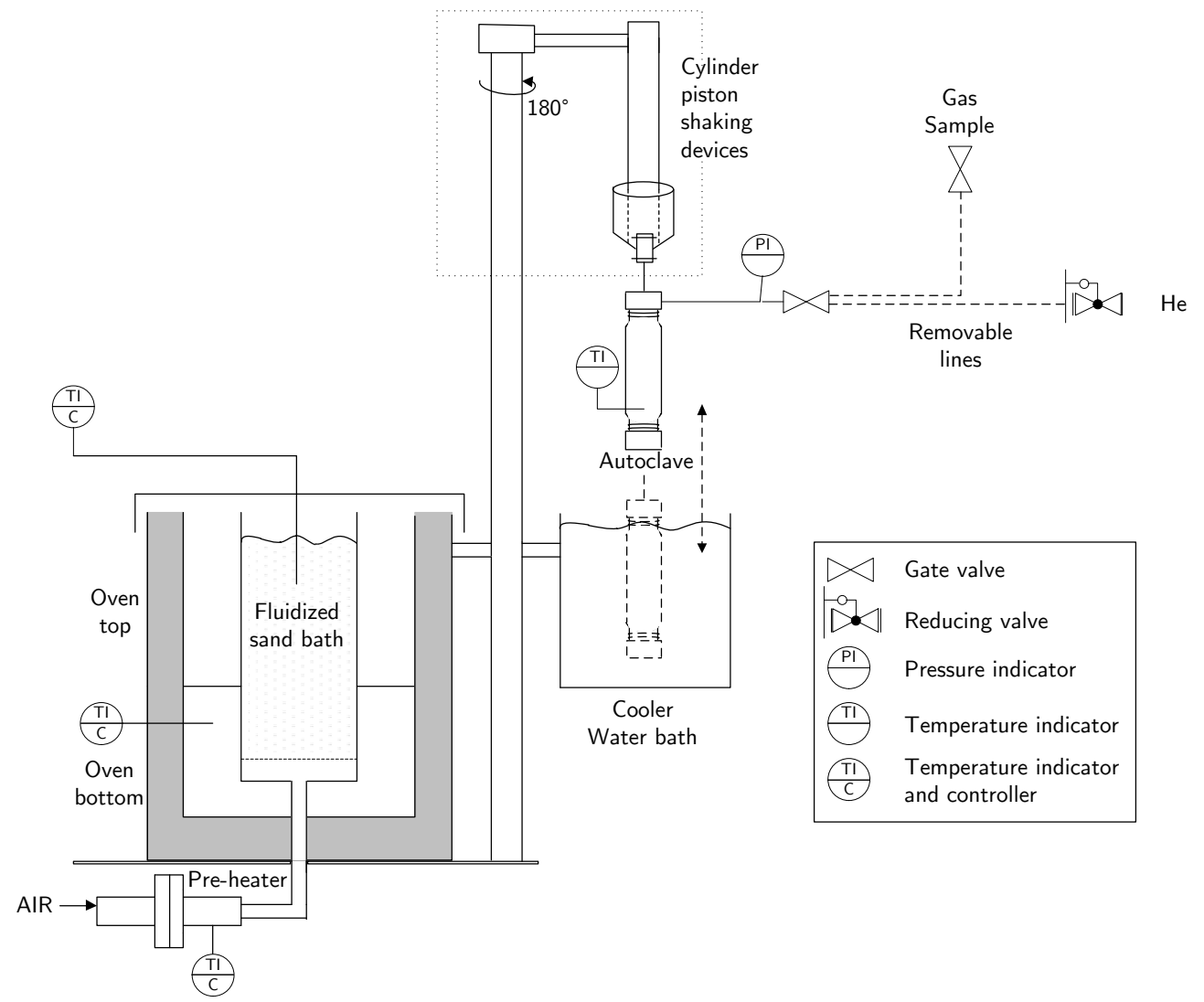

Figure 2. Schematic diagram of the HTT set-up (adapted from de Miguel Mercader et al. ${ }^{30}$ )

The reactor was displaced by $180^{\circ}$ to be located on top of the fluidized sand bed by activating the rotating arm. Then it was lowered into the sand bed using the pneumatic piston, and immediately after that, the shaking was activated for mixing purposes. After the desired reaction time at the chosen temperature, the autoclave was lifted and immersed into a water bath to achieve fast quenching. Upon heating, a rapid increase of the pressure inside the autoclave was observed; mainly due to the vapor pressure of the water at the specific temperature. The final pressure of gas produced was recorded after cooling the reactor to room temperature. Before depressurizing the system, a gas sample was taken for analysis.

Figure 3 shows the subsequent product collection and separation steps. First, the product was collected after disconnecting and opening the autoclave. Afterwards, extraction with a solvent was required to collect the maximum amount of product, which was often stuck to the walls of the reactor. Dichloromethane (Sigma-Aldrich, 99\% purity) was used for that purpose by first adding $20 \mathrm{ml}$ and shaking vigorously connecting once more the 
reactor to the pneumatic shaker (for 5 to $10 \mathrm{~min}$ ). Then the autoclave was rinsed five times (using $10 \mathrm{ml}$ of solvent each time). Next, other parts of the system (reactor lid and gas pipe) were flushed with acetone to collect any other algae residue by evaporation of the acetone.

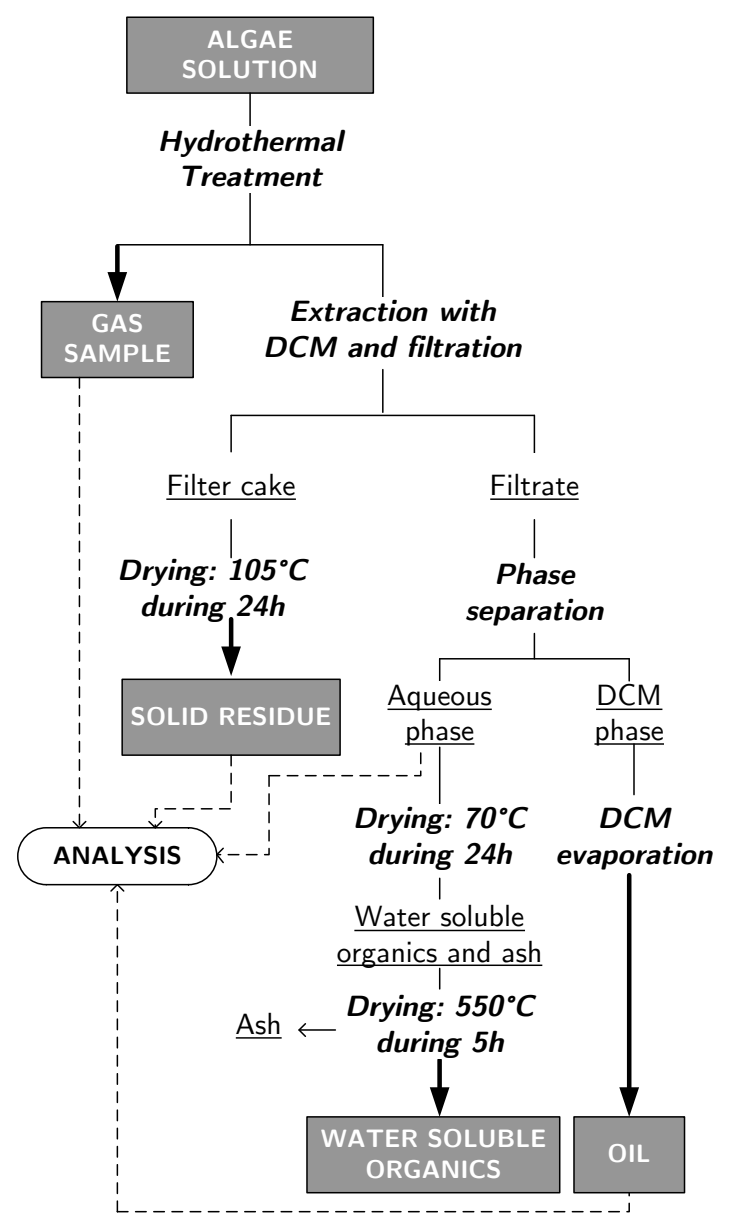

Figure 3. Product recovery and separation procedure

The sample mixture was vacuum filtrated (glass microfiber filter, Whatman GF/B, $1 \mu \mathrm{m}$ pore size) with a simple system consisting of a Buchner funnel, a side arm flask and a pump. After filtration of the whole mixture, the small residue still present in the glass vessel previously containing the sample was washed with $20 \mathrm{ml}$ of dichloromethane. At the same time, this amount of solvent helped to wash the filter, ensuring that the filter cake consisted of just solid residue. To remove any residual solvent, the solids were dried at $105^{\circ} \mathrm{C}$ over $24 \mathrm{~h}$ and finally quantified. After filtration, the filtrate was centrifuged at $7500 \mathrm{rpm}$ for $15 \mathrm{~min}$ to optimize the separation between the dichloromethane bottom phase and the aqueous top phase which was subsequently recovered by aspiration using a syringe. The water-soluble organics were quantified in the following way: first, $2 \mathrm{ml}$ of aqueous phase were evaporated at around $70^{\circ} \mathrm{C}$ for $24 \mathrm{~h}$ obtaining a mixture of organics 
and ash soluble in water; after that, this mixture was treated at $550^{\circ} \mathrm{C}$ for $5 \mathrm{~h}$ to quantify only the ash which was subtracted from the previous grams of organics and ash. In a similar way, the dichloromethane was removed by evaporation and recovered by using a cold trap system. The recovered dichloromethane solvent was as transparent as in its pure form and it could be reused for other extractions. Finally, to obtain an oil product totally free of solvent, the sample was placed under continuous flushing with $\mathrm{N}_{2}$ as carrier gas for $24 \mathrm{~h}$, in order to prevent possible lipids oxidation. The loss of some light compounds from both evaporation steps (for the aqueous and the dichloromethane phases) is likely and it should be considered.

After carrying out all these steps, four products were obtained: oil (crude from dichloromethane soluble fraction), gas, water soluble organics and solid residue. The oils and solids were analyzed for their elemental composition. The aqueous phases were analyzed for their nutrients concentration using a spectrophotometer. In addition, visualization of the solid residue was performed using a scanning electron microscope in order to determine if the algae cells were still present in this residual product. A specific biological dehydration technique was used to dry the solid residue samples before imaging and is described in detail in Section 2.4.

In addition, a dichloromethane extraction at room temperature was performed as control experiment. The microalgae was subjected to the same solvent amount $(70 \mathrm{ml})$ and product recovery procedure (Figure 3) used for the thermal experiments. After dichloromethane evaporation and $24 \mathrm{~h}$ of flushing with nitrogen, the extracted oil was obtained and analyzed.

\subsection{Analyses}

Elemental analysis (EA): the elemental composition in terms of nitrogen, carbon and hydrogen (in wt\%) in the oil product and solid residue was determined using an elemental analyzer (EA, Thermo Scientific Flash 2000). Oxygen was calculated by difference. In a different configuration of the elemental analyzer, the real weight percentage of oxygen was determined experimentally for some of the oils. The measured ('real') oxygen values were in close range to the ones calculated by difference. Duplicate analyses were conducted for each sample and if the difference was within $\pm 1 \%$, the results were accepted and the average values were taken.

Calorimeter: the higher heating value (HHV) of selected oil samples was measured using a bomb calorimeter (IKA® Calorimeter System, C 2000 basic, C 2000 control). The HHV 
was also estimated by Boie's formula (see Section 2.5) and compared with the experimental HHV measured with the calorimeter in order to ensure its reliability.

Gas chromatograph (GC): after each experiment, a gas sample was analyzed using a gas chromatograph (Varian Micro GC CP-4900) containing two analytical columns: Molsieve 5A (10 m) and PPQ (10 m), using helium as carrier gas. For the experiments performed at high temperatures (above $375^{\circ} \mathrm{C}$ ) where a high production of hydrogen was obtained, a micro-GC with three analytical columns (Varian CP-4900, $10 \mathrm{~m}$ Molsieve 5A Ar, $10 \mathrm{~m}$ Molsieve 5A He, $10 \mathrm{~m}$ PPQ He, $8 \mathrm{~m}$ Sil-5CB He) was used with helium and argon as carrier gases.

Spectrophotometer: the nutrients concentrations (in their ionic and cationic form) in the aqueous phases were determined using a spectrophotometer (DR 5000, HACH Coorporation). The compounds quantified were (purchased from HACH LANGE): Total nitrogen (Laton, LCK 238), nitrate (LCK 339), nitrite (LCK 541), ammonium (LCK 305), total phosphorus (LCK 349) and phosphate (LCK 349).

Scanning electron microscopy (SEM): the algal cells (in the solid residue) visualization was carried out using a scanning electron microscope (SEM, JEOL TSM 5600), allowing for high-resolution images showing details undetectable by a common optical microscope. The samples were sputtered with a thin gold layer (Baltzers Union SCD 40) before placing them inside the SEM.

Gel permeation chromatography (GPC): we obtained an indication of the molecular weight distribution of the oils using an Agilent 1200 series HPLC system with 3 GPC PLgel3micrometer MIXED-E columns connected in series. The column temperature was $40^{\circ} \mathrm{C}$, with a flow of $1 \mathrm{ml} / \mathrm{min}$ and tetrahydrofuran (THF) was used as solvent. Calibration was carried out using polystyrene standards with a molecular weight ranging from 162 to $29510 \mathrm{~g} / \mathrm{mol}$. It should be taken into account that the separation mechanism of GPC is based on volume exclusion and not by weight and thus it serves only for mutual comparison between different samples. ${ }^{31}$

\subsection{Sample preparation for scanning electron microscopy (SEM)}

In SEM, wet samples such as microalgae (or solid residues) must be dried prior to analysis. We found that with a common air drying process using an oven, the cells appear completely flat and deflated. Therefore, other methods were tested to avoid the damaging effect of the surface tension forces of water while dehydrating the samples. The 
critical point drying (CPD) technique, as described below, was found to give excellent results.

First, a fixing liquid (Formalin, formaldehyde in water solution) was added to the algae cake. Formalin is commonly used as a fixative to preserve and fix tissue in microscopic slides. After washing out this liquid, the water present in the algae sample was replaced by ethanol by increasing stepwise the ethanol concentration. Once the algae were in a mixture with $100 \%$ ethanol, they were placed inside the critical point dryer (Baltsers $\mathrm{CPD}$ 030) where the ethanol was replaced for $\mathrm{CO}_{2}$ liquid at low temperature $\left(6^{\circ} \mathrm{C}\right)$ and high pressure (50 bar). After the removal of all the ethanol, the pressure chamber in the CPD was heated up to $40^{\circ} \mathrm{C}$, which forced the $\mathrm{CO}_{2}$ to become directly supercritical without evaporating. Finally, the $\mathrm{CO}_{2}$ was released leaving the cells in their intact status.

\subsection{Definitions}

As stated in the previous section, various products were obtained after HTT of Desmodesmus sp. and their yields ( $Y_{i}$ (d.a.f.), in wt\%) have been calculated as:

$\mathrm{Y}_{\mathrm{i}}($ d.a.f $)=\frac{\mathrm{m}_{\mathrm{i}}}{\mathrm{m}_{\text {algae }}} \cdot 100 \%$

where $i$ represents the oil, gas, solid residue or water soluble organics and $m_{i}$ the total mass of product.

The organic dry weight in the algal feed $\left(m_{\text {algae }}\right)$ was defined as:

$\mathrm{m}_{\text {algae }}=\mathrm{m}_{\text {algae solution feed }}-\mathrm{m}_{\text {moisture }}-\mathrm{m}_{\text {ash }}$

In Section 2.1, the determination of the total mass of moisture $\left(m_{\text {moisture }}\right)$ and ash $\left(m_{\text {ash }}\right)$ in the algal solution feed was described.

The amount of each gas produced (expressed in mol or g) was determined using the gas composition (obtained by GC analysis) and the final pressure and temperature inside the reactor (after cooling down) by the ideal gas law.

After elemental analysis of the oils, the weight percentages of carbon, hydrogen and oxygen were used to calculate the molar $H / C$ and $O / C$ ratios, and the higher heating value (HHV) using Boie's formula (Eq. 5). 
molar $\mathrm{H} / \mathrm{C}=\frac{\mathrm{H} w t \% \cdot \mathrm{Mw}_{\mathrm{C}}}{\mathrm{Cwt} \% \cdot \mathrm{Mw}_{\mathrm{H}}}$

molar $\mathrm{O} / \mathrm{C}=\frac{\mathrm{O} w t \% \cdot \mathrm{Mw}_{\mathrm{C}}}{\mathrm{Cwt} \% \cdot \mathrm{Mw}_{\mathrm{O}}}$

$\mathrm{HHV}_{\text {Boie }}=0.3516 \cdot \mathrm{C}+1.16225 \cdot \mathrm{H}-0.1109 \cdot \mathrm{O}+0.0628 \cdot \mathrm{N}$

To calculate the energy recovery (in \%) of the algae conversion process, the total energy content of the algal feedstock should be compared to the energy content of the oil product. Therefore, this value was calculated as follows:

Energy Recovery $(\%)=\frac{\mathrm{HHV}_{\mathrm{oil}} \cdot \operatorname{mass}_{\text {oil }}}{\mathrm{HHV}_{\text {algae feed (d.a.f.) }} \cdot \mathrm{mass}_{\text {algae feed (d.a.f.) }}} \cdot 100 \%$

It should be taken into account that the external work applied to the reaction system (such as e.g. energy for heating) is not included in this energy recovery value.

Finally, the nitrogen distribution between the product streams was determined as the ratio of their nitrogen mass to the initial nitrogen mass of the algal feed:

$\mathrm{Y}_{\mathrm{N}, \mathrm{i}}=\frac{\mathrm{m}_{\mathrm{N}, \mathrm{i}}}{\mathrm{m}_{\mathrm{N} \text { in algae feed }}} \cdot 100 \%$

The remaining fraction allowed estimation of the nitrogen content in the gaseous stream and in losses. The nitrogen in the water fraction of the feed (growth medium) was not included in this definition, but did not contribute to more than around $5 \%$ of the total nitrogen in the whole algal slurry.

\section{Results and discussion}

We investigated the influence of experimental temperature $\left(175-450^{\circ} \mathrm{C}\right)$ and reaction time (5-60 min) on the products distribution (yields) giving a closer look to the quality of the oil -in terms of elemental and molecular composition- and the behavior of the cells. A wide range of these two reaction parameters was applied in order to understand the process and identify the most suitable reaction conditions (towards maximum oil yield and improved quality). Additional HTT experiments $\left(200\right.$ and $300^{\circ} \mathrm{C}$ for 5 and $60 \mathrm{~min}$ ) 
using a slurry with higher dry algae concentrations (up to $17.4 \mathrm{wt} \%$ ) were performed. The increase in algae concentration did not show significant effects on the product distribution and their properties. This fact was also reported in other studies (e.g. by Jena et al. ${ }^{32}$ with algae concentrations ranging between 10 to $50 \mathrm{wt} \%$ ) and, hence, this parameter was not further investigated.

\subsection{Effect of temperature and reaction time}

\subsubsection{Products distribution}

Figure 4 shows the effect of temperature on the product yields on dry ash free algae basis (oil, water soluble organics, gas and solid residue) after HTT of Desmodesmus sp. from 175 to $450^{\circ} \mathrm{C}$ with two reaction times of 5 (Figure $4(\mathrm{a})$ ) and $60 \mathrm{~min}$ (Figure 4(b)). The effect of reaction time (excluding the heating time of 6-7 min to reach final temperature) on the product yields was also studied over the range of 5 to $60 \mathrm{~min}$ for two temperature series: $200^{\circ} \mathrm{C}$ (Figure $5(\mathrm{a})$ ) and $300^{\circ} \mathrm{C}$ min (Figure $5(\mathrm{~b})$ ). To our knowledge, there has been no previous study reporting reaction time influence at temperatures around $200^{\circ} \mathrm{C}$.

In Figures 4(a) and 4(b), the same trends were observed: an increase in temperature led to an increase in oil and gas yields and a decrease in water soluble organics and solid residue. The maximum oil production was reached at $375^{\circ} \mathrm{C}$ for both reaction times being slightly higher for the experiment performed over $5 \mathrm{~min}$ (49.4 wt\%). Therefore, by comparing these two series of experiments we conclude that the HTT batch process can be optimized to shorter reaction times (order of minutes) at temperatures higher than $300^{\circ} \mathrm{C}$. The maximum yield of water soluble organics was reached in the range between $225-250^{\circ} \mathrm{C}$ for both series of experiments.

The product yield trends from HTT of our microalgae species increasing temperature are similar to those reported by Anastasakis et al. ${ }^{33}$ and Zhou et al. ${ }^{24}$ for hydrothermal processing of macroalgae. The maximum oil yield in the studies by Zhou et al. ${ }^{24}$ and Brown et al. ${ }^{23}$ was, however, obtained at a temperature somewhat lower than $375^{\circ} \mathrm{C}$ $\left(300^{\circ} \mathrm{C}\right.$ with $30 \mathrm{~min}$ reaction time and $350^{\circ} \mathrm{C}$ with 60 min reaction time, respectively). In conclusion, the oil production showed a strong dependency on the liquefaction temperature in accordance with recent studies in literature. ${ }^{8,32,34}$ 

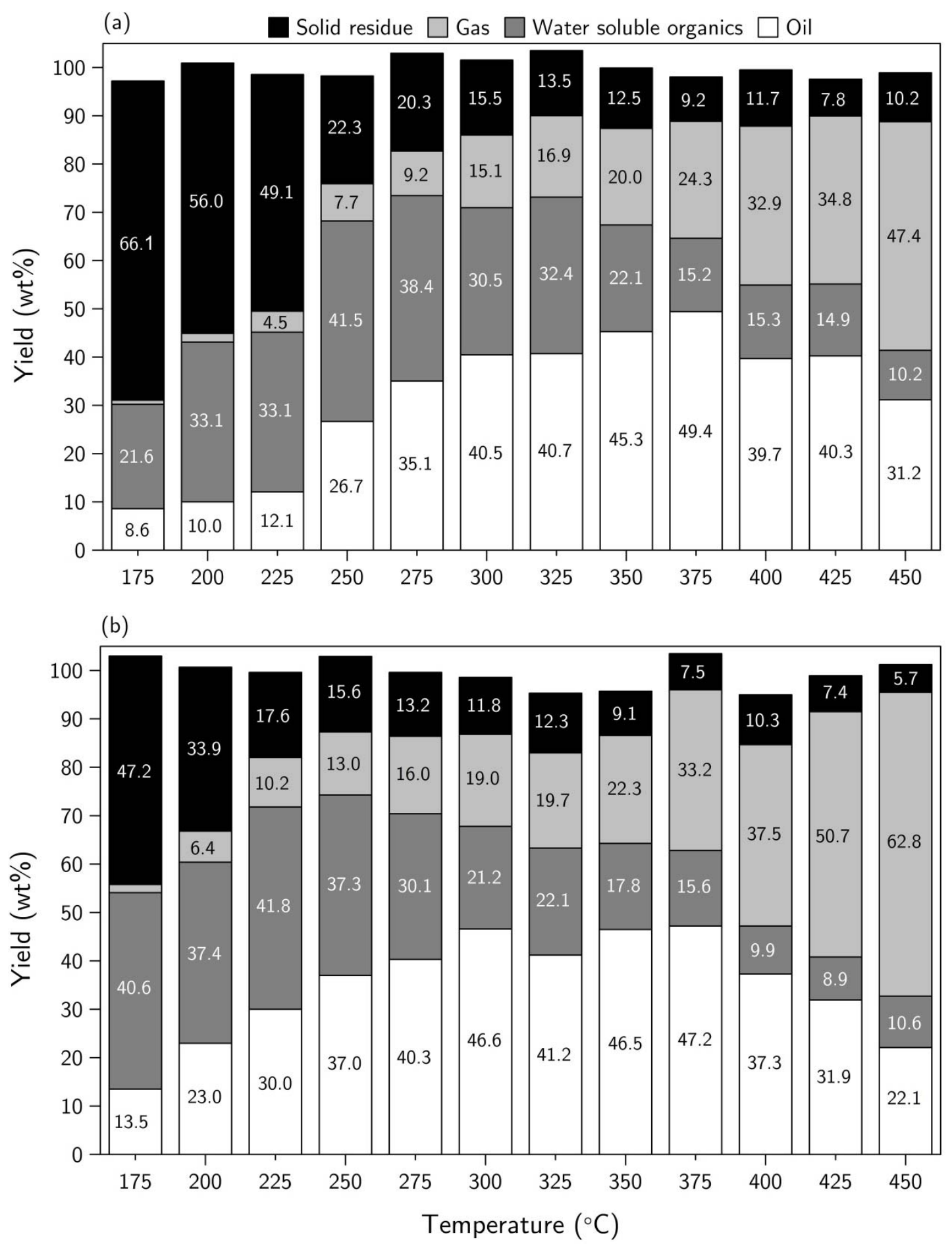

Figure 4. Effect of reaction temperature on the product yield distribution after HTT of Desmodesmus sp. for (a) 5 min reaction time and (b) 60 min reaction time

Generally, the oil yields obtained were higher than those reported in other investigations, even though the lipid content of the microalgae used in the present work is lower. As an example, the yield obtained from the experiment at $350^{\circ} \mathrm{C}$ and 60 min reaction time (47.2 wt\%) almost doubles the yields reported by Ross et al. ${ }^{10}$ adding $\mathrm{Na}_{2} \mathrm{CO}_{3}$ to the 
reaction mixture (27.3 wt\% for Chlorella vulgaris and $20.0 \mathrm{wt} \%$ for Spirulina); it is slightly higher than the values presented by Biller et al. ${ }^{35}$ (35.8 wt\% for Chlorella vulgaris and $34.3 \mathrm{wt} \%$ for Nannochloropsis oc.); and comparable to the yield obtained by Brown et al. ${ }^{23}$ (43 wt\% using Nannochloropsis sp.). All the yields reported in the previous studies were obtained applying the same reaction conditions $\left(350^{\circ} \mathrm{C}\right.$ and $60 \mathrm{~min}$ reaction time, with slight differences in algae concentration). Likely, the different biochemical composition of each type of microalgae was the reason for this broad variation, although we must also consider that the reactor system from each study can differ in heating rate and mixing mode, which could give an additional effect on the final product yield and distribution. Considering that the lipid based oil content of this microalgae strain is only $12.2 \mathrm{wt} \%$ (measured by B\&D extraction explained in Section 2.1), the oil will consist merely of compounds originating from the non-lipid fractions of the algal cell (such as carbohydrates, proteins and algaenan).
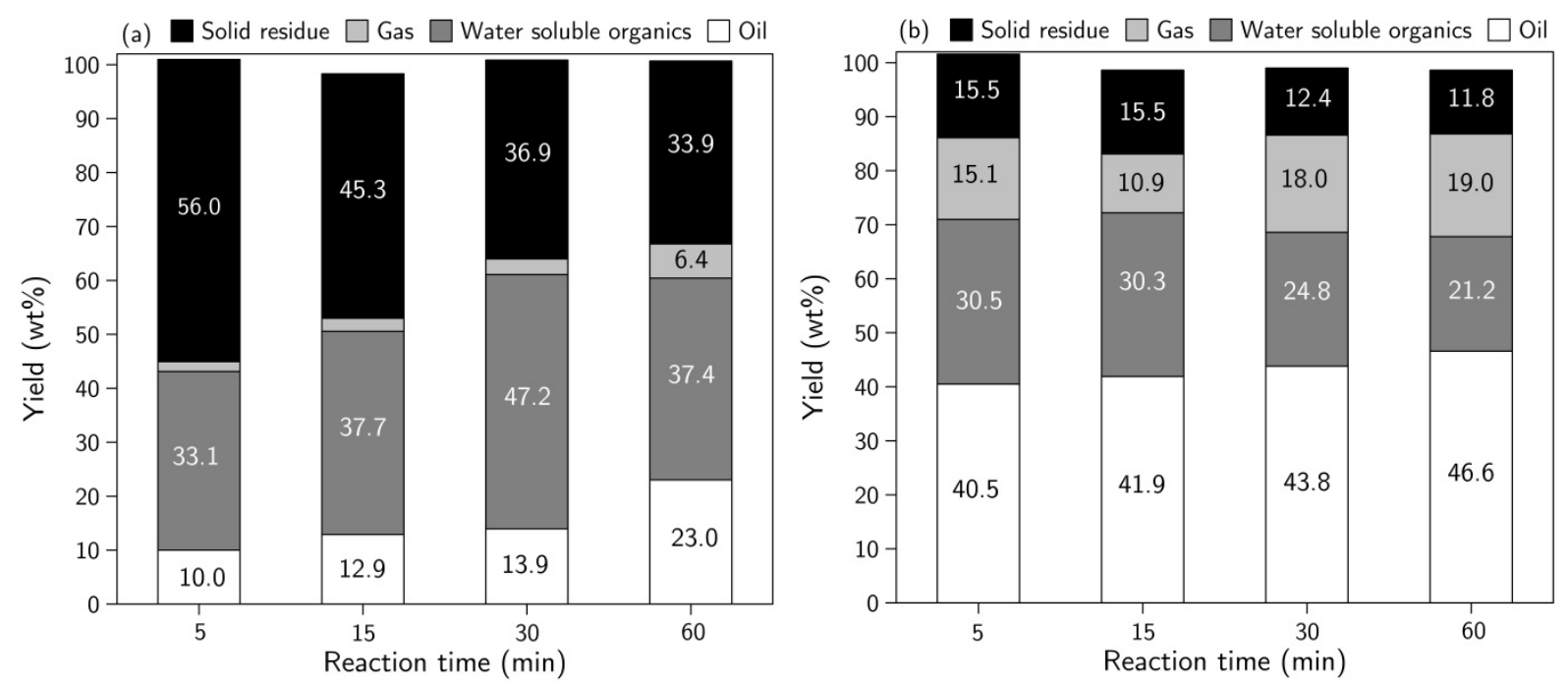

Figure 5. Effect of reaction time on the product yield distribution after HTT of Desmodesmus sp. at (a) $200^{\circ} \mathrm{C}$ and (b) $300^{\circ} \mathrm{C}$

As can be observed in Figures 4 and 5, the effect of reaction time on the product distribution is more pronounced at lower temperatures. From 175 to $275^{\circ} \mathrm{C}$ the difference between the products yields of 5 and $60 \mathrm{~min}$ was significantly larger than at higher temperatures. At temperatures above $275^{\circ} \mathrm{C}$, the product distribution does not change much after 5 min reaction time. Apparently, the initial HTT reactions of algae are fast at these temperatures, fact that was also observed by Knežević et al. ${ }^{13}$ after the thermal treatment of glucose, pyrolysis oil and wood. At increased reaction severity (high temperature or/and long reaction time) more water soluble organics were converted ending into the oil or gas. Above $375^{\circ} \mathrm{C}$, the oil yield even started decreasing mainly due 
to the further decomposition of unstable oil molecules towards gaseous products and, possibly, to some extent polymerization of intermediates to high molecular weight compounds remaining in the solid residue. On basis of our data, polymerization cannot be confirmed, however, earlier studies proved that polymerization of sugars (compounds also present in algae) commonly occurs during subcritical hydrothermal processing. ${ }^{13}$

In several publications $\mathrm{s}^{8,24,36,37}$ biomass liquefaction is described as:

1) Hydrolysis of biomass macromolecules (lipids, proteins and carbohydrates in case of algal biomass) into smaller fragments;

2) Conversion of these fragments by e.g. dehydration into other, smaller compounds;

3) Rearrangement via condensation, cyclisation and polymerization producing new components.

It should be noticed that some of the above mentioned liquefaction mechanisms from literature were reported for experiments in which woody biomass was used as feedstock. The different biochemical composition of the microalgal biomass, compared to that of terrestrial biomass in other studies, may play a role here.

The yield of each product depends on the reaction conditions, influencing the competition between depolymerization, cracking and repolymerization reactions (resulting in larger molecules and aggregates). As suggested by Yuan et al., ${ }^{37}$ after investigating the process of straw liquefaction, hydrolysis dominates during low temperature liquefaction (below $220^{\circ} \mathrm{C}$ ) and after that, the competition between hydrolysis and repolymerization starts when the temperature ranges between 220 and $300^{\circ} \mathrm{C}$. Finally, repolymerization becomes predominant with a further increase in temperature, increasing the oil and gas production.

Similar trends could be hypothesized from our results by observing Figure 4 . From $175^{\circ} \mathrm{C}$ to around $225-250^{\circ} \mathrm{C}$ and short reaction time hydrolysis into smaller fragments (soluble in water) dominated, as indicated by the increase in water soluble organics and the low oil yields obtained. In the range of $250-375^{\circ} \mathrm{C}$ oil production increased and reached a maximum, while the organic content in water decreased. This might indicate the promotion of oil-forming reactions converting water soluble products into oil and gas together with the release of oily compounds from the cells. From $375^{\circ} \mathrm{C}$, the oil yield decreased again, while significantly more gas was produced. The change in product spectrum with temperature as found in this study may be partially related to the 
changing properties of (hot compressed-) water with increasing temperature. ${ }^{38}$ The transition to supercritical water conditions might promote the radical-induced cracking reactions in this regime. On the other hand, it is however not clear if the three main HTT reactions (hydrolysis, cracking and polymerization) are taking place consecutively or in parallel and neither at which stage the molecules that configure the oil matrix are formed.

\subsubsection{Oil composition}

Table 3 shows the elemental composition and higher heating value (HHV) of the oils obtained after HTT of Desmodesmus sp. at each temperature over the two different reaction times (5 and $60 \mathrm{~min}$ ). In addition, the molar $O / C$ and $H / C$ ratios and the percentage of energy recovery in the oil are shown. The HHV of the oil obtained after $\mathrm{HTT}$ at $375^{\circ} \mathrm{C}$ and 5 min reaction times was experimentally measured using a bomb calorimeter. The value obtained was $36.0 \mathrm{MJ} / \mathrm{kg}$, close to the calculated value by Boie's formula $(35.4 \mathrm{MJ} / \mathrm{kg})$. This equation was found to be suitable for several different samples and hence the heating value for the rest of the oils was calculated using that expression. Table 4 shows the same features as in Table 3, but for the case of reaction time effect at two different temperatures $\left(200\right.$ and $\left.300^{\circ} \mathrm{C}\right)$.

Table 3. Oil elemental composition and properties after HTT of Desmodesmus sp. increasing temperature over $5 \mathrm{~min}$ and $60 \mathrm{~min}$ reaction time

\begin{tabular}{|c|c|c|c|c|c|c|c|c|c|c|c|c|c|c|c|c|}
\hline \multirow[t]{2}{*}{$\begin{array}{c}\mathrm{T} \\
\left({ }^{\circ} \mathrm{C}\right)\end{array}$} & \multicolumn{2}{|c|}{$\begin{array}{c}\mathrm{N} \\
(\mathrm{wt} \%)\end{array}$} & \multicolumn{2}{|c|}{$\begin{array}{c}\mathrm{C} \\
(\mathrm{wt} \%)\end{array}$} & \multicolumn{2}{|c|}{$\begin{array}{c}\mathrm{H} \\
(\mathrm{wt} \%)\end{array}$} & \multicolumn{2}{|c|}{$\begin{array}{c}\mathrm{O} \\
(\mathrm{wt} \%)\end{array}$} & \multicolumn{2}{|c|}{$\begin{array}{c}\text { HHV } \\
(\mathrm{MJ} / \mathrm{kg})\end{array}$} & \multicolumn{2}{|c|}{$\begin{array}{c}\text { Molar } \\
\mathrm{O} / \mathrm{C}\end{array}$} & \multicolumn{2}{|c|}{$\begin{array}{c}\text { Molar } \\
\text { H/C }\end{array}$} & \multicolumn{2}{|c|}{$\begin{array}{c}\text { Energy } \\
\text { recovery } \\
(\%)\end{array}$} \\
\hline & 5 & 60 & 5 & 60 & 5 & 60 & 5 & 60 & 5 & 60 & 5 & 60 & 5 & 60 & 5 & 60 \\
\hline 175 & 0.4 & 1.0 & 64.2 & 68.1 & 9.0 & 9.2 & 26.5 & 21.7 & 30.1 & 32.3 & 0.31 & 0.24 & 1.69 & 1.62 & 11 & 19 \\
\hline 200 & 1.6 & 3.7 & 67.1 & 69.2 & 9.1 & 9.6 & 22.2 & 17.5 & 31.8 & 33.7 & 0.25 & 0.19 & 1.63 & 1.66 & 14 & 33 \\
\hline 225 & 3.5 & 4.8 & 73.2 & 70.6 & 9.7 & 9.2 & 13.6 & 15.4 & 35.7 & 34.1 & 0.14 & 0.16 & 1.58 & 1.56 & 18 & 44 \\
\hline 250 & 4.1 & 5.9 & 71.5 & 72.8 & 8.9 & 8.9 & 15.4 & 12.4 & 34.0 & 34.9 & 0.16 & 0.13 & 1.49 & 1.46 & 39 & 55 \\
\hline 275 & 5.7 & 6.0 & 71.3 & 73.9 & 8.6 & 8.8 & 14.3 & 11.3 & 33.9 & 35.3 & 0.15 & 0.11 & 1.45 & 1.43 & 51 & 61 \\
\hline 300 & 6.3 & 6.0 & 72.4 & 75.0 & 9.0 & 8.8 & 12.3 & 10.2 & 34.9 & 35.8 & 0.13 & 0.10 & 1.49 & 1.40 & 60 & 71 \\
\hline 325 & 6.3 & 6.0 & 72.9 & 74.4 & 8.6 & 8.8 & 12.2 & 10.8 & 34.6 & 35.6 & 0.13 & 0.11 & 1.42 & 1.42 & 60 & 62 \\
\hline 350 & 6.2 & 6.0 & 73.1 & 75.8 & 8.6 & 9.1 & 12.1 & 9.1 & 34.8 & 36.6 & 0.12 & 0.09 & 1.41 & 1.43 & 67 & 73 \\
\hline 375 & 6.3 & 5.7 & 74.5 & 75.4 & 8.6 & 8.9 & 10.5 & 9.9 & 35.4 & 36.2 & 0.11 & 0.10 & 1.39 & 1.42 & 75 & 73 \\
\hline 400 & 5.8 & 5.5 & 74.5 & 76.5 & 9.0 & 9.1 & 10.7 & 8.9 & 35.8 & 36.9 & 0.11 & 0.09 & 1.44 & 1.43 & 61 & 59 \\
\hline 425 & 6.4 & 5.9 & 74.2 & 75.3 & 8.8 & 8.4 & 10.6 & 10.5 & 35.5 & 35.4 & 0.11 & 0.10 & 1.42 & 1.34 & 61 & 48 \\
\hline 450 & 6.5 & 6.8 & 75.5 & 75.7 & 8.6 & 7.5 & 9.4 & 9.9 & 35.9 & 34.7 & 0.09 & 0.10 & 1.37 & 1.19 & 48 & 33 \\
\hline
\end{tabular}


In Table 3, we can observe that the composition of the oil varied similarly with temperature for both reaction time series. Increasing temperature led to an increase in carbon content (from $64.2 \mathrm{wt} \%$ up to $75.5 \mathrm{wt} \%$ with 5 min reaction time) resulting in higher heating values (increasing from around 30 to around $36 \mathrm{MJ} / \mathrm{kg}$ ). The maximum energy recovery into oil of $75 \%$ was obtained at $375^{\circ} \mathrm{C}$ for 5 min reaction time with the following carbon distribution ( $\mathrm{C}$ in product / $\mathrm{C}$ in dry ash free feedstock): $70.9 \mathrm{wt} \%$ in oil, $13.2 \mathrm{wt} \%$ in gas, $4.7 \mathrm{wt} \%$ in solid residue and $11.2 \mathrm{wt} \%$ in aqueous fraction (estimated by difference). The hydrogen content was nearly constant over the temperature range and decreased when reaching $450^{\circ} \mathrm{C}$. A remarkable increase in nitrogen content was observed from 175 to $275^{\circ} \mathrm{C}$ and thereafter it maintained constant at around $6 \mathrm{wt} \%$. This nitrogen content variation was more pronounced than reported in other studies. ${ }^{23,32}$ Finally, the oxygen content decreased significantly with increasing temperature (more pronounced with the $60 \mathrm{~min}$ reaction time) showing that deoxygenation occurs. The carbon and hydrogen contents of the oil obtained were significantly higher than that of the original dry algal biomass and with the continuous drop of oxygen level in the oil by increasing temperature, HTT resulted in a significant energy densification from $22 \mathrm{MJ} / \mathrm{kg}$ for the algal biomass to around $36 \mathrm{MJ} / \mathrm{kg}$ for the oil obtained. The molar $O / C$ and $H / C$ ratios of the algal oils were close to that of biodiesel (FAME and FAEE) and heavy crude oil. Furthermore, a reduction in the H/C ratio increasing temperature was also observed, likely with increasing content of aromatic compounds in the oil.

Table 4. Oil elemental composition and properties after HTT of Desmodesmus sp. with increasing reaction time at $200^{\circ} \mathrm{C}$ and $300^{\circ} \mathrm{C}$

\begin{tabular}{ccccccccc}
\hline \multicolumn{7}{c}{ Temperature $\mathbf{2 0 0}^{\circ} \mathbf{C}$} \\
\hline $\begin{array}{c}\text { R. t. } \\
(\mathbf{m i n})\end{array}$ & $\begin{array}{c}\mathbf{N} \\
(\mathbf{w t} \%)\end{array}$ & $\begin{array}{c}\mathbf{C} \\
(\mathbf{w t} \%)\end{array}$ & $\begin{array}{c}\mathbf{H} \\
(\mathbf{w t} \%)\end{array}$ & $\begin{array}{c}\mathbf{O} \\
(\mathbf{w t} \%)\end{array}$ & $\begin{array}{c}\mathbf{H H V} \\
(\mathbf{M J} / \mathbf{k g})\end{array}$ & $\begin{array}{c}\text { Molar } \\
\mathbf{O} / \mathbf{C}\end{array}$ & $\begin{array}{c}\text { Molar } \\
\mathbf{H} / \mathbf{C}\end{array}$ & $\begin{array}{c}\text { Energy } \\
\text { recovery }\end{array}$ \\
\hline $\mathbf{5}$ & 1.6 & 67.1 & 9.1 & 22.2 & 31.8 & 0.25 & 1.63 & 13.6 \\
$\mathbf{1 5}$ & 2.1 & 66.6 & 8.6 & 22.8 & 31.0 & 0.26 & 1.55 & 17.0 \\
$\mathbf{3 0}$ & 2.0 & 68.1 & 9.5 & 20.5 & 32.8 & 0.23 & 1.67 & 19.5 \\
$\mathbf{6 0}$ & 3.7 & 69.2 & 9.6 & 17.5 & 33.7 & 0.19 & 1.66 & 33.1 \\
\hline & & & \multicolumn{7}{c}{ Temperature $\mathbf{3 0 0}{ }^{\circ} \mathbf{C}$} & & & \\
\hline $\mathbf{5}$ & 6.3 & 72.4 & 9.0 & 12.3 & 34.9 & 0.13 & 1.49 & 60.3 \\
$\mathbf{1 5}$ & 6.0 & 72.9 & 9.0 & 12.0 & 35.2 & 0.12 & 1.48 & 62.8 \\
$\mathbf{3 0}$ & 5.9 & 73.5 & 8.9 & 11.7 & 35.2 & 0.12 & 1.45 & 65.8 \\
$\mathbf{6 0}$ & 6.0 & 75.0 & 8.8 & 10.2 & 35.8 & 0.10 & 1.40 & 71.2 \\
\hline
\end{tabular}


In Table 4, as observed with the product distribution results, increasing the reaction time had more effect on the oil composition and its heating value at $200^{\circ} \mathrm{C}$ in comparison with the $300^{\circ} \mathrm{C}$ series. Noticeable is the nitrogen content of the $200^{\circ} \mathrm{C}$ oil, which showed a remarkable rise from 1.6 to $3.7 \mathrm{wt} \%$, particularly from 30 to $60 \mathrm{~min}$, while at $300^{\circ} \mathrm{C}$ it was already at high level after 5 minutes. Oil deoxygenation was also achieved by increasing reaction time.

The high nitrogen content of the oil produced (mainly due to the large protein content of the aquatic organism used) is an important drawback in view of fuel applications, as undesirable $\mathrm{NO}_{\mathrm{x}}$ emissions will occur during combustion. For co-feeding of HTT algal oil to existing crude oil-based refineries, for the production of transportation fuels, the high nitrogen content may also be prohibitive. Similarly, some deoxygenation might be necessary, although recent work by de Miguel Mercader et al. ${ }^{39}$ showed that full deoxygenation is not required for successful co-processing of hydrodeoxygenated pyrolysis oil along with a heavy oil petroleum fraction in a lab scale simulated FCC unit. Pyrolysis oil from terrestrial biomass has the advantage over algal oil obtained by HTT, that it contains a very low fraction of nitrogen (e.g. $<0.1$ wt\% using pine wood $^{40}$ ). However, the energy density of the HTT algal oil can be twice as high as that of pyrolysis oil (14-24 $\mathrm{MJ} / \mathrm{kg}$ using pine wood $\left.^{41}\right)$ and more close to that of petroleum crude oil $(42 \mathrm{MJ} / \mathrm{kg})$.

Table 5. Elemental composition of oils obtained after HTT at the same reaction conditions $\left(350^{\circ} \mathrm{C}\right.$ and 60 min reaction time) using different microalgal feedstock

\begin{tabular}{|c|c|c|c|c|c|c|c|}
\hline Reference & Algae & $\begin{array}{c}\mathrm{N} \\
(\mathrm{wt} \%) \\
\end{array}$ & $\begin{array}{c}\mathrm{C} \\
(\mathrm{wt} \%) \\
\end{array}$ & $\begin{array}{c}\mathrm{H} \\
(\mathrm{wt} \%) \\
\end{array}$ & $\begin{array}{c}\mathrm{O} \\
(\mathrm{wt} \%) \\
\end{array}$ & $\begin{array}{c}\mathrm{S} \\
(\mathrm{wt} \%) \\
\end{array}$ & $\begin{array}{c}\text { HHV } \\
(\mathrm{MJ} / \mathrm{kg})\end{array}$ \\
\hline Present work & Desmodesmus sp. & 6.0 & 75.8 & 9.1 & 9.1 & n.d. ${ }^{a}$ & $36.6^{\mathrm{b}}$ \\
\hline Brown $2010^{23}$ & Nannochloropsis sp. & 3.9 & 76.0 & 10.3 & 9.0 & 0.9 & 39.0 \\
\hline \multirow[t]{2}{*}{ Biller $2010^{18}$} & $\begin{array}{c}\text { Porphyridium } \\
\text { cruentum }\end{array}$ & 5.4 & 72.8 & 8.5 & 13.3 & 0.3 & 35.7 \\
\hline & Spirulina & 7.0 & 73.3 & 9.2 & 10.4 & 0.0 & 36.8 \\
\hline Duan $2010^{19}$ & Nannochloropsis sp. & 4.2 & 75.3 & 10.2 & 9.2 & 0.8 & 38.5 \\
\hline \multirow[t]{2}{*}{ Biller $2011^{35}$} & $\begin{array}{c}\text { Nannochloropsis } \\
\text { oculata }\end{array}$ & 4.1 & 68.1 & 8.8 & 18.9 & 0.0 & 34.5 \\
\hline & Chlorella vulgaris & 5.9 & 70.7 & 8.6 & 14.8 & 0.0 & 35.1 \\
\hline
\end{tabular}

${ }^{a}$ Not determined. ${ }^{\text {b }}$ Calculated with Boie's formula

In view of the oil upgrading methods, especially for denitrogenation, a preliminary identification of the oil chemical nature and the nitrogen containing compounds in the oil is required. An additional complexity was found, as, by contrasting various studies, the 
influence of the type of microalgal feedstock on the composition of the oil became evident. Oils produced after thermal processing of different microalgae species at $350^{\circ} \mathrm{C}$ and 60 min reaction time in absence of a catalyst were compared (see Table 5) and a considerable variation in the nitrogen content could be observed. Spirulina, having the highest protein content ${ }^{18}$ (65 d.a.f. \%), showed also the highest nitrogen content in the oil.

\subsubsection{Aqueous phase, solid residue and gas analyses}

As previously mentioned, a constant decrease of the water soluble organics was noticed with increasing temperature. This was clearly visible when observing the color of the aqueous phases recovered from the various temperature treatments. At higher temperatures, transparency increased and gradually the solution became lighter in color suggesting the conversion of the dissolved organics into gaseous and oil products. Identification, on a molecular level, of these organic compounds soluble in water would provide more information about the reaction mechanisms occurring. Zhou et al. ${ }^{24}$ (processing macroalgae) found acetic acid as the most abundant compound in the aqueous phase followed by glycerol, suggesting its formation from hydrolysis of lipids during HTT. They also detected several organic nitrogen-containing compounds. Other studies $^{10,32}$ reported the presence of acetates and formates (likely from carbohydrates hydrolysis). The amounts of these organic constituents varied per study, pointing out once again the influence of the type of algal biomass used. Spectrophotometry analyses of the aqueous phases were performed in order to evaluate the potential of nutrients recycling for further cultivation of algae. The thermal treatment of microalgae led to the formation of $\mathrm{NH}_{4}^{+}$(dissolved in the aqueous phase), which was not present as nitrogen nutrient in the initial growth medium (containing mainly $\mathrm{NO}_{3}{ }^{-}$and $\mathrm{PO}_{4}{ }^{3-}$ ) prior to HTT. Ammonium was most likely formed by thermal decomposition of proteins yielding initially amino acids which were further hydrolyzed (deamination) producing ammonia and after accepting a proton from water it produced $\mathrm{NH}_{4}{ }^{+}$and $\mathrm{OH}^{-}$. The formation of ammonium was previously reported in other investigations. ${ }^{25,26}$ The $\mathrm{NH}_{4}{ }^{+}$concentration increased with increasing temperature while $\mathrm{NO}_{3}{ }^{-}$decreased but to a lesser extent. The

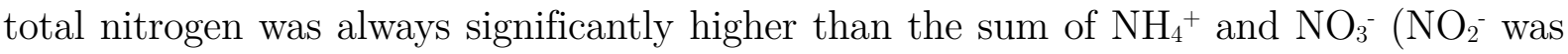
below detection limits) indicating the presence and formation of organic nitrogen containing compounds being the major constituents in the aqueous fraction together with $\mathrm{NH}_{4}{ }^{+}$. After HTT, all the phosphorous in the aqueous phase was present in the form of $\mathrm{PO}_{4}^{3-}$, as evidenced by total phosphorous and $\mathrm{PO}_{4}{ }^{3-}$ determination being nearly equal. However, an important decline in the phosphorous recovery via the aqueous phase with 
increasing HTT temperature was noticed. Depending on the possibilities to recover phosphorus from the other product fractions (solid residue and oil phase), an appropriate HTT operating temperature must be selected in order to minimize losses of phosphorus. In conclusion, the basic inorganic $\mathrm{N}$ and $\mathrm{P}$ nutrients necessary for Desmodesmus sp. growth were found in the aqueous product phase. In addition, organic carbon and nitrogen compounds were also found, which could be beneficial (as carbon source for heterotrophic growth) but could also adversely inhibit growth. Also some toxic metals (e.g. $\mathrm{Ni}$ and $\mathrm{Cr}$ ) from the reactor wall could be dissolved in the aqueous phase leading to the same inhibitory effect. Preliminary results from growth studies with the algae species used in this work, and using recycled HTT aqueous phase are very promising and will be published in the near future.

From the elemental analysis results, we could observe a steady decrease of carbon (5 min: 48.9-19.3 wt\%; $60 \mathrm{~min}$ : 49.9-19.4 wt\%), nitrogen (5 min: 6.3-1.3 wt\%; $60 \mathrm{~min}: 7.1-1.8$ wt\%) and hydrogen (5 min: 7.0-1.8 wt\%; $60 \mathrm{~min}$ : 6.8-1.2 wt\%) contents in the solid residue with increasing temperature. The fraction estimated by difference (100-C-H-N), which could be oxygen but also other products such as ashes, increased (5 min: 37.7-77.6 wt\%; $60 \mathrm{~min}$ : 36.3-77.6 wt\%). At low temperatures (175 and $\left.200^{\circ} \mathrm{C}\right)$, the elemental composition and HHV of the solid residues were close to that of the initial algal feedstock (in Table 2).

The main compound in the gas phase was $\mathrm{CO}_{2}$ (99.7-66.9 mol\% with 5 min and 98.5-58.1 mol\% with 60 min reaction time), the amount of which remained nearly constant from 175 to $350^{\circ} \mathrm{C}$ and from there it started decreasing while small hydrocarbons $\left(\mathrm{CH}_{4}\right.$ and $\mathrm{C}_{2}-$ $\mathrm{C}_{3}$ ) and $\mathrm{H}_{2}$ began to increase significantly. Very small amounts of $\mathrm{CO}$ and $\mathrm{H}_{2}$ were formed suggesting that deoxygenation occurs mainly via decarboxylation rather than by decarbonylation. Besides the gaseous carbon, earlier studies also identified nitrogen containing compounds in the gas fraction. Duan et al. ${ }^{19}$ reported the presence of $\mathrm{N}_{2}$ (excluding its origin from residual air) suggesting its formation from ammonia decomposition. Components such as $\mathrm{NO}_{2}, \mathrm{~N}_{2} \mathrm{O}, \mathrm{HCN}$ and $\mathrm{NH}_{3}$ were found after FTIR analysis of the gas product in the work by Ross et al., ${ }^{10}$ although a catalyst was used in those experiments. The GC equipment used in this work was able to measure $\mathrm{N}_{2}$, but we could not distinguish if its origin was from traces of air or from the algal biomass itself. Therefore, the fraction of nitrogen ending in the gas was estimated from an elemental balance with the nitrogen content of the oil, aqueous phase and solid phase products (calculation method can be found in Section 2.5). However, the total nitrogen test (see Section 2.3.) employed for the aqueous phase may give an underestimated value of 
nitrogen content. It is known that this oxidative method may not be able to completely digest all organic $\mathrm{N}$ compounds (e.g. pyridine) and would thus yield lower values for the nitrogen content of the aqueous phase.

Figure 6 shows the nitrogen distribution over the product phases with increasing HTT reaction temperature. As observed in other investigations ${ }^{10,18}$ reporting a nitrogen balance, a significant fraction of nitrogen ended up in the gas phase and this fraction seemed to increase with temperature.

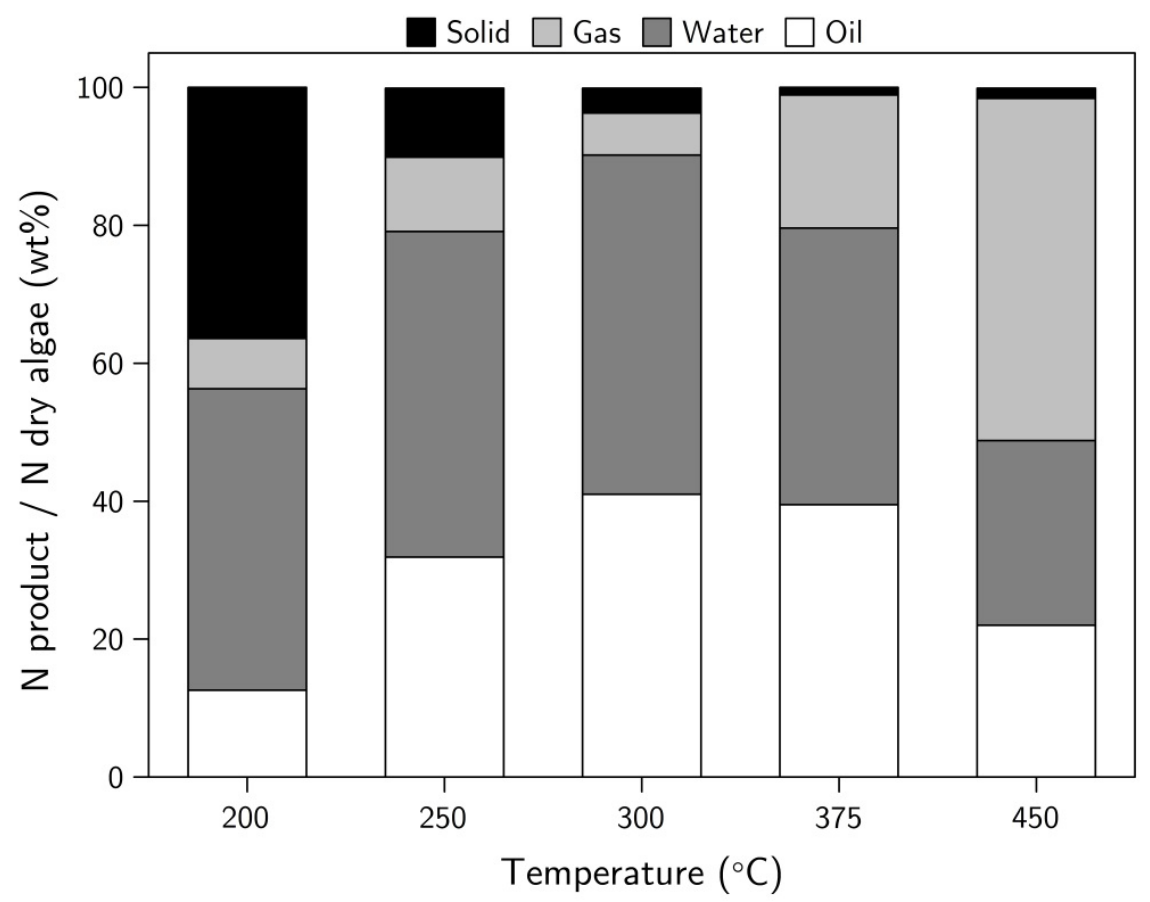

Figure 6. Effect of reaction temperature on the nitrogen distribution between the products obtained after HTT of Desmodesmus sp. with 60 min reaction time

For the experiments changing reaction time (Figure 5), analyses of the other product fractions (gas, solid residue and aqueous phase) were also performed. In general, when the reaction time for $\mathrm{HTT}$ at $200^{\circ} \mathrm{C}$ was increased, the properties of the oil and the other product fractions varied in similar manner as when the temperature was raised at a constant reaction time.

\subsubsection{Algae cell observations after hydrothermal treatment}

As can be seen in Figure 4, the most remarkable increase in oil yield and decrease in solid residue occurred in the temperature range from 225 to $250^{\circ} \mathrm{C}$ for the 5 min reaction time series (49.1-22.3 wt\% and 12.1-26.7 wt\% respectively). It was anticipated that this could be related to a change in the morphology of the algal cells, considering that 
intracellular compounds may be more accessible if the cell wall is broken, which may have increased the overall oil yield. At the same time, if the cell wall material itself is thermally degraded into oil, the total yield would increase even more. However, such an abrupt change was not observed for the $60 \mathrm{~min}$ reaction time series. Therefore, SEM analysis were performed (Figure 7) to visually inspect the cells in the solid residue after HTT with increasing temperature in order to investigate the possible link between yields and cell structure.

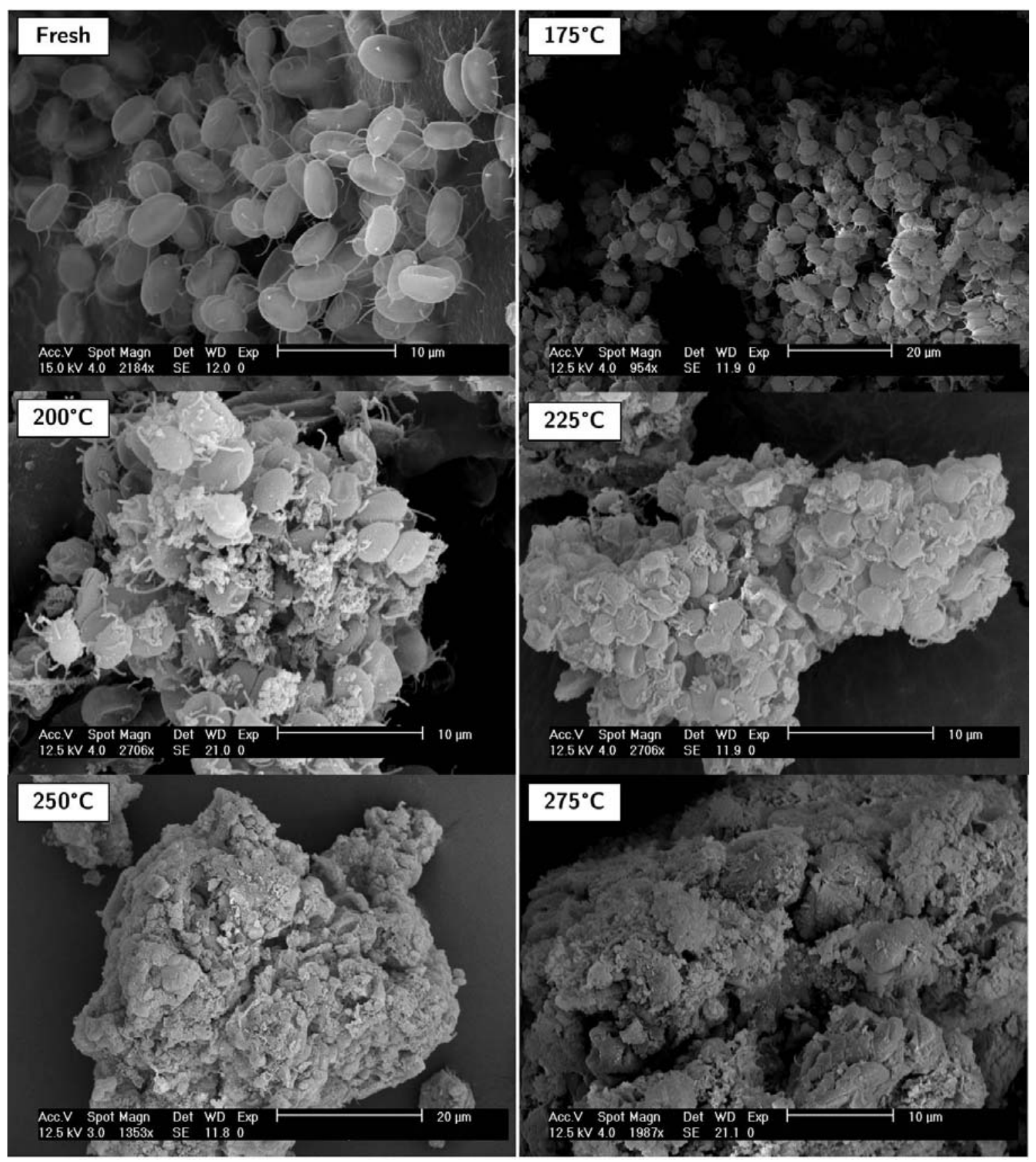

Figure 7. Effect of reaction temperature on the algal cells after HTT of Desmodesmus sp. for 5 min reaction time 
These micrographs clearly showed that no major cell wall breakage took place below $250^{\circ} \mathrm{C}$ and 5 min reaction time, since the cell wall remained visually intact, confirming the strong resistance of Desmodesmus sp. physical structure to temperature. After HTT at $175^{\circ} \mathrm{C}$, the cells appeared unbroken and their appearance was similar to that of the fresh microalgae. Cell wall breakage was not achieved after $\mathrm{HTT}$ at $200^{\circ} \mathrm{C}$, although the presence of some type of residue/precipitate was visible on the outer surface of the cells. This solid material could be debris from a minor decomposition of the cell walls regarding the slight decrease in solid yield from 175 to $200^{\circ} \mathrm{C}$, or intracellular compounds released from the cells. HTT at $225^{\circ} \mathrm{C}$ led to a strong cell clustering effect where the cells appeared deformed and closely packed with again some solid residue detected on them. Finally, while the cell wall was still visually unbroken after $\mathrm{HTT}$ at $225^{\circ} \mathrm{C}$, the following increase in temperature to $250^{\circ} \mathrm{C}$ led to a major breakage of the cells. Above $250^{\circ} \mathrm{C}$, no individual cells were recognizable and only a compact mass of cells debris was visible. In conclusion, the significant difference between the product yields from 225 to $250^{\circ} \mathrm{C}$ coincided with the breakage of the cells occurring in that temperature range with 5 min reaction time.

A possible explanation for the cells clustering and adhesion observed after $\mathrm{HTT}$ at $225^{\circ} \mathrm{C}$ could be the result of precipitation or coagulation of released proteins by their thermal denaturation during the whole heating process. Zepka et al. ${ }^{42}$ studied the influence of drying conditions on the biochemical composition of algal biomass and reported that such a protein degradation process could be expected. Denaturation can be promoted by various agents such as heat, $\mathrm{pH}$, salts and surface effects. Denaturation in microalgae can also lead to the formation of viscous gels by applying heat. ${ }^{43}$ These gels could promote the adhesion of the cells. Another possibility for the observed clustering could be the occurrence of Maillard reaction producing brown nitrogenous polymers or melanoidins. Peterson et al. ${ }^{44}$ reported system plugging problems caused by this product during hydrothermal processing at $250^{\circ} \mathrm{C}$ of glucose, as a model carbohydrate, and glycine, as model protein of biomass. Finally, aggregation of the cells could have happened since their polar cell wall is surrounded by water that behaves like an organic non polar solvent due to the lowering in dielectric constant at those reaction conditions.

Nevertheless, the high stability of the cell wall over temperature is the most noteworthy feature of Desmodesmus sp. This thermal resistance could be related to the presence of certain biomacromolecules, termed algaenans, in the cell wall of many algae from the Chlorophyta division (green algae) such as Desmodesmus sp. The building blocks of algaenans consist of linear $\mathrm{C}_{22}$ to $\mathrm{C}_{34}$ even numbered carbon chains with functional 
groups cross-linking the monomers with ether and ester bonds. ${ }^{45}$ Salmon et al. ${ }^{46}$ reported a very low algaenan (isolated from Botryococcus Braunii) conversion (3-9\%) by means of heating from 200 to $300^{\circ} \mathrm{C}$ and $9 \mathrm{~h}$ reaction time. These insoluble, non-hydrolysable biopolymers seem to have a particular resistance to temperature.

A related question arose when noticing the unbreakable behaviour of the cells wall below $250^{\circ} \mathrm{C}$ : Do the intracellular constituents remain intact during HTT at those operating conditions? To answer that question, first the oil yield and elemental composition of the oils obtained after HTT at 175,200 and $225^{\circ} \mathrm{C}$ were compared to that of the oils obtained from two different solvent extractions: the common Bligh and Dyer (B\&D) extraction method (described in Section 2.1) without the washing step with aqueous $\mathrm{Na}_{2} \mathrm{SO}_{4}$ solution; and a dichloromethane extraction (DCM) using the same amount of solvent as for the recovery of the products after HTT (in total $70 \mathrm{ml}$ ) and applying the same product recovery procedure (Figure 3). Both room temperature extractions were applied to the same amount of algae solution $(\sim 20 \mathrm{~g})$ with a dry algae concentration of 7 $8 \mathrm{wt} \%$, as used in the HTT experiments. The results are shown in Table 6 .

Table 6. Yield and elemental analysis of oils obtained after Bligh and Dyer extraction (B\&D), dichloromethane extraction (DCM) and HTT at 175,200 and $225^{\circ} \mathrm{C}$ with 5 min reaction time

\begin{tabular}{cccccc}
\hline Experiment & Oil yield (wt\%) & $\mathbf{N}(\mathbf{w t} \%)$ & $\mathbf{C ~ ( w t \% )}$ & $\mathbf{H ~ ( w t \% )}$ & O (wt\%) \\
\hline $\mathrm{B} \& \mathrm{D}$ & 16.5 & 1.4 & 74.0 & 9.8 & 14.7 \\
$\mathrm{DCM}$ & 7.1 & 0.8 & 75.5 & 10.5 & 13.3 \\
$175^{\circ} \mathrm{C}, 5 \mathrm{~min}$ & 8.6 & 0.4 & 64.2 & 9.0 & 26.5 \\
$200^{\circ} \mathrm{C}, 5 \mathrm{~min}$ & 10.0 & 1.6 & 67.1 & 9.1 & 22.2 \\
$225^{\circ} \mathrm{C}, 5 \mathrm{~min}$ & 12.5 & 3.5 & 73.2 & 9.7 & 13.6 \\
\hline
\end{tabular}

The first aspect to highlight is that it was possible to obtain an oily product extracted with the use of only DCM as solvent. This indicates that the oil obtained after HTT might be a mixture of compounds obtained from the hydrothermal processing and other compounds extracted by the DCM used afterwards for product recovery purposes from the reactor. The same phenomenon was observed by Brown et al. ${ }^{23}$ On the other hand, slight differences between yields of solvent extracted oils and HTT oils at low temperatures became apparent as well as in elemental composition particularly for the nitrogen and carbon content. Therefore, GPC analysis of the extracted and HTT oils were performed in order to compare their molecular weight distribution. The results are represented in Figure 8 where the diagrams were cut at low molecular weight $(\sim 100$ $\mathrm{g} / \mathrm{mol}$ ) to remove the peak corresponding to the degradation products of the GPC-eluent 
THF. The differential distribution $d_{\text {total mass }} / d_{\log \text { molar mass }}(W(\log M))$ is plotted versus the molar mass $(\mathrm{M})$.

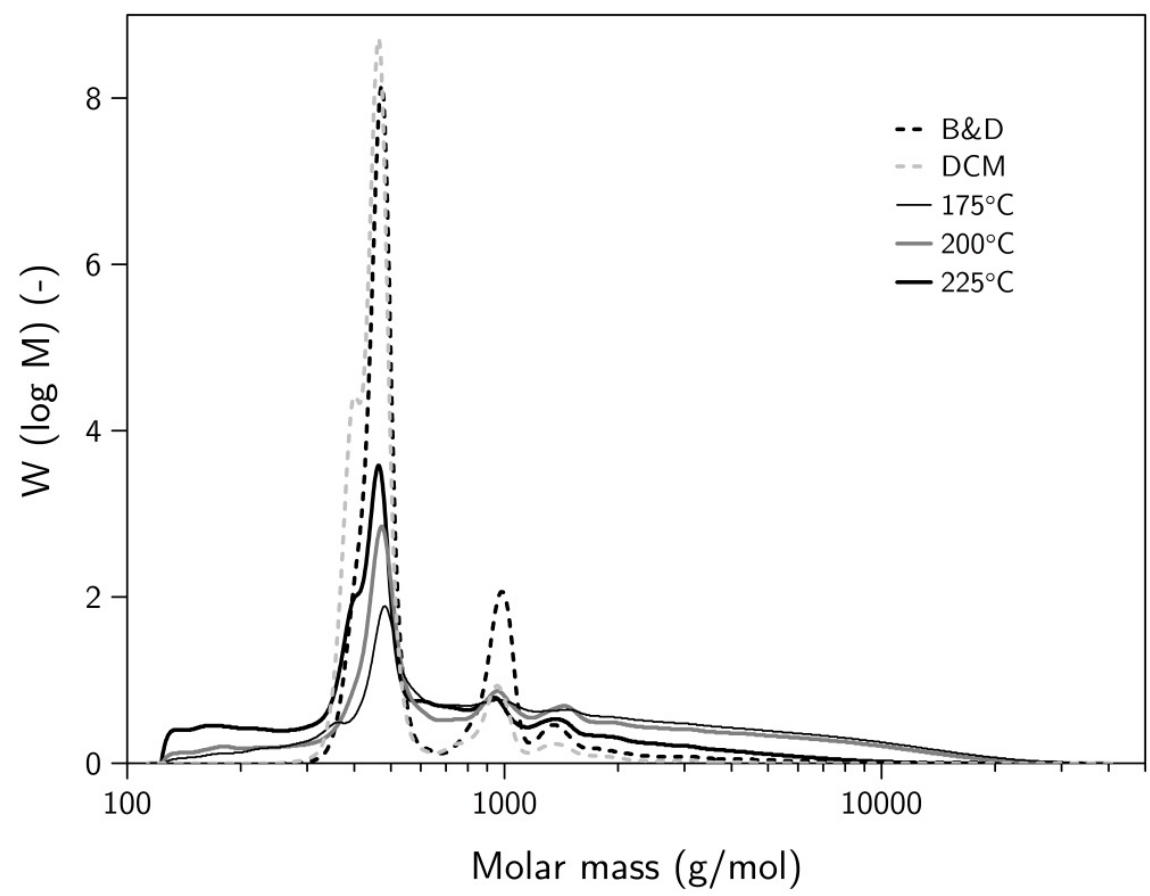

Figure 8. Molecular weight distribution by GPC analysis of oils obtained after Bligh and Dyer extraction (B\&D), dichloromethane extraction (DCM) and HTT at 175, 200 and $225^{\circ} \mathrm{C}$ with 5 min reaction time

As can be seen in Figure 8, the molecular weight distribution of the HTT oils was significantly different from that of the extracted oils (B\&D and DCM). The HTT oils had a wider molecular weight distribution where more heavy compounds were produced (tailing observed from 2000 up to more than $10000 \mathrm{~g} / \mathrm{mol}$ ), most probably related to the presence of hydrophobic polypeptides or proteins, as well as some lighter components (from 100 to $300 \mathrm{~g} / \mathrm{mol}$ ) which had minor presence in the extracted oils. The peak observed at around 400 to $500 \mathrm{~g} / \mathrm{mol}$ was most likely related to the presence of lipids. These results lead to the conclusion that at relatively low temperatures the cellular compounds already undergo thermal degradation, even though the outer algal cell wall remains visually non broken.

In addition, the DCM extracted oil and the oil from $\mathrm{HTT}$ at $200^{\circ} \mathrm{C}$ and 5 min reaction time were analysed in terms of fatty acid composition (detailed information of these analyses is reported in the Appendix of this chapter). It was found that while only bounded fatty acids (representing the esterification products from mono-, di-, triglycerides, phospholipids, saccharolipids, etc.) were present in the DCM extracted oil, the $200^{\circ} \mathrm{C}$ HTT oil also contained free fatty acids (FFA). This indicates that already at 
those less severe HTT conditions, hydrolysis into FFA occurred. This reduces the possibilities of obtaining an oil rich in lipids with intact molecular structure (e.g. triglycerides) at relatively low HTT temperatures. Moreover, the presence of FFA in the oil could be detrimental if transesterification of this oil using an homogeneous basic catalyst (most commonly used) is performed as undesirable soaps would be produced by neutralisation of the FFA, decreasing the biodiesel yield. ${ }^{47}$ In contrast, FFA would not cause difficulties if hydrotreating of the oil for upgrading purposes is preferred. More general, as in some studies a method termed thermolysis (or also autoclaving) is applied as mild heat pre-treatment (around 70 to $120^{\circ} \mathrm{C}$ ) for cell breakage prior to extraction, ${ }^{48,49}$ it is now clear that such a thermal treatment could already (undesirably) modify the molecular structure of the intracellular compounds.

As shown in Figure 5, a rise in oil production was obtained by increasing reaction time at $200^{\circ} \mathrm{C}$. Again, the observed relatively large increase in oil yield (specially from 30 to 60 min reaction time) was compared with visual inspection of the solid residue after HTT at different reaction times and $200^{\circ} \mathrm{C}$ (Figure 9) by SEM analysis.

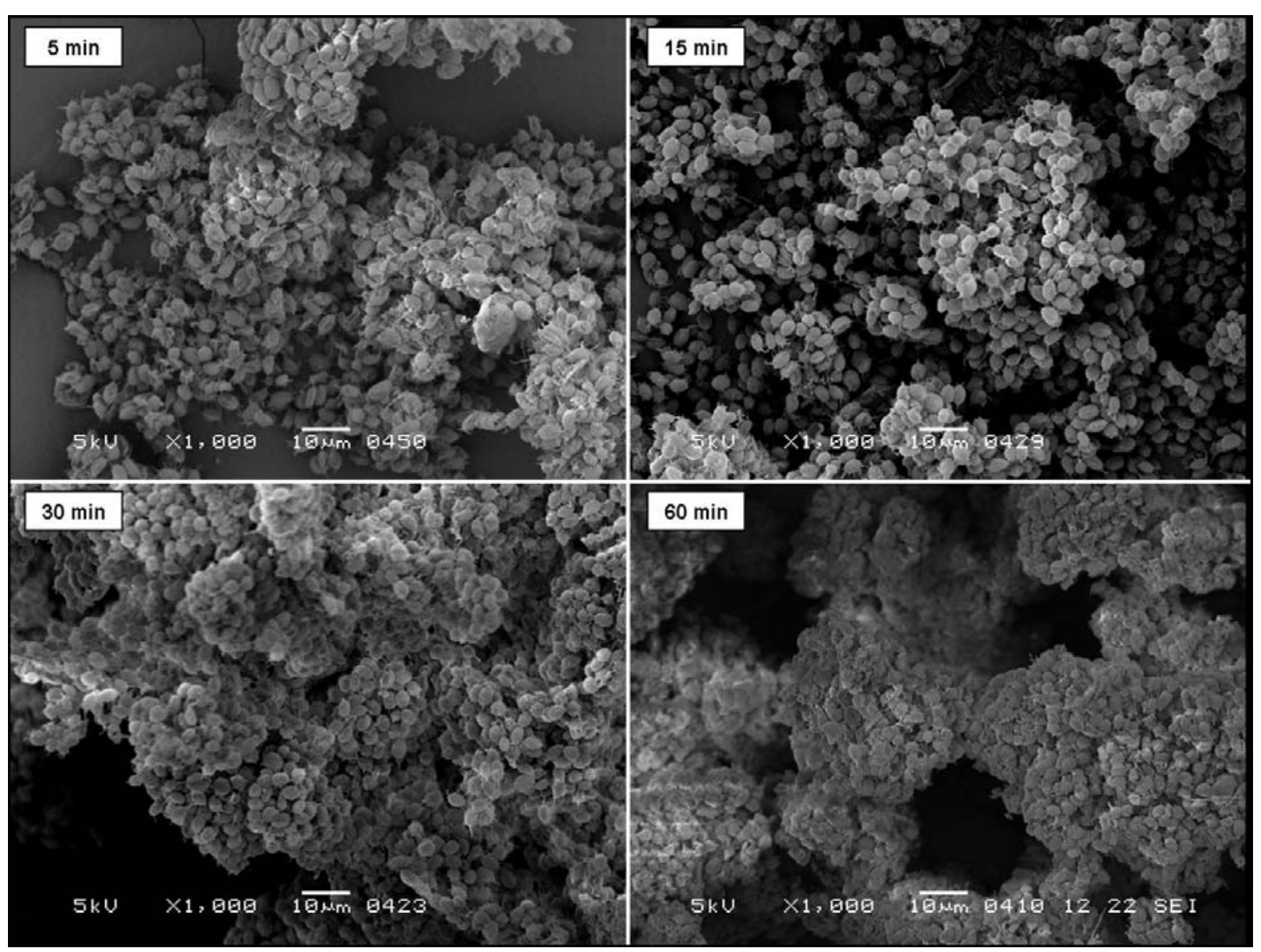

Figure 9. Effect of reaction time on the algal cells after HTT of Desmodesmus sp. at $200^{\circ} \mathrm{C}$ 
With the temperature series experiments we could observe that one of the main characteristics of Desmodesmus sp. is that they possess a very strong cell wall. In these new series of experiments, this feature was confirmed, since after $200^{\circ} \mathrm{C}$ HTT during 5 and $15 \mathrm{~min}$, all the cells were still visually unbroken and no major cell differences could be detected between both. However, from 30 min reaction time onwards, the cells started compacting and clustering, although still keeping the cell wall non ruptured. Therefore, we could prove again that at relatively low temperatures $\left(200^{\circ} \mathrm{C}\right)$ longer reaction times resulted in a thermal degradation of the cellular compounds without breaking the cell wall since the oil yield increased as well as the $\mathrm{N}$ and $\mathrm{C}$ content. HTT experiments at $200^{\circ} \mathrm{C}$ over even longer reaction time will be performed in following research to ensure if the breakage of the cells takes place at a certain point in time at such low temperature.

Moreover, now we observed that the previous reported cells breakage from 225 to $250^{\circ} \mathrm{C}$ and 5 min reaction time (observed in Figure 7) was not the only phenomenon causing the sudden increase in oil yield since $\mathrm{HTT}$ at $200^{\circ} \mathrm{C}, 60$ min reaction time and visually unbroken cells led to an oil yield (23 wt\%) comparable to the one obtained with HTT at $250^{\circ} \mathrm{C}, 5 \mathrm{~min}$ reaction time and major breakage of cells (26 wt\%).

\subsection{Comparison of HTT algal oils produced under different reaction conditions}

In order to give an idea on the difference between "milder" and "harsher" HTT, in terms of molecular composition of the product, chemical features of two different oils obtained at 250 and $375^{\circ} \mathrm{C}$ (both over 5 min reaction time) were here shown. Two oils were analysed in terms of their molecular weight distribution (by GPC), fatty acid composition and yield of (categories of-) chemical compounds (by stepwise Py-SPMEGC-MS combined with TGA). Complete information about the new developed analytical techniques applied as well as the results of the extensive study on the chemical nature of the oils can be found in the Appendix of this chapter.

The following oil features were identified and quantified by GC-FID analysis (after applying esterification): total amount of fatty acids (TFA) and its yield from algae (TFA yield); the amount of free fatty acids (FFA) and the total FFA yield calculated from the total yield of oil. The amount of bounded fatty acids (BFA) and yield is then calculated by difference $(\mathrm{BFA}=$ TFA-FFA). These results are shown in Table 7 . 
Table 7. Total fatty acid (TFA), free fatty acid (FFA) and bounded fatty acid (BFA) content of the oils obtained after $\mathrm{HTT}$ at 250 and $375^{\circ} \mathrm{C}$ with 5 min reaction time

\begin{tabular}{|c|c|c|c|c|c|c|c|}
\hline \multirow[b]{2}{*}{$\begin{array}{c}\mathrm{T} \\
\left({ }^{\circ} \mathrm{C}\right)\end{array}$} & \multirow[b]{2}{*}{$\begin{array}{c}\text { Oil yield } \\
\left(\% \mathrm{w} / \mathrm{w}_{\text {algae }}\right)\end{array}$} & \multirow[b]{2}{*}{$\begin{array}{c}\text { TFA in } \\
\text { oil } \\
\left(\% \mathrm{w} / \mathrm{w}_{\text {oil }}\right)\end{array}$} & \multirow[b]{2}{*}{$\begin{array}{l}\text { TFA yield } \\
\left(\% \mathrm{w} / \mathrm{w}_{\text {algae }}\right)\end{array}$} & \multicolumn{4}{|c|}{$\mathrm{TFA}=\mathrm{FFA}+\mathrm{BFA}$} \\
\hline & & & & $\begin{array}{c}\text { FFA in } \\
\text { oil } \\
\left(\% \mathrm{w} / \mathrm{w}_{\text {oil }}\right)\end{array}$ & $\begin{array}{l}\text { FFA yield } \\
\left(\% \mathrm{w} / \mathrm{w}_{\text {algae }}\right)\end{array}$ & $\begin{array}{c}\text { BFA in } \\
\text { oil } \\
\left(\% \mathrm{w} / \mathrm{w}_{\text {oil }}\right)\end{array}$ & $\begin{array}{l}\text { BFA yield } \\
\left(\% \mathrm{w} / \mathrm{w}_{\text {algae }}\right)\end{array}$ \\
\hline 250 & 26.7 & $28.0 \pm 4.0$ & 7.5 & $12.5 \pm 0.4$ & 3.3 & 15.5 & 4.1 \\
\hline 375 & 49.4 & $4.8 \pm 1.8$ & 2.4 & $3.4 \pm 0.2$ & 1.7 & 1.4 & 0.7 \\
\hline
\end{tabular}

In the oil from $\mathrm{HTT}$ at $250^{\circ} \mathrm{C}, 45 \%$ of TFA were in the form of FFA. The residual $55 \%$ were BFA (as mainly mono-, di-, triglycerides and phospholipids present in the oil matrix after HTT of microalgae). On the other hand, at $375^{\circ} \mathrm{C}$ the maximum oil yield was achieved but producing a lower amount of TFA due to the thermal degradation of these compounds into smaller fragments. The "non-lipid" fraction of this oil accounted for the largest portion of this product. In addition, FFA represented the major part of TFA content of this oil $(71 \%)$ which might indicate that at higher temperatures acylglicerols and more complex lipids were thermally decomposed (likely by hydrolysis) into free fatty acids.

An indication of the molecular weight distributions of both oils, as determined by GPC analyses, are shown in Figure 10.

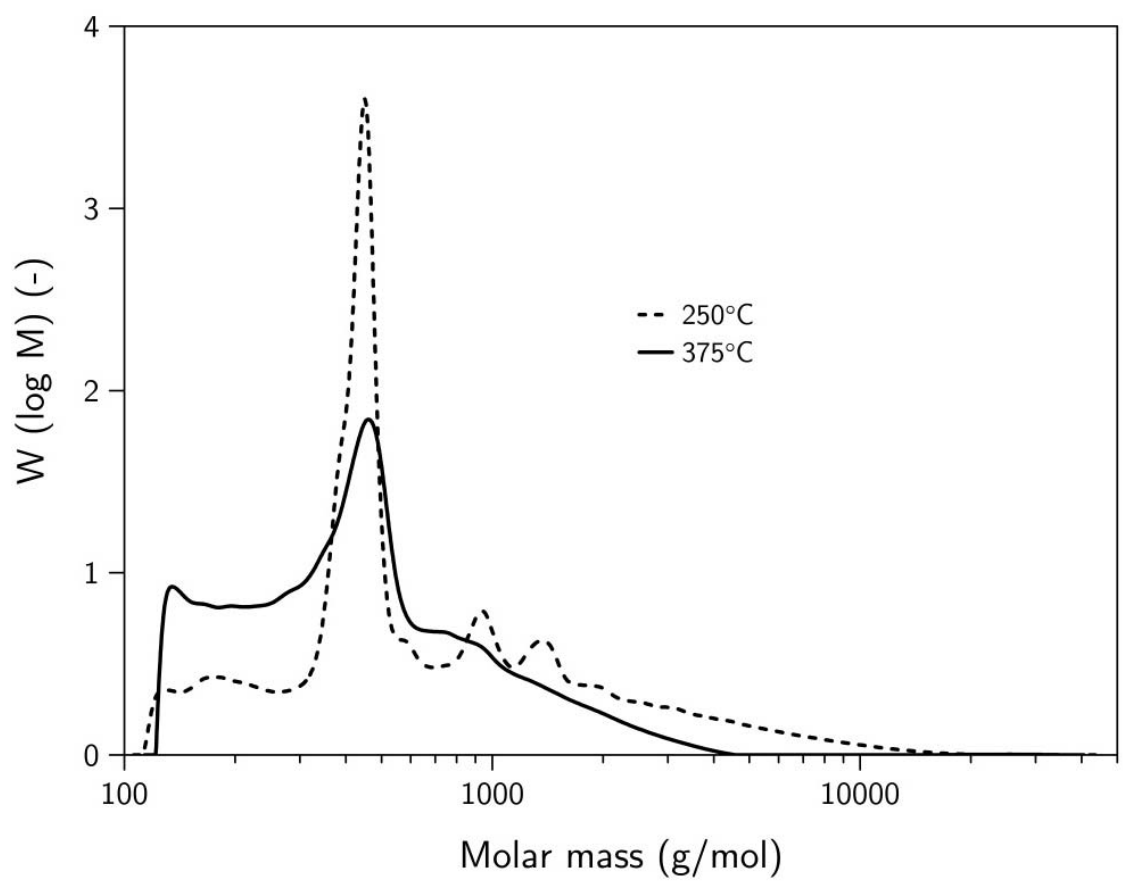

Figure 10. Molecular weight distribution obtained by GPC analysis of the oils obtained after HTT at 250 and $375^{\circ} \mathrm{C}$ over 5 min reaction time 
This result confirmed that the two products have a different chemical composition. The MW distribution of the $\mathrm{HTT} 250^{\circ} \mathrm{C}$ oil showed more pronounced peaks (at least 3, the first one also observed in Figure 8 being related to the presence of lipids) while the HTT $375^{\circ} \mathrm{C}$ oil showed a wider distribution which could be related to the a larger mixture of compounds with different molecular weight. The tailing observed from the $250^{\circ} \mathrm{C}$ oil indicated that it contains heavier compounds than the oil from $\mathrm{HTT}$ at $375^{\circ} \mathrm{C}$. This might point out that when the temperature is increased, more molecules (proteins, carbohydrates, lipid macromolecules and algaenan derivatives) are broken down into smaller and lighter fragments.

Finally, the characterization of the oils in a molecular level was performed with an alternative analytical technique using Stepwise Py-SPME-GC-MS combined with TGA. With this, we could successfully identify and quantify over $80 \%$ of the oil. Several GC detectable compounds found in previous studies ${ }^{10,23,24}$ were also identified in this work (e.g. fatty acids, nitrogen heterocycles such as pyrroles and indole derivatives, long chain alkanes, etc.). To our knowledge, until now a clear picture of the substances forming the non-GC detectable fraction in the HTT oil is lacking in the literature. This issue is addressed in the work presented in the Appendix (collaboration with the University of Bologna) and for the oils presented in Table 7 the identified compounds were divided into several chemical classes and combined with the HTT oil yields in Figure 11. In the case of the 250 and $375^{\circ} \mathrm{C}$ oils the different chemical classes were further rearranged into six groups: Asphaltene-like materials (non-volatile matter after pyrolysis at $500^{\circ} \mathrm{C}$ ); Lipid derivatives (sterols, FFA, BFA, carotenoid derivatives, phytols, etc.); Nitrogen (N-) containing compounds (mainly protein derivatives); algaenan derivatives (long chain hydrocarbons and sporopollenin derivatives); hydrocarbons (only when $\mathrm{nC}<20$ ); and not yet identified compounds.

As can be seen, the largest fraction of the $250^{\circ} \mathrm{C}$ oil was the "lipid derivatives" fraction. More than $50 \%$ of this oil consisted of low-nitrogen, high energy value compounds such as lipids, algaenans and hydrocarbons, whereas the $375^{\circ} \mathrm{C}$ oil only contained $29 \%$ of that type of compounds. However, the overall oil yield was significantly inferior for HTT at low temperature.

With increasing temperature, an increase of "N-containing compounds" fraction could be observed which was in agreement with the increase of nitrogen content (from $4.1 \mathrm{wt} \%$ for $250^{\circ} \mathrm{C}$ HTT to $\left.6.3 \mathrm{wt} \% 375^{\circ} \mathrm{C} \mathrm{HTT}\right)$. That fraction of the oil contained significant amounts of cyclic dipeptides (diketopiperazines), pyrroles, pyrrolidinones, indole derivatives and various peptides produced by means of thermal degradation of algal 
proteins. As a result, a simultaneous effect of oil yield and nitrogen content increase occurred. At the same time, an important decrease of the "lipid derivatives" fraction took place. Most likely, these compounds broke down into smaller fragments via thermal degradations reactions or reacted with other compounds (e.g. reaction between ammonia and fatty acids producing fatty acid amides). Finally, we could observe a large increase in algaenan derivatives (mainly alkyl based polymers) and asphaltenes (heavy polymeric matter, similar to asphaltenen or maltene, which could also be partially produced during the analytical method used). The latter fraction was likely formed by polymerization reactions and charring of earlier formed oily compounds.

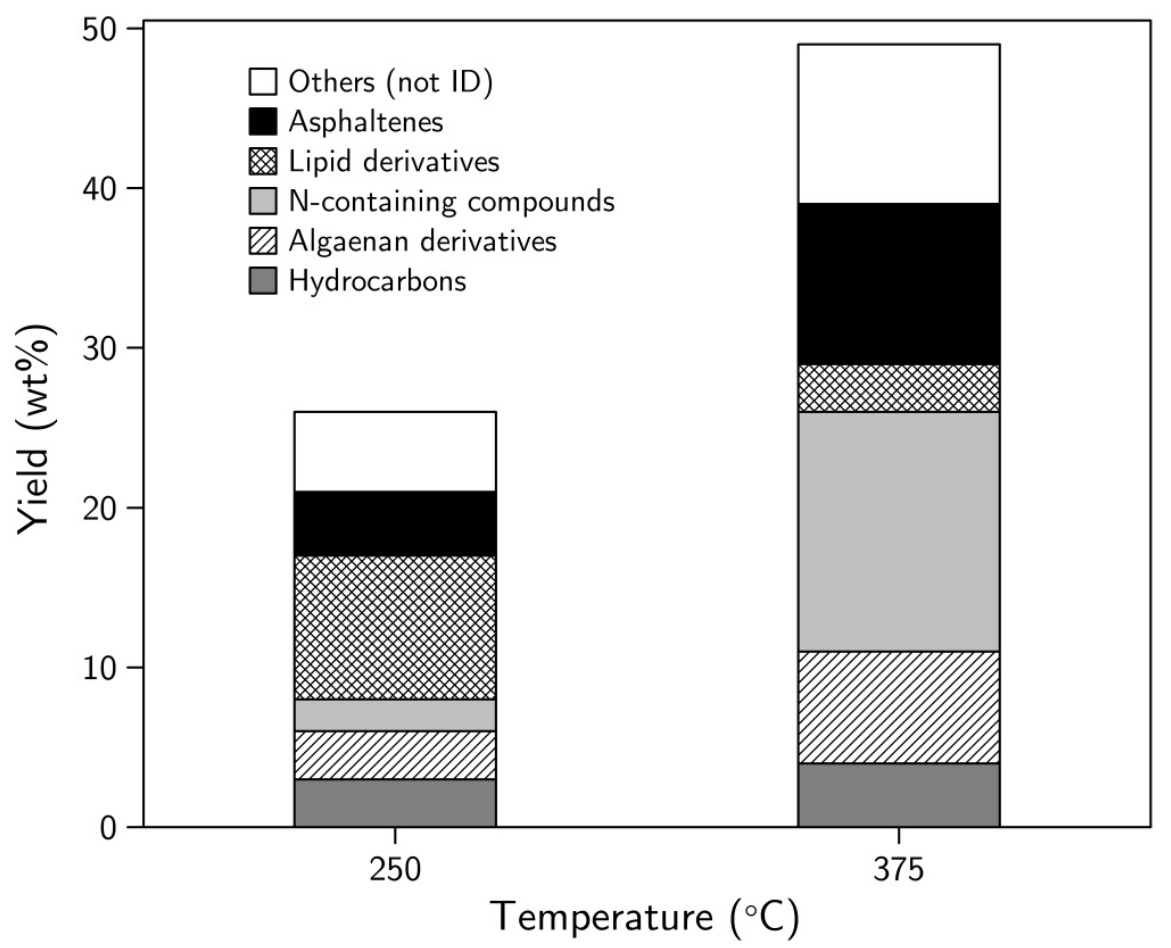

Figure 11. Yield of different chemical categories for the oils obtained from $\mathrm{HTT}$ at $250^{\circ} \mathrm{C}$ and $375^{\circ} \mathrm{C}$ with 5 min reaction time

In short, the oil product obtained by $250^{\circ} \mathrm{C}$ and 5 min reaction time HTT was comparable to a lipid-extracted oil, enriched with a contribution of energy valuable compounds like algaenan derivatives and hydrocarbons. The highest oil yield was obtained at $375^{\circ} \mathrm{C}$ HTT with a significant contribution of hydrocarbon-like compounds. However, this product was largely composed by less desirable substances such as asphaltenes and compounds produced via thermal degradation of the algal protein fraction, hence reducing its quality. 


\section{Discussion and conclusions}

Hydrothermal treatment of Desmodesmus sp. was studied extensively over a wide range of reaction conditions $\left(175-450^{\circ} \mathrm{C}\right.$ and reaction times up to $\left.60 \mathrm{~min}\right)$. The product yield distribution over oil-, gas-, aqueous phase and solid residue was determined with proper mass balance closure. Up to $75 \%$ of the calorific value of the algal biomass could be retrieved in the oil phase after $\mathrm{HTT}$ at $375^{\circ} \mathrm{C}$ and 5 min reaction time. However, high oil yields were accompanied by high nitrogen content in the oil (up to 6\%).

The temperature resistance of the cell wall of Desmodesmus sp. was confirmed in this study. However, it became evident that the intracellular compounds already suffered from thermal degradation at temperatures below those where the cell wall breaks, reducing the possibilities of obtaining an oil rich in lipids with intact molecular structure (e.g. triglycerides), even at relatively low HTT temperatures. A SEM visualization of the algal cells after HTT increasing temperature over 5 min reaction time showed a significant rise of oil yield in the temperature region where the cells seem to be thermally broken.

The recycling of the inorganic $\mathrm{N}$ and $\mathrm{P}$ nutrients dissolved in the HTT aqueous product phase to cultivate a next generation of Desmodesmus sp. seems possible. Yet it is necessary to study experimentally the influence of the (dissolved) organic fraction and metals on the growth.

A conclusion from investigating the chemical nature of the HTT oils $\left(250\right.$ and $\left.275^{\circ} \mathrm{C}\right)$ is that the desired product specifications of the final liquid fuel are the key factor for the selection of a specific algal species to be used as feedstock. Many algae species, especially from the green algae division, contain low lipid content but considerable amounts of algaenans. ${ }^{45}$ Therefore, we could think of an algae biorefinery (incorporating heat integration and nutrients recycling) using two different algal feedstock (growing in different systems): 1) lipid (triglycerides)-rich microalgae for biodiesel production; and 2) algaenan-rich microalgae with low lipid content to produce a hydrocarbon rich fuel as refinery feedstock. The downstream residues from both algae routes could be mixed and further treated to e.g. produce other energy valuable products which could be integrated into the same biorefinery system.

The selection of the HTT operating conditions to be used might depend on the further application of the produced oil. If a lipid-rich oil is preferable, temperature should not exceed $250^{\circ} \mathrm{C}$; for a crude-like oil, the temperature can be as high as to $375^{\circ} \mathrm{C}$ to obtain 
the maximum amount of that product. It is important to remember, however, that these temperature indications are possibly specific for this type of microalgae.

From HTT at high temperatures and maximum bio-crude yield, a complex oil matrix with a large variety of compounds was obtained. This oil was highly viscous and had a high nitrogen content, mainly due the presence of protein derivatives. Upgrading methods for denitrogenation and viscosity reduction will be required for the further processing of this oil. If HTT of microalgae is used as a direct conversion method to produce a liquid fuel, the following upgrading methods could be considered: denitrogenation methods after HTT (via extraction or hydrodenitrogenation); catalytic hydrothermal treatment for in situ denitrogenation; and hydro-liquefaction. However, some of those methods were applied in other studies and the nitrogen content was not significantly reduced. Besides that, in an algae biorefinery system such processes would add costs (use of catalyst and hydrogen), increase energy consumption and imply losing high valuable products (such as certain proteins with, for instance, pharmaceutical characteristics). Therefore, from our point of view, these deleterious aspects could be overcome by using HTT of microalgae as post-treatment of the residual microalgae after wet extraction of proteins within an algae biorefinery concept, unless a suitable and economic (catalytic-) process for denitrogenation is found.

\section{Acknowledgements}

The authors thank the financial support by IMPACT - Institute for energy and resources of the University of Twente (The Netherlands).

\section{Bibliography}

[1] G.W. Huber, S. Iborra, A. Corma, Synthesis of Transportation Fuels from Biomass: Chemistry, Catalysts, and Engineering, Chem. Rev., 106(9), 4044-4098, 2006.

[2] E.S. Umdu, M. Tuncer, E. Seker, Transesterification of Nannochloropsis oculata microalga's lipid to biodiesel on $\mathrm{Al}_{2} \mathrm{O}_{3}$ supported $\mathrm{CaO}$ and $\mathrm{MgO}$ catalysts, Bioresour. Technol., 100(11), 2828-2831, 2009.

[3] K. Vijayaraghavan, K. Hemanathan, Biodiesel Production from Freshwater Algae, Energy Fuels, 23(11), 5448-5453, 2009.

[4] R. Halim, B. Gladman, M.K. Danquah, P.A. Webley, Oil extraction from microalgae for biodiesel production, Bioresour. Technol., 102(1), 178-185, 2011.

[5] W. Peng, Q. Wu, P. Tu, Effects of temperature and holding time on production of renewable fuels from pyrolysis of Chlorella protothecoides, J. Appl. Phycol., 12(2), 147-152, 2000. 
[6] X. Miao, Q. Wu, C. Yang, Fast pyrolysis of microalgae to produce renewable fuels, J. Anal. Appl. Pyrolysis, 71(2), 855-863, 2004.

[7] A. Hirano, K. Hon-Nami, S. Kunito, M. Hada, Y. Ogushi, Temperature effect on continuous gasification of microalgal biomass: theoretical yield of methanol production and its energy balance, Catal. Today, 45(1-4), 399-404, 1998.

[8] S. Zou, Y. Wu, M. Yang, C. Li, J. Tong, Thermochemical catalytic liquefaction of the marine microalgae dunaliella tertiolecta and characterization of bio-oils, Energy Fuels, 23(7), 3753-3758, 2009.

[9] L. Xu, D.W.F. Brilman, J.A.M. Withag, G. Brem, S. Kersten, Assessment of a dry and a wet route for the production of biofuels from microalgae: Energy balance analysis, Bioresour. Technol., 102(8), 5113-5122, 2011.

[10] A.B. Ross, P. Biller, M.L. Kubacki, H. Li, A. Lea-Langton, J.M. Jones, Hydrothermal processing of microalgae using alkali and organic acids, Fuel, 2010.

[11] C. Elliott Douglas, L.J. Sealock, R.S. Butner, Product analysis from direct liquefaction of several high-moisture biomass feedstocks, American Chemical Society, 1988.

[12] A.A. Peterson, F. Vogel, R.P. Lachance, M. Fröling, M.J. Antal Jr, J.W. Tester, Thermochemical biofuel production in hydrothermal media: A review of sub- and supercritical water technologies, Energy Environ. Sci., 1(1), 32-65, 2008.

[13] D. Knežević, W. van Swaaij, S. Kersten, Hydrothermal conversion of biomass. II. conversion of wood, pyrolysis oil, and glucose in hot compressed water, Ind. Eng. Chem. Res., 49(1), 104-112, 2009.

[14] Y. Dote, S. Sawayama, S. Inoue, T. Minowa, S.-y. Yokoyama, Recovery of liquid fuel from hydrocarbon-rich microalgae by thermochemical liquefaction, Fuel, 73(12), 1855-1857, 1994.

[15] P. Metzger, C. Largeau, Botryococcus braunii: a rich source for hydrocarbons and related ether lipids, Appl. Microbiol. Biotechnol., 66(5), 486-496, 2005.

[16] H. Huang, X. Yuan, G. Zeng, J. Wang, H. Li, C. Zhou, X. Pei, Q. You, L. Chen, Thermochemical liquefaction characteristics of microalgae in sub- and supercritical ethanol, Fuel Process. Technol., 92(1), 147-153, 2011.

[17] T. Matsui, A. Nishihara, C. Ueda, M. Ohtsuki, N. Ikenaga, T. Suzuki, Liquefaction of micro-algae with iron catalyst, Fuel, 76(11), 1043-1048, 1997.

[18] P. Biller, A.B. Ross, Potential yields and properties of oil from the hydrothermal liquefaction of microalgae with different biochemical content, Bioresour. Technol., 102(1), 215-225, 2011.

[19] P. Duan, P.E. Savage, Hydrothermal liquefaction of a microalga with heterogeneous catalysts, Ind. Eng. Chem. Res., 50(1), 52-61, 2010.

[20] C. Yang, L. Jia, C. Chen, G. Liu, W. Fang, Bio-oil from hydro-liquefaction of Dunaliella salina over Ni/REHY catalyst, Bioresour. Technol., 102(6), 4580-4584, 2011.

[21] T. Minowa, S. Yokoyama, M. Kishimoto, T. Okakura, Oil production from algal cells of Dunaliella tertiolecta by direct thermochemical liquefaction, Fuel, 74(12), 1735-1738, 1995.

[22] Y.F. Yang, C.P. Feng, Y. Inamori, T. Maekawa, Analysis of energy conversion characteristics in liquefaction of algae, Resour. Conserv. Recycl., 43(1), 21-33, 2004.

[23] T.M. Brown, P. Duan, P.E. Savage, Hydrothermal liquefaction and gasification of Nannochloropsis sp, Energy Fuels, 24(6), 3639-3646, 2010.

[24] D. Zhou, L. Zhang, S. Zhang, H. Fu, J. Chen, Hydrothermal liquefaction of macroalgae enteromorpha prolifera to bio-oil, Energy Fuels, 24(7), 4054-4061, 2010.

[25] T. Minowa, S. Sawayama, A novel microalgal system for energy production with nitrogen cycling, 
Fuel, 78(10), 1213-1215, 1999.

[26] U. Jena, N. Vaidyanathan, S. Chinnasamy, K.C. Das, Evaluation of microalgae cultivation using recovered aqueous co-product from thermochemical liquefaction of algal biomass, Bioresour. Technol., 102(3), 3380-3387, 2011.

[27] Deparment of Energy (DOE), National algal biofuels technology roadmap, 2010.

[28] E.G. Bligh, W.J. Dyer, A rapid method of total lipid extraction and purification, Can. J. Biochem., 37(8), 911-917, 1959

[29] A. Hara, N.S. Radin, Lipid extraction of tissues with a low-toxicity solvent, Anal. Biochem., 90(1), 420-426, 1978.

[30] F. de Miguel Mercader, P.J.J. Koehorst, H.J. Heeres, S.R.A. Kersten, J.A. Hogendoorn, Competition between hydrotreating and polymerization reactions during pyrolysis oil hydrodeoxygenation, AlChE J., 3160-3170, 2011.

[31] E. Hoekstra, S.R.A. Kersten, A. Tudos, D. Meier, K.J.A. Hogendoorn, Possibilities and pitfalls in analyzing (upgraded) pyrolysis oil by size exclusion chromatography (SEC), J. Anal. Appl. Pyrolysis, 91(1), 76-88, 2011.

[32] U. Jena, K.C. Das, J.R. Kastner, Effect of operating conditions of thermochemical liquefaction on biocrude production from Spirulina platensis, Bioresour. Technol., 102(10), 6221-6229, 2011.

[33] K. Anastasakis, A.B. Ross, Hydrothermal liquefaction of the brown macro-alga Laminaria Saccharina: Effect of reaction conditions on product distribution and composition, Bioresour. Technol., 102(7), 4876-4883, 2011.

[34] M. Aresta, A. Dibenedetto, M. Carone, T. Colonna, C. Fragale, Production of biodiesel from macroalgae by supercritical $\mathrm{CO}_{2}$ extraction and thermochemical liquefaction, Environ. Chem. Lett., 3(3), 136-139, 2005.

[35] P. Biller, R. Riley, A.B. Ross, Catalytic hydrothermal processing of microalgae: Decomposition and upgrading of lipids, Bioresour. Technol., 102(7), 4841-4848, 2011.

[36] A. Demirbas, Mechanisms of liquefaction and pyrolysis reactions of biomass, Energy Convers. Manage., 41(6), 633-646, 2000.

[37] X.Z. Yuan, J.Y. Tong, G.M. Zeng, H. Li, W. Xie, Comparative studies of products obtained at different temperatures during straw liquefaction by hot compressed water, Energy Fuels, 23(6), 3262$3267,2009$.

[38] N. Akiya, P.E. Savage, Roles of water for chemical reactions in high-temperature water, Chem. Rev., 102(8), 2725-2750, 2002.

[39] F. de Miguel Mercader, M.J. Groeneveld, S.R.A. Kersten, N.W.J. Way, C.J. Schaverien, J.A. Hogendoorn, Production of advanced biofuels: Co-processing of upgraded pyrolysis oil in standard refinery units, Appl. Catal., B, 96(1-2), 57-66, 2010.

[40] F. de Miguel Mercader, M.J. Groeneveld, S.R.A. Kersten, R.H. Venderbosch, J.A. Hogendoorn, Pyrolysis oil upgrading by high pressure thermal treatment, Fuel, 89(10), 2829-2837, 2010.

[41] R.J.M. Westerhof, D.W.F. Brilman, M. Garcia-Perez, Z. Wang, S.R.G. Oudenhoven, W.P.M. van Swaaij, S.R.A. Kersten, Fractional condensation of biomass pyrolysis vapors, Energy Fuels, 25(4), 18171829, 2011.

[42] L.Q. Zepka, E. Jacob-Lopes, R. Goldbeck, M.I. Queiroz, Production and biochemical profile of the microalgae Aphanothece microscopica Nägeli submitted to different drying conditions, Chem. Eng. Process., 47(8), 1305-1310, 2008. 
[43] I.S. Chronakis, Gelation of edible blue-green algae protein isolate (Spirulina platensis strain pacifica): Thermal transitions, rheological properties, and molecular forces involved, J. Agric. Food. Chem., 49(2), 888-898, 2001.

[44] A.A. Peterson, R.P. Lachance, J.W. Tester, Kinetic evidence of the maillard reaction in hydrothermal biomass processing: Glucose-glycine interactions in high-temperature, high-pressure water, Ind. Eng. Chem. Res., 49(5), 2107-2117, 2010.

[45] G.J.M. Versteegh, P. Blokker, Resistant macromolecules of extant and fossil microalgae, Phycol. Res., 52(4), 325-339, 2004.

[46] E. Salmon, F. Behar, F. Lorant, P.G. Hatcher, P. Metzger, P.-M. Marquaire, Thermal decomposition processes in algaenan of Botryococcus braunii race L. Part 1: Experimental data and structural evolution, Org. Geochem., 40(3), 400-415, 2009.

[47] G. Vicente, M. Martínez, J. Aracil, Integrated biodiesel production: a comparison of different homogeneous catalysts systems, Bioresour. Technol., 92(3), 297-305, 2004.

[48] J. Lee, C. Yoo, S. Jun, C. Ahn, H. Oh, Comparison of several methods for effective lipid extraction from microalgae, Bioresour. Technol., 101(1, Supplement 1), S75-S77, 2010.

[49] K. Kita, S. Okada, H. Sekino, K. Imou, S. Yokoyama, T. Amano, Thermal pre-treatment of wet microalgae harvest for efficient hydrocarbon recovery, Appl. Energy, 87(7), 2420-2423, 2010. 


\section{Appendix A}

Hydrothermal treatment (HTT) of microalgae:

Detailed molecular characterization of HTT oil in view of HTT mechanism elucidation 


\begin{abstract}
The understanding of the chemical nature of the oil is important for both the optimization of the process and the design of up-grading strategies for further use as energy carrier or towards transportation fuels. Hydrothermal treatment (HTT) oil is a complex matrix, whose composition is strongly affected by the feedstock type and by the HTT experimental conditions. In the present work, HTT oil from Desmodesmus sp. was subjected to a detailed chemical analysis. Various characterization techniques (silica gel chromatography, methanolysis, size exclusion chromatography, analytical pyrolysis, elemental analysis and thermogravimetric techniques) were coupled to gather a clearer information on the chemical nature of HTT oil obtained at different reaction times, temperatures and different slurry concentrations. Special attention was paid to the fate of $\mathrm{N}$ in the HTT process and the nature of the N-containing species in the oil. By cross-checking results from the chemical characterization of the oil with process data, it was finally possible to identify some different competitive reactions involved in the formation of HTT oil at different conditions. Results show that main compounds obtained at low temperature are still classifiable as lipids, which are extractable without the HTT, together with some short chain algaenan and some hydrophobic protein fragments that end up in organic solvent phase. At higher temperature $\left(300-375^{\circ} \mathrm{C}\right)$, proteins and cellulose started to break down, giving cyclic dipeptides and amino-acids side chains (by pyrolysis-like reactions), carbohydrates derivatives (e.g. furans) and products from the cross reaction of proteins and carbohydrates (e.g. formation of alkyl-pyrrolidinones, pyrazines, pyrroles and melanoidin-like materials). This phenomenon is responsible of the observed increase in oil mass yield with increasing processing temperature, as well as the increase in nitrogen content of the oil. Optimization of the production of fuels and fuel precursors by HTT should be done in conjunction with evaluation of downstream processing options and/or the possibility to recycle unconverted material to the algae cultivation.
\end{abstract}




\section{A.1 Introduction}

Various conversion and extraction routes can be used for the production of liquid fuels from microalgae and biomass. For highly lipidic algae, lipid extraction, eventually combined with transesterification (bio-diesel production), allows the obtainment of a lipid extract (almost similar to fossil derived fuels) and a non-lipid byproduct. Alternatively, especially for microalgae with low lipid content (or for residues from lipid extraction), thermo-chemical processes such as pyrolysis (on dry matter) and hydrothermal treatment (HTT, on wet material) can produce liquid fuels by exploiting all biomass constituents (lipids, carbohydrates, proteins and algaenan).

Because microalgae are generally collected as wet matrix, HTT is considered one of most interesting ways for producing fuels from aqueous microalgal slurry by means of heat application followed by a phase separation. This process allows the obtainment of an oily phase (containing lipids and hydrophobic products from HTT), a variable amount of gas and water-soluble substances (which could be recycled back to the microalgae growth plant) and a solid residue. As shown in Section 3 (Chapter 2), the HTT oil yield is higher than that obtainable by solvent extraction and the liquid fraction is characterized by an acceptable higher heating value (HHV, similar to biodiesel) and a relatively low oxygen content. Nevertheless, especially with high temperature HTT, the high nitrogen content can be a problem for the direct utilization of the oil as fuel (due to increased $\mathrm{NO}_{\mathrm{x}}$ emission), and at the same time it determines a net loss of nitrogen from the cultivation system (nitrogen burnt in the oil cannot be recycled to the culture).

Although knowledge about the composition of the HTT oils is an important issue for both process optimization (which requires understanding of the HTT mechanism) and upgrading of the fuel, only limited information (mainly by GC-MS and elemental analysis) on the chemical nature of HTT oil is currently available. ${ }^{1-4}$ The objective of this work is therefore to identify the chemical nature of the compounds in the oils, as obtained by hydrothermal treatment for the microalgae Desmodesmus sp., with a particular focus on the nitrogen containing compounds responsible of the high nitrogen content of the oil, and to obtain (where possible) information about the liquefaction mechanism.

A partial characterization of HTT oils can be obtained by means of direct gas chromatography (GC) analysis of the oil, which is dissolved in a suitable solvent and subsequently derivatized and analyzed. There are a large number of studies that reported the GC-MS analysis of HTT oils obtained under different conditions from various 
microalgal species. ${ }^{1,2}$ Nevertheless, even if various derivatization procedures are used, a large part of the oil cannot be analyzed by means of conventional GC techniques. ${ }^{3}$ Some additional information on this non GC-amenable fraction can be gathered by means of high pressure liquid chromatography (HPLC), ${ }^{5}$ fourier transform infrared (FTIR), ${ }^{1} \mathrm{H}-$ NMR $,{ }^{6}{ }^{13} \mathrm{C}-\mathrm{NMR},{ }^{4}$ size exclusion chromatography (SEC) or gel permeation chromatography (GPC), ${ }^{5}$ previously successfully applied in the characterization of the unknown fraction of pyrolysis oil from lignocellulose. ${ }^{7}$ However, as mentioned before, nowadays a clear idea of the molecular constituents in microalgal HTT oil is still lacking. Analytical pyrolysis coupled to gas chromatography (Py-GC-MS) is widely used for the characterization of high molecular weight organic materials, especially if it is refractory to standard chemical analysis, and it is probably one of the best techniques employed so far for this purpose. This is mainly due to its speed (no time-consuming sample pretreatment is needed) in investigating the macromolecules in terms of pyrolysis products. For this reason, Py-GC-MS has been often applied for characterization of refractory materials derived from thermal or environmental degradation of natural occurring substrates as kerogen-like materials, ${ }^{8,9}$ algaenan, ${ }^{10}$ humic substances, ${ }^{11}$ soots $^{12}$ and black carbon. ${ }^{13}$ Py-GC-MS can be considered one of the few techniques able to provide general information on molecular structures in complex mixtures of compounds or materials that are not susceptible to hydrolysis, methanolysis or other derivatization techniques.

However, in spite of its proven utility and well-established nature, the method may face some limitations when implemented in a conventional way using commercial systems. There is strong evidence that flash pyrolysis produces an important amount of non-GC detectable matter (due to foaming and aerosols droplets formation) that condenses into cool spots and determines mass transfer problems, together with systematic "memory effect", which hampers the analysis. ${ }^{14,15}$

However, if the pyrolysis is slow, heat transfer is good, the sample is small and a nondiscriminating technique is used, almost all the compounds produced and volatilized can be considered GC amenable. ${ }^{16}$ It follows that, combining semiquantitative data from analysis of pyrolysis vapors and quantitative data (e.g. weight loss from thermogravimetric analysis (TGA)), it is possible to obtain an absolute quantification of different molecular constituents (e.g. GC detectable compounds) and polymeric materials (e.g. asphaltene, protein like material and algaenan).

By mean of pyrolysis coupled with solid phase microextraction (Py-SPME-GC-MS), as a result of decoupling pyrolysis and analysis, it is possible to partially overcome the concerns typical of Py-GC-MS. In Py-SPME-GC-MS, the analytes are collected on a 
SPME fiber (used in dynamic sampling mode). ${ }^{17}$ This sampling method was successfully applied before in the analysis of volatiles evolved from flash pyrolysis of biomass, ${ }^{18}$ micropollutants arising from catalytic biomass pyrolysis ${ }^{19}$ and in artwork resins characterization studies. ${ }^{20}$

By means of this approach, and performing a stepwise process, it is possible to obtain information on both GC detectable and non-GC-detectable substances in the oil samples from HTT of microalgae. For this reason, in this work, pyrolysis was used in combination to other conventional techniques to identify the chemical nature of HTT oil, with a particular focus on the compounds responsible of the high nitrogen content of the oil. Among various fit-for-purpose techniques available, column fractionation, SEC, PySPME-GC-MS, TGA and stepwise Py-SPME-GC-MS were applied as source of complementary information.

\section{A.2 Materials and methods}

\section{A.2.1 HTT oil characterization: overall conceptual scheme}

In view of a better understanding of molecular composition of HTT oils from microalgae, oil samples obtained from HTT of Desmodesmus sp. were subjected to the conceptual analytical scheme shown in Figure A.1.

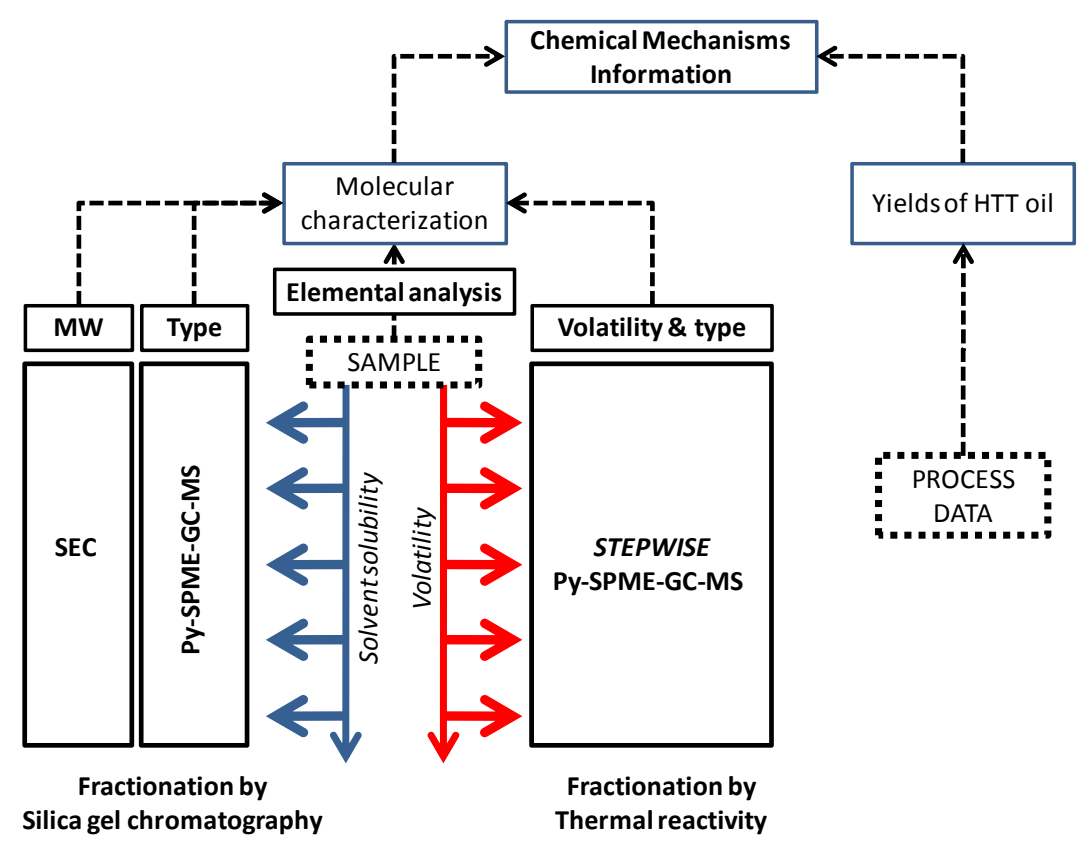

Figure A.1. Overall characterization scheme used for the elucidation of HTT mechanism of Desmodesmus sp. under different reaction conditions 
Information obtained by silica gel chromatography, SEC, Py-SPME-GC-MS, TGA and stepwise Py-SPME-GC-MS were evaluated together. In particular, the decoupling of analysis and pyrolysis through SPME was exploited for the analysis of pyrolysis vapours in a slow stepwise pyrolysis. Subsequently, the semi-quantitative (\% of GC area) information was coupled to quantitative data (weight loss) from TGA. By means of these approaches, a first impression of the overall chemical nature (on molecular basis) of the oil was gathered. Moreover, by coupling the information on composition (chemical analysis) and on HTT yields (from HTT experiments), it was possible to obtain some information on the HTT mechanism from a molecular point of view.

\section{A.2.2 Microalgal HTT oil sample obtainment and bulk analysis}

The HTT procedure and sample obtainment were fully described in Section 2.2 (Chapter 2 ) as well as the main features of the microalgae used in Section 2.1 (Chapter 2). In this work, the oil samples selected for analysis from the previous work (Chapter 2) are shown in Table A.1

Table A.1. Characteristics of samples analyzed.

\begin{tabular}{|c|c|c|c|c|c|c|c|c|}
\hline \multirow{2}{*}{$\begin{array}{c}\text { Sample } \\
\text { no. }\end{array}$} & \multirow{2}{*}{$\begin{array}{c}\mathrm{T}^{\mathrm{a}} \\
\left({ }^{\circ} \mathrm{C}\right)\end{array}$} & \multirow{2}{*}{$\begin{array}{l}\mathrm{RT}^{\mathrm{b}} \\
(\min )\end{array}$} & \multirow{2}{*}{$\begin{array}{l}\text { Slurry conc. } \\
\text { (wt\%) }\end{array}$} & \multirow{2}{*}{$\begin{array}{l}\text { Oil yield } \\
(w t \%)\end{array}$} & \multicolumn{4}{|c|}{ Elemental composition (wt\%) } \\
\hline & & & & & $\mathrm{C}$ & $\mathbf{H}$ & $\mathbf{N}$ & $\mathrm{O}$ \\
\hline 1 & 200 & 5 & 7.87 & 10 & 67.1 & 9.1 & 1.6 & 22.2 \\
\hline 2 & 200 & 60 & 7.87 & 23 & 69.2 & 9.6 & 3.7 & 17.5 \\
\hline 3 & 300 & 5 & 7.87 & 40 & 72.4 & 9.0 & 6.3 & 12.3 \\
\hline 4 & 300 & 60 & 7.87 & 47 & 75.0 & 8.8 & 6.0 & 10.2 \\
\hline 5 & 300 & 60 & 17.43 & 43 & 73.5 & 9.0 & 6.4 & 11.2 \\
\hline 6 & 250 & 5 & 7.87 & 27 & 71.5 & 8.9 & 4.1 & 15.4 \\
\hline 7 & 375 & 5 & 7.87 & 49 & 74.5 & 8.6 & 6.3 & 10.5 \\
\hline $\mathrm{DCM}_{\text {Extract }} \mathrm{d}^{\mathrm{d}}$ & 25 & - & 7.87 & 7.1 & 75.5 & 10.5 & 0.8 & 13.3 \\
\hline
\end{tabular}

${ }^{a}$ Reaction temperature of HTT. ${ }^{\mathrm{b}}$ Reaction time of HTT. ${ }^{\mathrm{c}}$ Algae concentration in the sample submitted to HTT. ${ }^{\text {d }}$ Oil obtained with cold dichloromethane (DCM) extraction on fresh microalgal slurry.

\section{A.2.3 Analysis of total fatty acids and free fatty acids}

The determination of the total fatty acids (TFA) in each sample was performed following a procedure slightly modified from the literature: ${ }^{21}$ the sample $(5 \mathrm{mg})$ was dissolved in 0.4 $\mathrm{ml}$ of dimethylcarbonate containing $0.048 \mathrm{mg}$ of tridecanoic acid, then $0.07 \mathrm{ml}$ of 2,2dimethoxypropane were added. Potassium methoxide $(0.2 \mathrm{ml})$ was added, and the sample 
was placed in an incubator at $90^{\circ} \mathrm{C}$, with shaking at $300 \mathrm{rpm}$ for $30 \mathrm{~min}$. The sample was cooled for $5 \mathrm{~min}$ to room temperature and $0.2 \mathrm{ml} \mathrm{BF}_{3}$ methanol were added before repeating the incubation for $30 \mathrm{~min}$. After cooling for $5 \mathrm{~min}$ to room temperature, $2 \mathrm{ml}$ of brine and $1 \mathrm{ml}$ of hexane containing $0.07 \mathrm{mg}$ of methylnonadecanoate were added before centrifuging at $4000 \mathrm{rpm}$ for $1 \mathrm{~min}$. The upper hexane-dimethylcarbonate layer, containing TFA, was transferred to vials for GC-FID. Each analysis was repeated in quadruplicate.

The determination of free fatty acids (FFA) was done by means of silylation: a sample (5 $\mathrm{mg}$ ) was dissolved in $0.1 \mathrm{ml}$ of acetonitrile containing $0.048 \mathrm{mg}$ of tridecanoic acid and $0.07 \mathrm{mg}$ of methylnonadecanoate, then $0.1 \mathrm{ml}$ of bis-trimethylsilyltrifluoroacetamide (BSTFA) containing $1 \%$ of tri-methylchlorosilane (TMCS) and $0.1 \mathrm{ml}$ of pyridine was added. The sample was placed in an incubator at $60^{\circ} \mathrm{C}$ for $1 \mathrm{~h}$. The solution containing FFA was transferred to vials for GC-FID analysis. Each analysis was repeated in quadruplicate. For both TFA and FFA analysis, GC analysis were performed with a Varian 2000 GC-FID system equipped with a HP-5 fused-silica capillary column (stationary phase poly[5\% diphenyl/95\% dimethyl]siloxane, $30 \mathrm{~m}, 0.25 \mathrm{~mm}$ i.d., $0.25 \mathrm{~mm}$ film thickness). Injector temperature was $280^{\circ} \mathrm{C}$ and the following thermal program was used: from $50^{\circ} \mathrm{C}$ to $200^{\circ} \mathrm{C}$ at $50^{\circ} \mathrm{C} / \mathrm{min}$, then from $200^{\circ} \mathrm{C}$ to $310^{\circ} \mathrm{C}$ at $5^{\circ} \mathrm{C} / \mathrm{min}$.

\section{A.2.4 Silica gel chromatography}

An aliquot of each oil (about $150 \mathrm{mg}$ ) was fractionated on a column (1 cm diameter 15 $\mathrm{cm}$ length) packed with silica gel using $150 \mathrm{ml}$ of hexane, $150 \mathrm{ml}$ of hexane/dichloromethane 1:1 (v/v), $150 \mathrm{ml}$ of dichloromethane, $200 \mathrm{ml}$ of ethyl acetate/dichloromethane $1: 1(\mathrm{v} / \mathrm{v}), 100 \mathrm{ml}$ of ethyl acetate, $200 \mathrm{ml}$ of ethyl acetate/methanol 1:1 (v/v), and $200 \mathrm{ml}$ of methanol, in this order. The solvent of each fraction was evaporated under vacuum and the resulting pure fraction was weighted; pure fractions eluted with various solvents were analyzed by SEC and were subjected to pyrolysis. The same procedure was also accomplished on dichloromethane extract (about $100 \mathrm{mg}$ ). Procedure and more results concerning the cold dichloromethane extraction can be seen Section 2 (Chapter 2).

\section{A.2.5 Size exclusion chromatography (SEC)}

An indication of the molecular weight distribution of the fraction from silica gel chromatography was obtained with an Agilent 1200 series HPLC system with 3 GPC 
PLgel $3 \mu \mathrm{m}$ MIXED-E columns connected in series. The column temperature was $40^{\circ} \mathrm{C}$, with a flow of $1 \mathrm{ml} / \mathrm{min}$ of tetrahydrofuran as solvent. Calibration was carried out using polystyrene standards with a molecular weight ranging from 162 to $29510 \mathrm{~g} / \mathrm{mol}$.

\section{A.2.6 Thermogravimetric analysis (TGA)}

TGA data was obtained with NETZSCH STA 449F3, using a thermal program starting from $30^{\circ} \mathrm{C}$ to $700^{\circ} \mathrm{C}$ with a temperature increase of $10^{\circ} \mathrm{C} / \mathrm{min}$. Samples $(7-8 \mathrm{mg})$ were analyzed using $\mathrm{Al}_{2} \mathrm{O}_{3}$ crucibles.

\section{A.2.7 Stepwise Py-SPME-GC-MS}

A series of five pyrolysis runs, each for 10 minutes at different set temperatures $\left(200^{\circ} \mathrm{C}\right.$, $300^{\circ} \mathrm{C}, 400^{\circ} \mathrm{C}, 500^{\circ} \mathrm{C}, 600^{\circ} \mathrm{C}$ ) were performed on the same sample. Actual inner temperatures $\left(200^{\circ} \mathrm{C}, 280^{\circ} \mathrm{C}, 350^{\circ} \mathrm{C}, 425^{\circ} \mathrm{C}, 500^{\circ} \mathrm{C}\right)$ were measured with a thermocouple on an empty quartz tube. Temperature ramp to the set temperature was $0.01^{\circ} \mathrm{C} / \mathrm{ms}$, which is the lowest ramp available with the CDS pyroprobe system. The sample was placed in the quartz tube and kept under $100 \mathrm{ml} / \mathrm{min}$ nitrogen stream for the duration of the experiment. SPME sampling was performed with a carboxen/PDMS in "exposed mode" as showed in previous work ${ }^{19}$ with an adaption concerning the distance between the fiber and the end quartz tube (Figure A.2), which was kept to $1 \mathrm{~mm}$ to eliminate all mass discrimination effects and to raise collection effectiveness. The gross correspondence of TGA and step-wise pyrolysis losses was checked by weighing the quartz tube before and after each step, and given the greater precision of TGA data, this was used for all calculations.

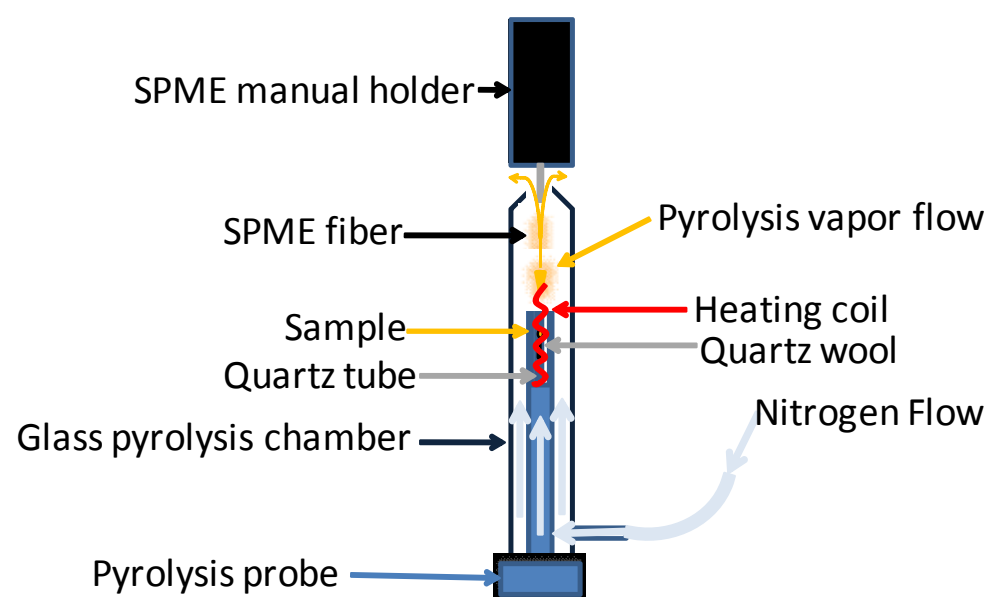

Figure A.2. Experimental apparatus used for this work 
After pyrolysis, the SPME fiber was desorbed in the injector port of the GC-MS system (Agilent 6851 equipped with 5668 quadruple detector) lined with SPME low expansion volume liner. Desorption was performed in splitless mode at $300^{\circ} \mathrm{C}$, and pyrolysis products were separated over a DB-5HT fused-silica capillary column (stationary phase poly $[5 \%$ diphenyl $/ 95 \%$ dimethyl]siloxane, $30 \mathrm{~m}, 0.25 \mathrm{~mm}$ i.d., $0.1 \mathrm{~mm}$ film thickness, upper temperature limit of $400^{\circ} \mathrm{C}$ ) with the following thermal program: $35^{\circ} \mathrm{C}$ for 5 minutes, then $10^{\circ} \mathrm{C} / \mathrm{min}$ until $350^{\circ} \mathrm{C}$, held for $10 \mathrm{~min}$.

The resulting chromatogram was totally integrated and divided into 401 detectable peaks. The absolute yield of compounds in each step, as example see Figure A.6, was calculated by multiplying the percentage of total GC-MS areas due to the analyte by the corresponding total mass loss due to the thermal step calculated by TGA, using the following formula:

$$
\mathrm{PP}\left(\mathrm{T}_{\mathrm{i}}\right)=\left[\mathrm{m}\left(\mathrm{T}_{\mathrm{i}}\right)-\mathrm{m}\left(\mathrm{T}_{\mathrm{i}-1}\right)\right] \cdot\left(\frac{\mathrm{A}_{\text {compound }}}{\mathrm{A}_{\text {tot }}}\right)
$$

Where $P P\left(T_{i}\right)$ is the absolute amount of the pyrolysis product produced by the thermal step (expressed as $\mathrm{m} / \mathrm{m}_{\text {sample }}$ ), $m\left(T_{i}\right)-m\left(T_{i-1}\right)$ is the mass loss due to the thermal step, and $A_{\text {compound }} / A_{\text {tot }}$ is the relative area of the analyte.

\section{A.2.8 Absolute quantification of compounds/polymers in the HTT oils: rationale and procedure}

For the absolute quantitation, data from stepwise pyrolysis were interpreted similarly to previous works dealing with kerogen characterization, ${ }^{22}$ and the qualitative data (from interpretation of chromatogram) were combined to quantitative data concerning the mass loss at a set temperature.

Basically, the TGA gives information about the mass loss caused by each thermal step, and the SPME analysis gives information on the relative amount of GC detectable compounds produced during that thermal step. Table A.2 shows the attribution of different compounds evolved in stepwise Py-SPME-GC-MS. All the detectable GC-MS peaks (also small peaks and overlapped peaks) were included in the data handling. Some compounds were identified (through NIST library and comparison with literature spectra) with certainty; for other compounds only the chemical class was attributed, and for about one hundred peaks no identification was possible (they were accounted as "not 
ID" compounds). The first two thermal steps $\left(200^{\circ} \mathrm{C}\right.$ and $\left.270^{\circ} \mathrm{C}\right)$ were attributed to the thermal desorption of GC detectable compounds. For steps performed at temperature higher than $270^{\circ} \mathrm{C}$, the detected compounds were interpreted as fragments produced from thermal degradation of larger polymers. For example, hydrocarbons (e.g. $\mathrm{C}_{4}-\mathrm{C}_{32}$ alkanes and alkenes with one double bond) evolved in the first two steps were interpreted as "hydrocarbons", whereas the same hydrocarbons revealed at higher temperature steps were considered as some pyrolysis products from hydrocarbon polymers (namely Desmodesmus sp. algaenan). The basic assumption was that, by following the pyrolysis with an extremely low temperature gradient and by using a robust GC column and high temperature program, almost all compounds evolved from slow thermal desorption/pyrolysis were GC-detectable or, at least, that the GC-MS compounds were representative of the overall composition of evolved pyrolysis products.

Therefore, for each step, the overall area percentage $\left(A / A_{\text {tot }}\right)$ of the compounds (for light compounds able to be desorbed) and of the pyrolysis derivatives (for heavy compounds) multiplied for the mass loss of the step could give a quantitative measure of the analyte. Therefore, for all desorbed analytes, the concentrations were then calculated as follows:

$\mathrm{X}_{\text {compound }} \approx \mathrm{PP}_{\text {compound }}\left(270^{\circ} \mathrm{C}\right)+\mathrm{PP}_{\text {compound }}\left(200^{\circ} \mathrm{C}\right)$

Where $X$ is the concentration $\left(\mathrm{g} / \mathrm{g}_{\text {sample }}\right)$ of the analyte, $P P_{\text {compound }}\left(270^{\circ} \mathrm{C}\right)$ and $P P_{\text {compound }}\left(200^{\circ} \mathrm{C}\right)$ are the absolute yields of the compound in first two pyrolysis temperature steps (see Section A.2.7). For macromolecular substances (e.g. peptides and algaenans), the overall abundance was calculated as follows:

$\mathrm{X} \approx \Sigma \mathrm{PP}_{\mathrm{i}}\left(350^{\circ} \mathrm{C}\right)+\Sigma \mathrm{PP}_{\mathrm{i}}\left(420^{\circ} \mathrm{C}\right)+\Sigma \mathrm{PP}_{\mathrm{i}}\left(500^{\circ} \mathrm{C}\right)$

Where $\Sigma P P_{i}$ indicats the summed absolute yields of all markers attributed to the macromolecule decomposition (see Table A.2) in high temperature steps. Finally the amount of "asphaltene" was described as the material which is not breakable at $500^{\circ} \mathrm{C}$ $\left(m_{500^{\circ} \mathrm{C}} / m_{0}\right)$. 
Table A.2. Scheme of the conceptual assumptions used for interpretation of stepwise pyrolysis and quantification of various classes of compounds

\begin{tabular}{|c|c|c|c|c|c|}
\hline \multirow{2}{*}{ Compounds revealed } & \multicolumn{5}{|c|}{ Pyrolysis step } \\
\hline & $200^{\circ} \mathrm{C}$ & $280^{\circ} \mathrm{C}$ & $350^{\circ} \mathrm{C}$ & $425^{\circ} \mathrm{C}$ & $500^{\circ} \mathrm{C}$ \\
\hline Hydrocarbons & \multicolumn{2}{|c|}{ Desorption } & \multicolumn{3}{|c|}{ Algeneans (polymethylens) } \\
\hline Cyclic dipeptides (DKP) & \multicolumn{2}{|c|}{ Desorption } & \multicolumn{3}{|c|}{ Proteins } \\
\hline Pyrroles & \multicolumn{2}{|c|}{ Desorption } & \multirow{4}{*}{\multicolumn{3}{|c|}{ Melanoidin-like polymers }} \\
\hline Pyrazines & \multirow{3}{*}{\multicolumn{2}{|c|}{$\begin{array}{l}\text { Desorption } \\
\text { Desorption } \\
\text { Desorption }\end{array}$}} & & & \\
\hline Pyridines & & & & & \\
\hline Piperidines & & & & & \\
\hline Alkylnitriles & \multirow{2}{*}{\multicolumn{2}{|c|}{$\begin{array}{l}\text { Desorption } \\
\text { Desorption }\end{array}$}} & \multirow{2}{*}{\multicolumn{3}{|c|}{ Bounded fatty amides $/$ Bounded fatty acids }} \\
\hline Alkylamides & & & & & \\
\hline Sterols & \multicolumn{2}{|c|}{ Desorption } & \multicolumn{3}{|c|}{ Bounded sterols and sterol esters } \\
\hline Polyarenes & \multicolumn{2}{|c|}{ Desorption } & \multicolumn{3}{|c|}{ Polyaromatic materials and pyrogenic PAHs } \\
\hline Indole derivatives & \multirow{2}{*}{\multicolumn{2}{|c|}{$\begin{array}{l}\text { Desorption } \\
\text { Desorption }\end{array}$}} & \multirow{2}{*}{\multicolumn{3}{|c|}{ Proteins }} \\
\hline Phenols & & & & & \\
\hline Benzene and alkyl benzenes & \multicolumn{2}{|c|}{ Desorption } & \multicolumn{3}{|c|}{$\begin{array}{l}\text { Algeneans and sporopollenins derivatives } \\
\text { (partially aromatic) }\end{array}$} \\
\hline Imidazoles & \multicolumn{2}{|c|}{ Desorption } & \multicolumn{3}{|c|}{ Proteins } \\
\hline Pyrrolidines & \multicolumn{2}{|c|}{ Desorption } & \multicolumn{3}{|c|}{ Fatty amido acids/proteins } \\
\hline Oxygenated compounds & \multicolumn{2}{|c|}{ Desorption } & \multicolumn{3}{|c|}{ Carbohydrates } \\
\hline Free fatty acids ${ }^{\mathrm{a}}$ & \multicolumn{2}{|c|}{ Desorption $^{a}$} & \multicolumn{3}{|c|}{ Bounded fatty acids ${ }^{\mathrm{a}}$} \\
\hline Sulfur containing compounds & \multicolumn{2}{|c|}{ Desorption } & \multicolumn{3}{|c|}{ Not found } \\
\hline Aromatic amines and amides & \multirow{2}{*}{\multicolumn{2}{|c|}{$\begin{array}{l}\text { Desorption } \\
\text { Desorption }\end{array}$}} & \multirow{2}{*}{\multicolumn{3}{|c|}{ Melanoidin-like polymers }} \\
\hline Other heterocyclic aromatic & & & & & \\
\hline "Carotenoids" derivatives and phytol & \multicolumn{2}{|c|}{ Desorption } & \multicolumn{3}{|c|}{ Sporopollenins } \\
\hline Others revealed not ID & \multicolumn{5}{|c|}{ Others revealed not ID } \\
\hline
\end{tabular}

a Data not used for final quantification (standard FFA and TFA analysis was used).

\section{A.3 Results and discussion}

\section{A.3.1 Analysis of TFA and FFA in the HTT oil samples}

Table A.3 shows total fatty acids and free fatty acid contents of analyzed samples, measured with a slightly modified procedure that is usually applied to vegetable oil, as described in Section A.2.3. Total fatty acids (TFA) accounted for more than $60 \%$ of the microalgae cold-extracted oil, and no free fatty acids (FFA) were observed. In comparison 
to the cold extract, TFA became less important mass contributors in the HTT oils samples (5-28\% of the weight depending on the sample), and especially for HTT oils obtained at high temperature and longer reaction time conditions, the "non-acyls" part of the oil could be considered to be the most important portion. Additionally, the amount of FFA largely increased as consequence of thermal treatment. In fact, they represented a large part of TFA in HTT oils, from $42 \%$ of samples obtained at $200^{\circ} \mathrm{C}$ for $60 \mathrm{~min}$, to $71-72 \%$ of samples obtained at $200^{\circ} \mathrm{C}$ for $5 \mathrm{~min}$ and $375^{\circ} \mathrm{C}$ for $5 \mathrm{~min}$.

Table A.3. Total fatty acids (TFA) and free fatty acids (FFA) content $\left(\% \mathrm{w} / \mathrm{w}_{\text {oil }}\right)$ of samples

\begin{tabular}{|c|c|c|c|c|c|c|c|c|c|c|c|c|}
\hline $\begin{array}{c}\mathrm{T}^{\mathrm{a}} \\
\left({ }^{\circ} \mathrm{C}\right) \\
\end{array}$ & $\begin{array}{c}\mathrm{RT}^{\mathrm{b}} \\
(\mathrm{min})\end{array}$ & $\begin{array}{c}\text { TFA } \\
(\mathrm{wt} \%) \\
\end{array}$ & $\begin{array}{c}\text { FFA } \\
(w t \%)\end{array}$ & $\begin{array}{c}\text { FFA/TFA } \\
(\%)\end{array}$ & $16: 4$ & $16: 3$ & $16: 0$ & $18: 4$ & $18: 2$ & $18: 1$ & $18: 3$ & 18:0 \\
\hline $25^{\mathrm{c}}$ & - & $62 \pm 10^{\mathrm{d}}$ & - & - & 2.5 & 14 & 3.3 & 10 & 4.3 & 2.1 & 3.4 & 1.3 \\
\hline 200 & 5 & $12.2 \pm 3.0$ & $8.8 \pm 2.2$ & 72 & - & - & 8.2 & - & - & 3.5 & - & 0.9 \\
\hline 200 & 60 & $24.7 \pm 5.8$ & $10.4 \pm 0.6$ & 42 & 3.1 & 1.2 & 4.7 & 0.6 & 1.5 & 3.1 & 7.9 & 0.6 \\
\hline 250 & 5 & $28.0 \pm 4.0$ & $12.5 \pm 0.4$ & 45 & 3.7 & 1.8 & 7.3 & 0.8 & 1.6 & 3.9 & 9.1 & 1.2 \\
\hline 300 & 5 & $12.8 \pm 0.5$ & $5.8 \pm 0.5$ & 45 & 0.4 & 0.9 & 4.6 & - & 3.4 & 3.0 & 1.1 & 1.1 \\
\hline 300 & 60 & $7.5 \pm 0.9$ & $3.8 \pm 0.1$ & 51 & - & - & 2.6 & - & - & 1.9 & 0.9 & 0.8 \\
\hline $300^{\mathrm{e}}$ & $60^{\mathrm{e}}$ & $5.5 \pm 1.9$ & $3.1 \pm 0.6$ & 56 & - & - & 2.2 & - & - & 1.9 & 0.9 & 0.7 \\
\hline 375 & 5 & $4.8 \pm 1.8$ & $3.4 \pm 0.2$ & 71 & - & - & 2.1 & - & - & 0.7 & - & 0.6 \\
\hline
\end{tabular}

${ }^{\mathrm{a}}$ HTT reaction temperature. ${ }^{\mathrm{b}}$ Reaction time of HTT. ${ }^{\mathrm{c}}$ Cold dichloromethane extract. ${ }^{\mathrm{d}}$ Mean \pm standard deviation. ${ }^{\mathrm{e}} 17 \% \mathrm{w}_{\text {microalgae }} / \mathrm{w}_{\text {slurry }}$ algae concentration in the sample submitted to HTT.

The samples obtained at intermediate conditions $\left(200^{\circ} \mathrm{C}\right.$ for $60 \mathrm{~min}$ and $250^{\circ} \mathrm{C}$ for $5 \mathrm{~min}$ ) were the ones with the highest TFA content (42\% e $45 \%$ respectively) and the lowest FFA content.

Looking at fatty acid composition (Table A.3), high temperature samples and, noticeably, samples from milder $\mathrm{HTT}\left(200^{\circ} \mathrm{C}\right.$ for $\left.5 \mathrm{~min}\right)$ were characterized by a lower content of polyunsaturated fatty acids (PUFA) than samples obtained at intermediate conditions $\left(200^{\circ} \mathrm{C}\right.$ for $60 \mathrm{~min}$ and $250^{\circ} \mathrm{C}$ for $5 \mathrm{~min}$ ), as well as the dichloromethane extract. Whereas the reduced amount of PUFA in high temperature samples can be related to higher reactivity (e.g. polymerization) of these FA, the absence of PUFA in sample from $200^{\circ} \mathrm{C} \mathrm{HTT}$ for $5 \mathrm{~min}$ is counterintuitive, but it could be related to a complex mechanism involving the hydrolysis of less polar PUFA containing BFA (e.g. tryacylclicerols), saponification and poor recovery of soaps in the sample obtained at $200^{\circ} \mathrm{C}$ for 5 min. This hypothesis will be discussed more in detail in the section devoted to the interpretation of the results in view of possible chemical pathways (Section A.3.7). 
The influence of reaction time on TFA concentration was different depending on the temperature of the hydrothermal treatment: at $200^{\circ} \mathrm{C}$, TFA concentration strongly increased from 5 to $60 \mathrm{~min}$, whereas at $300^{\circ} \mathrm{C}$, it was possible to observe a slight decrease from 5 to 60 min.

\section{A.3.2 Py-SPME-GC-MS: qualitative analysis}

Py-GC-MS and one-step Py-SPME-GC-MS $\left(700^{\circ} \mathrm{C}, 0.01^{\circ} \mathrm{C} / \mathrm{ms}\right)$ were tested in a preliminary way on HTT samples, mainly to obtain more information on the chemical nature of the unsaponifiable and non-GC-detectable matter. Pyrograms were characterized by a large number of overlapping peaks, of which only a portion was tentatively or certainly identified. 401 GC-MS peaks were quantified; among them, 274 compounds were totally or partially identified (partially means an identification of the chemical class to which the compounds belong) in the chromatograms obtained by PySPME-GC-MS. The compounds identified are grouped into the following chemical classes:

1. Pyrazines: GC detectable products of Maillard reaction between sugars and amino acids or amines (formed by amino acids decomposition) and pyrolysis products of Melanoidin-like polymers; ${ }^{23}$

2. Pyridines: pyrolysis products of "strongly" charred/coked proteins ("black nitrogen") and pyrolysis products of Melanoidin-like polymers $;{ }^{23}$

3. Pyrroles: pyrrole derivatives (pyrrole and various alkyl-pyrroles), markers of melanoidin polymers ${ }^{24}$, also from the pyrolysis/thermal degradation of chlorophylls (porphyrin) and proteins ("black nitrogen") $;^{25}$

4. Alkylnitriles and fatty-amides: HTT products from condensation of ammonia and fatty acids or pyrolysis products formed by the high temperature $\left(>350^{\circ} \mathrm{C}\right)$ reaction between ammonia (from proteins pyrolysis) and fatty acids $;{ }^{26}$

5. Indoles, phenols, aromatic amides: pyrolysis products that can be produced by side chains of amino acids (such as phenylalanine, tryptophan and tyrosine); ${ }^{27}$

6. PAHs: polycyclic aromatic hydrocarbons with two or more aromatic rings, mainly in polyalkylated form, which can be formed from various biological precursors in HTT or pyrolysis of biomass; ${ }^{28}$

7. Cyclic dipeptides: diketopiperazines (DKP) which are typical pyrolysis products of peptides, formed through depolymerization and cyclization; $;^{27}$ 
8. FFA: free fatty acids dissolved in the oil and formed from bounded fatty acids released by mean of pyrolysis; ${ }^{29}$

9. Hydrocarbons: hydrocarbons with variable length (alkanes and alkenes) produced by TFA and FFA pyrolysis/decarboxylation or $\beta$-cleavage, ${ }^{29}$ or by algaenans (hydrocarbon containing polymers) pyrolysis;

10. Carotenoids fragments and phytol derivatives: fragmentation products of both carotenoids (low temperature) and sporopollenins (high temperature), another refractory polymer typical of microalgae;

11. Pyrrolidinones: HTT products obtainable from thermal degradation of DKP or peptides; $;^{30}$

12. Piperidines: compounds which can be obtained from both HTT of proteins as well as from pyrolysis of melanoidin ${ }^{23}$

13. Oxygenated compounds (pyrolysis-oil-like products): pyrolysis products of cellulose or other carbohydrates;

14. Sulphurated compounds: small sulphur containing compounds dimethyl-disulfide and dimethyl-trisulfide, which are probably originated from disulfide bonds in proteins and/or by thiol-disulfide exchange reactions;

15. Alkyl-aromatics: xylene, ethylbenzene, styrene and other alkyl aromatics, cyclization pyrolysis products of small hydrocarbons or fatty acids. ${ }^{11}$ They can be a byproduct of thermal decomposition of algaenans, lipids or proteins ${ }^{31}$ or they can indicate the presence of aromatic structures in the algaenan-like material.

\section{A.3.3 Preliminary characterization through column chromatography/SEC/Py-SPME-GC-MS}

In the preliminary characterization of the samples, the HTT oils and DCM extract were fractionated by means of column chromatography. Different fractions were obtained by elution with solvents with increasing polarity (starting from hexane until pure methanol). The isolated fractions were then subjected to Py-SPME-GC-MS and SEC to gather more information on their composition. Figure A.3 shows the mass percentage eluted sequentially by means of increasing polar solvent. More than $90 \%$ of oil mass was eluted with a solvent mixture, which was more polar than dichloromethane/ethylacetate (fractions 4, 6 and 7), indicating that the oil was mainly formed by heavy and/or polar compounds. The DCM extracted oil eluted almost totally (more than $60 \% \mathrm{w} / \mathrm{w}$ ) with 
dichloromethane/ethylacetate and in a minor amount with ethylacetate/methanol. Noticeably, all the HTT oils except the one obtained at $200^{\circ} \mathrm{C}$ for 5 min showed a similar mass distribution, with most of mass eluted with dichloromethane/ethylacetate, ethylacetate/methanol and a lot of mass retained on the silica. Because the oils were obtained through a solvent/water partition (thus, we could exclude the presence of strongly polar substances), this could indicate the presence of a high amount of high molecular weight compounds. Among the tested samples, the oil obtained at $200^{\circ} \mathrm{C}$ for 5 min was peculiar, because it was characterized by low amount of oil mass retained on the silica and a larger amount of oil eluted with methanol (last fraction of the column chromatography).

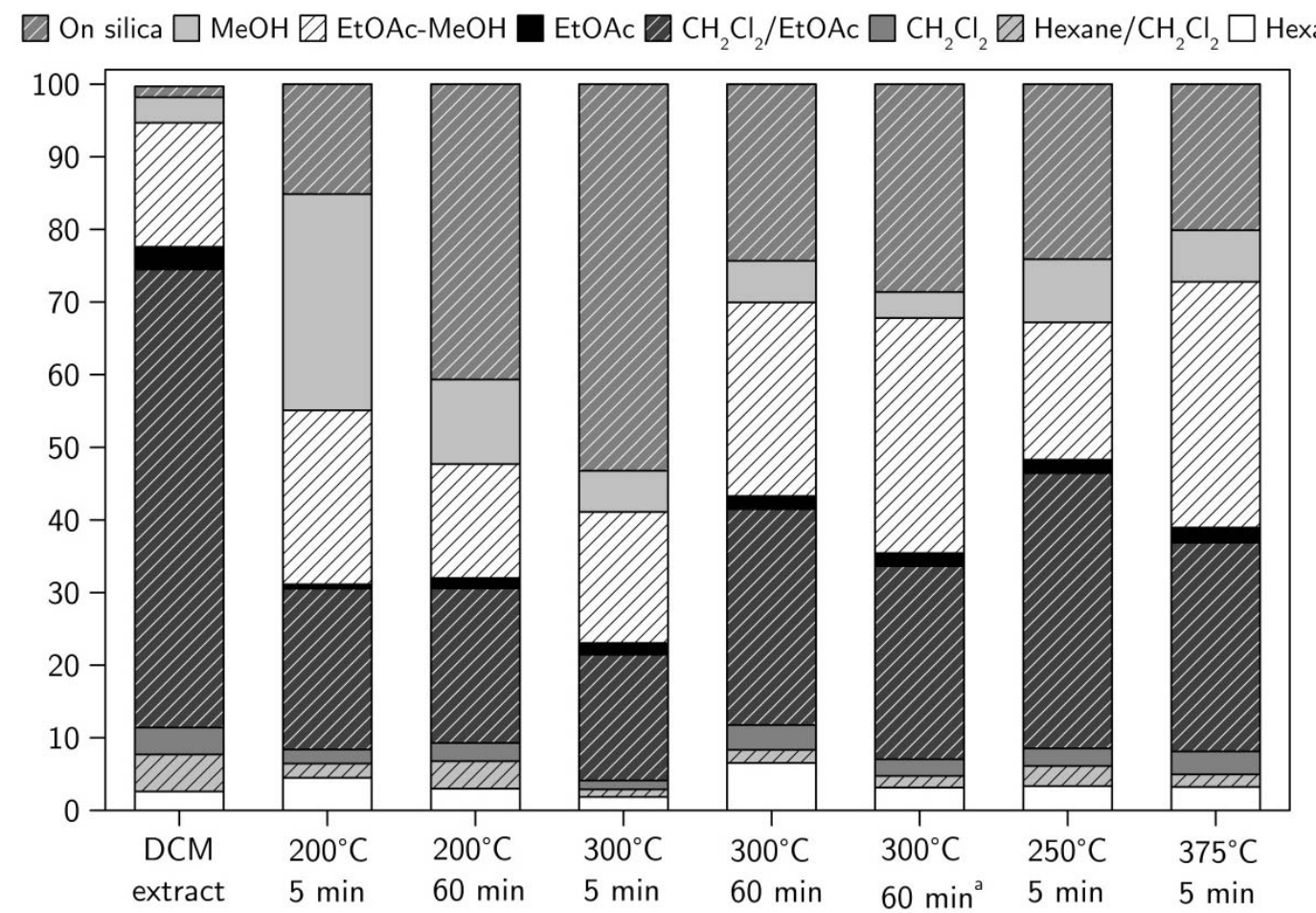

Figure A.3. Mass fraction eluted sequentially by silica gel column chromatography. ${ }^{\text {a }} 17 \% \mathrm{w}_{\text {microalgae }} / \mathrm{w}_{\text {slurry }}$ algae concentration in the sample submitted to HTT

Interestingly, the fractions eluted with the same solvent mixtures from different oils revealed a roughly identical pyrogram and similar SEC chromatogram, indicating a similarity of the same fractions obtained from different oil samples. This means that HTT produced a variable relative amount of the various chemical categories, but "qualitatively" the products were similar. 
As a typical example, Figure A.4 shows a chromatogram obtained by Py-SPME-GC-MS and size exclusion chromatography (SEC) of the most important fractions (4,6 and 7) of sample obtained at $200^{\circ} \mathrm{C}$ for 5 min reaction time.

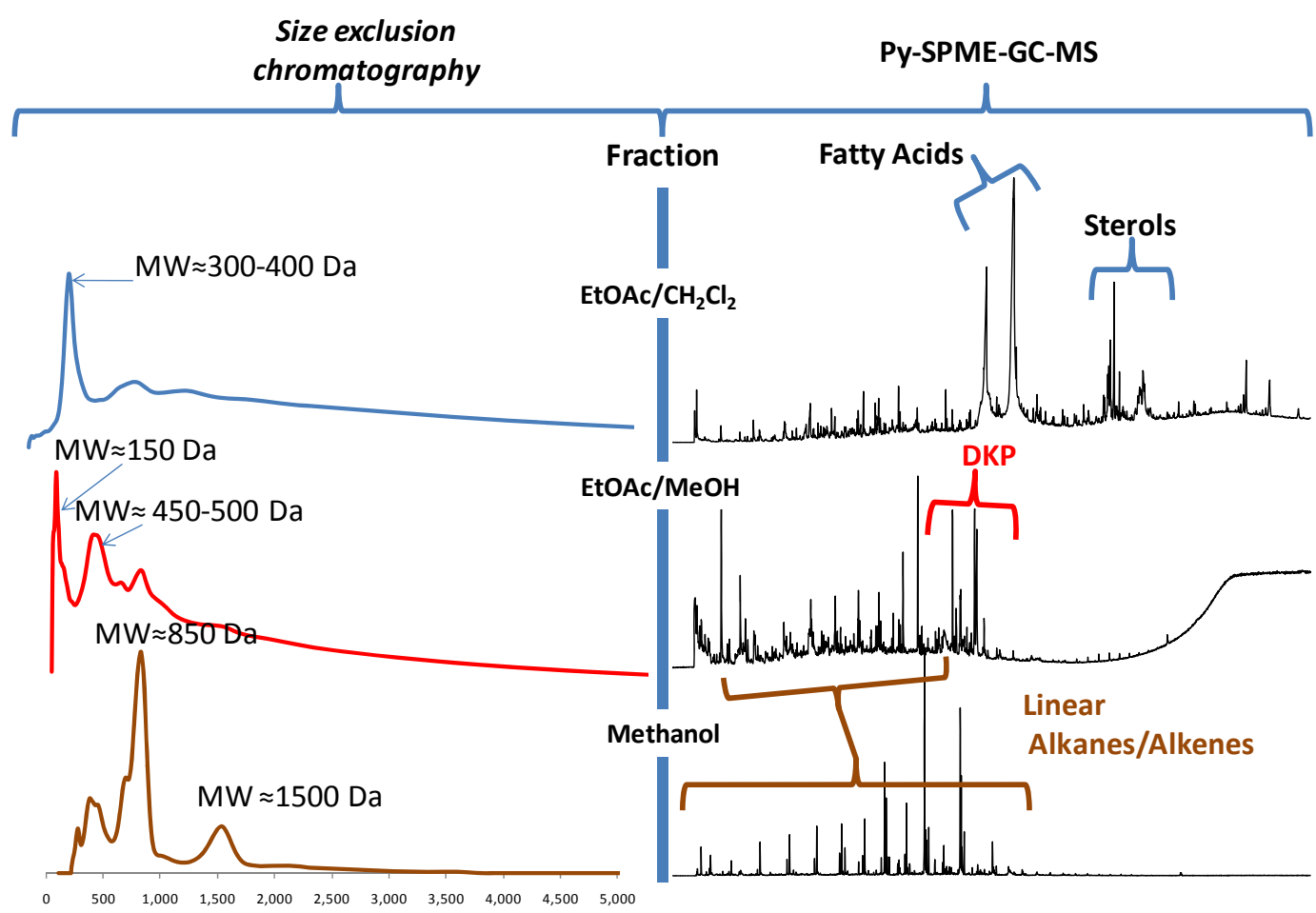

Figure A.4. Typical SEC chromatograms (left) and pyrograms from $700^{\circ} \mathrm{C}\left(\mathrm{ramp} 10^{\circ} \mathrm{C} / \mathrm{s}\right)$ Py-SPME-GCMS (right) of fractions 4, 6 and 7 of the column chromatography (eluted with EtOAc/ $\mathrm{CH}_{2} \mathrm{Cl}_{2}$, EtOAc/MeOH and $\mathrm{MeOH}$ respectively). Data are obtained from fractionation and analysis of the HTT oil obtained at $200^{\circ} \mathrm{C}$ for $5 \mathrm{~min}$

Pyrolysis of fraction $4\left(\mathrm{AcOEt} / \mathrm{CH}_{2} \mathrm{Cl}_{2}\right)$ revealed a large amount of fatty acids (FA) and sterols in the pyrogram, as well as a large SEC peak at about 300-400 Da, indicating the presence of large amount of lipid derived materials in this fraction. On the contrary, upon pyrolysis, fraction $6(\mathrm{AcOEt} / \mathrm{MeOH})$ showed no FA but a remarkable release of DKP and "algaenan-derived" linear hydrocarbons, ${ }^{32}$ which indicated the presence of a certain amount of DKP/peptides and algaenans, respectively. SEC of fraction 6 showed a small group of peaks with relatively low molecular weight (130-190 Da), probably attributable to the presence of hydrophobic DKP and/or dipeptides. In fraction 7 (methanol), SEC revealed three broad peaks at 400-500 Da, 800-900 Da and around 1500 Da, which could be interpreted (because a large amount of long linear hydrocarbons is observed in the pyrogram) as monomers, dimers and trimers of the basic structural unit of trilaminar outer wall of Scenedesmus and other green microalgae. ${ }^{32,33}$ 
The fact that these large molecular aliphatic substances, even if characterized by low polarity, eluted so difficulty from silica gels is not that intuitive and could be related to the dimensions and strong packing of these large saturated aliphatic structures.

In conclusion, column chromatography coupled with Py-SPME-GC-MS and SEC revealed the presence of three large classes of compounds (separated by column fractionation) that accounted for most of the oil mass. One was formed by lipid derived compounds (containing FA, sterols and some derivatives of these compounds). The second fraction revealed the presence of a considerable amount of protein derived materials (mainly DKP, peptides and/or cyclic peptides) and another formed by poorly soluble material not easily eluted, probably consisting of algaenan derivatives (which was revealed by both pyrolysis and by SEC of fraction 7) or/and formed by compounds that are not completely breakable upon pyrolysis (e.g. asphaltenes ).

\section{A.3.4 Stepwise Py-SPME-GC-MS: quantitative analysis}

As a result of the large complexity of the oil matrix and difficulties in chromatographic separations, a different column-free separation approach, by means of differential thermal reactivity, was undertaken.

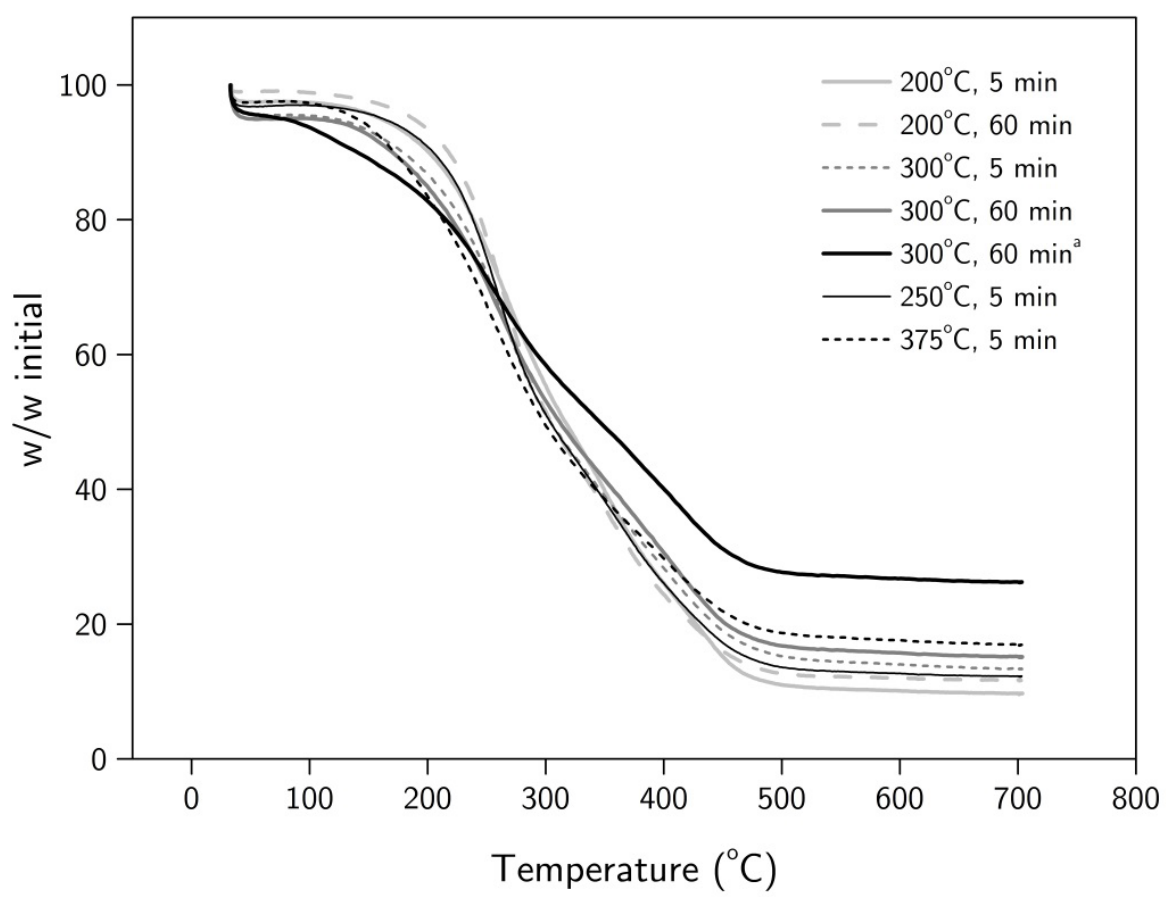

Figure A.5. Thermogravimetric analysis of seven HTT oil samples obtained under different conditions. ${ }^{\mathrm{a}} 17 \% \mathrm{w}_{\text {microalgae }} / \mathrm{w}_{\text {slurry }}$ algae concentration in the sample submitted to HTT 
Therefore, to obtain a clearer result, stepwise pyrolysis was added in the analytical scheme and thermogravimetric data (TGA) were coupled to semiquantitative data obtained through Py-SPME-GC-MS. Figure A.5 shows the thermogravimetric plot obtained from the oil samples, and Table A.4 shows the mass losses measured for each pyrolysis step.

Table A.4. Mass loss of each step calculated from TGA

\begin{tabular}{|c|c|c|c|c|c|c|c|c|}
\hline \multirow[b]{2}{*}{ Sample no. } & \multirow{2}{*}{$\begin{array}{c}\mathbf{T}^{\mathrm{a}} \\
\left({ }^{\circ} \mathbf{C}\right)\end{array}$} & \multirow{2}{*}{$\begin{array}{c}\mathrm{RT}^{\mathrm{b}} \\
(\mathrm{min})\end{array}$} & \multicolumn{5}{|c|}{ Pyrolysis Step $\left({ }^{\circ} \mathrm{C}\right)$} & \multirow{2}{*}{$\begin{array}{c}\text { Total } 500^{\circ} \mathrm{C} \\
\text { weight loss } \\
\text { w/ } / \mathrm{w}_{0} \%\end{array}$} \\
\hline & & & 200 & 280 & 350 & 425 & 500 & \\
\hline 1 & 200 & 5 & 11 & 26 & 24 & 19 & 10 & 90 \\
\hline 2 & 200 & 60 & 7 & 32 & 24 & 17 & 7 & 85 \\
\hline 3 & 300 & 5 & 14 & 27 & 20 & 15 & 8 & 82 \\
\hline 4 & 300 & 60 & 16 & 24 & 18 & 17 & 7 & 81 \\
\hline 5 & $300^{\mathrm{c}}$ & $60^{c}$ & 18 & 19 & 14 & 14 & 8 & 72 \\
\hline 6 & 250 & 5 & 10 & 31 & 21 & 16 & 8 & 85 \\
\hline 7 & 375 & 5 & 18 & 26 & 18 & 13 & 7 & 80 \\
\hline
\end{tabular}

a Reaction temperature of HTT. ${ }^{\mathrm{b}}$ Reaction time of HTT. ${ }^{\mathrm{c}} 17 \mathrm{wt} \%$ algae concentration in the sample submitted to HTT.

In general, when HTT conditions became harsher, the mass losses were shifted towards the lower temperature, and this could indicate that, by raising the temperature of HTT, the volatility and/or the thermal reactivity of compounds in the oil increased. For all samples, it should be noticed that, even by using long desorption/pyrolysis time (10 min each step), only a part (less than $50 \% \mathrm{w} / \mathrm{w}_{\text {sample }}$ ) of the sample volatilized under $280^{\circ} \mathrm{C}$. As suggested by other authors, ${ }^{3,22}$ the steps below $280^{\circ} \mathrm{C}$ could be considered as the maximum operational range for the effective evaporation in a $\mathrm{GC}$ injector port, making only a part of the oil directly detectable by GC-MS.

Therefore, because the rest of the oil (more than $50 \%$ ) required higher temperatures to be volatilized, it was argued that it was formed by polymers or other high molecular weight compounds. From TGA data, this fraction resulted to be formed partially by thermal breakable materials (around 40-50\%) and by not volatile materials, even at $500^{\circ} \mathrm{C}$ (between $28 \%$ and $10 \%$ of the oil weight), and thus, it was probably formed by asphaltene-like or maltene-like material (still stable to $500^{\circ} \mathrm{C}$ pyrolysis conditions). As for column fractionation, the pyrogram obtained for each Py-SPME-GC-MS step (as the pyrogram of each fraction) is simpler than the one obtained by one-step $500^{\circ} \mathrm{C}$ pyrolysis and allowed to conduct a more in-depth investigation of the HTT oil samples. A typical 
example of the results obtained (in this case from the sample obtained at $300^{\circ} \mathrm{C}$ for 60 min) is shown in Figure A.6. As example, Figure A.7 graphically shows the results obtained by combining TGA and Py-SPME-GC-MS data. This plot reveals that the TGA losses were a complex sum of different pyrolysis products volatilization. All samples were performed in duplicate, and the temperature of release of each compound was in all cases almost constant, indicating a good reproducibility of the method.

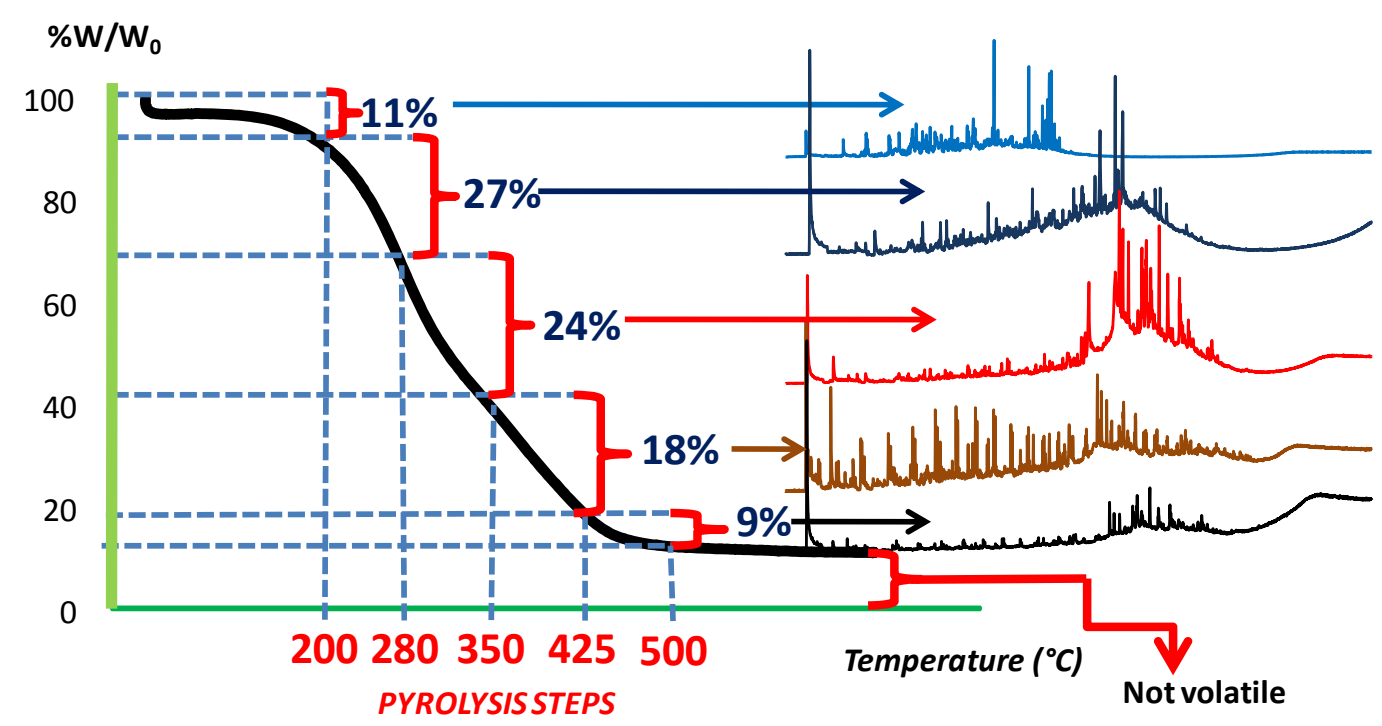

Figure A.6. Example of the five chromatograms resulting from 5 steps Py-SPME-GC-MS coupled with thermogravimetric data. Analysis of the sample obtained at $300^{\circ} \mathrm{C}$ for $60 \mathrm{~min}$

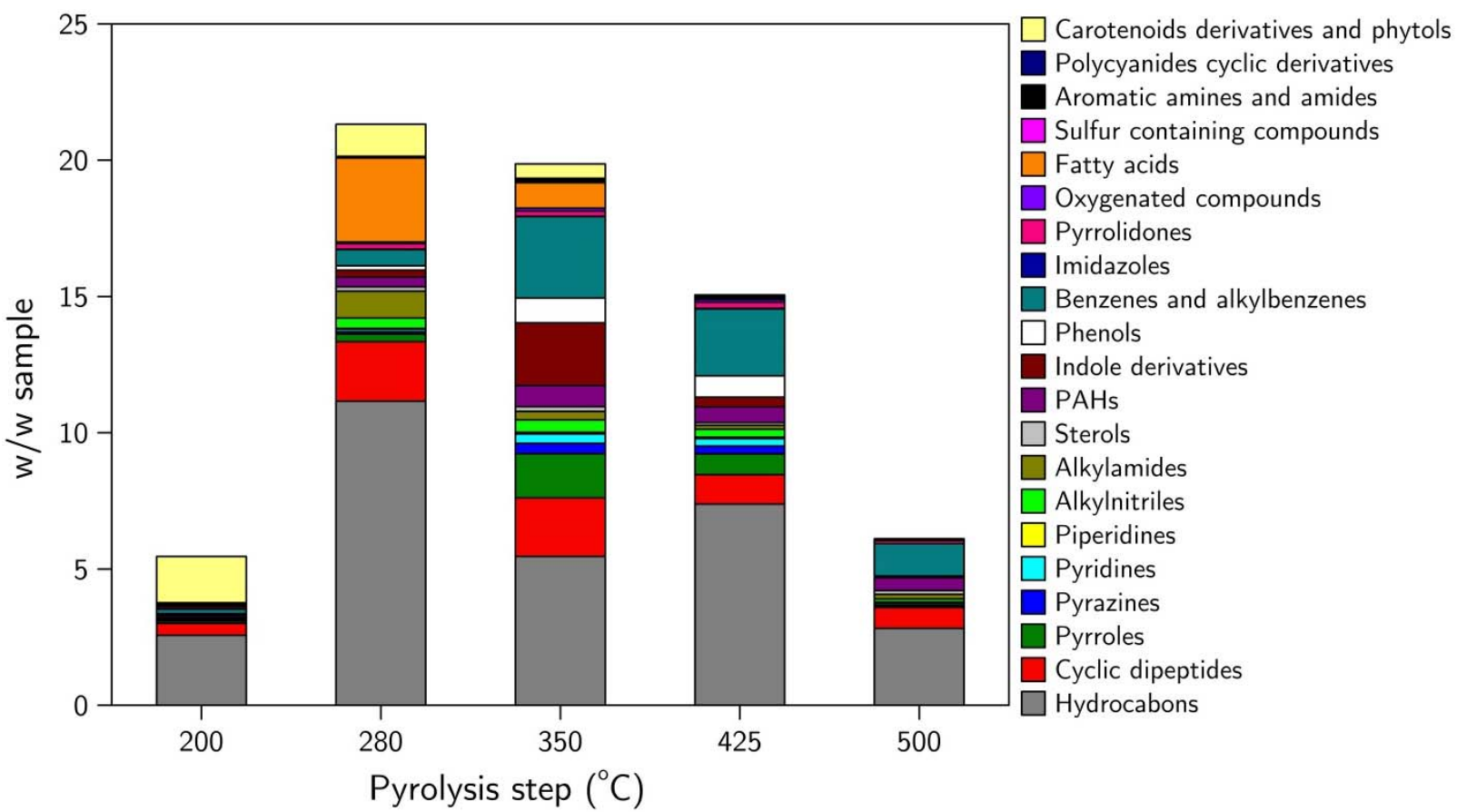

Figure A.7. Example of different species evolution during stepwise Py-SPME-GC-MS experiments on sample 2 (HTT at $200^{\circ} \mathrm{C}$ for $60 \mathrm{~min}$ ). The height of the bar represents the overall mass loss and the colored portions represent the relative amount of each class in the pyrogram produced by the step 
Qualitatively, in the first two steps $\left(200^{\circ} \mathrm{C}\right.$ and $\left.280^{\circ} \mathrm{C}\right)$ there was a simple volatilization of products that are usually GC analyzable, and the chromatograms closely resembled to those obtained by means of simple GC-MS injection of the HTT oil solubilised in dichloromethane. Compounds detected in these pyrolysis steps consisted mainly of hydrocarbons (in particular $\mathrm{C}_{15} \mathrm{H}_{32}$ and $\mathrm{C}_{17} \mathrm{H}_{36}$ ), pyrazines, diketopiperazines (DKP), alkyl-pyrroles and pyrrolidones. The last three compounds classes were usually found in pyrolysis oils obtained with flash pyrolysis of protein-containing materials. ${ }^{34}$ These compounds occurred in the highest concentration in samples from HTT performed above the supercritical point $\left(375^{\circ} \mathrm{C}\right.$ for $\left.5 \mathrm{~min}\right)$.

Compounds revealed during desorption steps $\left(200^{\circ} \mathrm{C}\right.$ and $\left.280^{\circ} \mathrm{C}\right)$ were similar to those revealed by GC-MS analysis and similar to those detected by other authors using direct GC-MS analysis of HTT obtained from similar feedstock. ${ }^{1,4}$ During subsequent steps performed at higher temperatures $\left(420-500^{\circ} \mathrm{C}\right)$, the chromatograms became featured by pyrolysis products of polymers or, more in general, by volatile products from thermal degradation of materials that are not usually GC-amenable. In particular, in the $350^{\circ} \mathrm{C}$ $420^{\circ} \mathrm{C}$ steps, the pyrogram was characterized by the presence of peptides, pyrolysis products and alkyl-pyrroles (e.g. the sample from HTT at $300^{\circ} \mathrm{C}$ for $60 \mathrm{~min}$ ), fatty acids (e.g. the sample from HTT at $250^{\circ} \mathrm{C}$ for $5 \mathrm{~min}$ ), as well as some linear and branched polyenes hydrocarbon chains (probably from degradation of carotenoids-like compounds). In the last steps $\left(420-500^{\circ} \mathrm{C}\right)$, the chromatogram was almost totally formed by signals of aliphatic alkanes and monounsaturated alkenes up to $\mathrm{C}_{30}$, with typical distribution also observed by Derenne et al. and Allard et al. for Chlorella fusca and Scenedesmus communes (which are very similar to desmodesmus sp.) algaenans. ${ }^{32,33}$

Looking at the evolution of different pyrolysis products among subsequent temperature steps, the stepwise pyrolysis of HTT oil can be interpreted as an evaporation of GCdetectable substances followed by thermal degradation of peptide-like materials and/or melanoidin-like polymers and, finally, at temperatures higher than $420^{\circ} \mathrm{C}$, the breaking of $\mathrm{C}-\mathrm{C}$ bonds and the release of algaenan fragments.

\section{A.3.5 Py-SPME-GC-MS and TGA: Evaluation of the final composition of HTT oil}

The use of pyrolysis products distribution for establishing the exact type of substances/polymers mixture in the pyrolyzed sample is not an easy task. Different authors showed that, in the case of well-known mixtures (e.g. lignin + cellulose), by 
means of semiquantitative analysis of pyrolysis product relative abundances, it is possible to predict the composition of the starting materials..$^{35}$ Nevertheless, when this approach is applied to a totally unknown matrix, formed by thermally altered materials, the main problem is that some pyrolysis products can be primary pyrolysis products (a fragment of the polymer) of a polymer, secondary pyrolysis products of another, or they can be thermal degradation products already present in the material. ${ }^{11}$ A typical example is alkyl-pyrroles, which can be considered primary pyrolysis products of pyrrole containing macromolecules (melanoidin or chlorophyll), as well as secondary pyrolysis products of proteins (from thermal decomposition of DKP or amino acids).

By means of stepwise pyrolysis, it is possible to partially separate the degradation of the polymers with different chemical reactivity. Therefore, it is possible to observe if the pyrolytic formation/evaporation of two substances is linked to the same material (if two stepwise pyrolysis peaks reasonably correspond) or to two different polymers (if the volatilization of two compounds is in different temperature ranges). Following the classification summarized in Table A.2, pyrolysis fragments produced in different steps were attributed to different starting materials in the HTT oil. Far from giving this approach as being the most accurate, it could be considered as a simple method for the investigation of both GC amenable and "heavy" fraction of HTT oil (Table A.5). Moreover, it allowed us to attempt a quantitative analysis of the HTT oil composition, targeted to understand relative changes due to different HTT reaction conditions.

All quantifications were performed in duplicate, showing a really good repeatability for compounds normally detectable in direct GC-MS, with relative standard deviation (RSD) below 10\%, and an acceptable repeatability (for the purpose of a fact-finding study) for "polymeric" substances (RSD below 30\%). Finally, the quantification of compounds/polymers with low concentration was generally flawed by a higher RSD than quantification of the most abundant HTT oil constituents. 
Table A.5. Concentrations of various classes of compounds in HTT samples

\begin{tabular}{|c|c|c|c|c|c|c|c|c|}
\hline Temperature $\left({ }^{\circ} \mathrm{C}\right)$ & 25 & 200 & 200 & 250 & 300 & 300 & $300^{\mathrm{a}}$ & 375 \\
\hline Reaction time (min) & - & 5 & 60 & 5 & 5 & 60 & $60^{\mathrm{a}}$ & 5 \\
\hline Hydrocarbons & 3 & 12 & 9.8 & 11 & 6.9 & 6.3 & 6.0 & 8.0 \\
\hline Cyclic dipeptides & - & 0.3 & 1.9 & 0.5 & 4.3 & 1.5 & 3.1 & 3.2 \\
\hline Pyrroles & - & 1.0 & 0.3 & 0.8 & 1.1 & 3.5 & 3.2 & 3.9 \\
\hline Pyrazines & - & 1.0 & 0.1 & 1.0 & 0.9 & 2.1 & 2.6 & 1.2 \\
\hline Pyridines & - & 0.8 & 0.1 & 0.3 & 0.4 & 0.8 & 0.6 & 1.0 \\
\hline Piperidines & - & 0.1 & - & 0.1 & 0.4 & 0.6 & 0.5 & 0.5 \\
\hline Alkylnitriles & - & 0.7 & 0.3 & 0.2 & 1.3 & 0.7 & 0.6 & 1.6 \\
\hline Alkylamides & - & 0.4 & 0.7 & 0.2 & 0.8 & 1.0 & 1.4 & 1.2 \\
\hline Sterols & 5 & 0.3 & 0.4 & 0.2 & 0.1 & 0.2 & 0.1 & 0.1 \\
\hline Polyarenes & - & 2.3 & 0.3 & 1.7 & 0.6 & 1.2 & 0.7 & 1.0 \\
\hline Indole derivatives & - & 0.3 & 0.2 & 0.4 & 1.0 & 2.0 & 1.4 & 1.9 \\
\hline Phenols & - & 0.6 & 0.1 & 0.2 & 0.5 & 0.9 & 1.4 & 2.6 \\
\hline Benzenes and alkyl benzenes & - & 1.1 & 0.6 & 0.6 & 1.2 & 2.7 & 2.0 & 1.7 \\
\hline Imidazoles & - & - & - & 0.1 & - & 0.1 & - & 0.1 \\
\hline Pyrrolidinones & - & 0.6 & 0.2 & 0.3 & 1.1 & 2.6 & 2.1 & 3.0 \\
\hline Oxygenated compounds & - & 0.2 & 0.1 & 0.4 & 0.4 & 1.1 & 0.8 & 0.7 \\
\hline Free fatty acids & - & 8.8 & 10 & 13 & 5.8 & 3.8 & 3.1 & 3.4 \\
\hline Sulfur containing compounds & - & - & - & - & - & - & - & 0.1 \\
\hline Aromatic amines and amides & - & 0.3 & 0.1 & 0.7 & 0.2 & 0.6 & 0.5 & 0.4 \\
\hline Polycyanides cyclic derivatives & - & 0.1 & - & 0.1 & 0.1 & 0.2 & 0.1 & 0.2 \\
\hline Carotenoids derivatives and phytol & 11 & 2.3 & 2.1 & 3.5 & 0.1 & 0.1 & - & 0.1 \\
\hline Carbohydrates & - & 0.1 & 0.2 & 0.1 & 0.2 & 0.3 & 0.2 & 0.4 \\
\hline Peptides & 2.5 & 4.8 & 6.5 & 2.6 & 7.4 & 4.2 & 4.6 & 4.1 \\
\hline Sporopollenins & - & 1.7 & 0.4 & 3.0 & 0.1 & 0.2 & 0.2 & 0.4 \\
\hline Algaenan (polymethylens) & 5.2 & 16 & 11.2 & 5.7 & 9.5 & 11 & 6.7 & 10 \\
\hline Algaenan derivatives ${ }^{\mathrm{b}}$ & - & 3.1 & 4.8 & 2.4 & 4.9 & 4.9 & 4.2 & 4.8 \\
\hline Melanoidin & - & 3.3 & 3.0 & 0.8 & 2.5 & 2.8 & 2.2 & 2.3 \\
\hline Breakable aromatic polymers & - & 2.1 & 1.3 & 0.6 & 0.8 & 0.6 & 0.4 & 0.8 \\
\hline Bounded fatty acids & 62 & 3.4 & 14 & 16 & 7.0 & 3.7 & 2.4 & 1.4 \\
\hline Sum of identified by GC-MS & 89 & 68 & 69 & 66 & 60 & 60 & 51 & 60 \\
\hline Asphaltene $^{c}$ & 2 & 10 & 13 & 14 & 16 & 17 & 28 & 19 \\
\hline Not ID & 6 & 22 & 18 & 20 & 24 & 23 & 20 & 21 \\
\hline $\mathrm{w}_{\text {oil }} / \mathrm{w}_{\text {biomass }}$ & 7.1 & 10 & 23 & 27 & 40 & 47 & 47 & 43 \\
\hline
\end{tabular}

${ }^{\mathrm{a}} 17 \% \mathrm{w} / \mathrm{w}$ slurry concentration. ${ }^{\mathrm{b}}$ Alkyl aromatic compounds, which are evolved at high temperature, and then were attributed to algaenans thermal alteration. ${ }^{\mathrm{c}}$ Not volatile at $500^{\circ} \mathrm{C}$. 
Because one of the main targets of the analysis was to identify the molecular form in which nitrogen (which can be a problem for the downstream processing of the oil) was transferred to HTT oil, the method consistency was checked especially on the nitrogen containing compounds. Figure A.8 shows the relationship between N content calculated from the composition obtained with Py-SPME-GC-MS and the real N content obtained with elemental analysis. Besides a relative imprecision of the analysis, especially for samples with low concentration of nitrogen (e.g. sample 6), it was possible to notice a close linear relationship between the $\mathrm{N}$ content of the sample (as measured by elemental analysis) and the sum of the fractions of the $\mathrm{N}$ containing compounds/fragments detected. Considering the $\mathrm{N}$ content of identified pyrolysis products of Py-SPME-GC-MS analysis, it was possible to account about $70 \%$ of nitrogen present in the oil. The remaining $30 \%$ was probably retained in the large number of not identified compounds, in the non-volatile part of the HTT oil $\left(\right.$ coked at $\left.500^{\circ} \mathrm{C}\right),{ }^{36}$ or eliminated as ammonia (non-quantified) during pyrolysis. By assuming that "not identified" compounds had grossly the same average elemental composition of identified compounds, more than $92 \%$ of the nitrogen can be detected in pyrolysis fragments, indicating a general consistence of the stepwise Py-SPME-GC-MS approach.

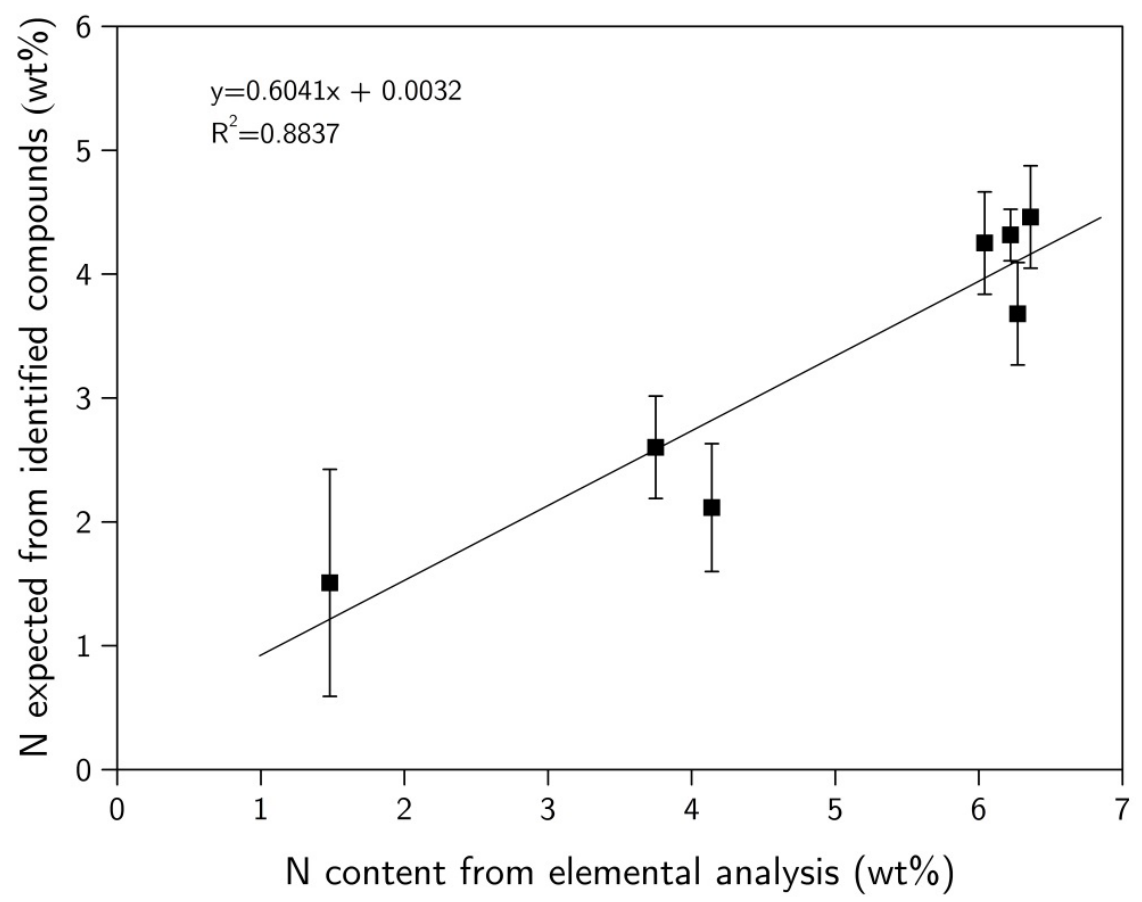

Figure A.8. Scattering graph of expected N starting from Py-SPME-GC-MS analysis (composition shown in Table A.5) and $\mathrm{N}$ of the sample obtained by means of elemental analysis 


\section{A.3.6 Influence of HTT conditions on overall yields and oil composition}

By multiplying the composition (obtained by the elaboration of stepwise Py-SPME-GCMS data) and the mass yields of HTT oils (Table A.1) it was possible to attempt a tentative elucidation of the overall effect of HTT conditions on the "formation" of various compounds and chemical categories.

Others $\square$ Asphaltenes-like materials $\square$ HMW Maillard reaction ${ }^{\mathrm{a}} \square$ LMW Maillard reaction $^{\mathrm{b}} \square$ AA side chains

Cyclic dipeptides (DKP) $\square$ Polypeptides $\square$ Pyrrolidones $\square$ Alkylamides and Alkylnitriles $\square$ Lipids and algaenan derivatives
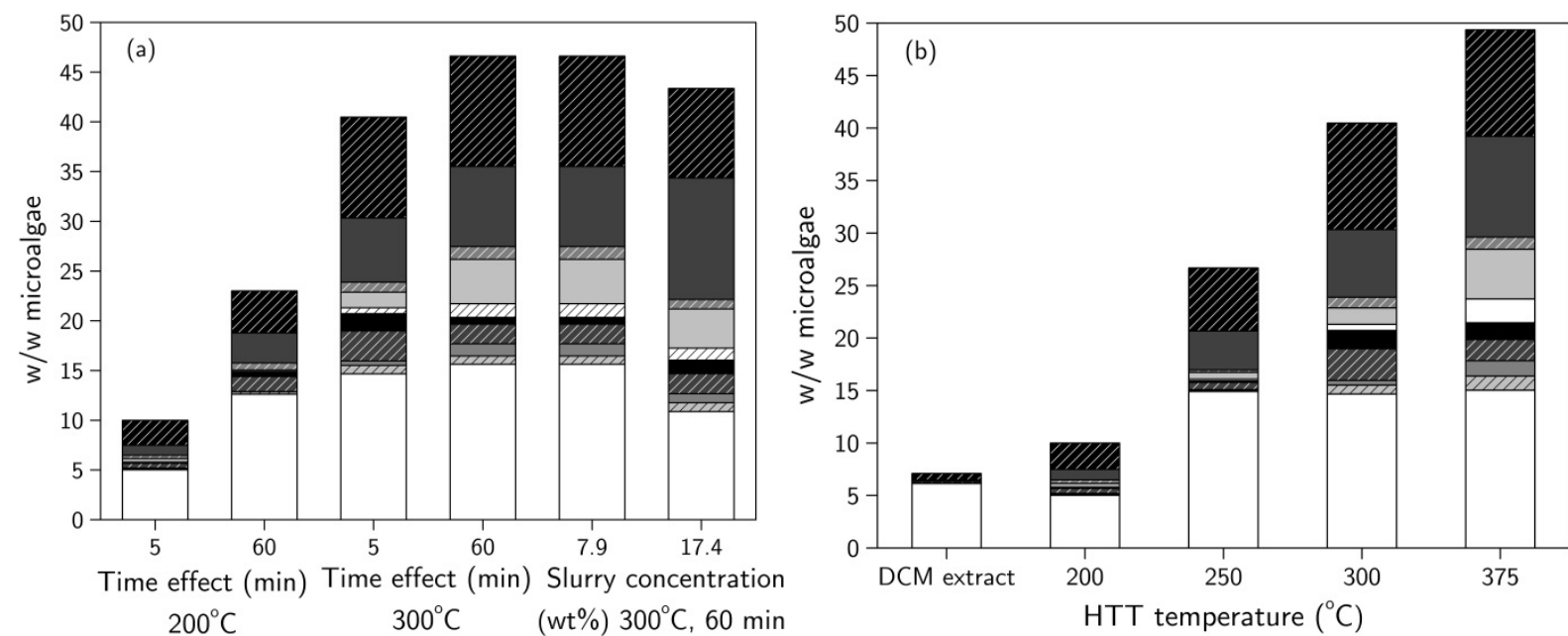

Figure A.9. (a) Effect of reaction time and slurry concentration and (b) Effect of temperature (5 min reaction time) on the yield of major chemical categories in HTT conversion of microalgal slurry. ${ }^{a}$ High molecular weight products of Maillard reaction (e.g. melanoidin like materials). ${ }^{\mathrm{b}}$ Low molecular weight products of Maillard reaction (e.g. pyrroles, pyrazine, pyridines and piperidines). ${ }^{\mathrm{c}}$ Amino acids side chains: indole, phenol, imidazole and methyl derivatives

Figures A.9(a) and A.9(b) give a glance of the overall effect of change in experimental parameters, showing the yields of all different chemical categories (chemical classes and compounds of Table A.2 were further grouped into major categories). Figure A.9(a)

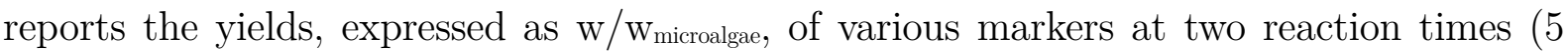
and $60 \mathrm{~min})$ for two temperatures $\left(200\right.$ and $\left.300^{\circ} \mathrm{C}\right)$ and with different microalgal slurry concentration ( 8 and $17 \% \mathrm{w}_{\text {microalgae }} / \mathrm{w}_{\text {slurry }}$ ), while Figure A.9(b) shows the products yields obtained by 5 min HTT at different reaction temperature. According to the stepwise PySPME-GC-MS, the main feature of the HTT at $200^{\circ} \mathrm{C}$ for $5 \mathrm{~min}$ (in comparison to cold extraction) was the appearance of about $5 \% \mathrm{w} / \mathrm{w}_{\text {microalgae }}$ of unsaponifiable matter, mainly consisting in algaenan fragments and a small amount of peptides (less than 1\% $\left.\mathrm{w} / \mathrm{w}_{\text {microalgae }}\right)$. 
When lower temperature and time of reaction $\left(200^{\circ} \mathrm{C}\right.$ and $250^{\circ} \mathrm{C}$ for $\left.5 \mathrm{~min}\right)$ were used, the HTT process grossly resembled a normal lipid extraction, with an additional important contribution of some algaenan fragments (linear alkanes polymers) and hydrophobic peptides (polypeptides and cyclic dipeptides which could formed during partial depolymerisation of proteins), probably driven by the drop in water polarity (dielectric constant drops from $78.5 \mathrm{~F} / \mathrm{m}$ at $25^{\circ} \mathrm{C}$ to $27.1 \mathrm{~F} / \mathrm{m}$ at $250^{\circ} \mathrm{C}$ ). ${ }^{37}$ Moreover, under these conditions, a certain degree of hydrolysis (with formation of FFA and probably also water-soluble nitrogen containing compounds) and formation of linear alkanes (mainly $\mathrm{C}_{15} \mathrm{H}_{32}$ and $\mathrm{C}_{17} \mathrm{H}_{36}$ ) was observed.

The "extraction" phenomenon seems to be roughly completed using milder conditions $\left(200^{\circ} \mathrm{C}\right.$ for $60 \mathrm{~min}$ or $250^{\circ} \mathrm{C}$ for $\left.5 \mathrm{~min}\right)$. At higher temperature, the contribution from thermal induced reactions seemed to play a crucial role in the process. These thermal induced events mainly consists in proteins depolymerisation/pyrolysis and interaction between proteins and carbohydrate HTT products (mainly through Maillard reaction). Therefore, the increase in the oil yield observed at temperature higher than $250^{\circ} \mathrm{C}$ is mainly determined by the increase in hydrophobic compounds originated from non-lipidic material. The most important contributors are, in particular, polypeptides, cyclic dipeptides, melanoidin if milder conditions (e.g. $300^{\circ} \mathrm{C}$ for $5 \mathrm{~min}$ ) and small products from Maillard reactions (e.g. pyrroles, pyrazines) for harsher conditions (e.g. $375^{\circ} \mathrm{C}$ for 5 min or $300^{\circ} \mathrm{C}$ for $\left.60 \mathrm{~min}\right)$. In particular, when supercritical conditions $\left(\mathrm{T}>374^{\circ} \mathrm{C}\right)$ are applied for short time, the HTT process produces a liquid that resembled the hydrophobic fraction of pyrolysis oil obtainable from proteinaceous material (e.g. rich in DKP or pyrroles). ${ }^{34}$

Looking to the effect of slurry concentration, the most noticeable effect of higher slurry concentration was the enhanced polymerization and charring of these fragments, with formation of a larger amount of polymeric substances (melanoidin-like materials), DKP and compounds that did not break even if pyrolyzed at temperature higher than $500^{\circ} \mathrm{C}$. Because of its thermal resistance to rupture or volatilization, this fraction was probably formed by relatively large polyarenes or alkyl-polyarenes ${ }^{36}$ still soluble in organic solvents (and, in fact, extractable by DCM) with thermal reactivity similar to petroleum asphaltene or maltene. Because this fraction is not vaporizable or breakable, it could probably be one of the most problematic to treat in a downstream upgrading process. In that case, HTT of too concentrated algal slurries should be avoided in order to keep asphaltene levels low. 
Looking at the "kinetics" of formation and/or extraction of various HTT products at two temperatures $\left(200^{\circ} \mathrm{C}\right.$ and $\left.300^{\circ} \mathrm{C}\right)$, it is possible to obtain some information on the main chemical mechanisms that are involved in the HTT processing of original microalgal macromolecules. Figure A.10 shows the change in yields (expressed as $\% \mathrm{w} / \mathrm{w}_{\text {microalgae}}$ ) of most relevant products, typical of different macromolecules present in the feedstock, produced during the HTT at $200^{\circ} \mathrm{C}$ and $300^{\circ} \mathrm{C}$ for different times. For the FA derivatives (Figure A.10(a)) the main processes at $200^{\circ} \mathrm{C}$ are "extraction" and hydrolysis, as evidenced by the increasing amounts of both BFA and FFA. The mechanism for the production of the odd number alkanes and alkenes (probably originated from FA) found needs further research as, in general, non-catalytic decarboxylation is not occurring below $300^{\circ} \mathrm{C} .38$
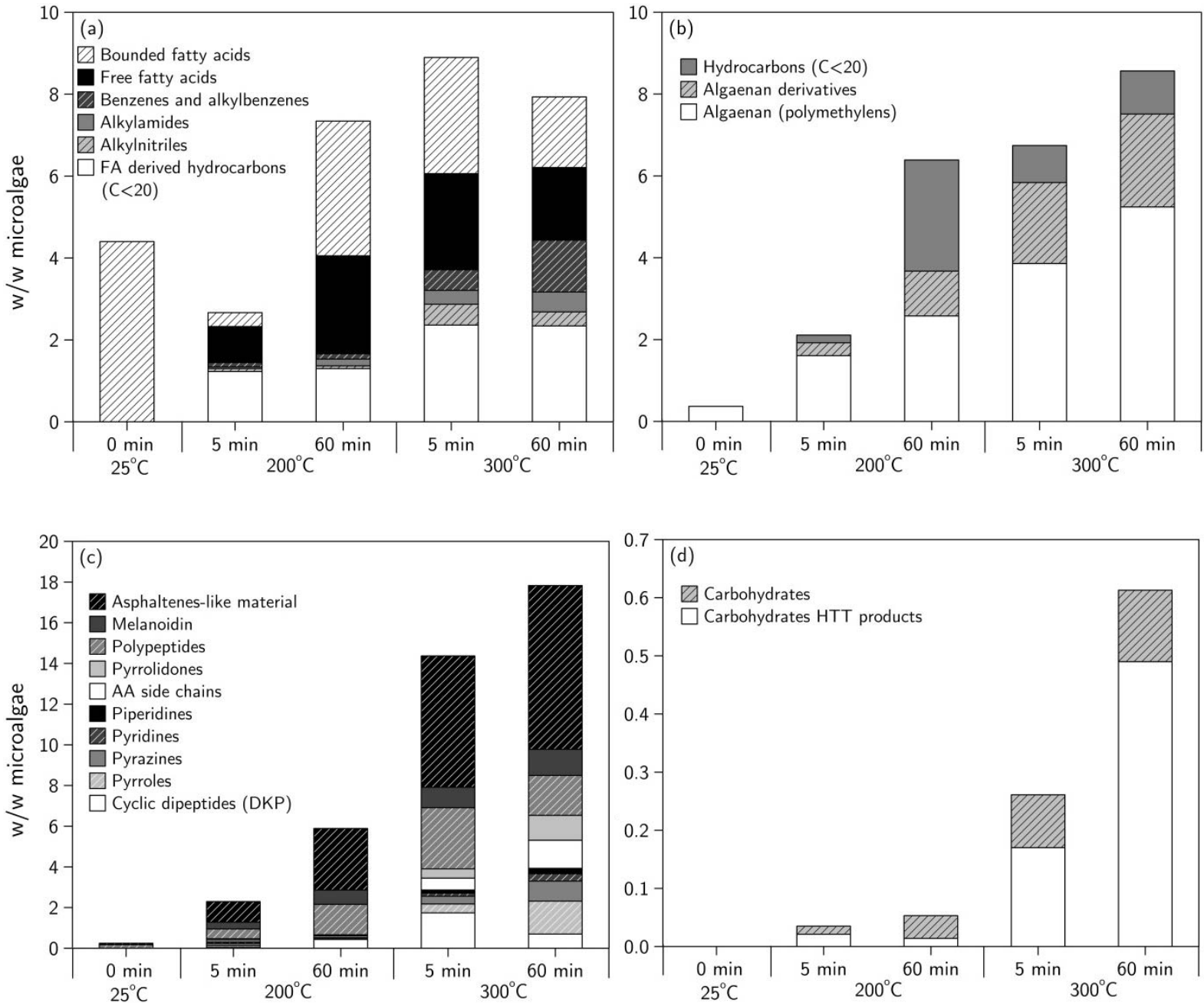

Figure A.10. Effect of reaction time on the yields (expressed as \% w/ $\mathrm{w}_{\text {microalgae }}$ ) of various markers from fatty acids, proteins and algaenans 
It is interesting to notice that, in comparison to cold DCM extraction (the slurry is extracted without HTT), the HTT performed at $5 \mathrm{~min} 200^{\circ} \mathrm{C}$ resulted in a slight decrease (from $4.3 \%$ to $2.7 \% \mathrm{w} / \mathrm{w}_{\text {microalgae }}$ ) in the yield of TFA derivatives (BFA, FFA and FA derived hydrocarbons). This finding can be explained by considering that the most extractable part of the TFA (afforded by the cold extraction) was triacylglicerols (TAG) which, upon thermal degradation, were hydrolyzed to FFA. Because FFA are more polar and can form soaps, they are less extractable (can be retained by the solid residue) than the TAG originally present. When the $200^{\circ} \mathrm{C}$ HTT was conducted for longer time (60 min), the yield of TFA derivatives was increased again to $7.3 \%$. This increase is probably driven by a large extent of extraction/hydrolysis of polar structural lipids. This phenomenon (initial TAG hydrolysis, saponification and progressive structural BFA extraction) could also explain the variable fatty acids composition among various sample shown in the Section A.3.1. At $300^{\circ} \mathrm{C}$, the extraction of $\mathrm{FA}$ derivatives seems almost complete after $5 \mathrm{~min}$, as testified by the reaching of a maximum for overall FA derivatives yield $(8.9 \%)$. In comparison with low temperature, likely, the most important additional process is the conversion of BFA and FFA to fatty amides, alkylnitriles and a certain amount of long chain alkylaromatic compounds, which can be formed by intermolecular cyclization and aromatization through hydrogen loss.

Concerning algaenan (Figure A.10(b)), at both $200^{\circ} \mathrm{C}$ and $300^{\circ} \mathrm{C}$, there is a monotonic increase in extraction (with only minor depolymerisation to small hydrocarbons) which slows down at around 8-9\% yield. The maximum yield of algaenan derivatives obtained was $8.6 \%$, which is close to the typical order of magnitude for expected concentrations of algaenan in green algae and approaches the "fiber" value (provided by the supplier) of the feedstock. Therefore, the effectiveness of algaenan recovery by mean of $300^{\circ} \mathrm{C} \mathrm{HTT}$ should be considered to be relatively high. ${ }^{33}$

On the side of nonlipid material (proteins and carbohydrates, Figure A.10(c) and A.11(d)) the contribution to the $200^{\circ} \mathrm{C}$ HTT oil is low and mainly consists of the production and/or extraction of hydrophobic peptides (3\% yield after $60 \mathrm{~min}$ ) and in melanoidin formation (1.5\% yield after $60 \mathrm{~min}$ ). When HTT was performed at higher temperature $\left(300^{\circ} \mathrm{C}\right)$, the qualitative effect of reaction time on the relative composition of the nonlipid derived oil was more pronounced. Because the 5 min HTT products formed are similar to those obtained at lower temperature, the primary HTT products are probably the same for both the $200^{\circ} \mathrm{C}$ and $300^{\circ} \mathrm{C}$ cases. Nevertheless, as the reaction time became longer (60 min), it was possible to observe a strong increase of typical secondary HTT products such as pyrazines, indoles derivatives, phenols and 
pyrrolidinones, which were not present in the feedstock. This indicated that, at $300^{\circ} \mathrm{C}$, a longer time favours both the Maillard reaction (e.g. reaction between hydroxy-aldehydes and amino acids) and thermal degradation of peptides (cleavage of side chains and pyrrolidinones formation) via pyrolysis-like reactions. When increasing reaction time during $300^{\circ} \mathrm{C} \mathrm{HTT}$, the absolute yields of peptides were markedly decreased and DKP were slightly decreased, possibly as a result of progressive hydrolysis of peptide bond, with formation of amino acids or smaller and more hydrophilic peptides and/or the Maillard reactions with carbohydrate derived compounds.

Given the importance in determining the nitrogen fate, the reactivity of protein and carbohydrates during HTT is a crucial point. In general, protein and carbohydrates can interact between themselves and/or with lipids through various ways (summarized in Figure A.11). The reaction scheme could be really complex and, for this reason, the experimental conditions can have a large effect on the final amount and type of solvent hydrophobic HTT products. According to our results, at low temperature $\left(200^{\circ} \mathrm{C}\right)$, the main chemical route for the protein conversion is probably the depolymerization (as suggested by the production of a significant amount DKP) by mean of hydrolysis and by thermal depolymerization. As demonstrated by Meetani et al., peptide depolymerization can be described as a progressive cyclization with the formation of gradually smaller cyclic oligopeptides, until the formation of DKP. ${ }^{39}$

This phenomenon could explain both the presence of DKP (especially under harsher conditions) and the presence of longer oligopeptides (revealed in Section A.3.3 by SEC and pyrolysis).

Even at temperatures as low as $200^{\circ} \mathrm{C}$, carbohydrates and proteins can be fragmented into smaller products (e.g. amines and aldehydes), which might be able to form melanoidin-like materials as well as certain amount of the asphaltene-like materials. According to this study, all these reactions seem to proceed steadily at $200^{\circ} \mathrm{C}$, with a constant increase as the reaction time was increased. At higher temperature $\left(300^{\circ} \mathrm{C}\right)$, the reactivity of proteins and carbohydrates is qualitatively and quantitatively more important for the HTT oil formation. In fact, as the reaction proceeds, we see a strong increase in "pyrolysis-like" products produced from thermal fragmentation of proteins and carbohydrates (e.g. amino acids side chains or 2-methyl-cyclopentenone) and of smaller product from Maillard reactions (e.g. pyrroles), at the expense of peptides and DKP, which are probably converted to amino acids and/or to other byproducts. 


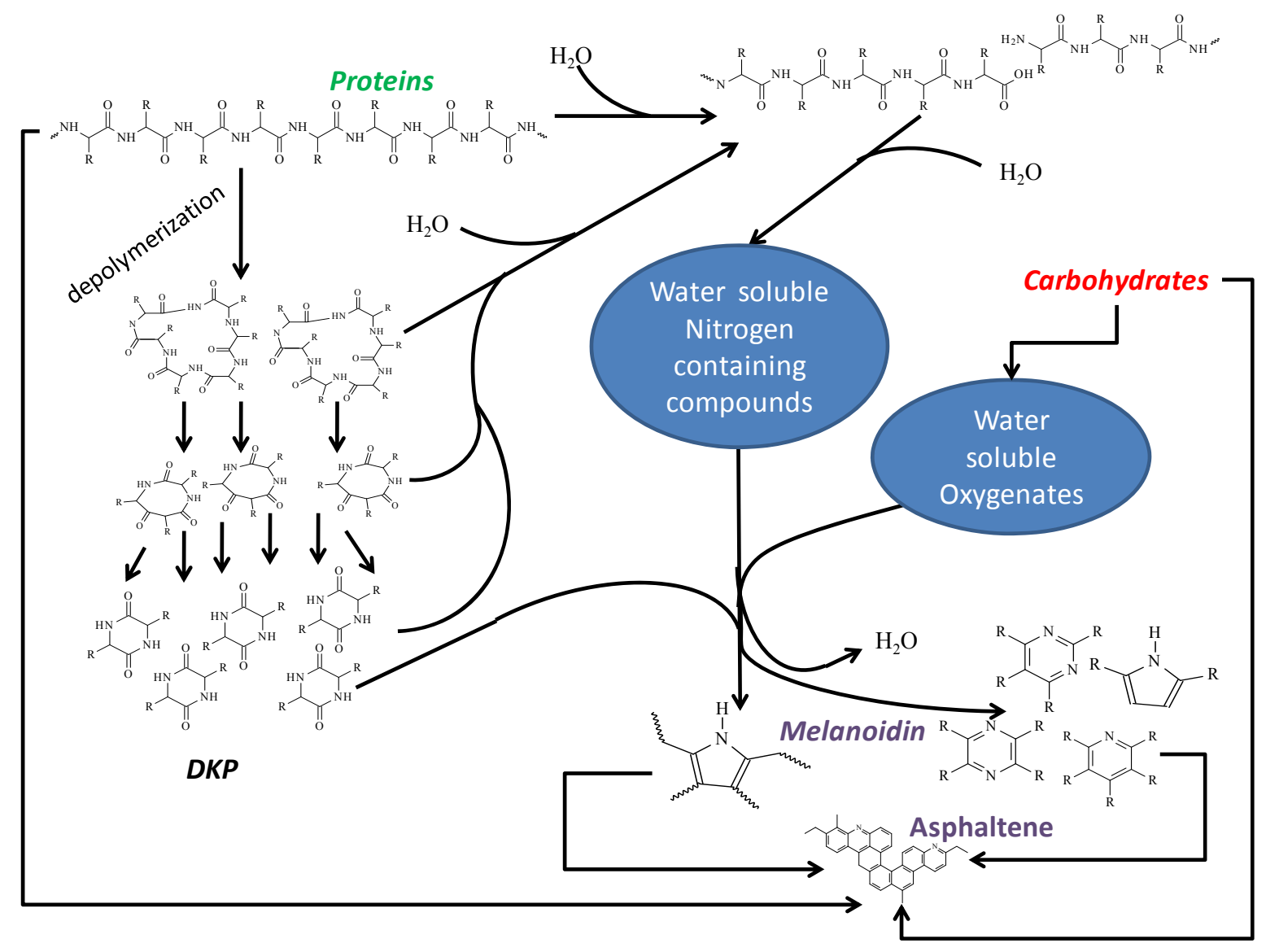

Figure A.11. Possible mechanisms for HTT oil formation from proteins/carbohydrates macromolecules

\section{A.4 Conclusions}

From the molecular point of view, the HTT oil is a mixture of a huge number of compounds and macromolecular constituents, whose polarity ranges from peptides to long chain hydrocarbons coming from thermal degradation of algaenan. Stepwise PySPME-GC-MS, SEC and column fractionation represent relatively simple techniques for disclosing more information on the portion not susceptible to conventional chemical analysis. In particular, Py-SPME-GC-MS analysis was applied for the first time in a factfinding of the chemical nature of HTT oil from microalgae.

Despite the extreme complexity of the solvent matrix, the molecular characterization results obtained are consistent with the overall oil elemental composition, and the applied technique allowed us to obtain a satisfactory closure of the mass balance of about $80 \%$.

From a relative comparison between the oil samples obtained from different HTT process conditions, an initial understanding of the reactions involved in HTT was obtained. 
First of all, HTT at relatively low temperature $\left(200-250^{\circ} \mathrm{C}\right)$ allows the extraction of the solvent soluble part of the cell constituents. Therefore lipids (extractable also without HTT), some short chain algaenans and some hydrophobic protein fragments end up in organic solvent phase. In this case, most of proteins and carbohydrates were not transformed to water insoluble products. Below $250^{\circ} \mathrm{C}$, the process was accompanied by a certain degree of thermal degradation (e.g. hydrolysis of BFA), but the phenomenon can be seen as a sort of improved extraction of lipids as well as hydrophobic resistant polymers (algaenan).

As the treatment becomes harsher $\left(300-375^{\circ} \mathrm{C}\right)$, proteins and cellulose started to break down, giving DKP and amino-acids derivatives (by pyrolysis-like reaction), carbohydrates derivatives (e.g. furans), products from cross reaction of those (e.g. formation of alkylpyrrolidinones, pyrazines, pyrroles and melanoidin like materials) and a certain amount of asphaltene-like material. Proteins degradation drastically increased the oil yields, but the hydrophobic portion of protein-carbohydrate derived materials drove up the nitrogen content of the oil. This means that, even if a high yield of oil can be obtained from lipidpoor microalgae, the oil obtained at high temperature may not be useable directly as fuel and, from its compositional complexity, it may not be easily upgradable without a downstream complete chemical dissociation (catalytic cracking or gasification). Work in this area is recommended.

This also suggests, looking to the entire process, that it may be more convenient to use relatively "milder" conditions (e.g. $250^{\circ} \mathrm{C}$ for $5 \mathrm{~min}$ ), in order to maximize the yield of lipid and algaenan derivatives partition in the oil and/or to stress (by some pretreatment or catalyst) the conversion of carbohydrates and proteins to water soluble compounds that could be recycled back to the culture or anaerobically digested to methane.

\section{Bibliography}

[1] S. Zou, Y. Wu, M. Yang, C. Li, J. Tong, Bio-oil production from sub- and supercritical water liquefaction of microalgae Dunaliella tertiolecta and related properties, Energy Environ. Sci., 3(8), 1073$1078,2010$.

[2] U. Jena, K.C. Das, J.R. Kastner, Effect of operating conditions of thermochemical liquefaction on biocrude production from Spirulina platensis, Bioresour. Technol., 102(10), 6221-6229, 2011.

[3] A.B. Ross, P. Biller, M.L. Kubacki, H. Li, A. Lea-Langton, J.M. Jones, Hydrothermal processing of microalgae using alkali and organic acids, Fuel, 89(9), 2234-2243, 2010. 
[4] S. Zou, Y. Wu, M. Yang, C. Li, J. Tong, Thermochemical catalytic liquefaction of the marine microalgae dunaliella tertiolecta and characterization of bio-oils, Energy Fuels, 23(7), 3753-3758, 2009.

[5] P. Biller, R. Riley, A.B. Ross, Catalytic hydrothermal processing of microalgae: Decomposition and upgrading of lipids, Bioresour. Technol., 102(7), 4841-4848, 2011.

[6] D. Zhou, L. Zhang, S. Zhang, H. Fu, J. Chen, Hydrothermal liquefaction of macroalgae enteromorpha prolifera to bio-oil, Energy Fuels, 24(7), 4054-4061, 2010.

[7] D. Mohan, C.U. Pittman Jr, P.H. Steele, Pyrolysis of wood/biomass for bio-oil: A critical review, Energy Fuels, 20(3), 848-889, 2006.

[8] S.R. Larter, A.G. Douglas, A pyrolysis-gas chromatographic method for kerogen typing, Phys. Chem. Earth., 12(C), 579-583, 1980.

[9] A.D. Czaja, A.B. Kudryavtsev, G.D. Cody, J.W. Schopf, Characterization of permineralized kerogen from an Eocene fossil fern, Org. Geochem., 40(3), 353-364, 2009.

[10] R.B. Kodner, R.E. Summons, A.H. Knoll, Phylogenetic investigation of the aliphatic, non-hydrolyzable biopolymer algaenan, with a focus on green algae, Org. Geochem., 40(8), 854-862, 2009.

[11] C. Saiz-Jimenez, Analytical pyrolysis of humic substances: Pitfalls, limitations, and possible solutions, Environ. Sci. Technol., 28(11), 1773-1780, 1994.

[12] E.M. Fitzpatrick, J.M. Jones, M. Pourkashanian, A.B. Ross, A. Williams, K.D. Bartle, Mechanistic aspects of soot formation from the combustion of pine wood, Energy Fuels, 22(6), 3771-3778, 2008.

[13] J. Kaal, C. Rumpel, Can pyrolysis-GC/MS be used to estimate the degree of thermal alteration of black carbon?, Org. Geochem., 40(12), 1179-1187, 2009.

[14] Z. Parsi, T. Górecki, J. Poerschmann, Advances in non-discriminating pyrolysis, J. Anal. Appl. Pyrolysis, 74(1-2), 11-18, 2005.

[15] C. Torri, M. Reinikainen, C. Lindfors, D. Fabbri, A. Oasmaa, E. Kuoppala, Investigation on catalytic pyrolysis of pine sawdust: Catalyst screening by Py-GC-MIP-AED, J. Anal. Appl. Pyrolysis, 88(1), 7-13, 2010.

[16] P.R. Patwardhan, D.L. Dalluge, B.H. Shanks, R.C. Brown, Distinguishing primary and secondary reactions of cellulose pyrolysis, Bioresour. Technol., 102(8), 5265-5269, 2011.

[17] S. Risticevic, V.H. Niri, D. Vuckovic, J. Pawliszyn, Recent developments in solid-phase microextraction, Anal. Bioanal.Chem., 393(3), 781-795, 2009.

[18] C. Torri, D. Fabbri, Application of off-line pyrolysis with dynamic solid-phase microextraction to the GC-MS analysis of biomass pyrolysis products, Microchem. J., 93(2), 133-139, 2009.

[19] D. Fabbri, A. Adamiano, C. Torri, GC-MS determination of polycyclic aromatic hydrocarbons evolved from pyrolysis of biomass, Anal. Bioanal.Chem., 397(1), 309-317, 2010.

[20] S. Prati, G. Sciutto, R. Mazzeo, C. Torri, D. Fabbri, Application of ATR-far-infrared spectroscopy to the analysis of natural resins, Anal. Bioanal.Chem., 399(9), 3081-3091, 2011.

[21] M.J. Griffiths, R.P. Van Hille, S.T.L. Harrison, Selection of direct transesterification as the preferred method for assay of fatty acid content of microalgae, Lipids, 45(11), 1053-1060, 2010.

[22] J.S. Leventhal, Stepwise pyrolysis-gas chromatography of kerogen in sedimentary rocks, Chem. Geol., 18(1), 5-20, 1976.

[23] V.A. Yaylayan, E. Kaminsky, Isolation and structural analysis of Maillard polymers: Caramel and melanoidin formation in glycine/glucose model system, Food Chem., 63(1), 25-31, 1998.

[24] R. Tressl, G.T. Wondrak, L.A. Garbe, R.P. Krüger, D. Rewicki, Pentoses and hexoses as sources of new melanoidin-like maillard polymers, J. Agric. Food. Chem., 46(5), 1765-1776, 1998. 
[25] J.M. Bracewell, G.W. Robertson, Quantitative comparison of the nitrogen-containing pyrolysis products and amino acid composition of soil humic acids, J. Anal. Appl. Pyrolysis, 6(1), 19-29, 1984.

[26] B.R.T. Simoneit, A.I. Rushdi, M.R.B. Abas, B.M. Didyk, Alkyl amides and nitriles as novel tracers for biomass burning, Environ. Sci. Technol., 37(1), 16-21, 2003.

[27] G. Chiavari, G.C. Galletti, Pyrolysis-gas chromatography/mass spectrometry of amino acids, J. Anal. Appl. Pyrolysis, 24(2), 123-137, 1992.

[28] T.M. McCollom, B.R.T. Simoneit, E.L. Shock, Hydrous pyrolysis of polycyclic aromatic hydrocarbons and implications for the origin of PAH in hydrothermal petroleum, Energy Fuels, 13(2), 401-410, 1999.

[29] D. Fabbri, V. Bevoni, M. Notari, F. Rivetti, Properties of a potential biofuel obtained from soybean oil by transmethylation with dimethyl carbonate, Fuel, 86(5-6), 690-697, 2007.

[30] V.A. Basiuk, J. Douda, Pyrolysis of poly-glycine and poly-L-alanine: Analysis of less-volatile products by gas chromatography/Fourier transform infrared spectroscopy/mass spectrometry, J. Anal. Appl. Pyrolysis, 55(2), 235-246, 2000.

[31] E.B. Higman, I. Schmeltz, W.S. Schlotzhauer, Products from the thermal degradation of some naturally occurring materials, J. Agric. Food. Chem., 18(4), 636-639, 1970.

[32] P. Blokker, S. Schouten, H. Van den Ende, J.W. De Leeuw, P.G. Hatcher, J.S. Sinninghe Damsté, Chemical structure of algaenans from the fresh water algae Tetraedron minimum, Scenedesmus communis and Pediastrum boryanum, Org. Geochem., 29(5-7 -7 pt 2), 1453-1468, 1998.

[33] S. Derenne, C. Largeau, C. Berkaloff, B. Rousseau, C. Wilhelm, P.G. Hatcher, Non-hydrolysable macromolecular constituents from outer walls of Chlorella fusca and Nanochlorum eucaryotum, Phytochemistry, 31(6), 1923-1929, 1992.

[34] C. Torri, C. Samorì, A. Adamiano, D. Fabbri, C. Faraloni, G. Torzillo, Preliminary investigation on the production of fuels and bio-char from Chlamydomonas reinhardtii biomass residue after bio-hydrogen production, Bioresour. Technol., 102(18), 8707-8713, 2011.

[35] R. Fahmi, A.V. Bridgwater, S.C. Thain, I.S. Donnison, P.M. Morris, N. Yates, Prediction of Klason lignin and lignin thermal degradation products by Py-GC/MS in a collection of Lolium and Festuca grasses, J. Anal. Appl. Pyrolysis, 80(1), 16-23, 2007.

[36] H. Groenzin, O.C. Mullins, Molecular size and structure of asphaltenes from various sources, Energy Fuels, 14(3), 677-684, 2000 .

[37] S.S. Toor, L. Rosendahl, A. Rudolf, Hydrothermal liquefaction of biomass: A review of subcritical water technologies, Energy, 36(5), 2328-2342, 2011.

[38] M. Snåre, I. Kubičková, P. Mäki-Arvela, K. Eränen, D.Y. Murzin, Heterogeneous catalytic deoxygenation of stearic acid for production of biodiesel, Ind. Eng. Chem. Res., 45(16), 5708-5715, 2006.

[39] M.A. Meetani, O.K. Zahid, J. Michael Conlon, Investigation of the pyrolysis products of methionineenkephalin-Arg-Gly- Leu using liquid chromatography-tandem mass spectrometry, J. Mass Spectrom., 45(11), 1320-1331, 2010. 



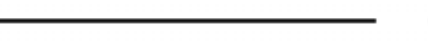

rar 


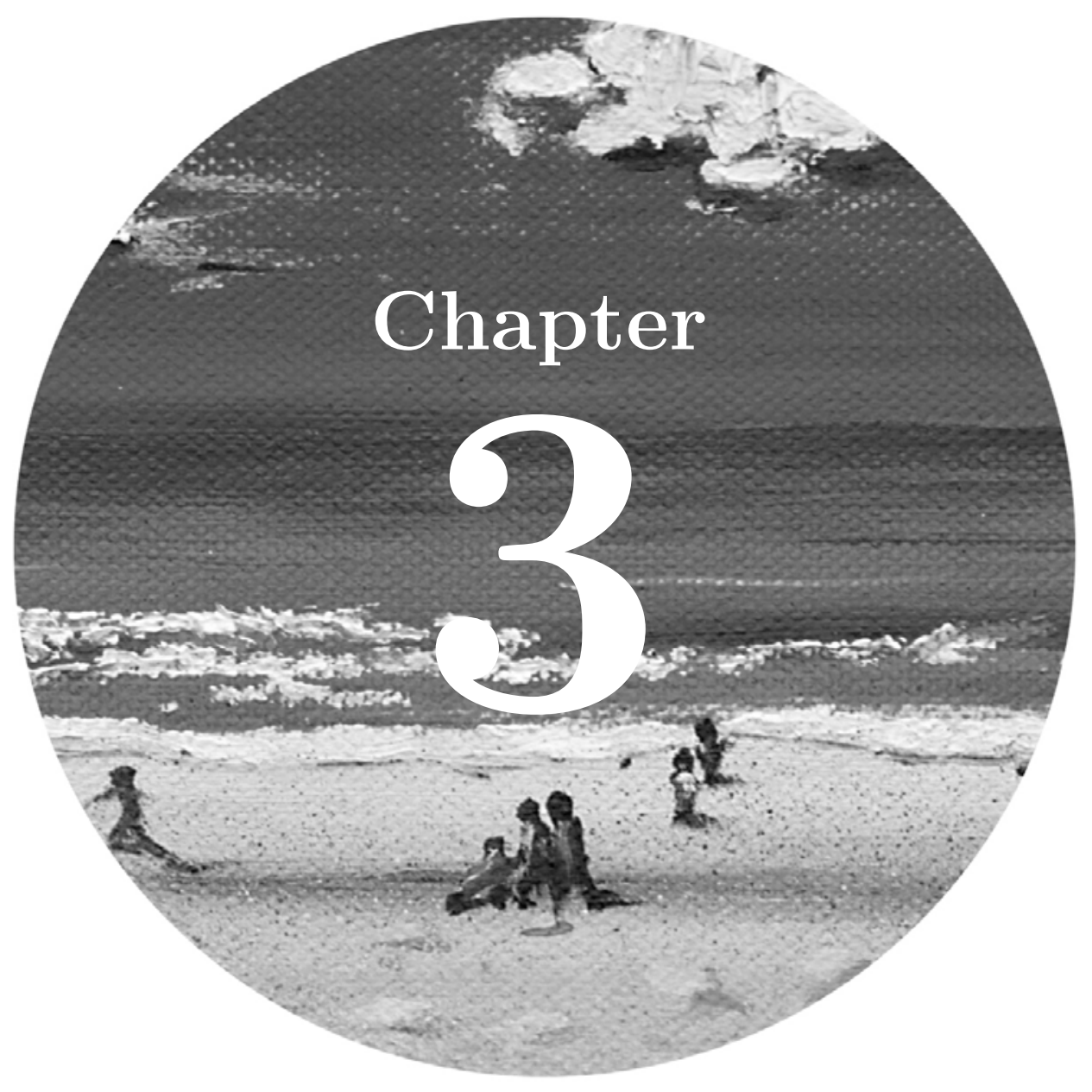

Hydrotreatment of hydrothermal liquefaction oil from microalgae: Preliminary results 


\begin{abstract}
In the present work, the hydrotreatment process was studied as upgrading method to improve the quality of oils produced from hydrothermal liquefaction (HTL) of microalgae (Desmodesmus sp.). Various operating conditions (temperature, catalyst loading and initial $\mathrm{H}_{2}$ pressure) were investigated. In addition, two oils (in dry form) produced at different HTL temperatures $\left(250\right.$ and $\left.350^{\circ} \mathrm{C}\right)$ were treated to evaluate the influence of initial HTL oil composition on the product after hydrotreatment. Temperature was found to be the most important process parameter with respect to the oil composition. With higher temperatures and catalyst loading higher reduction of nitrogen and oxygen was achieved. As a result, the oils produced had a higher heating value (up to $44 \mathrm{MJ} / \mathrm{kg}$ ) and reduced viscosity when compared to the crude HTL oil feed. The difference in composition of the HTL oils produced at 250 and at $350^{\circ} \mathrm{C}$ had an influence on the final compositions of the corresponding hydrotreated oils. Further research and more comprehensive analyses are required to understand the reaction networks and mechanisms leading to that observed effect. Furthermore, the future applicability of the hydrotreated oils was also assessed showing that their co-processing with long residue oil in the FCC unit of standard refineries is likely to be possible and even coprocessing with middle distillates might be also an option. About 20 wt\% of the hydrotreated HTL algal oils is a directly useful fraction of diesel-range hydrocarbons indicating the possible use of this fraction as drop-in fuel.
\end{abstract}




\section{Introduction}

In an algae biorefinery configuration where liquid transportation fuels are the main products, energy and cost intensive processes cannot be afforded, as those bio-liquids will have to compete with the still low crude oil price. Hydrothermal liquefaction (HTL) is considered a suitable process for the conversion of wet biomass such as microalgae. In contrast with other conversion technologies such as pyrolysis, it circumvents the need for complete water removal and the associated energy costs. In Chapter 2, we found that a high oil yield can be obtained ( $50 \mathrm{wt} \%)$, recovering $75 \%$ of the algal calorific value into the oil and an energy densification from 22 to $36 \mathrm{MJ} / \mathrm{kg}$. However, the primary oil produced has a limited end-user applicability for liquid transportation fuels production.

Due to the high protein content of microalgae $(\sim 38-44 \mathrm{wt} \%$ for the algae used in this study), HTL oil has a high nitrogen content which can be up to $7 \mathrm{wt} \%$ (see Table 1 in Chapter 2) being much higher than e.g. the maximum allowed for biodiesel (B100) (ASTM D 6751-07a). Undesirable $\mathrm{NO}_{\mathrm{x}}$ emissions will be produced upon combustion of such oil. The oxygen content, although lower than that of pyrolysis oil, leads to a lower heating value. Furthermore, it is a highly viscous and tar-like material with very poor flowing characteristics at room temperature, which will complicate transport through pipelines. Finally, HTL oils appear to be relatively acidic. Duan and Savage ${ }^{1}$ performed total acid number (TAN) measurements on their HTL oils and found them to be much higher than the biodiesel standard according to ASTM D 6751-07a. Although being a less critical feature, acidic bio-oils may lead to corrosion of equipment used for their storage, transport and processing.

Clearly, an upgrading process that is able to minimize the above mentioned adverse properties is required. The desired end product that we are aiming for would be an algalbased hydrocarbon rich oil either directly suitable as diesel-type fuel or, at least, suitable for co-processing in standard existing refinery units. For that purpose, a preliminary study was carried out where different methods were tested:

- Pre-treatment of fresh algae by protein extraction prior to HTL;

- Extractive techniques to separate the nitrogen containing compounds from the oil obtained after HTL of fresh algae;

- Catalytic cracking of the HTL oil (from fresh algae) using a zeolite catalyst (collaborative work presented $\mathrm{in}^{2}$ ). 
The main criteria for comparison were: oil yield, reduction of nitrogen and oxygen contents, overall oil composition and quality. The methods were either rather inefficient regarding the first two criteria or having a composition less suitable for the desired linear hydrocarbon rich fuel targeted in this work. Therefore, they were discarded for further investigation. Some details of that study are available in the Appendix of this chapter.

Treatment of the HTL crude oil by thermal means is another possible upgrading path. Roussis et al. $^{3}$ demonstrated a substantial removal of oxygen (49-96\% reduction relative to the original oil produced from HTL of Nannochloropsis sp.) by only applying high temperature $\left(350,400\right.$ and $\left.450^{\circ} \mathrm{C}\right)$ without the use of $\mathrm{H}_{2}$ and/or a catalyst. However, despite the high $\mathrm{O}$ removal achieved, the $\mathrm{N}$ content was not reduced at all. An alternative, catalytic hydrotreatment, is a well-known and widely applied technology for upgrading of petroleum-derived products (e.g. diesel, paraffin, naphtha) or bio-liquids like pyrolysis oil. Investment costs for such a technology are expected to be relatively low, as it can utilize existing infrastructure, which is readily available in petroleum refineries. Moreover, $\mathrm{H}_{2}$ at high pressure is also accessible in refinery where hydrodesulfurization and hydrodenitrogenation of petroleum oil are common practice.

Hydrotreatment with heterogeneous catalysts directly on the algal biomass (thus, not on the oil as in this study), sometimes named 'hydroliquefaction', was tested by Duan and Savage. ${ }^{4}$ Slightly higher oil yields were obtained and, with some of the catalysts tested (e.g. noble metals), the viscosity was improved. However, no significant reduction of the heteroatoms content $(\mathrm{O}, \mathrm{N}$ and $\mathrm{S})$ was achieved. Later, the same authors reported the catalytic hydrotreatment of crude HTL oil from Nannochlorpsis sp. in supercritical water (SCW) and studied the effect of different operating conditions and catalysts type. ${ }^{1,5,6}$ Their results were very promising; the upgraded oil had a lower acid number, lower $\mathrm{O}$ and $\mathrm{N}$ content (with sulphur even below detection limits) and appeared as a freely flowing liquid (for the catalytically treated oils) with a high alkane content. Therefore, in this work, we decided to further study hydrotreatment as upgrading method for HTL algal oils.

In Chapter 2, using the same microalgal feedstock as in this work, we identified two temperature regions with differences with respect to the oil product: the highest oil yield (49.4 wt\%) was obtained in the range of $350-375^{\circ} \mathrm{C}$, while at $250^{\circ} \mathrm{C}$ a lower amount of oil was produced (26.7 wt\%), but with the highest total fatty acid (TFA) content of $28.0 \pm 4.0 \mathrm{wt} \%$ (produced after esterification of the lipid derivatives present in the oil) among all the other oils. The higher temperature oil contained a larger fraction of nitrogen containing compounds and algaenan derivatives having a TFA content of 
$4.8 \pm 1.8 \mathrm{wt} \%$. Hence, both oils were produced (HTL at $250^{\circ} \mathrm{C}$ and $350^{\circ} \mathrm{C}$ with $5 \mathrm{~min}$ reaction time) to be the feedstock for hydrotreatment.

Duan and Savage ${ }^{1}$ suggested that, after HTL and solid residue separation, the aqueous phase/crude oil mixture could be used directly, as is, in a hydrotreatment step to upgrade the oil. In such a concept, the organics dissolved in the aqueous phase will, however, also consume a fraction of the $\mathrm{H}_{2}$ meant for treating the oil. At the same time, at these high temperatures, more small molecules produced from cracking reactions will be lost to the aqueous phase, which otherwise might remain in the oil. A further drawback could be a hydrogen partial pressure limitation, as the water vapor pressure will occupy an increasing fraction of the allowable operating pressure at increasing temperature. Also, catalyst supports and -stability is an issue when operating under supercritical water conditions (like possible phase transition from $\gamma-\mathrm{Al}_{2} \mathrm{O}_{3}$ to $\alpha-\mathrm{Al}_{2} \mathrm{O}_{3}$ leading to a reduction in surface area). ${ }^{7}$

Therefore, we decided to study hydrotreatment using the 'dry' oil, recovered after separation of the aqueous phase from HTL. At the time of experimentation, no similar work on hydrotreatment of dry HTL algal oils was available in literature. However, during the course of preparation of this chapter, a related work appeared where also dry HTL oil was hydrotreated in the presence of HZSM- $5 .{ }^{8}$ The results of that study are taken into consideration, however, a direct comparison might be difficult as zeolite catalysts are commonly employed in the petrochemical industry for fluid catalytic cracking and hydrocraking and not directly used as hydrogenation catalyst.

Two types of HTL crude oils with different composition were used as feedstock in order to investigate the influence of the HTL oil composition on the products obtained after hydrotreatment. To our knowledge, so far upgrading methods have only been applied to oils obtained under more severe hydrothermal conditions with temperatures in the range of $320-350^{\circ} \mathrm{C}$ HTL. ${ }^{1,5,6}$ Finally, for the oils obtained, a preliminary assessment of the quality of the oils will be made. Through simple tests and analyses, a first impression is obtained, trying to identify a realistic applicability of this product. 


\section{Experimental}

\subsection{Materials}

Freshwater microalgae Desmodesmus sp. were purchased from Ingrepro B.V. (The Netherlands). The proximate and ultimate analysis of the biomass are listed in Table 1. More details on the approximate biochemical composition of the algae used can be seen in Chapter 2 as well as the methods to determine the moisture and ash contents of the algal slurry.

Table 1. Microalgae Desmodesmus sp. properties

\begin{tabular}{cccc}
\hline \multicolumn{2}{c}{ Algal slurry properties (wt\%) } & \multicolumn{2}{c}{ Elemental composition (wt\%) } \\
\hline Moisture content & 82.5 & $\mathrm{C}$ & 43.9 \\
Organic content & 13.9 & $\mathrm{H}$ & 6.25 \\
Ash content & 3.60 & $\mathrm{~N}$ & 7.09 \\
& & $\mathrm{O}+\mathrm{ash}^{\mathrm{a}}$ & 42.8 \\
& & $\mathrm{HHV}(\mathrm{MJ} / \mathrm{kg})$ & 19.8 \\
\hline
\end{tabular}

${ }^{a}$ Calculated by difference as $100-(\mathrm{C}+\mathrm{H}+\mathrm{N})$

The same ash determination method applied to the algae was used for the HTL and treated oils. X-Ray Fluorescence spectrometry (XRF) was performed on the ash recovered from the oils. The $\mathrm{C}, \mathrm{H}$, and $\mathrm{N}$ contents of the dry algae were measured in duplicate using an elemental analyzer (Thermo Scientific Flash 2000, CHN-S). Maximum allowable difference between duplicates was $\pm 1 \%$, and if higher, the measurement was repeated. For the algae heating value, the equation proposed by Channiwala and Parikh ${ }^{9}$ was used as it is known to have a higher accuracy for the HHV estimation of biomass.

Duan and Savage ${ }^{7}$ studied the activity of a wide range of catalyst towards hydrothermal hydrodenitrogenation (HDN) of pyridine as model nitrogen containing compound of HTL algal oil. A total of 31 different types of catalyst were tested, ranging from noble metals to traditional HDN catalysts (e.g. sulfided $\mathrm{CoMo} / \mathrm{\gamma}-\mathrm{Al}_{2} \mathrm{O}_{3}$ ), with the highest activity and best performance for the $5 \% \mathrm{Pt} / \mathrm{\gamma}-\mathrm{Al}_{2} \mathrm{O}_{3}$. Therefore, we selected that catalyst for our hydrotreatment experimental study. In addition, $\mathrm{Pt}$ and $\mathrm{Pd}$ based catalyst are very effective for hydrothermal deoxygenation of fatty acids, even without $\mathrm{H}_{2} \cdot{ }^{10}$ Nobel metal catalyst show good performance in hydrodeoxygenation of pyrolysis oil $^{1}{ }^{11}$ and oxygenated compounds like guaiacol. ${ }^{12}$ 
$\mathrm{Pt} / \mathrm{Al}_{2} \mathrm{O}_{3}$ was purchased from Sigma-Aldrich (product number 205974) with a metal loading of $5 \mathrm{wt} \%$ (total BET surface area of $95.46 \pm 0.18 \mathrm{~m}^{2} / \mathrm{g}$ ). The catalyst was not pre-reduced (with $\mathrm{H}_{2}$ ) prior to usage as, under the hydrotreatment conditions applied, the catalyst is most probably rapidly activated (with the $\mathrm{H}_{2}$ initially present) already during the heating period to the operational temperature. Moreover, as Duan and Savage $^{7}$ pointed out, in an oxidizing environment (as in our case since HTL algal oil contains oxygenated compounds but also $\mathrm{H}_{2} \mathrm{O}$ can be potentially produced during hydrogenation), the catalyst should also show a certain degree of activity.

$\mathrm{H}_{2}$ and $\mathrm{N}_{2}$ (both with purity of $99.9 \%$ ) were obtained from the high pressure network in the laboratory. Dichloromethane (99\% purity), used to recover the products from the reactor (for both HTL and hydrotreatment), was obtained from Sigma-Aldrich. Several hydrocarbons (hexane, heptane, octane, undecane, dodecane and hexadecane), purchased from Sigma-Aldrich, were used as gas chromatography standards. Commercial Shell VPower diesel was used for the blending tests.

\subsection{Algae hydrothermal liquefaction}

To produce sufficient HTL oil as (the same) starting material for several hydrotreatment experiments, a batch reactor system with an intensively stirred (hollow shaft stirrer) autoclave (from Autoclave Engineers) with $0.6 \mathrm{~L}$ internal volume was used. The heating rate of this autoclave was ca. 17 times slower than that for the $45 \mathrm{ml}$ autoclave used in Chapter 2. Although different product yields were obtained (i.e. more oil was produced), heating rate did not have a significant effect on the composition of the oils. Duan and Savage ${ }^{1}$ and Zhang et al. ${ }^{13}$ also arrived at the same conclusions.

The autoclave, located in a safety chamber and controlled and monitored from the outside, was surrounded by an electric oven (jacket with a temperature controller) and had a thermocouple inside to record the inner temperature. The pressure was also monitored with a pressure transmitter located on a gas pipe connected to the autoclave. A schematic representation of the set-up can be found elsewhere, ${ }^{14}$ showing also a hydrogen supply line connected to the autoclave. Hydrogen was not used for HTL and hence that gas line was detached from the reactor.

The experimental procedure was initiated by filling the autoclave with $300 \mathrm{~g}$ of algal slurry (with the water content shown in Table 1). After that, the reactor was closed tightly and a leak test was conducted with $\mathrm{N}_{2}$. If the reactor was leak free, the nitrogen was vented, flushing at the same time the air initially present inside. After placing the 
electrical oven around the autoclave, stirring $(20 \mathrm{~Hz})$ and heating were started (outside the safety chamber). The heating rate to the experimental temperature was approximately $2.7^{\circ} \mathrm{C} / \mathrm{min}$. After $5 \mathrm{~min}$ reaction time at the corresponding temperature, the heating was stopped allowing the system to cool down $(\sim 3 \mathrm{~h})$. The final pressure at room temperature of gas produced was recorded, a gas sample was taken for analysis and, finally, the reactor was totally depressurized. The gas product was analyzed by means of gas chromatography (Varian Micro GC CP-4900 containing two analytical columns, Molsieve 5A (10 m) and PPQ $(10 \mathrm{~m})$, using helium as carrier gas). With the gas composition, the final pressure and temperature inside the reactor (after cooling) and the gas volume (total reactor inner volume minus the volume occupied by the algal slurry), the amount of gas produced could be calculated. After depressurization, the product collection with dichloromethane (DCM) and separation procedure described in detail in Chapter 2 was applied to separate and quantify the rest of the HTL products (oil or crude in DCM, solid residue and water soluble organics). For these HTL experiments, more oil was produced (as more algae was processed) requiring a larger quantity of DCM to properly recover the whole product. Yet the ratio DCM/water (in the $300 \mathrm{~g}$ algal slurry) was kept equal to that reported in the earlier HTL work. The definitions used to calculate the product yields in order to find the mass balance closure can be seen in Chapter 2. The HTL product yields are presented on dry algae ash free basis.

\subsection{Oil hydrotreatment}

The two HTL algal oils (named as $250^{\circ} \mathrm{C}$ HTL oil and $350^{\circ} \mathrm{C}$ HTL oil along this chapter) were submitted to different hydrotreatment operation conditions; changing temperature $\left(300\right.$ and $400^{\circ} \mathrm{C}$ ), catalyst loading (no catalyst, 20 and $50 \%$ relative to the HTL oil initially fed into the reactor) and initial $\mathrm{H}_{2}$ pressure at room temperature (10 and 15 $\mathrm{MPa}$ ). A reaction time of $1 \mathrm{~h}$ was maintained for all the experiments. Temperatures above $400^{\circ} \mathrm{C}$ were not investigated because, as shown in related studies, ${ }^{3,6,8}$ less oil is recovered while mainly containing a mixture of aromatic hydrocarbons. Assuming that all $\mathrm{N}$ and $\mathrm{O}$ atoms in the oil are removed in the form of $\mathrm{NH}_{3}$ and $\mathrm{H}_{2} \mathrm{O}, 57.6 \mathrm{mmol}$ of $\mathrm{H}_{2}$ would be the stoichiometric requirement for complete removal (calculated for the experiment with the largest amount oil used, $3.8 \mathrm{~g}$, and according to the elemental composition of the $350^{\circ} \mathrm{C}$ HTL oil). Hydrogen was added in excess (10 MPa at room temperature, corresponding to $160 \mathrm{mmol}$ of $\mathrm{H}_{2}$ ) to avoid hydrogen supply limitations. 
The set-up employed for these experiments was nearly identical to the one presented in Chapter 2 which was used, in that case, for HTL of algae. As only difference, the $45 \mathrm{ml}$ stainless steel autoclave (in-house made) was provided of a hollow shaft stirrer in order to improve mass transfer while, for HTL, a shaken reactor (with the same inner volume) was used. Also, an extra gas inlet was connected to the autoclave for $\mathrm{H}_{2}$ supply. A detailed description of this system was reported in Chapter 2 together with a schematic representation of it.

The experimental procedure was initiated by filling the reactor with 2-4 g of HTL oil and the desired catalyst amount (if added). Since the crude HTL oils were highly viscous, pre-heating to around $50^{\circ} \mathrm{C}$ was required to enhance their fluidity and be able to pour the product into the reactor. The autoclave was then closed firmly and connected to the moving arm. After a leakage test with $\mathrm{H}_{2}$, the reactor was purged to displace the air and re-filled with the desired starting $\mathrm{H}_{2}$ pressure. When the desired amount of gas was totally loaded, the reactor valve was securely tightened and the gas pipe disconnected. Stirring was started prior to heating to pre-saturate the liquid. Heating was provided by immersion in a hot fluidized sand bed heated by two electric ovens with two temperature controllers respectively. After $1 \mathrm{~h}$ reaction time, the autoclave was lifted and immersed into a water bath to achieve fast quenching ( 1-2 min). All these maneuvers were monitored and controlled outside the safety chamber, where the set-up was placed. The heating time to the experimental temperature for all the experiments was 6 to 7 min. The total pressure initially increased due to the increase in temperature, until the $\mathrm{H}_{2}$ started to dissolve and/or react (faster than the pressure rise by temperature) resulting in steady decrease of pressure.

After cooling to room temperature, the final pressure was recorded and, after taking a gas sample for analysis, the reactor was completely depressurized. The gas samples were analyzed in a gas chromatograph (micro-GC Varian CP-4900 with three analytical columns: $10 \mathrm{~m}$ Molsieve 5A Ar, $10 \mathrm{~m}$ Molsieve 5A He, $10 \mathrm{~m}$ PPQ He, 8 m Sil-5CB He using helium and argon as carrier gases).

To recover the final product, $50 \mathrm{ml}$ of dichloromethane was added, scraping the reactor walls with a metal bar to recover as much contents as possible. The collected liquid sample was vacuum filtrated (glass microfiber filter, Whatman GF/B, $1 \mu \mathrm{m}$ pore size) to recover the mixture of catalyst (if added) and solid residue possibly formed. The filtration system used is described elsewhere (in Chapter 2). To remove any residual solvent, the solids were dried at $105^{\circ} \mathrm{C}$ over $24 \mathrm{~h}$ and finally quantified by weighing. The amount of solid residue produced was calculated by subtracting the amount of catalyst 
initially added from the total residue mixture recovered. Dichloromethane was removed by continuously flushing with $\mathrm{N}_{2}$ at room temperature in a fume hood until a constant weight of oil was obtained. The yields of the various products (treated oil, produced solid residue and gas) were calculated as their mass divided by the mass of HTL oil initially loaded into the reactor.

With some of the oils obtained after hydrotreatment, a blending test with diesel was performed by mixing equal amounts of each of them (by weight). The mixture was intensively shaken for about 1 minute and left for $24 \mathrm{~h}$ to settle. After centrifugation, the diesel fraction and the oil residue were separated and weighted. The measurements were performed in duplo. The amount of oil dissolved in diesel was expressed as:

Oildissolved $(\mathrm{wt} \%)=\left(1-\frac{\text { residue }(\mathrm{g})}{\text { oil initiallyadded }(\mathrm{g})}\right) \cdot 100 \%$

\subsection{Oil analyses}

The elemental composition (Thermo Scientific Flash 2000, CHN-S) and the molecular weight $(\mathrm{Mw})$ distribution (Agilent 1200 series HPLC system with 3 GPC PLgel $3 \mu \mathrm{m}$ MIXED-E columns connected in series) of the HTL oils and the oils obtained after hydrotreatment were determined. More details about the equipment and their analytical procedure can be found in Chapter 2. Non-normalized $\mathrm{Mw}$ distributions will be presented. Therefore, the oil solutions $(0.040 \mathrm{~g}$ of oil in $4.00 \mathrm{ml}$ tetrahydrofuran; the solvent was able to dissolve all the oil) for SEC (size exclusion chromatography) analysis were prepared as accurate as possible to exclude differences on sample size among the oils analysed. Elemental analysis was performed in duplicate; if the difference between duplicates was within $\pm 1 \%$, the results were accepted and the average values were taken. With the elemental composition, the higher heating value of the oils was estimated using Boie's formula. $^{9}$

The result obtained from a micro carbon residue test (MCRT) provides an indication of the coke forming tendency of the fuel under degradation conditions. The oils were analysed for that using an ACR-M3 Micro Carbon Residue Tester (Tanaka Scientific) according to ASTM standard D4530-07. The test results are equivalent to the Conradson Carbon Residue test.

A characterization of the oils in a molecular level was performed with a gas chromatograph equipped with a mass spectrometer (GC 7890A MS 5975C, Agilent 
Technologies) and a capillary column (Varian CP9154 $60 \mathrm{~m}$ ). Samples were dissolved in acetone ( $5 \mathrm{wt} \%$ of oil in solution) and filtered before analysis (Whatman 0.2 um filter). While complete dilution of the treated oils was achieved with acetone, a small fraction of the HTL oils remained insoluble being, most probably, a mixture of very heavy compounds that, most likely, would not elute from the GC anyway. An amount of $1 \mu \mathrm{L}$ of sample was injected in a split ratio of 20:1. The column was operated in a constant flow mode using $2 \mathrm{ml} / \mathrm{min}$ of helium as a carrier gas. The injection port temperature was $250^{\circ} \mathrm{C}$. The following thermal program was used: $45^{\circ} \mathrm{C}$ for $4 \mathrm{~min}$, from $45^{\circ} \mathrm{C}$ to $280^{\circ} \mathrm{C}$ at $3^{\circ} \mathrm{C} / \mathrm{min}$ and then $310^{\circ} \mathrm{C}$ during $20 \mathrm{~min}$. Identification of each compound was based on retention times and our inspection of the MS fragmentation patterns and their best matches with mass spectra stored in the spectral library (NIST Mass Spectral Library, Version 08), therefore, their molecular identity should be considered as tentative. For some hydrocarbons, standard solutions were used for 'spiking' to ensure their identity in the oil chromatograms. For that, a known amount of a hydrocarbon was added into an oil and the chromatogram obtained was compared to that of the oil without the added hydrocarbon in order to see the peak area increase. The hydrocarbons tested are listed in Section 2.1. For hexadecane calibration, standard solutions were prepared to find an equation relating hexadecane concentration to peak area. For the rest of the compounds, a minimum of $90 \%$ match with the identity given by the spectral library was used as criterion.

For the determination of functional groups in the oils, FT-IR (Fourier transform infrared spectroscopy; Bruker Tensor 27; $1 \mathrm{~cm}^{-1}$ resolution, 16 scans and range of 4000-650 $\mathrm{cm}^{-1}$ ) analyses were performed. An amount of 2-5 $\mathrm{LL}$ of oil was placed on a ZnSe micro-plate. Using the Bruker OPUS data Collection and analysis Program, the observed peaks were correlated to functional groups.

\section{Results and discussion}

This section is divided in two parts. First the effect of operating conditions on the oil composition will be discussed. In the second part, the quality of the hydrotreated oil will be evaluated through simple tests to assess its applicability for further co-processing as refinery feed or as drop-in fraction. 


\subsection{Effect of hydrotreatment operating conditions}

This section focuses on the results regarding the characterization of both the HTL oil feed and the treated oils obtained under different hydrotreatment conditions. In addition, only a short discussion about the product yields will be given.

Table 2 compares the elemental composition (dry ash free) of the initial HTL crude oils and their corresponding treated oils produced. From the elemental composition, indicative parameters as the higher heating value can be calculated and the realized reduction in oxygen and nitrogen content can be seen. In addition, from GC-MS analysis, the content of alkanes in the oils is also shown in Table 2 expressed as the integrated peak area in the total ion chromatogram per mass of oil analysed. This feature will be discussed later after evaluating the changes in elemental composition.

Table 2. Elemental composition of HTL and treated oils

\begin{tabular}{|c|c|c|c|c|c|c|c|c|}
\hline \multicolumn{3}{|c|}{ Experiments } & \multicolumn{4}{|c|}{$\begin{array}{c}\text { Elemental composition } \\
(\mathrm{wt} \%)\end{array}$} & \multirow{2}{*}{$\begin{array}{c}\text { HHV } \\
(\mathrm{MJ} / \mathrm{kg})\end{array}$} & \multirow{2}{*}{$\begin{array}{c}\text { Alkanes } \\
\left(\text { peak area } \cdot 10^{-7} / \mathrm{g} \text { oil }\right)\end{array}$} \\
\hline $\begin{array}{c}\mathrm{P} \\
(\mathrm{MPa}) \\
\end{array}$ & $\begin{array}{c}\mathrm{T} \\
\left({ }^{\circ} \mathrm{C}\right) \\
\end{array}$ & $\begin{array}{c}\text { Cat. } \\
(\mathrm{wt} \%)\end{array}$ & $\mathbf{N}$ & $\mathrm{C}$ & $\mathbf{H}$ & $\mathrm{O}$ & & \\
\hline \multicolumn{3}{|c|}{ HTL $250^{\circ} \mathrm{C}$} & 6.38 & 70.56 & 8.12 & 14.93 & 33.0 & 26.2 \\
\hline 10 & 300 & no & 4.24 & 76.63 & 9.76 & 9.37 & 37.5 & 59.8 \\
\hline 10 & 300 & 20 & 5.89 & 76.09 & 10.70 & 7.33 & 38.7 & 55.5 \\
\hline 15 & 300 & 20 & 5.91 & 78.35 & 10.63 & 5.11 & 39.7 & 59.6 \\
\hline 15 & 400 & no & 3.19 & 80.93 & 10.86 & 5.03 & 40.7 & 1121.9 \\
\hline 15 & 400 & 20 & 1.74 & 82.14 & 11.93 & 4.18 & 42.4 & 1504.9 \\
\hline 15 & 400 & 50 & 0.99 & 79.78 & 11.50 & 7.73 & 40.6 & 1645.3 \\
\hline \multicolumn{3}{|c|}{ HTL $350^{\circ} \mathrm{C}$} & 5.84 & 71.65 & 8.45 & 14.06 & 33.8 & 7.3 \\
\hline 10 & 300 & no & 4.01 & 74.98 & 8.82 & 12.19 & 35.5 & 190.8 \\
\hline 10 & 300 & 20 & 3.98 & 72.32 & 9.90 & 13.79 & 35.7 & 578.5 \\
\hline 15 & 300 & 20 & 4.39 & 74.05 & 9.49 & 12.07 & 36.0 & 174.8 \\
\hline 15 & 400 & no & 2.68 & 84.54 & 11.29 & 1.22 & 42.9 & 1117.5 \\
\hline 15 & 400 & 20 & 1.30 & 84.72 & 12.24 & 1.74 & 43.9 & 1312.6 \\
\hline 15 & 400 & 50 & 1.83 & 85.21 & 12.25 & 0.71 & 44.2 & 1517.7 \\
\hline
\end{tabular}

High degrees of deoxygenation and denitrogenation were achieved, especially at the higher temperature $\left(400^{\circ} \mathrm{C}\right)$. For the experiments with the $250^{\circ} \mathrm{C} \mathrm{HTL}$ oil, the reduction in nitrogen content is similar to the one for the $350^{\circ} \mathrm{C} \mathrm{HTL}$ oil, but the remaining oxygen content is significantly higher $\left(\right.$ at $400^{\circ} \mathrm{C}$ ). For all the experiments, the treated oil had a lower nitrogen and oxygen content and a higher carbon and hydrogen content than that 108 
of the HTL oil. As a result, the upgraded oils had a higher fuel grade by exhibiting higher energy densities going up to $44.2 \mathrm{MJ} / \mathrm{kg}$ for the $350^{\circ} \mathrm{C} \mathrm{HTL}$ oil treated with 50 wt\% catalyst. Even without adding a catalyst, the $\mathrm{O}$ and $\mathrm{N}$ levels were reduced showing that temperature, the presence of $\mathrm{H}_{2}$ or the combination thereof, already promotes heteroatoms removal. For those experiments without catalyts, a fraction of the hydrogen was consume, since the final gas pressure after cooling down to room temperature was always lower than the that of the $\mathrm{H}_{2}$ initially fed to the reactor.

An oil yield of 80-90 wt\% (on HTL oil basis) was obtained for both HTL oils treated at $300^{\circ} \mathrm{C}$ and it when down to 50-60 wt $\%$ when the temperature was increased to $400^{\circ} \mathrm{C}$. Although a decrease in oil yield with temperature was expected, as generally more gas and coke is produced, ${ }^{6,8}$ the reduction was too pronounced. Since also the mass balance at those temperatures could not be closed, the explanation should be found in mass losses during the solvent removal step in the standard experimental procedure for oil recovery. In fact, just by simple visual inspection we observed that the $400^{\circ} \mathrm{C}$ oils were particularly volatile and, as it will be shown later, an increased fraction of low boiling point compounds was clearly observed in the total ion chromatogram of those oils. Therefore, at $400^{\circ} \mathrm{C}$, thermal and/or catalytic hydro-/cracking reactions were promoted, resulting in an oil with smaller and more volatile compounds, part of which were likely lost during the solvent (dichloromethane) evaporation step. We believe that more optimized separation methods are needed for a better recovery of the upgraded oil, treated at $400^{\circ} \mathrm{C}$. Related studies ${ }^{5,8}$ showed that $70-80 \mathrm{wt} \%$ oil yield can be achieved at that temperature. When assuming a typical $75 \mathrm{wt} \%$ oil yield for the treatment at $400^{\circ} \mathrm{C}$ and using the experimentally determined carbon content of the HTL and treated oils, the carbon recovery in treated oil ranged from 83 to $90 \%$ (for the whole range of hydrotreatment conditions tested).

For the non-catalytic solid residue (i.e. coke), slightly more was produced for the treatment at $400^{\circ} \mathrm{C}$, with no clear trends observed for the change of the other operation conditions (catalyst loading and $\mathrm{H}_{2}$ pressure). Water formation has been reported for hydrogenation of pyrolysis oil. ${ }^{15}$ However, we were not able to detect any water in the final product, likely due to the small amount of oil used (meaning that even less water would be produced) and the fact that, if formed, it is likely to be eliminated through the oil recovery procedure (dichloromethane evaporation at almost $40^{\circ} \mathrm{C}$ plus drying under $\mathrm{N}_{2}$ gas for more than $24 \mathrm{~h}$ ).

The major compounds in the gas phase were unreacted and/or produced $\mathrm{H}_{2}, \mathrm{CH}_{4}$ and $\mathrm{CO}_{2}$. Similar gas compositions were reported in the related HTL oil hydrotreatment 
studies. ${ }^{1,6,8}$ For most of the experiments, $\mathrm{CH}_{4}$ production was higher than that of $\mathrm{CO}_{2}$. In addition, small hydrocarbons, as $\mathrm{C}_{2}-\mathrm{C}_{3}$ hydrocarbons, were produced with $\mathrm{C}_{2} \mathrm{H}_{6}$ being the most abundant. A clear increase of $\mathrm{C}_{2}-\mathrm{C}_{3}$ hydrocarbons in the gas occurred by increasing temperature thereby suggesting again that cracking reactions were favored at higher temperatures. In some of the experiments, more nitrogen than calculated from residual air was present in the gas phase. While in the studies using SCW no nitrogen was detected in the gas (only in the water as $\mathrm{NH}_{3}$ ), ${ }^{5,6} \mathrm{Li}$ and Savage ${ }^{8}$ reported the presence of $\mathrm{NH}_{3}$ in the gas when the oils were hydrotreated in dry form.

Compared to the hydrotreatment studies in $\mathrm{SCW}$ at $400^{\circ} \mathrm{C},{ }^{1,5,6}$ we obtained higher levels of deoxygenation and denitrogenation in the hydrotreated $350^{\circ} \mathrm{C} \mathrm{HTL}$ oils. As oils obtained at lower HTL temperature were not investigated in the referred studies, no reference is available for the $250^{\circ} \mathrm{C}$ oil. For the treatment at $400^{\circ} \mathrm{C}$, even without catalyst and 3 to $5 \mathrm{~h}$ shorter reaction time (compared to the $4 \mathrm{~h}$ in ${ }^{1,5}$ and $6 \mathrm{~h}$ in ${ }^{6}$ respectively) we achieved a comparable level of nitrogen reduction and higher oxygen reduction than that in SCW.

Compared to the hydrotreatment at $400^{\circ} \mathrm{C}$ and $4 \mathrm{~h}$ reaction time on dry oil with HZSM5 , as reported by $\mathrm{Li}$ and Savage, ${ }^{8}$ we obtained again similar level of nitrogen reduction but again a more favorable, oxygen reduction with only 1 hour of reaction time. It is speculated here that the improved heteroatom removal in this study is caused by the higher partial pressure of $\mathrm{H}_{2}$ used in this work. As stated by Duan et al., ${ }^{7}$ an increase in $\mathrm{H}_{2}$ pressure can enhance the main two mechanisms where this gas participates: saturating double bonds and hydrocracking. In their study they showed how higher $\mathrm{H}_{2}$ pressures can lead to higher conversion of pyridine into $\mathrm{NH}_{3}$. At higher pressures, a higher solubility of hydrogen in the oil is achieved and thereby resulting in a higher availability of hydrogen in the vicinity of the catalyst and increasing the rate of the reactions occurring during hydrotreatment. ${ }^{16}$

The hydrotreatment also improved the viscosity of the oils. We could observed very clear physical changes. Only visual examination could be performed, as the amount of oil was not sufficient for viscosity measurements in available equipment. Both temperature and catalyst loading increase showed a positive effect on viscosity, with the effect of temperature being more noticeable. At the same time, non-catalytic treatment had little effect on the viscosity. Therefore, although a certain reduction in $\mathrm{N}$ and $\mathrm{O}$ content by non-catalytic hydrotreatment (as shown in Table 2) can be achieved, a certain amount of catalyst seems required for viscosity reduction. With the highest temperature and catalyst loading, a freely flowing liquid was produced from both HTL oils, which were 
initially a highly viscous tar-like product not flowing at room temperature. Figure 1 shows the changes observed for the $250^{\circ} \mathrm{C}$ HTL oil (Figure 1(a)) and the same oil hydrotreated at $400^{\circ} \mathrm{C}$ and $50 \mathrm{wt} \%$ catalyst (Figure $1(\mathrm{~b})$ ).

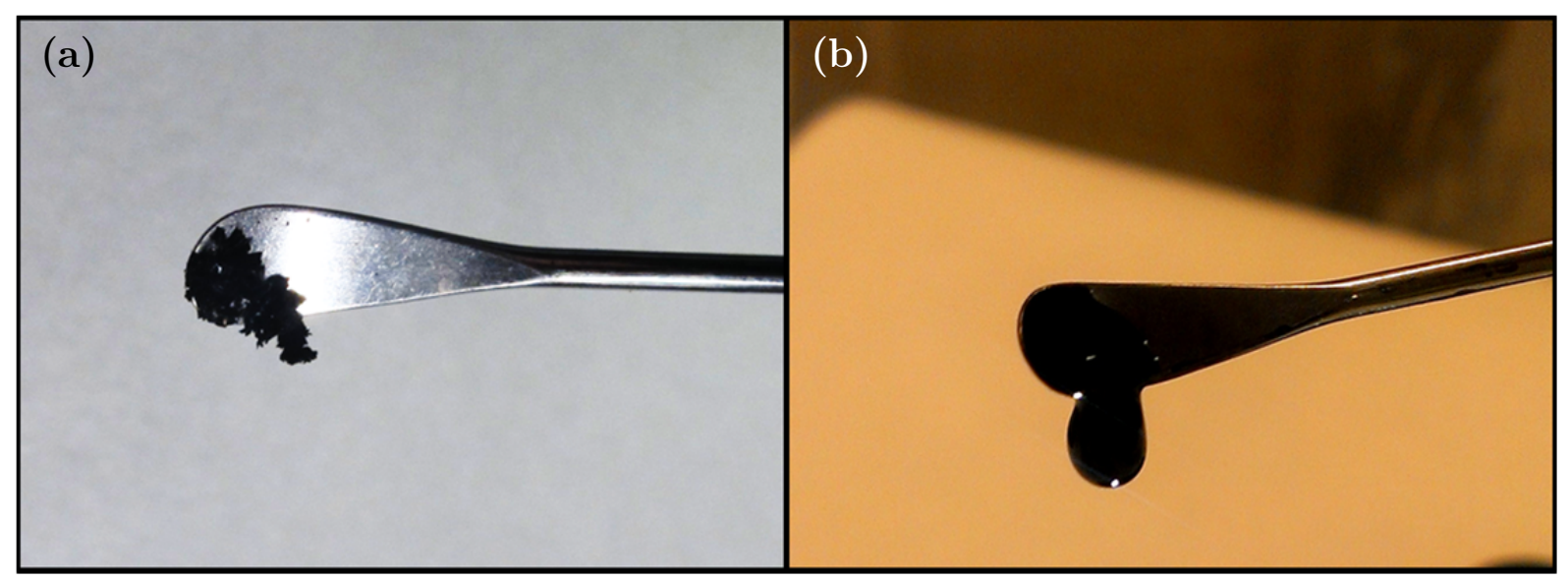

Figure 1. Pictures illustrating the change in viscosity with (a) the $250^{\circ} \mathrm{C}$ HTL oil and (b) the corresponding oil hydrotreated at $400^{\circ} \mathrm{C}$ and $50 \mathrm{wt} \%$ catalyst

Another aspect is related to the ash found in the oil. All treated oils had ash with the higher contents present in the initial HTL oils. From X-Ray Fluorescence spectrometry (XRF) of the recovered ash, Fe and $\mathrm{Cr}$ appeared to be the most abundant elements. While the origin of Fe could be from the biological algae components and their growth medium $\left(\mathrm{COMBO}^{17}\right)$, the source of $\mathrm{Cr}$ can only be the reactor wall material (INCOLOY 825). This indicates that a certain degree of reactor wall damage occurred during HTL and, possibly, also during hydrotreatment as high pressure $\mathrm{H}_{2}$ can also cause corrosion. ${ }^{18}$ Therefore, those metals could have also partially induced catalyzing effects during hydrotreatment.

The molecular weight $(\mathrm{Mw})$ distribution of the treated oils was compared to that of the initial HTL oils. The results are represented in Figure 2 where the curves were cut at low molecular weight $(\sim 100 \mathrm{~g} / \mathrm{mol})$ to remove the peaks corresponding to the degradation products of the GPC-eluent THF. The differential distribution $d_{\text {total mass }} / d_{\log \text { molar mass }}(\mathrm{W}(\log$ $\mathrm{M})$ ) is plotted versus the molar mass (M). Only the effect of temperature is shown as it was the parameter causing more remarkable changes with respect to elemental composition, viscosity and molecular weight distribution. The results reported are only for the $250^{\circ} \mathrm{C} \mathrm{HTL}$ oil, as similar molecular weight changes were observed for the $350^{\circ} \mathrm{C}$ HTL oil series. 


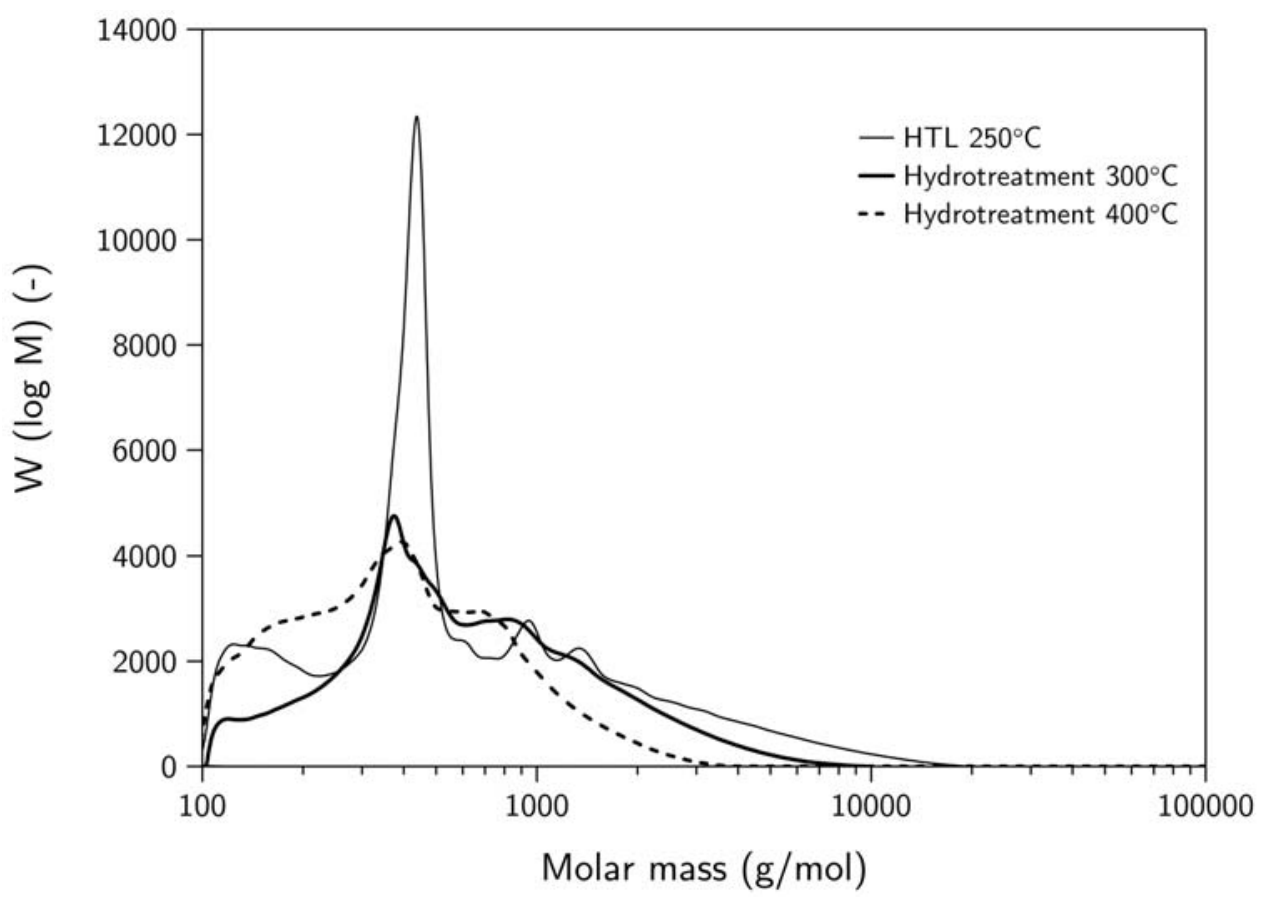

Figure 2. Molecular weight distribution of the $250^{\circ} \mathrm{C}$ HTL oil and the hydrotreated oils at different temperatures (20 wt\% catalyst and $15 \mathrm{MPa} \mathrm{H}_{2}$ )

In the work presented in Appendix of Chapter 2, a $250^{\circ} \mathrm{C} \mathrm{HTL}$ oil produced under the same liquefaction conditions and from the same algal feedstock was analyzed by means of combining column chromatography fractionation and size exclusion chromatography (SEC). The results showed that the large SEC peak at about 300-500 g/mol in Figure 2 corresponds to the presence of a large amount of lipid derived materials. The significant decrease of that peak in the treated oils indicates that, in the best case, the hydrotreatment promoted the conversion of those lipid derivatives (e.g. triglycerides, fatty acids, sterols, etc.) into other molecules such as hydrocarbons; however it could also indicate their reaction with other compounds. In Figure 2, the shift of the tailing of the distribution towards lower molecular weight (when going from the $250^{\circ} \mathrm{C}$ HTL oil to the oil treated at $300^{\circ} \mathrm{C}$ and at $400^{\circ} \mathrm{C}$ ) indicates the decrease in heavy compounds. In parallel, an increase can be observed in the region of lighter compounds (between 100 and $300 \mathrm{~g} / \mathrm{mol}$ ), which indicates again that the formation of smaller and lighter molecules is favored at higher temperatures.

Molecular characterization of the HTL and treated oils was performed by means of GCMS analysis. Although it is likely, and realized, that not all the compounds appeared in the chromatogram, as light substances could have been lost during solvent evaporation and very heavy substances are unlikely to elute from the GC column, the results give an indication of the overall changes in composition. 
The main chemical groups that could be identified in the oils were long-chain saturated hydrocarbons (alkanes), aromatic hydrocarbons (benzene derivatives) and nitrogen containing compounds. In the Appendix, GC-MS spectra of the HTL oils and the hydrotreated oils and a comprehensive table of the compounds identified with their tentative molecular identity and peak area per mass of oil analysed can be found. Also there, the results of the FT-IR analyses, aiming to observed possible changes in terms of functional groups, are reported (the results were in accordance with the observations that will be presented below).

Whereas the HTL oils were found to be a complex mixture (with the complexity of the composition increasing with temperature, see Appendix of Chapter 2) with a large fraction of $\mathrm{N}$ containing compounds, the treated oils were, in contrast, primarily a mixture of alkanes and a smaller fraction of aromatic hydrocarbons. As shown in Appendix of Chapter 2, the total fatty acid composition (accounting for both acylglycerols and fatty acids) of the HTL oils consisted of $\mathrm{C}_{16}$ to $\mathrm{C}_{18}$ fatty acids with different degrees of unsaturation (from saturated up to 4 double bonds).

The most interesting composition change produced by the hydrotreatment was the high fraction of alkanes. Their amount, as indicated by the GCMS response, increased both with temperature and catalyst loading, but clearly temperature had more effect. In Figure 3, the total ion chromatograms of HTL oils and treated oils illustrate the significant effect of temperature on the product composition. The abundance (y-axis) of all chromatograms presented has the same scale showing the clear increase of all peaks intensity and hence their area. The same increase in alkanes fraction can be seen in Table 2.

The $400^{\circ} \mathrm{C}$ treated oils chromatograms showed many and very well regularly spaced peaks (see Figure 3) corresponding to the series of n-alkanes ranging from from $\mathrm{C}_{8}$ (octane) to $\mathrm{C}_{28}$ (octacosane). Pentadecane $\left(\mathrm{C}_{15}\right)$, hexadecane $\left(\mathrm{C}_{16}\right)$, heptadecane $\left(\mathrm{C}_{17}\right)$ and octadecane $\left(\mathrm{C}_{18}\right)$ were the most abundant straight chain alkanes. That seems consistent with the TFA distribution of the initial HTL oils, since decarboxylation of $\mathrm{C}_{16}$ to $\mathrm{C}_{18}$ fatty acids would yield $\mathrm{C}_{15}$ and $\mathrm{C}_{17}$ alkanes, but also reduction reactions could occur forming n-alkanes with the same length as the related fatty acid (hence $\mathrm{C}_{16}$ and $\mathrm{C}_{18}$ ). The linear hydrocarbons smaller than $\mathrm{C}_{15}$ in the treated oils were probably formed through (hydro-)cracking reactions of longer chain alkanes since we could only identify heptadecane $\left(\mathrm{C}_{17}\right)$ in the original HTL oils, which was also identified in our previous study (Chapter 2 and its Appendix) together with pentadecane $\left(\mathrm{C}_{15}\right)$ and, as mention 
before, the shortest total fatty acid chain (from total fatty acid analysis) found in the HTL oils was $\mathrm{C}_{16}$.

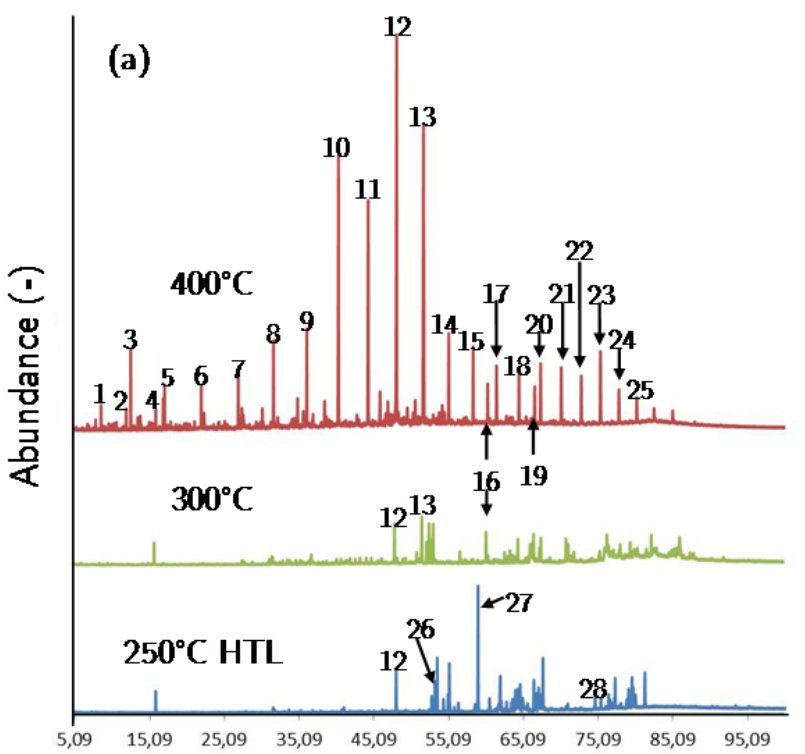

Time (min)

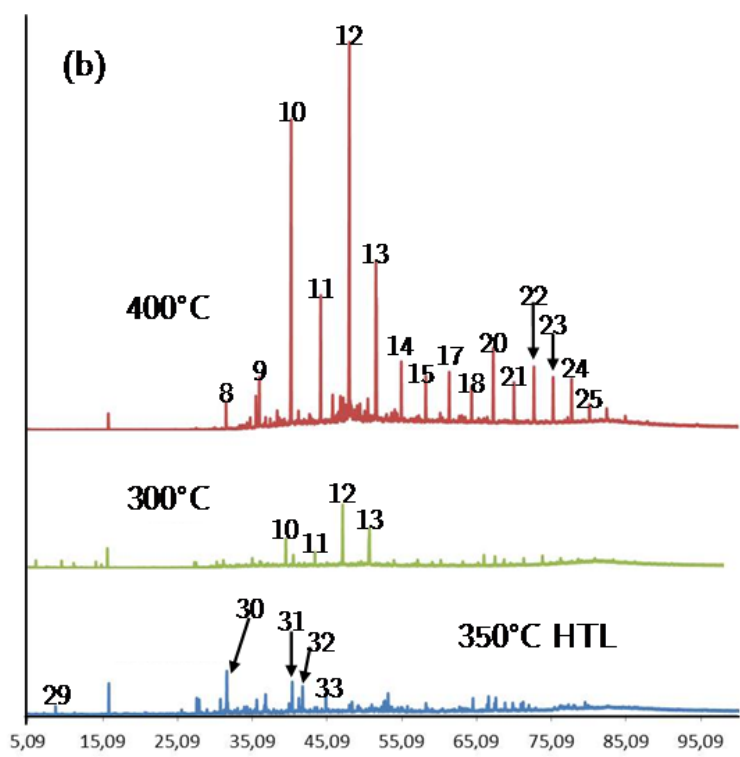

Time (min)
1: Toluene
8: Tridecane
15: Eicosane
22: Pentacosane
29: Pyrazine
2: Nonane
9: Tetradecane
16: Hexadecanenitrile
23: Hexacosane
30: 4-methyl-
3: Ethyl-benzene
10: Pentadecane
17: Heneicosane
24: Heptacosane
phenol
4: Decane
11: Hexadecane
18: Docosane
25: Octacosane
31: 1-pentadecene
5: Propyl-benzene
12: Heptadecane
19: Heptadecanenitrile
13: Octadecane
20: Tricosane
26: 2-phytene
32: Indole
6: Undecane
14: Nonadecane
21: Tetracosane
33: 3-methyl-1H-
indole

Figure 3. Effect of temperature (20 wt\% catalyst and $15 \mathrm{MPa} \mathrm{H}_{2}$ ) for (a) $250^{\circ} \mathrm{C}$ series and (b) $350^{\circ} \mathrm{C}$ series experiments with all the chromatograms having the same $\mathrm{x}$-axis scale

Again, HTL initial oil composition effects on the alkanes distribution were observed (see Figure 3). For the $250^{\circ} \mathrm{C}$ HTL oils treated: $\mathrm{C}_{8}-\mathrm{C}_{14}$ and $\mathrm{C}_{19}-\mathrm{C}_{28}$ were only identified in the treated oil at $400^{\circ} \mathrm{C}$. For the $350^{\circ} \mathrm{C}$ HTL oils series, $\mathrm{C}_{8}-\mathrm{C}_{12}$ were not identified (except for the oil treated with $50 \mathrm{wt} \%$ catalyst containing a small amount of $\mathrm{C}_{11}$ and $\mathrm{C}_{12}$ ). Above $\mathrm{C}_{13}$, all alkanes were detected in both $300^{\circ} \mathrm{C}$ and $400^{\circ} \mathrm{C}$ treated oils.

Aromatic hydrocarbons (excluding aromatic heteroarenes which have at least one $\mathrm{C}$ atom replaced by one heteroatom $\mathrm{O}, \mathrm{N}$ or $\mathrm{S}$; e.g. $\mathrm{N}$-aromatics), mainly alkyl substituted benzenes, polycyclic aromatic hydrocarbons (e.g. naphthalene derivative) and phenolic derivatives, were produced. For the $250^{\circ} \mathrm{C}$ hydrotreatment series, an increase of that chemical group occurred with increasing temperature and catalyst loading and most of them were alkyl substituted benzenes. On the other hand, only phenolic derivatives were 
identified in the $350^{\circ} \mathrm{C} \mathrm{HTL}$ oil and their amount decreased by hydrotreatment indicating deoxygenation. Further research and more detailed analyses are required to have better understanding of the effect of HTL oil composition on that of the oil obtained after hydrotreatment.

\subsection{Hydrotreated HTL algal oil for transportation fuels: applicability discussion}

Overall, nitrogen, oxygen and viscosity were substantially reduced by the hydrotreatment while obtaining an oil rich in n-alkanes, ranging from $\mathrm{C}_{8}$ to $\mathrm{C}_{28}$. The question is if the achieved oil quality is good enough to consider them for liquid fuel applications. Here only the hydrotreated oils obtained by treating with $50 \mathrm{wt} \%$ catalyst loading and at a temperature of $400^{\circ} \mathrm{C}$ will be evaluated, as they had the best combined quality in terms of $\mathrm{N}$ and $\mathrm{O}$ content, viscosity and alkane content.

In Table 3, several properties of the HTL oils and their corresponding treated oils are compared to that of various biomass-based (pyrolysis oil, hydrotreated pyrolysis oil and biodiesel) and fossil-based (long residue oil and diesel) liquid fuels.

Table 3. Liquid fuels comparison

\begin{tabular}{|c|c|c|c|c|c|c|}
\hline \multirow{2}{*}{ Product } & \multicolumn{4}{|c|}{ Elemental composition (wt\%) } & \multirow{2}{*}{ HHV (MJ/kg) } & \multirow{2}{*}{ MCRT (wt\%) } \\
\hline & $\mathbf{N}$ & $\mathrm{C}$ & $\mathbf{H}$ & $\mathrm{O}$ & & \\
\hline $250^{\circ} \mathrm{C}$ HTL oil & 6.4 & 70.6 & 8.1 & 14.9 & 33.0 & 12.0 \\
\hline Hydotreated $250^{\circ} \mathrm{C} \mathrm{HTL} \mathrm{oil}{ }^{\mathrm{a}}$ & 1.0 & 79.8 & 11.5 & 7.7 & 40.6 & 0.33 \\
\hline $350^{\circ} \mathrm{C}$ HTL oil & 5.8 & 71.7 & 8.4 & 14.1 & 33.8 & 16.8 \\
\hline Hydotreated $350^{\circ} \mathrm{C} \mathrm{HTL} \mathrm{oil}{ }^{\mathrm{a}}$ & 1.8 & 85.2 & 12.2 & 0.7 & 44.2 & 0.93 \\
\hline Pyrolysis oil $^{\mathrm{b}}$ & 0.0 & 54.3 & 7.0 & 38.7 & 17.4 & 26.2 \\
\hline Hydotreated Pyrolysis oil ${ }^{\mathrm{b}}$ & 0.0 & 74.4 & 10.0 & 15.6 & 35.1 & 5.57 \\
\hline Biodiesel $^{c}$ & n.s. ${ }^{\mathrm{c}}$ & 77.0 & 11.9 & 9.4 & 39.8 & $0.04^{\mathrm{e}}$ \\
\hline Diesel $^{\mathrm{c}}$ & n.s. & 86.1 & 13.9 & 0.0 & 46.2 & $0.04^{\mathrm{e}}$ \\
\hline Long residue oil ${ }^{\mathrm{d}}$ & 0.0 & 86.6 & 12.8 & 0.6 & 45.3 & 2.04 \\
\hline
\end{tabular}

${ }^{a}$ From hydrotreatment experiment with $50 \mathrm{wt} \%$ catalyst loading and $400^{\circ} \mathrm{C}$ temperature. ${ }^{\mathrm{b}}$ Data taken from ${ }^{15}$; Pyrolysis oil from forest residue hydrotreated under $310^{\circ} \mathrm{C}, 290 \mathrm{~min}$ and a starting $\mathrm{H}_{2}$ pressure of 19 $\mathrm{MPa}$ at room temperature; HHV calculated with Reed's formula with the dry elemental composition and the water content; MCRT on dry basis as the results were corrected for the water content. ${ }^{c}$ Data presented by Lin et al. ${ }^{19}$; commercial biodiesel produced from soybean oil and diesel from the Chinese Petroleum Company in Taiwan; n.s.= not specified. ${ }^{\mathrm{d}}$ Reported by de Miguel Mercader et al. ${ }^{15}$ with data given by Shell Global Solutions. ${ }^{e}$ For diesel, maximum according to ASTM D 975-11b; for biodiesel, maximum according to ASTM D 7467-10. 
The reduction of the nitrogen content by hydrotreatment at $400^{\circ} \mathrm{C}$ is more than significant. However, the lowest nitrogen content reached was $1 \mathrm{wt} \%$, which is actually still too high. The nitrogen content in crude oil ranges from 0.1-1.5 wt\% and the produced fossil-based diesel ends up having only traces or free of nitrogen. ${ }^{20}$ Therefore, unless this nitrogen content is further reduced by process optimization (which was outside the scope of this work), the total hydrotreated algal oil cannot be used as such for fuel applications, but requires further fractionation or co-processing in existing petroleum refineries.

The $\mathrm{C}, \mathrm{H}$ and $\mathrm{O}$ contents of the hydrotreated $250^{\circ} \mathrm{C} \mathrm{HTL}$ oil are similar to that of biodiesel, thereby showing comparable heating values. With respect to those same properties, the hydrotreated $350^{\circ} \mathrm{C}$ HTL oil and the long residue are even closer. When comparing with the lignocellusic-based oils (pyrolysis oil and its upgraded form by hydrodeoxygenation), the higher quality of the algae-based fuels becomes evident. The micro carbon residue (in MCRT tests), which provides an indication of the coke forming tendency under degradation/cracking conditions in refinery operations for the HTL algal oils, was significantly reduced by the hydrotreatment process and is comparable to (if not better than) the value for long residue oil.

In the work by de Miguel Mercader et al. ${ }^{15,21}$ hydrotreated pyrolysis oil was successfully co-processed with fossil long residue (both shown in Table 3) in a lab-scale FCC unit (catalytic cracking) with no operational problems and showing comparable products and yields to those obtained using fossil feed only. For such co-processing options, good miscibility with refinery products and a low MCRT value are relevant requirements. A significant increase of undesired products (coke and dry gas) was obtained when only hydrotreated pyrolysis oil was processed by catalytic cracking. Judging from the properties comparison shown in Table 3, both the HTL and corresponding hydrotreated algal oils can most likely be successfully co-processed. Even processing pure hydrotreated algal oil in the FCC unit might lead to positive results, as their oxygen content and MCRT value is much lower. The oxygen content of HTL oils is already at the level of hydrotreated pyrolysis oil, but their MCRT might be still high for the pure processing option. The oxygen content of hydrotreated HTL oils goes even below $1 \mathrm{wt} \%$ with a MCRT considerably lower than that of pyrolysis oil, hydrotreated pyrolysis oil and long residue.

The use of the algal-oils (both HTL and hydrotreated) might not be only limited to coprocess with the streams going to the FCC unit. Processing with middle distillates (or Straight-run gas oils; SRGO) used for the production of diesel and domestic fuel-oils 
should be further investigated. Co-processing of hydrotreated pyrolysis oil with straight run gas oil (SRGO) in a HDS unit showed an increase of the sulphur content of the product due to the competition between hydrogeoxygenation and hydrodesulfurization. ${ }^{14}$ Our oils have much lower oxygen content, specially the hydrotreated ones, therefore that competition would be significantly reduced. Nevertheless, co-processing with HTL oil (non-hydrotreated) seems less likely as the MCRT is still high.

Another option would be to extract the fraction of the oil that is valuable for direct drop-in. Figure 4 shows the aliphatic hydrocarbon distribution (as percentage of total area of all aliphatic hydrocarbon peaks) for the hydrotreated algal oils evaluated in this section. By 'spiking' the hexadecane peak (as indicated by the GCMS internal library) was confirmed and by varying the amount of additional hexadecane, a calibration for the response was performed. With this calibration, it was determined that the concentration of hexadecane in the treated oils, as shown in Figure 4, was 4.0 and 5.1 wt\% of the total treated oil. Assuming that the sensitivity towards hexadecane can be also used to quantify the rest of the n-alkanes, the range from $\mathrm{C}_{15}-\mathrm{C}_{18}$ hydrocarbons (the most abundant fraction) would account for almost $20 \mathrm{wt} \%$ of the oil.

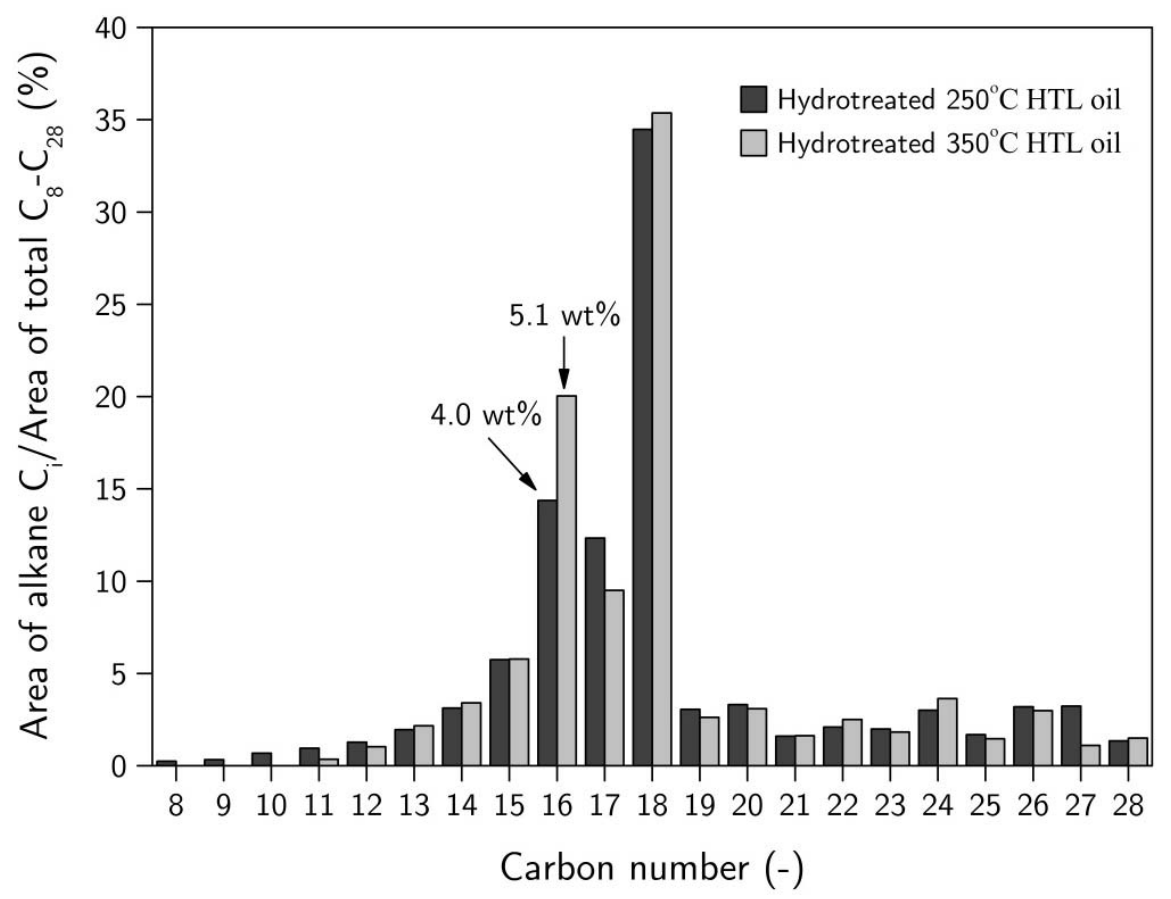

Figure 4. Alkanes distribution of hydrotreated oils (as percentage of total area of all alkane peaks)

Overall, it seems that already a significant fraction of the oil is made of diesel-range hydrocarbons ${ }^{20}$ which can possibly be considered for direct co-feeding in a refinery stream for the production of diesel or might be even directly miscible with diesel. 
The alkane yield (20 wt\% on hydrotreated HTL oil basis) can also be calculated on algae intake basis using the initial HTL oil yields $\left(31.9 \mathrm{wt} \%\right.$ for $250^{\circ} \mathrm{C}$ HTL and $56.5 \mathrm{wt} \%$ for $350^{\circ} \mathrm{C} \mathrm{HTL}$ ) and assuming that a hydrotreatment oil yield of $75 \mathrm{wt} \%$ is achieved at $400^{\circ} \mathrm{C}$ hydrotreatment by an improved product recovery. That leads to an alkane yield of $4.8 \mathrm{wt} \%$ (on algae dry weight basis) for the $250^{\circ} \mathrm{C}$ route and $8.6 \mathrm{wt} \%$ for the $350^{\circ} \mathrm{C}$ route. Therefore, although the $250^{\circ} \mathrm{C}$ HTL oil has a higher lipid content, it is preferable to perform HTL at higher temperatures and produce more oil in order to end up with a larger amount of diesel-range alkanes.

Miscibility with fossil based fuel streams is for co-processing and/or use via direct drop-in of (a fraction of the-) hydrotreated algal oil into diesel essential. Therefore, a blending experiment was performed where equal amounts (by weight) of both products were mixed. After intensive mixing, only one single phase could be observed, with a significant increase of viscosity compare to that of the pure diesel. The blend was left for $24 \mathrm{~h}$ and, after that, it was centrifuged. Thereby two clear phases were obtained: a pellet of diesel insolubles (residue in Eq. 1) and a liquid of hydrotreated algal oil compounds soluble in diesel. After separation of the phases, the following results were obtained: $67.3 \pm 1.4 \mathrm{wt} \%$ of the hydotreated $250^{\circ} \mathrm{C}$ HTL oil was soluble in diesel while, for the hydotreated $350^{\circ} \mathrm{C}$ HTL oil, the soluble fraction accounted for a $36.9 \pm 2.7 \mathrm{wt} \%$. Higher solubility for the hydrotreated $250^{\circ} \mathrm{C}$ HTL oil was expected as it is produced from an HTL oil feedstock with lower compositional complexity than that of the $350^{\circ} \mathrm{C}$ HTL, which has higher amounts of heavy compounds. It is important to point out that this was only a fast test to shortly assess the blending capabilities of the upgraded algal oils. Aspects like stability of the blend over time should further evaluated. However, the results already show their potential in refinery and, considering the dilutions achieved in diesel (i.e. the end product), hydrotreated HTL algal oil will probably blend even better with middle distillates prior to hydrotreatment.

\section{Conclusions}

Hydrothermal liquefaction oil obtained from microalgae is not directly suitable as transportation fuel replacement, considering especially the high nitrogen and oxygen content and viscosity. The high nitrogen content cannot effectively be reduced by solvent extraction without losing a more than proportional amount of oil, since the $\mathrm{N}$ is present in the oil in the form of organic molecules. 
In this study, the feasibility of hydrotreatment of the dry HTL oil was investigated and was found to result in an oil with improved properties. Comparing with related studies performing hydrotreatment in SCW, it was found that processing the oil in its dry form leads to higher degrees of $\mathrm{N}$ and $\mathrm{O}$ reduction, probably enabled by the higher $\mathrm{H}_{2}$ pressures. Both $\mathrm{N}$ and $\mathrm{O}$ were substantially reduced and the oils produced were freely flowing at room temperature. The treatment reduced the amount of aromatic nitrogen containing compounds, lipid substances and oxygenated molecules while a significant increase in n-alkanes ranging from $\mathrm{C}_{8}$ to $\mathrm{C}_{28}$ was obtained. Recovery of this fraction from the treated oils could be an interesting option for valorization.

The increase in severity of operating conditions (especially temperature) for hydrotreatment led to oils with higher quality. Improved product recovery procedures should enable quantitative evaluation of consumption and allow to optimize reaction conditions as temperature, hydrogen pressure and time. In view of the differences observed between the hydrotreated oils starting from 250 and $350^{\circ} \mathrm{C} \mathrm{HTL}$ oils, the combination of HTL operating conditions with hydrotreatment conditions needs further investigation. Underlying reaction networks and -mechanisms are still far from understood. Also, clear methods to assess oil quality for anticipated applications are needed. This can only be realized through enhanced cooperation with refinery operation and scale up of (algae and oil) production. If the process is applied within the frame of an algae biorefinery, the high pressure $\mathrm{H}_{2}$ required can be produced from supercritical water gasification of the organics in the aqueous phase recovered after HTL. Finally, coprocessing of HTL algal oils in a fossil fuel refinery, with and without hydrotreatment, seems feasible and should be tested.

\section{Acknowledgements}

The authors are thankful for the financial support by the province of Overijssel via the Green Energy Initiative of the University of Twente (The Netherlands).

\section{Bibliography}

[1] P. Duan, P.E. Savage, Upgrading of crude algal bio-oil in supercritical water, Bioresour. Technol., 102(2), 1899-1906, 2011.

[2] C. Torri, D. Fabbri, L. Garcia Alba, D.W.F. Brilman, Upgrading of oils derived from hydrothermal treatment of microalgae by catalytic cracking over H-ZSM-5: A comparative Py-GC-MS study, J. Anal. 
Appl. Pyrolysis, 101(0), 28-34, 2013.

[3] S.G. Roussis, R. Cranford, N. Sytkovetskiy, Thermal treatment of crude algae oils prepared under hydrothermal extraction conditions, Energy Fuels, 26(8), 5294-5299, 2012.

[4] P. Duan, P.E. Savage, Hydrothermal liquefaction of a microalga with heterogeneous catalysts, Ind. Eng. Chem. Res., 50(1), 52-61, 2010.

[5] P. Duan, P.E. Savage, Catalytic hydrotreatment of crude algal bio-oil in supercritical water, Appl. Catal., B, 104(1-2), 136-143, 2011.

[6] P. Duan, P.E. Savage, Catalytic treatment of crude algal bio-oil in supercritical water: optimization studies, Energy Environ. Sci., 4(4), 1447-1456, 2011.

[7] P. Duan, P.E. Savage, Catalytic hydrothermal hydrodenitrogenation of pyridine, Appl. Catal., B, 108109(0), 54-60, 2011.

[8] Z. Li, P.E. Savage, Feedstocks for fuels and chemicals from algae: Treatment of crude bio-oil over HZSM-5, Algal Res., 2(2), 154-163, 2013.

[9] S.A. Channiwala, P.P. Parikh, A unified correlation for estimating HHV of solid, liquid and gaseous fuels, Fuel, 81(8), 1051-1063, 2002.

[10] J. Fu, X. Lu, P.E. Savage, Catalytic hydrothermal deoxygenation of palmitic acid, Energy Environ. Sci., 3(3), 311-317, 2010.

[11] J. Wildschut, F.H. Mahfud, R.H. Venderbosch, H.J. Heeres, Hydrotreatment of fast pyrolysis oil using heterogeneous noble-metal catalysts, Ind. Eng. Chem. Res., 48(23), 10324-10334, 2009.

[12] A. Gutierrez, R.K. Kaila, M.L. Honkela, R. Slioor, A.O.I. Krause, Hydrodeoxygenation of guaiacol on noble metal catalysts, Catal. Today, 147(3-4), 239-246, 2009.

[13] B. Zhang, M. Keitz, K. Valentas, Thermal effects on hydrothermal biomass liquefaction, Appl. Biochem. Biotechnol., 147(1-3), 143-150, 2008.

[14] F. de Miguel Mercader, M.J. Groeneveld, S.R.A. Kersten, C. Geantet, G. Toussaint, N.W.J. Way, C.J. Schaverien, K.J.A. Hogendoorn, Hydrodeoxygenation of pyrolysis oil fractions: process understanding and quality assessment through co-processing in refinery units, Energy Environ. Sci., 4(3), 985-997, 2011.

[15] F. de Miguel Mercader, M.J. Groeneveld, S.R.A. Kersten, N.W.J. Way, C.J. Schaverien, J.A. Hogendoorn, Production of advanced biofuels: Co-processing of upgraded pyrolysis oil in standard refinery units, Appl. Catal., B, 96(1-2), 57-66, 2010.

[16] P.M. Mortensen, J.D. Grunwaldt, P.A. Jensen, K.G. Knudsen, A.D. Jensen, A review of catalytic upgrading of bio-oil to engine fuels, Appl. Catal., A, 407(1-2), 1-19, 2011.

[17] S. Kilham, D. Kreeger, S. Lynn, C. Goulden, L. Herrera, COMBO: a defined freshwater culture medium for algae and zooplankton, Hydrobiologia, 377(1), 147-159, 1998.

[18] P. Kritzer, N. Boukis, E. Dinjus, Factors controlling corrosion in high-temperature aqueous solutions: a contribution to the dissociation and solubility data influencing corrosion processes, J. Supercrit. Fluids, 15(3), 205-227, 1999

[19] C.-Y. Lin, H.-A. Lin, Diesel engine performance and emission characteristics of biodiesel produced by the peroxidation process, Fuel, 85(3), 298-305, 2006.

[20] C. Song, C.S. Hsu, I. Mochida, Chemistry of Diesel Fuels, Taylor \& Francis Group, 2000.

[21] F. de Miguel Mercader, M.J. Groeneveld, S.R.A. Kersten, R.H. Venderbosch, J.A. Hogendoorn, Pyrolysis oil upgrading by high pressure thermal treatment, Fuel, 89(10), 2829-2837, 2010. 


\section{Appendix B}

Evaluation of different upgrading methods and additional analyses of hydrotreated oils 


\section{B.1 Evaluation of upgrading paths}

In this research, different upgrading routes (Figure B.1) were evaluated. For denitrogenation, two main paths can be considered: direct HTL of fresh algae followed by upgrading of the oil produced; or pre-treatment of fresh algae (route (1) Figure B.1), aiming to reduce the nitrogen content by extracting the proteins and, in this way, leading to an oil with lower nitrogen content. From the direct HTL path, the crude oil could be further treated by various methods. such as the ones shown in Figure B.1: extractive techniques (option (2) in Figure B.1) to separate the nitrogen containing compounds, catalytic cracking using a zeolite catalyst (route (3) in Figure B.1) and hydrotreatment with high pressure $\mathrm{H}_{2}$ with or without the use of a catalyst (option (4) in Figure B.1).

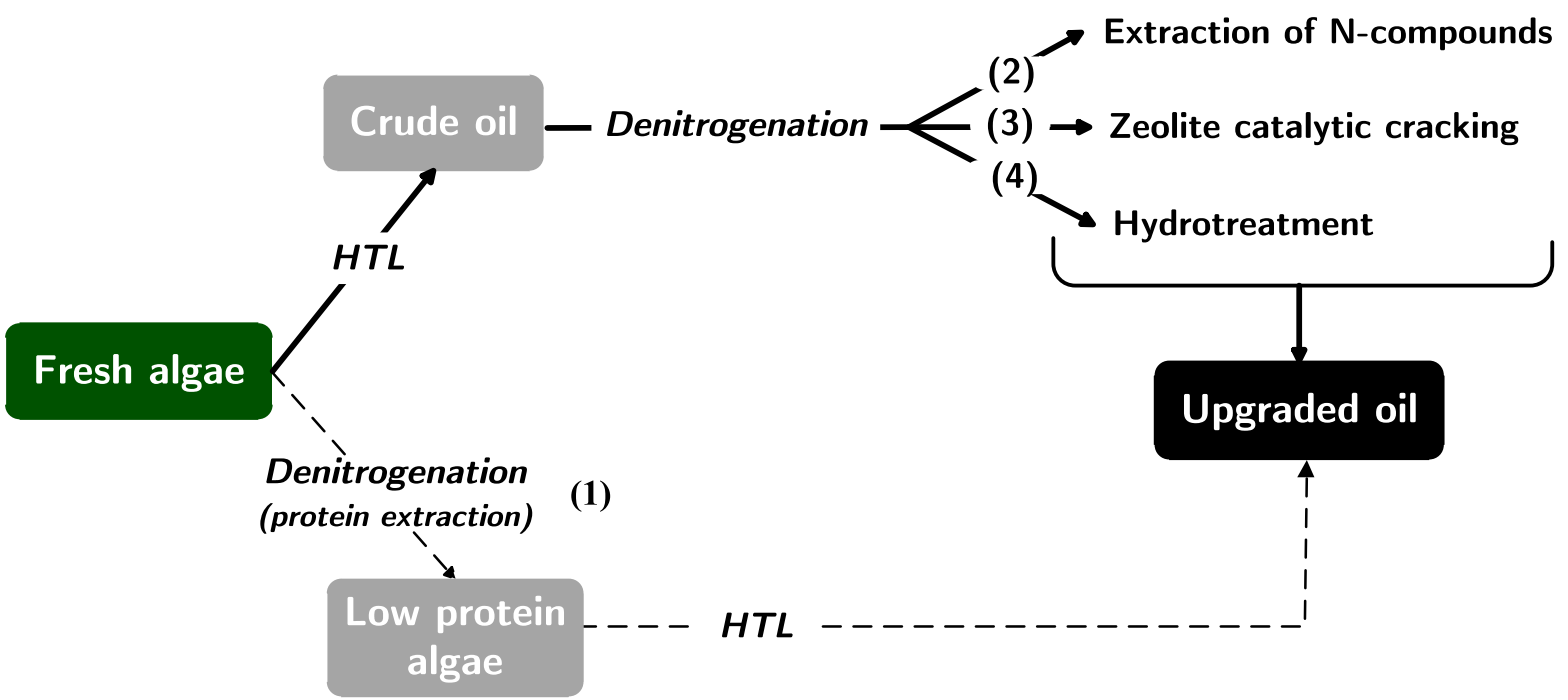

Figure B.1. Possible upgrading routes

In a preceding study on the detailed molecular characterization of HTL oils (produced from the same algal biomass as the one used here) presented in Appendix of Chapter 2 we found that the nitrogen fraction contains mostly cyclic dipeptides (diketopiperazines), pyrroles, pyrrolidinones, pyrazines, pyridines, piperidines, indole derivatives, aromatic amines and amides, and various peptides produced by means of thermal degradation of algal proteins. Several studies reporting methods for the elimination of these nitrogen substances (mainly heterocyclic compounds) from oils or fuels were found, most of them based on extraction and adsorption techniques:

- Extraction methods: with acidified water; ${ }^{1}$ with $2 \% \mathrm{H}_{2} \mathrm{SO}_{4}$ in ethanol; ${ }^{2}$ with water and methanol; ${ }^{3}$ by ion complexation; ${ }^{4}$ with ionic liquids; ${ }^{5}$ 
- Adsorption methods: with lithium-modified silica adsorbent $;{ }^{6}$ using $\mathrm{Cu}(\mathrm{I}) \mathrm{Y}$ zeolite. ${ }^{7}$

For denitrogenation of the HTL oil, extraction with acidified water and extraction with water and methanol were the methods showing shorter experimental time, easier manipulation and lowest price of the reagents used. Therefore, these two extraction methods were tested (route (2) in Figure B.1) on the HTL algal oils produced in the work presented in Chapter 2. Although a certain degree of denitrogenation was achieved (around 30\% N reduction), only an oil yield of about $37 \mathrm{wt} \%$ (mass of oil recovered after extraction divided by the initial mass oil treated) was obtained with still the same oxygen content and viscosity as that of the initial oil. These extraction methods showed therefore to be rather inefficient and were discarded for further investigation.

In another collaborative work with the University of Bologna, the catalytic cracking of HTL oil over HZSM-5 zeolite was studied (route (3) in Figure B.1), simulated with a PyGC-MS system, operated at $600^{\circ} \mathrm{C}$. All the experimental details and results of this work are reported elsewhere. ${ }^{8}$ Both $\mathrm{N}$ and $\mathrm{O}$ were substantially reduced (less than $0.2 \mathrm{wt} \%$ remaining for both $\mathrm{N}$ and $\mathrm{O}$ ), with yields on oil basis ( $\sim 50 \%$ mass yield and $\sim 60 \%$ carbon yield) slightly lower when compared to a long chain hydrocarbon model compound (hexatriacontane), but higher than those from cellulose and pyrolysis oil. The product contained mostly aromatic hydrocarbons but also their alkylated derivatives and small olefins. Hence, significant deoxygenation and denitrogenation was achieved and a highly aromatic product was obtained. Such a composition and corresponding high octane number, make it suitable for the production of gasoline, jet fuel and for (aromatics derived) chemicals production, but less suitable for the desired linear hydrocarbon rich fuel targeted in this work.

The presence of proteins in algae is known to be responsible for its high nitrogen content and, hence, protein removal prior to liquefaction is another approach to reduce the nitrogen content in the HTL product. Research on protein extraction from algal biomass has been carried out in the past, but reported separation techniques are mostly focused on effectively recovering the proteins in their active form. rather than aiming for full recovery. Moreover, no single study has been found reporting a method in which all the proteins were recovered. This is probably related to the high added value of technofunctional proteins (used as food ingredients and in the pharmaceutical industry), making the development of extraction methods with the aim of reducing the full algae $\mathrm{N}$ content less interesting. 
In this study physical and chemical treatments were implemented and tested for their efficacy to reduce completely the nitrogen content of the initial algae fed ((1) in Figure B.1). First, a physical treatment by milling (bead mill DYNO ${ }^{\circledR}$-Mill type MULTI LAB, Switzerland) was used to break the algal cell walls in order to release the water soluble proteins. This proved to be insufficient and a further hot alkaline chemical treatment, adapted from Meijer and Wijffels, ${ }^{9}$ was applied on the previous broken cells suspension to hydrolyze the amide bonds of the proteins into smaller fragments and molecules soluble in water. The $\mathrm{N}$ content of the algal debris was reduced by $50 \%$, leading to a $\mathrm{N}$ reduction in the $\mathrm{HTL}$ oil of $50 \%$ for the $250^{\circ} \mathrm{C}$ thermal treatment and $40 \%$ for $350^{\circ} \mathrm{C}$ thermal treatment (relative to the $\mathrm{N}$ in the HTL oil from fresh untreated algae). Although the nitrogen could be reduced, a dramatic drop in oil production was obtained. A reduction of more than $80 \%$ in oil yield was obtained, in comparison with the oil amount obtained from the initial algal mass without any pre-treatment. This is due to the low efficiency of the overall process including protein extraction. phase separation and HTL, both related to protein removal but also to operational and handling procedures involved. Therefore, unless a more optimized protein extraction method is developed aiming to obtain a low $\mathrm{N}$ content algal mass for HTL, this upgrading route seems less attractive. Meijer and Wijffels ${ }^{9}$ indicated that extremely low recoveries of proteins in Chlorella were caused by a poor extraction and not by the quantification method. However, a method optimal for one type of algal species can be far from optimal for another algal species and vice versa. ${ }^{9}$

\section{B.2 HTL and hydrotreated oils composition}

The individual compounds identified in HTL and hydrotreated oils are listed in Table B.1. In Table B.2. the individual compounds are classified in various chemical groups. Note that some compounds can be classified into two chemical classes (e.g. phenol as oxygen containing compound and aromatic hydrocarbon). 
Table B.1. Tentative identities of compounds identified in total ion chromatograms of HTL and hydrotreated oils

\begin{tabular}{|c|c|c|c|c|c|c|c|c|c|c|c|c|c|c|c|}
\hline \multirow[b]{2}{*}{ R.T. } & \multirow[b]{2}{*}{ Compound } & \multicolumn{14}{|c|}{ Experiments (peak area $\cdot 10^{-7} / \mathrm{g}$ oil) } \\
\hline & & $\begin{array}{c}\text { HTL } \\
250^{\circ} \mathrm{C}\end{array}$ & $\begin{array}{c}10^{\mathrm{a}}, \\
300^{\mathrm{b}} \\
\text { no }^{\mathrm{c}}\end{array}$ & $\begin{array}{c}10 \\
300 \\
20\end{array}$ & $\begin{array}{c}15 \\
300 \\
20\end{array}$ & $\begin{array}{c}15 \\
400 \\
\text { no }\end{array}$ & $\begin{array}{c}15 \\
400 \\
20\end{array}$ & $\begin{array}{c}15 \\
400 \\
50\end{array}$ & $\begin{array}{c}\text { HTL } \\
350^{\circ} \mathrm{C}\end{array}$ & $\begin{array}{c}10, \\
300, \\
\text { no }\end{array}$ & $\begin{array}{c}10 \\
300 \\
20\end{array}$ & $\begin{array}{c}15 \\
300 \\
20\end{array}$ & $\begin{array}{c}15 \\
400 \\
\text { no }\end{array}$ & $\begin{array}{c}15 \\
400 \\
20\end{array}$ & $\begin{array}{c}15 \\
400 \\
50\end{array}$ \\
\hline 7.7 & 1-Methyl-piperidine & - & - & 4.3 & 0.8 & - & - & - & - & - & - & 0.7 & - & - & - \\
\hline 7.8 & Octane & - & - & - & - & - & 5.7 & 4.0 & - & - & - & - & - & - & - \\
\hline 8.6 & Toluene & - & - & - & - & 1.2 & 7.3 & 14.3 & - & - & - & - & - & - & - \\
\hline 8.8 & Pyrazine & - & - & - & - & - & - & - & 4.4 & - & - & - & - & - & - \\
\hline 11.9 & Nonane & - & - & - & - & 1.6 & 8.4 & 5.4 & - & - & - & - & - & - & - \\
\hline 12.4 & Methyl-pyrazine & 4.7 & - & - & - & - & - & - & - & - & - & - & - & - & - \\
\hline 12.5 & Ethyl-benzene & - & - & - & - & 4.9 & 34.5 & 26.7 & - & - & - & - & - & - & - \\
\hline 16.5 & 2.5-Dimethyl-pyrazine & 4.1 & - & - & - & - & - & - & - & - & - & - & - & - & - \\
\hline 16.9 & Decane & - & - & - & - & 4.5 & 14.4 & 11.2 & - & - & - & - & - & - & - \\
\hline 17.1 & Propyl-benzene & - & - & - & - & 3.3 & 20.6 & 15.0 & - & - & - & - & - & - & - \\
\hline 20.1 & 2.4.6-Trimethyl-pyridine & - & 11.8 & - & - & - & - & - & - & - & - & - & - & - & - \\
\hline 20.7 & 2-Ethyl-6-methyl-pyrazine & 1.4 & & - & - & - & - & - & - & - & - & - & - & - & - \\
\hline 21.1 & 1.2.3-Trimethyl-benzene & - & - & - & - & 2.3 & 3.8 & 3.2 & - & - & - & - & - & - & - \\
\hline 22.0 & Undecane & - & - & - & - & 9.0 & 19.9 & 15.6 & - & - & - & - & - & - & 5.3 \\
\hline 22.4 & Butyl-benzene & - & - & - & - & 3.3 & 7.8 & 5.3 & - & - & - & - & - & - & - \\
\hline 26.9 & Dodecane & - & - & - & - & 16.0 & 26.8 & 20.8 & - & - & - & - & 1.1 & - & 15.6 \\
\hline 27.6 & Phenol & - & - & 4.0 & 3.6 & 10.1 & 13.1 & 8.8 & 14.9 & 4.2 & 7.3 & 5.2 & 3.1 & 1.1 & 6.9 \\
\hline 28.0 & 1-Methyl-2-pyrrolidinone & - & - & - & - & - & - & - & 10.1 & 2.5 & 3.2 & 4.8 & - & - & - \\
\hline
\end{tabular}


$28.9 \quad$ N-(2-methylpropyl)acetamide

30.8 1-Ethyl-2-pyrrolidinone

$31.6 \quad$ Tridecane

31.7 4-Methyl-phenol

$33.1 \quad$ 1-Ethyl-2.5-

pyrrolidinedione

Methylbutyl)acetamide

34.4

35.7

36.1

36.9

36.9

40.3

40.4

41.8

44.3

44.9

48.1

51.7

53.2

55.1

58.4

59.0

126
1-Acetyl-pyrrolidine

$$
\text { 4-Ethyl-phenol }
$$

Tetradecane

2-Piperidinone

1.2.3.4-Tetrahydro-1.1.6trimethyl-naphtalene

Pentadecane

1-Pentadecene

Indole

Hexadecane

3-Methyl-1H-indole

Heptadecane

Octadecane

2-Phytene

Nonadecane

Eicosane

Isophytol

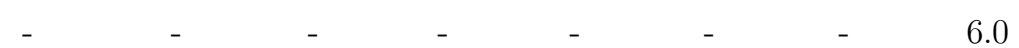

$-$

$-$

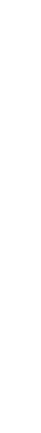

$\begin{array}{llll}- & 4.6\end{array}$

9.6

$\begin{array}{cccccc}2.7 & 2.5 & 4.5 & 2.3 & - & - \\ 1.6 & 6.0 & 7.2 & 17.9 & 17.3 & 32.9 \\ 5.9 & 6.2 & - & - & - & - \\ - & - & - & - & - & - \\ - & - & - & - & - & -\end{array}$




\begin{tabular}{|c|c|c|c|c|c|c|c|c|c|c|c|c|c|c|c|}
\hline 60.3 & Hexadecanenitrile & - & 3.5 & 13.5 & 18.5 & 20.9 & 22.8 & 34.5 & - & - & - & 2.8 & 36.8 & 5.1 & - \\
\hline 61.5 & Heneicosane & - & - & - & - & 29.6 & 38.1 & 26.2 & - & 4.6 & 11.0 & 4.6 & 30.7 & 39.1 & 24.7 \\
\hline 64.5 & Docosane & - & - & - & - & 30.7 & 35.4 & 34.5 & - & 3.4 & 5.8 & 3.4 & 29.1 & 26.9 & 38.1 \\
\hline 66.6 & Heptadecanenitrile & - & - & - & - & 16.4 & 24.9 & 41.8 & - & - & - & 2.3 & 28.4 & 4.0 & - \\
\hline 67.4 & Tricosane & - & - & - & - & 38.9 & 42.9 & 32.8 & - & 10.3 & 21.4 & 8.0 & 41.8 & 60.0 & 27.7 \\
\hline 68.9 & $\begin{array}{c}\text { 1-Methyl-9H-pyrido[3.4-b] } \\
\text { indole }\end{array}$ & - & 3.4 & 3.1 & 3.6 & - & - & - & 5.6 & 4.5 & 5.3 & 7.2 & - & - & - \\
\hline 69.9 & 9H-Pyrido[3.4-b] indole & - & - & - & - & - & - & - & 6.1 & - & - & - & - & - & - \\
\hline 70.2 & Tetracosane & - & - & - & - & 42.1 & 41.6 & 49.5 & - & 5.7 & 8.0 & 4.6 & 38.5 & 31.5 & 55.2 \\
\hline 70.9 & Dodecanamide & - & - & - & - & - & - & - & 9.1 & - & - & - & - & - & - \\
\hline 71.0 & Hexadecanamide & 6.3 & 4.1 & 14.7 & 16.5 & - & - & - & 5.6 & - & - & - & - & - & - \\
\hline 72.0 & N.N-dimethyl-octanamide & - & - & - & - & - & - & - & 8.2 & - & - & - & - & - & - \\
\hline 72.8 & Pentacosane & - & - & - & - & 34.5 & 32.4 & 27.8 & - & 9.6 & 12.8 & 4.6 & 33.3 & 42.1 & 22.1 \\
\hline 74.7 & $\begin{array}{c}\text { 3-Methyl-6-(phenylmethyl)- } \\
\text { 2.5-piperazinedione }\end{array}$ & 10.1 & - & - & - & - & - & - & - & - & - & - & - & - & - \\
\hline 75.4 & Hexacosane & - & - & - & - & 56.9 & 51.7 & 52.6 & - & 11.9 & 10.8 & 6.8 & 40.4 & 34.9 & 45.4 \\
\hline 76.5 & $\begin{array}{c}\text { 3-Benzyl-6-isopropyl-2.5- } \\
\text { piperazinedione }\end{array}$ & 10.4 & - & - & - & - & - & - & - & - & - & - & - & - & - \\
\hline 77.9 & Heptacosane & - & - & - & - & 29.0 & 26.9 & 53.1 & - & 12.7 & 11.9 & 5.0 & 27.6 & 35.6 & 16.8 \\
\hline 80.2 & Octacosane & - & - & - & - & 19.9 & 17.9 & 22.2 & - & 6.3 & 4.4 & 2.5 & 18.7 & 15.4 & 22.7 \\
\hline
\end{tabular}

${ }^{\mathrm{a}} \mathrm{H}_{2}$ pressure (MPa). ${ }^{\mathrm{b}}$ Temperature $\left({ }^{\circ} \mathrm{C}\right) .{ }^{\mathrm{d}}$ Catalyst loading $(\% \mathrm{w} / \mathrm{w}$ initial HTL oil amount) 
Table B.2. Compounds identified grouped into chemical categories

\begin{tabular}{|c|c|c|c|c|c|c|c|c|c|c|}
\hline \multicolumn{3}{|c|}{ Experiments } & \multicolumn{8}{|c|}{ Chemical categories (peak area $\cdot 10^{-7} / \mathrm{g}$ oil) } \\
\hline $\begin{array}{c}\mathrm{P} \\
(\mathrm{MPa})\end{array}$ & $\begin{array}{c}\mathrm{T} \\
\left({ }^{\circ} \mathrm{C}\right)\end{array}$ & $\begin{array}{l}\text { Cat. } \\
\text { (wt\%) }\end{array}$ & O-comp. & N-comp. & $\mathrm{N}$-aromatics & Aliphatic amides & Aliphatic nitriles & HC-aromatics & Alkanes & Others \\
\hline \multicolumn{3}{|c|}{ HTL $250^{\circ} \mathrm{C}$} & 109.8 & 39.0 & 32.7 & 6.3 & 0.0 & 0.0 & 26.2 & 101.7 \\
\hline 10 & 300 & no & 4.1 & 22.7 & 15.1 & 4.1 & 3.5 & 3.5 & 59.8 & 7.0 \\
\hline 10 & 300 & 20 & 18.7 & 39.5 & 11.3 & 14.7 & 13.5 & 12.7 & 55.5 & 25.3 \\
\hline 15 & 300 & 20 & 20.1 & 42.0 & 7.0 & 16.5 & 18.5 & 12.1 & 59.6 & 22.6 \\
\hline 15 & 400 & 50 & 8.8 & 78.1 & 1.8 & 0.0 & 76.2 & 80.2 & 1645.3 & 0.0 \\
\hline \multicolumn{3}{|c|}{ HTL $350^{\circ} \mathrm{C}$} & 119.2 & 116.5 & 82.9 & 33.5 & 0.0 & 57.4 & 7.3 & 33.9 \\
\hline 10 & 300 & no & 21.7 & 9.7 & 9.7 & 0.0 & 0.0 & 20.2 & 190.8 & 0.0 \\
\hline 10 & 300 & 20 & 29.5 & 11.1 & 11.1 & 0.0 & 0.0 & 29.1 & 578.5 & 0.0 \\
\hline 15 & 300 & 20 & 21.5 & 22.5 & 17.3 & 0.0 & 5.1 & 15.3 & 174.8 & 0.0 \\
\hline
\end{tabular}


The total ion chromatograms of the treated oils with increasing catalyst loading are shown in Figure B.2(a) for the $250^{\circ} \mathrm{C}$ oil series and Figure B.2(b) for the $350^{\circ} \mathrm{C}$ oil series under $400^{\circ} \mathrm{C}$ hydrotreatment; the y-axes of all chromatograms have the same scale. For both cases $\left(250\right.$ and $350^{\circ} \mathrm{C}$ HTL oils), the catalytic hydrotreatment produced an oil with higher amounts of all n-alkanes. An increase of their area can be seen in Figure B.2, when going from non-catalytic to 20 wt\% loading. From 20 to 50 wt\% catalyst loading, a reduction of $\mathrm{C}_{16}$ and $\mathrm{C}_{18}$ alkanes was observed while $\mathrm{C}_{15}$ and $\mathrm{C}_{17}$ increased. Yet it is difficult to elucidate a reaction mechanism affected by the catalyst loading which explains the previous observations. Again, temperature appeared as the process parameters that had more influence on the product composition. Already in the noncatalytically $400^{\circ} \mathrm{C}$ treated oil, a wide range of n-alkanes was formed.
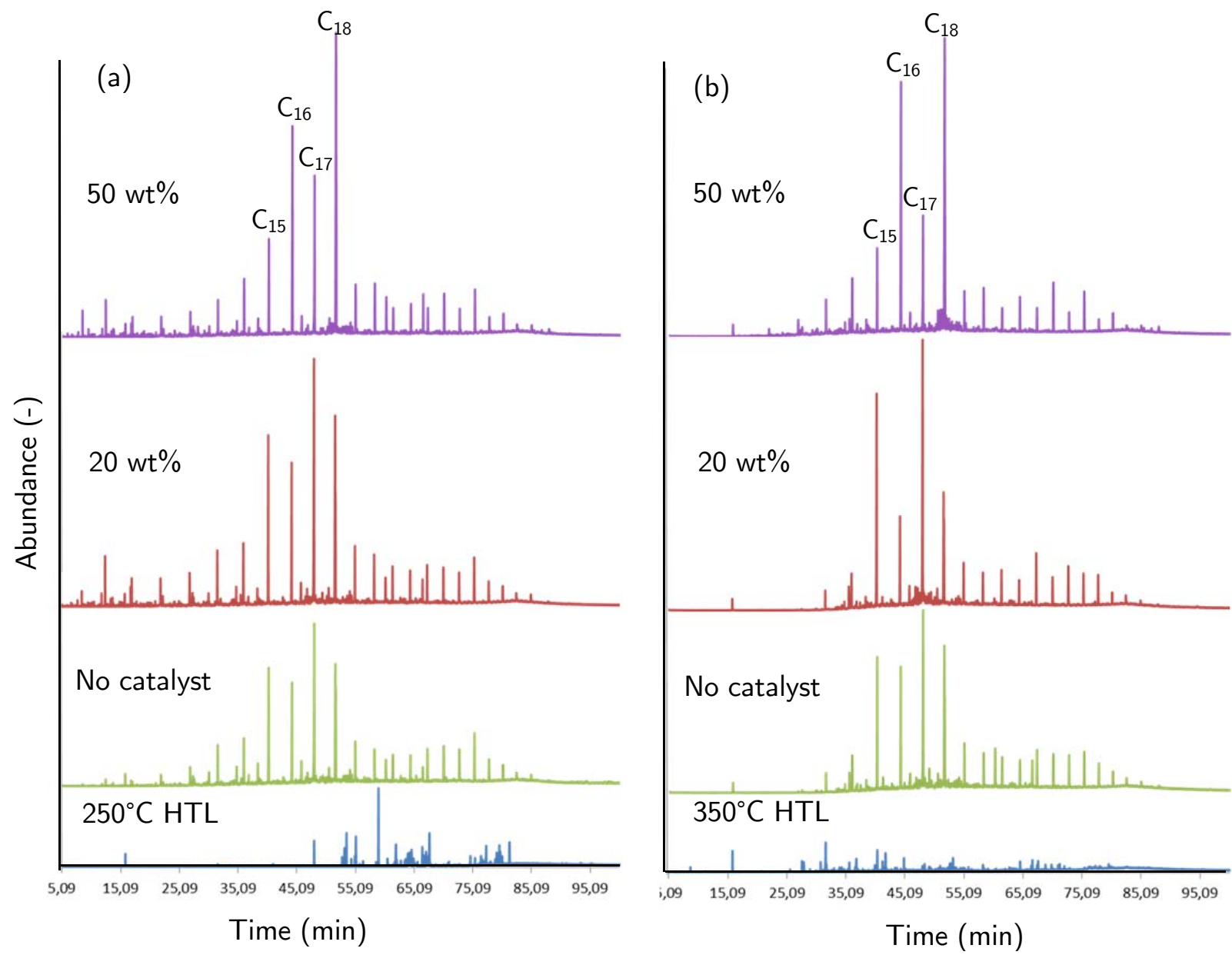

Figure B.2. Comparison of total ion chromatograms of the hydrotreated oils (at $400^{\circ} \mathrm{C}$ ) with increasing catalyst loading; (a) $250^{\circ} \mathrm{C}$ HTL series and (b) $350^{\circ} \mathrm{C}$ HTL series

FT-IR analysis were carried out to observe possible changes in terms of functional groups. We only show the results corresponding to the $250^{\circ} \mathrm{C}$ HTL oil series and changing hydrotreatment temperatures (See Figure B.3) as the rest of the oils had 
similar FT-IR spectra and, as mentioned before, temperature appeared to be the most relevant process parameter.

In all oils, the highest absorbance appeared in the region of 2800 to $300 \mathrm{~cm}^{-1}$ corresponding to the presence of alkanes and alkenes $(\mathrm{C}-\mathrm{H}$ and $=\mathrm{CH}$ groups respectively). That absorbance increased gradually with increasing hydrotreament temperature. A stretching in the region of $3300 \mathrm{~cm}^{-1}$ can be seen in the $250^{\circ} \mathrm{C} \mathrm{HTL}$ oil which could indicate the presence of $\mathrm{N}-\mathrm{H}$ and $\mathrm{OH}$ groups; the absorbance seems to be lowered in the treated oils. The stretching vibration corresponding to $\mathrm{C}=\mathrm{O}$ groups in carboxylic acids (and thus fatty acids) could be represented by the absorbance peak observed between 1650 to $1740 \mathrm{~cm}^{-1}$. Moreover, it could also represent the $\mathrm{C}=\mathrm{O}$ group in amides. As can be observed in Figure B.3, that absorbance peak is significantly reduced with temperature which, for the case of fatty acids, it might indicate their decarboxylation into n-alkanes. The absorbance peaks in the region between 1400-1600 $\mathrm{cm}^{-1}$ could represent $\mathrm{C}=\mathrm{C}$ groups in aromatic compounds while aromatics with $\mathrm{C}-\mathrm{H}$ groups could be indicated by the absorbance in the range of 715 to $800 \mathrm{~cm}^{-1}$. The FT-IR results seem to be in accordance with the previous observations (elemental composition, molecular weight distribution and GC-MS results) and with related studies. ${ }^{10-12}$

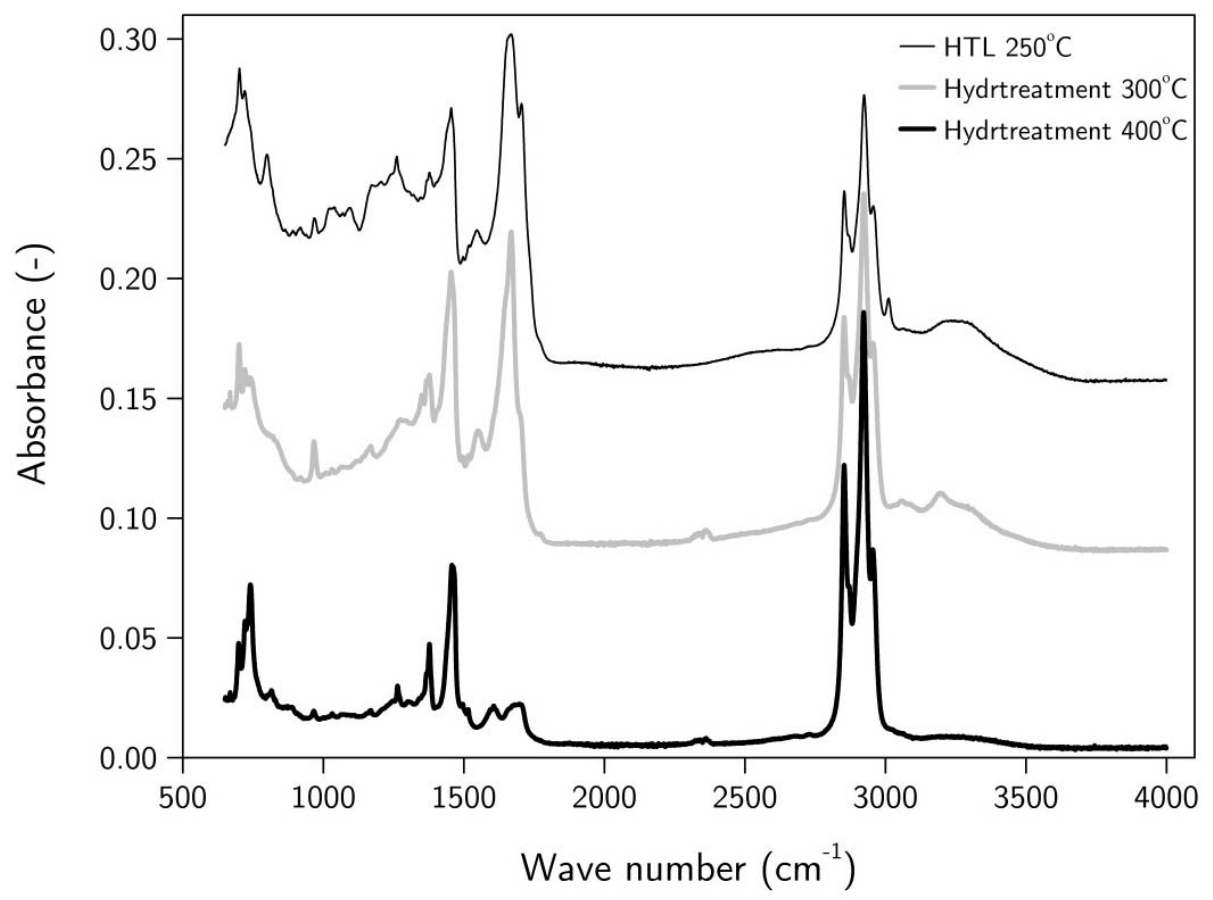

Figure B.3. FT-IR analyses of HTL $250^{\circ} \mathrm{C}$ oil and its corresponding treated oils from hydrotreatment at different temperatures (20 wt\% catalyst and $15 \mathrm{MPa} \mathrm{H}_{2}$ ) 


\section{Bibliography}

[1] D.D. Das, M.I. Schnitzer, C.M. Monreal, P. Mayer, Chemical composition of acid-base fractions separated from biooil derived by fast pyrolysis of chicken manure, Bioresour. Technol., 100(24), 6524-6532, 2009 .

[2] X. Cheng, T. Zhao, X. Fu, Z. Hu, Identification of nitrogen compounds in RFCC diesel oil by mass spectrometry, Fuel Process. Technol., 85(13), 1463-1472, 2004.

[3] Y. Kodera, K. Ukegawa, Y. Mito, M. Komoto, E. Ishikawa, T. Nakayama, Solvent extraction of nitrogen compounds from coal liquids, Fuel, 70(6), 765-769, 1991.

[4] J. Qi, Y. Yan, W. Fei, Y. Su, Y. Dai, Solvent extraction of nitrogen compounds from catalyticallycracked diesel oil by metal ion complexation, Fuel, 77(4), 255-258, 1998.

[5] S. Zhang, Q. Zhang, Z.C. Zhang, Extractive desulfurization and denitrogenation of fuels using ionic liquids, Ind. Eng. Chem. Res., 43(2), 614-622, 2003.

[6] A. Koriakin, K.M. Ponvel, C.-H. Lee, Denitrogenation of raw diesel fuel by lithium-modified mesoporous silica, Chem. Eng. J., 162(2), 649-655, 2010.

[7] D. Liu, J. Gui, Z. Sun, Adsorption structures of heterocyclic nitrogen compounds over Cu(I)Y zeolite: A first principle study on mechanism of the denitrogenation and the effect of nitrogen compounds on adsorptive desulfurization, J. Mol. Catal. A: Chem., 291(1-2), 17-21, 2008.

[8] C. Torri, D. Fabbri, L. Garcia Alba, D.W.F. Brilman, Upgrading of oils derived from hydrothermal treatment of microalgae by catalytic cracking over H-ZSM-5: A comparative Py-GC-MS study, J. Anal. Appl. Pyrolysis, 101(0), 28-34, 2013.

[9] E.A. Meijer, R.H. Wijffels, Development of a Fast, Reproducible and Effective Method for the Extraction and Quantification of Proteins of Micro-algae, Biotechnol. Tech., 12(5), 353-358, 1998.

[10] P. Duan, P.E. Savage, Upgrading of crude algal bio-oil in supercritical water, Bioresour. Technol., 102(2), 1899-1906, 2011.

[11] P. Duan, P.E. Savage, Catalytic hydrotreatment of crude algal bio-oil in supercritical water, Appl. Catal., B, 104(1-2), 136-143, 2011.

[12] P. Duan, P.E. Savage, Catalytic treatment of crude algal bio-oil in supercritical water: optimization studies, Energy Environ. Sci., 4(4), 1447-1456, 2011. 


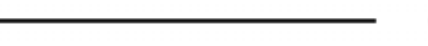

rar 


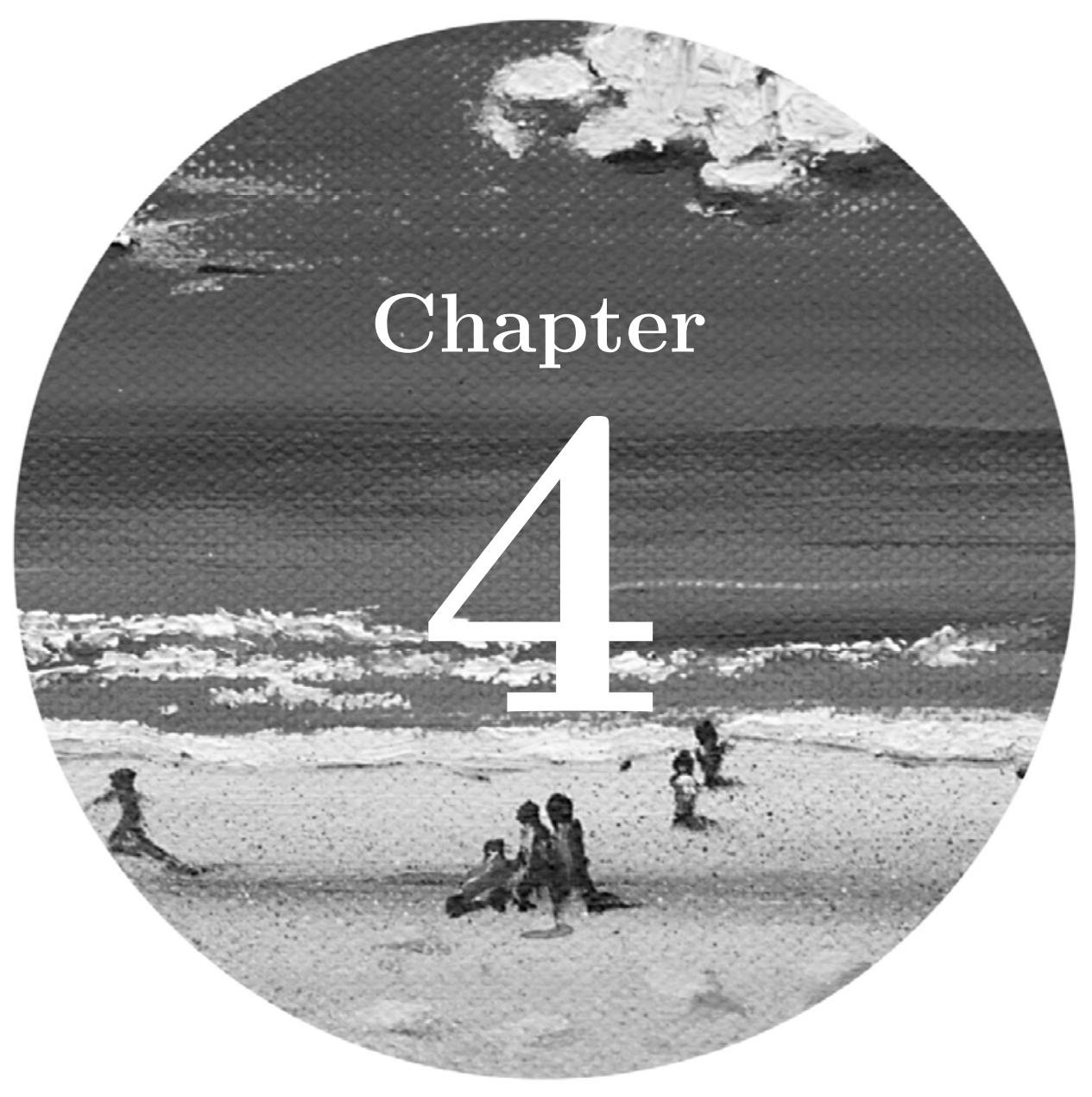

\section{Experimental and economical} evaluation of supercritical $\mathrm{CO}_{2}$ extraction of oil from microalgae 


\begin{abstract}
In this study, we performed an experimental and economical evaluation of supercritical $\mathrm{CO}_{2}\left(\mathrm{scCO}_{2}\right)$ extraction of lipid rich oil from microalgae. The purpose was to assess the feasibility of this option in an algae biorefinery configuration with bio-based diesel (biodiesel or green diesel) as one of the end products. Such a technical-economical evaluation has not been reported earlier. First, extraction experiments were performed using dry microalgae biomass in order to find the optimal extraction conditions in terms of maximized oil production and to determine extract composition ('quality'). At $50^{\circ} \mathrm{C}$ and a pressure of at least 35 $\mathrm{MPa}$ an oil with an acylglycerol content of more than $90 \mathrm{wt} \%$ (mainly triacylglycerols) was obtained, having a very low nitrogen content, moderate oxygen content and a $\mathrm{HHV}$ of $37 \mathrm{MJ} / \mathrm{kg}$. A detailed and complete analysis of the oils allowed us to divide the product into two fractions: a fraction containing compounds suitable for diesel fuels which are converted into fatty acid methyl esters (FAME) upon transesterification (the so-called FAME feed fraction) and a non-FAME feed fraction, containing compounds which could bring a certain profitability to the biorefinery system due to their economic value (e.g. sterols). $\mathrm{ScCO}_{2}$ extraction from wet microalgae was also assessed. Generally, the oil yields were lower for 'wet extraction' and showed a higher non-FAME feed content due to the increased polarity of the solvent mixture by the presence of water. Hence, $\mathrm{scCO}_{2}$ wet extraction has to overcome technical challenges to become as efficient as the dry extraction. Finally, a mathematical description based on our experimental data, together with simulations and process optimization, allowed us to find an approximate of the extraction costs including the energy consumption for dewatering. The approximated costs were in the range of 0.65 (for our dry feedstock with only $12 \mathrm{wt} \%$ extractable lipids in the microalgae) to $0.40 \$ / \mathrm{kg}$ oil (wet feedstock assuming a higher lipid content of $25 \mathrm{wt} \%$ in the microalgae) being in close range to that of conventional extraction using an organic solvent. Sustainability or environmental impact effects could favor the application of $\mathrm{scCO}_{2}$ under these comparable cost conditions.
\end{abstract}




\section{Introduction}

Nowadays, many on-going research is focusing on renewable energy sources that can help mankind to face future challenges related to fossil fuels scarcity and greenhouse gas emissions. Some examples of that are wind, solar energy (photovoltaic and thermal), hydro, geothermal and biomass, among others. Biomass based fuels like bio-ethanol, biodiesel (from lipid oil via transesterification) and green diesel (from lipid oil hydrogenation) can contribute to the gradual replacement of fossil fuels, especially in the transportation sector. ${ }^{1}$ Currently, bio-based diesel is primarily produced from vegetable oils (edible or non-edible). To that, several social and environmental impacts can be involved such as price increase of food-grade oils (as certain vegetable oils may be used for human consumption) and land competition. Animal fat or waste oils (e.g. greases) could also be used as feedstock but their available quantities cannot offset for the demand of diesel like fuels. ${ }^{1}$ Alternatively, microalgae, as source of lipid-rich oil, have the potential to become the most suitable feedstock for bio-based diesel with widely known advantages (e.g. fastest growing photosynthetic organisms). Indeed, microalgae (with 30 wt\% oil content) can produce 10 times more oil (in L/ha) than palm oil, considered to be one of the best performing oilseed crops. $^{2}$

Lipid recovery for diesel production has traditionally been performed by means of extraction using organic solvents (e.g. hexane, chloroform, etc.). However, several drawbacks related to the use of these chemicals, such as toxicity, generally high flammability and low selectivity towards neutral lipids (as triacylglycerols; more desirable for diesel like fuels), have been frequently mentioned. ${ }^{3-5}$ In addition, the energy consumption for solvent-solute separation is high and residual traces of the solvent often remain in both the extract and the feed (in the range of 500-1000 ppm ${ }^{6}$ ). Extraction using supercritical fluids (SFE) is an alternative to the use of organic solvents and, in particular, supercritical carbon dioxide shows great potential as it becomes supercritical at relatively low pressure $(7.38 \mathrm{MPa})$ and temperature $\left(31.06{ }^{\circ} \mathrm{C}\right) \cdot{ }^{5}$ Other advantages of $\mathrm{ScCO}_{2}$ that are frequently mentioned include ${ }^{4,5,7}$ :

- Environmentally friendly solvent with low toxicity, non-flammable, and high chemical inertness;

- Adjustable characteristics (e.g. density), hence the solvating power can be easily tuned;

- Low viscosity and high diffusivity. In close range to the compressibility and permeability potential of a gas, while exhibiting liquid-like solvating capability; 
- Suitable for extraction of compounds with low temperature stability;

- Higher selectivity towards neutral and non-polar lipids;

- The extract recovered is solvent free;

- The $\mathrm{CO}_{2}$ solvent itself can easily be recycled without creating any waste.

However, $\mathrm{scCO}_{2}$ extraction is also considered as a capital and energy intensive process due to the high pressures applied. ${ }^{6,8}$ Currently SFE extraction with $\mathrm{CO}_{2}$ is popular in several food and nutraceutical applications, where extra costs are compensated by the enhanced product quality and the associated added high value. For the fuel industry, lower margins are permissible on the products and therefore an optimized process is required where the cost should be minimized. Only few publications exist on $\mathrm{scCO}_{2}$ extraction of lipid rich oil from microalgae with the aim of producing liquid fuels. ${ }^{3,7}$

Most probably, the chance on successful implementation lies in the use of well-defined algae based biorefinery strategies where, together with the energy carrier products, the co-production of other high value-added products makes the overall concept profitable. In such integrated concepts, the biomass residue left after extraction can be further processed by means of e.g. hydrothermal liquefaction (when using a wet algal feedstock) producing a bio-crude like fuel (as refinery feed) and a $\mathrm{CO}_{2}$ rich gas, already available at high pressure which can potentially be recycled back to the extraction stage. Therefore, in this study, we aim for a complete evaluation of the process in terms of extraction efficiency, oil composition ('quality') and process economics within the frame of an algaebased biorefinery. $\mathrm{ScCO}_{2}$ extraction using dry microalgae has been investigated to find the optimal extraction conditions in order to maximize the yield and the quality of the oil extracted (in terms desired compounds for diesel-like fuels). After that, the extraction from wet algae was also assessed. Finally, we looked at the possible process optimization paths towards lower utility and investment costs.

\section{Experimental}

\subsection{Algal feedstock}

The commercially grown Desmodesmus sp. microalgae, purchased from Ingrepro B.V. (The Netherlands), was cultivated in open pond raceway system. The ash-free dry weight of all forms of biomass used was determined by first drying at $105^{\circ} \mathrm{C}$ for $24 \mathrm{~h}$ to determine the water content and, after that, the dry residue was further treated at $550^{\circ} \mathrm{C}$ 
under oxidizing conditions for $5 \mathrm{~h}$ to measure the ash content. All the results are presented on dry algae ash free (d.a.f.) basis, unless stated otherwise.

Different forms of feedstock obtained from the same algal species (all product samples obtained from Ingrepro B.V.) were used. Dry cylindrical pellets, with mean particle diameter of 2,5 $\mathrm{mm}$ and particle length of $3,8 \mathrm{~mm}$, were the first batch used to study the effect of various extraction conditions. The second batch, an algal slurry with a concentration of $7.66 \mathrm{wt} \%$ (dry ash free), was used to assess the supercritical $\mathrm{CO}_{2}$ extraction from wet biomass. This slurry was first centrifuged $(15 \mathrm{~min}$ at $8228 \times \mathrm{g}$ ), the supernatant decanted and the algal paste created was finally freeze dried obtaining a sample of dried whole cells. After that, these cells were mixed with different amounts of demineralized water to create various moisture contents. In addition, the algal slurry was also used "as-received" and from which water was removed by centrifugation in order to create the desired concentrations. Summarizing, three forms of algae were used in total: pellets; freeze dried whole cells (created from the algal slurry) mixed with water; and asreceived algal slurry. Furthermore, both pellets and freeze dried whole cells were crushed into a powder with a bead mill to evaluate if that would substantially improve the extraction efficiency.

Visual observations were performed using both optical (Carl-Zeiss microscope) and scanning electron microscopy (SEM; JEOL TSM 5600). A visual representation of the algal biomass used is given in Figure 1. As can be seen in the SEM picture of Figure $1(\mathrm{a})$, no intact (unbroken) cells were found in the pelletized algae.
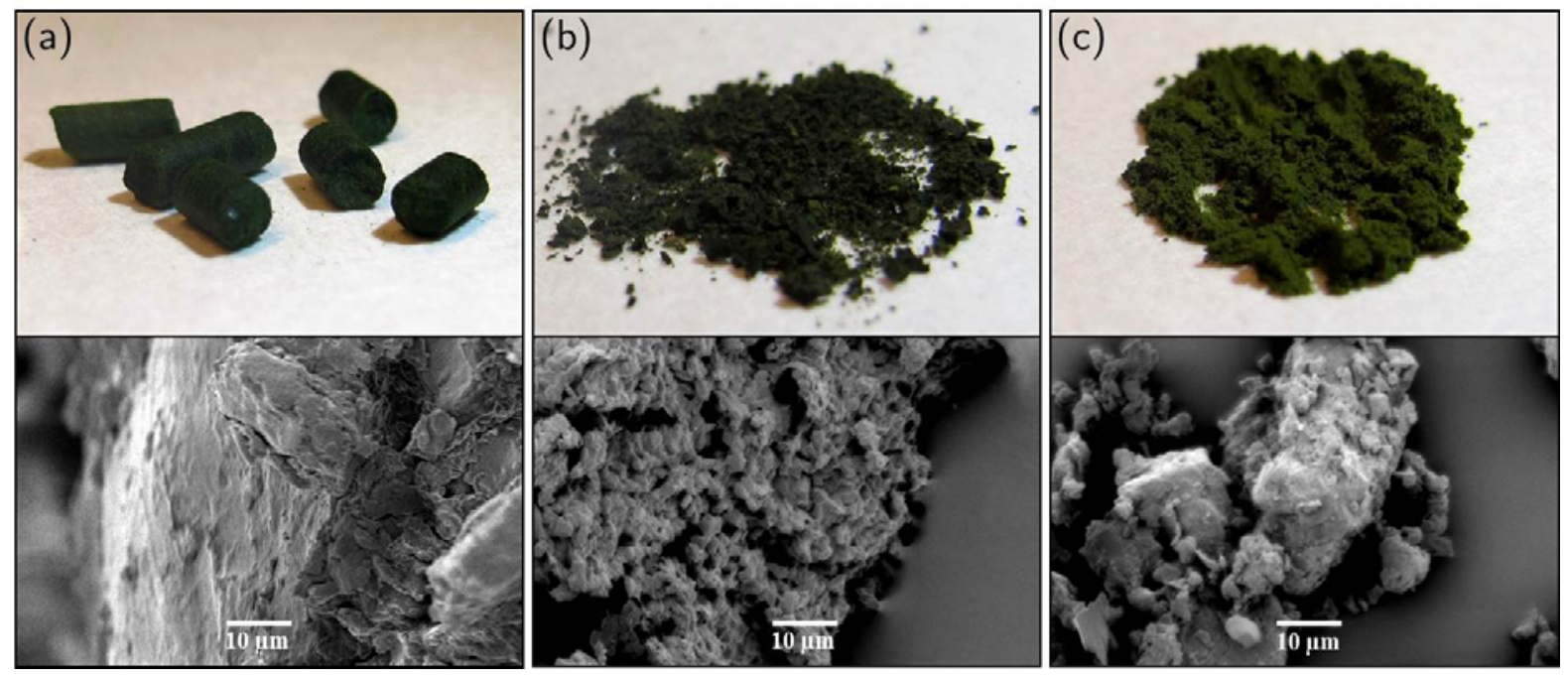

Figure 1. Three different forms of algae used: (a) pellets, (b) freeze dried whole cells (created from the algal slurry) which were later mixed with water; and (c) crushed freeze dried whole cells. SEM magnification: x1000 
The Bligh and Dyer (B\&D) method ${ }^{9}$ was applied as reference technique to extract the lipid oil from the algae and, after transesterification of the oil, the total fatty acid (TFA) or fatty acid methyl ester (FAME) content was found, see Section 2.3. Oil extracted by Bligh and Dyer (B\&D) is often used as reference for the maximum amount of extractable oil $^{3}$ and, therefore, this has been used here for comparison with other studies. However, it is important to point out that the various existing conventional extraction methods using organic solvents are not equivalent in terms of oil yield and composition. ${ }^{10} \mathrm{~B} \& \mathrm{D}$ is less selective to triacylglycerol (TAG) extraction than $\mathrm{scCO}_{2}$.

Table 1. Properties of the various microalgal feedstock used

\begin{tabular}{|c|c|c|}
\hline $\begin{array}{l}\text { Properties }^{\mathrm{a}} \\
\left(\mathrm{wt} \%, \text { a.r. }{ }^{\mathrm{b}}\right)\end{array}$ & Pellets & Freeze dried whole cells \\
\hline Moisture content & 4.13 & 1.33 \\
\hline Organic content & 82.6 & 83.5 \\
\hline Ash content & 13.3 & 15.1 \\
\hline \multicolumn{3}{|c|}{$\begin{array}{l}\text { Oil extracted by B\&D } \\
\text { (wt\%, d.a.f.) }\end{array}$} \\
\hline Lipid oil & 21.4 & 15.5 \\
\hline FAME & $9.89 \pm 2.2^{\mathrm{c}}$ & $5.99 \pm 1.0$ \\
\hline \multicolumn{3}{|l|}{$\begin{array}{c}\text { FA profile } \\
(w \mathrm{t} \% \text { of } \mathrm{FAME})\end{array}$} \\
\hline C16:0 & $17.2 \pm 0.3$ & $25.5 \pm 0.4$ \\
\hline $\mathrm{C} 16: 2$ & $1.5 \pm 0.3$ & $4.2 \pm 0.3$ \\
\hline C16:3 & $3.3 \pm 0.3$ & $12.4 \pm 0.5$ \\
\hline C18:0 & $2.8 \pm 0.5$ & $2.9 \pm 1.0$ \\
\hline C18:1 & $15.0 \pm 0.5$ & $12.2 \pm 0.3$ \\
\hline C18:2 & $52.0 \pm 0.9$ & $32.0 \pm 0.4$ \\
\hline C18:3 & $6.8 \pm 0.4$ & $6.9 \pm 0.3$ \\
\hline C18:4 & $1.5 \pm 0.4$ & $3.9 \pm 0.3$ \\
\hline
\end{tabular}

\begin{tabular}{ccc}
\hline $\begin{array}{c}\text { Elemental Composition } \\
\text { (wt\%, a.r.) }\end{array}$ & \\
\hline $\mathrm{C}$ & 48.9 & 44.1 \\
$\mathrm{H}$ & 7.08 & 6.26 \\
$\mathrm{~N}$ & 6.24 & 6.47 \\
$\mathrm{O}+\mathrm{ash}^{\mathrm{d}}$ & 37.8 & 43.2 \\
$\mathrm{HHV}(\mathrm{MJ} / \mathrm{kg})$ & 22.5 & 19.5 \\
\hline
\end{tabular}

a More information about these algal species can be found in Chapter 2 .

${ }^{\mathrm{b}}$ As received. ${ }^{\mathrm{c}}$ Mean \pm standard deviation. ${ }^{\mathrm{d}}$ Calculated by difference as $100-(\mathrm{C}+\mathrm{H}+\mathrm{N})$ 
The $\mathrm{C}, \mathrm{H}$, and $\mathrm{N}$ contents of the dry algae were measured in duplicate using an elemental analyzer (Thermo Scientific Flash 2000, CHN-S). If the difference between duplicates was within $\pm 1 \%$, the results were accepted and the average values were taken. The higher heating value (HHV) was calculated according Channiwala and Parikh's ${ }^{11}$ formula as it is known to have a higher accuracy for the HHV estimation of biomass. Table 1 summarizes the physical and chemical properties of both pellets and freeze dried whole cells obtained from the purchased algal slurry.

As can be observed in Table 1, the two algal feedstocks used had different B\&D lipid oil yield and FAME composition (obtained after transesterification of the oil). The fact that these two algal batches were from different harvesting times (i.e. different seasons) can explain those compositional variations. Those differences have no major effects, as pellets and whole cells were used for different purposes. It can be also observed that more than half of the oil extracted by B\&D was composed of substances that could not be converted into FAMEs, highlighting again the lower selectivity of this extraction method towards acylglycerols and free fatty acids (compounds converted into FAMEs). This makes the usefulness of the direct comparison of this method with other solvents such as $\mathrm{scCO}_{2}$ questionable. The $\mathrm{B} \& \mathrm{D}$ method it is known to co-extract other compounds such as chlorophyll along with the desired neutral lipids. ${ }^{12}$

\subsection{Supercritical $\mathrm{CO}_{2}$ extraction}

Liquid carbon dioxide $\left(\mathrm{CO}_{2}\right)$ with a purity of 99.996 vol\% was purchased from Praxair B.V. (The Netherlands). For the $\mathrm{scCO}_{2}$ extraction experiments, the experimental set-up shown in Figure 2 was used. All extractions were carried out in dynamic mode with constant $\mathrm{CO}_{2}$ mass flow (i.e. with a residence time depending on fluid density). Liquid $\mathrm{CO}_{2}$ was fed from the storage bottle (1 in Figure 2) into the extractor (5 and 6) with a high performance liquid chromatography (HPLC) pump (3). The feed pump was cooled, to prevent the $\mathrm{CO}_{2}$ entering the gas phase, using a circulating fluid cooler (2, fluid: coolant -38 NF from Kroon-Oil, The Netherlands). The pump heads were also cooled to remove any heat generated during compression and ensuring liquid $\mathrm{CO}_{2}$ conditions inside the pump.

Before entering the extractor, the liquid $\mathrm{CO}_{2}$ was heated by an electrical preheater (4). The extractor, 16 x $3 \mathrm{~mm}$ diameter and a total length of $625 \mathrm{~mm}$, was made of 316 stainless steel. The biomass sample (about $16 \mathrm{~g}$ per run) was placed in the upper-part of the extractor (6), suspended on a $5 \mu \mathrm{m} 316$ stainless steel filter and creating a bed of 415 
mm length. A second filter, identical to the first one, was placed on top of the biomass bed at the outlet of the extractor to prevent entrainment of the biomass sample out of the extractor. The lower-part of the extractor (5), upstream to the bed of algal biomass, was used to ensure supercritical conditions before the $\mathrm{CO}_{2}$ solvent reached the biomass sample. During the experiment, the entire extractor was kept at constant temperature using an electrical heater (Heater 1).

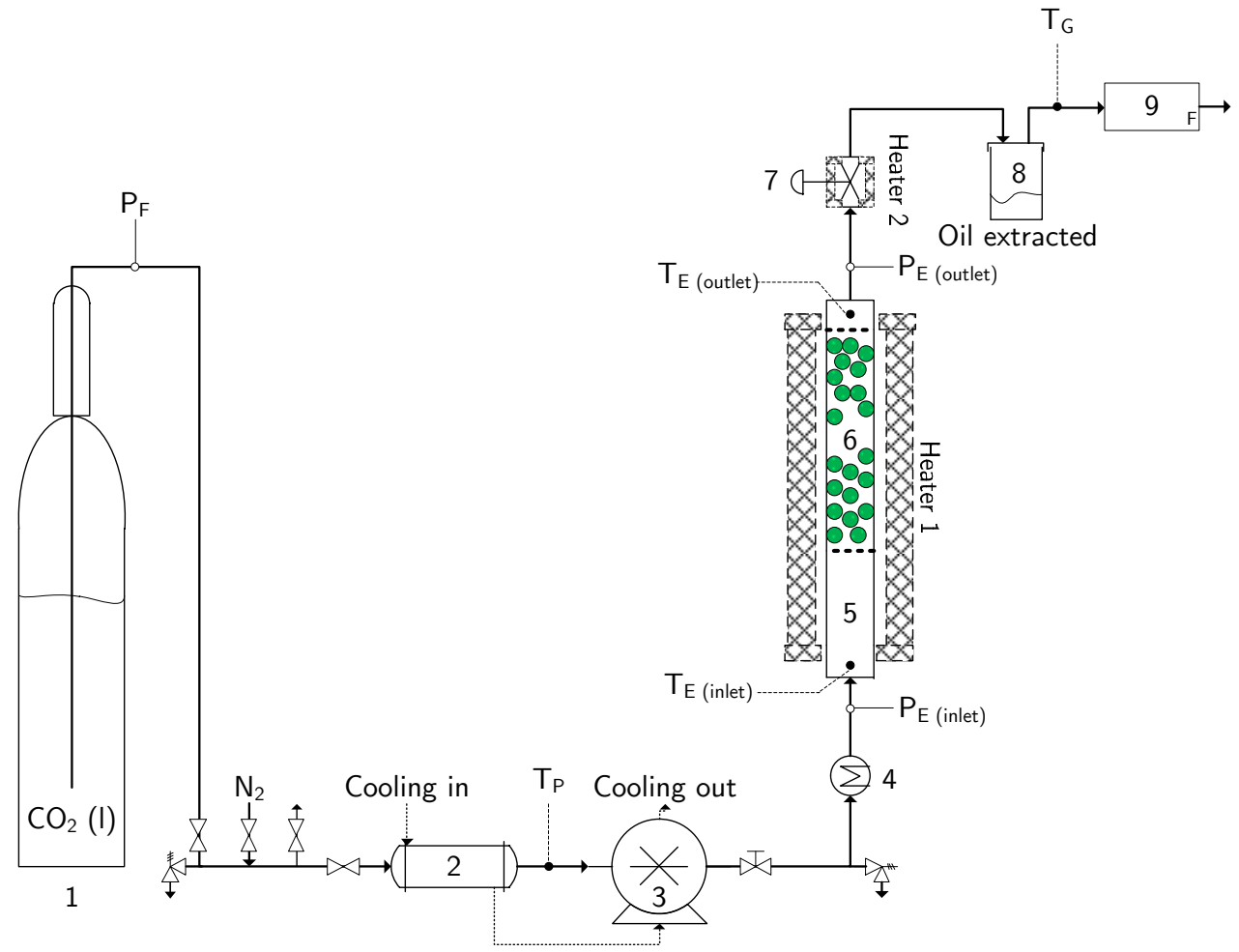

Figure 2. $\mathrm{ScCO}_{2}$ extractor scheme. 1) Gas flask with $\mathrm{CO}_{2}$ liquid, 2) $\mathrm{CO}_{2}$ pump feed cooler, 3) HPLC pump with cooled pump heads, 4) preheater, 5) extractor bottom-part, 6) extractor upper-part with biomass sample 7) BPR, 8) oil collector, 9) dry gas meter

$\mathrm{ScCO}_{2}$ conditions in the extractor were regulated by both temperature and pressure. The extraction temperature was regulated by the extractor heater and preheater. Temperature control for the extractor and preheater was done on basis of the measured extractor inlet temperature $\left(\mathrm{T}_{\mathrm{E}}\right.$, inlet $)$ and outlet temperature ( $\mathrm{T}_{\mathrm{E}}$, outlet $)$ using two thermocouples. The extraction pressure was regulated by a back pressure regulator (BPR) (7). Its pressure set-point was determined by measuring the extractor inlet pressure $\left(\mathrm{P}_{\mathrm{E}}\right.$, inlet $)$ and outlet pressure $\left(\mathrm{P}_{\mathrm{E} \text {, outlet }}\right)$. During an experiment, the BPR was heated with an electrical heater (Heater 2) to prevent solid formation inside the BPR due to adiabatic expansion of the $\mathrm{scCO}_{2}$ to $\mathrm{CO}_{2}$ gas. The extraction experiments were commenced when the system reached the desired pressure and temperature. 
After depressurization, the extracted oil precipitated in a glass collector (8) and was quantified gravimetrically. All the parts of the BPR, tubing connected to the collection vessel and the extracted algae were also weighed to ensure reliable mass balances that had to exceed always a $95 \%$ closure. Immediately after extraction and weighting, the oil samples were stored at $-20^{\circ} \mathrm{C}$ and in the dark prior to analysis.

The volumetric flow rate (adjusted by the HPLC pump speed) of the depressurized $\mathrm{CO}_{2}$ leaving the system was measured and controlled by a dry gas meter (9) under frequent time intervals. The temperature of the gas stream at the entrance of the gas meter was measured by a thermocouple $\left(\mathrm{T}_{\mathrm{G}}\right)$. Before every experiment the setup was tested for leakage at $4 \mathrm{MPa}$ using $\mathrm{N}_{2}$. For the extraction from wet algae biomass a different extractor, a stirred vessel of $45 \mathrm{ml}$ volume, was used instead of the fixed bed described above.

\subsection{Oil analyses}

For all the analytical methods described in this section, each analysis was repeated in triplicate. The determination of total fatty acid methyl esters (FAMEs, sum of free fatty acids, FFAs, and bounded fatty acids, BFAs) in both $\mathrm{B} \& \mathrm{D}$ and $\mathrm{scCO}_{2}$ oils was performed as described in ${ }^{13}$.

The amount of free fatty acids (FFAs) and sterols in $\mathrm{scCO}_{2}$ oils was determined by silylation. Samples $(5 \mathrm{mg})$ were dissolved in $\mathrm{CH}_{3} \mathrm{CN}(0.1 \mathrm{ml})$ containing tridecanoic acid $(0.02 \mathrm{mg})$ and methyl nonadecanoate $(0.02 \mathrm{mg})$; then bis-trimethylsilyltrifluoroacetamide $(0.1 \mathrm{ml})$ containing $1 \%$ of trimethylchlorosilane and pyridine $(0.1 \mathrm{ml})$ were added. The samples were placed in an incubator at $60^{\circ} \mathrm{C}$ for $1 \mathrm{~h}$ and then analyzed by GC-MS. With the FAME and FFA content, the amount of acylglycerols (bounded fatty acids, BFAs) in the oil was calculated by difference (BFAs = FAMEs-FFAs).

GC-MS analyses were performed by using a 6850 Agilent HP gas chromatograph connected to a 5975 Agilent HP quadrupole mass spectrometer. The injection port temperature was $280^{\circ} \mathrm{C}$. Analytes were separated by a HP-5 fused-silica capillary column (stationary phase poly[5\% diphenyl/95\% dimethyl]siloxane, $30 \mathrm{~m}, 0.25 \mathrm{~mm}$ i.d., $0.25 \mathrm{~mm}$ film thickness), with helium as carrier gas (at constant pressure, $33 \mathrm{~cm} / \mathrm{s}$ linear velocity at $\left.200^{\circ} \mathrm{C}\right)$. Mass spectra were recorded under electron ionization $(70 \mathrm{eV})$ at a frequency of $1 \mathrm{scan} / \mathrm{s}$ within the $12-600 \mathrm{~m} / \mathrm{z}$ range. 
For FAMEs analysis, the temperature of the column was increased from $50^{\circ} \mathrm{C}$ up to $180^{\circ} \mathrm{C}$ at $50^{\circ} \mathrm{C} / \mathrm{min}$, then from $180^{\circ} \mathrm{C}$ up to $300^{\circ} \mathrm{C}$ at $5^{\circ} \mathrm{C} / \mathrm{min}$. Tridecanoic acid and methyl nonadecanoate were utilized as internal standards for FAMEs quantification. The relative response factors used for the quantitation were obtained by injecting solutions of known amounts of methyl tridecanoate, methyl nonadecanoate and commercial FAMEs mixture.

For FFAs and sterols analysis, the temperature of the column was initially held at $50^{\circ} \mathrm{C}$ for $5 \mathrm{~min}$, then it was increased from $50^{\circ} \mathrm{C}$ to $350^{\circ} \mathrm{C}$ at $10^{\circ} \mathrm{C} / \mathrm{min}$, and finally held at $350^{\circ} \mathrm{C}$ for $10 \mathrm{~min}$. The relative response factor used for sterols quantitation was obtained by injecting solutions of known amounts of methyl nonadecanoate and cholesterol after silylation.

The quantitative analysis of glycolipids, determined on the basis of sugar content, was achieved by following the phenol-sulfuric acid method of Dubois, ${ }^{14}$ using glucose as standard for instrumental calibration. Each measurement was repeated in quadruplicate. Total protein content in the oils was estimated with the Folin Phenol reagent in accordance to the method of Lowry, ${ }^{15}$ using bovine serum albumin (BSA) as standard. Each measurement was repeated in duplicate.

The elemental composition of the oils was determined in the same manner as for the algal feedstock (see Section 2.1). With that, the HHV was calculated using Boie's equation. ${ }^{16}$ An Agilent 1200 series HPLC system with 3 GPC PLgel $3 \mu \mathrm{m}$ MIXED-E columns connected in series was used to determine the molecular weight distribution of the oils (size exclusion chromatography). Tetrahydrofuran (THF) was used as solvent with column temperature of $40^{\circ} \mathrm{C}$ and flow of $1 \mathrm{ml} / \mathrm{min}$. Polystyrene standards, with a molecular weight ranging from 162 to $29510 \mathrm{~g} / \mathrm{mol}$ were used for calibration.

\section{Methods}

\subsection{Definitions}

The oil yield was calculated as the weight of oil extracted (measured gravimetrically) per ash-free dry weight of algal biomass used (g oil / g algae dry ash free (d.a.f.)). After transesterification, the extracted oil could be divided into two fractions which were defined as: FAME feed fraction and non-FAME feed fraction (wt\% of oil). FFAs and BFAs in the oil are the compounds that can be converted into FAMEs and hence defined 
as the FAME feed fraction of the oil. The non-FAME feed fraction corresponds to the portion of the oil that could not be converted into FAMEs. By knowing the FAME composition of the oil, the FAME yield was defined in the same manner as that for the oil.

\subsection{Solubility}

To optimize the extraction and recovery conditions, and with this calculate the final cost, for a lipid extraction process using $\mathrm{scCO}_{2}$, an equation able to describe the change in solubility by changing $\mathrm{P}$ and $\mathrm{T}$ is required. The correlation derived in this work is, of course, specific for the algal biomass under consideration, but may provide a first indication for similar algal lipid oils. The correlation was found by fitting our experimental data to Chrastil's derived empirical relation, ${ }^{17}$ frequently used in extraction applications, using the surface fitting toolbox of MATLAB (version 7.11). The solubility, expressed as mass fraction, strongly depends on solvent density and is in inversely proportional to temperature:

$$
\ln (\mathrm{S})=(\mathrm{k}-1) \cdot \ln (\rho)+\frac{\mathrm{a}}{\mathrm{T}}+\mathrm{b}
$$

Where $S$ (in $\mathrm{kg} / \mathrm{kg} \mathrm{CO}_{2}$ ) is the solubility, $\rho$ (in $\mathrm{kg} / \mathrm{m}^{3}$ ) the solvent density, $T(\mathrm{~K})$ the temperature and $k$ (association number, average number of $\mathrm{CO}_{2}$ molecules in the solvato complex), $a$ (dependent on total heat of dissolution) and $b$ (dependent on molecular weight of solute and solvent and the association constant) are the parameters to be adjusted. ${ }^{18}$

In literature, most authors use the Redlich-Kwong-Aspen (and other derivatives) or Peng-Robinson based equations of state (EoS) to described oil- $\mathrm{CO}_{2}$ systems and to calculate the solvent density. ${ }^{19,}{ }^{20}$ The $\mathrm{scCO}_{2}$ densities, under different $\mathrm{P}$ and $\mathrm{T}$ conditions, were calculated with various EoS (Soave-Redlich-Kwong, Peng-Robinson, Redlich-Kwong-Aspen etc.) using the commercial process simulators UniSim ${ }^{\circledR}$ Design and Aspen Plus v12 (for Redlich-Kwong-Aspen). When comparing with the densities given by the NIST webBook, ${ }^{21}$ the Peng-Robinson-Stryjek-Vera (PRSV) EoS provided the closest approximation for the density values under the range of extraction conditions studied in this work (with in an average error of less than 2\%). That EoS was used to find the densities for both the data fitting with Chrastil equation and in the simulations described in the next section. 


\subsection{Economic evaluation}

All simulations were performed using the commercial UniSim ${ }^{\circledR}$ design suite process simulator, using the Peng-Robinson-Stryjek-Vera (PRSV) EoS. The process configuration considered (the selection process is later discussed in Section 4.5) is presented in Figure C.1 in the Appendix of this chapter. We assumed no pressure drop across the equipment.

All the required data related to cultivation (scale of operation) and algae properties are shown in Table 2. Algae growing rates and land area assumptions were obtained from $\mathrm{Xu}$ et al. ${ }^{22}$ As later explained in detail in Section 4.5, both dry and wet algae routes were evaluated with two different maximum extractable oil contents, 12 and 25 wt\%, leading to two different oil flow rates (71 and $149 \mathrm{~kg} / \mathrm{h}$, see Table 2). Although unlikely, both algae with different oil content were assumed to grow equally fast as we aimed to evaluate only the effect of oil content on costs.

Table 2. Microalgae related assumptions

\begin{tabular}{cccc}
\hline \multirow{2}{*}{ Item } & Unit & \multicolumn{2}{c}{ Algal feedstock } \\
\cline { 3 - 4 } & & Dry & Wet \\
\hline Annual productivity $^{\mathrm{a}}$ & metric ton/ha/yr & $50^{\mathrm{b}}$ & 50 \\
Open pond area & $\mathrm{ha}$ & 100 & 100 \\
Operating days per year & days & 350 & 350 \\
Algae mass flow rate & $\mathrm{kg} / \mathrm{h}$ & 595 & $1984^{\mathrm{c}}$ \\
Extractable oil content & $\mathrm{wt} \%$ & $12 / 25$ & $12 / 25$ \\
Oil flow rate & $\mathrm{kg} / \mathrm{h}$ & $71 / 149$ & $71 / 149$ \\
Density & $\mathrm{kg} / \mathrm{m}^{3}$ & $616^{\mathrm{e}}$ & $1000^{\mathrm{f}}$ \\
Porosity & - & 0.4 & 0 \\
\hline
\end{tabular}

${ }^{\text {a }}$ On dry algae basis. ${ }^{\mathrm{b}}$ As stated by $\mathrm{Xu}$ et al. ${ }^{22}$, assumed likeliest value in the range of usual performances of open raceways. ${ }^{c}$ On wet algae basis assuming a slurry with 30 wt\% dry algae concentration. ${ }^{\mathrm{d}}$ Calculated from the algae mass flow rate and the extractable oil content. ${ }^{e}$ Measured mean bed density of the algae pellets used experimentally. The value represents the bulk density hence including the void volume. ${ }^{\mathrm{f}}$ Slurry density assumed to be in close range to that of water.

$\mathrm{CO}_{2}$ losses related to (de-)pressurization of the vessel were not taken into account, as it was assumed most of the $\mathrm{CO}_{2}$ could be recovered and minor losses could be compensated by the $\mathrm{CO}_{2}$ produced at high pressure in other processes of the integrated algae biorefinery. 
Algae dewatering assumptions were adapted from the methods proposed by Xu et al. ${ }^{22}$ The cultivated algae is first concentrated to $2 \%$ dry weight (DW) by flocculation. After that, an advanced centrifugation technology was selected to achieve a dry content of 30 wt $\%$ in one stage from the previous $2 \mathrm{wt} \%$ slurry. The estimated duty, based on values given by the manufacturer $\left(\right.$ Evodos $^{23}$ ), was $0.85 \mathrm{kWh} / \mathrm{m}^{3}$ feed flow (significantly lower than the reported $8 \mathrm{kWh} / \mathrm{m}^{3}$ to reach $22 \%$ dry algae biomass with a continuous flow centrifuge system as reported by Dassey and Theegala $\left.{ }^{24}\right)$. To prevent underestimations, a safety margin (factor 1.5) was applied, hence a value of $1.28 \mathrm{kWh} / \mathrm{m}^{3}$ was used in the calculations. For the dry route, an additional thermal drying step is required to reach $>85 \%$ DW. For this, a drying technology was selected consuming $2 \mathrm{MJ}$ per $\mathrm{kg}$ of evaporated water. ${ }^{22}$ The process duties (electricity, heating and cooling at different levels), as obtained from the simulations in $\mathrm{UniSim}^{\circledR}$, were translated into operating costs, using the utility cost specifications presented in the Appendix (Table C.1).

To estimate investment costs, preliminary equipment design calculations were conducted. General assumptions on lifetime, operating hours and installation factors can be found in the Appendix (Table C.2). For supercritical $\mathrm{CO}_{2}$ extraction processes, the pressure vessels (extraction and separation) represent a major cost contribution. Heat-transfer equipment and pumps were evaluated in less detail and costs of valves and instrumentation are included in the installation factors applied. ${ }^{25}$

The working volume of the extraction vessel for extracting from dry algae biomass was calculated using Eq. 2 where $\rho$ is the mean bed bulk density in $\mathrm{kg} / \mathrm{m}^{3}, \Phi_{D W}$ is the dry algal feed flow in $\mathrm{kg} / \mathrm{h}$ and $t_{\text {switch }}$ is the switching time (in min) to empty/refill an extractor with fresh algal material. The switching time and related issues will be discussed in more detail in Results Section 4.5.

$$
\mathrm{V}_{\text {vessel }}=\frac{\varphi_{\mathrm{DW}}}{\rho\left[60 \cdot\left(1 / \mathrm{t}_{\text {siwtch }}\right)\right]}
$$

To find the optimal aspect ratio $(\mathrm{H} / \mathrm{D})$ for the column, a sensitivity study was performed, thereby varying the $\mathrm{H} / \mathrm{D}$ ratio for several extraction pressures and a fixed vessel volume. The optimum H/D ratio was found to be around 10, above which the cost stayed nearly constant. The minimum wall thickness, dependent on the extraction pressure, was calculated using a method described by Peters et al., ${ }^{25}$ see Appendix. For the extraction from wet algae, the extractor volume was calculated with Eq. 2 and the wet algae feed flow and slurry density as shown in Table 2. A void volume fraction of 0.4 
(same as for the bed of algae pellets) was added for $\mathrm{scCO}_{2}$ disengagement from the slurry.

For recovery of the extracted oil, the design method for vertical gas-liquid separators provided by Sinnott and Towler ${ }^{26}$ was used. With that method, the oil settling velocity, minimum diameter and length of the vessel were found. The density of the oil extract was assumed to be $900 \mathrm{~kg} / \mathrm{m}^{3}$, which is in the range of vegetable oils and the measured densities of extracted algal oil from Kumar et al. ${ }^{27}$ The liquid level height was calculated from a minimum liquid residence time of 10 minutes (typically used ${ }^{26}$ ), with a minimum of $0.3 \mathrm{~m}$ required for a level controller.

Both the extraction and separation vessels were assumed to be cylindrical with a hemispherical head at each end. The material of construction should be resistant to the acids present in the process (i.e. $\mathrm{CO}_{2}, \mathrm{FFA}$ ) and for this, stainless steel 304 was selected. The total costs of the vessels were calculated as:

Total cost vessel $=$ steel $\mathrm{price} \cdot \mathrm{m}_{\mathrm{vessel}} \cdot \mathrm{I}$

The calculations to obtain the total mass (cylinder +2 hemi-spherical heads) of the pressure vessels ( $m_{\text {vessel}}$, in $\mathrm{kg}$ of steel) are shown in the Appendix, together with all assumptions required and the installation factor used for pressure vessels ( $I$ in Eq. 3). For the steel price (in $\$ / \mathrm{kg}$ steel) an equation given by Peters et al. ${ }^{25}$, to calculate the carbon steel price was used together with a carbon steel to stainless steel correction factor (see Appendix).

Several heat-transfer units are required in the process. The costs for these (heater, cooler and pre-heater) are approximated by a simple estimation based on the required heat transfer area (in $\mathrm{m}^{2}$ ) calculated from the duty (in $\mathrm{W}$ ) obtained from the UniSim ${ }^{\circledR}$ process simulation and the assumed overall heat coefficient $\left(500 \mathrm{~W} / \mathrm{m}^{2} / \mathrm{K}\right)$ and by the mean temperature difference (i.e. the driving force; assumed to be $30 \mathrm{~K}$ ).

To find the cost of each piece of heat-transfer equipment, a correlation for the costs per unit of heat-transfer area was used, based on data for a multi-pipe (shell-and-tube) heat exchanger (Peters et al. $^{25}$ ). This was multiplied by the installation factor for heat exchangers and a pressure adjustment factor, see Appendix. The pump costs were roughly estimated from the graphs presented by Peters et al. ${ }^{25}$ for the required volume flow at a design pressure of $35 \mathrm{MPa}$. A centrifugal pump was selected as it can easily reach $35 \mathrm{MPa} .^{25}$ Costs including correction for pressure, stainless steel and installation 
factors were estimated to be: $100000 \$$ for the case with $12 \mathrm{wt} \%$ extractable oil content and 150000 \$ (as when more oil has to be extracted, a higher $\mathrm{CO}_{2}$ flow rate is required). The prices of the utilities were obtained for the year 2003 and the equipment cost assumptions dated from the year 2002. For the final cost estimation (to bring the costs to an estimate for 2013) a nominal inflation correction of $2 \%$ per year was assumed. Due to the intrinsic approximation of the adopted costing methods, the resulting final figure must be also considered as approximate.

\section{Results and discussion}

\subsection{Supercritical fluid extraction (SFE) system: performance evaluation}

The performance of the constructed extraction system was evaluated to ensure the reliability of the subsequent experimental results. First, various sets of two experiments under identical extraction conditions (duplo's) were performed to assess the repeatability of the extraction procedure in terms of oil yield. Seven sets were performed in total, testing different pressures $(25$ and $35 \mathrm{MPa})$, temperatures $\left(50\right.$ and $\left.80^{\circ} \mathrm{C}\right)$, flow rates $(2.9$ and $5.7 \mathrm{~g} / \mathrm{min}$ of $\mathrm{CO}_{2}$ ) and varying total amount of $\mathrm{CO}_{2}$ processed (156-1000 g, equivalent to total extraction time). The relative differences on the oil yields between the two experiments of each set ranged between 0.3 and $4.2 \%$. The results can be consider sufficiently close to allow trend detection on basis of single experiments per set of conditions.

Generally, three consecutive regions can be identified in a extraction curve: a linear region with constant extraction rate; a transition period; and a diffusion-controlled period where the extraction rate decreases due to increasing mass transfer resistances and depletion of solute in the solid matrix. ${ }^{8}$ If equilibrium is achieved (i.e. the solvent is saturated with the solute to be extracted), the slope of the initial linear region of the extraction curve corresponds to solubility (maximal solvent loading under the specific extraction conditions). However, when having only one yield curve, an initial linearity observed does not necessarily prove that equilibrium solubility conditions have been reached. The straight line could be also caused by a constant mass transfer resistance controlling the process. ${ }^{8}$ Hence, a change in $\mathrm{CO}_{2}$ flow rate is needed to evaluate that indeed the rate of extraction is limited by solute solubility. Figure 3 shows $\mathrm{scCO}_{2}$ extraction curves obtained after changing flow rate at constant extraction conditions of 
$25 \mathrm{MPa}$ and $50^{\circ} \mathrm{C}$. The curves were plotted in two different ways: extraction yield vs. amount of solvent (i.e. cumulative $\mathrm{CO}_{2}$ passed) in Figure 3(a) and extraction yield vs. extraction time in Figure 3(b).
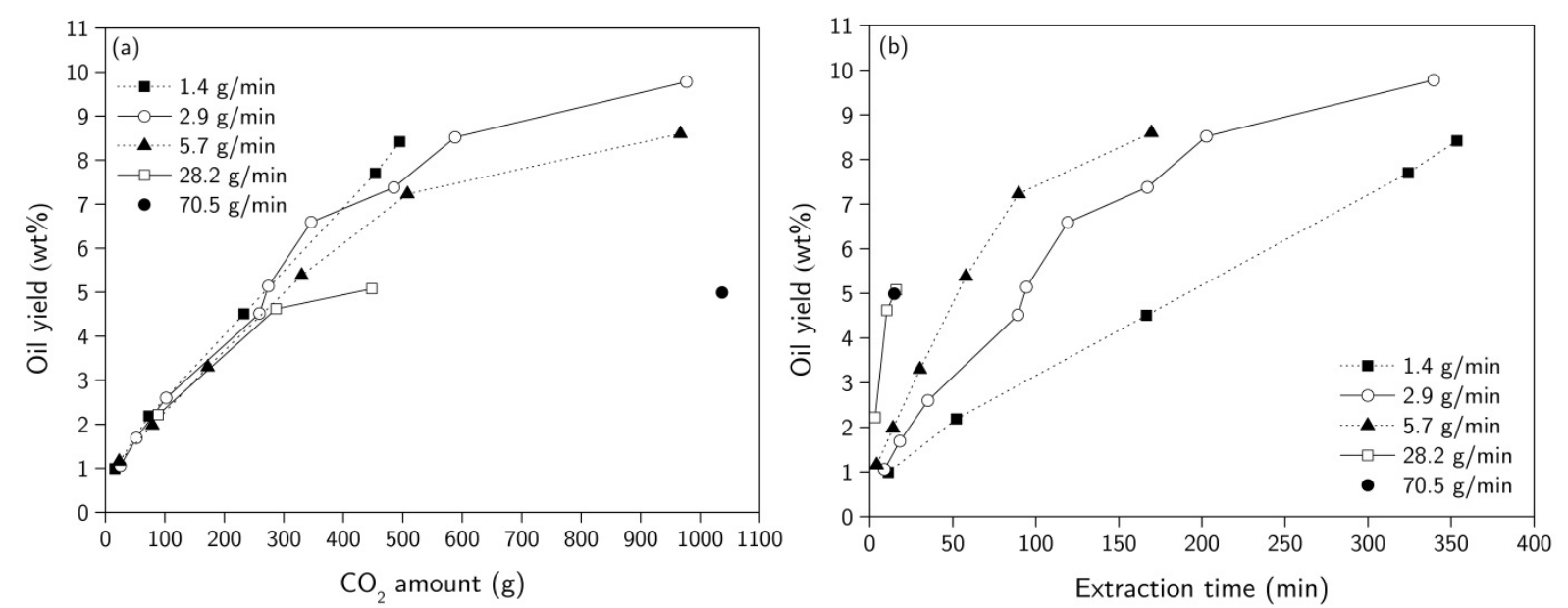

Figure 3. Cumulative extraction curves for $25 \mathrm{MPa}, 50^{\circ} \mathrm{C}$ operating conditions with (a) oil yield as function of amount of solvent and (b) oil yield as function of extraction time

As can be seen in Figure 3(a), all curves had a nearly identical (overlapping) initial linear period, until about $300 \mathrm{~g} \mathrm{CO}_{2}$. When representing the results as function of extraction time, it can be seen that the slope of the linear region increases nearly proportional to the increase in flow rate: e.g. the flow rate was approximately doubled from 1.4 to 2.9 $\mathrm{g} / \mathrm{min}$ corresponding to a doubling of slope from 0.021 to 0.041 . Consequently, the results indicate the existence of a solubility controlled region, in which a constant extraction rate was achieved for all the flow rates tested. In this region the slope can be used to determine the oil equilibrium solubility. The oil concentration in the $\mathrm{CO}_{2}$ stream exiting the column remains constant and the change in flow rate is the only parameter that leads to an increases in the slope. The length of the algae fixed bed seems to be sufficient to saturate the solvent leaving the system with oil. Reverchon and Poletto ${ }^{28}$ reported the same behavior in their extraction process from rose and tuberose concretes at constant extraction conditions of $8 \mathrm{MPa}$ and $40^{\circ} \mathrm{C}$.

It can be also observed that at higher flow rates the linear region ends earlier, shifting to the period where the extraction rate starts to decline. That can be seen clearly in the extraction curve of $28.2 \mathrm{~g} / \mathrm{min}$ flow rate where the linear region ends after $300 \mathrm{~g} \mathrm{CO}_{2}$ while for $1.4 \mathrm{~g} / \mathrm{min}$ the oil yield increases linearly even after $450 \mathrm{~g} \mathrm{CO}_{2}$. This could be explain by the fact that with a flow rate of $28.2 \mathrm{~g} / \mathrm{min}$, the residence time of the $\mathrm{CO}_{2}$ in the column $(\approx 0.81 \mathrm{~min}$ ) seems to be too short for a sufficient contact time of the solvent 
with the solid matrix. During the constant extraction rate phase, maximum solvent loading is still achieved by extracting the easy accessible solute and, after that, the less accessible solute (i.e. extract within the solid matrix) begins to be extracted but at a much lower rate due to the too short contact time reaching the diffusion-controlled phase earlier than for slower flow rates. As reference point, the oil yield obtained with an even faster flow rate, $70.5 \mathrm{~g} / \mathrm{min}$ is also plotted in Figure 3 which follows the trend earlier explained. For the subsequent experiments performed to study the effect of the various extraction parameters, a standard flow rate of $2.9 \mathrm{~g} / \mathrm{min}$ was selected and maintained.

\subsection{Effect of extraction pressure and $\mathrm{CO}_{2}$ amount (or extraction time)}

\subsubsection{Oil yield and FAME composition}

First, the effect of pressure was investigated in terms of oil yield (to evaluate extraction efficiency) and composition (to evaluate the selectivity of the process). Transesterification of the oil was performed to find out which fraction of the oil extracted can be converted into FAMEs (i.e. FAME feed fraction of the oil). With that and together with the FFA analysis, we can evaluate process conditions to maximize the extraction of BFAs (mainly triglycerides, TAGs) as those compounds are preferable for the further conversion into a diesel like fuel. The presence of FFAs in oil, on the other hand, are known to be disadvantageous for biodiesel production ${ }^{29}, 30$ and lipidic compounds like pigments (e.g. carotenoids) have deleterious effects on the catalyst during hydrogenation to produce green diesel. ${ }^{31}$ Therefore, regardless of which end diesel type product is targeted (biodiesel or green diesel), the production of acylglycerols (BFAs) should be enhanced during extraction.

Cumulative curves of oil yield as function of $\mathrm{CO}_{2}$ amount are shown in Figure 4 for three different extraction pressures, 20, 25 and $35 \mathrm{MPa}$ (the maximum operating pressure by our system), at constant temperature $\left(50^{\circ} \mathrm{C}\right)$ and at a $\mathrm{CO}_{2}$ feed flow rate of $2.9 \mathrm{~g} / \mathrm{min}$.

The oil yield increased with higher extraction pressure and solvent amount. This trend was expected since the solubility of a solute in a supercritical fluid is known to be largely a density-driven phenomenon. ${ }^{8}$ Since the density of $\mathrm{scCO}_{2}$ increases with pressure at constant temperature, higher extraction pressure would increase the $\mathrm{scCO}_{2}$ solvent strength, resulting in a higher oil yield, an effect that has been widely reported in literature. For $35 \mathrm{MPa}$, the two regions of the extraction curve are well differentiated. 
The oil is rapidly extracted at the beginning of the extraction and after about $200 \mathrm{~g} \mathrm{CO}_{2}$ the rate drastically decreases and it seems to level off at a plateau near $12 \mathrm{wt} \%$ oil yield. It is questionable if a further increase in pressure would lead to higher oil yields. In fact, several authors (using algae as feedstock) showed no significant increase at pressures above $30 \mathrm{MPa}$ and in the range of 50 to $60^{\circ} \mathrm{C} \cdot{ }^{5,7,32}$ Moreover, even if a higher pressure would lead to more extracted oil, it is still necessary to evaluate if that would also translate into an increase of the suitable FAME feed fraction of the oil. More insight into the composition of oil is therefore needed.

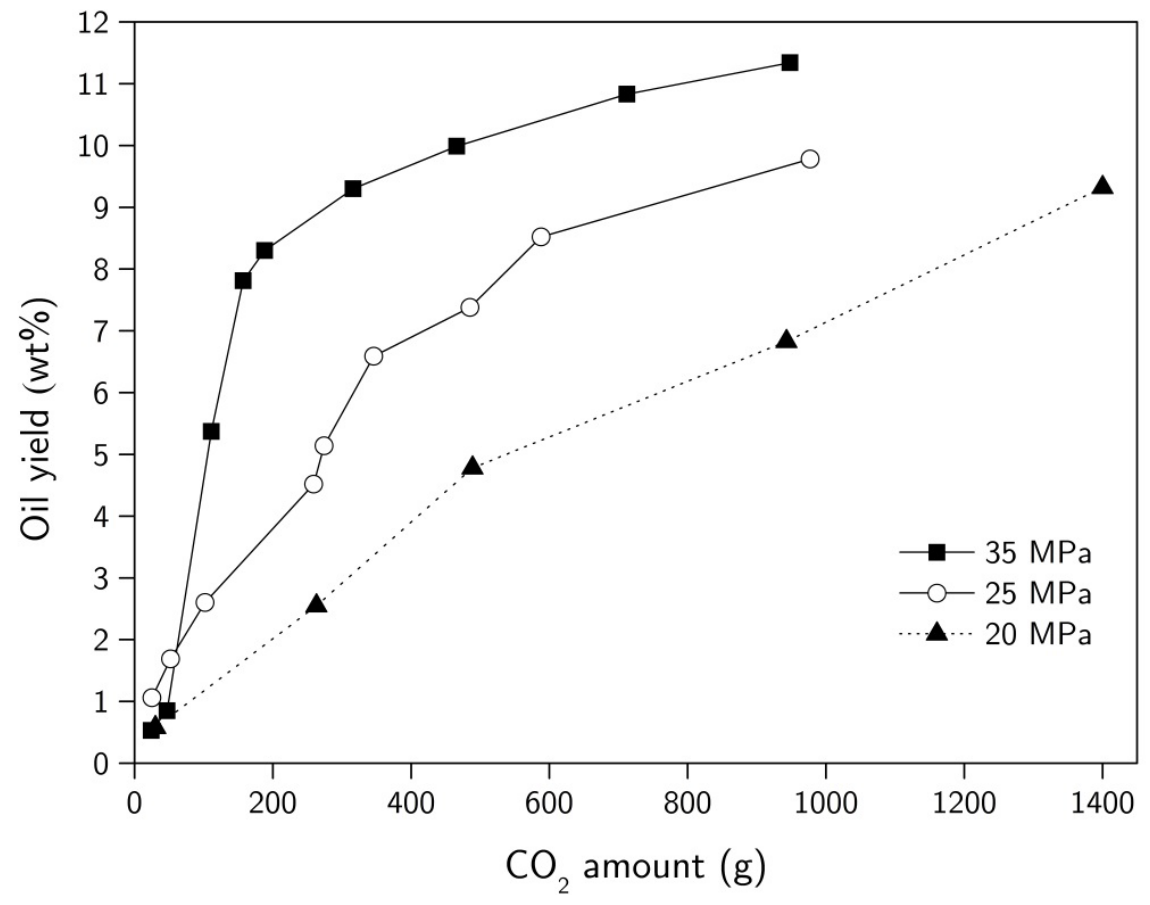

Figure 4. Yield of oil (d.a.f.) extracted at $50^{\circ} \mathrm{C}$ as function of $\mathrm{CO}_{2}$ amount at a feed rate of $2.9 \mathrm{~g} / \mathrm{min}$

Figures 5(a) and 5(c) show both the total oil and FAME yields (d.a.f. basis) obtained for the extractions at 25 and $35 \mathrm{MPa}$ respectively. Figures 5(b) and 5(d) show the oil composition change as both FAME and non-FAME feed fractions for the two pressures mentioned. Note that in Figure 5(a), no FAME yield is presented for the extractions using $25 \mathrm{~g}$ of $\mathrm{CO}_{2}$ because the amount of oil extracted was not sufficient for the transesterification and analysis procedures applied. The same occurred for extractions after 24 and $47 \mathrm{~g}$ of $\mathrm{CO}_{2}$ in Figure 5(c).

For the FFA content of the oil no clear trends were observed with respect to change in pressure and $\mathrm{CO}_{2}$ amount. Generally, FFAs have higher solubility than TAGs in $\mathrm{scCO}_{2}{ }^{33}$, 34 and their mass fraction on the extracted oil should decrease with extraction time. However, the FFA fraction of the oil was probably too small for trend detection. On 
average, the oils had only $2.2 \mathrm{wt} \%$ of FFA content (3 wt\% of total FAME and $0.5 \mathrm{wt} \%$ of algae). Therefore, it can be stated that the FAME fraction produced upon transesterification originates mostly from the acylglycerols fraction of the oil extracted (BFAs accounting for more than $95 \mathrm{wt} \%$ of total FAME).
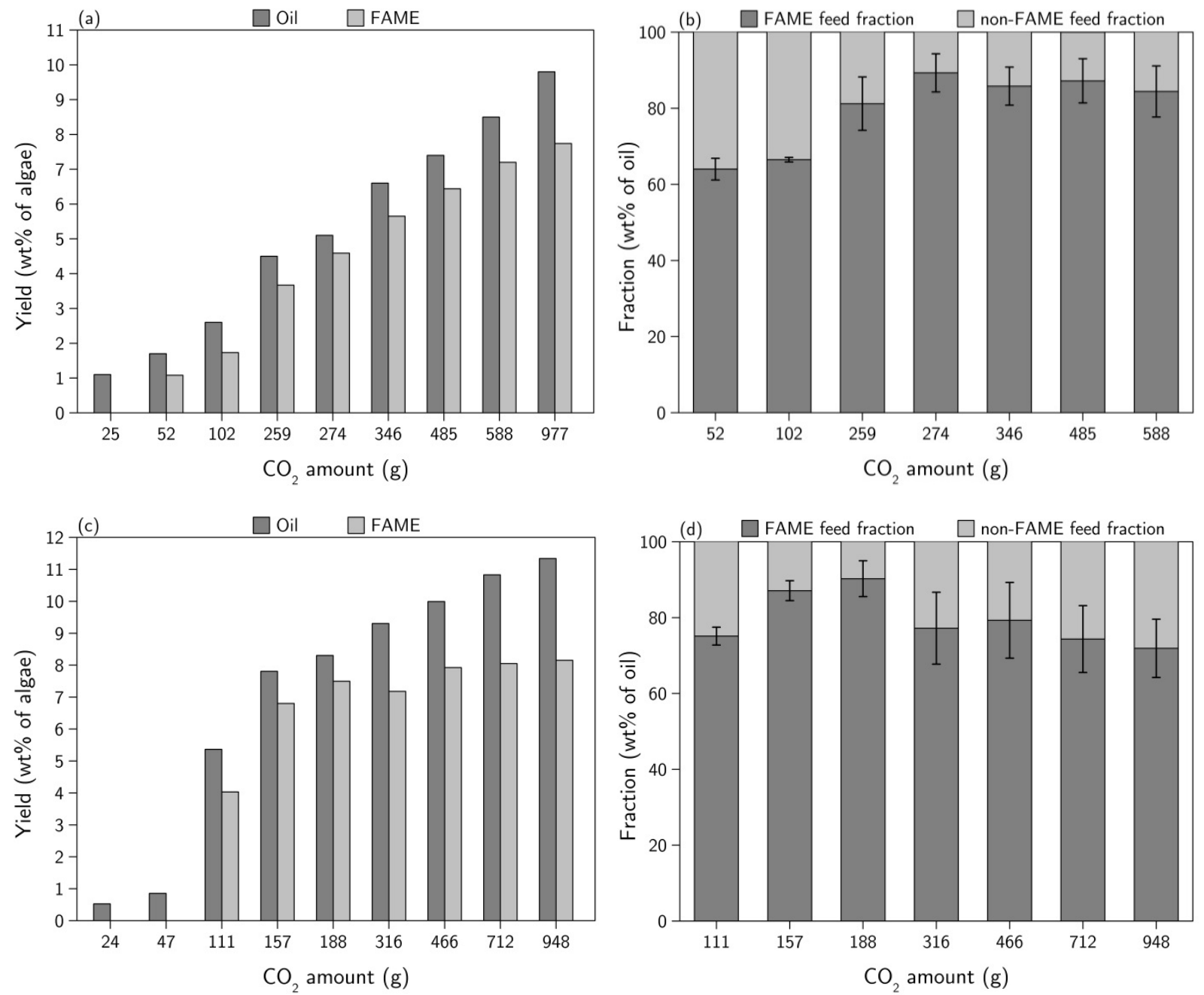

Figure 5. (a) total oil and FAME yields and (b) the oil composition change as both FAME and nonFAME feed fractions for the extractions at $25 \mathrm{MPa}$, (c) total oil and FAME yields and (d) the oil composition change as both FAME and non-FAME feed fractions for the extractions at $35 \mathrm{MPa}$

It can be clearly observed that for $25 \mathrm{MPa}$ (Figure 5(a)) the FAME yield increased along the extraction as does the total oil yield. When looking at the oil composition (Figure $5(\mathrm{~b})$ ), the maximum FAME feed content seems to be reached in the region of $300 \mathrm{~g} \mathrm{CO}_{2}$ and, above $588 \mathrm{~g}$, a slight increase of non-FAME feed fraction can be observed. For the $35 \mathrm{MPa}$ series the FAME yield (Figure 5(c)) increases until $188 \mathrm{~g} \mathrm{CO}_{2}$ and from that point it remains constant, which might indicate the full recovery of all FAME feed compounds under that specific solvating power. At $35 \mathrm{MPa}$, the $\mathrm{CO}_{2}$ solvent density is 
higher which leads to a higher solvating power. Therefore, the initial extraction rate is higher and the maximum amount of extractable FAME feed is reached earlier than at 25 $\mathrm{MPa}$. From $188 \mathrm{~g} \mathrm{CO}_{2}$ onwards, the total oil yield keeps rising, but only because of the increased extraction of non-FAME feed compounds (see Figures 5(c), (d)). Most likely, those substances are more polar and have higher molecular weight being less volatile and requiring a longer time to be extracted (lower extraction rate).

The residual pellets (after extraction in the same way as the original feedstock) were also analysed (in the same way as the original pellet feedstock) and, generally, the oils yielding low FAME amount after transesterification corresponded to the residual pellets with higher FAME content and vice versa.

For both extraction pressures studied, it appears that two types of "non-FAME feed fractions" might exist, both being a mixture of compounds, but with a, on average, very different solubility in $\mathrm{CO}_{2}$. Looking at Figures 5(b) and 5(d), the initial "non-FAME feed" content of the oil (highly soluble in $\mathrm{CO}_{2}$ ) is preferentially extracted during the initial extraction phase. As this fraction is exhausted in the pellet and the larger TGA fraction is still being extracted, a maximum in the FAME feed fraction is reached (see Figure 5(b) from 52 to $274 \mathrm{~g} \mathrm{CO}_{2}$ ). After that, the non-FAME feed content starts increasing again, now based on the slower extraction of the "non-FAME fraction" with a lower solubility/accessability than the FAME producing compounds. Instead of 'solubility' also differences in the 'location' of the various extract substances in the solid matrix could play a role. However, we believe that the pellets used have a random distribution of the substances within the whole matrix (i.e. FAME feed components can be both at the surface and inside the solid matrix) and that it is the differences in solubility of the extract components creating the trends observed. These results also indicate possibilities for, what is known as-, "fractional extraction" where fractions are collected as a function of time throughout the extraction. ${ }^{18}$ That, together with the possibilities of easily tuning the solvent power, allows us to maximize the purity of the oil and the value of the product, reaching BFA contents as high as $90 \%$.

In brief, it seems that in order to maximize both the oil yield and its content of FAME feed compounds, we should operate at high pressures (at least in the range of $35 \mathrm{MPa}$ or higher for the algal biomass used here) while the amount of $\mathrm{CO}_{2}$ used (or extraction time) can be optimized to lower values (compare Figure 5(b) and 5(c)) to enhance the purity (in terms of FAME feed) of the oil extracted which would imply a substantial reduction in costs. However, when extracting at higher pressures, co-extraction of other "non-FAME feed" compounds seems unavoidable. Therefore, the following aspects were 
studied in more detail: 1) composition of the "non-FAME fraction" (Section 4.2.2); their co-extraction might be positive if those substance have a certain economic value and; 2) study the effect of temperature to evaluate if the FAME yield can be further maximized (Section 4.3).

Before that, the effect of pressure and $\mathrm{CO}_{2}$ amount (or extraction time) on the fatty acid (FA) profile obtained after transesterification of the oil was evaluated (Figure 6). As shown in other studies, ${ }^{5,35}$ no significant changes of FAME composition were observed along the extraction (i.e. increasing $\mathrm{CO}_{2}$ amount). On average, the FAME fraction had the following fatty acid composition (as wt\% total FAME): $10.8 \mathrm{wt} \%$ of C16:0, $4.0 \mathrm{wt} \%$ of C18:0, 28.9 wt\% of C18:1 and 56.3 wt\% of C18:2. Linoleic acid (C18:2) was the most abundant, as that in the FA profile of the oil extracted by B\&D (see Table 1). However, $\mathrm{scCO}_{2}$ was clearly more selectively towards compounds producing a FAME mixture with lower degree of unsaturation. With the B\&D method, the FAME mixture contained highly unsaturated PUFAs (with 3 or more double bonds) which were not produced by the supercritical solvent. When aiming for biodiesel production, Pinzi et al. ${ }^{36}$ defined the following ideal composition: 1) high presence of monounsaturated fatty acids; 2) low content of polyunsaturated acids (to enhance oxidative stability) and; 3) controlled saturated fatty acids content (to reduce cold flow problems). According to that, supercritical $\mathrm{CO}_{2}$ appears to be a more suitable solvent to accomplish those requirements.
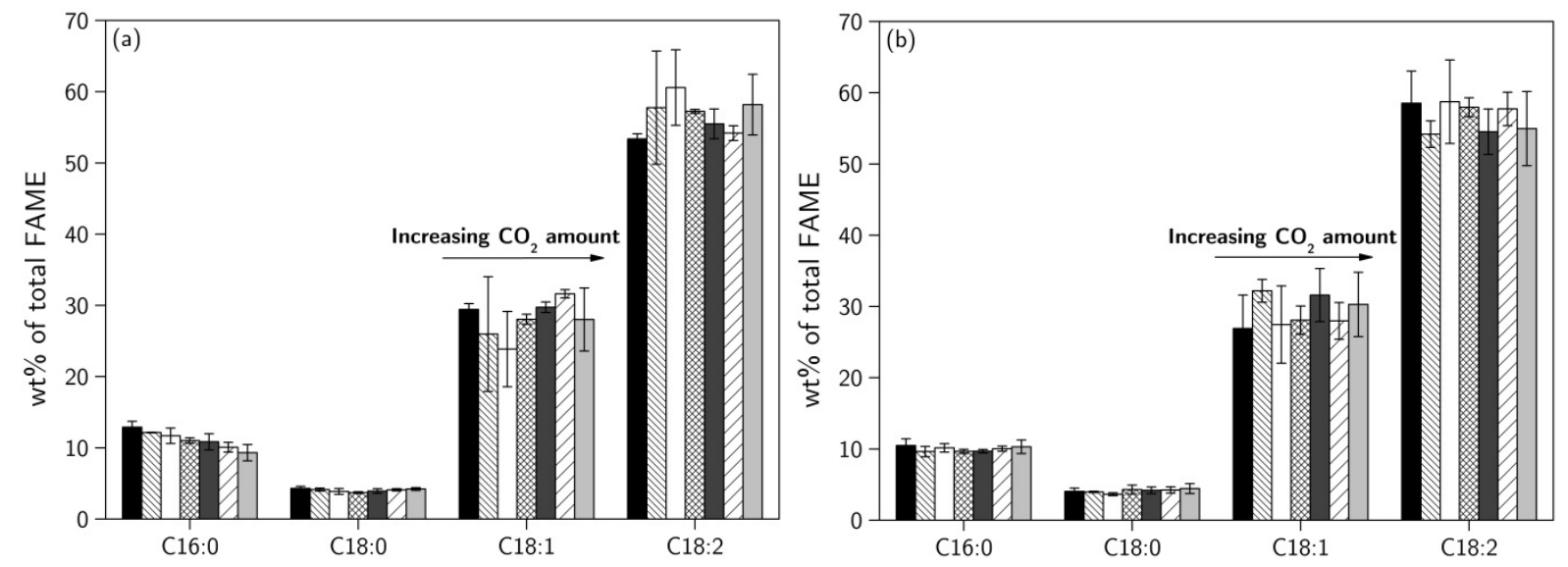

Figure 6. Fatty acid profile obtained after transesterification of the oils obtained at (a) $25 \mathrm{MPa}$ and increasing $\mathrm{CO}_{2}$ amount from 52-977 $\mathrm{g} \mathrm{CO}_{2}$; and at (b) $35 \mathrm{MPa}$ and increasing $\mathrm{CO}_{2}$ amount from 111-948 g $\mathrm{CO}_{2}$

Furthermore, the FA profile was also comparable for both 25 and $35 \mathrm{MPa}$ extraction pressures. However, greater differences were observed when comparing with the $20 \mathrm{MPa}$ 
oil extract. After $\sim 480 \mathrm{~g} \mathrm{CO}_{2}$ and $20 \mathrm{MPa}$, the following FA distribution was obtained (as wt\% of total FAME): $16.8 \pm 0.4$ wt\% of C16:0, $6.0 \pm 0.6$ wt\% C18:0, $51.5 \pm 0.6$ wt\% C18:1 and 25.7 \pm 0.3 wt\% C18:2. A decrease in C16:0 and C18:1 and increase in C18:2 was obtained with the rise in pressure. The results suggest that, with the increase in solvent power under isothermal conditions, the compounds with higher molecular weight and higher degree of unsaturation (i.e. BFAs and FFAs yielding C18:2 methyl esters) become more soluble and thereby their concentration in the FAME mixture become more prevalent. Some authors agree that solubility is more affected by molecular weight (e.g. $256.5 \mathrm{~g} / \mathrm{mol}$ for $\mathrm{C} 16: 0$ and $280.5 \mathrm{~g} / \mathrm{mol}$ for C18:2) than by the degree of unsaturation. ${ }^{34}$, 37, 38 The same trends were observed by Cheung ${ }^{39}$ for the extraction from red and brown seaweed.

\subsubsection{Non-FAME fraction composition}

Fats and oils are complex mixtures of not only FFAs and BFAs (mono, di and triglycerides) but also minor lipids that constitute the unsaponifiable fraction (e.g. sterols, pigments, etc.). ${ }^{18}$ In our extracted oils, the non-FAME feed fraction was found to contain sterols, glycolipids, less than 1\% apolar (lipo-)proteins and an unidentified fraction likely containing pigments (e.g. chlorophylls). Choi et al. ${ }^{40}$ identified also hydrocarbons in the oil extracted from Scenedesmus obliquus, an algal species similar to the one used in this study. Phospholipids are practically insoluble in $\mathrm{scCO}_{2},{ }^{3,41}$ hence their presence in the unidentified fraction seems unlikely.

With increasing extraction time and pressure, the oils changed from light yellow to darker yellow/green color, indicating the presence of chlorophyll, and became more viscous. This is probably due to the fact that at higher pressures, the solubility of more polar and heavier compounds like chlorophyll is enhanced. Its presence in microalgal oil extracted with $\mathrm{scCO}_{2}$ together with the color change towards green has been reported by several authors. ${ }^{3,7}$

Figure 7 shows the composition of the sterol fraction for the extraction at $35 \mathrm{MPa}$ and $50^{\circ} \mathrm{C}$ with increasing cumulative $\mathrm{CO}_{2}$ amount (below $157 \mathrm{~g} \mathrm{CO}_{2}$, there was not sufficient oil for sterols analysis). As can be seen, the extracted sterol mixture had the same composition along the extraction curve with stigmasterol as most abundant substance. Moreover, no clear trends were observed for quantitative analysis of sterols with respect to change in pressure. On average, the sterol oil content was $3.2 \mathrm{wt} \%$ (of total extracted 
oil). Mendes et al. ${ }^{42}$ also reported the presence of sterols in all the extracts from Botryococcus braunii at $40^{\circ} \mathrm{C}$ and from 12.5 to $30 \mathrm{MPa}$.

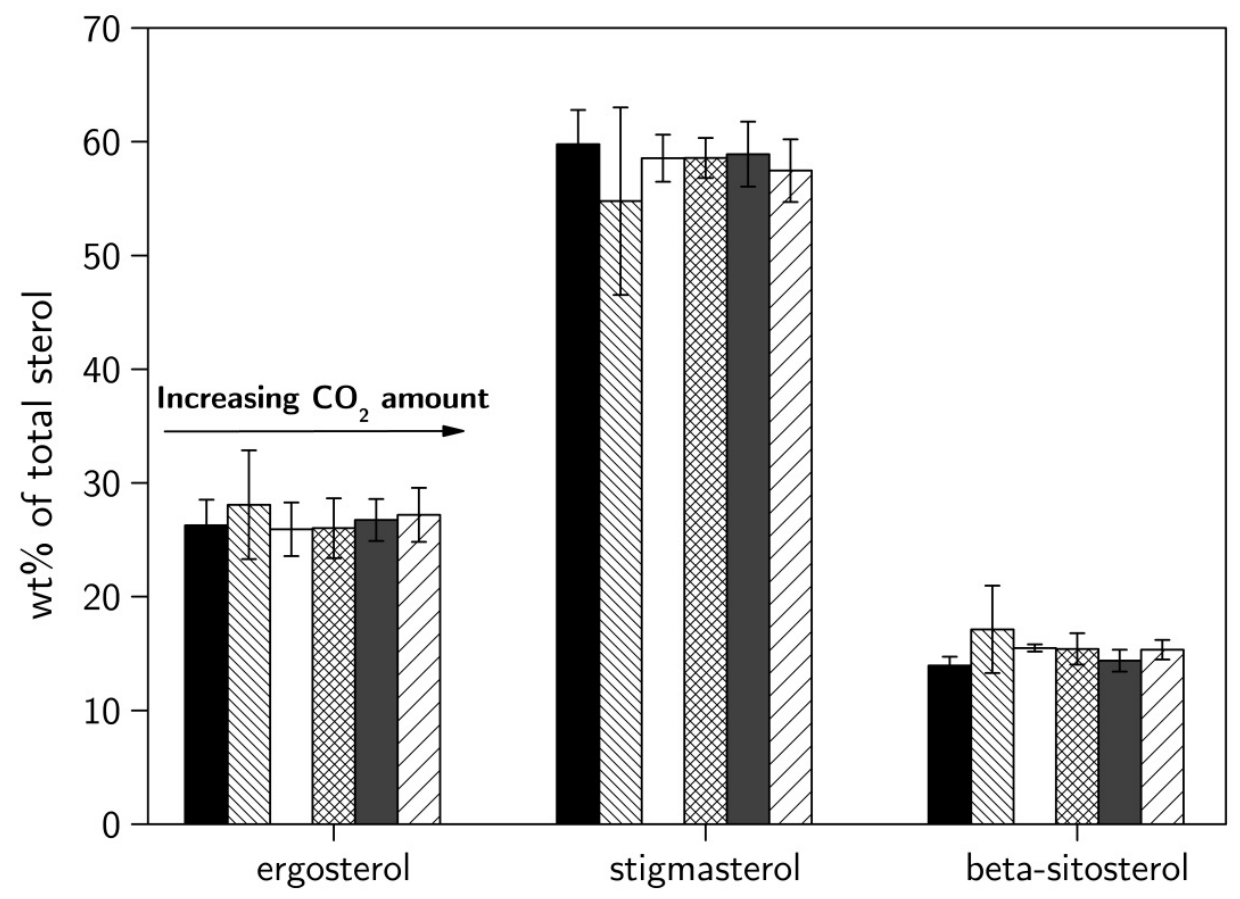

Figure 7. Sterols profile of the oils obtained $35 \mathrm{MPa}$ and increasing $\mathrm{CO}_{2}$ amount from 157-948 $\mathrm{g} \mathrm{CO}_{2}$

The high added value of the sterols extracted derives from their multiple range of uses: in the pharmaceutical and cosmetic industries for the production of drugs and hormones as well as in the food industry. ${ }^{18}$ Consequently, co-extraction of these type of compounds could be an interesting option depending on the further separation costs, their nature and required purity and isolation after $\mathrm{scCO}_{2}$ extraction. Moreover, the large difference in market volume between high added value products and fuels should not be overlooked. Their co-extraction should be strategically defined to avoid an immediate saturation of their market.

\subsubsection{Oil elemental composition and molecular weight distribution}

In terms of elemental composition, no significant differences were observed with increasing $\mathrm{CO}_{2}$ amount and within the range of pressures tested. The averaged composition was: $0.38 \mathrm{wt} \% \mathrm{~N}, 74.85 \mathrm{wt} \% \mathrm{C}, 10.36 \mathrm{wt} \% \mathrm{H}$ and $14.41 \mathrm{wt} \% \mathrm{O}$ (by difference). Its higher heating value (calculated by Boie's equation), $36.8 \mathrm{MJ} / \mathrm{kg}$, is in close range with that of a typical heavy fuel oil (40 MJ/kg) and sunflower oil (39.6 $\mathrm{MJ} / \mathrm{kg}$ ). That together with the very low nitrogen content (necessary to avoid $\mathrm{NO}_{\mathrm{x}}$ emissions during combustion) makes this algal oil a suitable candidate for the production 
of diesel like fuels. In addition, size-exclusion chromatography (SEC) analysis were performed to compare the molecular weight distribution of a $\mathrm{scCO}_{2}$ oil (extracted at 20 $\mathrm{MPa}, 50^{\circ} \mathrm{C}$ and $943 \mathrm{~g} \mathrm{CO}_{2}$ ) with that of sunflower oil, pure triolein and pure monoolein. The diagrams were cut at low molecular weight $(\sim 100 \mathrm{~g} / \mathrm{mol})$ to remove the peak corresponding to the degradation products of the GPC-eluent THF. The differential distribution $d_{\text {total mass }} / d_{\log \text { molar mass }}(W(\log M))$ is plotted versus the molar mass $(M)$.

As can be seen in Figure 8, there is an overestimation of the molecular weight of triolein $\left(\mathrm{M}_{\mathrm{w}}\right.$ real $\left.=885.4 \mathrm{~g} / \mathrm{mol}\right)$ and monoolein $\left(\mathrm{M}_{\mathrm{w}}\right.$ real $\left.=356.5 \mathrm{~g} / \mathrm{mol}\right)$. This aspect has been observed before, ${ }^{43-45}$ and it is related to the differences in hydrodynamic volume with the standard used for calibration (Polystyrene). Therefore SEC results should not be used for the determination of absolute molecular mass, but for comparison purposes as done here.

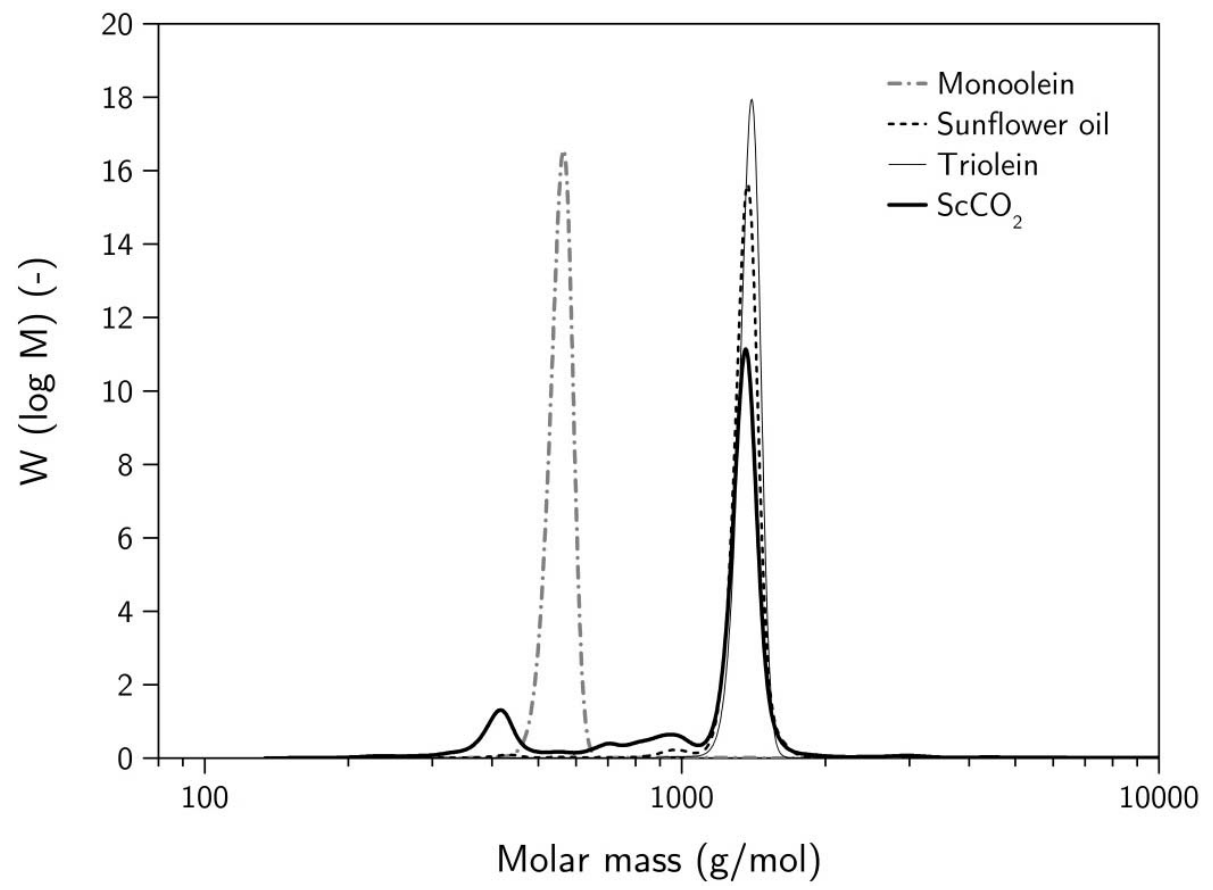

Figure 8. Molecular weight distribution of a $\mathrm{scCO}_{2}$ oil (extracted at $20 \mathrm{MPa}, 50^{\circ} \mathrm{C}$ and $943 \mathrm{~g} \mathrm{CO}_{2}$ ) with that of sunflower oil, pure triolein and pure monoolein

Two peaks can be clearly distinguished for the $\mathrm{scCO}_{2}$ oil, with the second peak (higher molecular weight) coinciding with the triolein and sunflower oil peaks. This indicates once again the presence of triglycerides as major constituents in the extracted oil. The similarities with sunflower oil were expected since this oilseed is mainly composed of triglycerides and its major fatty acids are the same ones as for our $\mathrm{scCO}_{2}$ oil (i.e. C16:0, C18:0, C18:1 and C18:2). ${ }^{8}$ The small peak at the beginning, with lower molecular weight than monoolein, could indicate the presence of sterols and/or FFAs. 


\subsection{Effect of extraction pressure and temperature}

\subsubsection{Oil yield and FAME composition}

The combined effect of pressure and temperature was evaluated aiming to find optimum conditions for the maximum oil amount and FAME feed content. All the experiments in this section were performed using a relatively low total $\mathrm{CO}_{2}$ amount, intending to be at the linear solubility-controlled region of the extraction curve (see Section 4.1.). In that way, results obtained under different extraction conditions can be compared without the need of creating a complete extraction curve (i.e. less time consuming) and are more relevant in view of the anticipated process. As stated by Güçlü and Temelli, ${ }^{18}$ when only the extraction yield/recovery achieved after a certain period of time (far from the initial linear region) or at the end of the extraction period is reported (common practice in many extraction studies), the effect of temperature and pressure on the different aspects of the extraction cannot be well distinguished.

In Table 3, extraction yield and solubility of the extract (proportional parameters in the solubility-controlled period) variations across the change in pressure (20-35 MPa) and temperature $\left(40-80^{\circ} \mathrm{C}\right)$ are presented. Also the change in solvent density (calculated by Peng-Robinson-Stryjek-Vera (PRSV) EoS) is shown for the various extraction conditions studied. Again, the significant increase in solubility with the isothermal increase of pressure can be observed.

From preliminary experiments performed at $20 \mathrm{MPa}$ and increasing temperature from 40 to $80^{\circ} \mathrm{C}$ (result not shown here), we observed a sharp decrease in extracted oil yield due to the decrease in solvent density, having as immediate consequence the drop in FAME feed components. However, that trend could change when increasing temperature at a higher constant pressure. Under isobaric conditions, two mechanisms can affect solubility when increasing temperature: the decrease in solvent density leading to a decrease in solubility and the increase in solute vapor pressure leading to an increase solubility. At the so-called crossover pressure $\left(\mathrm{P}^{*}\right)$, these competing effects will balance each other such that the solubility isotherms at various temperatures intersect on that point. Therefore, the extraction pressure selected will determine the dominant mechanism: at $\mathrm{P}<\mathrm{P}^{*}$ the decrease in density dominates (retrograde behavior) and at $\mathrm{P}>\mathrm{P}^{*}$ the increase in vapor pressure dominates (non-retrograde behavior). ${ }^{18}$ 
Table 3. Solubility change with both extraction temperature and pressure

\begin{tabular}{|c|c|c|c|c|c|c|}
\hline \multicolumn{2}{|c|}{ Process conditions } & \multirow{2}{*}{$\begin{array}{c}\text { PRSV density } \\
\left(\mathrm{kg} / \mathrm{m}^{3}\right)\end{array}$} & \multirow{2}{*}{$\begin{array}{l}\text { Oil yield } \\
(w t \%)\end{array}$} & \multirow{2}{*}{$\begin{array}{r}\mathrm{m}_{\mathrm{CO} 2} \\
(\mathrm{~g})\end{array}$} & \multirow{2}{*}{$\begin{array}{c}\mathrm{S} \\
(\mathrm{g} / \mathrm{kg})\end{array}$} & \multirow{2}{*}{$\begin{array}{l}S_{\text {mean }} \\
(\mathrm{g} / \mathrm{kg})\end{array}$} \\
\hline $\mathrm{T}\left({ }^{\circ} \mathrm{C}\right)$ & $\mathrm{P}(\mathrm{MPa})$ & & & & & \\
\hline \multirow{7}{*}{40} & \multirow{2}{*}{25} & \multirow{2}{*}{886} & 3.70 & 186 & 2.50 & \multirow{2}{*}{2.28} \\
\hline & & & 7.60 & 497 & 2.05 & \\
\hline & \multirow{3}{*}{30} & \multirow{3}{*}{929} & 3.36 & 94 & 4.78 & \multirow{3}{*}{4.78} \\
\hline & & & 4.49 & 134 & 4.54 & \\
\hline & & & 7.24 & 191 & 5.02 & \\
\hline & \multirow{2}{*}{35} & \multirow{2}{*}{963} & 4.26 & 108 & 5.27 & \multirow{2}{*}{5.64} \\
\hline & & & 5.93 & 131 & 6.01 & \\
\hline \multirow{7}{*}{50} & \multirow{2}{*}{20} & \multirow{2}{*}{767} & 2.55 & 263 & 1.30 & \multirow{2}{*}{1.30} \\
\hline & & & 4.78 & 489 & 1.30 & \\
\hline & \multirow{2}{*}{25} & \multirow{2}{*}{833} & 5.14 & 274 & 2.61 & \multirow{2}{*}{2.65} \\
\hline & & & 6.59 & 345 & 2.69 & \\
\hline & \multirow{3}{*}{35} & \multirow{3}{*}{922} & 5.47 & 110 & 6.79 & \multirow{3}{*}{6.59} \\
\hline & & & 5.37 & 111 & 6.38 & \\
\hline & & & 7.81 & 157 & 6.61 & \\
\hline \multirow{3}{*}{80} & 25 & 672 & 2.19 & 187 & 1.56 & 1.56 \\
\hline & \multirow{2}{*}{35} & \multirow{2}{*}{796} & 6.11 & 155 & 5.25 & \multirow{2}{*}{5.48} \\
\hline & & & 6.64 & 156 & 5.70 & \\
\hline
\end{tabular}

For all the pressures, the solubility decreased with increasing temperature but the decline was more notable for lower pressures. When comparing the extraction tests at $35 \mathrm{MPa}$, the solubility values are relatively close for the various temperatures tested. That might indicate that the crossover pressure of the overall oil mixture- $\mathrm{scCO}_{2}$ system has been reached (or we are nearby) where the effects of solute vapor pressure and solvent density have equilibrated. With a further increase in pressure, the solubility will then increase with temperature.

Bjornsson et al. ${ }^{5}$ reported a significant increase of oil yield (almost double) from 50 to $70^{\circ} \mathrm{C}$ at $35 \mathrm{MPa}$ and $270 \mathrm{~min}$ extraction time using Nannchloropsis granulate. Also Cheung, ${ }^{39,46}$ using red and brown seaweed, showed increased yields with temperature for $31 \mathrm{MPa}$ pressure. However at a similar pressure (30 MPa), Halim et al. ${ }^{7}$ obtained lower yields when rising from 60 to $80^{\circ} \mathrm{C}$ from Chlorococcum sp. Therefore, the crossover pressure it is a clear specific property of the solute-solvent system, which is different for each algal species. 
As mentioned before, if at $35 \mathrm{MPa}$ we are at or near the crossover of solubility isotherms, a further rise in pressure would lead to more oil when operating at high temperature. It is, however, not sure if this also would lead to an increased yield of FAME feed compounds. For the increase in temperature at $25 \mathrm{MPa}$ (from 40 to $80^{\circ} \mathrm{C}$ after $186 \mathrm{~g} \mathrm{CO}_{2}$ used) and at $35 \mathrm{MPa}$ (from 50 to $80^{\circ} \mathrm{C}$ after $156 \mathrm{~g} \mathrm{CO} 2$ used), Figure 9(a) shows the oil and FAME yields and Figure 9(b) shows the oil composition in terms of FAME and nonFAME feed fractions.
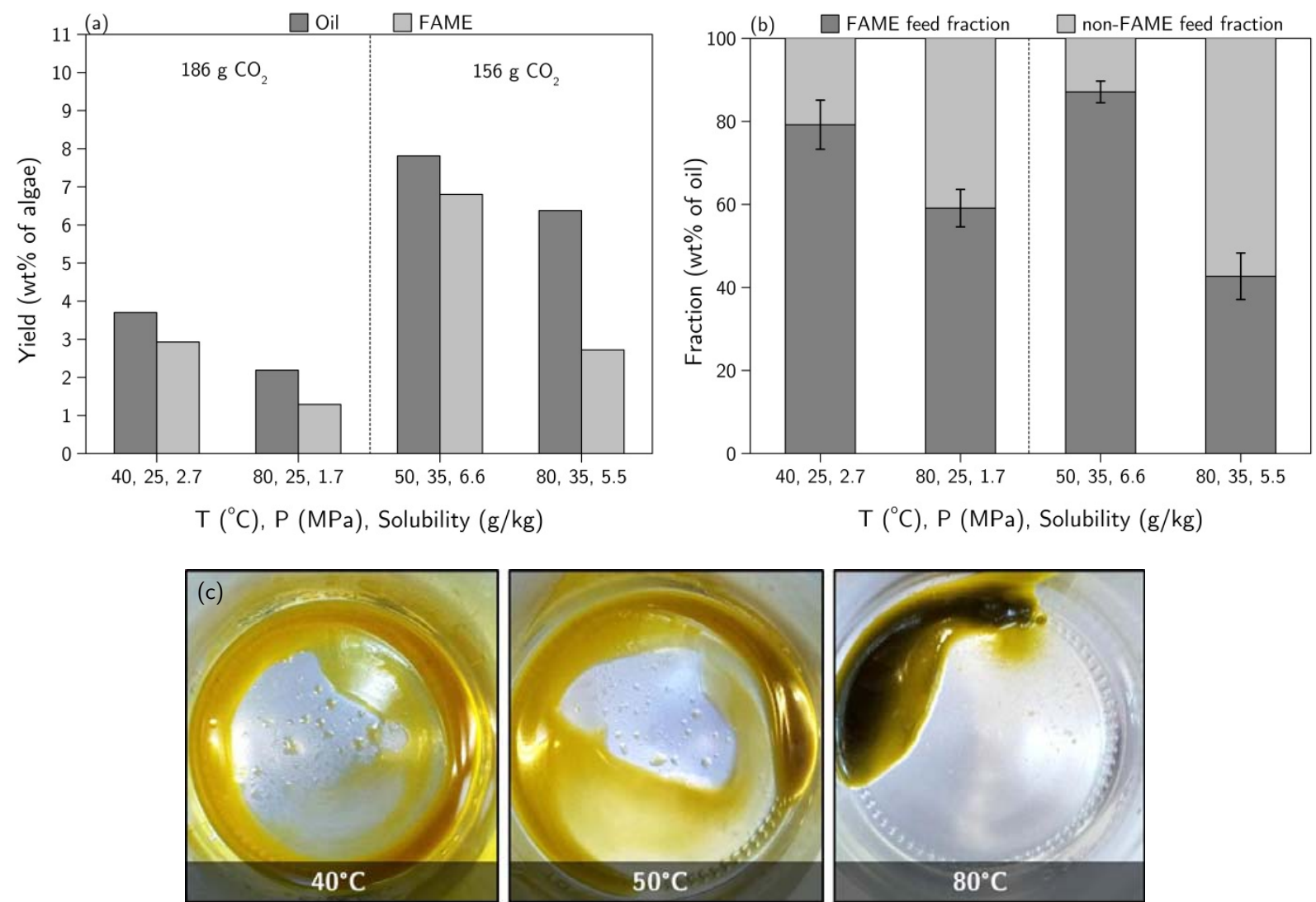

Figure 9. (a) total oil and FAME yields and (b) oil composition as FAME and non-FAME feed fractions for the extractions at $25 \mathrm{MPa}$ and $35 \mathrm{MPa}$ changing temperature; (c) change of oil color with extraction temperature

Looking at Figure 9(a), it can be clearly observed that the decrease in FAME yield with temperature is significantly larger than that of the whole oil yield. At $35 \mathrm{MPa}$, while the oil yields are relatively close $\left(7.81 \mathrm{wt} \%\right.$ at $50^{\circ} \mathrm{C}$ and $6.64 \mathrm{wt} \%$ at $\left.80^{\circ} \mathrm{C}\right)$, the oil from $80^{\circ} \mathrm{C}$ extraction contains 50\% less FAME feed compounds than the oil from $50^{\circ} \mathrm{C}$. Moreover, the decrease in FAME feed fraction with temperature seems more pronounced at higher pressure. These compositional changes were also foreseen already by observing the physical changes of the oils. As can be seen in Figure 9(c), the color of the oil became significantly darker and green at higher temperatures and also remarkably more viscous 
("butter" like) indicating the extraction of components which are heavier, likely more saturated and that can give green pigmentation like chlorophyll. The increase in viscosity could be also caused by presence of more solid substances; e.g. sterols like stigmasterol and $\beta$-sitosterol, and chlorophyll are solid under the extraction conditions tested ${ }^{8,18}$ (melting points: $\sim 160^{\circ} \mathrm{C}, \sim 140^{\circ} \mathrm{C}$ and $\sim 120^{\circ} \mathrm{C}$ respectively) while triolein is liquid $\left(5^{\circ} \mathrm{C}\right.$ melting point).

These observations could be related to differences in crossover pressure for the various classes of compounds within the overall oil mixture. Likely, the compounds producing the non-FAME feed fraction have a lower crossover pressure than the actual operating pressures (25 and $35 \mathrm{MPa}$ ) and, therefore, showing a non-retrograde behavior with their solubility increasing with temperature. As a result, their fraction in the oil becomes larger when operating at $80^{\circ} \mathrm{C}$. In fact, it has been reported that solid solutes (like stigmasterol, $\beta$-sitosterol, and chlorophyll) have a lower crossover pressure than liquids. ${ }^{8}$

${ }^{18}$ Acosta et al. ${ }^{47}$ reported a crossover pressure of stigmasterol at around $17 \mathrm{MPa}$, while Ozlem et al. ${ }^{18}$ mentioned much higher crossover pressures for acyglycerols (28 MPa for mono- and diolein and $31 \mathrm{MPa}$ for triolein, lipids that dominate in the FAME fraction of the oil).

In terms of FAME profile, a slight increase of saturated fatty acids (C16:0 and C18:0) was obtained at higher temperatures coinciding with the more waxy-like texture of the oils. The elemental composition was in close range to that found for the oils in Section 4.2. (extractions changing pressure). Only a slight decrease in carbon content (from ca. 75 to $71 \mathrm{wt} \%$ ) was observed when operating at $80^{\circ} \mathrm{C}$.

Summarizing, with these series of experiments in became clear that higher operating temperatures do not improve the extraction in terms of both oil yield and composition of FAME feed components and $50^{\circ} \mathrm{C}$ seems to be the optimal temperature within the conditions studied.

\subsubsection{Mathematical description of the experimental data by Chrastil equation}

The experimental data presented in Table 3 allowed us to find an equation describing the change in solubility under the range of extraction pressures and temperatures tested. For that, the experimental mean solubility values listed in Table 3 were fitted to Chrastil equation (using the MATLAB fitting toolbox, see Section 3.2). Chrastil relation is valid for solubilities below 100-200 g/L since, above that, the density of the solution (oil- $\mathrm{scCO}_{2}$ 
solvent) starts to significantly differ from that of the pure solvent. ${ }^{17}$ In this case, the solubility values are still low (with ca. $6 \mathrm{~g} / \mathrm{L}$ being the highest value for extraction at 35 $\mathrm{MPa}$ and $50^{\circ} \mathrm{C}$ ) and, hence, the density of the $\mathrm{CO}_{2}$ was assumed to be independent of its extracted solute content. The $\mathrm{scCO}_{2}$ densities used for the data fitting with the Chrastil equation were calculated by PRSV Equation of State, in order to have a consistent approach with process simulations in UniSim ${ }^{\circledR}$ Design in subsequent work. From the available Equations of State this EoS was found to describe best the experimental density data with less than $2 \%$ deviation in comparison with NIST data. For the oil solubility correlation, Table 4 shows the results obtained for the fitting parameters.

Table 4. Chrastil fitting parameters

\begin{tabular}{ccccc}
\hline \multirow{2}{*}{ Chrastil equation } & \multicolumn{4}{c}{ Parameters } \\
\cline { 2 - 5 } & $\mathbf{k} \pm \mathbf{c . i} .^{\mathrm{a}}$ & $\mathbf{- ( a} \pm \mathbf{c . i})$. & $\mathbf{- ( b \pm \mathbf { c . i } . )}$ & $\mathbf{R}^{2}$ \\
\hline $\ln (\mathrm{S})=(\mathrm{k}-1) \cdot \ln (\rho)+\frac{\mathrm{a}}{\mathrm{T}}+\mathrm{b}$ & $9.81 \pm 3.16$ & $4494 \pm 1729$ & $51.33 \pm 16.83$ & $0.96^{\mathrm{b}}$ \\
\hline
\end{tabular}

${ }^{\text {a }} 95 \%$ confidence interval for the mean. ${ }^{\mathrm{b}} \mathrm{R}$ : correlation coefficient.

The goodness of the solubility data fit was within an acceptable range, with a $\mathrm{R}^{2}$ of 0.96 and an improvement to correlations accepted and used elsewhere. ${ }^{17,48,49}$ The quality of the fit for the experimental findings at $40{ }^{\circ} \mathrm{C}, 50{ }^{\circ} \mathrm{C}$ and $80{ }^{\circ} \mathrm{C}$ is shown in Figure 10 .

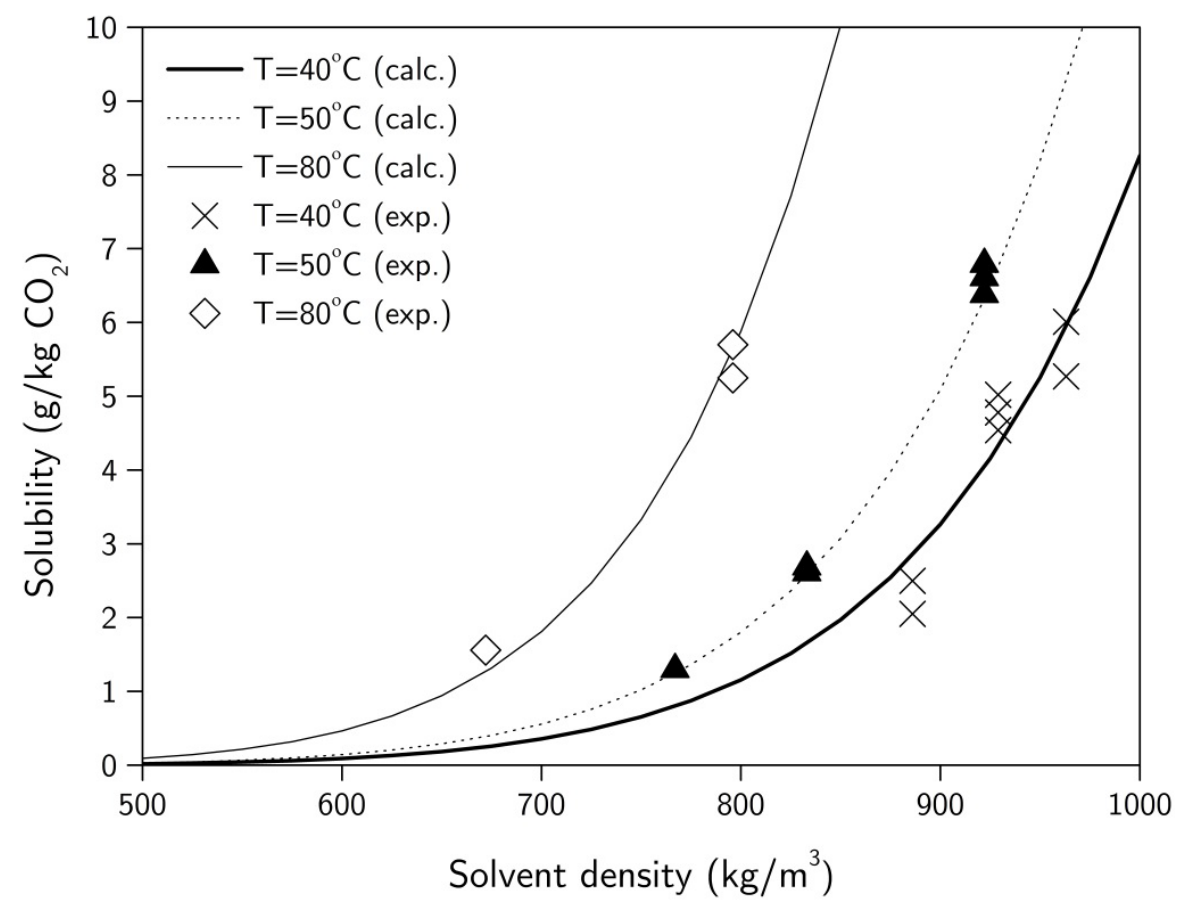

Figure 10. Calculated solubility and experimentally determined solubility 
The Chrastil equation is very sensitive to the density values used, yet its extrapolation capacity was found to be remarkably good. Chrastil ${ }^{17}$ evaluated the results only up to 25 $\mathrm{MPa}$ but compared the obtained equation with data from Stahl et al..$^{50}$ for experiments up to $200 \mathrm{MPa}$ and found a good fit. The Chrastil equation and parameters from Table 4 were used for the process optimization.

\subsection{Effect of algal feedstock}

Several authors indicated the importance of solid matrix pretreatment by grinding to enhance product yields. ${ }^{6}{ }^{51}$ Reverchon and de $\mathrm{Marco}^{6}$ stated that pretreatment is especially important for microalgae, as the strong polymeric structure of their cell walls can hinder extraction. By grinding a reduction of the particle size is achieved, thereby increasing the contact area and reducing the diffusion path length (i.e. enhances internal mass transfer, smaller intra-particle resistance to solute diffusion). However, with decreasing particle size and increasing pressure drop, channeling effects can occur leading to a poorer solvent-solute contact and thereby reducing the extraction efficiency. ${ }^{6}$

In this work $\mathrm{scCO}_{2}$ extraction was performed on both crushed pellets and crushed freeze dried whole cells (see Figure 1) under the same extraction conditions $\left(25 \mathrm{MPa}, 50^{\circ} \mathrm{C}\right.$ and $480 \mathrm{~g} \mathrm{CO}_{2}$ ). The results were compared to those from their equivalent extractions using non-crushed feedstocks. Surprisingly, no large differences were observed; there was only a slight increase in oil yield when using crushed algae ( $4 \%$ higher oil yield for pellets and $10 \%$ higher for cells). Despite the increase in oil amount, the FAME feed content of the oil remained, however, the same. In contrast, Mendes et al. ${ }^{51}$ reported an oil yield ca. 2.6 times higher with crushed algae. Consequently, the extraction enhancement by pretreatment seems to be relative to the algal species used and its cell wall characteristics. It should be realized that the high energy cost of such disruption methods cannot be overlooked when considering such pretreatment steps for $\mathrm{scCO}_{2}$ lipid-based fuels.

Additionally, the feasibility of $\mathrm{scCO}_{2}$ extraction from wet microalgae was assessed as that would circumvent the energy costs related to feedstock drying. The first extraction experiment was successfully performed in the fixed bed extractor, using wetted algal pellets with $25 \mathrm{wt} \%$ water content. However, we were unable to process pellets with a higher water content in a such a extractor configuration, as the algae were creating a clay like formation at the upper part of extractor right under the filter at the outlet. Such a clay layer was nearly impermeable causing filter plugging and eventually resulting in a 
pressure built-up which would trigger the pump to shut down for safety. Therefore, as next attempt in this work, a stirred vessel of $45 \mathrm{ml}$ was used as extractor with the algal slurry occupying about half of the volume. This time, freeze dried whole cells (instead of pellets) were mixed with water in order to resemble to an algal slurry paste coming from the cultivation/harvesting stage. An algal slurry with a (dry, ash free) concentration of $17.1 \mathrm{wt} \%$ (with $80 \mathrm{wt} \%$ water content) was created, which requires potentially less than $5 \%$ of the total energy costs required for complete (thermal) drying. ${ }^{22}$ All the wet extraction results in terms of oil yield and FAME feed mass fraction of the oil are listed in Table 5 together with the extraction conditions applied.

Table 5. Effect of water content on oil yield and its FAME composition

\begin{tabular}{cccccccc}
\hline Feed & Extractor & $\begin{array}{c}\mathbf{P} \\
(\mathbf{M P a})\end{array}$ & $\begin{array}{c}\mathbf{T} \\
\left({ }^{\circ} \mathbf{C}\right)\end{array}$ & $\begin{array}{c}\mathbf{C O}_{2} \\
(\mathrm{~g})\end{array}$ & $\begin{array}{c}\text { Water content } \\
(\text { wt\%) }\end{array}$ & $\begin{array}{c}\text { Oil yield } \\
(\text { wt\%) }\end{array}$ & $\begin{array}{c}\text { FAME } \\
\text { (wt\% of oil) }\end{array}$ \\
\hline pellets & fix bed & 30 & 50 & 950 & 4.13 & 10.32 & 77.1 \\
pellets & fix bed & 30 & 50 & 950 & 25.0 & 8.94 & 29.5 \\
\hline cells & stirred vessel & 25 & 50 & 500 & 1.33 & 6.87 & 76.6 \\
cells & stirred vessel & 25 & 50 & 500 & 80.0 & 1.44 & 57.5 \\
\hline
\end{tabular}

It is clear that a lower oil yield was obtained when increasing the water content of the mixture and it seems that the higher the water content, the larger is the yield difference between wet and nearly dry extraction. Note that the extraction efficiency is expected to be lower for the stirred vessel experiment, due to the lower pressure and the backmixed nature of the system (considering that the applied $500 \mathrm{~g} \mathrm{CO}_{2}$ is less than the amount needed for complete extraction at these conditions according to Figure 5). The FAME feed content of the oil also decreased with the increase in water content, which is probably related to the change in solvent polarity. In fact, addition of water as co-solvent to increase solvent polarity has been often applied. ${ }^{52,53}$ Due to the increased polarity, the solvent extracts more polar lipids (e.g. chlorophyll) which lead to an increase of the nonFAME feed fraction of the oil. Those results agree with visual observations of the oil color changing towards green, when extracting from wet algae (Figure 11).

Two opposing effects caused by water addition have been mentioned in the literature: ${ }^{18,52}$ 1) it can act as a barrier to diffusion; both for the $\mathrm{scCO}_{2}$ entering the matrix and as for the oil diffusing out of the matrix; 2) it can enhance the permeability of the cell wall by swelling effects thereby improving diffusion. Generally (for non-algal biomass; e.g. seeds) lipid recovery decreases with increasing water content, but exceptions to that have been found. For instance, Leeke et al. ${ }^{54}$ showed an increase of extraction yield of essential oils 
from a model herb with water addition (highest at $80 \%$ water content), while Dunford and Temelli52 reported the opposite from Atlantic mackerel. Therefore, it appears that there is no generalized effect of water as it seems to be highly dependent on the feedstock used. Although less effective than the dry case, the experiments with the freeze dried whole cells and water showed the potential use of $\mathrm{scCO}_{2}$ extraction from wet biomass (and a lower water content might yield higher amounts of oil). Extraction should, however, be evaluated using the real feedstock obtained after cultivation and dewatering in an algae biorefinery configuration; hence using the concentrated "as-received" algal slurry. Therefore, we processed that feedstock under several extraction conditions (including those applied to the slurry made with freeze dried whole cells and water) and $80 \%$ water content). Remarkably, no oil was recovered in any of the tests. However, clear changes on the algal slurry appearance were observed.

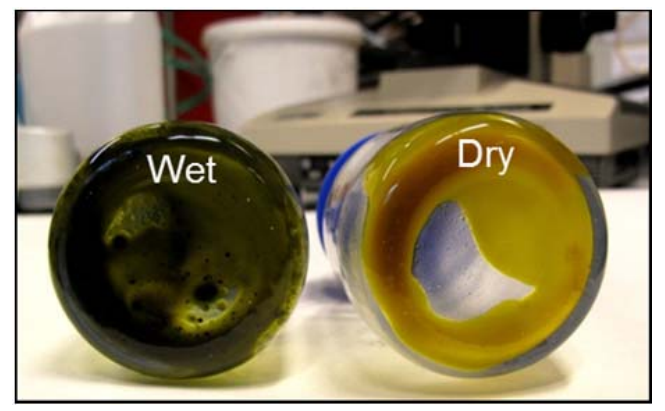

Figure 11. Oils extracted from dry and wet microalgae

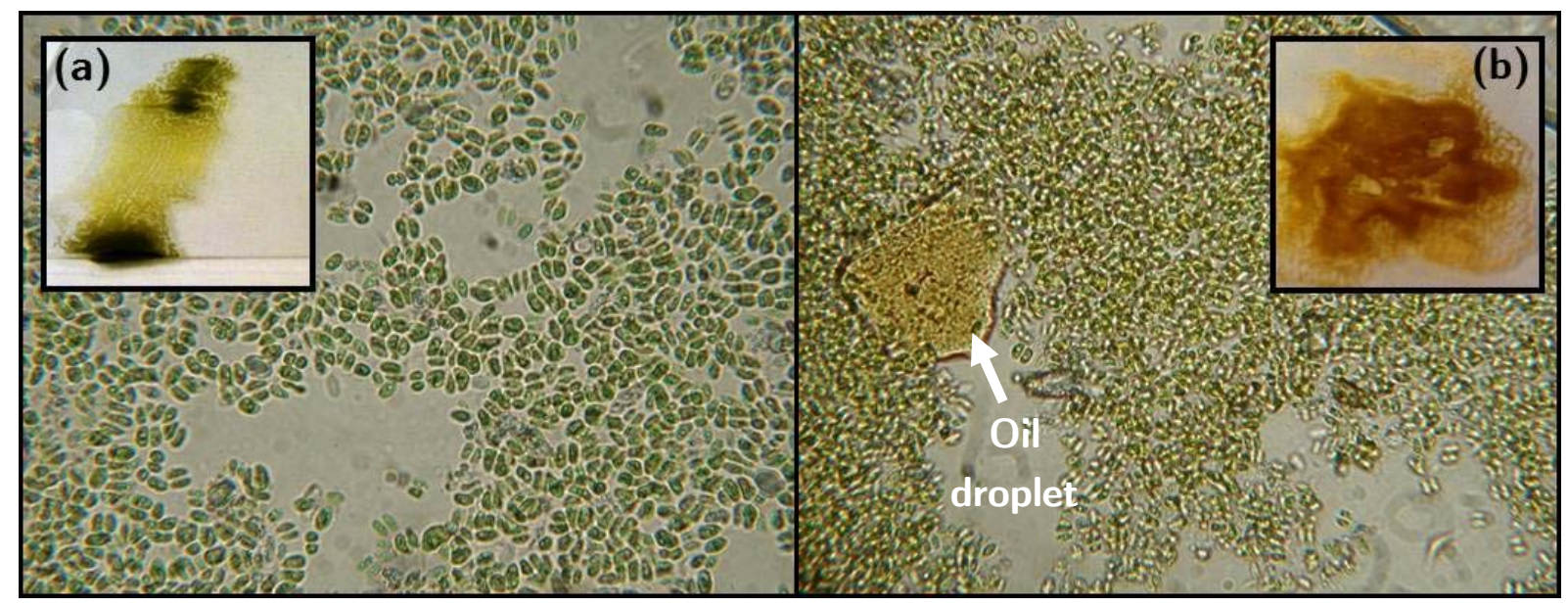

Figure 12. (a) fresh algal slurry in between two microscope plates as well as its optical microscope image and (b) the same as (a) but with the algal slurry after extraction

A picture of the fresh algal slurry in between two microscope plates as well as its optical microscope image are displayed in Figure 12(a). The same is shown in Figure 12(b) for the slurry after $\mathrm{scCO}_{2}$ extraction. A clear change in color can be observed; the algal 
slurry after extraction turned yellow in a similar tonality as that of the oil (see Figure 9). When observing the algae under the microscope, yellow oil droplets, as the one indicated in Figure 12(b), were identified all over the sample, around cells or groups of cells. It seems that extraction was achieved, but the solvent was not able to transport the oil out of the slurry mixture. Furthermore, after acetone extraction from the residual algal slurry shown in Figure 12(b), an oil was recovered having the same elemental composition, color and texture as that from $\mathrm{scCO}_{2}$ extraction of dry algae. Moreover, it was verified that such an oil phase was not recovered when performing a blank with acetone and the fresh feed algal slurry.

There is not much information in literature about the role of intra- and extracellular water for $\mathrm{scCO}_{2}$ extraction from algae. Lehotay ${ }^{55}$ stated that for solutes that are insoluble in water, the solutes precipitate onto matrix surfaces, and even though they may be very soluble in the extraction fluid, the excess water in the sample acts as a barrier in transfer of the solute to the fluid. In our case, for the case of freeze dried whole cells, despite the $80 \%$ extra-cellular water concentration, oil was extracted and transported out of the extractor. The absence of a high amount of intra-cellular water (cytoplasm) in the freeze dried cells, may have played a role here.

Remarkably, Halim et al. ${ }^{7}$ obtained even higher oil yields than from dry biomass when a clay like algal paste with $70 \mathrm{wt} \%$ water content was mixed with inert diatomaceous earth which absorbed water. More work on the role of water is however needed to improve understanding.

\subsection{Economic evaluation}

As stated in the introduction of this work, extraction costs should be minimized, especially when considering the production of fuels. Within the range of experimental conditions studied, optimal conditions, in terms of oil yield and quality, were found at high pressure (in the range of $35 \mathrm{MPa}$ ) and moderate temperature $\left(50^{\circ} \mathrm{C}\right)$. A further increase in pressure will reduce the $\mathrm{CO}_{2}$ requirement due to the increased solubility but, in parallel, will increased equipment constraints and material costs. Therefore, experimental findings are not sufficient for the complete evaluation of this process thereby requiring an additional economical assessment based on operating and investment costs.

$\mathrm{ScCO}_{2}$ extraction of lipids from microalgae in continuous counter-current operating columns appears to be challenging since it would require sophisticated solid handling, 
especially at high extraction pressures. ${ }^{56}$ In principle, a wet algal feedstock could be used in a continuous process, but at 20-30 wt\% solid content the slurry is already a highly viscous paste, leading to difficulties in pumping. A semi-continuous process seems to be a practical method for such extraction cases. $\mathrm{CO}_{2}$ is relatively easy to recycle and by clever use of a number of extraction vessels, a nearly continuous counter-current process can be achieved. A typical outline for a semi-continuous counter-current process consist of a certain number of extractors in operation while, in parallel, one extractor is being emptied and reloaded with fresh algal feed. ${ }^{49}, 56$ Switching schemes, except for solids exchange, are comparable to multibed pressure swing adsorption processes. Recirculated $\mathrm{CO}_{2}$, coming from a repressurization step, after a depressurization for lipid oil recovery extracted in the previous cycle, first enters the extractor with the most depleted algal biomass feed and then continues to a next bed of algal biomass with a shorter time on stream (fresher material). Such a process configuration was considered for this economical assessment.

A step wise strategy was followed in order to find the optimum process design. In the first step a fluid package was selected, basically to describe the density for (sc-) $\mathrm{CO}_{2}$ (as described in Section 3.2.). After that, the energy consumption for circulating $\mathrm{CO}_{2}$ between extraction conditions and lipid oil recovery conditions (lower pressure) was evaluated for several process configurations; here the focus was on minimizing the energy input. To the identified attractive configurations the experimentally found solubility data of our oil- $\mathrm{scCO}_{2}$ system was added (see Section 4.3.2.). Optimal extraction and separation conditions were obtained by sensitivity studies on design parameters (focusing on the extraction vessel as it is one of the major cost drivers) and on extraction/separation conditions. The final costs of the optimized extraction process were calculated considering both dry and wet algae as feed and with two different maximum extractable oil contents: $12 \mathrm{wt} \%$, representing our experimentally studied algae (as the oil yield appeared to approach towards that constant value, see Figure 4); and $25 \mathrm{wt} \%$, being a realistic high lipid content situation for e.g. algae cultivated under nitrogen starvation. The performance criterion (objective function) adopted for the optimization was the cost per unit of oil produced, expressed in $\$ / \mathrm{kg}$. All the assumptions related to microalgae cultivation and those used for costs calculations are described in Section 3.3.

To find the minimum extraction cost possible, calculations were performed assuming the case of an ideal extraction were the maximum solvent loading is always achieved and the maximum extraction rate achievable is continuously maintained until all the oil from the solid matrix is depleted. According to that, the $\mathrm{CO}_{2}$ flow rate required to extract all the 
oil from one fresh bed could be calculated using the oil flow rates in Table 2 and the solubility at the selected extraction conditions. If that minimal cost is already inconceivably high, this extraction process is unlikely to be a serious candidate for an algae biorefinery with liquid fuels as one of the main product streams.

Important to realize here is that the operating window for smooth operation in practice strongly depends on the chosen operation conditions and the available time to add a fresh charged vessel to the system. It is therefore more practical to evaluate the cost according to the so-called switching time required to depressurize, unload, load with fresh material and pressurize again an extraction unit. As explained in Section 3.3., the volume of the extraction vessel was calculated according that operational time and the microalgae production rate. Although Bravi et al. ${ }^{49}$ reported a minimum of $10 \mathrm{~min}$ for switching time, we selected a more conservative estimation of $20 \mathrm{~min}$ to allow for a more plausible and smoother operation. As stated by Bravi et al., ${ }^{49}$ a minimum of two independent extraction vessels are required to maintain continuous operation (one in operation and the other one in the unloading/loading phase). In this study we considered three extractors as the minimum amount possible, having two in operation (in series, to assure a saturated $\mathrm{CO}_{2}$ flow) and the third one in the unloading/loading phase.

The most optimal configuration leading to the lowest costs consisted of a combination of pressure and temperature swing between extraction and oil recovery, with heat integration between the $\mathrm{CO}_{2}$ rich streams. The process outline and the properties of each stream can be found in the Appendix. For the dry algae case, extraction at $35 \mathrm{MPa}$ and $50^{\circ} \mathrm{C}$ was found to be optimal, due to the higher solubility, hence less $\mathrm{CO}_{2}$ has to be recycled, which dramatically drops the total extraction costs by a large decrease in utility consumption. Those extraction conditions were also the preferred ones from experimental observations. Above $35 \mathrm{MPa}$, the total costs started rising as the decrease in utility costs (due to the decrease in $\mathrm{CO}_{2}$ flow rate) cannot offset for the increase in extractor cost (due to increase in wall thickness). The possible savings by pressure increase are limited by the switching time as with that, the extractor volume is calculated, resulting in the same volume for all pressures but with increasing wall thickness. Oil/ $\mathrm{CO}_{2}$ separation at elevated pressures and increasing temperature was found to be beneficial, as with a reduced pressure swing less energy is needed to recycle the $\mathrm{CO}_{2}$ back to its extraction conditions. Disadvantages of separation at higher pressure are the increased capital investment, mainly on the separation vessel. Due to a smaller density difference between the oil and the $\mathrm{CO}_{2}$, the settling velocity decreases, thereby a larger vessel is required at a higher pressure. The optimum was found at $25 \mathrm{MPa}$ and $100^{\circ} \mathrm{C}$. Although the oil 
solubility at those conditions is very low $\left(0.65 \mathrm{~g} / \mathrm{kg} \mathrm{CO}\right.$ ), the recycled $\mathrm{CO}_{2}$ still contains a small fraction of dissolved oil, thereby reducing the cyclic capacity from the theoretical maximum solubility. Therefore, the net, useful ('cyclic') solubility (solubility at extraction conditions minus that at separation conditions) was used for calculations as the actual solubility of the recycled stream.

For the wet algae case, the same separation conditions were applied. For extraction, 35 $\mathrm{MPa}$ was maintained but at $80^{\circ} \mathrm{C}$ as it has been reported that elevated temperatures at high pressures (i.e. harsher conditions) can lead to increased cell damage (similarly to cell disruption techniques) enhancing the extraction. ${ }^{3}$ The wet slurry was assumed to have an algae dry weight concentration of $30 \mathrm{wt} \%$ as, from cost analysis (sensitivity studies), that concentration was the most promising. It should be noted that, to identify the potential of this route, the wet extraction was assumed to be of the same efficiency as that for the dry algal feedstock. On the other hand, the only benefit of wet algae considered was that no thermal drying was necessary; other possible benefits during handling, like possibilities to pump it, were neglected. The final calculated costs are represented in Figure 13.

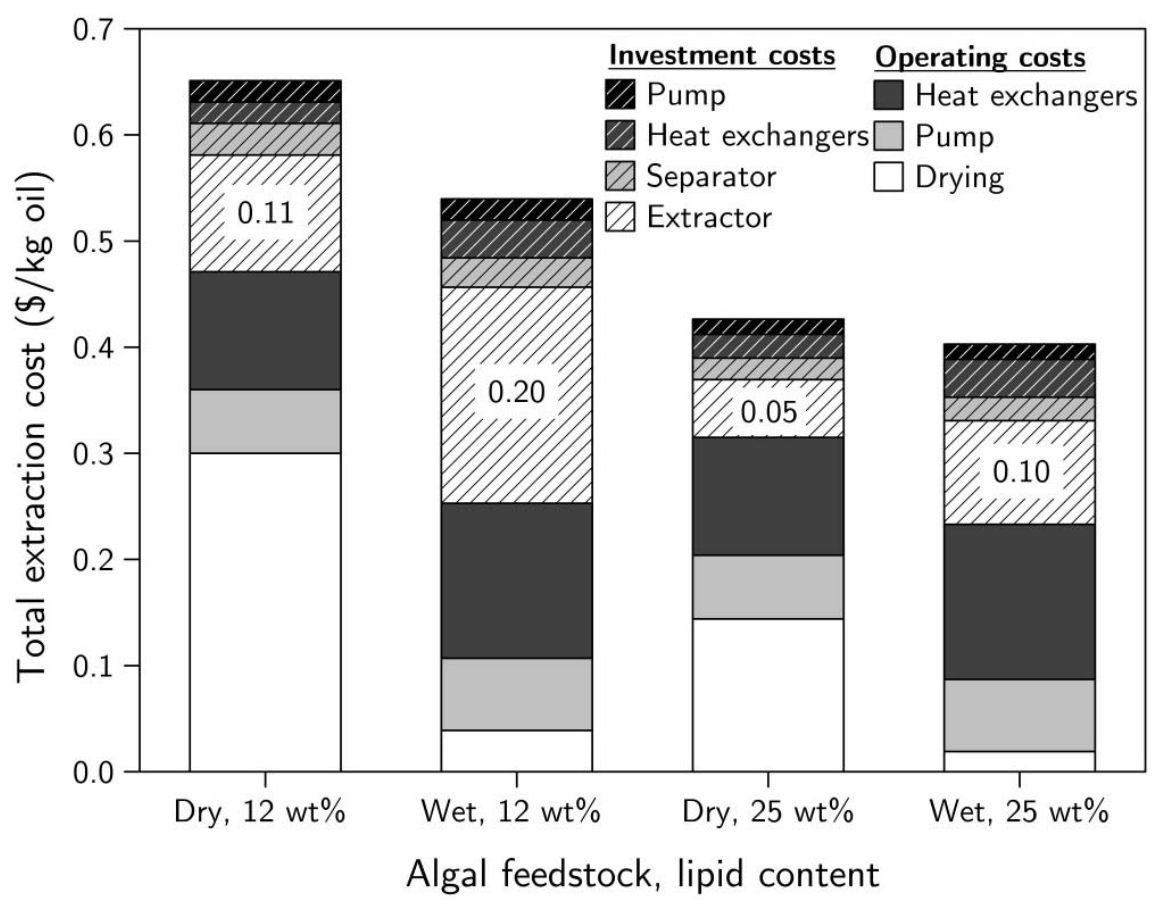

Figure 13. Final calculated costs

The configuration deemed optimal had extraction costs of $0.65 \$ / \mathrm{kg}$ oil for the dry feedstock and $0.54 \$ / \mathrm{kg}$ oil for the wet feedstock. When cultivating an algae with substantially higher extractable oil content the costs were reduce to $0.43 \$ / \mathrm{kg}$ oil (dry algae) and $0.40 \$ / \mathrm{kg}$ oil (wet algae). For the extraction from dry algae, cost could be 
slightly higher as drying is in general not $100 \%$ effective and moisture can also be absorbed by the solid matrix during storage. The extractor cost would increase by $2 \%$ if considering that $15 \mathrm{wt} \%$ moisture still remains after the algal feedstock is thermally dried (assuming a constant bed density).

High pressure equipment can become large cost drivers and, as can be seen in Figure 13, the extraction pressure vessel is here the major investment cost driver. Separation vessels are much less expensive and therefore if fractionation (for high value added lipids) could be profitable, addition of more separation vessels should be considered as $\mathrm{scCO}_{2}$ is nontoxic and therefore possibly lipid-based products can be sold easily to the food manufacturers. For wet extraction they might also be required to separate co-extracted polar lipids which are not desired for diesel like fuels production.

For the algae with $12 \%$ lipids, a reduction of almost $20 \%$ is obtained by the extraction from a wet algae slurry with 30 wt\% DW. Drying is clearly an important energy consumer; it covers more than $60 \%$ of all energy transferred into the system for the case with 12 wt\% oil. It is evident that the drying step needs to be improved to be energetically attractive. Improvement opportunities may involve energy from e.g. waste heat or sunlight. At the same time, extractor costs for the wet extraction under a similar performance is nearly double compared to the dry extraction due to the extra mass in the feedstock in the form of moisture. In brief, cost can be substantially reduced by processing wet algae, but its extraction performance still has to be proven for its technical feasibility.

Costs are however further, and more strongly, reduced when an algal feedstock with a higher extractable oil content is used. However, when more oil is available for extraction possibly a longer residence time is required which translates into the addition of more extraction vessels (assuming the volume is unchanged as it only depends on the algae flow rate and switching time). When considering four extractors (three in operation), the total costs would increase by $4 \%$ for the dry case and $8 \%$ for the wet case. Interestingly, the benefits of avoiding thermal drying are not that remarkable for the case with $25 \mathrm{wt} \%$ extractable oil.

The effect of increasing the algae productivity, assuming 100 metric ton/ha/yr, was also evaluated. All costs shown in Figure 13 decreased by ca. 6\% (for dry algae) and ca. 8$10 \%$ (for wet algae). The size of both pressure vessels became larger due to the higher algae flow rate but the price per $\mathrm{kg}$ of steel dropped due to the typical economy of scale. 
Although only utility and investment costs were taken into account, the final extraction price for both dry and wet algae seems promising. Bravi et al. ${ }^{49}$ evaluated the process economics of $\mathrm{scCO}_{2}$ extraction from sunflower oil using a similar cost calculation methodology. In their optimized process configuration, with no drying required, the cost of extraction were about $0.67 € / \mathrm{kg}$ oil; roughly the double of that for our extraction case without considering the drying costs $(0.34 \$ / \mathrm{kg}$ oil $)$. Brunner ${ }^{56}$ listed several product cost estimations (for extraction from solid matrixes) in which they found investment costs are typically around $30-50 \%$ of the total cost. For this study, the investment costs were also in that range of the total costs. According to our estimations, conventional solvent extraction using e.g. hexane is in the range of $0.3 \$ / \mathrm{kg}$ oil without considering the cost for algae dewatering. Supercritical $\mathrm{CO}_{2}$ extraction cost estimates (which include dewatering) are still higher but not far from those from conventional solvent extraction, especially for the case when a high oil content algae is processed. Moreover, cell disruption methods are generally required when using common organic solvents to improve diffusion into the inner cell. That would further increase the cost estimate for hexane extraction.

Oil hydrogenation into green diesel is the preferred conversion method in our envisaged algae biorefinery as the product shows better fuel properties than biodiesel (e.g. more stability) and hydrogen can be recovered from other process steps of the refinery setting. Davis et al. ${ }^{57}$ showed an increase of approximately $15 \%$ for the raw oil production cost extracted from algae when hydrogenation of the oil to diesel product was added to the costs. Including that $15 \%$ to our costs estimates, the costs in Figure 13 on algal diesel basis would range between $0.75 \$ / \mathrm{kg}$ (or $0.67 \$ / \mathrm{L}$, dry algae with $12 \mathrm{wt} \%$ extractable oil) and $0.50 \$ / \mathrm{kg}$ (or $0.45 \$ / \mathrm{L}$, wet algae with $25 \mathrm{wt} \%$ extractable oil). Dermibas et al. stated that a reasonable target price for microalgal oil is $0.48 \$ / \mathrm{L}$ for algal diesel to be cost competitive with petrodiesel. Therefore, the costs found, which do not include yet cultivation expenses, must be further optimized, but are still low enough to be considered in an algae biorefinery configuration. In fact, utilisation of the spent algal biomass by e.g. hydrothermal liquefaction into a crude bio-oil can further increase the profitability of the process. The key for the success of this extraction process can only be achieved within in a biorefinery based production strategy.

\section{Conclusions and outlook}

For the lipid oil extraction from Desmodesmus sp. microalgae with $\mathrm{scCO}_{2}$ as solvent, we found that an extraction temperature of $50^{\circ} \mathrm{C}$ and a pressure of at least $35 \mathrm{MPa}$ is 
required to maximize both the oil yield and its FAME feed content. At $35 \mathrm{MPa}$ the oil yield approached a constant value of $12 \mathrm{wt} \%$ after $950 \mathrm{~g}$ of $\mathrm{scCO}_{2}$ and $1 \mathrm{~h}$ of extraction time. However, after using only $20 \%$ of this total amount, the entire FAME feed fraction was already extracted and reached its maximum content in the oil, being more than $90 \%$ of the total oil. The results showed the possibilities of optimizing the $\mathrm{CO}_{2}$ amount and extraction time in order to enhance the purity of the oil extracted. The FAME fraction had very low FFA content since over $95 \mathrm{wt} \%$ of the oil was composed of BFA (mainly triacyglycerides); highly desired compounds in the feed for the production of both (transesterified) biodiesel and (hydrogenated) green diesel. The extracted oil had very low nitrogen content and a high HHV ( $\sim 37 \mathrm{MJ} / \mathrm{kg}$; only 7-8\% lower than most vegetable oils).

In the non-FAME feed fraction, other compounds of interest were found such as sterols and pigments. The extraction of those substances can be of importance in a biorefinery setting due to their high economical value resulting from their direct application in the pharmaceutical and food industries. By fractional extraction, where fractions are collected as a function of time throughout the extraction, or stepwise depressurization some degree of FAME/non-FAME feed separation could be achieved. As the separation vessel appeared to be relatively inexpensive compared to the extraction vessel, the stepwise depressurization seems to be a better option. From the process optimization study, it was further concluded that direct $\mathrm{scCO}_{2}$ extraction from the wet slurry significantly lowers the costs, compared to the dry extraction, for slurry concentrations equal or above $30 \mathrm{wt} \%$ and a lower lipid content of the algae. Technical challenges need to be overcome however to make it as efficient as the dry extraction. From the simulation studies, a combination of pressure and temperature swing was found to be the most optimal configuration (with minimal energy input and investment costs) with extraction at $35 \mathrm{MPa}$ and $50^{\circ} \mathrm{C}\left(80^{\circ} \mathrm{C}\right.$ for the wet algae case $)$ and separation at $25 \mathrm{MPa}$ and $100^{\circ} \mathrm{C}$. The approximated cost were in the range of 0.65 (dry feedstock, 12 wt\% extractable oil) to $0.40 \$ / \mathrm{kg}$ oil (wet feedstock, $25 \mathrm{wt} \%$ extractable oil). For a higher lipid content of the algal biomass (25 wt\% or more), the differences between dry and wet extraction are less pronounced, making dry extraction (based on current extraction efficiencies) a better candidate at only slightly higher costs.

\section{Acknowledgements}

The authors are thankful for the financial support by the province of Overijssel via the Green Energy Initiative of the University of Twente (The Netherlands). 


\section{Bibliography}

[1] T.M. Mata, A.A. Martins, N.S. Caetano, Microalgae for biodiesel production and other applications: A review, Renewable Sustainable Energy Rev., 14(1), 217-232, 2010.

[2] A. Singh, S.I. Olsen, A critical review of biochemical conversion, sustainability and life cycle assessment of algal biofuels, Appl. Energy, 88(10), 3548-3555, 2011.

[3] L. Soh, J. Zimmerman, Biodiesel production: the potential of algal lipids extracted with supercritical carbon dioxide, Green Chem., 13(6), 1422-1429, 2011.

[4] C. Crampon, G. Charbit, E. Neau, High-pressure apparatus for phase equilibria studies: solubility of fatty acid esters in supercritical $\mathrm{CO}_{2}$, J. Supercrit. Fluids, 16(1), 11-20, 1999.

[5] W.J. Bjornsson, K.M. MacDougall, J.E. Melanson, S.J.B. O'Leary, P.J. McGinn, Pilot-scale supercritical carbon dioxide extractions for the recovery of triacylglycerols from microalgae: a practical tool for algal biofuels research, J. Appl. Phycol., 24(3), 547-555, 2012.

[6] E. Reverchon, I. De Marco, Supercritical fluid extraction and fractionation of natural matter, J. Supercrit. Fluids, 38(2), 146-166, 2006.

[7] R. Halim, B. Gladman, M.K. Danquah, P.A. Webley, Oil extraction from microalgae for biodiesel production, Bioresour. Technol., 102(1), 178-185, 2011.

[8] A.E. Bailey, Y.H. Hui, Bailey's industrial oil and fat products: Industrial and consumer nonedible products from oils and fats, in, John Wiley \& Sons, Incorporated, 1996, pp. 397-423.

[9] E.G. Bligh, W.J. Dyer, A rapid method of total lipid extraction and purification, Can. J. Biochem., 37(8), 911-917, 1959.

[10] J.B. Guckert, K.E. Cooksey, L.L. Jackson, Lipid sovent systems are not equivalent for analysis of lipid classes in the microeukaryotic green alga, Chlorella, J. Microbiol. Methods, 8(3), 139-149, 1988.

[11] S.A. Channiwala, P.P. Parikh, A unified correlation for estimating HHV of solid, liquid and gaseous fuels, Fuel, 81(8), 1051-1063, 2002.

[12] S. Archanaa, S. Moise, G.K. Suraishkumar, Chlorophyll interference in microalgal lipid quantification through the Bligh and Dyer method, Biomass Bioenergy, 46(0), 805-808, 2012.

[13] C. Samori, D. Lopez Barreiro, R. Vet, L. Pezzolesi, D.W.F. Brilman, P. Galletti, E. Tagliavini, Effective lipid extraction from algae cultures using switchable solvents, Green Chem., 15(2), 353-356, 2013.

[14] M. Dubois, K.A. Gilles, J.K. Hamilton, P.A. Rebers, F. Smith, Colorimetric method for determination of sugars and related substances, Anal. Chem., 28(3), 350-356, 1956.

[15] O.H. Lowry, N.J. Rosebrough, A.L. Farr, R.J. Randall, Protein measurement with the Folin phenol reagent, J. Biol. Chem., 193(1), 265-275, 1951.

[16] W. Boie, Fuel technology calculations, Energietechnik, 3, 309-316, 1953.

[17] J. Chrastil, Solubility of solids and liquids in supercritical gases, J. Phys. Chem., 86(15), 3016-3021, 1982.

[18] Ö. Güçlü-Üstündağ, F. Temelli, Correlating the solubility behavior of minor lipid components in supercritical carbon dioxide, J. Supercrit. Fluids, 31(3), 235-253, 2004.

[19] C.S. Lim, Z.A. Manan, M.R. Sarmidi, Simulation modeling of the phase behavior of palm oilsupercritical carbon dioxide, J. Am. Oil Chem. Soc., 80(11), 1147-1156, 2003.

[20] I. Gracia, M.T. García, J.F. Rodríguez, M.P. Fernández, A. de Lucas, Modelling of the phase behaviour for vegetable oils at supercritical conditions, J. Supercrit. Fluids, 48(3), 189-194, 2009.

[21] NIST webbook, http://webbook.nist.gov/chemistry/, (accessed 13-4-2013). 
[22] L. Xu, D.W.F. Brilman, J.A.M. Withag, G. Brem, S. Kersten, Assessment of a dry and a wet route for the production of biofuels from microalgae: Energy balance analysis, Bioresour. Technol., 102(8), 5113$5122,2011$.

[23] Evodos, http://www.evodos.eu/, (accessed 13-4-2013).

[24] A.J. Dassey, C.S. Theegala, Harvesting economics and strategies using centrifugation for cost effective separation of microalgae cells for biodiesel applications, Bioresour. Technol., 128(0), 241-245, 2013.

[25] M. Peters, K. Timmerhaus, R. West, Plant design and economics for chemical engineers, McGraw-Hill Education, 2002.

[26] R.K. Sinnott, G. Towler, Chemical engineering design: SI edition, Elsevier Science, 2009.

[27] P. Kumar, M.R. Suseela, K. Toppo, Physico-chemical characterization of algal oil: a potential biofuel, Asian J. Exp. Biol. Sci., 2(3), 493-497, 2011.

[28] E. Reverchon, M. Poletto, Mathematical modelling of supercritical $\mathrm{CO}_{2}$ fractionation of flower concretes, Chem. Eng. Sci., 51(15), 3741-3753, 1996.

[29] H.J. Berchmans, S. Hirata, Biodiesel production from crude Jatropha curcas L. seed oil with a high content of free fatty acids, Bioresour. Technol., 99(6), 1716-1721, 2008.

[30] V.B. Veljković, S.H. Lakićević, O.S. Stamenković, Z.B. Todorović, M.L. Lazić, Biodiesel production from tobacco (Nicotiana tabacum L.) seed oil with a high content of free fatty acids, Fuel, 85(17-18), 26712675, 2006.

[31] S. Koritala, Selective hydrogenation of soybean oil: VII. Poisons and inhibitors for copper catalysts, J. Am. Oil Chem. Soc., 52(7), 240-243, 1975.

[32] G. Andrich, U. Nesti, F. Venturi, A. Zinnai, R. Fiorentini, Supercritical fluid extraction of bioactive lipids from the microalga Nannochloropsis sp, Eur. J. Lipid Sci. Technol., 107(6), 381-386, 2005.

[33] H. Sovova, M. Zarevucka, M. Vacek, K. Stransky, Solubility of two vegetable oils in supercritical $\mathrm{CO}_{2}$, J. Supercrit. Fluids, 20(1), 15-28, 2001.

[34] T. Walker, H. Cochran, G. Hulbert, Supercritical carbon dioxide extraction of lipids from Pythium irregulare, J. Am. Oil Chem. Soc., 76(5), 595-602, 1999.

[35] Y.-H. Chen, T.H. Walker, Fed-batch fermentation and supercritical fluid extraction of heterotrophic microalgal Chlorella protothecoides lipids, Bioresour. Technol., 114(0), 512-517, 2012.

[36] S. Pinzi, I.L. Garcia, F.J. Lopez Gimenez, M.D. Luque de Castro, G. Dorado, M.P. Dorado, The ideal vegetable oil-based biodiesel composition: A review of social, economical and technical Implications, Energy Fuels, 23(5), 2325-2341, 2009.

[37] Z.-R. Yu, B. Singh, S.S.H. Rizvi, J.A. Zollweg, Solubilities of fatty acids, fatty acid esters, triglycerides, and fats and oils in supercritical carbon dioxide, J. Supercrit. Fluids, 7(1), 51-59, 1994.

[38] V.K. Mishra, F. Temelli, B. Ooraikul, Extraction and purification of $\omega-3$ fatty acids with an emphasis on supercritical fluid extraction-A review, Food Res. Int., 26(3), 217-226, 1993.

[39] P.C.K. Cheung, Temperature and pressure effects on supercritical carbon dioxide extraction of n-3 fatty acids from red seaweed, Food Chem., 65(3), 399-403, 1999.

[40] K.J. Choi, Z. Nakhost, V.J. Krukonis, M. Karel, Supercritical fluid extraction and characterization of lipids from algae scenedesmus obliquus, Food Biotechnol., 1(2), 263-281, 1987.

[41] R.L. Mendes, B.P. Nobre, M.T. Cardoso, A.P. Pereira, A.F. Palavra, Supercritical carbon dioxide extraction of compounds with pharmaceutical importance from microalgae, Inorg. Chim. Acta, 356(0), 328334, 2003. 
[42] R.L. Mendes, H.L. Fernandes, J.A.P. Coelho, J.M.S. Cabral, A.M.F. Palavra, J.M. Novais, Supercritical carbon dioxide extraction of hydrocarbons from the microalga Botryococcus braunii, J. Appl. Phycol., 6(3), 289-293, 1994.

[43] S. Hansen, M. Myers, W. Artz, Nonvolatile components produced in triolein during deep-fat frying, J. Am. Oil Chem. Soc., 71(11), 1239-1243, 1994.

[44] S. Hansen, W. Artz, Supercritical fluid fractionation of thermally oxidized canola oil, J. Am. Oil Chem. Soc., 71(6), 615-618, 1994.

[45] E. Hoekstra, S.R.A. Kersten, A. Tudos, D. Meier, K.J.A. Hogendoorn, Possibilities and pitfalls in analyzing (upgraded) pyrolysis oil by size exclusion chromatography (SEC), J. Anal. Appl. Pyrolysis, 91(1), 76-88, 2011.

[46] P.C.K. Cheung, A.Y.H. Leung, P.O. Ang, Comparison of supercritical carbon dioxide and soxhlet extraction of lipids from a brown seaweed, sargassum hemiphyllum (Turn.) C. Ag.†, J. Agric. Food. Chem., 46(10), 4228-4232, 1998.

[47] J. Castaneda Acosta, A.W. Cain, N.H. Fischer, F.C. Knopf, Extraction of bioactive sesquiterpene lactones from magnolia grandiflora using supercritical carbon dioxide and near-critical propane, J. Agric. Food. Chem., 43(1), 63-68, 1995.

[48] B.M.C. Soares, F.M.C. Gamarra, L.C. Paviani, L.A.G. Gonçalves, F.A. Cabral, Solubility of triacylglycerols in supercritical carbon dioxide, J. Supercrit. Fluids, 43(1), 25-31, 2007.

[49] M. Bravi, R. Bubbico, F. Manna, N. Verdone, Process optimisation in sunflower oil extraction by supercritical CO2, Chem. Eng. Sci., 57(14), 2753-2764, 2002.

[50] E. Stahl, W. Schilz, E. Schütz, E. Willing, A quick method for the microanalytical evaluation of the dissolving power of supercritical gases, Angew. Chem., Int. Ed. Engl., 17(10), 731-738, 1978.

[51] R.L. Mendes, H.L. Fernandes, J. Coelho, E.C. Reis, J.M.S. Cabral, J.M. Novais, A.F. Palavra, Supercritical $\mathrm{CO}_{2}$ extraction of carotenoids and other lipids from Chlorella vulgaris, Food Chem., 53(1), 99$103,1995$.

[52] N.T. Dunford, F. Temelli, Extraction conditions and moisture content of canola flakes as related to lipid composition of supercritical $\mathrm{CO}_{2}$ extracts, J. Food Sci., 62(1), 155-159, 1997.

[53] S.M. Pourmortazavi, S.S. Hajimirsadeghi, Supercritical fluid extraction in plant essential and volatile oil analysis, J. Chromatogr. A, 1163(1-2), 2-24, 2007.

[54] G. Leeke, F. Gaspar, R. Santos, Influence of water on the extraction of essential oils from a model herb using supercritical carbon dioxide, Ind. Eng. Chem. Res., 41(8), 2033-2039, 2002.

[55] S.J. Lehotay, Supercritical fluid extraction of pesticides in foods, J. Chromatogr. A, 785(1-2), 289-312, 1997.

[56] G. Brunner, Gas extraction: an introduction to fundamentals of supercritical fluids and the application to separation processes, Steinkopff, 1994.

[57] R. Davis, A. Aden, P.T. Pienkos, Techno-economic analysis of autotrophic microalgae for fuel production, Appl. Energy, 88(10), 3524-3531, 2011. 


\section{Appendix C}

Data and assumptions used for the costs calculations 


\section{C.1 Utility costs and general equipment assumptions}

Utility costs are summarized below in Table C.1. ${ }^{1}$

Table C.1. Utility costs

\begin{tabular}{ccc}
\hline Utility & Description & Cost - 2003 (\$/GJ) \\
\hline Electricity & & 16.8 \\
Cooling water & $\mathrm{T}=20^{\circ} \mathrm{C}$ & 0.35 \\
Residual process heat & $\mathrm{T}=90^{\circ} \mathrm{C}$ & 0.35 \\
Low pressure steam (5 barg) & $\mathrm{T}=160^{\circ} \mathrm{C}$ & 6.08 \\
High pressure steam (41 barg) & $\mathrm{T}=254^{\circ} \mathrm{C}$ & 9.83 \\
Chilled water & $\mathrm{T}=5^{\circ} \mathrm{C}$ & 4.43 \\
Refrigerant & $\mathrm{T}=-20^{\circ} \mathrm{C}$ & 7.89
\end{tabular}

For cooling to $30^{\circ} \mathrm{C}$ and higher, cooling water was used while chilled water was used when cooling down to $15-30^{\circ} \mathrm{C}$. Refrigerant was used for cooling to lower temperatures (i.e. when evaluating condensation at $10^{\circ} \mathrm{C}$ ). For heating it was assumed that heat from elsewhere in the process can be recovered which could be used for heating up to $50^{\circ} \mathrm{C}$. The pre-heater was always utilized with steam for efficient heating. Heating up for all other cases was conducted with low pressure steam, with exception of heating up above $150^{\circ} \mathrm{C}$ (when required to evaluate the different extraction and separation possibilities) which was done by high pressure steam. Values given in Table C.1 are valid for the 2003. The costs were corrected for the yearly inflation for the final process design.

Assumptions related to equipment are shown in Table C.2.

Table C.2. General equipment assumptions

\begin{tabular}{ccc}
\hline Constant & Value & Unit \\
\hline Lifetime & 10 & years \\
Operating days & 350 & days \\
Stainless steel cost factor $^{\mathrm{a}}$ & 3.0 & - \\
Installation factor: pressure vessels $^{\mathrm{b}}$ & 4.0 & - \\
Installation factor: heat exchangers $^{\mathrm{b}}$ & 3.5 & - \\
Pump adiabatic efficiency & 75 & $\%$ \\
\hline
\end{tabular}

a Correction factor required as the equation to calculate the metal price is for carbon steel. Factor taken from ${ }^{2}$. b Taken from $^{3}$. 


\section{C.2 Pressure vessels calculations}

The design procedure for pressure vessels given by Peters et al. ${ }^{2}$ was followed. The pressure vessel minimum wall thickness ( $t$, in $\mathrm{m}$ ) was calculated by equations C.1 and C.2. The assumptions used for that are listed in Table C.3.

$$
\begin{array}{ll}
\mathrm{t}=\frac{\mathrm{P} \cdot \mathrm{r}_{\mathrm{i}}}{\mathrm{S} \cdot \mathrm{E}_{\mathrm{j}}-0.6 \cdot \mathrm{P}}+\mathrm{C}_{\mathrm{c}} & \mathrm{P} \leq 0.385 \cdot \mathrm{S} \cdot \mathrm{E}_{\mathrm{j}} \\
\mathrm{t}=\mathrm{r}_{\mathrm{i}} \cdot\left(\frac{\mathrm{S} \cdot \mathrm{E}_{\mathrm{j}}+\mathrm{P}}{\mathrm{S} \cdot \mathrm{E}_{\mathrm{j}}-\mathrm{P}}\right)^{\frac{1}{2}}-\mathrm{r}_{\mathrm{i}}+\mathrm{C}_{\mathrm{c}} & \mathrm{P}>0.385 \cdot \mathrm{S} \cdot \mathrm{E}_{\mathrm{j}}
\end{array}
$$

With,

$\begin{array}{ll}P & \text { Pressure }(\mathrm{kPa}) \\ r_{i} & \text { Vessel inside radius }(\mathrm{m}) \\ S & \text { Maximum allowable working stress }(\mathrm{kPa}) \\ E_{j} & \text { Joint efficiency, assumed to be } 1.0 \text { (fully radiographed) } \\ C_{c} & \text { Corrosion allowance }(\mathrm{m})\end{array}$

Table C.3. Pressure vessel assumptions

\begin{tabular}{ccc}
\hline Constant & Value & Unit \\
\hline Steel density & 7833 & $\mathrm{~kg} / \mathrm{m}^{3}$ \\
Maximum allowable working stress & 100000 & $\mathrm{kPa}$ \\
Corrosion allowance & 3 & $\mathrm{~mm}$ \\
Joint efficiency & 1 & - \\
\hline
\end{tabular}

The maximum allowable working stress is dependent on the temperature, and was assumed to be $100.000 \mathrm{kPa}$ for all temperatures during extraction and separation. ${ }^{2}$ The joint efficiency depends on the type of welding, if the joints are fully examined radiographed the efficiency can be assumed to be 1 . The corrosion allowance for 10 year lifetime is assumed to be $3 \mathrm{~mm}^{2}$

With the thickness, the inside radius and the total radius of the vessel can be determined. With that, the total mass of the vessel $\left(\mathrm{m}_{\text {vessel }}\right)$ was calculated $\mathrm{as}^{2}$ : 


$$
\begin{aligned}
& \mathrm{m}_{\text {vessel }}=\mathrm{m}_{\text {cylinder }}+\mathrm{m}_{\text {heads }} \\
& \mathrm{m}_{\text {cylinder }}=\left(\pi \cdot \mathrm{R}_{0}^{2}-\pi \cdot \mathrm{r}_{\mathrm{i}}^{2}\right) \cdot \mathrm{L} \cdot \rho_{\text {steel }} \\
& \mathrm{m}_{\text {heads }}=2 \cdot\left(2 \cdot \pi \cdot \mathrm{r}_{\mathrm{i}}^{2} \cdot t\right) \cdot \rho_{\text {steel }}
\end{aligned}
$$

With,

$$
\begin{array}{ll}
m_{\text {cylinder }} & \text { mass of cylinder }(\mathrm{kg}) \\
m_{\text {heads }} & \text { mass of top and bottom heads closing the cylinder }(\mathrm{kg}) \\
R_{0} & \text { Total radius being }\left(t+r_{i}, \text { in } \mathrm{m}\right) \\
\rho_{\text {steel }} & \text { Density of steel being (in Table C.3) } \\
L & \text { Length of the vessel (m) }
\end{array}
$$

With the total mass of the vessel (in $\mathrm{kg}$ ), the costs for pressure vessels were calculated. Cost for pressure vessels in January 2002 (including nozzles, manholes, saddles or skirts, but no internals such as trays or agitators) as dollars per kilogram weight of the fabricated unit f.o.b. with carbon steel as the cost basis is given by:

$$
\text { Cost }=73 \cdot\left(\mathrm{m}_{\mathrm{vessel}}\right)^{-0.34}
$$

This relation is applicable over a weight range from 400 to $50,000 \mathrm{~kg}$. the cost factor to convert from carbon Stainless Steel 304 is listed in Table C.2.

\section{C.3 Heat-transfer equipment}

Purchased costs for several areas were taken from data for a multi-pipe heat exchanger proposed by Peters et al. ${ }^{2}$ Those values were plotted resulting in a linear fit with the following equation:

$\mathrm{HT}_{\text {cost }}=200 \cdot \mathrm{A}$

With $H T_{\text {cost }}$ being the purchased cost for heat-transfer equipment (in $\$$ ) and $A$ the heattransfer area $\left(\right.$ in $\mathrm{m}^{2}$ ). The values used to obtain Eq. C.7 were valid up to $4 \mathrm{MPa}$ of pressure. Therefore, the obtained results had to be corrected as higher pressures than 4 $\mathrm{MPa}$ were used (both for extraction and separation) in our study. From data provided 
Peters et al., ${ }^{2}$ an approximate correction factor of 3 was found for the high extraction pressures evaluated in this work. That factor was assumed to be a proper first estimation.

\section{C.4 Process outline and stream properties}

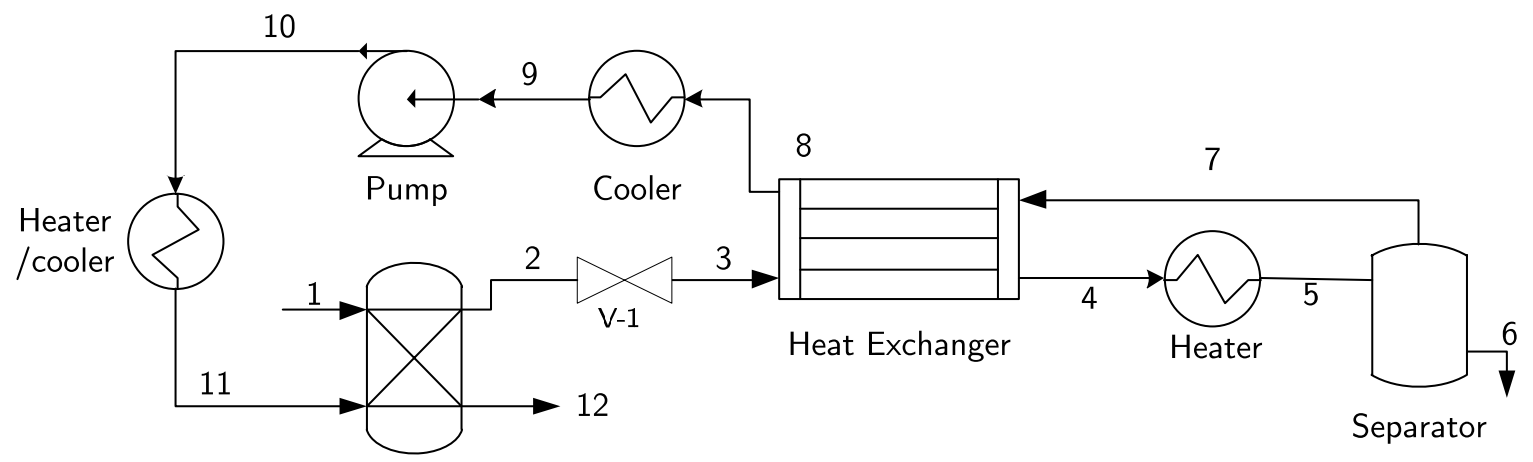

Extraction Vessel

Figure C.1. Process outline

Table C.4. Stream properties of the process depicted in Figure C.1

\begin{tabular}{|c|c|c|c|c|c|c|c|c|c|c|c|c|}
\hline \multicolumn{13}{|c|}{ Dry Extraction } \\
\hline Stream \# & 1 & 2 & 3 & 4 & 5 & 6 & 7 & 8 & 9 & 10 & 11 & 12 \\
\hline Phase & $\mathrm{SC}^{\mathrm{a}} / \mathrm{L}^{\mathrm{b}}$ & $\mathrm{SC}$ & $\mathrm{SC}+\mathrm{L}$ & $\mathrm{SC}+\mathrm{L}$ & $\mathrm{SC}+\mathrm{L}$ & $\mathrm{L}$ & $\mathrm{SC}$ & $\mathrm{SC}$ & $\mathrm{L} / \mathrm{SC}$ & $\mathrm{SC}$ & $\mathrm{SC}$ & $\mathrm{SC} / \mathrm{L}$ \\
\hline Temperature $\left({ }^{\circ} \mathrm{C}\right)$ & 50 & 50 & 46 & 70 & 100 & 100 & 100 & 76 & 30 & 41.3 & 50 & 50 \\
\hline Pressure (MPa) & 35 & 35 & 25 & 25 & 25 & 25 & 25 & 25 & 25 & 35 & 35 & 35 \\
\hline \multicolumn{13}{|c|}{ Wet extraction } \\
\hline Stream \# & 1 & 2 & 3 & 4 & 5 & 6 & 7 & 8 & 9 & 10 & 11 & 12 \\
\hline Phase & $\mathrm{SC} / \mathrm{L}$ & $\mathrm{SC}$ & $\mathrm{SC}+\mathrm{L}$ & $\mathrm{SC}+\mathrm{L}$ & $\mathrm{SC}+\mathrm{L}$ & $\mathrm{L}$ & $\mathrm{SC}$ & $\mathrm{SC}$ & $\mathrm{L} / \mathrm{SC}$ & $\mathrm{SC}$ & $\mathrm{SC}$ & $\mathrm{SC} / \mathrm{L}$ \\
\hline Temperature $\left({ }^{\circ} \mathrm{C}\right)$ & 80 & 80 & 72.4 & 90 & 100 & 100 & 100 & 82 & 30 & 41.3 & 80 & 80 \\
\hline Pressure (MPa) & 35 & 35 & 25 & 25 & 25 & 25 & 25 & 25 & 25 & 35 & 35 & 35 \\
\hline
\end{tabular}

${ }^{\text {a }}$ Supercritical. ${ }^{\text {b Liquid, }}$

\section{Bibliography}

[1] R. Turton, R.C. Bailie, W.B. Whiting, J.A. Shaeiwitz, D. Bhattacharyya, Analysis, synthesis and design of chemical processes, Prentice Hall PTR, 2012.

[2] M. Peters, K. Timmerhaus, R. West, Plant design and economics for chemical engineers, McGraw-Hill Education, 2002.

[3] G.P. Towler, R.K. Sinnott, Chemical engineering design: Principles, practice, and economics of plant and process design, Elsevier/Butterworth-Heinemann, 2012. 


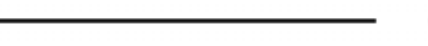

rar 


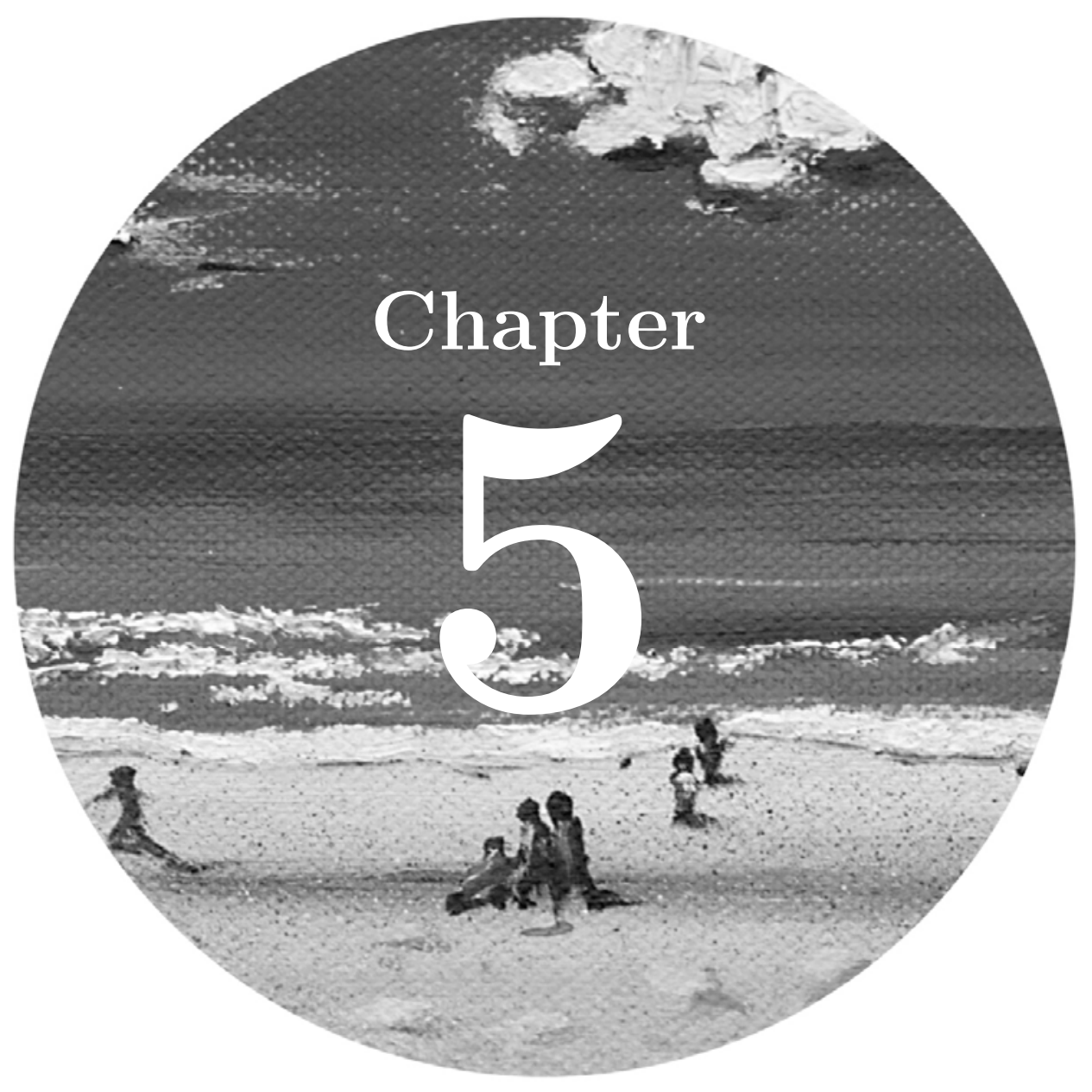

Microalgae growth on the aqueous phase from hydrothermal liquefaction of the same microalgae 


\begin{abstract}
Cultivation of Desmodesmus sp. microalgae in the recycled aqueous phase (AP) recovered after Hydrothermal Liquefaction (HTL) of the same microalgae was studied to evaluate the potential of nutrients recycling. The AP dilution ratio was systematically varied, using either water or water enriched with standard medium, while keeping the same $\mathrm{N}$ concentration (either as total- $\mathrm{N}$ or as sum of ammonia and nitrate) as that in the standard medium. More than $90 \%$ of the organic compounds in the AP were identified and quantified and potential growth inhibiting substances (e.g. phenols) were found. However, the combination of growth and analytical results showed that the lack of (macro-/micro)nutrients, other than $\mathrm{N}$ and $\mathrm{P}$, in the $\mathrm{AP}$ is the main cause of growth reduction rather than toxicity due to insufficient AP dilution, as pointed in previous investigations. Therefore, these (macro-/micro-)nutrients such as Mg should be supplied upon AP recycling. For this specific cultivation case, algae production costs related to nutrients consumption can be significantly reduced considering that a $50 \%$ N-replacement was achieved, while showing nearly identical growth as that in standard culture medium.
\end{abstract}




\section{Introduction}

Algae are the fastest growing photosynthetic organisms on earth and therefore an interesting resource for energy, as they are capable to store sunlight in the form of energy-rich organic compounds and do not directly compete for arable land. The productivity of algae could be 50 times higher than that of switchgrass, one of the fastest growing terrestrial plants. ${ }^{1}$ However, the current estimated costs for algae cultivation are significantly higher; 5-10 $\$ / \mathrm{kg}^{2}$ vs. $0.025-0.1 \$ / \mathrm{kg}^{3}$ for switchgrass. Moreover, the energy requirements associated with the production of nitrogen nutrients (e.g. nitrate) represent a significant part of the total energy inputs, comparable to the electrical requirements. ${ }^{4}$ Also phosphorus, another essential nutrient, is a non-renewable resource and current global reserves may be depleted in 50-100 years. ${ }^{5}$ In our research we aim to alleviate these issues for algae biorefinery concepts by recycling of these nutrients, from downstream processing back to the algae cultivation section.

In an algae biorefinery scheme, several extraction and conversion processes could be combined to co-produce high value-added products, feed/food ingredients and energy carrier products from microalgae. A promising conversion method hereto is Hydrothermal Liquefaction (HTL) to produce an algal bio-crude oil. This process circumvents the costs of energy intensive drying for complete dewatering. Several studies into the hydrothermal conversion of lignocellulosic biomass and algae have been performed in the past. ${ }^{6-8}$

The aqueous phase (AP) obtained from HTL of microalgae contains $\mathrm{N}$ and $\mathrm{P}$ constituents that, ideally, can be recycled, aiming to reduce cultivation costs and energy production costs induced by the continuous use of fresh make-up nutrients. Furthermore, the AP contains several oxygenated hydrocarbons that may be assimilated when a heterotrophic/mixotrophic algal strain is used. In previous work (Chapter 2) we showed that the molecular and elemental composition of the AP can significantly vary, in particular with HTL operating temperature. The largest N sources in the aqueous HTL product were always organic N-containing compounds and ammonia. Moreover, as shown in the work by Biller et al., ${ }^{9}$ AP composition can also vary depending on the algal strain used.

The desirable situation within a biorefinery framework would be performing HTL of algae (-debris) under the adequate process conditions in which all the $\mathrm{N}$ in the algae is transferred to the AP in the form of $\mathrm{NH}_{3} / \mathrm{NH}_{4}{ }^{+}$, while still producing enough crude biooil. In developing such a concept, the algal strain selected for growth might be of importance, as it must e.g. be able to persist within a medium containing organic 
constituents from recycled HTL process water and preferably assimilate all the nutrients offered via this HTL aqueous phase recycling.

This idea of nutrients recycling was first proposed and tested in a pioneering investigation of Minowa and Sawayama. ${ }^{10}$ In their study, Chlorella vulgaris was successfully cultivated (comparable to that in standard medium) in the recycled AP from catalytic gasification of the same algae when this one was diluted in nitrogen-less standard medium. Recently, Jena et al. ${ }^{11}$ and Biller et al. ${ }^{9}$ tested similarly the ability to grow algae in diluted aqueous phase from HTL. Both stressed the importance of AP dilution in view of inhibitory effects of potential toxic compounds at high concentrations. Biller et al. ${ }^{9}$ stated that heavy dilution of AP is required to avoid growth inhibition. Both observed that the algae in water diluted AP could not grow as fast as that in standard culture medium, even when sufficient amounts of $\mathrm{N}$ and $\mathrm{P}$ were present in the media. In this work, we also investigate the replacement/recycling of $\mathrm{N}$ and $\mathrm{P}$ containing nutrients in the growth medium. However, this was done by following a different approach. In our study we used mixtures of standard culture medium, AP and demineralized water with the same nitrogen concentration (either as total $\mathrm{N}$ or the sum of ammonia and nitrate) as that in standard growth medium. Consequently, the main differences between the tested media in this work were the concentrations of (macro-/micro-)nutrients and potentially toxic organic compounds.

\section{Experimental}

\subsection{Algal feedstock}

The freshwater microalgae used, Desmodesmus sp., was provided by Ingrepro B.V. (The Netherlands), where industrial cultivation is performed in raceway ponds and at high $\mathrm{pH}$ to maintain monoculture conditions. The batch obtained was centrifuged and maintained in the dark, cold and in COMBO $(C B)$ growth medium ${ }^{12}$ from which $\mathrm{KCl}$, $\mathrm{Na}_{2} \mathrm{SiO}_{3} \bullet 9 \mathrm{H}_{2} \mathrm{O}$, animal trace elements and later $\mathrm{NaHCO}_{3}$ (since $\mathrm{CO}_{2}$ gas was used as carbon source) were excluded. The constructed growth setup was inoculated with that maintained culture. The proximate and ultimate analyses and biochemical composition of this type of microalgae can be found in Chapter 2 . 


\subsection{Hydrothermal Liquefaction (HTL) and aqueous phase (AP) recovery}

From a batch of concentrated Desmodesmus sp. solution (7.66 wt\% of algae dry ash free), several batch HTL tests (using 20 g of feedstock each time) were performed using a 45 $\mathrm{ml}$ stainless steel autoclave, heated by immersion in a hot fluidized sand bed. These experiments were carried out with an inert atmosphere at $300^{\circ} \mathrm{C}$ for 5 min reaction time (excluding 6-7 minutes of heating time). After these $5 \mathrm{~min}$ of reaction time, fast quenching ( 1-2 min) was performed by inserting the autoclave in a water bath. After gas analysis, a subsequent product collection and separation procedure was carried out to obtain the other three products: oil (crude from dichloromethane soluble fraction), water soluble organics (aqueous phase AP), and solid residue. A detailed explanation of the setup, products separation procedures and typical mass balance closure can be found in Chapter 2. In the present study, however, additional detailed analyses on the obtained aqueous phase fractions were performed.

Nutrients concentration, $N i$ and COD (in $\mathrm{mg} / \mathrm{L}$ ): These compounds were quantified with standardized tests (HACH LANGE) and measured with a spectrophotometer (DR 5000, HACH Corporation). The compounds quantified were: total nitrogen (TN, LCK 238), nitrate $\left(\mathrm{NO}_{3}-\mathrm{N}\right.$, LCK 339), ammonia $\left(\mathrm{NH}_{4}-\mathrm{N}\right.$, LCK 305), phosphate ( $\left.\mathrm{PO}_{4}-\mathrm{P}, \mathrm{LCK} 349\right)$, $\mathrm{Ni}$ (LCK 337), and COD (chemical oxygen demand, LCK 414). Organic nitrogen (org-N) was calculated as: $\mathrm{TN}-\left(\mathrm{NO}_{3}-\mathrm{N}\right)-\left(\mathrm{NH}_{4}-\mathrm{N}\right)-\left(\mathrm{NO}_{2}-\mathrm{N}\right)$. Nitrite was below the detection limit for all the aqueous phases analyzed. In the $\mathrm{NH}_{4} \mathrm{~N}$ test, the total nitrogen coming from both $\mathrm{NH}_{3}$ and $\mathrm{NH}_{4}{ }^{+}$, which coexist in equilibrium depending on $\mathrm{pH}$, is determined.

Total Organic Carbon, TOC (in $\mathrm{mg} / \mathrm{L}$ ), was measured with a Shimadzu TOC/TIC analyzer.

$\mathrm{Na}, \mathrm{K}, \mathrm{Ca}, \mathrm{S}$ and $\mathrm{Mg}$ nutrients were determined by means of inductively coupled plasma optical emission spectroscopy (ICP-OES, Varian Vista MPX) and $C l$ by ion chromatography (IC, Dionex ICS 2100). Analyses were performed in duplicate.

AP organic compounds: Analyses of volatile compounds (acetic acid, ethanol and acetone) in $\mathrm{AP}$ were performed by acidifying the medium to $\mathrm{pH}=2$ with potassium bisulfate prior to $10 \mathrm{~min}$ SPME (Solid Phase Microextraction, polyacrilate coating) immersion sampling and direct GC-MS (equipped with MDN- 5S) and GC-FID (equipped with NUKOL ${ }^{\mathrm{TM}}$ capillary column) for acetic acid analysis. Quantitative analyses were done by calibration with solutions of known concentrations of pure 
standards. The non-volatile fraction of water soluble organics was quantified by evaporation of water, derivatization and GC-MS analysis. Derivatization and analysis were performed following a published procedure ${ }^{13}$ which was slightly modified in order to obtain complete silylation of cyclic dipeptides and polyhydroxylated compounds.

\subsection{Cultivation system}

A batch growth setup was designed, constructed and calibrated (Figure 1). The total reactor volume was distributed over 10 glass reactors (1 to 10 in Figure 1(a)), each having a volume of 2 L. Fluorescent artificial lighting (Philips MASTER TL-D Graphica 58W/9651SL) was used as light source in continuous mode. Light intensities and wavelength spectra inside the reactor compartment and individual reactors were determined using a USB4000 spectrophotometer (Ocean Optics). As a significant drop in received light was measured for reactors 1 and 10, located at both ends of the reactor compartment (see Figure 1(a)), these two reactors were not used.
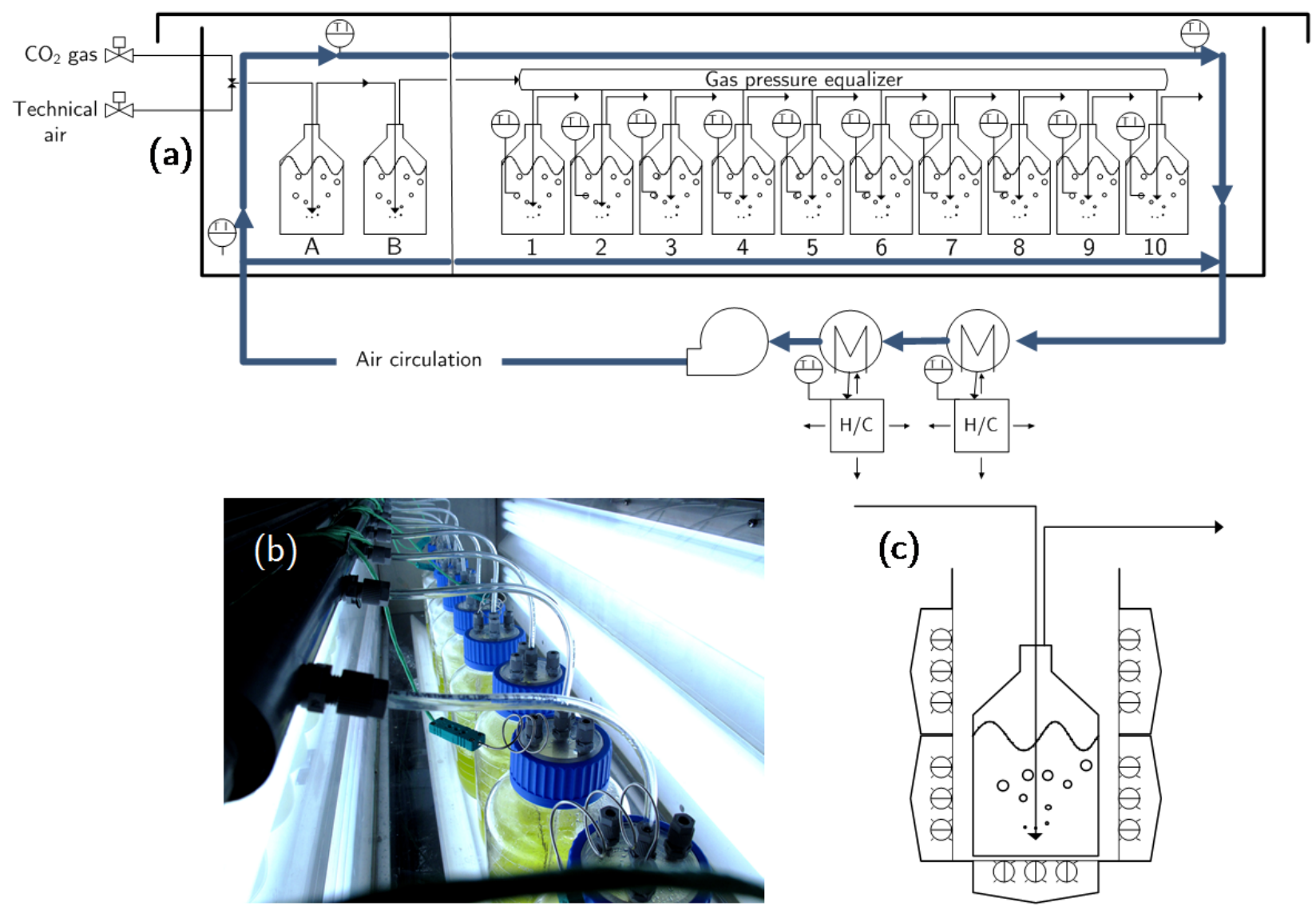

Figure 1. (a) Growth setup scheme, (b) Inoculated individual reactors and (c) lights configuration in the reactor compartment 
The temperature inside the reactor compartment was kept constant with max. \pm 1 difference throughout the reactor compartment by air circulation (blue arrow in Figure 1(a)). For mixing, oxygen stripping and carbon dioxide supply inside the reactors, aeration enriched with pure $\mathrm{CO}_{2}$ was used. This gas mixture was saturated with water through two saturators (In Figure 1(a)), vessels A, B containing demineralized water) to minimize evaporation losses. A gas pressure equalizer ensured equal inlet pressure for all connected reactors. After passing the reactors, the gas was emitted to the general compartment.

\subsection{Growth measurements and medium analyses}

Algae growth was followed by measuring optical density at $750 \mathrm{~nm}$ (OD750) with a spectrophotometer (DR 5000, HACH Corporation). The measured optical density was found to properly correlate with the algae dry weight content of the corresponding solution (after filtrating and drying at $105^{\circ} \mathrm{C}$ for $24 \mathrm{~h}$ ). Up to OD750 $=0.7$, a linear relationship was obtained with the following equation:

Algae concentration $(\mathrm{g} / \mathrm{L})=1.58 \cdot$ OD750

The constant parameter in Eq.1 (1.58) has a 95\% confidence interval of 1.56 to 1.61.

Specific growth rate $\left(\mathrm{h}^{-1}\right)$ of a microalgal population is determined from the exponential growth phase and automatic inline measurements of optical density can lead to a clear identification of this exponential phase. However, this becomes more difficult when taking OD750 measurements between intervals of 6-10h, as done in this work. Therefore, for our case, we chose total algae produced (in $\mathrm{g} / \mathrm{L}$ ) in tests of 68-96 $\mathrm{h}$ and maximum daily productivity as best indicators for comparing the growth between different culturing experiments. Maximum daily productivity found over the course of a growth test was defined as ' $\mathrm{P}_{\max }$ ' and calculated by a 3 point approximation of the maximum gradient.

Nutrients consumption was measured by determining the concentration of nutrients in the medium during growth using the same HACH LANGE tests used for aqueous phase analyses. For that, the samples were centrifuged ( $8000 \mathrm{rpm}, 5 \mathrm{~min})$ to separate the supernatant from the algal pellet. Nutrients analyses were performed on the recovered supernatant immediately after that separation.

A light intensity of $247 \mathrm{\mu mol} / \mathrm{m}^{2} / \mathrm{s}$ (measured at the reactor wall) was used for all growth experiments leading to an onset of light limitation at a concentration of about $0.3 \mathrm{~g} / \mathrm{L}$. 
That light limitation concentration was estimated first theoretically based on the BeerLambert law. Later, that algal concentration was reaffirmed by identifying the beginning of the linear region of the growth curves which generally occurs when a specific requirement such as light is limiting cell division. ${ }^{14}$ The initial algae concentration for all experiments was always lower to avoid light limitations.

\subsection{Algae adaptation}

The maintained fresh algae need a period of physiological adjustment due to changes in e.g. nutrients or culture conditions. ${ }^{14} \mathrm{~A}$ sample of maintained culture was centrifuged at $8000 \mathrm{rpm}$ for 5 min after which the cell pellet was collected, re-suspended and transferred to the individual growth reactors, each containing $1 \mathrm{~L}$ COMBO medium and aerated at $1.8 \mathrm{vvm}$ with an air- $\mathrm{CO}_{2}$ mixture containing $2 \mathrm{vol} \%$ of $\mathrm{CO}_{2}$ and showing an initial $\mathrm{pH}$ of 7.4. The culture was kept under this controlled environment inside the reactor compartment, where the temperature and light intensity were gradually increased up to a final value of 27.0 ( $\pm 1^{\circ} \mathrm{C}$ throughout the reactor compartment) and a light intensity of $247 \mathrm{mmol} / \mathrm{m}^{2} / \mathrm{s}$. Every day during this adaptation time, half of the culture volume was replaced by fresh medium to avoid light limited conditions due to high concentrations of algae. After a period of approximately 15 days, the algae seemed to have adapted to the new growth environment since a repeatable growth behavior was observed without fluctuations. By visual inspection using a microscope, the algal cells were the same as the initial feedstock without abnormal morphological characteristics. This algal batch was used as inoculum for the first growth test using aqueous phase (Section 2.6). Every time a new growth test was started, the algae were pre-adapted again (for at least 5 days diluting the culture every day), to the growth conditions as previously mentioned. Algae growth was not performed under axenic conditions, as in an envisaged algae biorefinery, outdoor cultivation under non-axenic conditions is preferable in order to reduce costs.

\subsection{Algae growth experiments}

The same inoculation method was used for all the growth experiments. In this method, the algae to be used as inoculum is first maintained and adapted to COMBO medium and the growth conditions mentioned in Section 2.5. After that, the culture (growing at exponential phase) is harvested and centrifuged at $8000 \mathrm{rpm}$ during $5 \mathrm{~min}$ after which the supernatant is removed. The cells pellet is used to inoculate the corresponding reactors until an initial algae concentration of $0.03-0.04 \mathrm{~g} / \mathrm{L}$. 
Three series of growth experiments were performed, with the only difference being the characteristics of the culture medium. Those differences can be seen in Table 1, where all the details concerning media preparation are shown.

The first growth experiment (expt. 1 in Table 1), used as control test, was performed in standard culture medium COMBO $(C B)$. Two reactors containing $1 \mathrm{~L}$ of the same medium were inoculated. The results of this test were used to compare with the growth experiments adding AP.

In the second growth experiment (expt. 2 in Table 1), performed as proof of concept, two reactors were inoculated containing a medium with a high concentration of AP. The AP was diluted in demineralized water for one reactor and in COMBO-medium for the other ( $W+A P \_20 x$ and $C B+A P \_20 x$ respectively). All the phosphorus in the aqueous phase was in the form of phosphate, at a concentration of $159 \mathrm{mg} / \mathrm{L}$. Therefore, since there was sufficient $\mathrm{P}$ supply via the aqueous phase, no extra $\mathrm{P}$ was added (see Table 1 phosphorous stock solution).

Finally, in the third growth experiment (expt. 3 in Table 1), the medium of all the reactors had the same initial nitrogen concentration (either as total-N including organic $\mathrm{N}$ or as $\mathrm{NO}_{3}-\mathrm{N}+\mathrm{NH}_{4}-\mathrm{N}$ ) and equal to that in the standard COMBO medium (approx. 13 $\mathrm{mg} / \mathrm{L}$ of $\mathrm{N}$ ). To reach that, different amounts of AP were added. For each growth configuration of experiment series no.3 two reactors were used (as duplo). Hence, each data point in the graphs presented represents the mean value between the results obtained from both reactors, along with their corresponding error bars. Four reactors contained only water-diluted aqueous phase ( $W+A P-1$ and $W+A P-2)$ and the other four supplemented with COMBO $(C B+W+A P-1$ and $C B+W+A P-2)$. Furthermore, in all media $\mathrm{P}$ was supplied to the same final concentration as in COMBO medium (by adding specific amounts of $\mathrm{P}$ stock solution, see Table 1), as the purpose of this test was to look at the influence of only the N-substances. 
Table 1. Media preparation of the various growth experiments

\begin{tabular}{|c|c|c|c|c|c|c|c|}
\hline \multirow{2}{*}{ Expt. } & \multirow{2}{*}{ Reference name } & \multicolumn{4}{|c|}{ wt\% of total medium ${ }^{a}$} & \multirow{2}{*}{$\begin{array}{l}\mathrm{AP} \\
\mathrm{DF}^{\mathrm{f}}\end{array}$} & \multirow{2}{*}{ Final $\mathbf{N}$ concentration in medium created from... } \\
\hline & & $\mathrm{CB}^{\mathrm{b}}$ & $\mathbf{W}^{\mathrm{c}}$ & $\mathbf{A} \mathbf{P}^{\mathrm{d}}$ & $\mathbf{P}^{\mathrm{e}}$ & & \\
\hline 1 & CB & 100 & 0.00 & 0.00 & 0.00 & - & $\left(\right.$ from $\left.\mathrm{NO}_{3}-\mathrm{N}\right)$ in $\mathrm{CB}$ \\
\hline \multirow[b]{2}{*}{2} & $\mathrm{~W}+\mathrm{AP} \_20 \mathrm{x}$ & 0.00 & 95.0 & 5.00 & 0.00 & 20 & $\left(\right.$ from $\mathrm{NO}_{3}-\mathrm{N}+\mathrm{NH}_{4}-\mathrm{N}+$ Org- $\left.\mathrm{N}^{g}\right)$ in only $\mathrm{AP}$ \\
\hline & $\mathrm{CB}+\mathrm{AP} \_20 \mathrm{x}$ & 95.0 & 0.00 & 5.00 & 0.00 & 20 & $\begin{array}{c}8 \% \mathrm{~N}\left(\text { from } \mathrm{NO}_{3}-\mathrm{N}\right) \text { in } \mathrm{CB}+92 \% \mathrm{~N}\left(\text { from } \mathrm{NO}_{3}-\mathrm{N}+\mathrm{NH}_{4}-\mathrm{N}+\text { Org- }\right) \\
\text { in } \mathrm{AP}\end{array}$ \\
\hline \multirow{4}{*}{$3^{\mathrm{i}}$} & $\mathrm{W}+\mathrm{AP}-1$ & 0.00 & 99.28 & 0.62 & 0.10 & 160 & $\left(\right.$ from $\left.\mathrm{NO}_{3}-\mathrm{N}+\mathrm{NH}_{4}-\mathrm{N}\right)$ in only $\mathrm{AP}$ \\
\hline & $\mathrm{W}+\mathrm{AP}-2$ & 0.00 & 99.58 & 0.32 & 0.10 & 311 & $\left(\right.$ from $\mathrm{NO}_{3}-\mathrm{N}+\mathrm{NH}_{4}-\mathrm{N}+$ Org- $\left.\mathrm{N}\right)$ in only $\mathrm{AP}$ \\
\hline & $\mathrm{CB}+\mathrm{W}+\mathrm{AP}-1$ & $50.0^{\mathrm{h}}$ & 49.64 & 0.31 & 0.05 & 320 & $50 \% \mathrm{~N}\left(\right.$ from $\left.\mathrm{NO}_{3}-\mathrm{N}\right)$ in $\mathrm{CB}+50 \% \mathrm{~N}\left(\right.$ from $\left.\mathrm{NO}_{3}-\mathrm{N}+\mathrm{NH}_{4}-\mathrm{N}\right)$ in $\mathrm{AP}$ \\
\hline & $\mathrm{CB}+\mathrm{W}+\mathrm{AP}-2$ & 50.0 & 49.79 & 0.16 & 0.05 & 623 & $\begin{array}{c}50 \% \mathrm{~N}\left(\text { from } \mathrm{NO}_{3}-\mathrm{N}\right) \text { in } \mathrm{CB}+50 \% \mathrm{~N}\left(\text { from } \mathrm{NO}_{3}-\mathrm{N}+\mathrm{NH}_{4}-\mathrm{N}+\text { Org- } \mathrm{N}\right) \\
\text { in } \mathrm{AP}\end{array}$ \\
\hline
\end{tabular}

${ }^{\text {a }}$ Total Amount of medium $=1$ L. ${ }^{\mathrm{b}}$ COMBO standard growth medium. ${ }^{\mathrm{c}}$ Demineralized water. ${ }^{\mathrm{d}}$ Aqueous phase recovered from HTL experiment. ${ }^{\mathrm{e}}$ Phosphorous stock solution. ${ }^{\mathrm{f}}$ DF: Dilution Factor. ${ }^{\mathrm{g}}$ Organic nitrogen. ${ }^{\mathrm{h}}$ As an example: $1 \mathrm{~L}$ medium with $500.0 \mathrm{ml} \mathrm{CB}, 496.4 \mathrm{ml} \mathrm{W}, 3.1 \mathrm{ml}$ AP and $0.5 \mathrm{ml} \mathrm{P} .{ }^{\mathrm{i}}$ Final target of nitrogen concentration being $13 \mathrm{mg} / \mathrm{L}$ for all the growth media of expt. 3 . 


\section{Results and discussion}

The AP fractions were produced by performing HTL experiments all under the same reaction conditions, $300^{\circ} \mathrm{C}$ and 5 min reaction time (excluding heating time). Similar results as those reported in Chapter 2 were obtained, including a mass balance closure of almost $100 \%$ and oil yield of $40 \mathrm{wt} \%$ with a $\mathrm{HHV}$ for the oil of $35 \mathrm{MJ} / \mathrm{kg}$. Further analyses performed on the oil, gas and residual solids fractions are not presented here, as they are very similar to that shown in Chapter 2 and since the focus of this work was on the usage of the aqueous phase for algae production.

\subsection{Proof of concept: algae growth using HTL-AP}

First, as a proof of concept, the non-optimized (i.e. regardless of the final nutrients concentration in the medium) growth of Desmodesmus sp. in a medium containing a significant fraction of AP obtained from HTL of the same microalgae was tested. The main objective was to test if the presence of a large amount of AP would lead to significant adverse effects on growth.

The initial concentrations of $\mathrm{NO}_{3}-\mathrm{N}$ and $\mathrm{NH}_{4}-\mathrm{N}$ for each medium as well as the amount of organic nitrogen can be seen in Table 2. The difference in $\mathrm{NO}_{3}-\mathrm{N}$ between the $W+A P \_20 x$ and $C B+A P \_20 x$ media is the amount of $\mathrm{NO}_{3}-\mathrm{N}$ coming from COMBO addition (12.4 mg from $\mathrm{CB}$ and $5.7 \mathrm{mg}$ from $\mathrm{AP}$ ). The same occurring for the phosphorous supply, having a higher initial concentration of $\mathrm{P}$ in the $C B+A P \_20 x$ medium due to the double contribution from the AP and from COMBO.

Table 2. Initial media composition, total algae produced and maximum daily productivity in standard COMBO $(\mathrm{CB})$ and in media with aqueous phase (AP) co-feeding 20x diluted

\begin{tabular}{|c|c|c|c|c|c|c|}
\hline \multirow{2}{*}{$\begin{array}{c}\text { Experiment } \\
\text { reference }^{a}\end{array}$} & \multicolumn{4}{|c|}{ Initial composition in medium $(\mathrm{mg} / \mathrm{L}$ of $\mathrm{N}$ and $\mathrm{P})$} & \multirow{2}{*}{$\begin{array}{l}\operatorname{Algae}^{b} \\
(g / L)\end{array}$} & \multirow{2}{*}{$\begin{array}{c}P_{\max }{ }^{c} \\
(\mathrm{~g} / \mathrm{L} / \mathrm{d}\end{array}$} \\
\hline & $\mathrm{NO}_{3-\mathrm{N}}$ & $\mathrm{NH}_{4}-\mathrm{N}$ & Org-N & $\mathrm{PO}_{4}-\mathrm{P}$ & & \\
\hline $\mathrm{CB}$ & 13.0 & 0.00 & 0.00 & 1.55 & $0.81^{\mathrm{d}}$ & 0.70 \\
\hline $\mathrm{W}+\mathrm{AP} \_20 \mathrm{x}$ & 5.70 & 47.8 & 91.7 & 8.00 & $0.21^{\mathrm{e}}$ & 0.14 \\
\hline $\mathrm{CB}+\mathrm{AP} \_20 \mathrm{x}$ & 18.1 & 47.8 & 91.7 & 9.40 & $0.74^{\mathrm{e}}$ & 0.62 \\
\hline
\end{tabular}

${ }^{a}$ For abbreviations refer to Table 1. ${ }^{\mathrm{b}}$ Total algae produced. Estimated error below 10\%. ${ }^{\mathrm{c}}$ Maximum daily

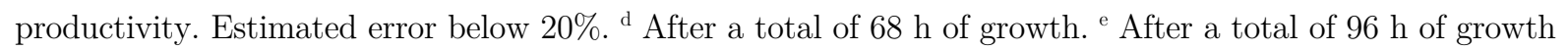

Also in Table 2, the total algae produced after $96 \mathrm{~h}$ of growth and the maximum daily productivity for the media with AP are shown and compared to those in standard COMBO medium (control test) after $68 \mathrm{~h}$ of growth. The growth with AP in COMBO 
was significantly faster than that with only AP in water. After 4 days of growth, a total of $0.74 \mathrm{~g} / \mathrm{L}$ of algae were produced, almost a factor four higher than that produced in $W+A P \_20 x$. At the same time, the growth results from $C B+A P \_20 x$ and $C B$ appeared comparable. In $C B+A P \_20 x$, a slightly longer lag phase was observed, thereby taking longer time to reach an algae concentration comparable to that in $C B$ after $68 \mathrm{~h}$ (see Table 2). However, their maximum daily productivities were in the same range. Therefore, the presence of a large amount of AP in COMBO is apparently not causing a major reduction in growth. The small difference could be related to the fact that the algae was, for the first time, in a medium with AP and at high concentration needing a longer time for adaptation. In $C B+A P \_20 x$ medium, the inoculum was transferred from one set of growth conditions to another (by adding AP) while the cells in COMBO were totally acclimated.

Growth could develop in the $W+A P \_20 x$ medium. However, it did not result in optimal algae growth showing a significantly lower productivity than that in COMBO. The few studies available, ${ }^{9-11}$ although having different strains; different growth reactor configurations; and different growth conditions, also showed a drop in growth rate when using only water-diluted AP. Biller et al. ${ }^{9}$ reported even no growth for Spirulina platensis in 50x diluted AP, while having a higher $\mathrm{N}$ concentration than that in standard medium. Jena et al. ${ }^{11}$ reported the same for Chlorella minutissima in a $10 \mathrm{x}$ water diluted AP with sufficient amounts of both $\mathrm{N}$ and $\mathrm{P}$.

In the section below, the possible explanations to the previous observations are discussed.

Ammonia is formed during HTL of microalgae. In the AP, free ammonia $\left(\mathrm{NH}_{3}\right)$ exists in equilibrium with ammonium $\left(\mathrm{NH}_{4}^{+}\right)$, depending on temperature and, especially, $\mathrm{pH}$. When the $\mathrm{pH}$ increases, the equilibrium shifts towards ammonia, which is the main toxic form for algae. ${ }^{15}$ In literature, it was demonstrated that the concentration at which $\mathrm{NH}_{3}$ inhibits the growth of a specific algal strain is fixed, and $\mathrm{pH}$ only affects how much $\mathrm{NH}_{3}$ is available via the degree of dissociation. ${ }^{15}$ The growth of Scenedesmus obliquus, an algal strain very similar to the one used in this work, was completely inhibited by $\mathrm{NH}_{3}$ concentrations above $2 \mathrm{mM} .{ }^{15}$ If, for the experiments shown in this section, all $\mathrm{N}$ coming from the equilibrium $\mathrm{NH}_{3} / \mathrm{NH}_{4}{ }^{+}$would be in the form of free $\mathrm{NH}_{3}$, its concentration would be $3.4 \mathrm{mM}$ which could be in the range of toxicity. However, both $W+A P \_20 x$ and $C B+A P \_20 x$ media had the same amount of initial $\mathrm{NH}_{4} \mathrm{~N}$ (total $\mathrm{N}$ in the form of $\mathrm{NH}_{3} / \mathrm{NH}_{4}{ }^{+}$) as they were equally diluted, and the initial $\mathrm{pH}$ of only $C B$ was similar to that of water. Therefore, ammonia toxicity occurring only in the $W+A P \_20 x$ medium is unlikely to have caused the lowered growth rate. 
Following the same reasoning, both media had the same concentrations of potential growth inhibitory compounds such as phenols, metals (e.g. Ni, from reactor wall corrosion) and fatty acids. In contrast with other studies reporting the importance of AP dilution $^{9,11}$ to avoid toxicity, this cultivation tests show that, at least for Desmodesmus algal strain, dilution is not likely to be the main limiting factor for growth.

Another explanation, alternative to the growth inhibition by poisoning, could be related to the nutrients ratio, rather than their absolute concentration. However, the $W+A P \_20 x$ and $C B+A P \_20 x$ media had comparable N:P ratio (e.g. 6.7 and 7.0 respectively when only counting for inorganic nitrogen $\mathrm{NO}_{3}-\mathrm{N}$ and $\mathrm{NH}_{4}-\mathrm{N}$ ), but were exhibiting large differences in growth.

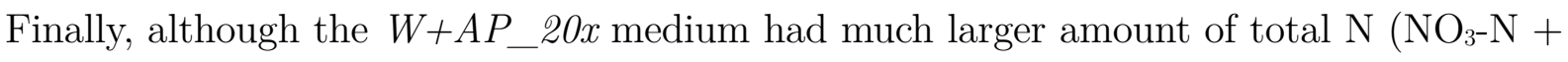
$\left.\mathrm{NH}_{4}-\mathrm{N}+\operatorname{org}-\mathrm{N}\right)$ and $\mathrm{P}$ nutrients than that in the standard medium (11 times more $\mathrm{N}$ and 5 times more $\mathrm{P}$ ), the maximum daily productivity was much lower. In preliminary experiments (results not shown here) we observed that cultivating with higher amounts of nitrate and phosphate than that in COMBO did not lead to higher growth rates, thus indicating that the concentrations in standard medium seem quite optimal for the $\mathrm{N}$ and $\mathrm{P}$ demand. Consequently, the form in which $\mathrm{N}$ is available might have played an important role instead. The lower amount of nitrate in the $W+A P \_20 x$ medium (compare to that in $C B$ ) could have had an effect. The supply of $\mathrm{NH}_{4}-\mathrm{N}$ might have not been sufficient to offset for the decline in $\mathrm{NO}_{3}-\mathrm{N}$, which could be due to the possible preference of the algae to this latter nutrient. To investigate this further, a new growth experiment (presented in the following section) was performed focusing on the participation of the various AP nitrogen sources during growth and to find out why AP enriched with COMBO exhibits a better growth performance.

\subsection{Analysing the participation of N-compounds in HTL-AP during growth}

New HTL AP was produced for these experiments and analyzed in detail. Its concentration of $\mathrm{N}$ containing compounds $\left(70 \mathrm{mg} / \mathrm{L} \mathrm{NO}-\mathrm{N}, 2012 \mathrm{mg} / \mathrm{L} \mathrm{NH}_{4}-\mathrm{N}\right.$ and 1964 $\mathrm{mg} / \mathrm{L}$ organic $\mathrm{N})$ as well as the chemical oxygen demand $\left(34897 \mathrm{mg} / \mathrm{L}\right.$ of $\left.\mathrm{O}_{2}\right)$ were slightly different compared to the AP composition in the earlier growth run, and in the same range of other studies. ${ }^{9}$ Nickel was also detected, most likely from corrosion or leaching from the reactor walls (INCOLOY 825) during HTL. Biller et al. ${ }^{9}$ also reported the presence of $\mathrm{Ni}$ in HTL aqueous phase (0-4 ppm). This type of toxic metal can affect 
almost every aspect of algal metabolism, growth and differentiation. ${ }^{14}$ However, in our AP, Ni concentration was still low $(1.9 \mathrm{mg} / \mathrm{L})$ and ended up even much lower in the growth media due to the dilution factors of more than 100 times. Therefore, no inhibitory effects were expected.

The purpose of this experiment was to learn about the participation of the different nitrogen-containing species during the growth of algae, upon recycling HTL aqueous phase. Knowing the HTL-AP composition, it was possible to control the initial amount of the various $\mathrm{N}$ constituents in the medium. Instead of adding a large amount of AP (as in the previous tests), we now added a specific amount of it, in order to reach the exact same $\mathrm{N}$ concentration (either as total- $\mathrm{N}$ or in a given form) as that in the standard COMBO medium. That procedure simultaneously resulted in media with different AP dilution factors. Again, we tested some reactors containing only water-diluted aqueous phase and others supplemented with COMBO.

All the details on media preparation can be found in Table 1 and the exact final concentration of the various $\mathrm{N}$ containing compounds in the initial media are shown in Table 3. In the media $W+A P-1$ and $C B+W+A P-1$, we targeted a final concentration of around $13 \mathrm{mg} / \mathrm{L}$ of $\mathrm{N}$ (as in COMBO), however here just in terms of inorganic nitrogen species $\left(\mathrm{NO}_{3}-\mathrm{N}+\mathrm{NH}_{4}-\mathrm{N}\right)$. Hence, in $C B+A P-1$ medium, $50 \%$ of the $\mathrm{N}$ (in the form of nitrate) in COMBO was replaced by $\mathrm{N}$ (in the form of $\mathrm{NO}_{3}-\mathrm{N}$ and $\mathrm{NH}_{4}-\mathrm{N}$ ) from the $\mathrm{AP}$. For $W+A P-2$ and $C B+W+A P-2$, the final $\mathrm{N}$ concentration of around $13 \mathrm{mg} / \mathrm{L}$ was targeted, but now also taken into account the organic nitrogen present in the AP. That was done to evaluate its possible consumption and thereby resulting in lower concentrations of $\mathrm{NO}_{3}-\mathrm{N}+\mathrm{NH}_{4}-\mathrm{N}$.

As can be seen, the design of this experiment allows for the evaluation of several aspects such as: dilution factor effect, water or water + COMBO diluted AP; organic nitrogen presence; and $\mathrm{NO}_{3}-\mathrm{N}$ vs. $\mathrm{NH}_{4}-\mathrm{N}$.

This time, the AP was much more diluted leading to lower initial concentrations of $\mathrm{NH}_{4}$ $\mathrm{N}$ than that in the medium of the previous growth experiment (Section 3.1.). The highest amount of $\mathrm{NH}_{4} \mathrm{~N}$ was therefore found in the $W+A P-1$ medium, but a concentration below the estimated toxicity limit for Scenedesmus and Desmodesmus strains. 
Table 3. Initial distribution of $\mathrm{N}$ substances, initial phosphorus concentration, total algae produced and maximum daily productivity in standard COMBO $(\mathrm{CB})$ and in different media configurations containing HTL aqueous phase (AP)

\begin{tabular}{|c|c|c|c|c|c|c|c|c|}
\hline \multirow{2}{*}{$\begin{array}{l}\text { Experiment } \\
\text { reference }^{\mathrm{a}}\end{array}$} & \multicolumn{5}{|c|}{$\begin{array}{l}\text { Initial N-composition in medium } \\
(\mathrm{mg} / \mathrm{L} \text { of } \mathrm{N})\end{array}$} & \multirow{2}{*}{$\begin{array}{l}\text { Initial } \\
\mathrm{PO}_{4}-\mathrm{P} \\
(\mathrm{mg} / \mathrm{L})\end{array}$} & \multirow{2}{*}{$\begin{array}{l}\operatorname{Algae}^{\mathrm{b}, \mathrm{c}} \\
(\mathrm{g} / \mathrm{L})\end{array}$} & \multirow{2}{*}{$\begin{array}{c}P_{\max }^{d} \\
(g / L / d)\end{array}$} \\
\hline & $\mathrm{NO}_{3^{-}} \mathrm{N}$ & $\mathrm{NH}_{4}-\mathrm{N}$ & Org-N & $\begin{array}{c}\mathrm{NO}_{3}-\mathrm{N}+ \\
\mathrm{NH}_{4}-\mathrm{N}\end{array}$ & $\begin{array}{c}\text { Total } \\
\text { N }\end{array}$ & & & \\
\hline $\mathrm{CB}$ & 13.0 & 0.00 & 0.00 & $\underline{13.0}$ & 13.0 & 1.55 & 0.81 & 0.70 \\
\hline $\mathrm{W}+\mathrm{AP}-1$ & 0.38 & 11.9 & 9.13 & $\underline{12.3}$ & 21.4 & 1.55 & 0.17 & 0.19 \\
\hline $\mathrm{W}+\mathrm{AP}-2$ & 0.22 & 6.09 & 5.79 & 6.31 & $\underline{12.1}$ & 1.55 & 0.18 & 0.19 \\
\hline $\mathrm{CB}+\mathrm{W}+\mathrm{AP}-1$ & 6.82 & 5.83 & 5.39 & $\underline{12.7}$ & 18.0 & 1.55 & 0.84 & 0.61 \\
\hline $\mathrm{CB}+\mathrm{W}+\mathrm{AP}-2$ & 6.59 & 2.97 & 3.04 & 9.56 & $\underline{12.6}$ & 1.55 & 0.73 & 0.66 \\
\hline
\end{tabular}

${ }^{\mathrm{a}}$ For abbreviations refer to Table 1. ${ }^{\mathrm{b}}$ Total algae produced. Estimated error below 10\%. ${ }^{\mathrm{c}}$ After a total of 68 h of growth. ${ }^{d}$ Maximum daily productivity. Estimated error below $20 \%$.

The growth curves for the various cultivation tests are shown in Figure 2, together with the reference run using COMBO, each for a period of nearly 3 days of growth.

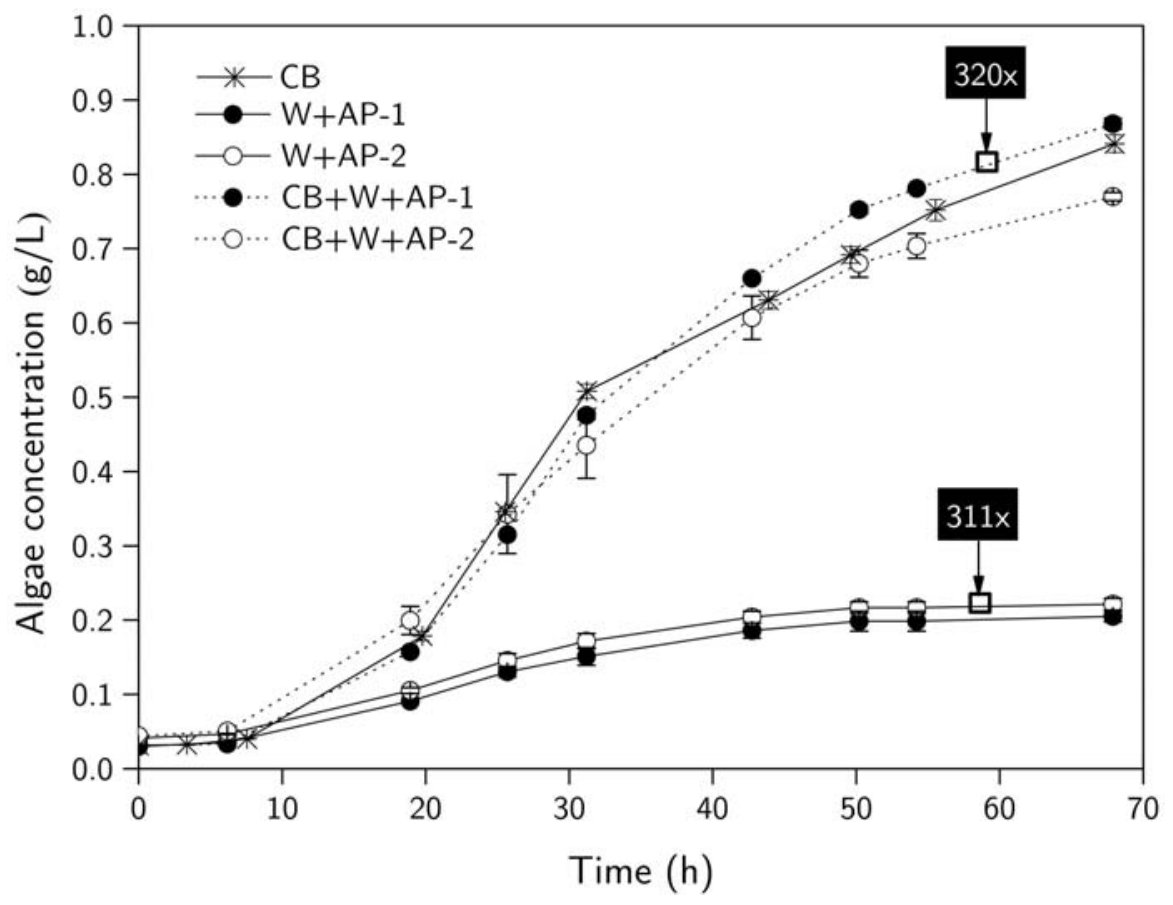

Figure 2. Algae growth in standard COMBO $(\mathrm{CB})$ and in different medium configurations containing HTL aqueous phase (AP)

Very clear trends were obtained with significant differences. The algae could grow much faster in the medium containing a mixture of $C B+W+A P-1$ than only in $W+A P-1$, despite having almost identical $\mathrm{N}$ concentration from the sum of $\mathrm{NO}_{3}-\mathrm{N}$ and $\mathrm{NH}_{4}-\mathrm{N}(12.3$ 
and $12.7 \mathrm{mg} / \mathrm{L}$ in Table 3$)$. At the same time, the growth curve for $C B+W+A P-1$ was very similar to that for COMBO alone. This is also reflected in Table 3, where comparable maximum daily productivities are shown producing nearly the same amount of algae after $68 \mathrm{~h}$ of growth. This already excludes the hypothesis that, in previous experiments, the lower amount of nitrate in the $W+A P \_20 x$ medium could have limited the growth, since now the amount of $\mathrm{NO}_{3} \mathrm{~N}$ in $C B+W+A P-1(6.82 \mathrm{mg} / \mathrm{L})$ of the new experiment was almost half of that in $C B$, while showing comparable algae growth performance. Moreover, it seems that the standard medium can be further optimized to lower $\mathrm{N}$ amounts as the growth rate in the medium $C B+W+A P-2$ (with lower inorganic nitrogen) was very similar to that in $C B$ alone.

Overall, algae in $C B, C B+W-A P-1$ and $C B+W+A P-2$ exhibited comparable growth with a total algae production of about $0.80 \mathrm{~g} / \mathrm{L}$ (see Table 3 ). The growth curves for $W+A P-1$ and $W+A P-2$ were even close to each other (see Figure 2), showing equal maximum daily productivity $(0.19 \mathrm{~g} / \mathrm{L} /$ day $)$ while reaching a total of $0.18 \mathrm{~g} / \mathrm{L}$ of algae. This indicates that the presence of organic nitrogen did not seem to have any (positive nor negative) effect on growth rate, suggesting that, most likely, this type of compounds are not toxic, but also not readily metabolized by the algae.

To investigate further which form of $\mathrm{N}$ is preferred during growth, several samples were taken during cultivation and analyzed for the $\mathrm{NO}_{3}-\mathrm{N}$ and $\mathrm{NH}_{4}-\mathrm{N}$ content in the medium. As ammonia stripping by the continuous flow of $\mathrm{CO}_{2}$ enriched air could also lead to a decrease of $\mathrm{NH}_{4}-\mathrm{N}$ during growth, first an experiment was performed where $9.4 \mathrm{ml}$ of $\mathrm{AP}$ was added to a $1 \mathrm{~L}$ demineralized water in a reactor vessel, contained into the same growth system. The same $\mathrm{CO}_{2}$-areation flow was used and after $68 \mathrm{~h}$ of continuous bubbling, still the same amount of $\mathrm{NH}_{4}-\mathrm{N}$ as the initial value was found in the aqueous solution, indicating that no significant ammonia stripping had occurred.

Figure 3 shows the $\mathrm{NO}_{3}-\mathrm{N}$ and $\mathrm{NH}_{4} \mathrm{~N}$ consumption results during growth in the media $W+A P-1$ and $C B+W+A P-1$. Algae growth was accompanied by decrease in nitrogen content in the medium, indicating that nitrogen removal was due to algal uptake and assimilation. For all media tested, complete $\mathrm{NO}_{3}-\mathrm{N}$ and $\mathrm{NH}_{4}-\mathrm{N}$ removal was accomplished after $30 \mathrm{~h}$ of growth, except for the $W+A P-1$ medium where the algae did not consume any nitrate (Figure 3(a)). There, the initial amount of $\mathrm{NH}_{4}-\mathrm{N}$ was much higher than $\mathrm{NO}_{3}-\mathrm{N}$ and after $68 \mathrm{~h}$ there was still $\mathrm{NH}_{4}-\mathrm{N}$ available and thus no need for the algae to consume nitrate. Possibly, after complete depletion of $\mathrm{NH}_{4}-\mathrm{N}$, the cells would have started consuming $\mathrm{NO}_{3}-\mathrm{N}$, as is e.g. observed in the uptake of $\mathrm{N}$ within the $C B+W+A P$ 1 medium where $\mathrm{NH}_{4}-\mathrm{N}$ was consumed faster than $\mathrm{NO}_{3}-\mathrm{N}$ and when all the $\mathrm{NH}_{4}-\mathrm{N}$ was 
finished (after $20 \mathrm{~h}$ ) the remaining $\mathrm{NO}_{3} \mathrm{~N}$ was consumed. Consequently, in all the culture media, the microalgal cells preferentially utilize $\mathrm{NH}_{4}-\mathrm{N}$, most likely because it is easier to convert into amino-acids than $\mathrm{NO}_{3}-\mathrm{N} .{ }^{16}$
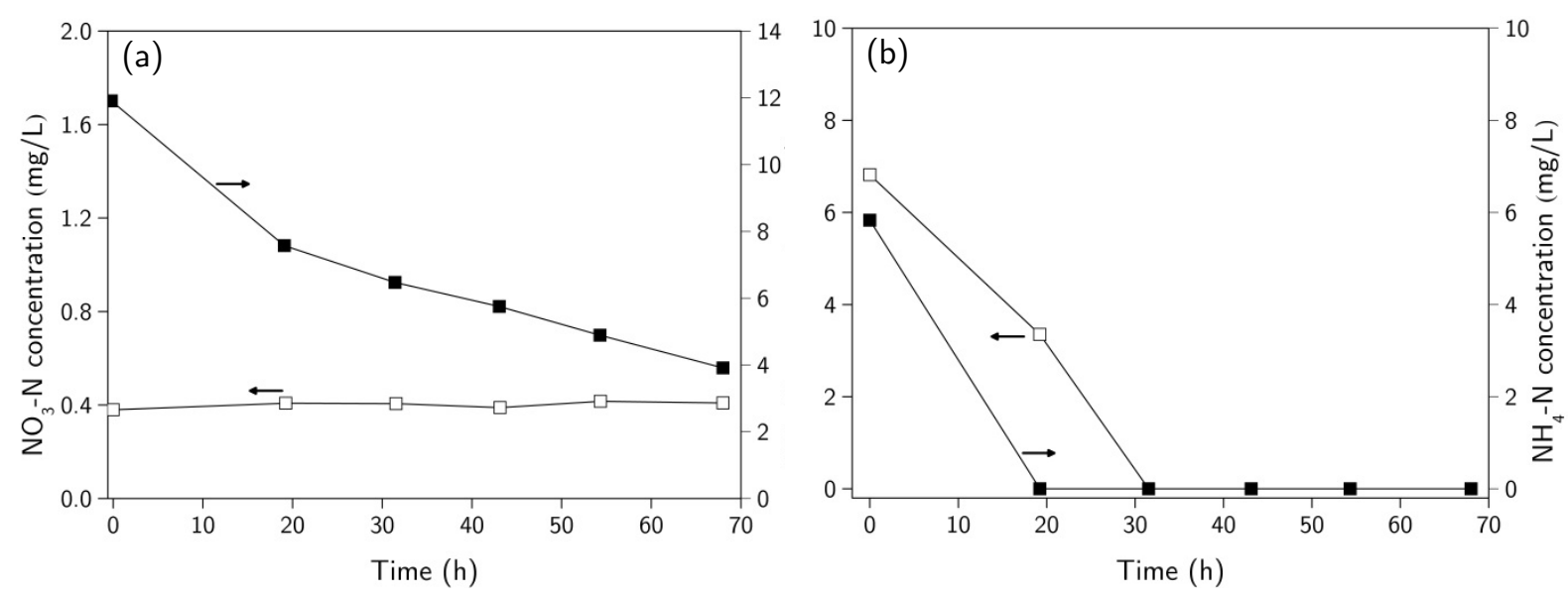

Figure 3. $\mathrm{NO}_{3}-\mathrm{N}$ and $\mathrm{NH}_{4}-\mathrm{N}$ consumption during growth in media (a) $W+A P-1$ and (b) $C B+W+A P-1$

Nitrogen depletion (and also $\mathrm{P}$ which was also depleted after $30 \mathrm{~h}$ ) seems to coincide with the decrease of growth rate. For all the reactors, from 20 to $30 \mathrm{~h}$ the biomass concentration was increasing at the highest growth rates and after that, a significant decrease of the growth rate occurred (see Figure 2, change of slope of the curve after 30 $\mathrm{h}$, coinciding with the full depletion of $\mathrm{N}$ and $\mathrm{P}$ ).

Additionally, the total nitrogen content was also monitored along the growth to study the possible consumption of organic nitrogen. For a period of almost 3 days of growth, the amount of org- $\mathrm{N}$ remained nearly constant in all the growth media. Hence, no evidence of consumption of organic nitrogen was found, at least over the time used for cultivation. Total organic carbon content was also monitored along the growth in all the cultures, but again no clear evidence of its consumption was found for the cultivation period of $68 \mathrm{~h}$. Therefore, no clear signs of mixotrophic growth were observed. Biller et al. ${ }^{9}$ reported consumption of organic carbon by Scenedesmus dimorphous (similar strain to the one used in this work) in AP, but the cells were not able to use it to the same extent as the other algal species tested. Mixotrophic growth could lead to higher carbon efficiencies for the combined HTL-cultivation loop. On the other hand, if an algal species with desirable properties is used but cannot assimilate any kind of organic source, still the carbon efficiency can be improved by extracting the energy-containing compounds from the AP by other methods as e.g. supercritical water gasification or anaerobic digestion of the AP prior to recycling to cultivation. These treatments would convert the 
organic matter into a high heating value gas while, most likely, producing more ammonia dissolved into the AP for further reuse.

The effect of the AP dilution factor by itself can be evaluated as a results of the adjustment of the various media for different nitrogen compositions. Again, and in contrast with earlier literature, ${ }^{9,11}$ the lowered growth does not seem to be caused by insufficient dilution related to toxicity of certain organic compounds. This is proven by comparing the media $C B+W+A P-1$ and $W+A P$-2 with comparable AP dilution $(320 \mathrm{x}$ and $311 \mathrm{x}$ respectively) and $\mathrm{NH}_{4}-\mathrm{N}$ concentration (the preferable $\mathrm{N}$ source) but with algae exhibiting completely different growth performance. Almost 5 times more algae were produced in the medium enriched with COMBO.

Although the results showed that the toxicity of organics can be excluded, still the AP was analyzed in detail to get a general picture of water soluble products of HTL. The dissolved organics fraction was mainly composed of small water soluble compounds, which became GC-detectable by adopting a derivatization procedure optimized for the determination of cyclic dipeptides. ${ }^{13}$ The quantitative analysis of the identified substances provided an exhaustive picture of the water soluble organic matter, with a mass closure of more than $90 \%$. Table 4 shows the concentration (in $\mathrm{mg} / \mathrm{L}$ ) of organic constituents, grouped into various categories for pure AP and initially in $W+A P-1$ medium. This medium had the lowest dilution factor $(160 \mathrm{x})$ and hence the highest concentration of potential inhibitory compounds.

Residual solvent, used for the oil recovery in the HTL experiment, was a prominent contaminant detected at a concentration of about $1 \%$ in the solution. Among the HTL generated substances, acetic acid was found to be the most abundant compound, followed by pyroglutamic acid, earlier detected in remarkable amount in HTL oil (see Appendix of Chapter 2). Expressed as mass yield, the water dissolved fraction of this compound corresponds to about $1.6 \%$ of the original microalgal biomass. Other water soluble HTL products with less important contribution (100-600 mg/L) were found to be sugar-like compounds, hydroxyacids, cyclic dipeptides, small fatty acids, amines and amino acids. Although detectable, only minor amount of phenols $(58 \mathrm{mg} / \mathrm{L})$ ended up in the water phase, even though larger amounts of these compounds were detected in the HTL oils (see Appendix of Chapter 2). Their concentration in the AP was almost identical to that in the HTL aqueous phase composition as reported by Jena et al. ${ }^{11}$ In our case, the $W+A P-1$ medium contained the highest phenol concentration, $0.3 \mathrm{mg} / \mathrm{L}$, but still too low to expect any kind of inhibitory effect. Hirooka et al. ${ }^{17}$ reported that nitrophenols and chlorophenols are known to be toxic to aquatic organisms within the 
range from 5 to $25 \mathrm{mg} / \mathrm{L}$. The $\mathrm{EC}_{50}$ for Scenedesmus quadricauda (similar to our algae) was $184 \mathrm{mg} / \mathrm{L}$ after $24 \mathrm{~h}$ exposure, ${ }^{18}$ a concentration 600 time higher than that in the $W+A P-1$ medium.

Table 4. Concentration of various organic compounds in the aqueous phase after HTL and in the medium $W+A P-1(160 \mathrm{x}$ diluted $)$

\begin{tabular}{|c|c|c|}
\hline Compounds $^{\mathrm{a}}$ & $\begin{array}{c}\text { In pure AP } \\
(\mathrm{mg} / \mathrm{L})\end{array}$ & $\begin{array}{c}\text { Initially in } W+A P-1 \text { medium } \\
(\mathrm{mg} / \mathrm{L})\end{array}$ \\
\hline Residual solvent $\left(\mathrm{CH}_{2} \mathrm{Cl}_{2}\right)$ & $13000 \pm 120$ & 81.3 \\
\hline Acetic acid & $3946 \pm 550$ & 24.7 \\
\hline Ethanol & $359 \pm 50$ & 2.24 \\
\hline Acetone & $208 \pm 25$ & 1.30 \\
\hline Polyols & $558 \pm 179$ & 3.49 \\
\hline Amines & $138 \pm 57$ & 0.86 \\
\hline$>\mathrm{C}_{2}$ fatty acids & $236 \pm 92$ & 1.48 \\
\hline Aminoacids & $152 \pm 35$ & 0.95 \\
\hline Cyclic dipeptides & $252 \pm 83$ & 1.58 \\
\hline Nitrogen aromatic & $223 \pm 121$ & 1.39 \\
\hline Hydroxyacids & $582 \pm 191$ & 3.64 \\
\hline Sugars & $136 \pm 66$ & 0.85 \\
\hline Pyrrolidones ${ }^{\mathrm{b}}$ & $1394 \pm 255$ & 8.71 \\
\hline Phenols & $58 \pm 14$ & 0.36 \\
\hline Dipeptides & $3 \pm 3$ & 0.02 \\
\hline Not identified & $762 \pm 352$ & 4.76 \\
\hline Sum $^{\mathrm{c}}$ & 9006 & 56.3 \\
\hline
\end{tabular}

The water soluble fraction, formed by a large number of constituents, was characterized by different degrees of toxicity or metabolic activity. Some (sugars, carboxylic acid, aminoacids and hydroxyacids) can definitely be considered non-toxic metabolites and potentially be used as carbon and nitrogen sources. Others, in particular nitrogen containing compounds like pyroglutamic acid, cyclic dipeptides and amines, can still be considered degradable or utilizable (upon hydrolysis they are converted to amino-acids), but they are characterized by well-known high biological activity. Finally, a considerable amount of "xenobiotics" (e.g. nitrogen containing aromatics) ended up in the water phase, with concentrations that ranged from $0.09 \%$ to $0.12 \%$. 
Pyroglutamic acid ${ }^{19}$ has antifouling activity against algal spore attachment and is one of the most abundant water soluble compounds produced. Cyclic dipeptides are often characterized by biological activity and some are produced by marine microorganisms ${ }^{20}$ as protection toward grazing, which can be beneficial in big scale cultivation. On the opposite, cyclo(Pro-Tyr) showed an herbicidal activity and, therefore, its toxicity could be expected at high concentration. Finally, various aromatic heterocycles (e.g. pyridine derivatives) and phenols detected also showed toxicity toward microalgae at relatively low concentration..$^{21,22}$

Relatively high concentrations (close to growth inhibition values) of toxic compounds were detected in the HTL-AP. Yet, due to the 160 times dilution factor, toxicity levels were not reached. Possible inhibition phenomena would occur only after many recycles (e.g about 100 recycles are needed for achieving the $\mathrm{IC}_{50}$ for pyroglutamic acid) and in absence of significant bio-degradation of these pollutants. Testing a series of several cycles of cultivation with liquefaction and AP recycling is however recommendable and activities in this direction are already initiated in our laboratories. Furthermore, the high concentration of the residual dichloromethane solvent (widely used in HTL laboratory studies) in the aqueous phase emphasizes the need of replacement for a greener solvent with low water solubility.

Finally, at this point, we excluded several aspects which, in our view, did not limit algae growth: $\mathrm{NH}_{4} \mathrm{~N}$ toxicity, differences in $\mathrm{N}: \mathrm{P}$ ratio and organic substances toxicity (by insufficient AP dilution). Instead, there are strong indications suggesting that something specific is missing in the AP, which is required to maintain an optimal growth speed. Besides N, P and C, other nutrients that are essential for algae growth are $\mathrm{S}, \mathrm{Ca}, \mathrm{Mg}$, $\mathrm{Na}, \mathrm{K}$ and $\mathrm{Cl}$. These nutrients are required in a relatively large amount. Micronutrients include $\mathrm{Fe}, \mathrm{B}, \mathrm{Mn}, \mathrm{Cu}, \mathrm{Mo}, \mathrm{V}, \mathrm{Co}, \mathrm{Ni}, \mathrm{Si}$ and Se. ${ }^{23}$ To evaluate that, inductively coupled plasma optical emission spectroscopy (ICP-OES) and ion chromatography (IC) analyses were performed on the HTL aqueous phase. Table 5 shows the main micronutrients in pure $\mathrm{AP}$, in the various cultures after addition of AP and in standard COMBO.

The lack of almost all these elements (except potassium) in the media with only water and AP became evident. Many of the trace elements are important in enzyme reactions and for the biosynthesis of many compounds, e.g. Co is essential for vitamin $B_{12}$ production, ${ }^{14}$ which on their turn can be essential cofactors for carboxylase enzymes involved in fatty acid synthesis. ${ }^{24}$ Richmond $^{14}$ indicated that autoclaving cultivation media (for sterilization) results in a certain degree of vitamins decomposition, which could also happen when performing HTL where more severe conditions are applied. 
Growth of Dunaliella tertiolecta in standard culture medium was compared to that in the same medium without the presence of $\mathrm{Fe}^{3+}, \mathrm{Co}^{2+}, \mathrm{Mn}^{2+}$ or $\mathrm{Mo}^{2+}$ by Chen et al. ${ }^{25}$ Algae growth was substantially reduced when iron was eliminated followed closely by elimination of cobalt, magnesium and molybdenum. Magnesium is essential for the synthesis of chlorophyll and photosynthesis. In conclusion, a lack of other essential nutrients (e.g. $\mathrm{Mg}$ ), other than $\mathrm{N}$ and $\mathrm{P}$, seems to be the primary cause of the growth reduction observed.

Table 5. Elements present in standard COMBO $(\mathrm{CB})$ and in different media configurations containing HTL aqueous phase (AP)

\begin{tabular}{ccccccc}
\hline \multirow{2}{*}{ Elements } & In pure AP & \multicolumn{5}{c}{ Initial concentration $(\mathbf{m g} / \mathbf{L})$ in medium $^{\mathrm{a}}$} \\
\cline { 3 - 7 } & $(\mathbf{m g} / \mathbf{L})$ & CB & W+AP-1 & W+AP-2 & CB+W+AP-1 & CB+W+AP-2 \\
\hline $\mathrm{Na}$ & $334.5 \pm 6.5$ & 28.4 & 2.09 & 1.08 & 15.3 & 14.7 \\
$\mathrm{~K}$ & $1290 \pm 120$ & 3.91 & 8.05 & 4.15 & 5.98 & 4.03 \\
$\mathrm{Ca}$ & $17.0 \pm 0.4$ & 10.0 & 0.11 & 0.05 & 5.06 & 5.04 \\
$\mathrm{Cl}$ & $62.0 \pm 0.0$ & 86.4 & 0.39 & 0.20 & 43.4 & 43.3 \\
$\mathrm{Mg}$ & $29.3 \pm 0.1$ & 3.65 & 0.18 & 0.09 & 1.91 & 1.87 \\
$\mathrm{~S}$ & $83.0 \pm 0.0$ & 7.39 & 0.52 & 0.27 & 3.95 & 3.83 \\
\hline
\end{tabular}

${ }^{a}$ For abbreviations refer to Table 1

Considering that $C B$ contained $13 \mathrm{mg} / \mathrm{L}$ of $\mathrm{NO}_{3}-\mathrm{N}$ and that $C B+W+A P-1$ had 6.59 $\mathrm{mg} / \mathrm{L}$ of $\mathrm{NO}_{3}-\mathrm{N}$ from $C B$ and $0.22 \mathrm{mg} / \mathrm{L}$ of $\mathrm{NO}_{3}-\mathrm{N}$ plus $5.83 \mathrm{mg} / \mathrm{L}$ of $\mathrm{NH}_{4}-\mathrm{N}$ from $\mathrm{AP}$, $50 \%$ of the $\mathrm{N}$ in $C B$ can be replaced by $\mathrm{N}$ in $\mathrm{AP}$ while having no detrimental effects on growth rate, thus reducing $\mathrm{N}$ nutrient costs by half. However, micronutrients should be supplied upon AP recycling and the dilution factor must be that one that supplies just enough $\mathrm{N}$ and $\mathrm{P}$ for the algae to grow at rates comparable to that in $C B$.

\section{Conclusions}

Cultivation of Desmodesmus sp. was performed to evaluate nutrients recycling via the AP from HTL of the same microalgae. This was done by systematically varying the AP dilution, using only water or a mixture of water and standard medium (CB), while keeping the same nitrogen concentration (either as total $\mathrm{N}$ or the sum of ammonia and nitrate) as that in standard growth medium. A substantial reduction in growth was observed when using water diluted AP. In contrast, comparable growth as that in standard culture medium was achieved when the mixture $\mathrm{W}+\mathrm{AP}$ was enriched with 
COMBO. Based on our results, differences in N:P ratio and toxicity by too high concentrations of $\mathrm{NH}_{4}-\mathrm{N}$ and/or organics compounds can be excluded as the cause of growth reduction. Algae in media with nearly equal AP dilution factor (thus same N:P ratio and same concentrations of organics and $\mathrm{NH}_{4} \mathrm{~N}$ ) exhibited completely different growth rates. Therefore, we have strong indications that the lack of essential (macro/micro-)nutrients (other than $\mathrm{N}$ and $\mathrm{P}$ ) in $\mathrm{AP}$, enhanced when diluting with water, is the major cause. These nutrients must be supplied upon AP recycling to avoid growth reduction.

\section{Acknowledgements}

The authors are thankful for the financial support by the province of Overijssel via the Green Energy Initiative of the University of Twente (The Netherlands).

\section{Bibliography}

[1] Y. Li, M. Horsman, N. Wu, C.Q. Lan, N. Dubois-Calero, Biofuels from microalgae, Biotechnol. Prog., 24(4), 815-820, 2008.

[2] J.W. Richardson, M.D. Johnson, J.L. Outlaw, Economic comparison of open pond raceways to photo bio-reactors for profitable production of algae for transportation fuels in the Southwest, Algal Res., 1(1), 93-100, 2012.

[3] R. Perrin, K. Vogel, M. Schmer, R. Mitchell, Farm-scale production cost of switchgrass for biomass, Bioenergy Res., 1(1), 91-97, 2008.

[4] L. Lardon, A. Helias, B. Sialve, J.-P. Steyer, O. Bernard, Life-cycle assessment of biodiesel production from microalgae, Environ. Sci. Technol., 43(17), 6475-6481, 2009.

[5] D. Cordell, J. Drangert, S. White, The story of phosphorus: Global food security and food for thought, Global Environ. Change, 19(2), 292-305, 2009.

[6] D. Knežević, W. van Swaaij, S. Kersten, Hydrothermal conversion of biomass. II. conversion of wood, pyrolysis oil, and glucose in hot compressed water, Ind. Eng. Chem. Res., 49(1), 104-112, 2009.

[7] A.A. Peterson, F. Vogel, R.P. Lachance, M. Fröling, M.J. Antal Jr, J.W. Tester, Thermochemical biofuel production in hydrothermal media: A review of sub- and supercritical water technologies, Energy Environ. Sci., 1(1), 32-65, 2008.

[8] P. Duan, P.E. Savage, Hydrothermal liquefaction of a microalga with heterogeneous catalysts, Ind. Eng. Chem. Res., 50(1), 52-61, 2010.

[9] P. Biller, A.B. Ross, S.C. Skill, A. Lea-Langton, B. Balasundaram, C. Hall, R. Riley, C.A. Llewellyn, Nutrient recycling of aqueous phase for microalgae cultivation from the hydrothermal liquefaction process, Algal Res., 1(1), 70-76, 2012.

[10] T. Minowa, S. Sawayama, A novel microalgal system for energy production with nitrogen cycling, 
Fuel, 78(10), 1213-1215, 1999.

[11] U. Jena, N. Vaidyanathan, S. Chinnasamy, K.C. Das, Evaluation of microalgae cultivation using recovered aqueous co-product from thermochemical liquefaction of algal biomass, Bioresour. Technol., 102(3), 3380-3387, 2011.

[12] S. Kilham, D. Kreeger, S. Lynn, C. Goulden, L. Herrera, COMBO: a defined freshwater culture medium for algae and zooplankton, Hydrobiologia, 377(1), 147-159, 1998.

[13] D. Fabbri, A. Adamiano, G. Falini, R. De Marco, I. Mancini, Analytical pyrolysis of dipeptides containing proline and amino acids with polar side chains. Novel 2,5-diketopiperazine markers in the pyrolysates of proteins, J. Anal. Appl. Pyrolysis, 95, 145-155, 2012.

[14] A. Richmond, Handbook of microalgal culture: biotechnology and applied phycology, John Wiley \& Sons, Ames, 2008.

[15] Y. Azov, J.C. Goldman, Free ammonia inhibition of algal photosynthesis in intensive cultures, Appl. Environ. Microbiol., 43(4), 735-739, 1982.

[16] C. Huang, J.-J. Hung, S.-H. Peng, C.-N.N. Chen, Cultivation of a thermo-tolerant microalga in an outdoor photobioreactor: Influences of CO2 and nitrogen sources on the accelerated growth, Bioresour. Technol., 112(0), 228-233, 2012.

[17] T. Hirooka, Y. Akiyama, N. Tsuji, T. Nakamura, H. Nagase, K. Hirata, K. Miyamoto, Removal of hazardous phenols by microalgae under photoautotrophic conditions, J. Biosci. Bioeng., 95(2), 200-203, 2003.

[18] T. Tišler, J. Zagorc-Končan, Comparative assessment of toxicity of phenol, formaldehyde, and industrial wastewater to aquatic organisms, Water, Air, Soil Pollut., 97(3-4), 315-322, 1997.

[19] J.-Y. Cho, J.-S. Choi, S.-E. Kang, J.-K. Kim, H.-W. Shin, Y.-K. Hong, Isolation of antifouling active pyroglutamic acid, triethyl citrate and di- $n$-octylphthalate from the brown seaweed Ishige okamurae, J. Appl. Phycol., 17(5), 431-435, 2005.

[20] R. Huang, X. Zhou, T. Xu, X. Yang, Y. Liu, Diketopiperazines from marine organisms, Chem. Biodiversity, 7(12), 2809-2829, 2010.

[21] A. Fiorentino, A. Gentili, M. Isidori, P. Monaco, A. Nardelli, A. Parrella, F. Temussi, Environmental effects caused by olive mill wastewaters: Toxicity comparison of low-molecular-weight phenol components, J. Agric. Food Chem., 51(4), 1005-1009, 2003.

[22] B.B. Singh, R. Chandra, Comparative chronic toxicity of pyridine, $\alpha$-Picoline, and $\beta$-Picoline to Lemna minor L. and Chlorella vulgaris B Bull. Environ. Contam. Toxicol., 75(3), 482-489, 2005.

[23] I. Suh, C.-G. Lee, Photobioreactor engineering: Design and performance, Biotechnol. Bioprocess Eng., $8(6), 313-321,2003$.

[24] M.T. Croft, M.J. Warren, A.G. Smith, Algae need their vitamins, Eukaryotic Cell, 5(8), 1175-1183, 2006 .

[25] M. Chen, H. Tang, H. Ma, T.C. Holland, K.Y.S. Ng, S.O. Salley, Effect of nutrients on growth and lipid accumulation in the green algae Dunaliella tertiolecta, Bioresour. Technol., 102(2), 1649-1655, 2011. 


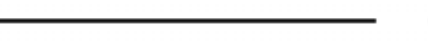

rar 


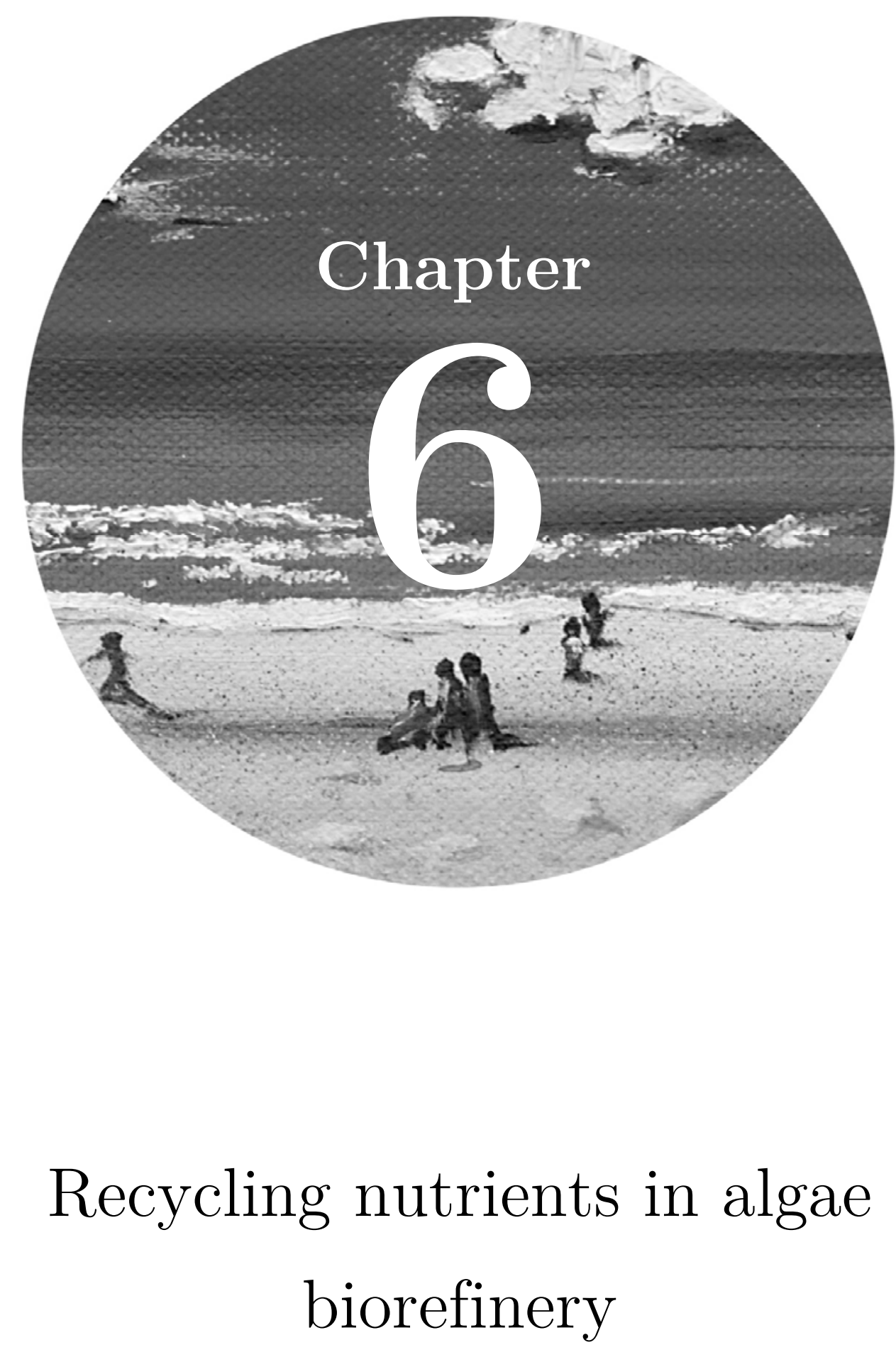




\begin{abstract}
In this study, the multiple recyclability potential of aqueous phase (AP) containing nutrients obtained from hydrothermal liquefaction (HTL) of microalgae is demonstrated. This has been done by carrying out, for the first time, a complete set of 4 continuous growth-HTL cycles where Desmodesmus sp. cells were grown 5 times and HTL of the biomass produced was performed 4 times. No detrimental effects on growth were observed, for cultivating at least 5 generations of algae, while converting $50 \%$ of the biomass (dry basis) into a crude oil (with an energy content of $33-35 \mathrm{MJ} / \mathrm{kg}$ ). Simultaneously, cultivation costs are reduced by the replacement of fresh make-up nitrogen and phosphorous nutrients via AP addition and recycling medium from the dewatering step (depleted medium). A comparable growth performance to that in standard medium was obtained in the last cycle, where the maximal accumulation of growth inhibitory compounds from the previous cycles was present. In that last generation of algae, $39.5 \%$ of $\mathrm{N}$ and $65.7 \%$ of $\mathrm{P}$ in the medium was replaced by that from AP. Moreover, a detailed characterization of the AP soluble organics was performed to identify possible accumulating compounds. Other aspects were observed that might be necessary to control upon the multiple times usage of HTL-AP: apparent cell morphology changes (if those would appear to be detrimental to e.g. growth performance), increase of potential toxic organic substances in the AP throughout the cycles and leaching of heavy metals from the reactor walls during HTL. Further reduction on nutrients costs can be achieved by recovering the phosphate from the HTL solid residue and converting the organic fraction of the AP into algal consumable substances (e.g. ammonia), next to the simultaneous production of an energy- and $\mathrm{CO}_{2}$ rich gas. This study brings us one step forward towards standalone operation of algae biorefineries.
\end{abstract}




\section{Introduction}

A growing awareness towards the environment and the depletion of fossil resources is increasing the demand for renewable energy carriers and fuels that can be incorporated into existing infrastructures. An interesting and potential source of bioenergy is the use of microalgae; organisms capable of harvesting energy from sunlight and store it in the form of energy-rich organic compounds, while exhibiting other outstanding advantages such as: being the fastest growing photosynthetic organisms on earth with higher photosynthetic efficiency than that of terrestrial biomass and without direct competition for arable land.

Possibly the main drawback, when considering algae, is the high costs of cultivation. The high energy requirements for the production of essential fertilizers as $\mathrm{N}$ and $\mathrm{P}$ contribute significantly to this. ${ }^{1}$ In parallel, $\mathrm{P}$ depletion is becoming a global concern that must be alleviated to secure food production, a fundamental factor to the world's prosperity. ${ }^{2}$ Therefore, recycling of these nutrients is, by any means, essential.

Hydrothermal liquefaction (HTL) can be applied to convert wet algal biomass into energy carriers (crude oil and gas). HTL has the advantage that it does not require complete dewatering and thermal drying of the algal feedstock, thereby saving on related energy costs. A HTL-crude oil is obtained which can be further used as (fossil fuel like) refinery feed, along with the possible co-production of other value-added products and feed/food ingredients from algae in a biorefinery setting. Next to the HTL-oil, an aqueous phase (AP) containing inorganic and organic N, P and C constituents is obtained. Ideally, this phase can be recycled and used as source of nutrients for algae growth to reduce cultivation costs.

For nutrients supply in large scale cultivation of microalgae, to be processed in a biorefinery concept as described above, there are essentially two options. The most often considered one is to cultivate algae on wastewater derived nutrients from municipal, agricultural and industrial activities. Here, algae provide a water cleaning treatment that otherwise would be conventionally performed by means of chemical processing or activated sludge. ${ }^{3}$ If the wastewater contains some, but not sufficient nutrients to maintain maximum growth rates, the (partial) recycling of the AP to the cultivation step can supply the missing fraction of nutrients required. Here, wastewater treatment and AP recycling are complementary synergetic processes. If nutrients recycling proves to be very successful, standalone operation can be considered and only a small make-up fraction of nutrients needs to be resupplied to the system, to compensate for the fraction 
of $\mathrm{N}$ and $\mathrm{P}$ lost as HTL product; e.g. in the form of artificial fertilizer or wastewater nutrients. Whatever biorefinery scheme including HTL is considered, an AP fraction will always be produced and this must be processed in order to keep the system in closed loop configuration as much as possible, without (significant) waste streams.

Few studies have shown the possibilities of growing algae in the recovered AP coproduct. $^{4-7}$ The most recent study by $\mathrm{Du}$ et al. ${ }^{8}$ investigated the feasibility of using recovered nutrients from hydrothermal carbonization (HTC) for cultivation of Chlorella vulgaris. In Chapter 5, we showed that diminished growth rates of Desmodesmus sp. microalgae do not seem to be related to the toxicity of specific compounds (AP organics), but rather result from missing essential minor nutrients, other than $\mathrm{N}$ and $\mathrm{P}$, in the AP. In that study, the concentration in the culture media of potential toxic compounds such as phenols was too low to inhibit growth. However, accumulation of those compounds could occur in a process configuration with continuous recycling of AP. Therefore, the multiple recyclability potential of AP-nutrients has been evaluated in this work. A test sequence was designed for a complete set of 4 continuous growth-HTL cycles where Desmodesmus sp. microalgae were grown 5 times using AP and HTL of the biomass produced was performed 4 times. With that, we aim to evaluate the following aspects: 1) feasibility of using, multiple times, the aqueous phase (AP) containing water soluble organic and inorganic compounds resulting from HTL for the algae cultivation; 2) effect of the possible accumulation of growth inhibitory compounds; 3) effect of not only AP recycling but also recycled algae and untreated culture medium water (from the dewatering step) on cultivation, HTL product yields and composition; and 4) potential of nutrients savings. With that, further improvements and/or requirements to sustain such a recycling configuration can be defined.

\section{Experimental}

\subsection{Algal feedstock, cultivation system and growth measurements}

In this work, microalgae from the species Desmodesmus sp. was used as inoculum (supplied by Ingrepro B.V., The Netherlands). The algal batch had comparable properties and similar elemental composition to the algal feedstock used in Chapters 2 and 5. Immediately after being delivered, the batch was maintained in standard COMBO medium $^{9}$ (from which $\mathrm{KCl}, \mathrm{Na}_{2} \mathrm{SiO}_{3} \bullet 9 \mathrm{H}_{2} \mathrm{O}$ and animal trace elements were excluded), in the dark and cold, prior to reactor inoculation. The cultivation system and the method 
to monitor algae growth by measuring optical density at $750 \mathrm{~nm}$ (OD750) are extensively described in our previous work (Chapter 5). Also in that work, the linear relationship correlating measured OD750 and algae concentration (in $\mathrm{g} / \mathrm{L}$ ) is shown.

\subsection{Algae adaptation}

A detailed description of the algae acclimatization procedure followed in this work can be found in Chapter 5 . The purpose of this procedure was to progressively adapt the cells to the growth conditions listed in Table 1, being the final settings used in all the growth tests. Briefly, the reactors containing $2 \mathrm{~L}$ of $\mathrm{COMBO}$ standard medium were first inoculated with a cell pellet of maintained fresh algae. After that, a gradual increase of the various settings (e.g. $\mathrm{T}$ and light intensity) was performed until the final growth conditions (Table 1) were reached.

Table 1. Growth conditions

\begin{tabular}{cc}
\hline Parameter & Value \\
\hline Temperature $\left({ }^{\circ} \mathrm{C}\right)$ & $25.0 \pm 1$ \\
Light intensity $\left(\mathrm{\mu mol} / \mathrm{m}^{2} / \mathrm{s}\right)^{\mathrm{a}}$ & 247 \\
$\mathrm{CO}_{2}$ in air mixture $(\mathrm{vol} \%)$ & 5 \\
Aeration $\left(\mathrm{vvm}^{\mathrm{b}}\right)$ & 1.8 \\
Initial $\mathrm{pH}$ & 7.8 \\
\hline${ }^{\mathrm{a}}$ Measured at the reactor wall. ${ }^{\mathrm{b}}$ Volume gas \\
per volume media per min $(\mathrm{L} / \mathrm{L} / \mathrm{min})$
\end{tabular}

Two out of the ten growth reactors located inside the illuminated chamber were used to continuously maintain the culture in standard COMBO medium during the whole experimental period. Regularly, during the adaptation period, half of the volume of the culture broth was replaced by fresh medium to avoid nutrients and light limited conditions due to high concentrations of algae. According to the light intensity applied (247 $\left.\mathrm{\mu mol} / \mathrm{m}^{2} / \mathrm{s}\right)$ during the test and the reactors dimensions, light was limiting at an algae concentration of approx. $0.3 \mathrm{~g} / \mathrm{L}$ for this specific cultivation system. As explained in Chapter 5, that light limitation concentration was estimated first theoretically based on the Beer-Lambert law. Later, that algae concentration was reaffirmed by identifying the beginning of the linear region of the growth curve (for the growth in standard culture medium explained in Section 2.3.) which generally occurs when a specific requirement such as light is limiting cell division. ${ }^{10}$ 


\subsection{Algae growth in standard culture medium}

This growth experiment, used as a control test, was performed in standard culture medium (COMBO) and the growth conditions listed in Table 1. For the AP-nutrients recycling experiment (see Section 2.4), we estimated that an amount of about 8-12 $\mathrm{g}$ of dry algae was sufficient, both for analyses and to create an algal slurry (after the dewatering step) for hydrothermal liquefaction with an 8-12 wt\% of algae concentration. Therefore, the target in this test was to grow until a concentration of about $0.8-1.2 \mathrm{~g} / \mathrm{L}$ in each reactor and a total volume of $10 \mathrm{~L}$.

A sample from the continuously maintained culture, adapted to the conditions in Table 1 was centrifuged at $8000 \mathrm{rpm}$ for $5 \mathrm{~min}$. The cell pellet obtained was used to inoculate five reactors, each containing $2 \mathrm{~L}$ of three times concentrated COMBO (but still keeping the same N:P ratio as in COMBO) until an initial algae concentration of $0.2 \mathrm{~g} / \mathrm{L}$ and, after that, growth was monitored by regularly measuring OD750. In previous research (Chapter 5) we observed that, when the exact concentrations of nutrients in COMBO were used, $\mathrm{N}$ and $\mathrm{P}$ were rapidly consumed in less than $24 \mathrm{~h}$ (for this specific algal strain and reactor configuration). Since we predicted that a growth longer than $24 \mathrm{~h}$ was required to achieve the targeted concentration of $0.8-1 \mathrm{~g} / \mathrm{L}$ per reactor, a three times more concentrated medium was used to avoid nutrients limitation and allowing us to follow nutrients uptake during the course of the whole growth period. Also in that way it was possible to calculate (for comparison purposes with AP recycling later on) how much $\mathrm{NO}_{3}-\mathrm{N}$ and $\mathrm{PO}_{4}-\mathrm{P}$ is consumed per gram of algae produced.

\subsection{Algae growth under AP-nutrients recycling}

\subsubsection{Experiment design}

The following experiment was designed aiming to evaluate the effect of recycling and reusing inorganic nutrients $(\mathrm{N}$ and $\mathrm{P}$ ) for growth multiple times as well as the fate of other recycled compounds (e.g. water soluble organics) resulting from HTL of the same cultivated microalgae. Figure 1(a) shows the overall experimental sequence where algae were grown 5 times and HTL was performed 4 times. Consequently, a total of 4 complete cycles (growth + HTL) were performed and, in the $5^{\text {th }}$ cycle, only growth was carried out. After harvesting and dewatering, HTL of the algal slurry was performed producing an aqueous phase that was recovered and added to the growth medium of the next cycle. 

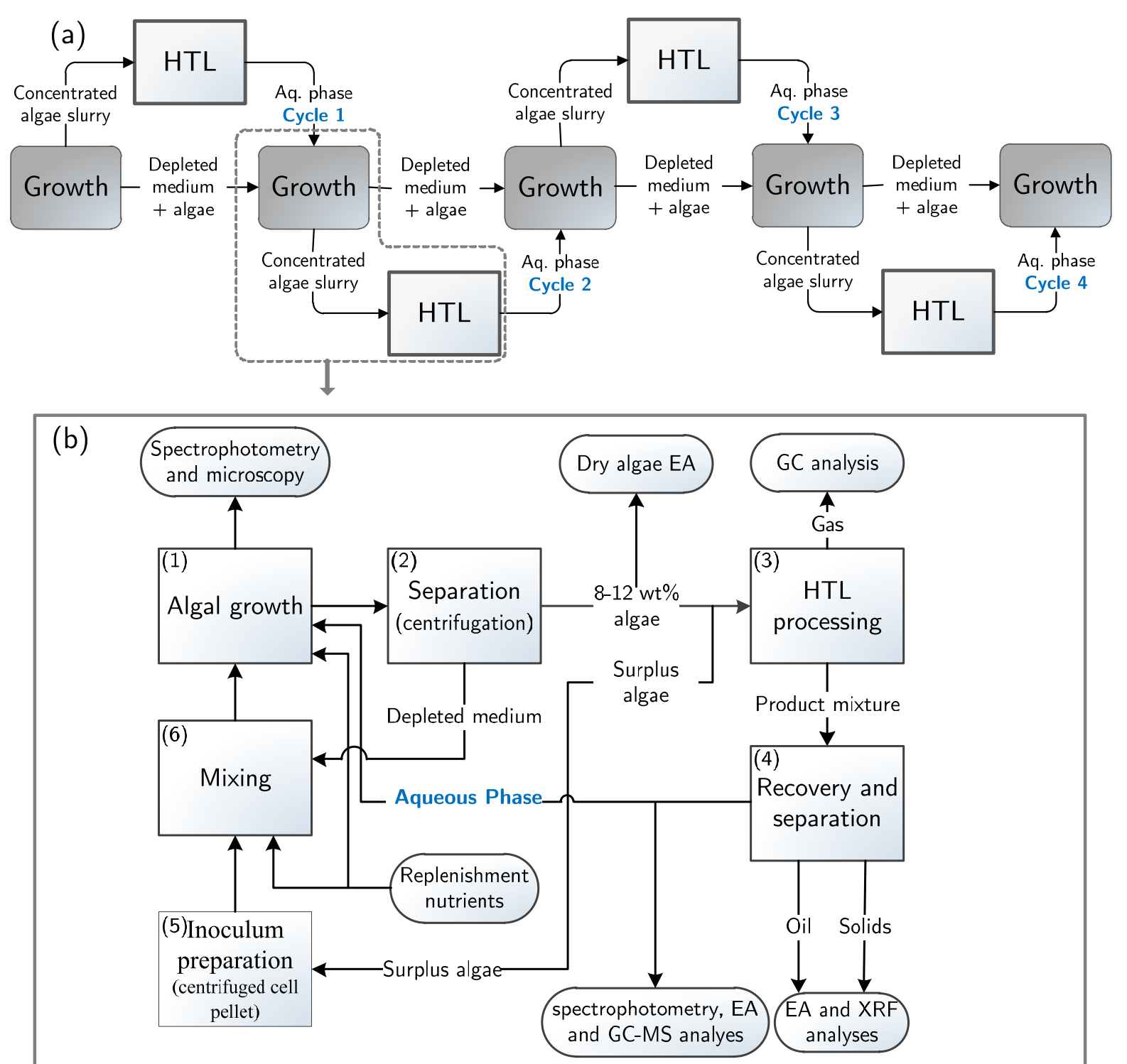

Figure 1. Multiple times recycling of aqueous phase, depleted medium and algae. (a) overall experimental sequences of 4 complete cycles with 5 times growth and 4 times HTL; (b) experimental procedure for every, single cycle

The experimental procedure for every, single cycle is shown in Figure 1(b). For all the cases, the initiation of algae growth denoted the beginning of a new cycle (Stage (1) in Figure 1(b)). Algae were grown under constant growth conditions (Table 1) using 5 reactors representing a total volume of about $10 \mathrm{~L}$. For the first cycle, three times concentrated standard COMBO medium was used (as that for the control test, Section 2.3.), inoculated with a cell pellet from the reactors containing continuously maintained algae (see Section 2.2.). When a biomass concentration of approximately $0.8-1.2 \mathrm{~g} / \mathrm{L}$ was reached, the culture was harvested and dewatered, by means of centrifugation at 8000 rpm for $5 \mathrm{~min}$, for concentrating purposes (Stage (2)). The medium (supernatant) 
separated from the algae (termed "depleted medium" in Figure $1(\mathrm{~b})$ ) was stored at $-23^{\circ} \mathrm{C}$ to be reused in the subsequent cycle (cultivation of the next 'generation' of algae). Dewatering was performed until a concentration of about 8-12 wt\% because the viscosity of the mixture is still acceptable and easy to handle during batch HTL processing (as in this work), but also in case of future continuous HTL operation. A small sample (less than $4 \mathrm{mg}$ ) from the concentrated algae solution was taken for analysis and the rest was used in the processing step (Stage (3)) where HTL was performed.

A total of three batch HTL tests (using $20 \mathrm{~g}$ of feedstock each time) at $300{ }^{\circ} \mathrm{C}$ for $5 \mathrm{~min}$ reaction time (excluding heating time of 6 to $7 \mathrm{~min}$ ) were performed using an autoclave reactor. The batch setup used in this work, the HTL experimental procedure and the products separation method are precisely described in Chapters 2 and 5, as well as the HTL mass balance closure calculations, the oil higher heating value (HHV) determination and the energy recovery of the conversion process to the oil. For the algae heating value, the equation proposed by Channiwala and Parikh ${ }^{11}$ was used as it is known to have a higher accuracy for the HHV estimation of biomass. ${ }^{12}$ As single difference, the total mass of algae produced per cycle (required to know the final concentration after dewatering and thus calculate product yields after HTL) was found in this case by measuring the optical density at the moment of harvesting.

The products obtained from HTL in Stage (3) - i.e. oil, gas, solid residue and water soluble organics- were recovered and separated in Stage (4) and analyzed. Afterwards, the growth medium of the next cycle (Stage (6)) was prepared with the depleted medium and inoculated with a cell pellet of surplus algae, prepared in Stage (5). This 'surplus algae' was the fraction of concentrated algae solution that was not processed during the HTL step of the previous cycle. That mixture (Stage (6)) was then replenished with COMBO nutrients as those were nearly consumed in the recovered depleted medium. They were also added to the culture during growth in amounts depending on the observed algae growth (e.g. when AP nutrients were consumed before reaching the target algae concentration). It is important to note that not only $\mathrm{N}$ and $\mathrm{P}$ nutrition is important but also the rest of COMBO macro- and micronutrients (e.g. K, Ca, S, Fe, etc.). In our previous work (Chapter 5), these nutrients (minimally present in the recovered HTL aqueous phase) were also found to be essential to obtain a growth speed comparable to that in standard medium. When the medium contained only water and aqueous phase (with sufficient $\mathrm{N}$ and $\mathrm{P}$ ), the growth was substantially reduced. The amount of make-up nutrients was always quantified and kept minimal (i.e. only added when needed). 
Harvesting, dewatering, HTL processing and growth medium preparation of the next cycle were all performed during a single day to prevent the influence of nutrients depletion on the concentrated algae solution and to minimize any possible related shock stress to the algae. The aqueous phase after HTL was stored at $-23^{\circ} \mathrm{C}$. About $50 \mathrm{ml}$ of AP were recovered each time from which $10 \mathrm{ml}$ were kept for analyses. When it was confirmed that the algae earlier inoculated were out of the lag phase and already at exponential growth or even further at the linear phase (which, as explained later, depended on HTL setup availability), the remaining $40 \mathrm{ml}$ of aqueous phase were added to the medium.

\subsubsection{Analytical methods}

During growth, samples were taken from the various reactors and centrifuged at 8000 rpm for 5 min to separate the medium (supernatant) and the cell pellet for analyses. With the medium samples, nutrients consumption during the cultivation period could be followed using standardized tests (HACH LANGE) and measured with a spectrophotometer (DR 5000, HACH Corporation also used for OD750 measurements). The compounds quantified were: nitrogen in nitrate $\left(\mathrm{NO}_{3}-\mathrm{N}\right.$, LCK 339), nitrogen in ammonia $\left(\mathrm{NH}_{4}-\mathrm{N}\right.$, LCK 305) and phosphorous in phosphate $\left(\mathrm{PO}_{4}-\mathrm{P}\right.$, LCK 349). With the cell pellets, visual observations were performed using both Optical (Carl-Zeiss microscope) and Scanning Electron Microscopy (SEM; JEOL TSM 5600). Prior to SEM visualization, the algae samples were freeze dried. All the losses of biomass for analysis purposes were quantified.

After dewatering (Stage (2)), a sample was taken from the concentrated algae solution and dried at $105^{\circ} \mathrm{C}$ for $24 \mathrm{~h}$ to determine the elemental composition in terms of nitrogen, carbon and hydrogen using an elemental analyzer (EA, Thermo Scientific Flash 2000). Oxygen was calculated by difference and the measurements were performed in duplicate. If the difference between duplicates was within $\pm 1 \%$, the results were accepted and the average values were taken.

After HTL, the gas product was analyzed by means of gas chromatography (Varian Micro GC CP-4900 containing two analytical columns: Molsieve 5A (10 m) and PPQ (10 $\mathrm{m})$, using helium as carrier gas). With the gas composition, the final pressure and temperature inside the reactor (after cooling) and the gas volume (total reactor inner volume minus the volume occupied by the algal slurry), the amount of gas produced could be calculated. The oil and solid residue products were analyzed for elemental 
composition (EA, Thermo Scientific Flash 2000). Measurements were performed in duplicate and if the difference was within $\pm 1 \%$, the results were accepted and the average values were taken. Also X-Ray Fluorescence spectrometry (XRF) was performed on the ash produced from the solid residue after being treated at $550^{\circ} \mathrm{C}$ for $5 \mathrm{~h}$ (under oxidizing conditions).

Several aqueous phase analyses were carried out. The same standardized tests (HACH LANGE) used for growth medium analysis were used in this case, but also including total nitrogen (TN, LCK 238), and COD (LCK 414 in mg/L $\mathrm{O}_{2}$ ). Organic nitrogen (org$\mathrm{N})$ was calculated by difference from: $\mathrm{TN}-\left(\mathrm{NO}_{3}-\mathrm{N}\right)-\left(\mathrm{NH}_{4}-\mathrm{N}\right)-\left(\mathrm{NO}_{2}-\mathrm{N}\right)$ (nitrite was below detection limits for all the aqueous phases analyzed). $\mathrm{NH}_{4}-\mathrm{N}$ refers to the total concentration of $\mathrm{N}$ in the form of $\mathrm{NH}_{3}+\mathrm{NH}_{4}{ }^{+}$coexisting in equilibrium in water. The methods used to identify and quantify the AP organic compounds (both volatile and non-volatile fractions) are extensively described in Chapter 5. The \% of identified organics was calculated as the sum of identified organic compounds (from the analysis) by the total amount of water-soluble organic substances. The quantification of the total amount of water-soluble organics can be found in Chapter 2.

\section{Results and discussion}

\subsection{Algae growth in standard culture medium}

The startup of the AP-nutrients recycling test (Section 3.2) was strongly dependent on achieving a total of 8 to $10 \mathrm{~g}$ of algae. That specific amount of algae was necessary in order to perform HTL and create enough aqueous phase for the subsequent cycle and for analyses. Therefore, this algae growth experiment in standard medium was performed in five reactors operated in parallel to ensure that this target could be met. It also served us to evaluate possible differences in growth patterns between the 5 reactors and as base case experiment for comparison.

Figure 2 shows the increase of algae concentration over time for a total growth period of $94 \mathrm{~h}$. As can be seen, nearly $0.9 \mathrm{~g} / \mathrm{L}$ of algae were produced in each reactor in about 4 days starting from a concentration of about $0.2 \mathrm{~g} / \mathrm{L}$ under the growth conditions in Table 1. Comparable growth curves were obtained from all the reactors. Therefore, althought a difference in temperature of approx. $1^{\circ} \mathrm{C}$ from reactors 1 to 5 was always existing, it did not cause a major effect on growth. Consequently, averaging the algae concentration data 
obtained from 5 reactors leads to a standard deviation between $\pm 0.002-0.021 \mathrm{~g} / \mathrm{L}$ which was considered negligible and also valid for the AP-nutrients recycling test.

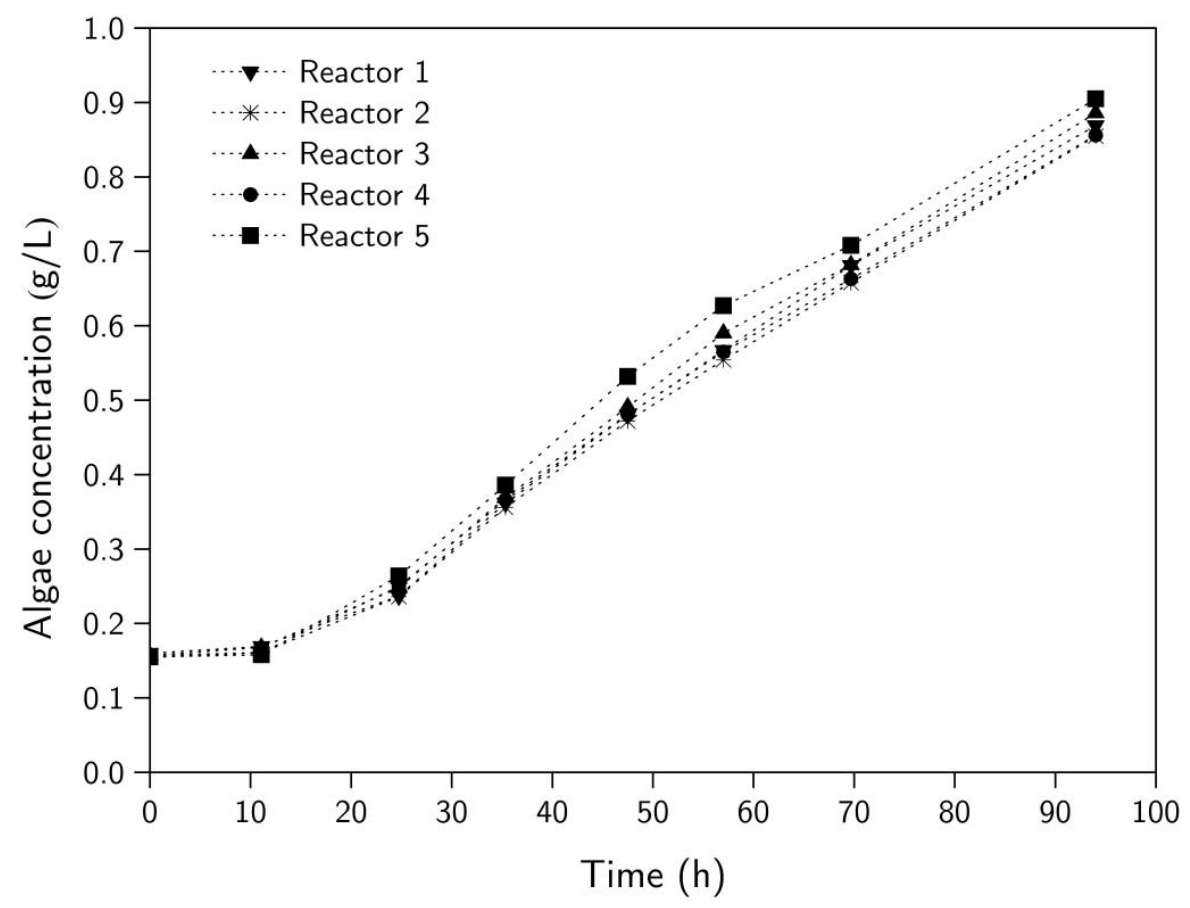

Figure 2. Algae growth in standard COMBO using 5 reactors of the illuminated chamber

\subsection{Algae cultivation under AP-nutrients recycling}

\subsubsection{Algae growth results}

A test sequence was designed, see Figure 1(a), where growth and HTL were combined into 4 cycles and the AP from the $4^{\text {th }}$ cycle was used again in the last cultivation trial. Figure 3 shows the growth response (in terms of algae concentration) per cycle as function of time. To reduce the number of lines, each data point represents the mean value of the measurements obtained from the 5 growth reactors (with their corresponding standard deviation) used in each cycle.

All cycles were successfully completed, regardless of the remarkable complexity of the experimental design. Eventually, an increase in cell density over time was observed in each of the cycles, indicating that the presence of AP cycled four times did not prevent growth. As can be seen, none of the cultures collapsed, at least not within the time frame studied. However, some significant differences and unusual behaviors (e.g. in cycles 2 and 3) can be observed in Figure 3, which will be explained in the following section. 


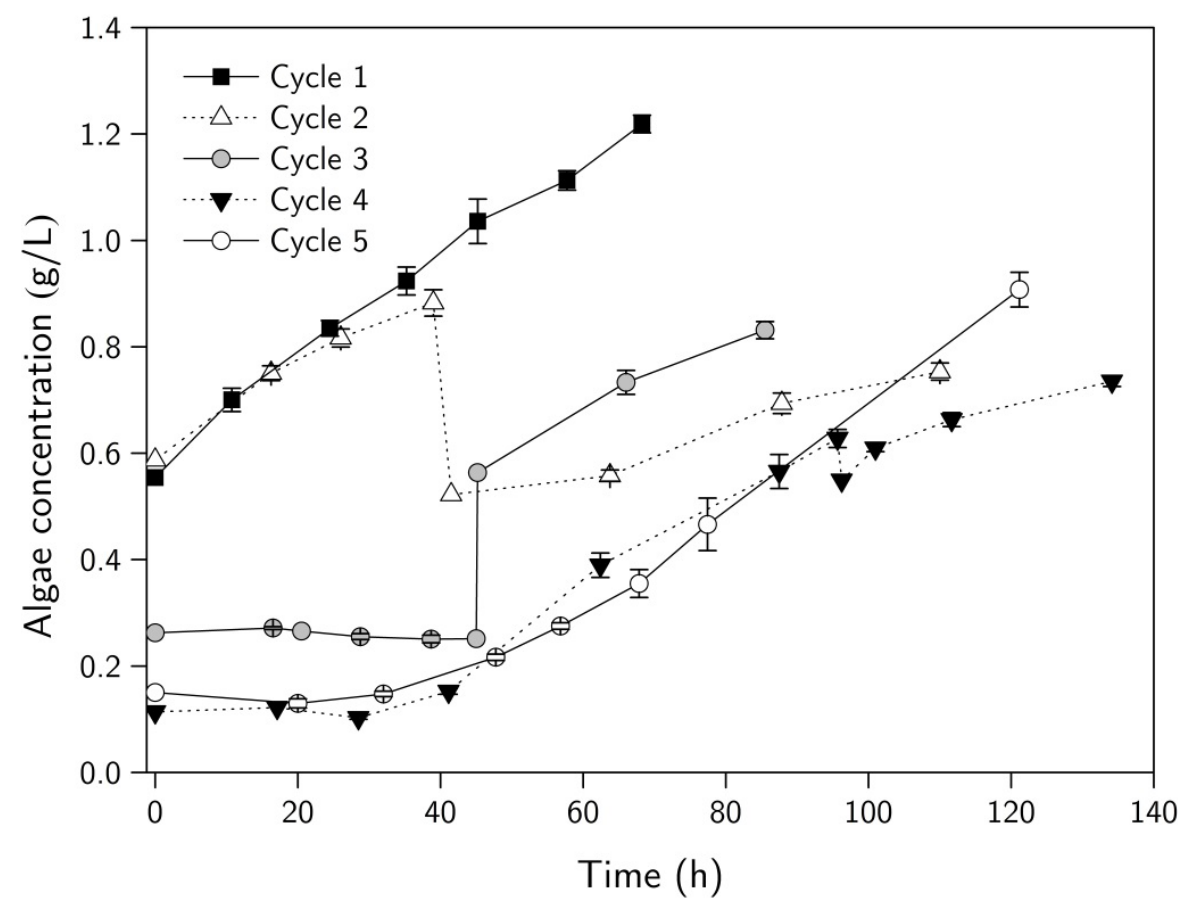

Figure 3. Algae growth per cycle. Error bars represent the standard deviation of the measurements obtained from the 5 growth reactors used

Because of a limited HTL setup availability, each cycle had different run times and, as consequence, in some of those tests growth was terminated before reaching the desired 1 $\mathrm{g} / \mathrm{L}$; e.g. in cycles $2(0.75 \mathrm{~g} / \mathrm{L})$, cycle $3(0.83 \mathrm{~g} / \mathrm{L})$ and cycle $4(0.74 \mathrm{~g} / \mathrm{L})$. These differences also explain why the initial concentration in cycles 3,4 and 5 is smaller than that of cycles 1 and 2. It is inherent to the test design, that the HTL setup availability affects the growth pattern in the cultivation tests, as all the algae unused for HTL were used as inoculum of the next cycle (in order to preserve all the mass in the system for mass balance purposes). For instance in cycle 2, less than $1 \mathrm{~g} / \mathrm{L}$ was obtained at the end of the growth period, therefore less surplus algae (see Figure 1(b)) was left after the HTL stage. That lesser amount of surplus algae, is later used as inoculum of cycle 3 , leading to a lower initial algae concentration.

Also related to HTL setup availability, the sudden drop in concentration noticeable in cycle 2 (at $39 \mathrm{~h}$ ) corresponds to a removal of algal biomass from the growth culture (by centrifugation). A significant increase in cell density was expected to happen reaching a very high algae concentration before being able to perform HTL. Therefore, prior to a possible collapse of the culture due to nutrients and/or light shortage caused by an envisaged high algae concentration, a certain amount of algae was deliberately removed and, after that, AP from cycle 1 was added. 
In contrasts with cycles 1 and 2, after $20 \mathrm{~h}$ of cultivation and adaptation, growth did not develop in cycle 3, even though having sufficient replenishing amounts of nutrients (see stage 6 in Figure 1(b)). Therefore, AP was added at that point intending to accelerate the growth. Also later (at $45 \mathrm{~h}$ ), the algae removed from cycle 2 were added to the culture, since in the whole experiment waste streams were undesired. That algae addition is reflected by the abrupt increase in concentration in cycle 3 (see Figure 3).

In cycle $4, \mathrm{AP}$ from cycle 3 was added after $96 \mathrm{~h}$ from the start of the test. That addition was done relatively late to ensure that the lag phase was terminated (recalling that a very long lag phase was observed in the previous cycle) and that harvesting time would fall in a day with full HTL equipment availability. Right before AP addition, a small drop in concentration can be observed, which corresponds to the addition of a small amount of demineralized water to offset medium evaporation. Finally, $56 \mathrm{~h}$ after starting cycle 5, AP was added to the medium. As can be seen in Figure 3, cycles 4 and 5 exhibited very similar algae growth patterns.

A relatively long lag phase was observed in cycles 3, 4 and 5 while that was not detected in cycles 1 and 2 . In cycle 1, a linear increase of concentration can be observed form the beginning of the growth indicating a constant growth rate. This generally occurs when a specific requirement such as light is limiting cell division. ${ }^{10}$ That appeared to be the case for cycle 1 since, after inoculation, the initial concentration was already well above the concentration where light becomes limiting $(0.3 \mathrm{~g} / \mathrm{L})$ and nutrients were in excess. The same seems to be happening for cycle 2 where again a large inoculum (volume of the inoculum compared to the volume of the medium) was used with non-existing/very short (as there is no measurement at e.g. $10 \mathrm{~h}$ ) lag phase. Andersen ${ }^{13}$ stated that, generally, the lag phase is short when, as in cycle 1 and 2, a large inoculum is used and the parent culture has been acclimated to similar growth conditions. On the other hand, the initial algae concentration in cycles 3,4 , and 5 was below the concentration limit towards the light-limiting region and using, as inoculum, algae from the previous cycle being at the linear growth phase. Hence, this could have been one of the causes for a longer lag phase, since a lag phase is unlikely to occur (or it is very short) when the inoculum comes from a exponentially growing culture. ${ }^{10}$ Lag phase can also be a response to several cell stress factors such as:

- The fact that the same algae was used repeatedly (i.e. no fresh algae added, the inoculum always being algae unused from the previous cycle). A fraction of the same cells were, several times, centrifuged and in high concentrated form (after 
dewatering) for relatively long time prior to inoculation (while HTL was being performed);

- Possible accumulation of non-degradable substances in the depleted medium (see Figure 1(b)) throughout the cycles. The cells might have needed longer time for adaptation to their presence.

Accumulation can occurred by means of AP addition, but also by excretion of auto inhibitory substances, as certain algae are able to produce and release a metabolite that, when accumulating, can be toxic to themselves. ${ }^{14}$ This process is known as autoinhibition and Nannochloropsis sp. ${ }^{15}$ and Chlorella vulgaris ${ }^{16}$ (from the green algae division as the species used in this work) have shown this behavior. The accumulation of substances is further evaluated in Section 3.2.3 after looking at the composition of the AP of each cycle.

In conclusion, algae could grow over 5 cycles of repeating cultivation and HTL, but exhibiting a period of lag phase in the later cycles, which is probably the result of a combination of several stress-related effects. Furthermore, the control experiment in COMBO standard medium (shown in previous section) was compared to the growth in cycle 5 (Figure 4). Both experiments were performed in the same batch-wise cultivation system and under the same growth conditions with almost identical initial algae concentration and total amount $\mathrm{N}$ and $\mathrm{P}$ added. The only difference was the presence of $\mathrm{AP}$ in cycle 5 and, yet more, the maximal accumulation possible of potential toxic compounds from the previous cycles.

As pointed out in our previous investigation (Chapter 5), a clear identification of the exponential growth phase is difficult without inline cell density measurements. Therefore, we chose the algae daily productivity (Prod. in Figure 4), as growth perfromace indicator, calculated from the slope of the linear phase of the growth curves in Figure 4. This linear region developed from the moment the algae cultivation was under light limiting conditions (above $0.3 \mathrm{~g} / \mathrm{L}$ ). In that region, nutrients were still in excess (thus no $\mathrm{N}$ or $\mathrm{P}$ limitation) and, in cycle 5 , AP was already added to the medium.

A longer lag phase can be observed for cycle 5 likely being the response to one or several stress related factors discussed above. Growth delay can be avoided when working with a continuous operating system for the complete sequence of growth, harvesting and HTL. From the moment cell division started, algae growth in cycle 5 showed a similar behavior to that in COMBO medium, exhibiting comparable algae productivities (0.20 and 0.25 
$\mathrm{g} / \mathrm{L} / \mathrm{d}$ ). For cycle 5, the productivity right after AP addition was even slightly higher, however the difference observed might not be statitically relevant.

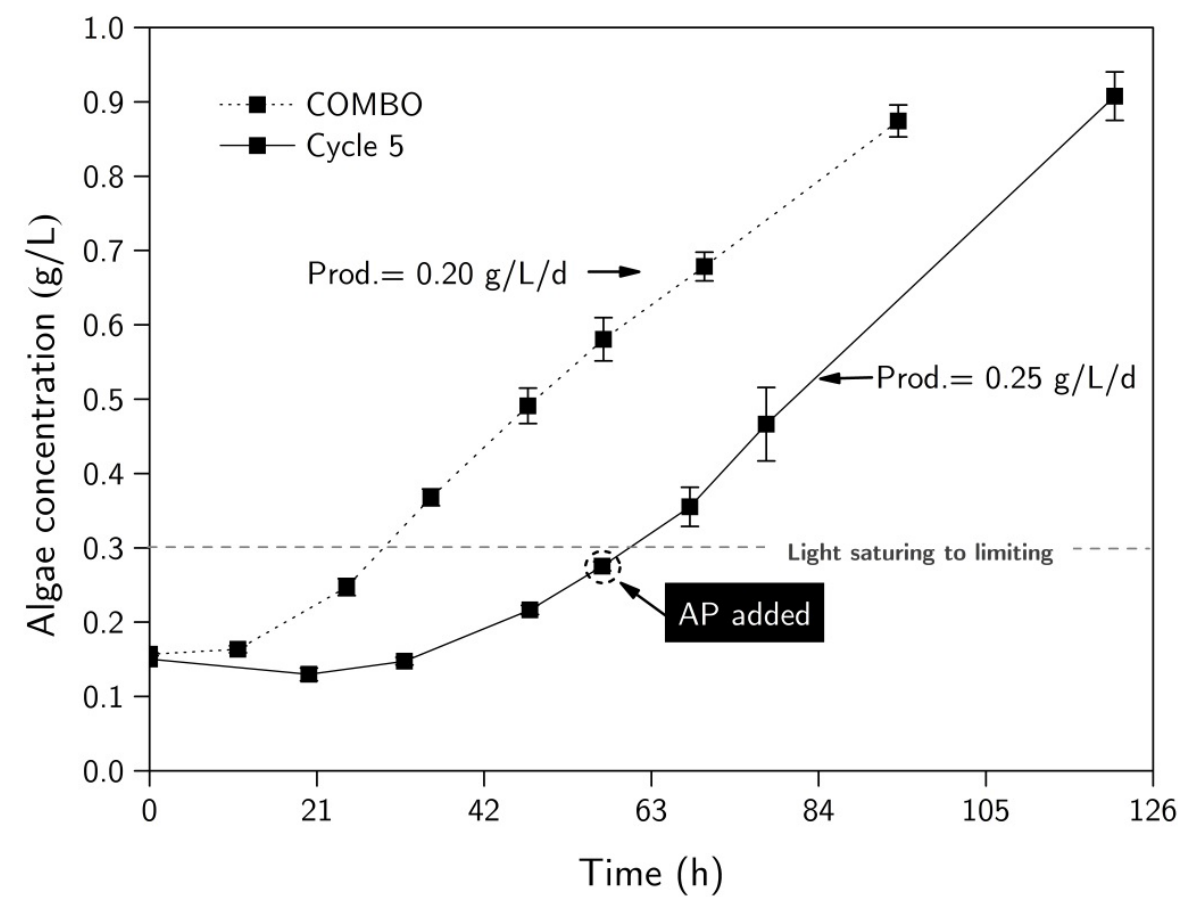

Figure 4. Algae growth in standard COMBO medium and in cycle 5. Error bars represent the standard deviation of the measurements obtained from the 5 growth reactors used

The comparison of cycle 5 with the COMBO control experiment also provides an indication of the overall potential on nutrients savings. From the total amount of nitrogen added in the $5^{\text {th }}$ cycle, $39.5 \%$ was replaced by $\mathrm{N}$ from recycled $\mathrm{AP}$ and depleted medium (nitrate, ammonia and org-N), while showing a comparable algae daily productivity in the linear growth phase to that in the control test. For phosphorus, 65.7 $\%$ of the total $\mathrm{P}$ was replaced by $\mathrm{P}$ from $\mathrm{AP}$ and depleted medium. For at least 5 cycles, nearly optimal growth (when compared to the growth in standard COMBO medium) was maintained, while recycling AP and reusing the untreated depleted medium (Figure 1(b)). Hence, no fresh algae nor water were added and thus, without reducing HTL-oil productivity, substantially savings in nutrients can be achieved.

\subsubsection{Algae cells observations}

During the test, a visual inspection of the cells was performed by SEM analysis. In Figure 5(a), the images of the algae at the start of cycles 1 (initial inoculum), 3 and at the end of cycle 5 are displayed. 


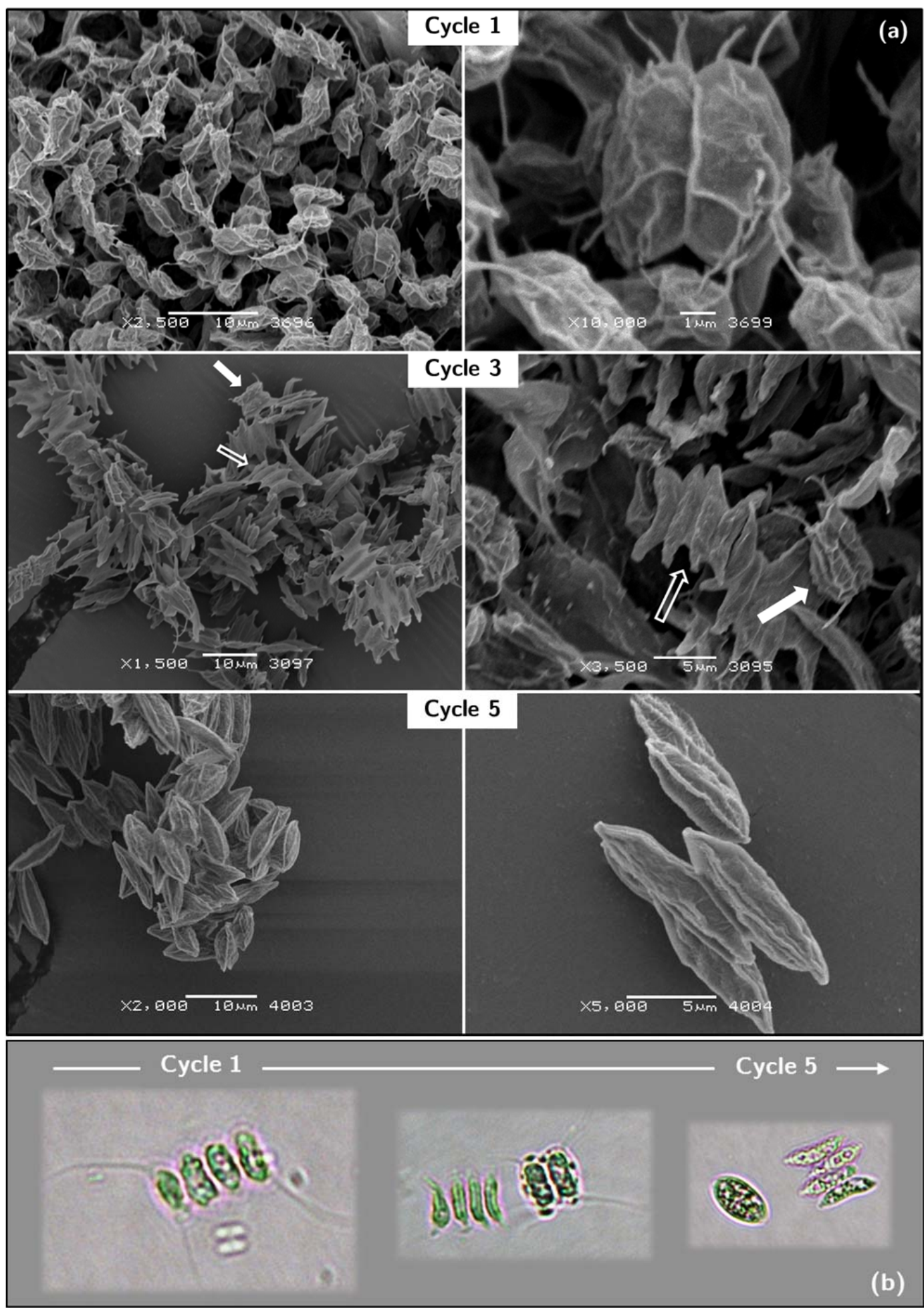

Figure 5. (a) SEM pictures of the cells in cycles 1, 3 and 5; (b) optical microscope images displaying the progress of the cells changes

To our surprise, clear differences were observed. The initial culture (cycle 1) consisted of Desmodesmus sp. cells with their familiar characteristic spines and oval shape (for reference, see Chapter 2). In cycle 3, part of the culture was still identical to the cells in 
cycle 1 (follow solid arrows in Figure 5(a)), however, another part of the cells already showed different morphology; having no spines and a more sickle-like shape (follow open arrows in Figure 5(a)). Finally, at the end of cycle 5, no cells with spines could be identified. Moreover, the culture now consisted of unicells, whereas in the previous cycles they were found in colonies of two or four cells. The optical microscope images displayed in Figure 5(b) clearly show the progress of the changes observed. Two possible explanations were considered for these changes:

- Contamination by the presence of another algae-like organism; or

- Cell morphological changes over the cycles.

Algae growth was not performed under axenic conditions, as in envisaged algae biorefinery configurations outdoor cultivation is preferable in order to reduce both capital investment and operational expenses. Considering that the algae was originally cultivated in an outdoor pond, possibly competing species were already present in the purchased culture, and could have aroused due to the changing environment throughout the cycles. However, in prior growth experiments where various parameters (light, $\mathrm{pH}, \mathrm{NH}_{4}-\mathrm{N}$ addition etc.) were drastically changed, no such changes were observed. In all those cases the cells seemed identical to those at the start, making this option less probable.

On the other hand, structural cell alterations due to environmental changes (e.g. presence of xenobiotic chemical substances) have been often described. ${ }^{17-19}$ The closest reference to our observations was given by El-dib et al. ${ }^{17}$ in their work on the effect of triazine herbicides on Scenedesmus sp. With increasing concentration of triazines in the medium, the cells showed clear morphological changes (nearly) identical to those described here. These nitrogen aromatic heterocycles (pyridine derivates) were found in the AP during our previous study (Chapter 5) and were confirmed to be present in the AP used in this test (as shown later in Section 3.2.3). Therefore, it was concluded that, in agreement with the results of El-dib et al. ${ }^{18}$, the continuous and direct exposure of our algae to these compounds is a very probable cause of the observed morphological changes.

\subsubsection{HTL products distribution and analyses}

The HTL products distribution (on algae dry basis) per cycle are shown in Figure 6. Table 2 shows the elemental composition and higher heating value (HHV) of both the cultivated algae and the oil produced after HTL. The energy recovery that compares the energy content of the algal feedstock to that of the oil is also presented in Table 2. 
Comparable product yields were obtained from all the cycles. About 5-6 mg of dry algae were processed each time yielding to $50 \mathrm{wt} \%$ oil with and energy content of $33.4 \mathrm{MJ} / \mathrm{kg}$. As can be seen in Table 2, the $\mathrm{C}$ and $\mathrm{H}$ contents of the oils were relatively similar but with larger difference on $\mathrm{N}$ content in line with the $\mathrm{N}$ composition of the algae. As reflected by comparing cycles 1 and 4, higher $\mathrm{N}$ content in the algae led to higher $\mathrm{N}$ content in the oil. The algae produced during cycle 4 had the highest amount of $\mathrm{N}$, as the growth period was longer and, hence, the medium received a larger amount of $\mathrm{N}$ nutrients (apart of AP, more fresh COMBO nutrients were added compare to that in other cycles).

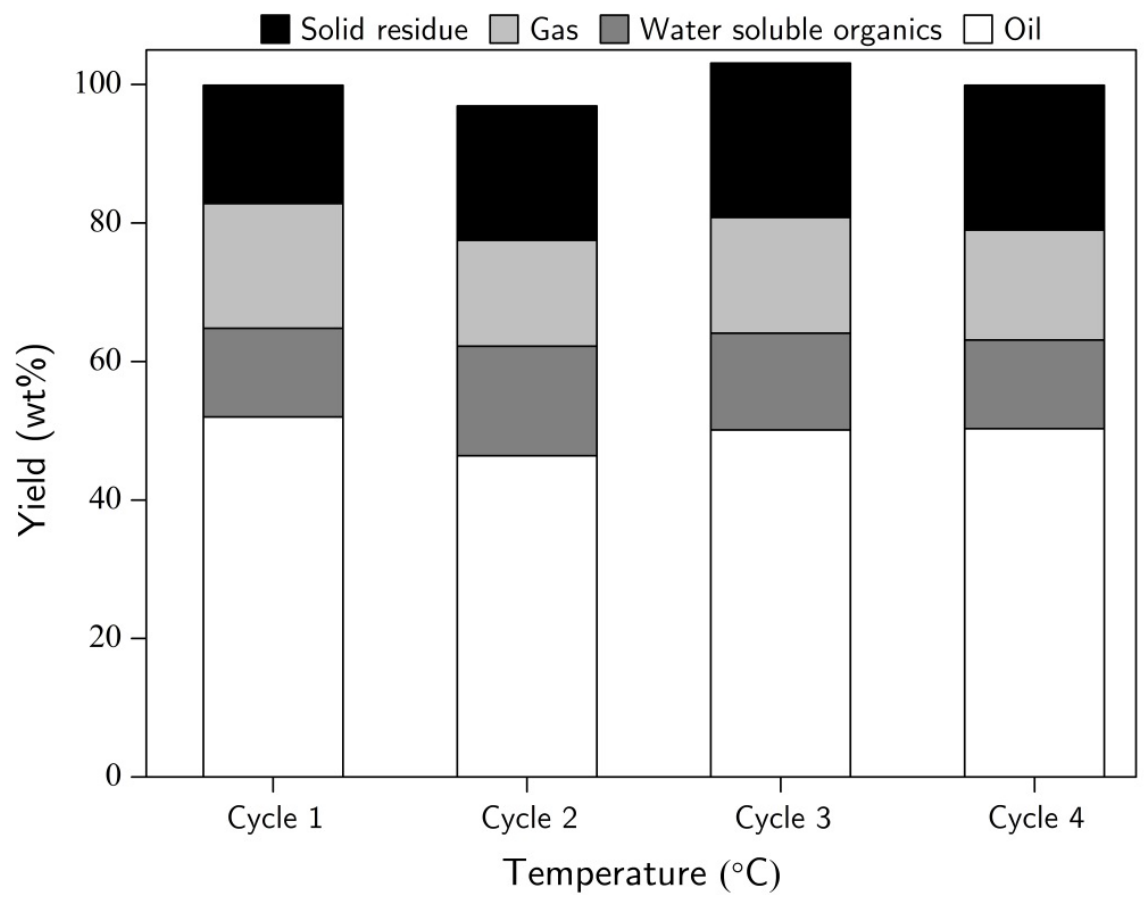

Figure 6. HTL product yield distribution

Table 2. Algae grown (A) and oil produced $(\mathrm{O})$ : elemental composition (on dry basis) and properties

\begin{tabular}{|c|c|c|c|c|c|c|c|c|c|c|c|}
\hline \multirow[t]{2}{*}{ Cycle } & \multicolumn{2}{|c|}{ N (wt\%) } & \multicolumn{2}{|c|}{ C (wt\%) } & \multicolumn{2}{|c|}{ H (wt\%) } & \multicolumn{2}{|c|}{$\mathrm{O}^{\mathrm{a}}(\mathrm{wt} \%)$} & \multicolumn{2}{|c|}{$\begin{array}{c}\text { HHV } \\
(\mathrm{MJ} / \mathrm{kg})\end{array}$} & \multirow{2}{*}{$\begin{array}{c}\text { Energy } \\
\text { recovery (\%) }\end{array}$} \\
\hline & A & $\mathrm{O}$ & A & $\mathrm{O}$ & A & $\mathrm{O}$ & A & $\mathrm{O}$ & A & $\mathrm{O}$ & \\
\hline 1 & 5.4 & 4.6 & 53.1 & 69.9 & 7.1 & 8.6 & 34.4 & 16.8 & 23.3 & 33.0 & 73.2 \\
\hline 2 & 5.1 & 4.2 & 51.0 & 72.1 & 7.2 & 9.3 & 36.8 & 14.4 & 22.4 & 34.8 & 71.9 \\
\hline 3 & 5.9 & 5.4 & 49.9 & 69.2 & 7.1 & 8.4 & 37.0 & 17.1 & 21.9 & 32.5 & 73.8 \\
\hline 4 & 7.5 & 6.3 & 50.4 & 69.3 & 7.1 & 8.8 & 34.9 & 15.6 & 22.2 & 33.2 & 74.3 \\
\hline
\end{tabular}

${ }^{a}$ By difference. For the oil, the values directly represent oxygen since no ash was found in that product. For the algae, a small amount of ash was present, thus the values represent oxygen + ash. 
The oil and water soluble organics yields were slightly different to those reported in our previous HTL study (Chapter 2), while the elemental composition and energy content of the oil were in the same range. Those differences can be related to the different characteristics of the feedstock (e.g. biochemical composition) used in each study, as cultivation was performed in significantly different systems (industrial scale outdoors pond in the previous work vs. lab scale batch system with closed reactors in this work). Also in this study and in accordance with our previous work, more oil was produced compared to the reported values in other investigations. ${ }^{20,21}$ However, direct comparison is difficult since, as reported in the review work by López et al. ${ }^{22}$ significant variations (experimental, data analysis, microalgae type, etc.) exists between investigations. The gas composition from the various cycles was also comparable consisting of mainly $\mathrm{CO}_{2}(\sim 70$ vol\%) and CO. Up to $73 \%$ of the calorific value of the algal biomass was retrieved in the oil phase. The total energy recovery value can be even higher when also the energy content of the gas is utilized. Furthermore, the gas produced could also be used as source of $\mathrm{CO}_{2}$ for algae growth or integrated into other processes of an algae biorefinery when recovered and maintained at high pressure (e.g. supercritical $\mathrm{CO}_{2}$ extraction). Similarly, the HTL solid residues of each cycle had a comparable elemental composition (on average, $6.8 \mathrm{wt} \% \mathrm{~N}, 61.6 \mathrm{wt} \% \mathrm{C}, 6.2 \mathrm{wt} \% \mathrm{H}$ and $25.5 \mathrm{wt} \% \mathrm{O}$ ).

Overall, the detected differences between the cycles were relatively small showing that the changes in algae morphology observed (and possibly also internal adjustments involving biochemical and physiological acclimation) and the possible accumulation of non-biodegradable compounds (via the depleted medium recycle) did not seem to affect the HTL yields and products elemental composition.

The HTL solid residue of each cycle was treated at $550^{\circ} \mathrm{C}$ for $5 \mathrm{~h}$ to recover the ash. After that, the resulting ashes were combined to create a sufficient amount of sample for XRF analysis (Table 3). The minerals concentrations of the combined ashes provide a reasonable approximation to that of a single cycle, as the ash content (7.7 wt\%), composition (N, C and $\mathrm{H}$ ) and yield of solids of each cycle were in the same range. In Table 3, the possible origin of each element is also indicated which could be: COMBO standard medium and AP $(C B+A P$ in Table 3$)$ or from corrosion or leaching of the reactors walls due to the relatively harsh conditions applied during HTL ( $I$ in Table 3 ). 
Table 3. Minerals concentration in ash from HTL solid residue fraction

\begin{tabular}{ccc}
\hline Elements & In ash (wt\%) & Present in \\
\hline $\mathrm{Ca}$ & 6.57 & $\mathrm{CB}+\mathrm{AP}{ }^{\mathrm{b}}$ \\
$\mathrm{Co}$ & 0.08 & $\mathrm{CB}+\mathrm{AP}$ \\
$\mathrm{Cu}$ & 0.30 & $\mathrm{CB}+\mathrm{AP}, \mathrm{I}$ \\
$\mathrm{Fe}$ & 19.7 & $\mathrm{CB}+\mathrm{AP}, \mathrm{I}$ \\
$\mathrm{K}$ & 2.30 & $\mathrm{CB}+\mathrm{AP}$ \\
$\mathrm{Mg}$ & 7.20 & $\mathrm{CB}+\mathrm{AP}$ \\
$\mathrm{Mn}$ & 0.37 & $\mathrm{CB}+\mathrm{AP}, \mathrm{I}$ \\
$\mathrm{Mo}$ & 0.38 & $\mathrm{CB}+\mathrm{AP}, \mathrm{I}$ \\
$\mathrm{P}$ & 12.0 & $\mathrm{CB}+\mathrm{AP}$ \\
$\mathrm{S}$ & 0.06 & $\mathrm{CB}+\mathrm{AP}$ \\
$\mathrm{Si}$ & 2.22 & $\mathrm{I}$ \\
$\mathrm{Zn}$ & 0.31 & $\mathrm{CB}+\mathrm{AP}$ \\
$\mathrm{Al}$ & 0.69 & $\mathrm{I}$ \\
$\mathrm{Cr}$ & 4.79 & $\mathrm{I}$ \\
$\mathrm{Ni}$ & 2.02 & $\mathrm{I}$ \\
$\mathrm{Sn}$ & 0.96 & - \\
$\mathrm{Ti}$ & 0.24 & $\mathrm{I}$ \\
\hline
\end{tabular}

${ }^{\text {a }}$ Not normalized for loss on ignition. ${ }^{\mathrm{b}} \mathrm{CB}+\mathrm{AP}$

$=$ from addition of $\mathrm{COMBO}$ and $\mathrm{AP} .{ }^{\mathrm{c}} \mathrm{I}=$

Present in INCOLOY 825.

Iron, the major compound in the ash, was present in the initial medium as COMBO nutrient in the form of $\mathrm{FeCl}_{3}$ and as chelating agent in the form of Fe-EDTA. However, the reactor wall material (INCOLOY 825 alloy) could also have been the source of it. Indeed, the presence of $\mathrm{Cr}$ and $\mathrm{Ni}$ in the solid residue indicates that a certain degree of reactor wall damaged occurred, as they could not come from any other source. Ni and $\mathrm{Cr}$ are, together with $\mathrm{Fe}$, the major constituents of that specific alloy (38-46\% Ni and 19.5$23.5 \%$ Cr). Those heavy metals might have ended up in the solid residue after precipitation and/or adsorption by the microalgae and, most likely, they were also present in solution during growth. Certainly, the presence of Ni in the HTL aqueous phase was shown in our previous investigation (Chapter 5), but at a concentration far below toxicity levels. However, a concentration increase of such compounds cannot be ruled out in this case, since it could be the result of accumulation along cycles. Elevated levels of these metal ions are toxic to most prokaryotic and eukaryotic organisms ${ }^{10}$, therefore, their control becomes a must in the frame of a recycling configuration. In addition, although the amount of silicon in the alloy used is very low, a significant 
amount of this substance was found in the ash. Most likely, traces of the glass microfiber filters (Whatman GF/B borosilicate glass) used for solid residue separation were the source of Si.

After iron, phosphorous was the second most prevalent compound in the ash. Consequently, the savings in $\mathrm{P}$ by recycling the aqueous phase can be further increased by regaining it also from the solid residue after HTL. Azuara et al. ${ }^{23}$ showed the possibilities of that by acid leaching from ashes of pig manure, but dedicated tests for HTL residues are recommendable. On the other hand, if complete solubilization of the solid residue in water could be achieved, it could be used as direct fertilizer containing a mixture of $\mathrm{C}, \mathrm{P}, \mathrm{N}$ and other nutrients.

\subsubsection{Aqueous phase composition}

At the time the repetitive recycling experiment was terminated, it was still not sure if more cycles would eventually lead to a slower growth due to accumulation of potential toxic compounds. For that reason the AP samples produced per cycle were analyzed to identify accumulating compounds. First, spectrophotometry analyses on the HTL process water were performed to identify and quantify the various $\mathrm{N}$ and $\mathrm{P}$ substances as well as to obtain an indication of the dissolved organic compounds by means of chemical oxygen demand (COD). Table 4 lists the concentrations of those compounds in the AP obtained from each cycle.

Table 4. Nitrogen, phosphorous and COD composition of the aqueous phase of each cycle (in mg/L)

\begin{tabular}{ccccc}
\hline Compounds & Cycle 1 & Cycle 2 & Cycle 3 & Cycle 4 \\
\hline $\mathrm{NO}_{3}-\mathrm{N}$ & 97 & 121 & 107 & 128 \\
$\mathrm{NH}_{4}-\mathrm{N}$ & 1402 & 745 & 1474 & 2180 \\
$\mathrm{Org}^{\mathrm{N}} \mathrm{N}^{\mathrm{a}}$ & 1531 & 1244 & 1599 & 2442 \\
$\mathrm{PO}_{4}-\mathrm{P}^{\mathrm{b}}$ & 180 & 180 & 590 & 778 \\
$\mathrm{COD}^{\mathrm{c}}$ & 29200 & 28200 & 35800 & 43000 \\
\hline${ }^{a}$ Organic nitrogen calculated as $\mathrm{TN}-\left(\mathrm{NO}_{3}-\mathrm{N}\right)-\left(\mathrm{NH}_{4}-\mathrm{N}\right)-$ \\
$\left(\mathrm{NO}_{2}-\mathrm{N}\right) ;$ nitrite always below detection limits. ${ }^{\mathrm{b}}$ Phosphorous \\
in the form of phosphate. ${ }^{\mathrm{c}}$ In $\mathrm{mg} \mathrm{O}_{2} / \mathrm{L}$.
\end{tabular}

An important fraction of $\mathrm{N}$ is contained in organic constituents, whose utilization by Desmodemus sp. proved to be difficult (see Chapter 5) under similar growth conditions applied in this study. Therefore, AP-organic N should be recovered through periodical conversion (which can also be done after several recycles) into ammonia by means of AP 
pretreatment (e.g. supercritical water gasification). This will also help in reducing the accumulation rate of other substances that could potentially inhibit growth. The highest concentration of $\mathrm{NH}_{4} \mathrm{~N}, 0.6 \mathrm{mM}$, was found in the AP of cycle 4. In Chapter 5 we proved that $\mathrm{NH}_{4}-\mathrm{N}$ concentration up to $3.4 \mathrm{mM}$ did not lead to growth inhibition (for the same microalgae), thus no ammonia toxicity was expected in any of the 5 cycles.

From Table 4, it can be observed when comparing the AP composition from cycles 1 and 4 that all concentrations increased. For both total-N and organic compounds (via COD value), the increase is around 50\%, whereas for $\mathrm{P}$ this is $300 \%$. Remarkably, the steep increase of $\mathrm{NH}_{4}-\mathrm{N}$ took place from cycle 3 to 4 , whereas for $\mathrm{P}$ this was already seen from cycle 2 to 3 . Total phosphorous determination was also performed on the AP, but the results obtained were nearly equal to those for $\mathrm{P}$ in the form of only $\mathrm{PO}_{4}{ }^{3-}$ indicating that phosphorous in the aqueous phase was mostly present in the form of $\mathrm{PO}_{4}{ }^{3-}$.

Nutrients analyses were also performed on the depleted medium obtained after the dewatering (Stage (2) in Figure 1(b)) of each cycle, showing only traces of unconsumed inorganic $\mathrm{N}$ and $\mathrm{P}$. Thus, same minor concentrations were present in the water of the concentrated algal slurry used for HTL. Consequently, the nitrate and phosphate as well as ammonia and the organic fraction in the AP were produced from the algal biomass itself.

The $\mathrm{N}$ content of the algae produced increased throughout the cycles (see Table 2), which might be related to the increase in $\mathrm{N}$ added during growth throughout the cycles; e.g. in cycle 2 a total amount of $279 \mathrm{mg}$ of $\mathrm{N}$ (from make-up nutrients and AP in total) was added while for cycle 4 it was $585 \mathrm{mg}$. Consequently, the increase in $\mathrm{N}$ content in the AP seems to be related to the increase of $\mathrm{N}$ in the algae, indicating that the conversion rate of $\mathrm{N}$ and $\mathrm{P}$ substances (from the algae to AP soluble compounds) during HTL depends on their concentration in the cells.

The same probably occurred for phosphorous, however, in contrast to the $\mathrm{N}$ supply, the total supply of $\mathrm{P}$ to cycles 2 to 4 was less than that in cycle 1 . In cycles 2 to 4 , the N:P ratio in the medium (by the supply of AP and make-up nutrients) was always higher than that in cycle 1 . That indicates possible $\mathrm{P}$ limiting conditions which could cause $\mathrm{P}$ storage within the cells in the form of polyphosphate, the so-called luxury uptake. Acidinsoluble polyphosphate is stored when the external phosphate concentration becomes limiting. ${ }^{24} \mathrm{P}$ limiting conditions could have been the cause of intracellular $\mathrm{P}$ increase throughout the cycles leading to the same gradual rise in $\mathrm{P}$ in the aqueous phase. Cycle 2 had the highest total N:P supply ratio of 14.5 compared to a ratio of 9.5 for cycle 1 
coinciding with the largest increase in $\mathrm{P}$ in the AP from cycle 2 to 3 . On the other hand, Jhon and Flynn et al. ${ }^{25}$ reported that not only $\mathrm{P}$ in the medium affects the $\mathrm{P}$ accumulation in the cells but also the N-status (in low or high concentration) and the different forms of $\mathrm{N}$. Above discussion illustrates the difficulty of explaining the observed results as, in a recycling configuration as used here, an AP is added that contains different forms of $\mathrm{N}$ and has a variable composition throughout the cycles.

Regardless of the AP composition changes along the cycles, the concentrations were, on average, very similar to those in the AP used in our previous study (Chapter 5) and in studies by others. ${ }^{6}$

To investigate the aqueous phase organics, GC-MS analyses were performed in order to identify potentially inhibiting compounds, and to evaluate the potential accumulation of these compounds. Table 5 shows the concentration (in $\mathrm{mg} / \mathrm{L}$ ) of the identified compounds, grouped in categories. In the Appendix of this work, examples of the GC-MS chromatograms obtained after analysis and the compounds present in each chemical class of Table 5 can be found.

Table 5. Concentration (in $\mathrm{mg} / \mathrm{L}$, mean of triplicate $\mathrm{RSD} \approx 30 \%, \mathrm{n}=3$ ) of organics in aqueous phase

\begin{tabular}{ccccc}
\hline Compound & Cycle 1 & Cycle 2 & Cycle 3 & Cycle 4 \\
\hline Acetic acid & 4441 & 4800 & 4150 & 3994 \\
Ethanol & 481 & 630 & 718 & 129 \\
Acetone & 281 & 421 & 478 & 26 \\
Polyols & 559 & 694 & 546 & 514 \\
Amines & 125 & 130 & 310 & 312 \\
$>$ C $_{2}$ carboxylic acid & 235 & 368 & 185 & 393 \\
Aminoacids & 133 & 150 & 162 & 463 \\
Cyclic dipeptides & 157 & 200 & 192 & 487 \\
Nitrogen aromatic & 867 & 780 & 1054 & 1210 \\
Hydroxyacids & 1454 & 1431 & 804 & 864 \\
Sugars & 615 & 496 & 541 & 367 \\
Pyrrolidones & 1492 & 1652 & 1531 & 2596 \\
Phenols & 76 & 57 & 49 & 71 \\
Sum of identified & 10916 & 11809 & 10720 & 11426 \\
Water soluble substances & 13063 & 13927 & 14328 & 14460 \\
\% Identified & $84 \%$ & $85 \%$ & $75 \%$ & $79 \%$ \\
\hline
\end{tabular}

${ }^{a}$ Pyroglutamic acid as most abundant compound in that category.

Chemical constituents observed in the first cycle resulted similar to those obtained in the AP of our previous study (Chapter 5) with acetic acid as the most abundant compound 
followed by pyroglutamic acid. Zhou et al. ${ }^{26}$ also found acetic acid as the most predominant AP constituent and Valdez et al. ${ }^{27}$ reported the presence of both.

Briefly, the water soluble fraction is formed by a large number of constituents. As explained in Chapter 5, three main groups of compounds are found in the AP:

- Substances that can be considered non-toxic metabolites which could be used by the algae as carbon and nitrogen sources (sugars, carboxylic acid, aminoacids and hydroxyacids);

- Compounds that can still be considered degradable (or consumable) but are known to exhibit biological activity. In particular, nitrogen containing compounds like pyroglutamic acid (0.1-0.2 wt\%), cyclic dipeptides (0.02-0.05 wt\%) and amines, and;

- "Xenobiotics" constituents such as nitrogen containing aromatics with concentrations in the AP ranging from 0.09 to $0.12 \mathrm{wt} \%$. These compounds have structural analogy with triazine and, due to relatively high concentration, could in fact cause some biological effect. ${ }^{17}$

The composition of the water phases obtained from the various cycles was rather similar, with an important increase of some organics, mainly nitrogen containing compounds, in the last cycle. That can be partially due to the observed increase of nitrogen in the $4^{\text {th }}$ generation of algae (see Table 2), to a certain bio-accumulation (concentration of the organics which are barely water-soluble onto microalgae) or, for cyclic dipeptides, also to a change in the proteome of the microalgae with, in instance, a change in the number of hydrophilic amino acids couples in the proteins of the microalgae (i.e. a biochemical composition change). In contrast, other compounds showed a decrease in concentration throughout the cycles, as is the case for hydroxyacids and sugars. That could also be related to the effects described above being mainly changes occurring at the cell level.

Other factors that could cause an influence on AP composition are: a) accumulation of non-degradable constitutes via the depleted medium (see Figure 1(b)), which after dewatering will also be present in the water of the algal slurry used for HTL; b) consumption of certain organics by the algae during growth. However, the contribution of those two mechanisms to the raise or decrease of a certain compound in the AP throughout the cycles is limited by the 1:250 dilution when AP (40 ml) is added to the 10L growth medium. 
A relative low degradation rate of xenobiotic potentially toxic compounds (in comparison to sugars of hydroxyacids) is expected. Therefore, formation of this classes of molecules during HTL can be indicated as the most problematic issue for the maintenance of the system under water/nutrient recycle regime. Moreover, the possible accumulation of compounds secreted by the algae itself can also become a problem in the longer term (autoinhibition). Both types of substances (supplied by AP or by algae itself) will accumulate in the exhaust depleted medium but, at the same time, the recycling of this water stream is a necessity in commercial microalgal plants to reduce production costs. Therefore, when toxic levels of accumulated compounds are reached (after a certain number of cycles), a process to convert them should be put in place. Options include supercritical water gasification or possibly anaerobic digestion. A further advantage would be that more ammonia would be produced.

Nitrogen aromatics accumulation can be given as an example. These compounds could only be delivered to the growth medium by means of direct addition of AP and by reusing the depleted medium, assuming no significant bio-degradation by the algae (thus accumulating in that water stream). According to that, nitrogen aromatics accumulation can be estimated using their found concentration in the AP of each cycle (Table 5), resulting in the following concentration in the mediums after AP addition: $3.3 \mathrm{mg} / \mathrm{L}$ for cycle $2,5.9 \mathrm{mg} / \mathrm{L}$ for cycle $3,8.2 \mathrm{mg} / \mathrm{L}$ for cycle 4 and $12.0 \mathrm{mg} / \mathrm{L}$ for cycle 5 . Nitrogen aromatics concentration increased by a factor $\sim 1.5$ from cycle to cycle and by a factor 3.6 for the overall experiment. The rapid increase throughout the cycles shows the importance of placing a water stream (AP) pretreatment prior to growth at a certain point of the recycling process.

\section{Discussion and overall conclusions}

A growth test sequence was performed where 4 cycles of algae cultivation-HTL conversion were completed and the recovered aqueous phase from the $4^{\text {th }}$ cycle was supplied, finally, to the $5^{\text {th }}$ subsequent cultivation step. The designed experiment consisted of a continuous sequence of several processes and accompanying analyses with all steps operating in batch mode. All the cycles were successfully completed and no major adverse effects on growth were observed. Although the HTL-AP, algae and water depleted medium (after dewatering) were being repeatedly cycled, algae grew in all the cycles. Cycle 5 had a comparable productivity to that in standard COMBO medium. 
Interestingly, clear differences were identified when observing and comparing the cultures of each cycle by SEM and optical microscopy, going from colonies of 2 or 4 cells with spines and an oval shape in cycle 1 to unicells with no spines and a more sickle-like shape in cycle 5. Most likely, morphological changes occurred as a result of acclimation to environmental changes (e.g. accumulations of toxic organics compounds) occurring throughout the cycles. A molecular characterization to obtain a DNA profile of the algae from each cycle would lead to more firm conclusions, as generally, morphological changes are accompanied with also internal adjustments affecting the biochemical composition of the related species. Those possible changes did not seem to affect the HTL product yields, and the oils from each cycle had a relatively similar elemental composition.

On the contrary, AP organic fraction showed significant changes along the cycles with increasing amounts for some of the compounds with potential inhibitory activity (e.g. pyrrolydones, phenols, cyclic dipeptides, etc.), which also retain large amount of AP nitrogen. The changes in AP composition are most likely related to bioaccumulation inside the organism and/or cell internal adjustments involving biochemical composition changes.The set of results obtained pointed towards the necessity of certain improvements to achieve a more optimized recycling configuration:

- Application of a pretreatment (e.g. supercritical water gasification, anaerobic digestion, reforming, etc.) to the AP and the depleted medium, either every cycle or when the concentration of non-biodegradable organics in the culture reaches undesirable levels detrimental to growth. Such levels were not yet reached in this study. The conversion of dissolved organics should be enhanced towards the production of usable nutrients such as ammonia;

- For research purposes at the lab scale, automation of measurements (for e.g. algae growth, nutrients, etc.) to be able to obtain more data and more accurate while working within a less demanding experimental design;

- Implementation of continuous operation systems for the combined sequence of growth, harvesting and HTL. The combination of automation and continuous operation (thus avoiding possible influence of storage of concentrated algae prior to HTL) would allow for cultivation at optimal growth rate with no lag phase delaying the targeted algae production.

Finally, algae response to recyclability of exhaust streams might become an important algae performance parameter when screening for the optimal algal species to a certain 
biorefinery configuration, as not all algal strains might behave in the same way upon growth in HTL-AP.

\section{Acknowledgements}

The authors are thankful for the financial support by the province of Overijssel via the Green Energy Initiative of the University of Twente (The Netherlands).

\section{Bibliography}

[1] L. Xu, D.W.F. Brilman, J.A.M. Withag, G. Brem, S. Kersten, Assessment of a dry and a wet route for the production of biofuels from microalgae: Energy balance analysis, Bioresour. Technol., 102(8), 51135122, 2011.

[2] D. Cordell, J. Drangert, S. White, The story of phosphorus: Global food security and food for thought, Global Environ. Change, 19(2), 292-305, 2009.

[3] J.K. Pittman, A.P. Dean, O. Osundeko, The potential of sustainable algal biofuel production using wastewater resources, Bioresour. Technol., 102(1), 17-25, 2011.

[4] U. Jena, N. Vaidyanathan, S. Chinnasamy, K.C. Das, Evaluation of microalgae cultivation using recovered aqueous co-product from thermochemical liquefaction of algal biomass, Bioresour. Technol., 102(3), 3380-3387, 2011.

[5] T. Minowa, S. Sawayama, A novel microalgal system for energy production with nitrogen cycling, Fuel, 78(10), 1213-1215, 1999.

[6] P. Biller, A.B. Ross, S.C. Skill, A. Lea-Langton, B. Balasundaram, C. Hall, R. Riley, C.A. Llewellyn, Nutrient recycling of aqueous phase for microalgae cultivation from the hydrothermal liquefaction process, Algal Res., 1(1), 70-76, 2012.

[7] K. Tsukahara, T. Kimura, T. Minowa, S. Sawayama, T. Yagishita, S. Inoue, T. Hanaoka, Y. Usui, T. Ogi, Microalgal cultivation in a solution recovered from the low-temperature catalytic gasification of the microalga, J. Biosci. Bioeng., 91(3), 311-313, 2001.

[8] Z. Du, B. Hu, A. Shi, X. Ma, Y. Cheng, P. Chen, Y. Liu, X. Lin, R. Ruan, Cultivation of a microalga Chlorella vulgaris using recycled aqueous phase nutrients from hydrothermal carbonization process, Bioresour. Technol., 126(0), 354-357, 2012.

[9] S. Kilham, D. Kreeger, S. Lynn, C. Goulden, L. Herrera, COMBO: a defined freshwater culture medium for algae and zooplankton, Hydrobiologia, 377(1), 147-159, 1998.

[10] A. Richmond, Handbook of microalgal culture: biotechnology and applied phycology, John Wiley \& Sons, Ames, 2008.

[11] S.A. Channiwala, P.P. Parikh, A unified correlation for estimating HHV of solid, liquid and gaseous fuels, Fuel, 81(8), 1051-1063, 2002.

[12] C. Sheng, J.L.T. Azevedo, Estimating the higher heating value of biomass fuels from basic analysis data, Biomass Bioenergy, 28(5), 499-507, 2005.

[13] R.A. Andersen, Algal culturing techniques, Elsevier/Academic Press, San Diego, 2005. 
[14] P.J. Harrison, P.A. Thompson, G.S. Calderwood, Effects of nutrient and light limitation on the biochemical composition of phytoplankton, J. Appl. Phycol., 2(1), 45-56, 1990.

[15] L. Rodolfi, G.C. Zittelli, L. Barsanti, G. Rosati, M.R. Tredici, Growth medium recycling in Nannochloropsis sp. mass cultivation, Biomol. Eng, 20(4-6), 243-248, 2003.

[16] G.E. Fogg, B. Thake, Algal cultures and phytoplankton ecology, University of Wisconsin Press, Madison, 1987.

[17] M.A. El-Dib, S. Shehata, H. Abou Waly, Response of freshwater alga Scenedesmus to triazine herbicides, Water, Air, Soil Pollut., 48(3-4), 307-316, 1989.

[18] M.A. El-Dib, S. Shehata, H. Abou-Waly, Response of freshwater algae (Scenedesmus spp.) to phenylurea herbicides, Water, Air, Soil Pollut., 55(3-4), 295-303, 1991.

[19] M. Lürling, E. Van Donk, Morphological changes in Scenedesmus induced by infochemicals released in situ from zooplankton grazers, Limnol. Oceanogr., 42(4), 783-788, 1997.

[20] A.B. Ross, P. Biller, M.L. Kubacki, H. Li, A. Lea-Langton, J.M. Jones, Hydrothermal processing of microalgae using alkali and organic acids, Fuel, 2010.

[21] P. Biller, R. Riley, A.B. Ross, Catalytic hydrothermal processing of microalgae: Decomposition and upgrading of lipids, Bioresour. Technol., 102(7), 4841-4848, 2011.

[22] D. López Barreiro, W. Prins, F. Ronsse, D.W.F. Brilman, Hydrothermal liquefaction (HTL) of microalgae for biofuel production: State of the art review and future prospects, Biomass Bioenergy, submitted for publication, 2013.

[23] M. Azuara, S.R.A. Kersten, A.M.J. Kootstra, Recycling phosphorus by fast pyrolysis of pig manure: Concentration and extraction of phosphorus combined with formation of value-added pyrolysis products, Biomass Bioenergy, 49(0), 171-180, 2013.

[24] N. Powell, A.N. Shilton, S. Pratt, Y. Chisti, Factors influencing luxury uptake of phosphorus by microalgae in waste stabilization ponds, Environ. Sci. Technol., 42(16), 5958-5962, 2008.

[25] E.H. John, K.J. Flynn, Growth dynamics and toxicity of Alexandrium fundyense (Dinophyceae): the effect of changing N:P supply ratios on internal toxin and nutrient levels, Eur. J. Phycol., 35(1), 11-23, 2000 .

[26] D. Zhou, L. Zhang, S. Zhang, H. Fu, J. Chen, Hydrothermal liquefaction of macroalgae enteromorpha prolifera to bio-oil, Energy Fuels, 24(7), 4054-4061, 2010.

[27] P.J. Valdez, M.C. Nelson, H.Y. Wang, X.N. Lin, P.E. Savage, Hydrothermal liquefaction of Nannochloropsis sp.: Systematic study of process variables and analysis of the product fractions, Biomass Bioenergy, 46(0), 317-331, 2012. 


\section{Appendix D}

Aqueous phase analytical results 
In Figure D.1, the typical chromatograms obtained after GC-MS analyses of the aqueous phase soluble organics from cycles 1, 2 and 3 are shown.

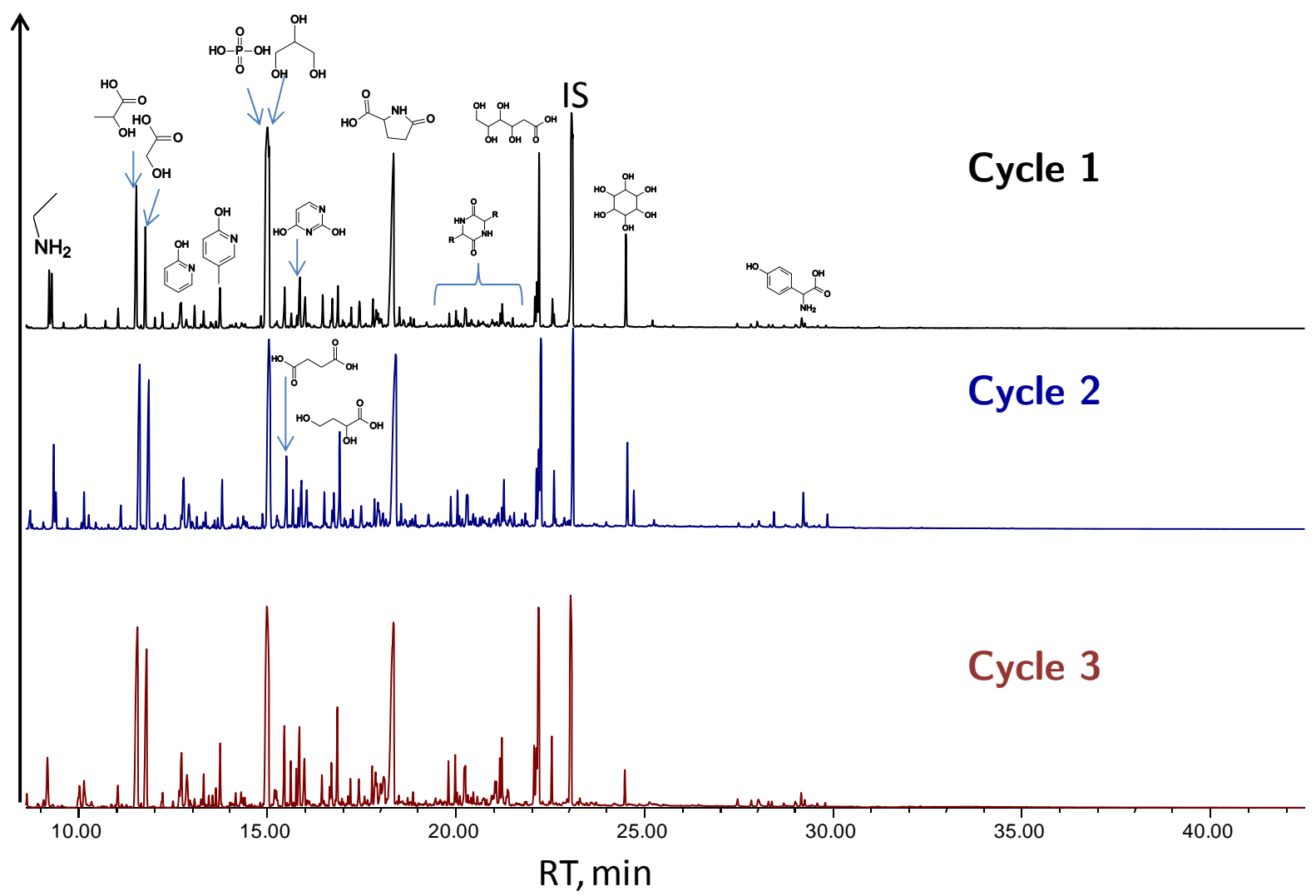

Figure D.1. GC-MS chromatograms of the water soluble organics in aqueous phase for cycles 1, 2 and 3

Below, the list of compounds identified in the mixture of AP soluble organics is shown. The compounds were divided in various chemical classes.

Table D.1. Compounds identified along the GC-MS chromatogram of aqueous phase from hydrothermal liquefaction of Desmodesmus sp.

\begin{tabular}{|c|c|c|c|}
\hline Chemical class & Name & $\begin{array}{c}\text { RT } \\
(\min )\end{array}$ & $\begin{array}{c}\text { Characteristic Ion } \\
\mathbf{m} / \mathbf{z}\end{array}$ \\
\hline \multirow{10}{*}{ Aminoacids } & alanine $\mathrm{TMS}^{\mathrm{a}}$ & 9.724 & 116 \\
\hline & glycine TMS & 12.501 & 102 \\
\hline & Valine TMS & 14.304 & 144 \\
\hline & proline derivative $\mathrm{TMS}^{\mathrm{b}}$ & 14.644 & 98 \\
\hline & proline TMS & 15.265 & 158 \\
\hline & norvaline TMS & 16.442 & 144 \\
\hline & glutamic acid TMS & 16.695 & 73 \\
\hline & allyl-alanine TMS & 19.195 & 156 \\
\hline & glutamine TMS & 19.382 & 246 \\
\hline & lysine derivative TMS & 20.694 & 174 \\
\hline
\end{tabular}




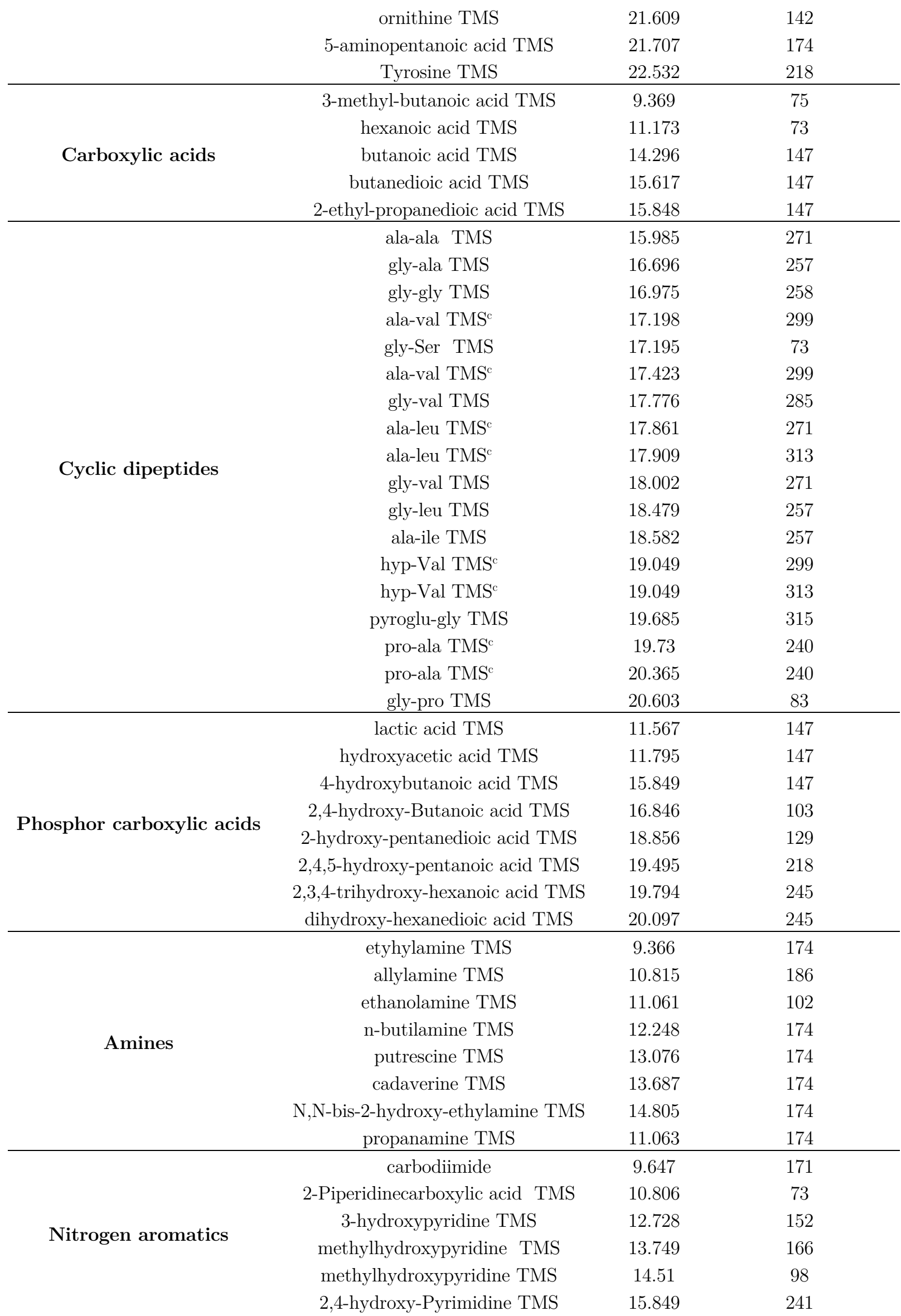




\begin{tabular}{|c|c|c|c|}
\hline & 1H-Indole-3-acetonitrile TMS & 16.446 & 213 \\
\hline & 5-methyl-2,4-hydroxypyridine TMS & 16.697 & 255 \\
\hline & hdyroxypyrazine TMS & 17.775 & 269 \\
\hline & 6-hydroxypurine TMS & 21.467 & 265 \\
\hline & purinic derivate $\mathrm{TMS}^{\mathrm{b}}$ & 22.124 & 264 \\
\hline & pyridoxine TMS & 23.279 & 73 \\
\hline & 2,6-dihdyroxypurine TMS & 23.659 & 353 \\
\hline \multirow{5}{*}{ Alcohols } & ethanediol TMS & 10.399 & 147 \\
\hline & 1,2 propanediol TMS & 10.398 & 117 \\
\hline & 2,3-butanediol TMS & 11.172 & 117 \\
\hline & unknown polyol TMS & 18.076 & 143 \\
\hline & glycerol TMS & 15.07 & 201 \\
\hline \multirow{7}{*}{ Phenols } & 3-methoxyphenol TMS & 13.54 & 181 \\
\hline & 2,4,6-Trimethoxyacetophenone TMS & 14.577 & 195 \\
\hline & unk phenol derivatives TMS & 14.577 & 195 \\
\hline & 3-hydroxybenzoic acid TMS & 18.716 & 267 \\
\hline & 2-hydroxy-cinnamic acid TMS & 19.004 & 193 \\
\hline & p-hydroxy-cinnamic acid TMS & 19.902 & 73 \\
\hline & parahydroxycinnamic acid TMS & 20.935 & 179 \\
\hline \multirow{3}{*}{ Pyrrolidones } & 2-pyrrolidinone TMS & 12.875 & 142 \\
\hline & pyroglutamic acid TMS & 18.345 & 156 \\
\hline & pyrrolidone derivative $\mathrm{TMS}^{\mathrm{b}}$ & 23.134 & 156 \\
\hline \multirow{5}{*}{ Sugars } & levoglucosan TMS & 20.45 & 204 \\
\hline & arabino-Hexonic acid TMS & 22.117 & 245 \\
\hline & sugar derivative $\mathrm{TMS}^{\mathrm{b}}$ & 22.531 & 73 \\
\hline & inositol TMS & 24.475 & 305 \\
\hline & 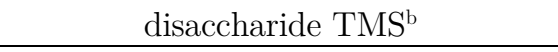 & 29.423 & 204 \\
\hline \multirow{4}{*}{ Others } & dipeptide Hyp-Lys TMS & 21.25 & 268 \\
\hline & $\mathrm{H}_{2} \mathrm{~S}$ TMS & 9.365 & 73 \\
\hline & boric acid TMS & 10.218 & 221 \\
\hline & 236phosphoric acid TMS & 15.071 & 299 \\
\hline
\end{tabular}

${ }^{a}$ Compound is in the per-trimethylsylilated form. ${ }^{\mathrm{b}}$ Tentatively identified. ${ }^{\mathrm{c}} \mathrm{S}$ and $\mathrm{R}$ derivatives. 



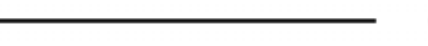

rar 


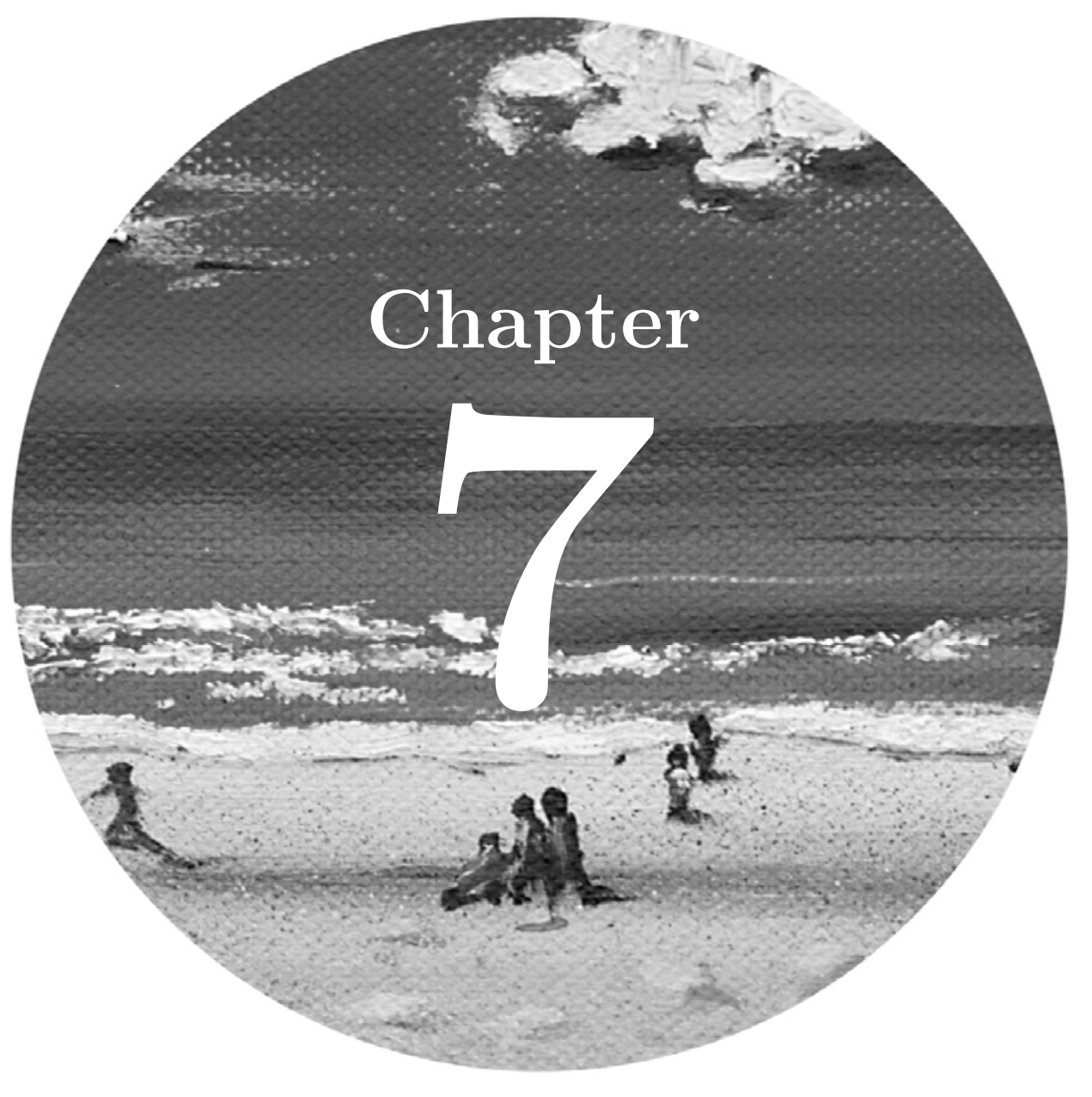

Summary and Outlook 


\begin{abstract}
The research described in this thesis aimed to evaluate the feasibility of various processes and concepts for their potential application in algae biorefinery configurations. This chapter presents a possible algae biorefinery concept which integrates all the features studied. Along with that, the work in this thesis will be summarized while pointing out the most promising aspects and the weaker points. From this all, trends and challenges for possible follow-up research are identified.
\end{abstract}




\section{Summary}

Mankind needs renewable resources to cover for the growing energy demand in a sustainable manner and thereby alleviate the environmental/socio/political issues driven by the use of fossil fuels. Biomass, a renewable source, is one of the sustainable alternatives that can contribute to both the production of energy carriers and chemicals. The use of microalgae has been investigated in this thesis for the production of energy in the form of liquid biofuels. The versatility and fast production rates of these microorganisms together with the possibilities of farming them without using valuable arable land, highlights their potential compared to other biomass sources.

In the context of algae to fuels, significant efforts have been made in the field of biology/biotechnology to find the most optimal cultivation system for efficient and cheap production of algae. Less has been done on evaluating the performance of the subsequent processes required to convert the algae into fuels. In many cases, little is known about their technical and economic feasibility. Therefore, with the work described in this thesis, we aimed to contribute on that and generate knowledge to scale-up operations in the field of algae processing. Several processes and concepts were evaluated for their potential to be integrated in a biorefinery setting.

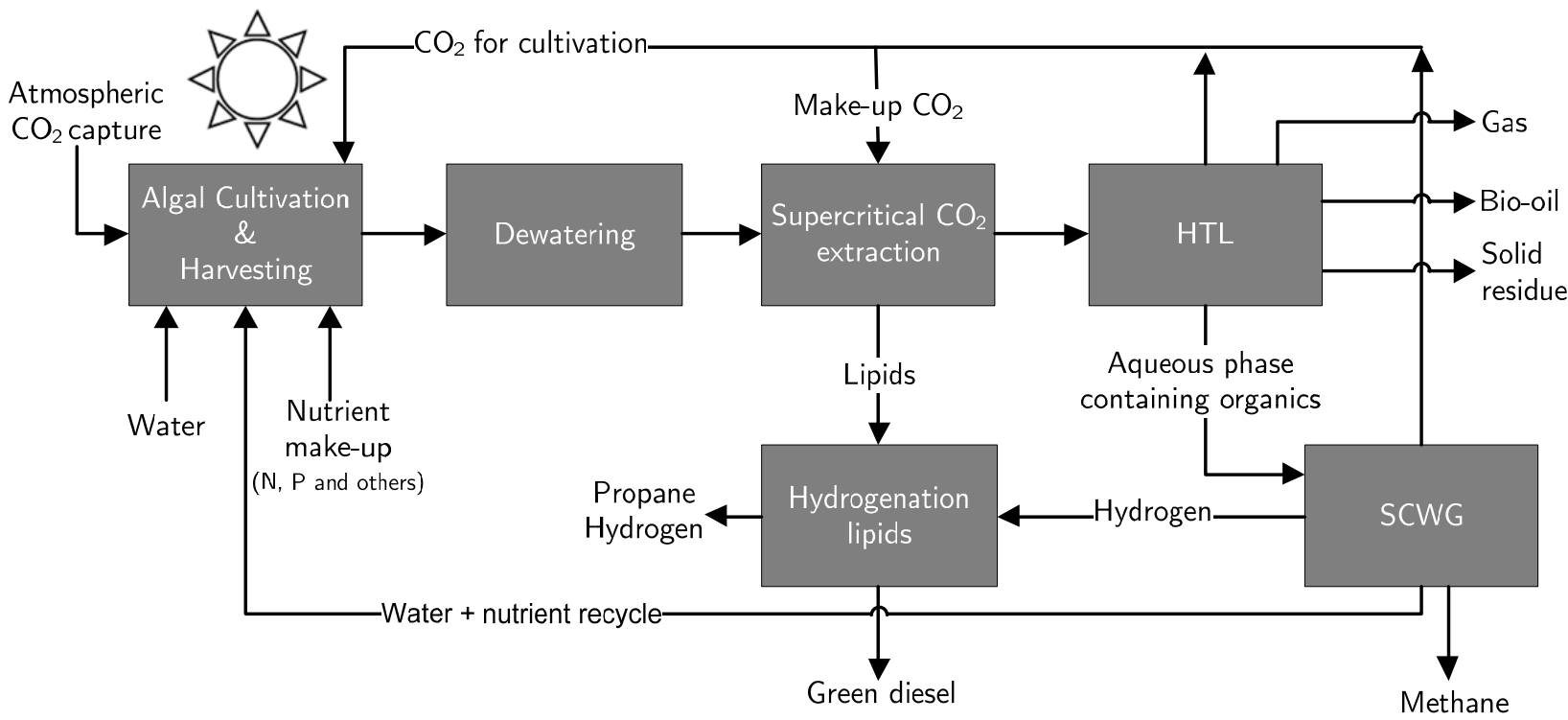

Figure 1. Algae Biorefinery concept for liquid fuels production

The biorefinery approach aims at the optimal utilization of biomass towards maximal economic and environmental benefits. Figure 1 illustrates an extended version of the simplified biorefinery scheme presented in Chapter 1, where all the processes and 
concepts evaluated in this thesis are integrated. The concept is focused on -, but not limited to, liquid transportation fuels. The co-production of valuable non-fuel products would lead to increased revenues which are likely to be required for the economic viability of the concept that has to compete with the still low prices of fossil-based fuels.

After algae cultivation and dewatering, the first processing step in the suggested biorefinery setting is supercritical $\mathrm{CO}_{2}\left(\mathrm{scCO}_{2}\right)$ extraction for the recovery of the lipids, which could be further hydrotreated into green diesel (or also known as renewable diesel). A techno-economical evaluation of this extraction technology was performed and assessed for both dry and wet microalgae. Firstly, with dry microalgae, the operating conditions were systematically changed aiming to maximize the oil yield and its quality. Extraction at high pressures (at least $35 \mathrm{MPa})$ and moderate temperatures $\left(50^{\circ} \mathrm{C}\right)$ was found to be the most optimal set of conditions according to those criteria and for the algal biomass used. From a broad set of analyses, the quality of the oil was assessed by identifying the fraction of the extracted oil that is suitable for diesel-based fuels (biodiesel and green diesel). $\mathrm{ScCO}_{2}$ extraction from wet microalgae was found to be less efficient, leading to lower oil yields with increasing amounts of more polar compounds undesirable for the targeted diesel product.

The experimental findings were used to estimate the minimum extraction costs (including both energy and capital costs) for different cases where also dewatering energy expenses were included. For the algal species used in this research with 12 wt\% lipid content, a value of $0.50 € / \mathrm{kg}$ oil extracted was found for extraction from dried algal biomass. Assuming a wet microalgae feedstock with $25 \mathrm{wt} \%$ extractable lipids and considering the wet extraction being as efficient as the dry extraction, the cost went down to $0.30 € / \mathrm{kg}$ oil extracted. Further research is needed to overcome the technical challenges of $\mathrm{scCO}_{2}$ wet extraction and realize this same efficiency. The costs for conventional extraction using organic solvents were found to be slightly lower, but the environmental impact related to the use of those solvents lessens their potential compared to $\mathrm{scCO}_{2}$.

Both fresh microalgae and the residual biomass left after $\mathrm{scCO}_{2}$ extraction could be the feedstock of the next processing step; hydrothermal liquefaction (HTL). A similar approach to that for $\mathrm{scCO}_{2}$ extraction was followed to evaluate the applicability of HTL as conversion method in a biorefinery setting. A wide range of reaction conditions was studied (esp. temperature and reaction time) on product distribution and composition, including a visual inspection of the treated algal cells after HTL, a feature that was not investigated earlier. At $375^{\circ} \mathrm{C}$, the maximum oil yield of almost $50 \mathrm{wt} \%$ was reached with 
a $75 \%$ recovery of the calorific value of the algae into the oil. Only 5 minutes of reaction time at the specific temperature were required to reach that maximum, implying that only small reactor volumes will be required for future continuous operations, which is beneficial in view of related capital costs.

HTL was found to be an efficient method to convert wet microalgae into a biocrude oil, while indirectly saving in dewatering costs as it circumvents the need for complete water removal and associated high energy cost for thermal drying (concentrating to 10-20 wt\% algae dry weight is still required). The increase in processing temperature led to higher amounts of oil. However, the higher the oil yield the higher is also its nitrogen content (going up to $6 \mathrm{wt} \%$ with the algal species used for this research). In a collaborative framework with the University of Bologna, the nature of the nitrogen containing compounds was investigated and thereby gaining more insight for further oil upgrading operations. That oil characterization also showed that the product composition can be tuned by the HTL operating conditions selected. For instance, if a lipid-rich oil is preferred, relatively low temperatures should be applied (below $250^{\circ} \mathrm{C}$ ), but this will be at the expenses of producing less oil.

To potentially consider the HTL oil as feedstock for a crude oil refinery, the nitrogen content must be reduced. For that purpose, several methods were evaluated: the removal of proteins (the cause of the high $\mathrm{N}$ in the HTL oil) prior to HTL; extractive techniques to separate the nitrogen containing compounds from the oil after HTL of fresh algae; and catalytic cracking of the HTL oil using a zeolite catalyst. The methods were either rather inefficient regarding oil yield and reduction of nitrogen and oxygen content or were producing an oil having a composition less suitable for the desired linear hydrocarbon rich fuel targeted. After that, another method was tested, hydrotreatment of HTL oil, and this was found to be effective in reducing not only the nitrogen- but also the oxygen content and the viscosity. Under the most severe hydrotreatment conditions tested $\left(400^{\circ} \mathrm{C}, 50 \mathrm{wt} \%\right.$ catalyst loading and $15 \mathrm{MPa}$ of initial $\mathrm{H}_{2}$ pressure), the highest levels of denitrogenation and deoxygenation were obtained. Even without adding a catalyst, a certain degree of heteroatoms removal was obtained (about 30\%). The oil shifted from a highly viscous tar-like product, not flowing at room temperature, to a freely flowing liquid rich in straight chain alkanes from which the diesel-range $\mathrm{C}_{15}-\mathrm{C}_{18}$ hydrocarbons was the most abundant fraction.

With simple characterization methods, indications on the future applicability of the hydrotreated HTL algal oil were obtained. The reduction of oxygen and nitrogen content (shown by elemental analysis) and in carbon-residue (shown by MCRT tests) appeared 
as promising result for future co-processing options. A blending test of hydrotreated HTL algal oil with fossil-based diesel showed that a relatively high fraction of the algal oil appears to be directly miscible in diesel. Although the direct use of this product in a diesel engine might still be far away, co-processing in standard refinery units seems feasible and even the use of a (hydrocarbon) fraction of this product as direct drop-in fuel cannot be excluded.

Besides the studies on algae processing, we also aimed to close the loops within the biorefinery. Recycling of water and nutrients (mainly N, P and C) is essential as well as the integration of heat and power where possible and the internal usage of other product streams (e.g. $\mathrm{H}_{2}$ ). All that would reduce environmental footprints and improve the mass and energy efficiency of the system. In this research, we especially studied the feasibility of recycling the nutrients containing aqueous phase (AP), which was recovered after hydrothermal liquefaction, to algae cultivation.

First, while keeping the same nitrogen concentration as that in the standard growth medium, the AP dilution factor was systematically varied by using only water or water enriched with standard medium. With that we demonstrated that the main cause of growth inhibition by AP addition was not its insufficient dilution leading to too high concentrations of toxic organics (a conclusion that was stated in earlier studies in literature). Instead, the lack of (macro-/micro-) nutrients, other than $\mathrm{N}$ and $\mathrm{P}$, in the AP caused that effect. In parallel, we proved that 50\% N-replacement of fresh intake, by direct recycling of the AP is possible, while showing a nearly identical growth behavior as that in the standard medium.

Finally, we went one step forward by evaluating the multiple recyclability potential of aqueous phase containing nutrients. A set of 4 continuous growth-HTL cycles was completed. Alternating, Desmodesmus sp. microalgae was cultivated (5 times) and HTL of the biomass produced was performed (4 times), while recycling the AP with nutrients from one cycle to the next cycle as well as the depleted medium from the intermediate dewatering step. The algal biomass growth rate (in the linear region) was comparable to that in standard medium. Despite the longer lag phase obtained throughout the cycles (which can be probably avoided by working with a continuous operation system for the combined sequence of growth, harvesting and HTL), the algae could eventually grow. The results showed the potential of developing algae-based biorefineries towards standalone operation. In addition, compounds accumulating in the AP were identified by a detailed chemical characterization of the AP soluble organics. Those results pointed out the relevance of placing a water stream treatment at a certain stage of the recycling 
process. If this is done by means of supercritical water gasification (as shown in Figure 1), a gas stream with hydrogen and $\mathrm{CO}_{2}$ and water stream containing more ammonia would be recovered for internal usage within in the biorefinery.

\section{Algae biorefinery: energy estimates and recommendations}

To have an indication on how the energy requirements are distributed within the biorefinery concept presented in Figure 1, we performed some rough calculations using real data and assumptions, most of which based on studies carried out within our research group: microalgae related assumptions presented in Chapter 4 (land area, algae productivity, etc.) using cultivation energy inputs from $\mathrm{Xu}$ et al. ${ }^{1}$ for a raceway pond (pumping, paddle wheel, etc.); energy calculations for dewatering and $\mathrm{scCO}_{2}$ extraction from Chapter 4 (assuming effective extraction from a slurry with 30 wt\% algae dry weight) and for HTL based on real data from Chapter 2; and using the work of Chakinala et al. ${ }^{2}$ as guidelines for SCWG of an organics rich aqueous phase.

In terms of energy costs, cultivation is one of the main bottlenecks in the biorefinery concept. For the system presented in Figure 1, cultivation accounted for about $43 \%$ of the total energy input (see Figure 2(a)). Since we assumed the case of extraction from a slurry with $30 \mathrm{wt} \%$ algae dry weight, the energy requirements for dewatering are relatively low and, hence, cultivation has a larger share in the total. As shown in various studies, ${ }^{1,3,4}$ cultivation (when considering the wet route) and cultivation together with drying (for the dry processing route) are major factors influencing the energy efficiency of algal biofuel based biorefineries.

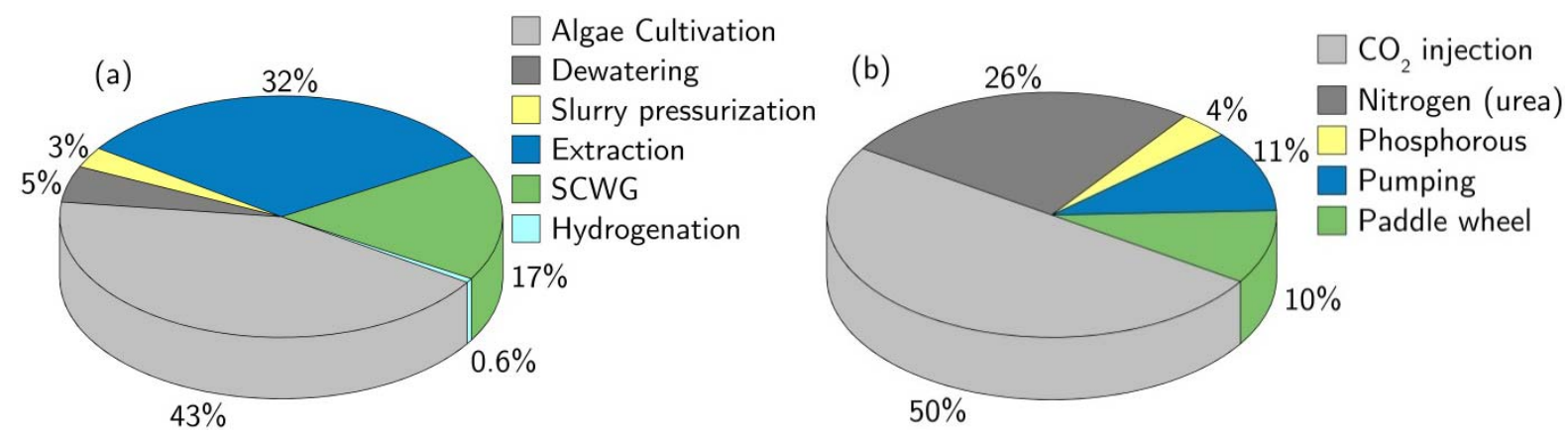

Figure 2. Distribution of energy requirements over the biorefinery concept of Figure 1; (a) total energy input (10.1 GJ/metric ton dry algae) and (b) cultivation energy distribution 
Within the cultivation energy expenses, depicted in Figure 2(b), the largest contributions are related to the use of nitrogen fertilizer (associated to its production costs) and $\mathrm{CO}_{2}$ supply; $26 \%$ and $50 \%$ respectively. Generally, in other techno-economic assessments and life cycle analyses, the energy demand of those two cultivation features have been reported similar. In Figure 2(b), we assumed (in line with Xu et al. ${ }^{1}$ ) a $15 \%$ recycling of nutrients, which increases the energy share for $\mathrm{CO}_{2}$ injection. As demonstrated in Chapter 5, even $50 \%$ nutrients replacement via recycling seems possible, while achieving the same growth rate as that in standard medium (at least for Demosdemus sp. microalgae). With that assumption, the N-fertilizer energy input shown in Figure 2(b) would go down to $18 \%$, but the overall cultivation costs in Figure 2(a), with respect to the whole biorefinery system, would be reduced only by $7.5 \%$.

The $\mathrm{CO}_{2}$ supply is energy demanding but also crucial to maintain reasonable algae productivities. When cultivating algae, $\mathrm{CO}_{2}$ dosing to enhance production rates is a common practice. To illustrate that, we performed various microalgae growth experiments in batch cultivation reactors, aerated with different $\mathrm{CO}_{2}$ concentrations (for more details see ${ }^{5}$, see Figure 3 .

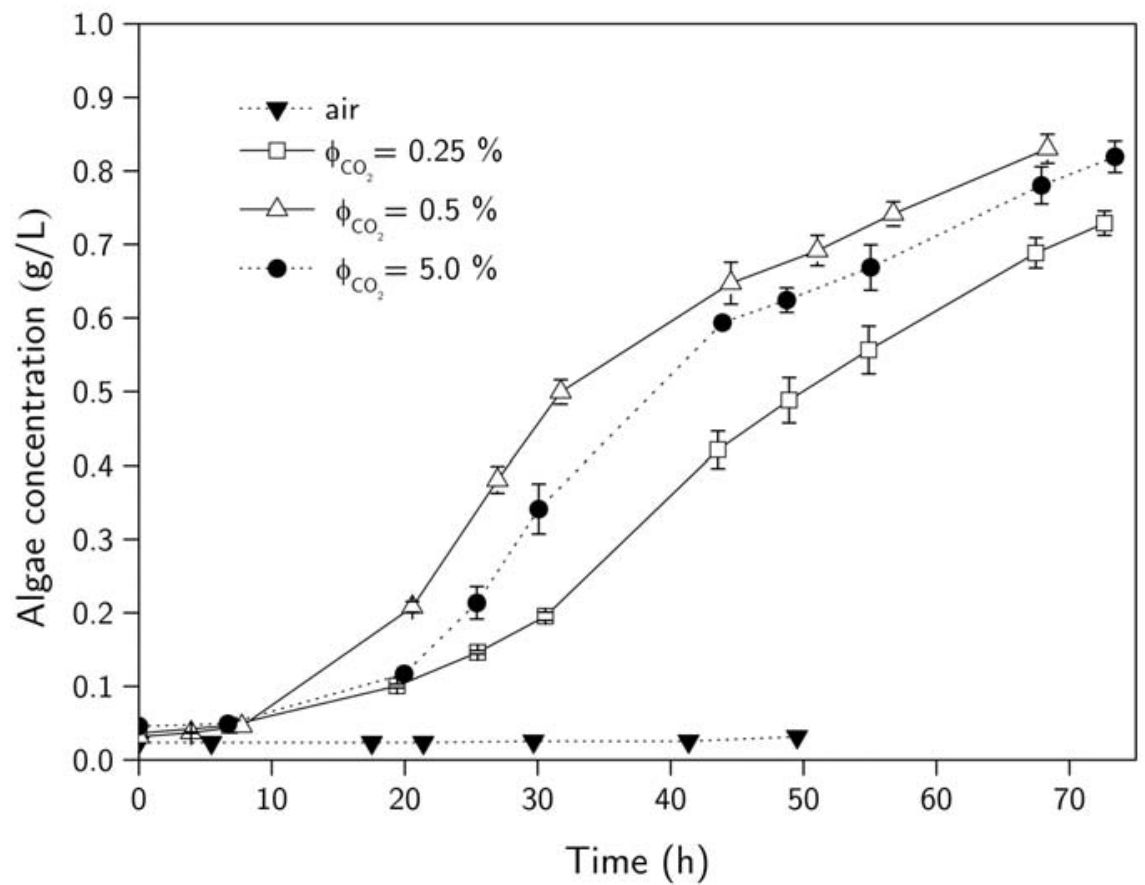

Figure 3. Algae growth under different concentrations of $\mathrm{CO}_{2}$ dosing (adapted from ${ }^{5}$ )

When producing carbon-containing products (as lipid- or HTL oil) in the biorefinery, a continuous intake of $\mathrm{CO}_{2}$ is required, next to internal recycling of $\mathrm{CO}_{2}$ generated in downstream processing (e.g. HTL) in the biorefinery. This $\mathrm{CO}_{2}$ is generally assumed to 
be supplied from large $\mathrm{CO}_{2}$ emitters like power plants and refineries, and this assumption was adhered in the above energy calculations. However, the use of atmospheric $\mathrm{CO}_{2}$ is an intriguing alternative that enables a sustainable carbon cycle avoiding fossil-based $\mathrm{CO}_{2}$ sources. Therefore, in a recent study ${ }^{5}$ we demonstrated the concept of combining atmospheric $\mathrm{CO}_{2}$ capture (using supported amine sorbents) and algae cultivation as alternative to the application of flue gas derived $\mathrm{CO}_{2}$. Such a concept adds to the flexibility of locating algae farms and to the sustainability of algae-derived products. The challenge is to develop this in an economic viable way of $\mathrm{CO}_{2}$ supply and this may be further supported by future discounts for using a non-fossil based source of carbon.

As mentioned earlier, the use of wet feedstock in algal biomass-based biorefineries can alleviate the large energy penalty associated with drying. For that reason, we selected technologies that can handle wet streams such as HTL and SCWG. The contribution of dewatering in Figure 2(a), referring to concentrating the algal slurry from cultivation to extraction conditions, is therefore limited. As displayed in Figure 1, we assumed $\mathrm{scCO}_{2}$ extraction for the recovery of the lipids from wet algae. In Chapter 4 it was shown that the 'wet extraction $\mathrm{scCO}_{2}$ ' technology still has to overcome technical challenges to become as efficient as the dry extraction. On the other hand, the alternative to $\mathrm{scCO}_{2}$ often considered, being wet extraction with organic solvents like hexane, has not been proven at large scale yet. ${ }^{6}$ Another, promising, novel alternative is the use of switchable solvents, ${ }^{7}$ which is still in a very early stage of development.

Supercritical $\mathrm{CO}_{2}$ extraction is an energy intensive process and the second largest share of the total energy demand of the suggested biorefinery of Figure 1, accounting for a $32 \%$ of the total (see Figure 2(a)). This energy requirement, and the rest of the energy estimates, was calculated for the algae used in this research having only about 12 wt\% lipid content. An algal species having a better combination of biomass productivity and lipid content, such as Nannochloropsis sp., may be more beneficial. That strain can naturally accumulate about 25-30 wt\% lipids (no stress conditions required), while showing a reasonable biomass productivity in an open system (almost 40 ton/ha/year, as reported by Jorquera et al. ${ }^{8}$ for our case a 50 ton/ha/year was assumed). We used the same type of calculations considering those microalgae as feedstock, while assuming the same lipid oil solubility in $\mathrm{scCO}_{2}$ as that shown in Chapter 4 and the same HTL products distribution as that shown in Chapter 2. For the microalgae with $12 \mathrm{wt} \%$ lipids, $62 \%$ of the total energy output reports to the HTL bio-oil and only $18 \%$ to green diesel. For the case with 25 wt $\%$ lipids, that changed to $44 \%$ and $42 \%$ respectively. The difference in overall costs will depend mainly on the capital costs. For extraction with 
$\mathrm{scCO}_{2}$, the option with higher lipid content algae would benefit most from lower capital costs since, as shown in Chapter 4, the contribution of the extractor costs is significant. However, other aspects related to strain selection which could affect the costs were not considered here. For instance, Nannochloropsis cells are small and with a thick cell wall, which might cause more difficulties in harvesting and further processing. ${ }^{9}$

An additional benefit of $\mathrm{scCO}_{2}$ extraction is that its output streams are already at elevated temperature and pressure for the subsequent processes. This may circumvent the need for separate pressurization steps and result in significant savings in electric and thermal energy requirements for HTL, SCWG and hydrogenation steps. Moreover, possible $\mathrm{CO}_{2}$ losses during extraction could be covered by the $\mathrm{CO}_{2}$ produced in the other process steps.

Next to the lipids, the other algal building blocks (proteins and carbohydrates) can be also extracted and used for a broad diversity of applications such as pharmaceuticals, nutrition, etc. In that context, more research is needed to evaluate if and how the order in which the targeted compounds (or fractions) are extracted affect their yields and composition. For instance, Sari et al. ${ }^{10}$ showed a higher protein recovery (by enzyme assisted protein extraction) from algae that had lipid oil removal in advance. Subsequently, the effect of a changed feed composition on HTL and SCWG should be checked.

After extraction, the algal residue could be further processed by means of hydrothermal liquefaction. In Figure 2(a), the energy consumption for HTL is negligible, since pressurization is done upfront extraction and heat integration with the SCWG unit allows for operation without additional heat supply. In Chapter 2, an extensive HTL study was performed using unextracted fresh microalgae. In view of future biorefinery development, it would be interesting to compare those results to that obtained from the defatted and low protein content algae equivalent. Recently, Vardon et al. ${ }^{11}$ compared for the first time the thermochemical conversion of raw and defatted Scenedesmus sp. Comparable yields for the product fractions were obtained, however the oil from the defatted algae had a significant different composition with (obviously) a lower content of fatty acids, esters and hydrocarbons. The different composition of the defatted algae will affect the reaction paths during HTL and, further downstream, the oil form defatted algae will probably have an influence on the reaction paths occurring during the subsequent oil upgrading method(s) applied. 
Due to the broad diversity of microalgae and their composition, several biorefinery platforms have been proposed. Hitherto the most common configuration, among all techno-economical evaluations and life cycle analyses, is the concept where hexane extraction (both 'wet' and 'dry' extraction are being considered) is applied for lipids recovery and, after that, the algal residues are anaerobically digested to a methane rich biogas available for heat and power integration. A fraction of the liquid digestate can be recirculated to the algae growth system. Despite some advantages of anaerobic digestion for residue conversion, clear technical obstacles remain, such as incomplete conversion, the presence of $\mathrm{N}$ in the algal debris that can be converted into ammonia and inhibit anaerobic microorganisms ${ }^{6}$ and the fact that it is an slow process (generally in the order of days $\left.{ }^{12}\right)$.

On the other hand, hydrothermal liquefaction produces a crude oil with a significantly higher energy density than biogas and within a processing time in the order of minutes. According to our estimations, no net energy input would be required for the HTL step separately, after heat integration with the SCWG unit. This type of integration makes the energy requirement for the concept presented in Figure 1 relatively low. The capital investment is likely to be higher than that for the biogas biorefinery concept as more process steps are involved.

From HTL, an aqueous phase containing dissolved organics is produced. In Chapters 5 and 6 we showed that, when recycling nutrients, accumulation of some toxic organics occurs, showing the importance of placing an aqueous phase pretreatment prior to growth at a certain point of the recycling process. That could be achieved by means of supercritical water gasification ( $\mathrm{SCWG}$ ), while producing at the same time a $\mathrm{H}_{2}$ rich gaseous product which could be used for both lipids hydrogenation and for upgrading of the HTL oil. The energy requirements for SCWG in Figure 2(a) refer to energy needed for preheating the feed stream, coming from HTL, to reaction conditions and include the heat of reaction at reaction conditions.

Preliminary estimates were performed to calculate the amount of $\mathrm{H}_{2}$ produced. For the SCWG, we assumed an aqueous phase containing only acetic acid (the major compound in that fraction as shown in Chapters 5 and 6) and nitrogen in the form of ammonia. The product would be a gas mixture mainly containing $\mathrm{CO}_{2}$ and $\mathrm{H}_{2}$. The reaction conditions selected were $600^{\circ} \mathrm{C}$ and $25 \mathrm{MPa}$ and the gasification efficiency was set to $66 \%$ (for a non-catalytic process). With the use of a catalyst, a near complete gasification efficiency may be achieved in relatively short reaction times. ${ }^{2}$ For estimating the hydrogen consumption during hydrogenation of lipids, triolein was used as model 
compound of algal oil and the specific hydrogen consumption, needed to convert this compound into $\mathrm{C}_{17}$ alkanes, was calculated. Optimal conditions for the hydrogenation, as found by Peng, ${ }^{13}$ were $270^{\circ} \mathrm{C}$ and $4 \mathrm{MPa}$. According to our calculations, from the initial $\mathrm{H}_{2}$ produced by gasification of the aqueous phase, around $7 \mathrm{~kg}$ of $\mathrm{H}_{2}$ per metric ton of dry algae is left after hydrogenation of the lipids. Li and Savage ${ }^{14}$ reported for hydrotreatment of HTL algal oil (in dry form) a consumption of $5 \mathrm{~kg}$ of $\mathrm{H}_{2}$ per metric ton of dry algae (assuming an oil yield of $45 \mathrm{wt} \%$ from HTL). Therefore, it seems that sufficient $\mathrm{H}_{2}$ is produced from SCWG to cover the $\mathrm{H}_{2}$ requirements to hydrotreat both the lipids and the HTL oil. However, less hydrogen would be available when using algae with higher lipid content than that of the species used in this research (12 wt\%).

However, the possible economic benefit of using our own source of $\mathrm{H}_{2}$ should be evaluated. Two possible options could be considered: 1) having a complete standalone biorefinery with green diesel and upgraded HTL oil as output streams reducing costs by using self-produced $\mathrm{H}_{2}$; in that case capital costs would be higher due to the required new infrastructure for hydrogenation; 2) having a decentralized biorefinery producing non-treated HTL crude oil and lipids which would be later transported to conventional refineries (with ready infrastructure and $\mathrm{H}_{2}$ available) for further treatment. In the latter case, the waste streams could be used for e.g. heat and power. If the use of self-produced $\mathrm{H}_{2}$ appears to be more economical, hydrogenation with a non-pure $\mathrm{H}_{2}$ flow should be studied to avoid the addition of more process steps for hydrogen purification purposes.

Overall, for the biorefinery concept presented in this thesis, the total and best case scenario energy costs (still assuming the wet extraction efficiency equal to that for the dry case) calculated for processing the algae both into hydrogenated lipids and HTL oil and based on the current price of natural gas in the Netherlands being $0.044 \epsilon_{/} / \mathrm{kWh}$ (for industrial consumers), would be approximately $0.12 € / \mathrm{kg}$ dry algae (equivalent to 1.43 $€ / \mathrm{kg}$ green diesel and $0.30 € / \mathrm{kg}$ HTL oil) for the algal strain with $12 \mathrm{wt} \%$ lipid content. In case of an algae strain with $25 \mathrm{wt} \%$ lipid content, the processing costs change to 0.51 $€ / \mathrm{kg}$ green diesel and $0.35 € / \mathrm{kg}$ HTL oil.

\section{Outlook}

In this thesis we have demonstrated that algae processing to liquid biofuels is technically feasible with reasonable yields. However, the products separation steps that will be required afterwards can weaken the so far conceived relative easy processing (e.g. 
separation of water and oil after HTL). Future research carried by the engineering side of the algae field should focus on those separation issues as well as on developing improved lipid extraction methods from wet microalgae when a diesel-based fuel is one of the desired end products.

Microalgae cultivation remains as the main challenge (at least in terms of energy costs) for the success of the algae-to-fuels biorefinery concept. Algae production has to be proven at commercial scale cultivating a species that is able to naturally produce a reasonable amount of lipids (when aiming for a bio-based diesel product), while growing fast in a cheap (most likely: open-configuration) system and being easy to harvest. That ideal/cheap algae might not exist, which highlights the importance of co-production of bulk chemicals, food and feed ingredients that can bring additional revenues to offset for the high cultivation costs. In that same context, nutrients recycling is essential and, in this thesis, its feasibility has been shown.

The current urge for very high lipid content microalgae might be questionable. Refinery opportunities of the biocrude obtained from hydrothermal liquefaction should be tested. If co-processing and/or direct drop-in fuel fractions appear as successful options, while covering a large fraction of the energy demand in the form of liquids, the need to maximize the algal lipids for diesel biofuels might become less crucial. As consequence, the biorefinery setting could be simplified by having less unit operations (cheaper) and being able to produce low lipid, robust, fast growing algae to reduce cultivation costs.

Finally, for the production of fuels, the overall costs of future algae-based biorefinery developments will also have to be compared with that of non-algae-based concepts. A clear example of that is the production of methanol from captured atmospheric $\mathrm{CO}_{2}$ and $\mathrm{H}_{2} \mathrm{O}$. Methanol can be further converted into electrical power, dimethyl ether (a diesel fuel substitute), light olefins and even longer hydrocarbons. For the sole purpose of solar energy harvesting and -storage, these non-algal sustainable approaches should not be overlooked.

Many challenges are still on the path towards a sustainable and economically viable algae-to-fuels commercial process. Yet we should use the momentum of the current "algae fever" to launch research initiatives towards that aim. The recently (2012) announced quadruple counting of algae based biofuels by the European Commission in the EU biofuels policy is another supporting instrument, underlining the importance of the developments targeted in this thesis. 


\section{Bibliography}

[1] L. Xu, D.W.F. Brilman, J.A.M. Withag, G. Brem, S. Kersten, Assessment of a dry and a wet route for the production of biofuels from microalgae: Energy balance analysis, Bioresour. Technol., 102(8), 51135122, 2011.

[2] A.G. Chakinala, D.W.F. Brilman, W.P.M. van Swaaij, S.R.A. Kersten, Catalytic and non-catalytic supercritical water gasification of microalgae and glycerol, Ind. Eng. Chem. Res., 49(3), 1113-1122, 2009.

[3] D.L. Sills, V. Paramita, M.J. Franke, M.C. Johnson, T.M. Akabas, C.H. Greene, J.W. Tester, Quantitative uncertainty analysis of life cycle assessment for algal biofuel production, Environ. Sci. Technol., 47(2), 687-694, 2012.

[4] C. Beal, R. Hebner, M. Webber, R. Ruoff, A.F. Seibert, The Energy Return on Investment for Algal Biocrude: Results for a Research Production Facility, Bioenergy Res., 5(2), 341-362, 2012.

[5] W. Brilman, L. Garcia Alba, R. Veneman, Capturing atmospheric $\mathrm{CO}_{2}$ using supported amine sorbents for microalgae cultivation, Biomass Bioenergy, (0).

[6] L. Lardon, A. Helias, B. Sialve, J.-P. Steyer, O. Bernard, Life-cycle assessment of biodiesel production from microalgae, Environ. Sci. Technol., 43(17), 6475-6481, 2009.

[7] C. Samori, D. Lopez Barreiro, R. Vet, L. Pezzolesi, D.W.F. Brilman, P. Galletti, E. Tagliavini, Effective lipid extraction from algae cultures using switchable solvents, Green Chem., 15(2), 353-356, 2013.

[8] O. Jorquera, A. Kiperstok, E.A. Sales, M. Embiruçu, M.L. Ghirardi, Comparative energy life-cycle analyses of microalgal biomass production in open ponds and photobioreactors, Bioresour. Technol., 101(4), 1406-1413, 2010.

[9] D. López Barreiro, W. Prins, F. Ronsse, W. Brilman, Hydrothermal liquefaction (HTL) of microalgae for biofuel production: State of the art review and future prospects, Biomass Bioenergy.

[10] Y.W. Sari, M.E. Bruins, J.P.M. Sanders, Enzyme assisted protein extraction from rapeseed, soybean, and microalgae meals, Ind. Crops Prod., 43(0), 78-83, 2013.

[11] D.R. Vardon, B.K. Sharma, G.V. Blazina, K. Rajagopalan, T.J. Strathmann, Thermochemical conversion of raw and defatted algal biomass via hydrothermal liquefaction and slow pyrolysis, Bioresour. Technol., 109(0), 178-187, 2012.

[12] A. Singh, S.I. Olsen, A critical review of biochemical conversion, sustainability and life cycle assessment of algal biofuels, Appl. Energy, 88(10), 3548-3555, 2011.

[13] B. Peng, Transformation of triglycerides and fatty acids into biofuels with sulfur-free catalysts, $\mathrm{PhD}$ thesis, Technische Universitat Munchen, Munchen, 2012.

[14] Z. Li, P.E. Savage, Feedstocks for fuels and chemicals from algae: Treatment of crude bio-oil over HZSM-5, Algal Res., 2(2), 154-163, 2013. 


\section{Samenvatting}

De samenleving heeft hernieuwbare grondstoffen nodig om op een duurzame manier te voldoen aan de groeiende vraag naar energie en tevens kunnen bijdragen aan het oplossen van milieu- en sociaal-politieke vraagstukken rondom het gebruik van fossiele brandstoffen. Biomassa is een hernieuwbare energiebron en kan daarom dienen als grondstof voor de duurzame productie van chemicaliën en brandstoffen. Dit proefschrift richt zich op de productie van energie, in de vorm van vloeibare biobrandstoffen, uit microalgen. Deze veelzijdige micro-organismen hebben vergeleken met andere vormen van biomassa een hoge groeisnelheid. Daarnaast hoeft er voor algenteelt geen beroep te worden gedaan op het beschikbare landbouwareaal, hetgeen voor veel andere soorten biomassa wel essentieel is. Dit maakt microalgen in potentie erg geschikt voor de productie van biobrandstoffen.

De laatste jaren richtte onderzoek zich vooral op de ontwikkeling van een efficiënte en goedkope manier om op grote schaal algen te kunnen produceren. Er is echter veel minder bekend over de efficiëntie van de processtappen die nodig zijn om uit de geproduceerde microalgen een bruikbare biobrandstof te produceren. In veel gevallen is er daarnaast weinig kennis over de technische en economische haalbaarheid van de productie van biobrandstoffen uit microalgen. Het werk, zoals gerapporteerd in dit proefschrift, richt zich daarom op de evaluatie van dit proces en op het vergaren van kennis met betrekking tot het opschalen van dergelijke systemen. Verschillende procesconfiguraties zijn geëvalueerd om geïntegreerd te kunnen worden in een microalgen bio-raffinaderij.

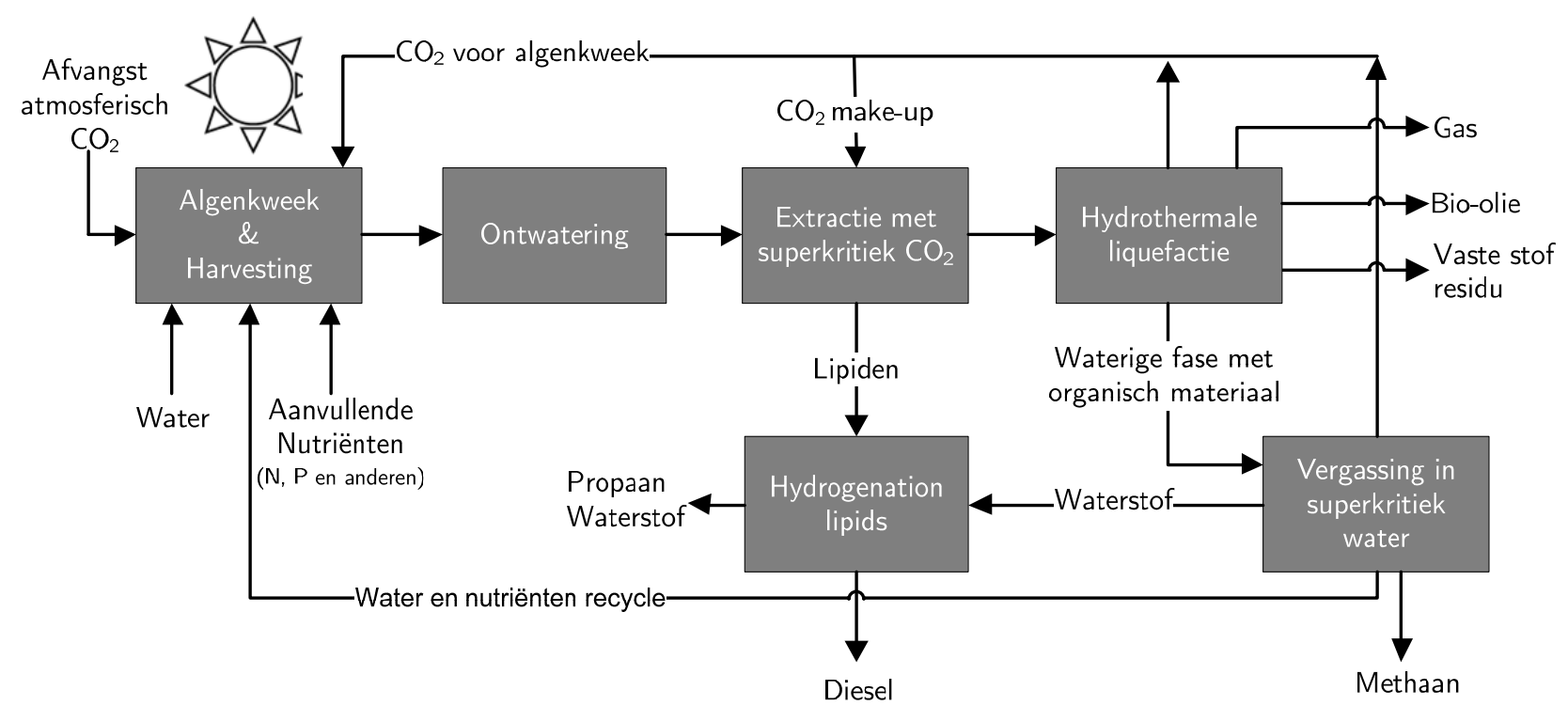

Figuur 1. Algen Bioraffinage Concept, gericht op de productie van biobrandstoffen 
In een bio-raffinaderij tracht men biomassa op een efficiënte, duurzame en economische manier om te zetten in energie, chemicaliën en biobrandstoffen. In Figuur 1 is het processchema van een algen bio-raffinaderij afgebeeld, met daarin opgenomen de verschillende processtappen die in dit proefschrift bestudeerd zijn. In dit concept staat de productie van biobrandstoffen centraal, maar laat ook ruimte voor de coproductie van meer waardevolle producten en (bulk) chemicaliën. De productie van hoogwaardigere producten is hoogstwaarschijnlijk nodig om het proces economisch rendabel te maken, aangezien dit proces (voorlopig) moet concurreren met de brandstoffen gemaakt uit relatief goedkope fossiele grondstoffen.

De eerste processtap in het voorgestelde bio-raffinage proces, na het groeien en ontwateren van de algen, is de extractie van lipiden uit de microalgen met behulp van superkritiek $\mathrm{CO}_{2}$. Deze lipiden kunnen na hydrogenering gebruikt worden als (bio)dieselcomponent. Deze extractiestap is uitgebreid geëvalueerd voor zowel droge als natte algen biomassa (Desmodesmus sp.). Voor gedroogde microalgen is getracht, door de extractiecondities te variëren, de olieopbrengst en oliekwaliteit te optimaliseren. Uit het experimentele werk bleek dat extractie onder hoge druk (ten minste $35 \mathrm{MPa}$ ), gecombineerd met temperaturen rond $50^{\circ} \mathrm{C}$ tot optimale resultaten leidt. Met behulp van verschillende analysetechnieken is bepaald hoeveel van de geëxtraheerde olie geschikt is voor omzetting naar biodiesel. De directe extractie van lipiden uit een geconcentreerde microalgen slurry in water bleek minder efficiënt. Dit resulteerde in lagere olie opbrengsten en de geëxtraheerde olie bevatte meer polaire verbindingen die niet meteen bijdragen aan de productie van diesel.

Het type microalg dat in dit werk gebruikt is, Desmodesmus sp., bevat $12 \%(\mathrm{~m} / \mathrm{m})$ lipiden. Op basis van de experimentele resultaten zijn de (minimale) kosten, gebaseerd op de som van het energieverbruik en de investeringskosten, voor de extractie afgeschat. De kosten voor ontwateren en drogen van de microalgenslurry zijn in deze evaluatie meegenomen. De berekende kosten, voor de extractie van lipiden uit droge microalgen, zijn € 0.50 per kilogram geëxtraheerde olie. Als aangenomen wordt dat de natte microalgen $25 \%(\mathrm{~m} / \mathrm{m})$ extraheerbare lipiden bevatten en dat natte extractie even efficiënt is als droge extractie, dan dalen de kosten voor lipide extractie uit natte microalgen biomassa naar $€ 0.30$ per kilogram geëxtraheerde olie. Er is echter meer onderzoek nodig om de efficiëntie van de extractie van olie uit een microalgenslurry te verbeteren en zo de extractie kosten te verlagen. De kosten voor extractie met conventionele organische oplosmiddelen zijn iets lager dan kosten voor extractie met superkritiek $\mathrm{CO}_{2}$. Ten opzichte van de conventionele extractiemethoden heeft extractie 
met superkritiek $\mathrm{CO}_{2}$ echter als bijkomend voordeel dat er in het proces geen toxische oplosmiddelen nodig zijn.

$\mathrm{Na}$ een eventuele extractie van lipiden, ondergaan de microalgen een hydrothermale liquefactie-('vervloeiings-') stap (HTL). Zowel vers geoogste microalgen als de biomassa resterend van de extractiestap kan als grondstof gebruikt worden in dit HTL proces. Wederom zijn de proces condities systematisch gevarieerd om zo de efficiëntie van HTL als omzettingsmethode te evalueren. Hierbij is met name gekeken naar het effect van verblijftijd en reactietemperatuur op de productverdeling en de productsamenstellingen. Tevens is met optische technieken visueel gekeken naar de toestand van de resterende algencellen na HTL behandeling, een aspect dat niet eerder bekeken is. De olie-opbrengst bleek maximaal ca. $50 \%(\mathrm{~m} / \mathrm{m})$, bij een reactietemperatuur van $375^{\circ} \mathrm{C}$ en een verblijftijd van 5 minuten. Bij deze condities bevat de geproduceerde olie ca. $75 \%$ van de calorische waarde van de oorspronkelijke microalgen als uitgangsmateriaal. Aangezien de verblijftijd die nodig is om de algen om te zetten relatief kort is, zal bij opschalen van het proces het benodigde reactorvolume relatief klein zijn, hetgeen gunstig is voor de investeringskosten.

Er kan geconcludeerd worden dat HTL een efficiënt proces is om microalgen om te zetten in soort ruwe olie; biocrude. Bovendien is het niet noodzakelijk om de algen volledig te drogen, wat resulteert in lagere energiekosten; het volstaat om de algenslurry uit de kweek te concentreren tot zo'n 10-20\%(m/m) op droge stof basis. Een hogere reactietemperatuur gedurende HTL resulteert in hogere olie-opbrengsten. Echter, een hogere opbrengst gaat gepaard met een hoger stikstofgehalte in de geproduceerde olie, tot wel $6 \%(\mathrm{~m} / \mathrm{m})$, gelijk aan dat in de algensoort gebruikt in dit onderzoek. In samenwerking met de Universiteit van Bologna is onderzocht in welke vorm, chemisch gezien, stikstof aanwezig is in de olie. Er is kennis vergaard over de samenstelling van de olie, welke kan worden gebruikt bij onderzoek naar het verwijderen van stikstof van de geproduceerde olie tijdens verdere opwerkingsprocessen. Uit dit onderzoek blijkt dat door de procescondities te variëren, de productsamenstelling sterk kan worden beïnvloed. Voor een lipide-rijke olie is het bijvoorbeeld beter om de temperatuur beneden de $250^{\circ} \mathrm{C}$ te houden, al gaat dit wel ten koste van de totale opbrengst aan HTL-biocrude.

Het stikstofgehalte van de HTL-olie is te hoog om direct bijgemengd te worden in een aardolieraffinaderij. Er zijn verschillende manieren onderzocht om het stikstofgehalte te verlagen waaronder; 1 : het verwijderen van het proteïnemateriaal voorafgaand aan HTL; 2: het verwijderen van de stikstofhoudende componenten uit de HTL-olie door middel van extractie; en 3: het kraken van de HTL-olie met behulp van een zeoliet katalysator. Deze methoden bleken ofwel inefficiënt in het selectief verwijderen van stikstof en 
zuurstof uit de olie ofwel het resulteerde in olie met een verminderde kwaliteit voor toepassing in diesel.

Hydrogenering van de HTL olie bleek wel een efficiënte manier om zowel het stikstofgehalte, het zuurstofgehalte als de viscositeit van de olie te verlagen. Onder de meest extreme condities die hier getest zijn $\left(400^{\circ} \mathrm{C}, 50 \%(\mathrm{~m} / \mathrm{m})\right.$ katalysatordosering en 15 MPa initiële $\mathrm{H}_{2}$ druk) was de verwijderingsgraad voor zuurstof en stikstof het hoogst. Zelfs zonder katalysator, bleek het mogelijk om bijna 30\% van de zuurstof en stikstof uit de olie te verwijderen. De olie veranderde van een visceus teerachtig product dat niet vloeide bij kamertemperatuur, in een vloeibaar product met een hoog gehalte aan onvertakte $\mathrm{C}_{15}-\mathrm{C}_{18}$ alkanen.

Met behulp van simpele analysetechnieken is getracht de toepasbaarheid van de verkregen olie te evalueren. De afname in het zuurstofgehalte en in het stikstofgehalte (gemeten met elementanalyse) en de afname in het koolstofresidu (gemeten met MCRT) van de olie na de hydrogenering, vergroot de mogelijkheden voor het bijmengen van de olie in een raffinaderij. Daarnaast bleek dat de gehydrogeneerde HTL-olie direct mengbaar is met diesel. Hoewel de olie op zichzelf (nog) niet te gebruiken is als brandstof voor een dieselmotor, lijkt het bijmengen van de olie in een olieraffinaderij haalbaar en is het direct gebruik van de alkaanfractie uit de behandelde olie, als bijmengcomponent in brandstof niet uitgesloten.

Naast het experimentele werk op het gebied van algenomzetting, is ook getracht de water- en nutriëntenkringloop in het bio-raffinage concept te sluiten. Het recyclen van proceswater en de nutriënten (met name N, P en C) is essentieel, evenals de integratie van warmte en het efficiënt gebruik van bijproducten zoals bijvoorbeeld $\mathrm{H}_{2}$. Dit resulteert in een lagere milieubelasting ('environmental footprint'), een hogere massa- en energieefficiëntie en uiteindelijk een kostenvoordeel. We hebben ons vooral gericht op het recyclen en het hergebruiken van de nutriënten, die achterblijven in de waterige fase (WF) uit het HTL-proces.

In groei-experimenten hebben we verschillende hoeveelheden WF bijgemengd bij het standaard groeimedium voor de algen en bij gewoon water. Daarbij hebben we de totale concentratie aan stikstof in het groeimedium constant gehouden. Uit deze experimenten is gebleken dat de algengroei niet verstoord werd door de mogelijke toevoeging en ophoping van toxische organische componenten in de WF uit de HTL, zoals vermeld in de literatuur. Door systematisch de samenstelling te variëren is aangetoond dat de groei werd verstoord door de afwezigheid van essentiële (macro-/micro-) voedingstoffen, anders 
dan $\mathrm{N}$ en $\mathrm{P}$. Uit het experimentele werk blijkt dat door recyclen van HTL-proceswater het verbruik van verse nutriënten in de kweekstap met $50 \%$ verminderd kan worden, zonder dat dit de groei, noch de groeisnelheid, van de algen belemmerd.

Dit hebben we verder onderzocht door meerdere malen de cyclus van algenkweek, gevolgd door HTL van de geproduceerde algen en recyclen van de WF naar de algenkweek te doorlopen. Afwisselend zijn de stappen van Desmodesmus sp. algenkweek (vijf keer) en HTL (vier keer) doorlopen, waarbij de WF met nutriënten uit de HTL-stap én het, grotendeels uitgeputte, resterende groeimedium uit de algencentrifugestap (tussen algenkweek en HTL) is teruggevoerd naar de algenkweek voor de volgende generatie algen. De groeisnelheid van de microalgen in het gecombineerde groeimedium bleek vergelijkbaar met de groeisnelheid in het standaard groeimedium. Hoewel de groeicurve voor de algenkweek in de experimenten met gerecycled medium een iets langere opstartcq. acclimatiseringsperiode te zien gaf, bleek de alg ook op gerecycled medium te groeien. In een continu opererend systeem, met continue algenoogst en WF-recycle, speelt dit effect van de opstartfase waarschijnlijk geen rol. De resultaten tonen aan dat de ontwikkeling van een geïntegreerde, 'stand alone' algenkweek en bio-raffinaderij wellicht tot de mogelijkheden behoort. De organische moleculen die zich, over de kweek-HTL cycli heen, ophopen in het groeimedium worden geïdentificeerd door middel van gedetailleerde analyse van de WF. Deze resultaten geven aan dat een water-reinigingsstap nodig is in het gepresenteerde algenraffinage-schema om verregaande ophoping van giftige componenten te voorkomen. Indien de waterreiniging is uitgevoerd door middel van vergassing in superkritiek water (zoals weergegeven in Figuur 1), dan wordt er een gasstroom verkregen die de nuttige componenten $\mathrm{H}_{2}$ en $\mathrm{CO}_{2}$ bevat die binnen het schema een toepassing vinden. Daarnaast zou de waterstroom meer ammoniak bevatten, welke als stikstofhoudend nutriënt voor algenkweek kan dienen.

De weg naar een duurzaam, economisch rendabel proces, waarin algen worden gekweekt en omgezet naar een biobrandstof is nog lang. De huidige "algenkoorts" kan helpen om het momentum te creëren voor doelgericht onderzoek hiertoe. De recent afgekondigde maatregel (2012) van de Europese Commissie om op algen gebaseerde biobrandstoffen viervoudig mee te tellen voor het bereiken van de doelstellingen in de EU Biofuels policy, is daarbij een illustratief voorbeeld van een beleidsinstrument dat het belang van de ontwikkelingen, zoals beschreven in dit proefschrift, onderschrijft. 



\section{Resum}

La humanitat necessita recursos renovables per cobrir la creixent demanda d'energia d'una manera sostenible, i així alleujar els problemes ambientals, socials i polítics derivats de l'ús dels combustibles fòssils. La biomassa, font d'energia renovable, és una de les alternatives sostenibles que poden contribuir tant a la producció de combustibles, com a l'obtenció de productes químics. En aquesta tesi s'investiga l'ús de les microalgues per a la producció d'energia en forma de biocombustibles líquids. La seva versatilitat i la ràpida velocitat de creixement, juntament amb la possibilitat de ser cultivades en terres no llaurables, són alguns dels factors que destaquen el potencial d'aquests microorganismes en comparació amb altres tipus de biomassa.

En el context de les algues per produir combustibles, la majoria dels esforços s'han dirigit cap al camp de la biologia/biotecnologia amb l'objectiu de trobar el sistema de cultiu més òptim per a la producció d'algues de la manera més eficient i econòmica. En canvi, s'ha dut a terme molta menys investigació sobre el rendiment dels processos posteriors, necessaris per convertir les algues en combustibles. En molts casos, poc se sap sobre la seva viabilitat tècnica i econòmica. Per tant, amb el treball descrit en aquesta tesi, pretenem contribuir en aquest camp i generar més coneixements per l'ampliació de futures operacions en el camp del processament d'algues. En aquesta tesi, hem avaluat el potencial de diversos processos i conceptes per a la seva integració en un esquema de biorefineria.

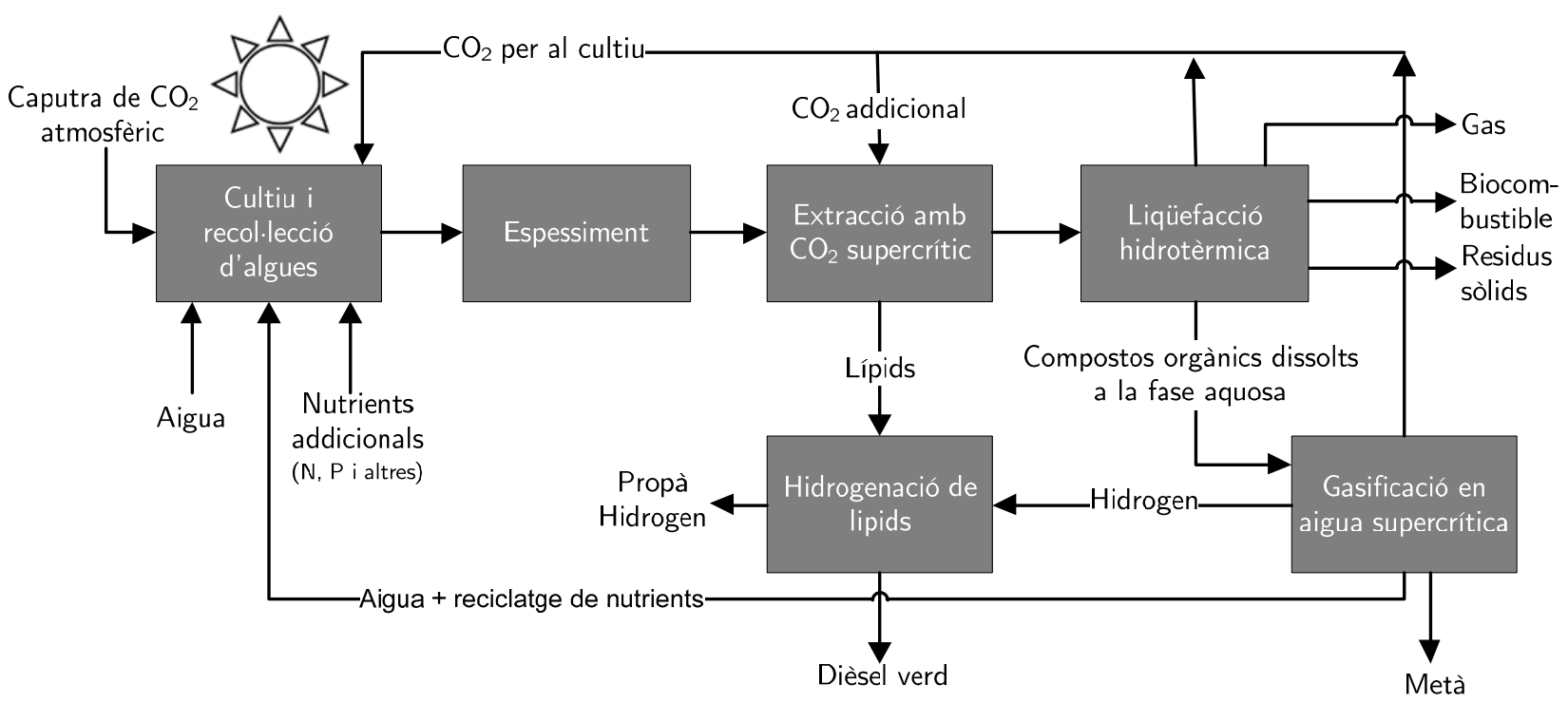

Figure 1. Concepte de biorefineria d'algues per a la producció de combustibles líquids. 
L'estratègia de biorefineria té com a objectiu principal l'òptima utilització de la biomassa per aconseguir un benefici econòmic i ambiental màxim. A la Figura 1 s'il · lustra una versió estesa de l'esquema simplificat de biorefineria presentat al Capítol 1, on s'integren tots els processos i conceptes avaluats en aquesta tesi. El concepte es centra en els combustibles líquids per al transport, però no està limitat només a això. La co-obtenció de productes d'alt valor afegit podria donar lloc a majors ingressos, el quals probablement acabin sent necessaris per a la viabilitat econòmica del concepte de biorefineria, el qual ha de competir amb els preus encara baixos dels combustibles d'origen fòssil.

Després de cultivar les algues i del seu espessiment (destinat a concentrar la suspensió d'algues), la primera etapa de processament en la configuració de biorefineria suggerida és l'extracció de lípids amb $\mathrm{CO}_{2}$ supercrític. Els lípids, localizats a l'interior de les cèl · lules de les algues, poden ser posteriorment convertits en un combustible de tipus dièsel (anomenat dièsel verd o dièsel renovable; green diesel a la Figura 1) mitjançant un procés d'hidrogenació. En aques treball d'investigació, hem realizat una avaluació tècnico-econòmica d'aquesta tecnologia, aplicada tan a les microalgues seques ("dry microalgae") com a la suspensió de microalgues (es a dir, en presència d'aigua o "wet microalgae"). En primer lloc, amb microalgues seques, les condicions d'extracció van ser modificades sistemàticament amb l'objectiu de maximitzar el rendiment d'oli (lípids) i la seva qualitat. D'acord a aquests criteris i per a aquesta espècie d'algues en concret, es va trobar com a conjunt de condicions de l'operació més òptim, l'extracció a altes pressions (almenys $35 \mathrm{MPa})$ i temperatures moderades $\left(50^{\circ} \mathrm{C}\right)$. A partir d'un ampli conjunt d'anàlisi, es va avaluar la qualitat del producte obtingut, identificant la fracció de l'oli adequada per combustibles dièsel. Per altra banda, l'extracció a partir d'algues amb un alt contigut d'humitat va resultar ser menys eficaç, ja que produïa una disminució del rendiment de l'oli amb una quantitat creixent de compostos més polars, els quals serien indesitjables per al producte dièsel final.

Els resultats experimentals van ser utilitzats per estimar els costos mínims d'extracció (incloent tan els costos l'energia com els de capital) per diferents casos on es van incloure també les despeses d'energia de l'etapa d'espessiment de les algues. Per a l'espècie d'algues utilitzada en aquesta investigació, amb un contingut en lípids del $12 \%$ en pes i utilizant com a matèria primera la biomassa seca, els costos d'extracció van resultar en $0.50 € / \mathrm{kg}$ d'oli extret. En el cas de l'extracció a partir de la suspensió de microalgues en aigua, suposant que s'aconsegueix una extracció tan eficient com en el cas de les algues seques i suposant un contingut en lípids del $25 \%$ en pes, els costos es van poder reduir a 
$0.30 € / \mathrm{kg}$ d'oli extret. Per tant, per ser capaços d'aconseguir aquests costos més reduits, necessitem més investigació en aquest camp per tal de superar els reptes tècnics de l'extracció a partir d'algues en aigua. Per altra banda, es va trobar que els costos de l'extracció convencional amb dissolvents orgànics són lleugerament inferiors, però l'impacte ambiental en relació amb l'ús d'aquests dissolvents disminueix el seu potencial en comparació a l'extracció amb $\mathrm{CO}_{2}$ supercrític.

Tan les microalgues recent cultivades com la biomassa residual d'algues que queda després de l'extracció amb $\mathrm{CO}_{2}$ supercrític podrien ser la matèria primera de la següent etapa de processament en l'esquema de biorefineria, la liqüefacció hidrotèrmica (HTL or hydrothermal liquefaction en anglès). Un enfocament similar a l'utilitzat per a l'extracció supercrítica va ser seguit per avaluar l'aplicabilitat del procés HTL com a mètode de conversió de les algues a biocombustible dins de la configuració de biorefineria. Es va estudiar una àmplia gamma de condicions de reacció (canviant la temperatura i el temps de reacció) i el seu efecte en la distribució dels diferents productes i la seva composició, incloent una inspecció visual de les cèl lules d'algues després del tractament d'HTL (aspecte que no ha sigut investigat amb anterioritat). A $375^{\circ} \mathrm{C}$, es va assolir un rendiment màxim d'oli de gairebé el $50 \%$ en pes amb una recuperació del $75 \%$ del poder calorífic de les algues en l'oli. Només 5 minuts de temps de reacció a la temperatura específica van ser requerits per tal d'arribar a aquest màxim, la qual cosa implica que, en futures operacions d'HTL en continu, el volum del reactor pot ser disminuït considerablement, sent això beneficiós en relació als costos de capital.

Els resultats experimentals obtinguts, van demostrar l'eficàcia de la liqüefacció hidrotèrmica com a mètode que permet la conversió de biomassa amb alt contingut d'humitat en biocombustible. Indirectament, a més, s'estalvia en alts costos energètics relacionats amb l'assecatge tèrmic, ja que l'eliminació completa d'aigua no és necessària (la suspensió d'algues tractada via HTL conté 10-20\% en pes sec d'algues). L'augment de la temperatura de processament va resultar en majors quantitats d'oli. Tanmateix, paral · lelament a aquest argument, el contingut de nitrogen en l'oli també va augmentar (arribant fins a un valor del 6\% en pes en l'oli per a les espècies d'algues utilitzades en aquesta investigació). En col · laboració amb la Universitat de Bolonya, la naturalesa dels compostos nitrogenats va ser investigada, obtenint amb això una visió més clara de la composició de l'oli i més informació útil per els processos requerits posteriorment per reduir el nitrogen i, en definitiva, millorar la qualitat de l'oli. Aquesta caracterització de l'oli també va demostrar que la composició d'aquest producte es pot ajustar per mitjà d'una preselecció de les condicions d'operació de la liqüefacció hidrotèrmica. Per exemple, 
si es prefereix un oli ric en lípids, s'ha d'aplicar una temperatura relativament baixa (per sota dels $250^{\circ} \mathrm{C}$ ), tenint en compte que això suposarà una menor producció d'oli.

Per potencialment considerar el processament de l'oli de liqüefacció (HTL-oli) en una refineria convencional (i així disminuir l'ús de petroli), el seu contingut en nitrogen ha de ser primerament reduit. Per aquest propòsit, diferents mètodes van ser avaluats: l'extracció de les proteïnes en les algues (la causa de l'alt contingut de $\mathrm{N}$ en l'oli) abans de ser processades a través d'HTL; tècniques d'extracció per separar el nitrogen de l'oli un cop HTL s'ha dut a terme a partir d'algues fresques; i craqueig catalític de l'oli de liqüefacció usant un catalitzador de zeolita. Els mètodes van resultar ser, o ineficients pel que fa al rendiment d'oli i la reducció del seus continguts de nitrogen i oxigen, o van acabar produint un oli amb menys nitrogen i oxigen però amb una composició final que no era la desitjada, sent aquesta un biocombustible ric en alcans d'estructura molecular lineal. Seguidament, un altre mètode va ser investigat, hidrogenació de l'HTL-oli, amb el qual vam ser capaços de reduir no només el nitrogen, sinó també el contingut d'oxigen i la viscositat de l'oli. Sota les condicions més severes d'hidrogenació investigades $\left(400^{\circ} \mathrm{C}\right.$, $50 \%$ en pes de catalitzador i $15 \mathrm{MPa}$ de pressió inicial d' $\mathrm{H}_{2}$ ), es van poder assolir els nivells més alts de desnitrogenació i desoxigenació. Fins i tot, es va poder obtenir un cert grau d'eliminació de N i O (aproximadament 30\%) sense addició de catalitzador. L'oli va passar a ser d'un producte semblant al quitrà i altament viscós sense poder fluir a temperatura ambient, a un líquid que pot fluir lliurement compost majoritàriament d'alcans d'estructura molecular lineal amb el rang d'hidrocarburs de tipus dièsel $\mathrm{C}_{15}-\mathrm{C}_{18}$ com a fracció més abundant en l'oli.

Amb un seguit de mètodes de caracterització simples, es van obtenir indicacions sobre la futura aplicabilitat de l'HTL-oli d'algues hidrogenat. La reducció del contingut d'oxigen i nitrogen (mesurat mitjançant anàlisi elemental) juntament amb la notable reducció en la tendència a la formació de carbó de coc (mesurat a partir de MCRT anàlisi, Micro Carbon Residue Test) són alguns dels resultats que van indicar l'alt potencial d'aquests olis en futures opcions de co-processament. Per al possible co-processament, l'oli hidrogenat produit a partir de les algues s'ha de poder barrejar en cert grau amb combustibles d'origen fòssil. Experiments d'aquest tipus van ser duts a terme amb els quals es va demostrar que una fracció relativament alta del biocombustible d'algues és directament miscible en dièsel. Tot i que l'ús directe d'aquest producte en un motor dièsel és encara limitat (ja que el nitrogen no s'ha pogut eliminar completament), el processament conjunt amb petroli (o productes del petroli) a la refineria sembla factible. Fins i tot, es podria considerar la possibilitat d'extreure la fracció de l'oli formada pels 
hidrocarburs del rang $\mathrm{C}_{15}-\mathrm{C}_{18}$ i afegir aquesta fracció de manera directa al combustible dièsel.

A més de la investigació en el processament d'algues per produir combustibles, en aquesta tesi també hem estudiat les possibilitats de tancar el cicle de la biorefineria presentada a la Figura 1. El reciclatge d'aigua i nutrients (principalment N, P i C) és essencial, així com la integració dels diferents corrents del sistema on sigui possible per tal de generar la pròpia energia elèctrica i tèrmica i, finalment, l'ús intern d'altres corrents de subproductes (per exemple $\mathrm{H}_{2}$ ). D'aquesta manera es pot assolir una major reducció de les futures empremtes ambientals, així com millorar la massa i l'eficiència energètica del sistema. En aquesta context, la investigació realitzada es va centrar a estudiar les possibilitats de reciclar els nutrients dissolts a la fase aquosa (aqueous phase en anglès, AP) que s'obté després de la liqüefacció hidrotèrmica per al cultiu d'algues.

En primer lloc, mantenint la mateixa concentració de nitrogen que hi hauria al medi de cultiu estàndard, vam variar el factor de dilució de l'AP d'una manera sistemàtica. L'AP va ser dissolta amb aigua o aigua enriquida amb medi de cultiu estàndard. Amb aquests experiments es va demostrar que la causa principal de la inhibició del creixement de les algues per addició d'AP no era la presència d'altes concentracions de compostos orgànics tòxics degut a un insuficient dilució de l'AP (conclusió indicada en estudis anteriors en la literatura científica). En canvi, la manca de (macro-/micro-) nutrients, altres que el $\mathrm{N}$ i $\mathrm{P}$, va ser identificada com la raó principal d'aquesta reducció de velocitat de creixement. Al mateix temps, amb aquest estudi es va demostrar que un 50\% del nitrogen necessari per al cultiu pot ser substituit pel nitrogen present a l'AP obtenint una velocitat de creixement de les algues indèntica a la que s'obtindria en el cas de ser cultivades al medi de cultiu estàndard.

Finalment, vam decidir donar un pas més endavant i avaluar les possibilitats de reciclar els nutrients de l'AP múltiples vegades. Amb aquesta finalitat, vam ser capaços de completar un conjunt de 4 cicles de creixement i liqüefacció hidrotèrmica de les algues en continu. Les microalgues (de l'espècie Desmodesmus) van ser cultivades 5 vegades i convertides en biocombustible per mitjà de liqüefacció hidrotèrmica 4 vegades, i al mateix temps, reciclant l'AP amb els nutrients d'un cicle al següent cicle i també l'aigua eliminada durant l'etapa d'espessiment. La velocitat de creixement de la biomassa d'algues (a la regió lineal) obtinguda en aquest experiment en continu va resultar ser comparable a la que succeirïa en el medi de cultiu estàndard. Tot i haver observat un retard inicial del creixement a través dels cicles (fet que probablement es pot evitar si s'utilitza un sistema de funcionament continu per a la seqüència combinada de les etapes 
de cultiu, recol lecció i HTL), eventualment les cèl·lules d'algues van començar a duplicar-se. Aquests resultats van demostrar les possibilitats de desenvolupar biorefineries d'algues les quals poden funcionar de manera autònoma. Per altra banda, es va realitzar una caracterització química detallada de l'AP per tal d'identificar aquells compostos orgànics solubles i potencialment tòxics per les algues que podrien arribar a accumular-se a l'AP al llarg dels cicles. Els resultats d'aquests anàlisis van indicar la importància d'aplicar un procés de tractament de l'AP capaç d'eliminar aquests compostos orgànics quan la seva acumulacio assoleix concentracions massa altes. Un possible procés de tractament a considerar és la gasificació en aigua supercrítica. En aquest procés, els compostos orgànics no desitjats són convertits en un gas que conté principalment hidrogen i $\mathrm{CO}_{2}$, i en amoníac que es dissol a la fase aquosa. Aquests productes poden ser utilitzats per als altres processos dins de la biorefineria (per exemple, l'hidrogen pot ser utilitzat per la hidrogenació dels lípids).

Encara existeixen molts obstacles a superar en el camí cap al desenvolupament d'un procés a mida comercial, on les algues són convertides en biocombustibles d'una manera sostenible i econòmicament viable. No obstant això, hem d'aprofitar l'actual impuls i interès en aquest camp per posar en marxa iniciatives d'investigació cap a aquest objectiu de comercialització. Recentment (2012), la Comissió Europea va anunciar, en el marc de les regulacions sobre biocombustibles a l'UE, que d'ara endavant, el contigut energètic dels biocombustibles produits a partir d'algues haurà de comptar per quatre. Aquest fet, subratlla la importància dels diferents estudis realitzats en aquesta tesi. 


\section{List of publications}

L. Garcia Alba, C. Torri, C. Samorì, J.J. van der Spek, D. Fabbri, S.R.A. Kersten, D.W.F. Brilman, Hydrothermal treatment (HTT) of microalgae: Evaluation of the process as conversion method in an algae biorefinery concept, Energy and Fuels, 26 (1), 642 - 657, 2012.

C. Torri, L. Garcia Alba, C. Samorì, D. Fabbri, D.W.F. Brilman, Hydrothermal treatment (HTT) of microalgae: Detailed molecular characterization of HTT oil in view of HTT mechanism elucidation, Energy and Fuels, 26(1), 658-671, 2012.

C. Torri, D. Fabbri, L. Garcia Alba, D.W.F. Brilman, Upgrading of oils derived from hydrothermal treatment of microalgae by catalytic cracking over H-ZSM-5: A comparative Py-GCMS study, Journal of Analytical and Applied Pyrolysis, 101(0), 28-34, 2013.

D.W.F. Brilman, L. Garcia Alba, R. Veneman, Capturing atmospheric $\mathrm{CO}_{2}$ using supported amine sorbents for microalgae cultivation, Biomass and Bioenergy (Article in press), 2013.

L. Garcia Alba, C. Torri, D. Fabbri, S.R.A. Kersten, D.W.F. Brilman, Microalgae Growth on the Aqueous Phase from Hydrothermal Liquefaction of the Same Microalgae, Chemical Engineering Journal (Article in press), 2013.

L. Garcia Alba, M. Vos, C. Torri, D. Fabbri, S.R.A. Kersten, D.W.F. Brilman, Recycling Nutrients in Algae Biorefinery, ChemSusChem (Accepted), 2013.

L. Garcia Alba, J. de Weerdt, A.M. Verschoor, W.P.M. van Swaaij, D.W.F. Brilman, Energy from algae: Growth optimization and algae-to-fuel conversion routes, $18^{\text {th }}$ European Biomass Conference and Exhibition Proceedings (OE1.4), 249-254, 2010.

D.W.F. Brilman, L. Garcia Alba, M. Vos, R. Veneman, (2012) Capturing Atmospheric CO $\mathrm{CO}_{2}$ for Microalgae Cultivation, 20 $0^{\text {th }}$ European Biomass Conference and Exhibition Proceedings (1BO.2.1), 77-84, 2012

\section{In preparation:}

L. Garcia Alba, F. Hoppenbrouwer, C. Samorì, A.G.J. van der Ham, E. Tagliavini, S.R.A. Kersten, D.W.F. Brilman, Experimental and economical evaluation of supercritical $\mathrm{CO}_{2}$ extraction of oil from microalgae.

L. Garcia Alba, L. Wang, S.R.A. Kersten, D.W.F. Brilman, Hydrotreatment of hydrothermal liquefaction oil from microalgae: Preliminary results. 



\section{About the Author}

Laura Garcia Alba was born on September $26^{\text {th }} 1985$ in Tarragona, Catalonia, Spain. She started her degree in Chemical Engineering and completed the first year at the University Rovira $i$ Virgili (URV) in the Higher Technical School of Chemical Engineering (Tarragona). Afterwards, she continued her studies at the University of Barcelona (UB). In 2008, she enrolled to the Erasmus-Socrates program and for 9 months she worked in the Sustainable Process Technology group (former Thermo Chemical Conversion of Biomass group) at the University of Twente (The Netherlands). There she completed her Master Thesis (projecte final de carrera) on "Upgrading of pyrolysis oil. Fundamental study using Model Compounds" supervised by dr. Ferran de Miguel Mercader and dr.ir. Kees Hogendoorn. With that, she graduated in 2008 (by the University of Barcelona) and started her PhD in February 2009 in the same research group under the supervision of dr.ir. Wim Brilman and prof.dr. Sascha Kersten. During her PhD research, she worked on the concept of Algae Biorefinery focusing on liquid fuels production and nutrients recycling. The results of this research are described in this thesis. 



\section{Acknowledgements}

Almost 5 years ago, I had to make a choice: going back to Tarragona or taking a huge opportunity that was given to me, staying in Enschede for a $\mathrm{PhD}$. After all my complaints about weather and food (among others), now I am immensely happy to know that I made the right decision. The amount of things I learnt through this journey is impossible to measure and all that made me grow both at the scientific and personal levels. Yet meeting all of you was by far what made this decision so enormously right! And also, of course, meeting the most important person in my life, but I will talk about him a little bit later $(;-))$.

First, I would like to start with my PhD mentors, my guidance and those from which I could never stop learning: Prof.dr.ir. Michiel Groeneveld, Prof.dr. Sascha Kersten and Dr.ir. Wim Brilman. Sascha, thanks for always challenging me and saying "ok, this is another Laura style paper, long veeery long, but I know you can do more". It is that "I know you can do more" which I kept repeating in my mind and helped me to move forward. Thanks for your wise comments that will for sure help me in my future professional life and for telling me to be always critical (and perhaps less emotional) with my work. I would also like to apologize for my comments on your flower shirts... I think they are awesome!. Wim, not even a million thanks would be enough to compensate for everything you have done for me. You were always positive and trying to find the good side of everything when I was deeply negative, you were bringing brilliant ideas when I was out of ideas, and in short, you were always providing that boost of motivation and energy whenever I was needing it. I think we both believe that believing is important, therefore, thank you very much for believing in me, and more importantly, thank you for make me believe a little bit more on myself. A very special thanks to my initial promotor, Michiel Groeneveld. Unfortunately, life only gave us two months as promotor-PhD student, but in that short time you managed to give all the strength and enthusiasm for the research that was about to start. I will never forget the last words you told me: "the world is turning very fast and you should keep working".

Furthermore, I would like to thank the rest of the committee members. It is a great honor to have you in my defense committee and I hope, that day, we have a nice and fruitful (and hopefully gentle $\odot$ ) discussion.

There are four main factors without which this thesis would have never existed: Everybody from Ingrepro and specially Anthony Verschoor; my favorite Italians; all the students that worked with me and the best technicians ever.

Anthony, thank you very much for providing endless amounts of algae to us and for sharing your endless biology knowledge which we needed many times. It has been a great pleasure to work with you. Also many thanks to Henny for always being there ready to give us another bottle of algae. I will now take the chance to thank my tinny beautiful algae for growing when I needed you to do so.

My favorite Italians, and as Wim and I say, the other half of this thesis. Cristian, the luck decided to put our posters next to each other in the biomass conference in Lyon and I could not be more happy about that. It has been amazing to work with you! Every time I asked for another extra last analysis (which was never the last one...) you always said yes. And it was not only about performing analysis, you were also giving a lot of input and sharing your incredible knowledge on everything. Then, after meeting you, I became doubly lucky, because you introduced me to your super scientist and amazing partner, Chiara, thank you very much for all your work and huge input, the countless replicates of samples and the encouragement you gave every time I told you "Chiara, I am so stressed...". Also thank you for the picture you sent me, I had it always on my desk :- Cristian and Chiara, I am very glad to know that beyond research partners we also became friends. Completing the list of best Italians, prof. dr. D. Fabbri. Daniele, thank you for reading and reviewing my work with such an enthusiasm and for all your useful 
comments to improve it. It was a great pleasure to work with all of you and, hopefully, this successful collaboration continues for very long time.

All the students had a very important contribution to this work, and for that and all your efforts and patience: THANK YOU VERY VERY MUCH! Sander, Jaapjan, Jan, Chiel, Tom, Maria, Piotr, Mathijs, Nurcan, Lei and Frederik, I wish you a life full of success.

A very special gratitude to that strong foundation that support our group, the technicians. Benno,

Robert, Karst and Johan, I really appreciate all your help and effort to make everything work perfect! Thanks for the countless times that you had to repair my autoclave or find a leak. Benno and Robert, thank you also for all your support and cheering me up when I was very down. Erna, thanks for all the help and for telling every time you saw me a bit stressed "Laura, don't worry, I will change the column for you". I would also like to thank Yvonne, for being always ready to help no matter how busy you were, for having many times candy on your desk to make us a little bit happier and for that final touch to my Samenvatting.

Having a good environment at work is something crucial and all of you, my TCCB friends, made that very easy to me. The old PhD TCCBers: Ferran, Dragan, Prasad, José (aka Mr. la Bomba), Elly, Agnes, Mariken, Roel, Antal, Anand, Magdalena and Pavlina. The current PhD and post-doc SPTers: Maria, Rens, Stijn, Michal, Samuel, Cindy, Xiaohua, Shushil and Annemarije. Those who were once TCCBers or SPTers: Roma, Xavier (my brother $($ )), Diego, Maarten, Nancy, David and many more. And all the staff members: Guus, Louis, Boelo, Wim van Swaaij, Henk and JeanPaul. Thank you very much for all the fun, support and amazing borrels. I had an amazing time with all of you!. Speaking of borrels (or crazy borrels depending on the level of destruction of our Meander bar and its surroundings), and to keep the tradition initiated in the acknowledgments of my lovely husband, I would like to thank Bongo Botrako with their Chipiron hit and La pegatina with their Mari Carmen record for making us jump, dance, sing and make our Saturdays unforgettably painful.

Next, I would like to address a few words to some special people starting with my brother/sister(paranymphs). Rens, my Dutch brother, thank you very much for making me less workaholic and little bit more alcoholic :). You tried really hard! Even pulling my chair with me on it when I was sitting in front of the computer on a Friday at $7 \mathrm{pm}$. In all my hard times, somehow you always managed to make me laugh, thanks a lot for that!. And as all my family said after the wedding in Tarragona, Marije (or the girl with the green dress as they said), you are as amazing as your sometimes very annoying boyfriend. Pavlina, my $1 / 2$ Greek $1 / 2$ Dutch sister, you have been a very important person to me here in Enschede and the perfect definition of a friend. You have always been there for me and that is why I chose you to be part of every important moment of my life (wedding, defense and many more to come). Remember this: "Life is not about waiting for the storm to pass, it's about learning to dance in the rain". Dimitris, godfather of my future child, thank you for being such a good friend, for your honesty, for telling everyone that I looked like Rihanna when I had short hair and for our endless fights that made our moments together much more lively. Yet, Lady gaga is definitely better than Madonna.

Ferran, des del moment en que vaig arribar a Enschede i vaig començar a treball com a estudiant teu, tu et vas convertir en el meu example. Sempre t'he tingut com a referència, i segurament, gràcies a això, mira on estic ara! Gràcies per ensenyar-me tant, sobretot al principi quan no parlava ni una paraula d'anglès i era una mica "pardilla" :- Michael, thanks for bringing a little bit of art (of any kind; music, paiting, cocktails making, salsa, etc.) to this bunch of nerds tha we are and thank you for all your input on Can's wedding suit. Thanks to you, he really was the most handsome and stylish groom I have ever seen (after you, of course ;-) ).

Roel (Rulo), thanks for listening to all my problems and being soooo wise! In many situations we behave very similarly, we in general worry too much about everything. Yet you learned how to handle it and you also tried to teach me that. Thanks also for paying attention to my clothing style suggestions, I 
hope you are not wearing a shirt with lines and a jacket also with lines on top in Canada! Also I would like to thank your "it is hard to believe that he is not your child" Stijn. Thanks for bringing fancy cheese and bitterballen when I complained about the quality of the food in the borrels. Also, I appreciate a lot our last discussions about refinery for one of my last chapters and all your help with stukjes. Prasad (Rago), thanks for your support and for your company on Sundays in Meander. On Sundays, it was very easy to feel alone, but you were always there to talk, gossip and work (just a little bit ;-) )

Guusio took the challenge of sitting next to me after Roel when he came back from Canada, and yes I say challenge because, well...you know... I complain a lot. You also kept listening to my problems, and help me with everything, especially with my often computer problems. Thanks for that and for being sometimes soooo "pesado", thanks to that I ate very interesting things which I thought I would never eat. Rebeca, gracias por tus consejos sobre la maternidad y enseñar-me que no es tan fàcil como sale en las películas - . Pero no te preocupes, que no me has asustado del todo! Eres una ejemplo de mami perfecta y super woman en lo profesional al mismo tiempo y espero poder ser un poquito como tú en el futuro.

Because my husband is probably one of the most social fellows of the whole University of Twente, I had the chance to meet a lot more amazing people from other research groups, with whom I shared many special moments. The majority were and are, of course, from the world of membranes and microfluidics: Rob and Cindy, Jigar and Falguni (thanks for sharing your love for Lady Gaga with me), John Heeks (the strongest person on earth), Hakan and Tugba, Katja and Christoph, Karina, Maik, Joao, Ikenna, Didi, Nicolas and Caroline, Harro and Imre (thanks for being always ready to help on anything as the day you sent the dress to Spain, thanks for being there), Antoine, Harmen, Enver (thanks for all the pictures you have taken for us for a variety of purposes. You are a true artist!), Anne Corine, Yali, Mayur (399 neighbor) and Tina, Anna Banana, Damon (together with Eric, thanks for making my husband a very hot rock star) and Saghar (I deeply envy your music skills, you are amazing!), Shishkule, Eric (I know we will meet pukki one day) and Olga (ex-concuñada, gracias por brindarnos con ese maravilloso consultorio del amor móvil. Después de un largo y duro día de trabajo, la vuelta a casa siempre era más divertida contigo en el coche. Gracias por esa sonrisa que nunca pierdes).

I would like to mention more special and cool people from other research groups and those who I met in the past at the university and that are now somewhere in the world: Gor, Burak, Trionfetti, Janet, Denis, Anil, Abdullah Can (aka "el guapo Can dos" according to my family and specially my (girl)friends ;-)), Marijana, Arturo, Cristina and Roger. Berta, la de l'Empordà i la persona que anyora la meva terra tant com jo, no perdis mai aquesta força que sempre he vist en tu i continua sent tal com ets, una tia de cordons ;-). Gregory, thanks for being such a good friend and teaching Can the art of French crepes. I know your true feelings about Eurovision; don't worry! France will win once, and we will celebrate all night. Celio, aka papi chulo, gràcies per visitar-me tantes vegades a la oficina i donarme tants ànims, tot i que jo encara no t'he visitat al teu nou grup (no tinc perdó... et prometo que ho faré abans de deixar Enschede).

My super tall and very handsome volleyball dudes: Dimitris (Greek brother-in-law), Leon (when are you going to propose to Irina? Come on man!), Jona, Wim, Frank, Tjeerd and Jesper. Thank you for taking care of my man, trying to keep him on shape (despite the tons of food he usually eats) and for your friendship. Tjeerd or, as I was always calling him: "my favorite player", thanks for introducing me to haring for the first time, I will never forget that. You will always be the number 10.

All of you, thank you for making me feel like a friend and not only the girlfriend of Can $:$;.

Matias, mil millones de gracias por tu amistad, por cuidarme tanto, por dejar que sea el árbitro del increíble juego boob-de-boul, por bailar tantas veces conmigo, por tener esa familia tan maravillosa que tienes, por todo. Siempre he sentido que me cuidabas un poquito como a una hermana pequeña, sobretodo cuando vivíamos juntos, algo que realmente necesitamos aquellos que añoramos tanto a nuestras familias. Solo puedo decirte un cosa: gracias. Gert, you are such a sweet and caring person! 
The day before our wedding in Tarragona you told me "Laura, don't worry, we will take care of everybody", thanks for that and for being there always ready to help.

Now, I would like to say a few words to my very special Turkish and very handsome brothers, Erman and Kadir. To me, both of you are the real definition of a friend because, for you guys, friendship is much more than calling every now and then, it means demonstrating that you are always there. Erman, I am the wife of your best friend but you never made me feel only like that! I always felt like your best friend as well, thank you for treating me in that away. I am also extremely happy that you work in the fashion business and I love those surprise gifts from you (you really know my clothing style ()). Kadir, thank you for your visits to Enschede or any place we were (for you, the distance is not an excuse). Thanks for bringing with you that essence of Turkey that makes me miss it as much as you do (BBQs drinking raki and singing). Thanks for being my thin plan B, you already have the looks of Can and you are also an awesome person ;-).

Romito, Carlos i Guilera, com em va dir el Roma en un dels seus últims emails: Quin orgull haver estudiat amb vosaltres!. M'heu fet riure tant! no us podeu imaginar com us vaig anyorar un cop vau tornar a casa... Gràcies per estar presents en tots els meus moments importants (ejem, menys la defensa...pero tranquils que no estic enfadada, no tingueu por ;-)). Angelita, escribirte un email después de haver visto la foto de tu boda, fue una de las mejores decisiones que he tomado nunca, porque con eso te pude recuperar y eso me hace increiblemente feliz. A pesar de los años, esa conexión entre nosotras sigue ahí y espero que siga para siempre. También quería decirte que junto a Cañi, soys de las personas mas fuertes que conozco. Gracias por ser tan buenos amigos conmigo.

Muchas gracias a toda la gente del Disc 45, a mi grupo, y en especial a mis tres magnificas, Vane, Camús y Espinosa. Gracias por no olvidaros nunca de mí y por esforzaros en que, de una manera u otra, no haya dejado nunca el disc a pesar de la distancia. Tenemos esa conexión especial de estar bailando y sin poder evitarlo, ir siempre acompasadas, espero que el futuro de cada una de nosotras también vaya acompasado $(-)$. Y a mi hermana, Vanessa, gracias por estar siempre a mi lado. Sé que hay una flor allá arriba que está muy orgullosa de ti y espero que hoy también lo este un poquito de mí. Moltes gràcies a les meves 6 outes. Com alguna vegada ha dit la Núria, per a mi també sou un pilar en la meva vida, i espero que puguem compartir juntes tots els moments importants que ens queden. He de donar les gràcies a un tal Brian Acton i Jan Koum per crear el whatsapp! Desde la seva existència, se en cada momento on esteu, com esteu, cotilleos imporants... pero sobretot he pogut rebre, d'una manera instantànea, el vostre suport cada vegada que ho he necessitat. Em compartit molts anys juntes, i se que ens queden molts mes per compartir. Cadascuna de vosaltres m'aporta coses molt importants que espero que no em faltin mai. Gràcies per estar sempre al meu costat. (Anna, em pots corretgir tot això que acabo d'escriure? ;-) ).

I would also like to thank my Turkish and second family. To all the members of the Aran/Aytekin family, thank you very much for making me feel like your daughter, sister, granddaughter, niece and cousin. Cenk, thank you very much for creating this beautiful design for my thesis, nobody could have done it better than you. Special words of thanks go to Necla, Ahmet, Betul, Elif, Bugra and also my sweet nieces (according to Spanish family rules) Lale and Melis. Thanks for taking care of me, for all your support and encouragement and for reducing the spicy level of the food and understand my issues with Ayran. Necla thank you for being such sweet and caring mother and incredibly strong woman (I hope to be a bit like you in the future). Ahmet, thank you for being my perfect teacher, I could listen at your wise comments over and over again. Betul, all my family is in love with you and it is because of your calmness, sweet an caring character. I will always appreciate the chance I had to meet an amazing person like Taner. Elif and Bugra thanks for opening the doors of your house every time we came to Amsterdam and show us how good parents you are.

Y ahora a vosotros, mi familia. Me cuesta escribir esto sin llorar, y ahora mismo estoy en la oficina, así que intentaré que no se me note $(-)$. A todos mis tios, tias y primos: gracias por echarme tanto de 
menos demostrándolo con esos abrazos infinitos que me dais cada vez que vengo a Tarragona. Gracias por no perderos ni un momento importante de mi vida y por todo vuestro apoyo. Gracias por aceptar a Can y quererle tanto. Us estimo moltíssim a tots. A mis heroínas, los fundamentos de nuestra familia y a las que le debemos todo, mi yaya Carmen y mi yaya Paz. Gracias por cuidarnos tanto, por vuestra comida inigualable, por vuestro amor que no tiene límites y per ser tan y tan bonitas. A aquellos que me faltan tanto... mis yayos. Yayo Jacinto, gracias por transmitir tanto sin decir nada, te echo de menos. Y a ti, el de la contraportada de la tesis, aquel de mirada triste que se convertía en brillo cada vez que me veía, a mi fuerza secreta, a mi yayo Francisco. He conseguido esto gracias a ti, he llegado tan lejos por ti y lo he hecho para que estés orgulloso de mi, por eso eres mi fuerza secreta. Seguro que me estás viendo, y celebro que por fin estés bien. A mi brother, Xavier, gracias por ser como eres y por tener todo aquello de lo que debo aprender tanto. Estoy increíblemente orgullosa de ti y tengo muchísimas suerte de tenerte como hermano. Una día sé que volveremos a aquellas rocas rojizas que nos cautivaron (con chinos en el bus a poder ser $($ )). Has tenido la suerte de conocer a una persona increíble, Cristina, esfuérzate para no perderla nunca. Y a vosotros, a los que les dedico esta tesis por todo el amor y apoyo incondicional, a mi padres Dulce (la persona más fuerte del mundo) y Angel (mi ángel de la guarda). Sois sin duda los mejores padre que uno podría desear, gracias por todos los sacrificios que habéis hecho por mí, por toda vuestra paciencia (no he sido fácil mucho veces...) y todos los valores que me habéis enseñado. Soy lo que vosotros habéis creado y no he podido tener más suerte. No creo que os pueda pagar nunca por todo lo que habéis hecho por mí, solo puedo desear ser, en un futuro, unos padres tan perfectos como vosotros lo habéis sido conmigo.

Last but by far not the least, my other half, mi pasión Turca, my soul mate, my boyfriend-now husbandand forever best friend, mi halilo and sometimes halilbe, the funniest person on earth and, definitely, MY LIFE, Halil Can Aran. One day I saw you in the elevator of Meander and I thought: what a handsome Italian boy. Latter I learnt that you were actually a Turkish guy named Can and I thought: OMG how exotic that is and what a weird name. One night in Lunatic was enough to realized how perfect you were. Because of that, you became my main reason to stay in Enschede, a decision that I will never ever regret. I love you for so many reasons...and I will start with those that will probably sound familiar to you: for trying to lose the $15 \mathrm{~kg}$ of bonus Can that you gained after meeting me, for taking the head of my prawns and every piece of food I am not able to finish, for not being angry because I only know 10 words of Turkish (perhaps 5 is a better approximation), for the way you love my family and your family, for the way you say "hugueame" and every single Spanish word with Z, for accepting several things: to do a salsa course, my sometimes a bit visible moustache (this only happened few times!) and my feminist (in some occasions too radical) side. Thanks Wim for taking him to Lunatic on that night, thank you for inviting me to your private toilet upstairs that, out of pure coincidence, was next to your room, thank you for never giving up during that month running after me and finally, after 3.5 years inviting me to walk with you to the Mediterranean balcony and ask me to marry you, the man of my life. I love many more things: your smile, the way you sing, your weird but very funny dance moves, the way you wake me up in the mornings (usually very early) and everything else. In the last period of my $\mathrm{PhD}$, you have done every single thing for me trying always to make me feel happy, thank you so much for that. I cannot wait for the future with you. $\infty \mathrm{x} \infty \mathrm{x} \infty \mathrm{x} \infty \mathrm{x} \infty$, no és suficient com per descriure el meu amor per tu. 